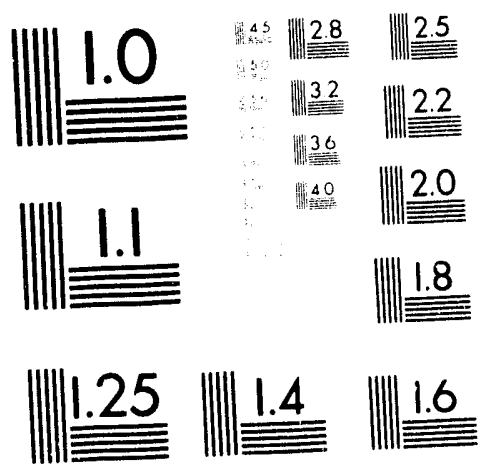



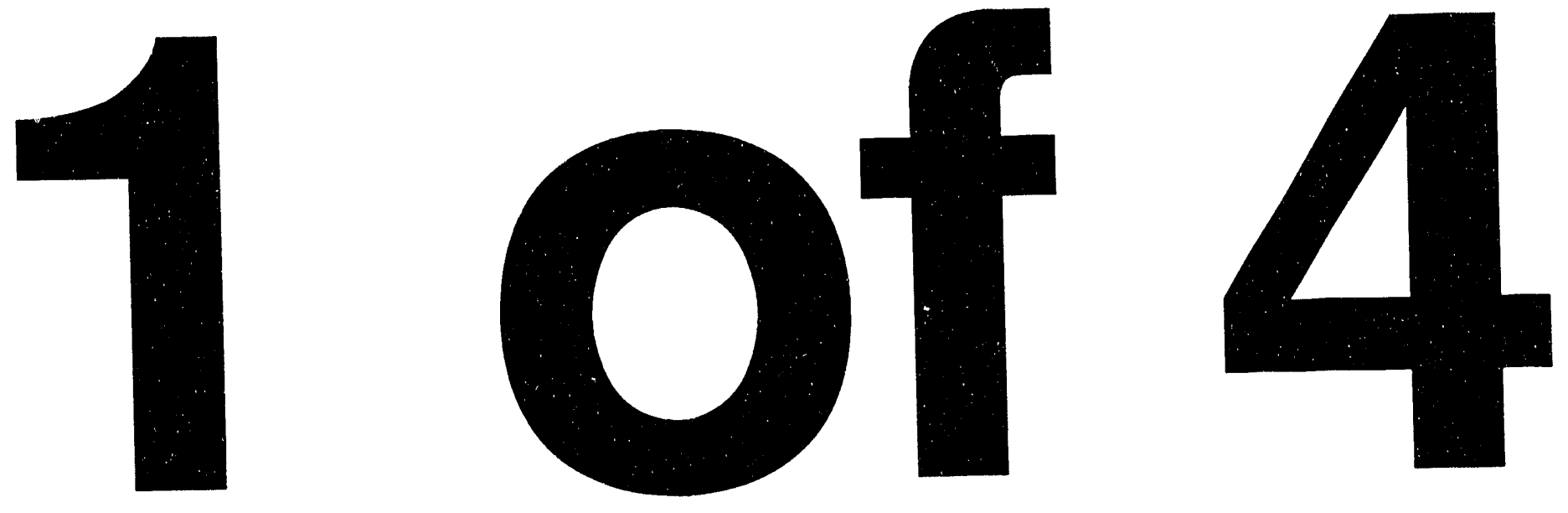


\title{
MOLTEN-CAUSTIC-LEACHING (GRAVIMELT) SYSTEM INTEGRATION PROJECT
}

\author{
FINAL REPORT
}

CONTRACT NUMBER DE-AC22-86-PC91257

MARCH 1993

PREPARED BY APPLIED TECHNOLOGY DIVISION TRW SPACE AND ELECTRONICS GROUP ONE SPACE PARK REDONDO BEACH, CA 90278

PREPARED FOR

U.S. DEPARTMENT OF ENERGY

PITTSBURGH ENERGY TECHNOLOGY CENTER

P. O. BOX 10940

PITTSBURGH, PA 15236 


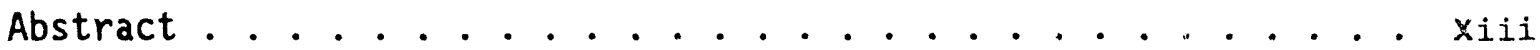

1. INTRODUCTION AND SUMMARY ............. . . 1-1

1.1 PROCESS DESCRIPTION ............. . . . . . . .

1.2 TEST COALS. . . . . . . . . . . . 1-8

1.3 TEST RESULTS AND CONCLUSIONS. . . . . . . . 1-10

1.4 ECONOMIC ANALYSIS .............. 1-12

1.5 RECOMMENDATIONS ............... 1-14

1.6 REPORT OUTLINE. . . . . . . . . . . . . . 1-14

2. TEST CIRCUIT DESCRIPTION ................. 2-1

2.1 ROTARY KILN REACTOR SYSTEM (SECTION 100). . . . . . 2-7

2.2 WATER WASHING SYSTEM (SECTION 200). . . . . . 2-22

2.3 ACID WASHING SYSTEM (SECTION 300) . . . . . 2-34

2.4 CAUSTIC REgENERATION SYSTEM (SECTION 400) . . . . 2-39

2.5 CAUSTIC CONCENTRATION SYSTEM (SECTION 500). . . . . 2-44

2.6 WASTE WATER TREATMENT SYSTEM (SECTION 600). . . . 2-53

2.7 DATA ACQUISITION AND CONTROL SYSTEM . . . . . . 2-60

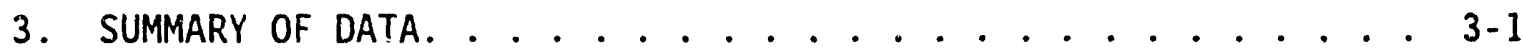

3.1 TEST MATRIX AND RUN CONDITION SUMMARY . . . . . . . 3-1

3.2 SULFUR AND ASH REMOVAL. . . . . . . . . . 3-2

3.3 VOLATILES AND HEAT CONTENT. . . . . . . . . . 3-2

3.4 PRODUCT COAL. . . . . . . . . . . . . . 3-2

4. TEST RESULTS ..................... . . . . .

4.1 COAL PRODUCT QUALITY. ............... 4-3

4.1.1 Sulfur Removal ........... . 4-4 
TABLE OF CONTENTS (CONYINUED)

Page

4.1.1.1 Pittsburgh No. 8 (Powhatan); 50:50

$\mathrm{KOH} / \mathrm{NaOH}$. . . . . . . . . . 4-4

4.1.1.2 Pittsburgh No. 8 (Powhatan); NaOH Only. 4-13

4.1.1.3 Kentucky No.9. ............ 4-18

4.1.1.4 Pittsburgh No. 8 (Blacksville); $\mathrm{NaOH}$ Only 4-21

4.1.2 Ash Removal. ................ 4-23

4.1.2.1 Pittsburgh No. 8 (Powhatan); 50:50

$\mathrm{KOH} / \mathrm{NaOH}$. . . . . . . . . . . 4-23

4.1.2.2 Pittsburgh No. 8 (Powhatan); $\mathrm{NaOH}$ Oniy. 4-27

4.1.2.3 Kentucky No.9. . . . . . . . 4-33

4.1.2.4 Pittsburgh No. 8 (Blacksville); $\mathrm{NaOH}$ Only 4-35

4.1.3 Heating Value. ... . . . . . . . . . . . 4-38

4.1.4 Volatiles Content. . . . . . . . . . . . . 4-39

4.1.5 Moisture Content .. . . . . . . . . . . . . 4-39

4.1.6 Trace Element Analysis............ 4-40

4.2 SULFUR FORMS ANALYSIS . . . . . . . . . . . . . . 4-42

4.3 KIIN GAS. . . . . . . . . . . . . . . . . . . 4-49

4.4 HUMIC ACID AND PHENOLICS FORMATION. . . . . . . . . 4-52

4.5 CARBONATE FORMATION ................. 4-61

4.5.1 Carbonate Formation During Run 11. . . ...... 4-63

4.5.2 Effect of Caustic/Coal Ratio on Carbonate

4.5.3 Effect of Kiln Temperature on Carbonate Formation. 4-71

4.5.4 Effect of Coal Type on Carbonate Formation . . . . 4-75

4.5.5 Effect of Caustic Type on Carbonate Formation. . . 4-76

4.5.6 Kiln Discharge Versus Spent Caustic Data... . 4-77

4.5.7 Suppression of Carbonate Formation . . . . . . 4-79

4.6 PARTICLE SIZE DISTRIBUTION. . . . . . . . . . . . 4 4-80

4.7 PROCESS LIQUID ANALYSIS ............... 4-89

4.7.1 Partition of Aluminum, Silicon and Iron for

Shakedown Through Run 9............ 4-89

4.7.2 Partition of Aluminum, Silicon ard Iron for Run 11 4-93

4.7.3 Regeneration of Caustic Solution . . . . . . . . 4-95

4.7.4 Treatment of Acid Wash Water. . . . . . . . . 4-100

4.8 WASH TRAIN PERFORMANCE. . . . . . . . . . . . . . 4-103 
TABLE OF CONTENTS (CONTINUED)

Page

4.9 MATERIAL BALANCE. ................... 4-109

4.9. I Coal Material Balance for Runs 1 through 9... . 4-109

4.9.2 Coal Material Balance for Run 11......... 4-112

4.9.3 Sulfur Material Balance. . . . . . . . . . 4-113

4.10 CORROSION/EROSION DATA. . . . . . . . . . . . . 4-117

5. EQUIPMENT EVALUATION/MODIFICATION/OFF-LINE TESTING . . . . . 5-1

5.1 REACTOR SECTION CRITICAL MODIFICATIONS. . . . . . 5-2

5.1.1 Installation of Breaker Bar in Kiln Discharge

Chute. . . . . . . . . . . . . . 5-2

5.1.2 Modification of the Kiln Gas Scrubbing System. . . 5-3

5.2 VACUUM FILTRATION SYSTEM CRITICAL MODIFICATIONS . . . . 5-3

5.2.1 Modification of Vacuum Filter Plumbing to Allow

for High Feed Flow Rates... . . . . . . . . 5-4

5.2.2 Automation of the Vacuum Filtration System . . . 5-6

5.3 WASHING SECTIONS CRITICAL MODIFICATIONS . . . . . . 5-8

5.3.1 Modification of the Wash Water Feed System to

Allow for Higher Temperatures. . . . . . . . . 5-8

5.3.2 Modification of the Anti-Foam Injection System . 5-8

5.4 EVAPORATION SYSTEM CRITICAL MODIFICATIONS . . . . . 5-8

5.4.1 Addition of Caustic Preheater. . . . . . . . . 5-9

5.4.2 Upgrade of the Heating and Insulation for Operation With $\mathrm{NaOH}$ Only as the Caustic Feed Material. . . 5-10

5.4.3 Modification of the Steam Condensing System. . . 5-10

5.4.4 Reduction of Evaporator Scaling Potential. . . . 5-11

5.5 OFF-LINE TESTING. . . . . . . . . . . . . 5-11

6. PROCESS AND REACTION MODELS. . . . . . . . . . . . 6-1

6.1 PROCESS MODEL .................... 6-1

6.2 REACTION MODEL................. 6-. 6 . . . 12

APPENDIX A. MCL RUN DATA SUMMARY. . . . . . . . . . . . . . . A-1

APPENDIX B. PRODUCT COAL ANALYSIS DATA. . . . . . . . . . B B-I 


\section{LIST OF FIGURES}

Page

Figure 1-1. Block Diagram of the MCL Process. . . . . . . . . . 1-5

Figure 1-2. Schematic of the MCL Process. . . . . . . . . . . 1-7

Figure 1-3. MCL Integrated Test Circuit Product Data for the Week of May 29, 1989 (Run 9)............ 1-13

Figure 2-1. Block Diagram of the MCL Process. . . . . . . . 2-3

Figure 2-2. Rotary Kiln Reaction System - Phase I. . . . . . . 2-11

Figure 2-3. Rotary Kiln Reaction System - Phase II. . . . . . . 2-12

Figure 2-4. Pneumatic Feed System . . . . . . . . . . . . 2-14

Figure 2-5. Feed Bag House Filter Above Kiln. . . . . . . . . 2-15

Figure 2-6. Feed End of Rotary Kiln... . . . . . . . . . 2-16

Figure 2-7. Discharge End of Rotary Kiln. . . . . . . . . . 2-18

Figure 2-8. Kiln Control Panel and Power Supply . . . . . . . . 2-20

Figure 2-9. Dissolver/Quench Tank in Trench Below Kiln....... 2-21

Figure 2-10. Water Washing System . . . . . . . . . . 2-23

Figure 2-11. Water and Acid Washing Process Area During
Groundbreaking . . . . . . . . . . . . . 2-27

Figure 2-12. Water and Acid Washing Process Equipment . . . . . 2-29

Figure 2-13. Vacuum Filter on Stand ............... . 2-30

Figure 2-14. Water Washing Centrifuge on Stand. . . . . . . . 2-31

Figure 2-15. Vacuum Filtration System Piping and Control Schematic. 2-33

Figure 2-16. Acid Washing System. . . . . . . . . . . . 2-36

Figure 2-17. Caustic Regeneration System. . . . . . . . . . 2-40

Figure 2-18. Caustic Regeneration System Equipment. . . . . . . 2-41

Figure 2-19. Caustic Concentration System - Phase I. . . . . 2-45

Figure 2-20. Caustic Concentration System - Phase II. . . . . . 2-46

Figure 2-21. Evaporator During Delivery to Site . . . . . . . 2-48 
Figure 2-22. Installed Evaporation System . . . . . . . . . 2-49

Figure 2-23. Evaporator Control Panel . . . . . . . . . . 2-50

Figure 2-24. Flaker............... . . 2-54

Figure 2-25. Water Treatment System - Phase I . . . . . . . . 2-56

Figure 2-26. Water Treatment System - Phase II. . . . . . . . . 2-58

Figure 2-27. Water Treatment System Equipment . . . . . . . . . ?-59

Figure 2-28. Data Acquisition System During Installation. . . . 2-61

Figure 3-1. Sulfur and Ash Data for Kiln and Product Coal as a . . 3-21

Figure 3-2. Volatiles, Sulfur and Heat Content Data for Product
Coal as a Function of Coal Exit Time. . . . . . . . . 35

Figure 4-1. Effect of Temperature and Caustic/Coal Ratio (R) on Sulfur Removal: Pittsburgh No. 8 (Powhatan); $\mathrm{KOH} / \mathrm{NaOH}=1 . . \cdot . \cdot . \cdot . \cdot . \cdot . \cdot . \cdot . . .44-10$

Figure 4-2. Comparison of Predicted and Experimental Vaiues for Sulfur Content of MCL Coal Product: Pittsburgh No. 8 (Powhatan); $\mathrm{KOH} / \mathrm{NaOH}=1$.......... 4-12

Figure 4-3. Effect of Temperature and Caustic/Coal Ratio (R) on Sulfur Removal: Pittsburgh No. 8 (Powhatan); $\mathrm{KOH} / \mathrm{NaOH}=0 . . . . . . . .44-15$

Figure 4-4. Comparison of Predicted and Experimental Values for Sulfur Content of $\mathrm{MCL}$ Coal Product: Pittsburgh No. 8 (Powhatan); $\mathrm{KOH} / \mathrm{NaOH}=0 . . . . . . . .4$ 4-17 Figure 4-5. Effect of Temperature and Caustic Type on Sulfur
Removal: Kentucky No. 9 Coal. . . . . . . . . 4-20

Figure 4-6. Kiln Temperatures and Sulfur Content for Run 11: . . . 4-22

Figure 4-7. Effect of Temperature and Caustic/Coal Ratio (R) on Ash Removal: Pittsburgh No. 8 (Powhatan);

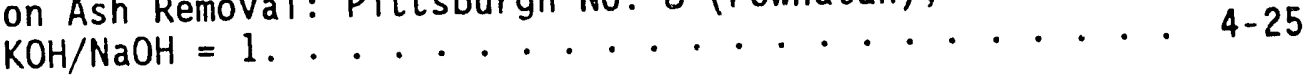
Figure 4-8. Comparison of Predicted and Experimental Values
for Ash Content of $\mathrm{MCL}$ Coal Product: Pittsburgh No. 8 (Powhatan); $\mathrm{KOH} / \mathrm{NaOH}=1$........... 4-26 


\section{LIST OF FIGURES (CONTINUED)}

Page

Figure 4-9. Correlation Between Pittsburgh No. 8 (Powhatan) Coal

Product Sulfur and Ash Content. . . . . . . . . 4-28

Figure 4-10. Effect of Temperature and Caustic/Coal Ratio (R) on Ash Removal: Pittsburgh No. 8 (Powhatan);

$\mathrm{KOH} / \mathrm{NaOH}=0 . . . . . . . . . . . . . .44-30$

Figure 4-11. Comparison of Predicted and Experimental Values for

Ash Content of MCL Coal Product: Pittsburgh No. 8

(Powhatan); $\mathrm{KOH} / \mathrm{NaOH}=0 . . . . . . . . . . .44-31$

Figure 4-12. Effect of Temperature and Caustic Type on Ash

Removal: Kentucky No. 9 Coal........... 4-34

Figure 4-13. Product Coal Ash During Run 11. . . . . . . . . 4-37

Figure 4-14. Coal Product Moisture vs. Ash Content: Pittsburgh

No. 8 Coal (Powhatan).............. 4-41

Figure 4-15. Comparison of Sulfate Sulfur in Kiln Discharge

Coal and Washed Coal Product .......... . 4-47

Figure 4-16. Effect of Leaching Temperature on Conversion to Humics 4-54

Figure 4-17. Humics Balance for Run 11... . . . . . . . . . 4-59

Figure 4-18. Carbonate Balance for Run 11.......... . . 4-69

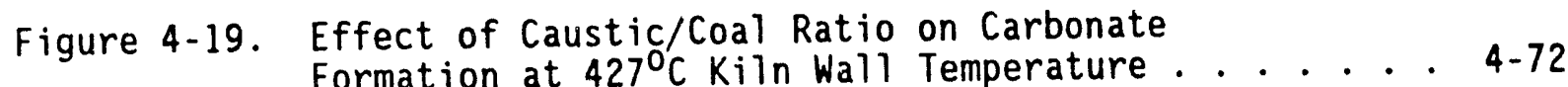

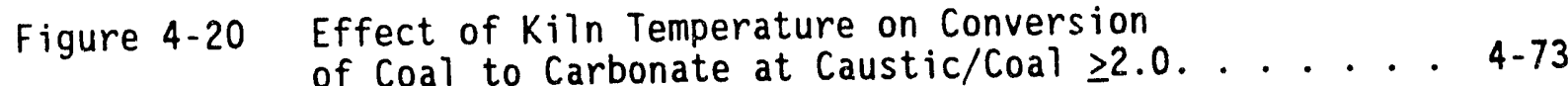

Figure 4-21. Effect of Kiln Temperature on Conversion

of Caustic to Carbonate at Caustic/Coal $\geq 2.0$.... 4-74

Figure 4-22. Comparison of Carbonate Formation in Kiln
Discharge and Spent Caustic Samples. . . . . . . 4-78

Figure 4-23. Particle Size Distribution for Feed Coal and Kiln
Discharge Coal: Pittsburgh No. 8 (Powhatan). . . . 4-83

Figure 4-24. Particle Size Distribution for Feed Coal and Coal

Product from Shakedown: Pittsburgh No. 8 (Powhatan). 4 4-84

Figure 4-25. Particle Size Distribution for Feed Coal and Washed

Coal Product: Pittsburgh No. 8 (Powhatan). . . . . 4-85 
Figure 4-26. Particle Size Distribution for Feed Coai and Washed Coal Product: Pittsburgh No. 8 (Blacksville) . . . 4-86

Figure 5-1. First Stage Rotary Drum Vacuum Filter Piping and Control System................ 5-5

Figure 5-2. Modified Vacuum Filtration System . . . . . . . . 5-7

Figure 6-1. Block Diagram of the MCL Process. . . . . . . . 6-2

Figure 6-2. Water Washing System. . . . . . . . . . . 6-6

Figure 6-3. Acid Washing System .............. 6-10 
LIST OF TABLES

Page

Table 1-1. Coal Feed to MCL Circuit . . . . . . . . . . 1-9

Table 2-1. MCL Design Material Balance. .......... 2-4

Table 2-2. Major Equipment. . . . . . . . . . 2-8

Table 2-3. Water Washing Design Material Balance. . . . . . . 2-24

Table 2-4. Acid Washing Design Material Balance ....... 2-37

Table 3-1. Test Matrix: Variable-by-Variable. . . . . . . 3-4

Table 3-2. Run Condition Summary. . . . . . . . . 3-8

Table 3-3. Quantities and Characteristics of Coal Products

Collected from MCL Operations. . . . . . . . . . 3-59

Table 3-4. MCL Product Coal Shipped at End of Phase I . . . . . 3-62

Table 4-1. Coal Product Quality for Tests With Pittsburgh

No. 8 Coal (Powhatan No. 6 Mine) and 50:50 KOH/NaOH. . 4-5

Table 4-2. Coal Product Quality For Tests With Pittsburgh

No. 8 Coal (Powhatan No. 6 Mine) and NaOH. ...... 4-6

Table 4-3. Coal Product Quality for Tests With Kentucky No. 9 Coal. 4-7

Table 4-4. Coal Product Quality For Tests With Pittsburgh

No. 8 Coal (Blacksville No. 2 Mine) and $\mathrm{NaOH}$.... 4-8

Table 4-5. Quantitative Cation and Anion Analysis ........ 4-43

Table 4-6. Removal of Minor and Trace Elements From Coal

Semi-Quantitative Emission Spectrographic

Analysis, $\mathrm{mg} / \mathrm{g}$ dmf.............. 4-. 4-. 44

Table 4-7. Sulfur Forms Analysis Results. . . . . . . . . . 4-45

Table 4-8. Kiln Gas Analysis Results. . . . . . . . . 4-50

Table 4-9. Formation of Humic Acid for Shakedown Through Run 9. . . 4-53

Table 4-10. Humics Analysis for Run 11.......... 4-58

Table 4-11. Carbonate Formation for Shakedown Through Run 9 . . 4-64

Table 4-12 Carbonate Analysis for Run 11.......... 4-68

Table 4-13. Coal Particle Size Distribution . . . . . . . . 4-81 


\section{LIST OF TABLES (CONIINUED)}

Page

Table 4-14. Partition Of Aluminum, Silicon and Iron for Shakedown

Through Run 9................. 4-91

Table 4-15. Partition of Aluminum, Silicon and Iron for Run 11. . 4-94

Table 4-16. Concentration Levels of Aluminum, Silicon and Iron

In Spent and Regenerated Caustic for Runs 2 Through 8 . 4-96

Table 4-17. Concentration Levels of Aluminum, Silicon and Iron

In Spent and Regenerated Caustic for Runs 10 and 11 . 4-98

Table 4-18. Concentration Levels of Aluminum, Silicon,

Iron, Sodium and Potassium In Acid Wash Water. . . . 4-101

Table 4-19. Centrifuge Centrate pH Measurements . . . . . . . 4-105

Table 4-20. Coal Fines in $\mathrm{C}-201$ Centrate. . . . . . . . . . 4-106

Table 4-21. Centrifuge Centrate and Slurry Characteristics. . . . 4-107

Table 4-22. Moisture Content of Centrifuge Cake . . . . . . . 4-108

Table 4-23. Coal Material Balance for Runs 1 Through 9. . . . . . 4-110

Table 4-24. Coal Material Balance for Run 11............ 4-114

Table 4-25. Concentration Levels of Sulfur In Spent and

Regenerated Caustic . . . . . . . . . . 4-115

Table 4-26. Corrosion/Erosion Rates in MCL Test Loop. . . . . . 4-118

Table 4-27. Visual Inspection of Erosion Coupons. . . . . . . . . 4-119

Table 6-1. MCL Design Material Balance. . . . . . . . . . 6-3

Table 6-2. Water Washing Design Material Balance. .. . . . . . 6-7

Table 6-3. Acid Washing Design Material Balance . . . . . . . . 6-11

Table 6-4. MCL Process Model Equations. . . . . . . . . . . 6-13

Table 6-5. Comparison of Reaction Model and Experimental Sulfur and Ash Contents of MCL Coal Product . . . . . . . 6-17 


\section{ABSTRACT}

The objectives of this program were to design, construct, shakedown and operate an integrated $\mathrm{MCL}$ test circuit to demonstrate the technical capability of the process for producing a demineralized and desulfurized coal that meets New Source Performance Standards (NSPS), to test process conditions aimed at lower costs, and to deliver product coal. These objectives were met by the procurement, construction, and operation of the integrated test circuit. Shakedown and a 63-test process matrix resulted in the production of about 3,700 pounds of treated coal. Product $\mathrm{MCL}$ coal may be used to displace oil in some turbine and diesel engines and may be used in the retrofit of oil-fired boilers. Two high sulfur, high ash coals and one medium sulfur, high ash coal representative of the Eastern United States coal production were processed: Pittsburgh No. 8 (Powhatan No. 6 mine), Kentucky No. 9, and Pittsburgh No. 8 (Blacksville No. 2 mine). Although mild kiln operating conditions ( 325 to $415^{\circ} \mathrm{C}$ and 1 to 2.3 hours residence time) and low caustic to coal ratios $(1: 1$ to $3: 1)$ were used, the combination of continuous operation and rigorous exclusion of air from the system allowed the production of MCL coal that had product sulfur content was well below NSPS standards, very low carbonate production, very little volatile losses, and low alkali retention by the product MCL coal. Optimization testing resulted in a product coal containing 0.2 to 0.4 percent sulfur $(0.26$ to 0.6 1 bs $\mathrm{SO}_{2} / \mathrm{million} \mathrm{Btu}$ ) and 0.15 to 0.5 percent ash with more than 90 percent organic sulfur removal, -95 percent $\mathrm{SO}_{2}$ reduction from run-of-mine coal, -91 percent $\mathrm{SO}_{2}$ reduction from precleaned process feed coal, and with heat content of about 14,000 Btu per pound. 


\section{INTRODUCTION AND SUMMARY}

This final report summarizes the work performed by the Applied Technology Division of the TRW Space and Electronics Group for the U.S. Department of Energy, Pittsburgh Energy Technology Center for the Molten-Caustic-Leaching (MCL or Gravimelt) process for the desulfurization and demineralization of coal. This work was performed under Contract No. DE-AC22-86-PC91257. The contract period of performance extended from 1986 through March 1993 and was divided into two phases.

The Gravimelt process was first conceived and laboratory tested at TRW in the late 1970's. The Pittsburgh Energy Technology Center of the DOE then sponsored a detailed laboratory study (1979-1981); an initial bench-scale project (1981-1983) to evaluate the technology; and a modular test circuit which was operated on a sequential but not simultaneous basis (1983-1986). Under Phase I (1986-1990) of the current program, an integrated test circuit was constructed and operated. The test circuit was refurbished, modified and operated for a second time under Phase II (1990-1993). The two phases of the current program are described in the following paragraphs.

\section{Phase I}

One of the objectives of Phase I of the current program was to construct. a continuous integrated 20 pounds of coal per hour MCL test circuit which incorporated as much as possible the already existing Government-owned modular test circuit from the previous program (U.S. DOE Contract No. $D E-A C 22-83-P C 63032)$. That is, Phase I involved the modification of the existing facility and the procurement and installation of additional equipment to upgrade the test circuit, inciuding the installation of an evaporator and multiple stages of countercurrent washing capability.

The remaining Phase I objectives were to operate the integrated MCL test circuit on a real-time long-term steady-state basis: 1) to demonstrate the technical capability of the process for producing a demineralized and desulfurized coal that meets New Source Performance Standards (NSPS), 2) to 
determine the range of effective process operation, 3) to test process conditions aimed at significantly lower costs, 4) to obtain scaleup data, and 5) to deliver product coal. These objectives were met by the procurement, construction, shakedown, and operation of the integrated $\mathrm{MCL}$ test circuit which produced product coal with a sulfur content well below the New Source Performance Standards.

The MCL integrated test circuit was operated during Phase I in a shakedown mode for approximately 100 hours in December 1988, followed by nine series of operational tests (Runs 1 through 9) covering a 54 -test process matrix during the January to June 1989 time period, resulting in the production of about 3,000 pounds of treated coal suitable for further testing and evaluation. A 50/50 potassium hydroxide/sodium hydroxide mixture and pure sodium hydroxide were used as the leaching agents. Two high sulfur, high ash feed coals representative of the Eastern United States coal production were processed during Phase I: Pittsburgh No. 8 (Powhatan No. 6 mine) and Kentucky No. 9. The Pittsburgh No. 8 coal was used for most of Phase I testing. The feed coal sizes were 14 mesh (for most of the testing) and 6 mesh. The rotary kiln was operated at reaction temperatures of 325 to $415^{\circ} \mathrm{C}$ (the kiln wall temperatures were higher) and at coal/caustic residence times of approximately one to two hours. Caustic-to-coal ratios of $1: 1$ to $3: 1$ were used.

During Phase I, optimization testing was performed resulting in a product coal containing 0.4 percent sulfur $\left(0.6 \mathrm{lbs} \mathrm{SO}_{2} / \mathrm{mill}\right.$ ion $\left.\mathrm{Btu}\right)$ and 0.15 percent ash with more than 90 percent organic sulfur removal, 95 percent $\mathrm{SO}_{2}$ reduction from run-of-mine coal, and 91 percent $\mathrm{SO}_{2}$ reduction from precleaned process feed coal. The results indicate that high sulfur Eastern coals can be treated to meet NSPS standards for sulfur oxides control while providing a product coal with heat content of about 14,000 Btu per pound and an ash content as low as a tenth of a percent. An assessment of equipment performance during Phase I indicated that the MCL circuit needed certain modifications in order to be capable of integrated, continuous, long-duration operational testing. 
Phase II

A follow-on to the original program was awarded in order that the test circuit could be modified and refurbished and that operations could be resumed. This added scope is referred to in this document as the Phase II program. The specific objectives of the Phase II program were: 1) to repair, refurbish and modify the existing $\mathrm{MCL}$ test circuit to allow for off-line unit operation testing followed by continuous long-term integrated test operations, 2) to determine the optimal or economic operating parameters for each the unit operation in the test circuit, 3) to obtain the necessary engineering and operational data for scaleup to a production unit, and 4) to produce product coal that meets NSPS stan_urds.

Phase II was completed without the addition of major capital equipment but rather with the replacement of parts and materials and with modification of existing equipment to upgrade to original performance levels consistent with commercial design requirements. The most critical modifications were those affecting the operation of the vacuum filters and the evaporator that were necessary for integrated operation.

After extensive off-line testing of the vacuum filters and the evaporator, two series of continuous around-the-clock operational tests (Runs 10 and 11) of the refurbished/modified MCL test circuit were performed covering a 9 -test process matrix and producing about 700 pounds of product coal. All sections of the plant (including the vacuum filters and the evaporator) were operated in an integrated fashion for extended periods of time with regenerated caustic recycle to the kiln. A medium sulfur, high ash Pittsburgh No. 8 coal fror a different mine (Blacksville No. 2 mine) was used during Run 11. A 21-hour period during Run 11 produced product coal containing 0.17 percent sulfur ( $0.26 \mathrm{lbs} \mathrm{SO}_{2} / \mathrm{mi} 11$ ion $\left.\mathrm{Btu}\right)$ with 83 percent organic sulfur removal, $94.3 \% \mathrm{SO}_{2}$ reduction from run-of-mine (ROM) coal, and $92.1 \% \mathrm{SO}_{2}$ reduction from precleaned process feed coal. This coal meets the most stringent U.S. standards. Product coal was produced with a sulfur content as low as 0.11 wt\% (0.17 lbs $\mathrm{SO}_{2} / \mathrm{million} \mathrm{Btu}$ or $198 \mathrm{mg} \mathrm{sO} 2 / \mathrm{Nm}^{3}$ ) which corresponds to a $96 \%$ reduction in sulfur from F.OM and which is one-half of the EC standard of $400 \mathrm{mg} \mathrm{SO} 2 / \mathrm{Nm}^{3}$. 
A new process configuration was tested in which the effluent from the acid wash train (without acid addition) was used as the water makeup for the water wash train, thus eliminating the one remaining aqueous process waste stream. This configuration resulted in product coal with low sulfur but moderate ash content.

The following sections of this Introduction and Summary contain a description of the integrated circuit, coal analyses and NSPS targets for the three project ccals, a summary of the test results and conclusions, an economic analysis, recommendations, and a report outline.

\section{I PROCESS DESCRIPTION}

The MCL process utilizes a melt of sodium hydroxide or mixtures of sodium and potassium hydroxides at reaction temperatures of 325 to $415^{\circ} \mathrm{C}$ to remove (at the high end of this temperature range) virtually all of the sulfur and mineral matter from coal. The process is a potential replacenent for the costly flue gas desulfurization systems contemplated for smaller utilities and industrial boilers. The product coal might also displace oil in some turbine and diesel engines and may be used in the retrofit of oil-fired boilers to coal-fired boilers. Product MCL coal may also have application as an inexpensive pre-activated or activated carbon for extraction of pollutants from waste water or to clean-up and concentrate radioactive or toxic waste.

The test circuit consists of six unit operations (Figure 1-1) which together provide a continuous system for leaching coal with caustic and then regenerating the reactant. These units are:

- A kiln for reacting molten caustic with coal (Section 100)

- A seven-stage water washing section for recovering caustic from the coal (Section 200)

- A three-stage acid washing section for removing residual metals and alkali and providing an ultra-pure coal product (Section 300)

- A regeneration section to provide purified aqueous caustic (Section 400)

- An evaporator section to provide molten or flaked caustic for recycle to the kiln reactor (Section 500) 


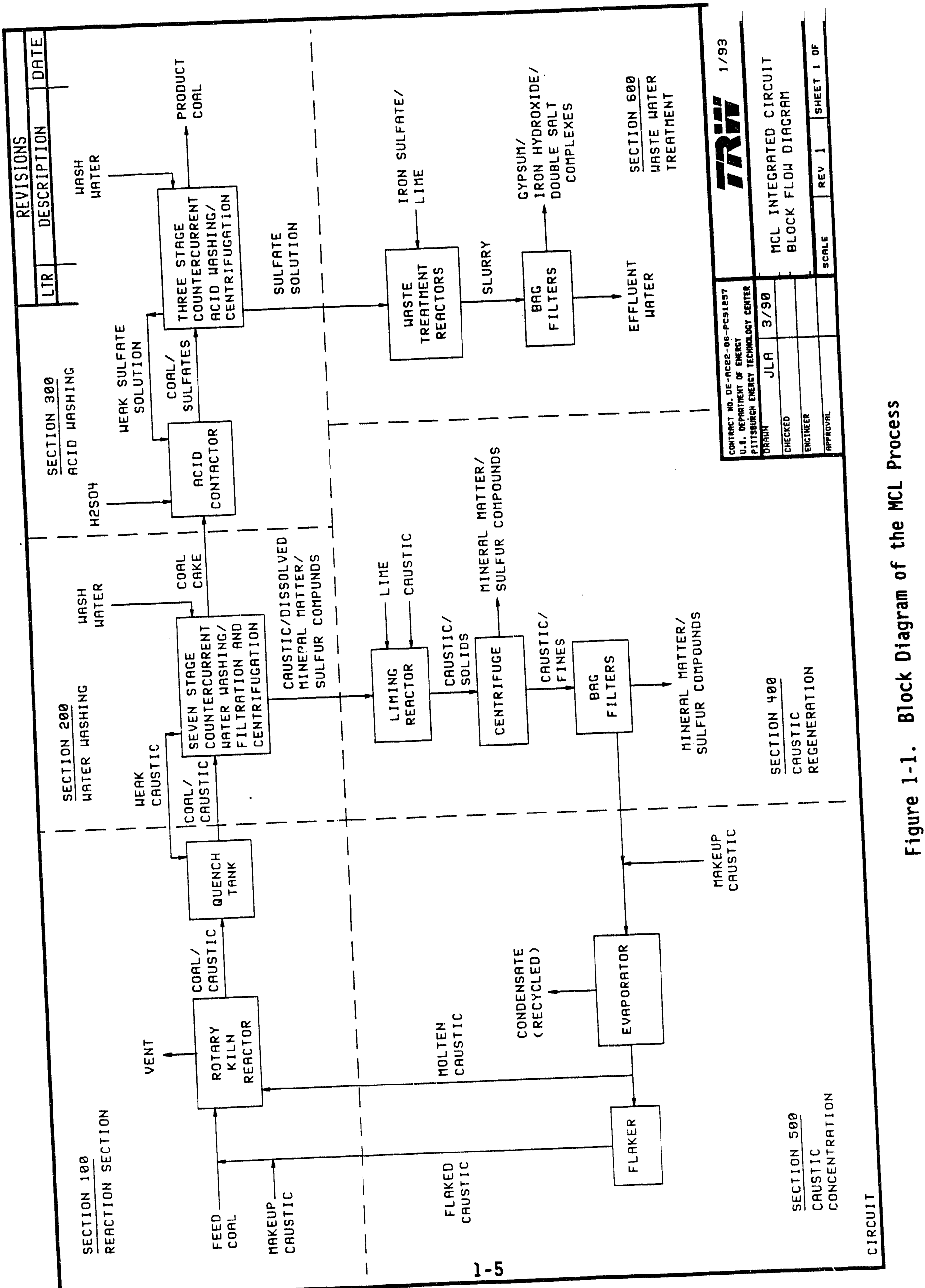


- A water treatment section to provide either dischargeable or recyclable water (Section 600).

The integrated test circuit facility that was constructed at the TRW San Juan Capistrano test site in California contains about 150 pieces of commercial equipment including filters, centrifuges, tanks, reactors, feeders, a kiln, and a rising film evaporator. It occupies 3700 square feet and is fitted with more than 6000 feet of piping, 425 valves, 80 instruments and controls as well as a computer control room and a data acquisition and reduction system.

A schematic of the MCL process is shown in Figure 1-2. Feed coal is premixed with anhydrous sodium hydroxide, or mixed sodium and potassium hydroxides, and then fed to a rotary kiln reactor (Section 100) where the mixture is heated to reaction temperatures of 325 to $415^{\circ} \mathrm{C}$ causing the caustic to: 1) melt and become sorbed in the coal matrix, 2) react with the coal sulfur and mineral matter, and 3) dissolve the reaction products containing sulfur and inorganic components.

The reaction mixture is cooled in the cooling section of the kiln such that the mixture exits the rotary kiln in the form of pellets that are then washed with water in a seven-stage countercurrent separation system consisting of two filters and five centrifuges (Section 200). As an example, four weights of wash water per weight of coal may be used resulting in a $50 \%$ aqueous reacted caustic solution exiting the first stage of filtration. A large part of the coal-derived iron and a small amount of sodium and/or potassium remain with the coal cake exiting this countercurrent washing system.

The coal cake is next washed to remove the residual iron and caustic in a countercurrent three-stage centrifuge system (Section 300). Sulfuric acid is added to the first stage to produce a pH of about 2 in order to dissolve the residual alkali and iron hydrcxide. As an example, three weights of wash water per weight of coal may be used resulting in an acid extract leaving the first stage containing 5,900 ppm of mixed sodium and potassium sulfates and $12,500 \mathrm{ppm}$ of iron sulfate. The product coal exiting this acid washing system contains about one weight of sorbed water per weight of coal. 


\section{WATER}

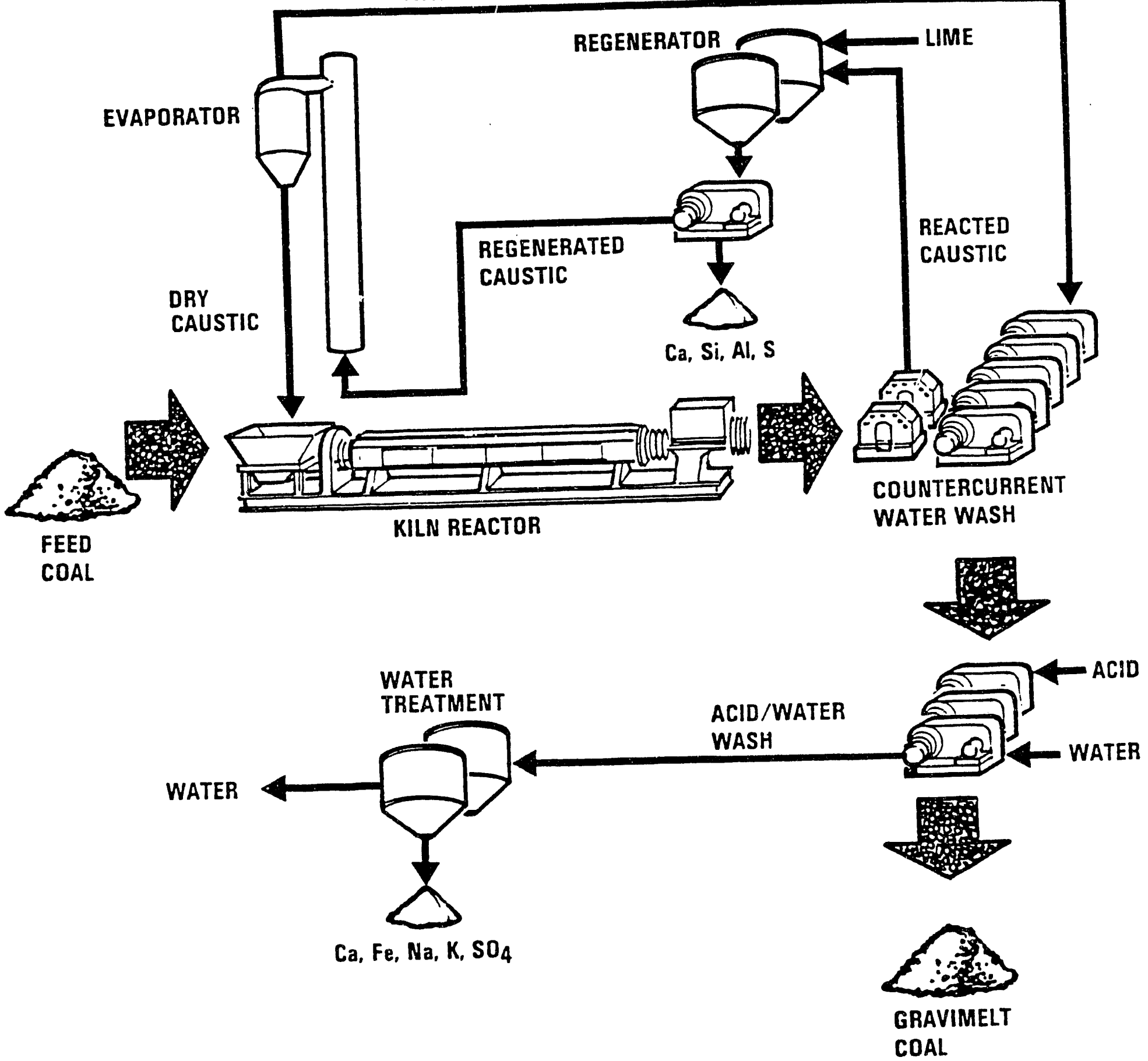

Figure 1-2. Schematic of the MCL Process 
All of the iron sulfate and a major portion of the alkali sulfates can be removed from the acid extract water by a sequence of heating and lime treatment (Section 600) to form insoluble minerals such as gypsum. This water can then be recycled to the water wash train. Under some (very carefully controlled) conditions, the precipitate formed can be a jarosite-like double salt which can be easily disposed of or land-filled.

A new process configuration, with potentially large cost savings to the process, was tested in which the effluent from the acid wash train (without acid addition) was used as the makeup wash water. for the water wash train, thus eliminating Section 600 . This configuration resulted in a product coa 1 with low sulfur anc moderate ash content suitable for those commercial applications that do not require low ash coal.

The aqueous caustic recovered from the first countercurrent washing circuit is limed to remove the coal-derived mineral matter, sulfur compounds, and carbonates (Section 400). The mixture is provided with sufficient residence time to permit precipitation of the impurities before being centrifuged. The purified liquid is preheated and sent to a caustic evaporator (Section 500) where the water is recovered for recycle to the first wash train, while producing anhydrous caustic (as either a molten liquid or as flakes) for reuse in the initial leaching of the coal in Section 100 .

\subsection{TEST COALS}

A major goal of the integrated $M C L$ project was to demonstrate reduction in sulfur to meet New Source Performance Standards. These standards are based on the sulfur content per million Btu, which is expressed in the form of 1 bs $\mathrm{SO}_{2} / 10^{6} \mathrm{Btu}$. This removal applies to the run-of-mine (ROM) coal analysis. The three coals tested in this program (Table 1-1) contain 4.5 to 11.1 ibs $\mathrm{SO}_{2} / 10^{6} \mathrm{Btu}$, which is the range that represents a majority of the coals mined east of the Mississippi, and fall within Category 3 of the New Source Performance Standards, which requires 90 percent reduction from ROM to 0.45 to 1.11 ibs $\mathrm{SO}_{2} / 10^{6} \mathrm{Btu}$. 
TABLE 1-1. COAL FEED TO MCL CIRCUIT

Coal

$\%$, w/w Dry Basis Sulfur Ash Vols

Heat Content,

Pittsburgh No. $8^{\text {a }}$

Run of Mine Cleaned

$5.08 \quad 34.90$

$4.27 \quad 11.57$

38.60

9144
13016

$-\cdot$

14719

Lbs $\mathrm{SO}_{2} / 10^{6} \mathrm{Btu}$ Btu/Lb

MF MAF Coal NSPS

Kentucky No. $9^{b}$

Run of Mine

$\begin{array}{rrrrrrr}4.70 & 17.00 & -- & 10525 & -- & 8.93 & \\ 3.41 & 9.25 & 40.53 & 13259 & 14610 & 5.14 & 0.89\end{array}$

Pittsburgh No. $8^{c}$

\section{Run of Mine}

Cleaned

$\begin{array}{rrrrrrr}2.80 & 16.90 & 35.2 & 12450 & 14982 & 4.50 & \\ 2.26 & 7.90 & 38.7 & 13910 & 15103 & 3.25 & 0.45\end{array}$

\footnotetext{
a Powhatan No. 6 Mine (Powhatan Point, Ohio), Nacco Division, North American Coal Company

b Pittsburgh \& Midway Coal Company

c Blacksville No. 2 Mine, Consolidation Coal Company (CONSOL)
} 
The data within the table show that although each coal is reduced in sulfur content per unit heat content by about 30-45 percent by physical cleaning at the mine, the remaining sulfur content is still about five to seven times that required by the New Source Performance Standards. There are two options for meeting New Source Performance Standards, either post-combustion removal (flue gas desulfurization) or front-end cleaning as represented by the Gravimelt (MCL) process.

\subsection{TESTS RESULTS AND CONCLUSIONS}

The integrated $\mathrm{MCL}$ test circuit was operated on an around-the-clock basis during 100 hours of shakedown operation in December 1988 and for nine operating weeks during the January to June 1989 time period during Phase I and for two weeks in June 1991 and May $199 \hat{c}$ during Phase II resulting in about 1000 hours of testing covering a 63-test matrix and producing about 3700 pounds of product coal. Analyses were obtained on process streams such as product coal, slurries, filtrates, centrates, kiln gas, and various effluent solids. These data were assessed in detail. An operations summary is as follows:

1. A 63-test main parameter operational matrix was performed with variation in average kiln wall temperature, 371 to $438^{\circ} \mathrm{C}$; kiln residence time, 1 to 2.3 nours; sodium hydroxide or 50:50 mixtures of sodium and potassium hydroxides; caustic to coal ratios of $1: 1$ to $3: 1$; feed coal particle size of 14 mesh and 6 mesh $\times 0$; three coals (Pittsburgh No. 8 [Powhatan No. 6 mine and Blacksville No. 2 mine] and Kentucky No. 9, with the two Pittsburgh No. 8 seams being emphasized); fresh versus recycled caustic; and nitrogen flow direction in kiln (cocurrent versus countercurrent).

2. As a part of the above test matrix, the plant was operated to produce $\mathrm{MCL}$ coal with virtually no loss of heat content, small loss of volatiles, very low carbonate formation, 1.1 to 2 weights of moisture per weight of coal in the caustic washing section, 0.78 to 1.46 weights of moisture to coal in the acid washing section (product coai), and which can utilize sodium hydroxide in place of mixed sodium and potassium hydroxides or 6 mesh $\times 0$ coal in place of 14 mesh $\times 0$ coal.

3. The plant produced about 3700 lbs (dry basis) of MCL coal varying in quality from 0.2 percent sulfur to 1.9 percent sulfur, 0.1 percent ash to 3.3 percent ash, 14,400 Btu/lb to $12,600 \mathrm{Btu} / 7 \mathrm{~b}$ (dry basis) and volatiles from 35 percent to 21 percent. 
4. Organic sulfur removal ranged from 57 percent at moderate temperatures, where small loss of volatiles and MAF heat content was observed, and maximized at over 90 percent removal where there was minimal MAF heat content loss in the product coal.

5. All three coals were reduced to NSPS compliance levels.

6. Optimization testing was performed on the high sulfur Powhatan Pittsburgh No. 8 coal (feed sulfur $4.27 \%$ ) with sodium hydroxide resulting in product coal containing 0.4 percent sulfur $(0.60$ ibs $\mathrm{SO}_{2} /$ million Btu) and 0.15 percent ash with more than 90 percent organic sulfur removal.

7. Optimization testing was performed on the medium sulfur Blacksville Pittsburgh No. 8 coal (feed sulfur $2.26 \%$ ) with sodium hydroxide resulting i product coal containing 0.17 percent sulfur $(0.26$ bs $\mathrm{SO}_{2} / \mathrm{milli}$ ion Btu) with 83 percent organic sulfur removal. Since a new process configuration was being tested and there was no acid wash (see No. 9 below), the ash content was $2.4 \%$.

8. Best overall coal products (Pittsburgh No. 8) were prepared with $\mathrm{NaOH}$ resulting in 0.2 to 0.77 percent sulfur; 0.15 to 0.64 percent ash; 13,400 to $14,200 \mathrm{Btu} / \mathrm{lb} ; 24$ percent volatiles; $0.26-0.60$ lbs $\mathrm{SO}_{2} /$ million Btu ( -95 percent $\mathrm{SO}_{2}$ reduction from $\mathrm{ROM}$ and -91 percent from precleaned process feed) to $1.08 \mathrm{lbs} \mathrm{SO}_{2} / \mathrm{milli}$ ion $\mathrm{Btu}$ ( 90 percent $\mathrm{SO}_{2}$ reduction from ROM and 84 percent from precleaned process feed).

9. For "moderate" ash (2 to $3 \%$ ) product coal, a new process configuration involving the elimination of acid washing and of the subsequent liming of the acid extract has the potential of significantly reducing the cost of the process for those commercial applications that do not require low ash coal (sulfur reduction was not affected).

The following process conclusions were drawn from the test information: 1) the combination of continuous operation, exclusion of air from the system, and mild conditions would allow the production of $\mathrm{MCL}$ coal which has very low carbonate or humics production or volatiles loss and which does not have the propensity to form excessively wet cakes in countercurrent washing, 2) the low moisture content of the cakes can significantly lower processing costs through the elimination of a number of stages of washing and the utilization of less wash water, 3) low alkali retention by $\mathrm{MCL}$ coal obtained from the water washing section indicated that alkali losses may be reduced to an insignificant level resulting in large cost savings to the process, 4) the use of one weight of caustic per weight of coal instead of 2 or 2-1/2 weights of caustic would result in additional savings of wash water and evaporation energy costs, 5) carbonate and humics formation may not be a problem in 
continuous test circuit operation, thereby saving lime costs and enhancing operation, and 6) elimination of acid wash (and acid waste water treatment) results in a product coal containing a "moderate" ash level and can result in significant cost savings.

A11 of the product sulfur and ash data obtained during Run 9 (high sulfur Powhatan Pittsburgh No. 8 coal) are presented in Figure 1-3. The run was made under a single set of conditions, varying only in the use of $\mathrm{NaOH}$ for the latter part of the week and mixed sodium and potassium hydroxides at the beginning of the week. Sulfur removal averaged 93 percent from ROM and 88 percent from mine cleaned coal. Ash reduction averaged 98 percent from the coal fed to the process. For several periods during the latter part of the week, the ash content was so low as to be essentially immeasurable by the standard ASTM technique.

\subsection{ECONOMIC ANALYSIS}

Utilizing TRW funding, design and economic studies were performed using the test results obtain during this program. At the end of testing, design and cost studies were performed for a plant producing 10,000 tons per day of product $\mathrm{MCL}$ coal. The total capital investment for the plant is estimated at $\$ 469$ million with cogeneration of electric power. If the plant fuel requirements are supplied by burning part (18\%) of the product MCL coal $(8200$ tons per day of net MCL coal), the thermal efficiency for the plant is $82.0 \%$. The annual operating costs are estimated at $\$ 151$ million which is equivalent to an upgrading cost of $\$ 56$ per ton of net product MCL coal.

In an alternate process configuration, the effluent from the acid wash train (without acid addition) is used as the makeup wash water for the water wash train, thus eliminating Section 600. This configuration resulted in a product coal with low sulfur and moderate ash content suitable for those commercial applications that do not require low ash coal. Eliminating the acid washing and the acid waste water treatment steps could result in a potential reduction in processing costs of nearly $25 \%$, reducing process cost forecasts to under $\$ 45 /$ ton $(17 \mathrm{mils} / \mathrm{kW}-\mathrm{hr}$ ) and $\mathrm{placing}$ the $\mathrm{MCL}$ Process in a significantly advantageous cost position relative to FGD for boilers and furnaces smaller than $200 \mathrm{MW}_{\mathrm{e}}$. 


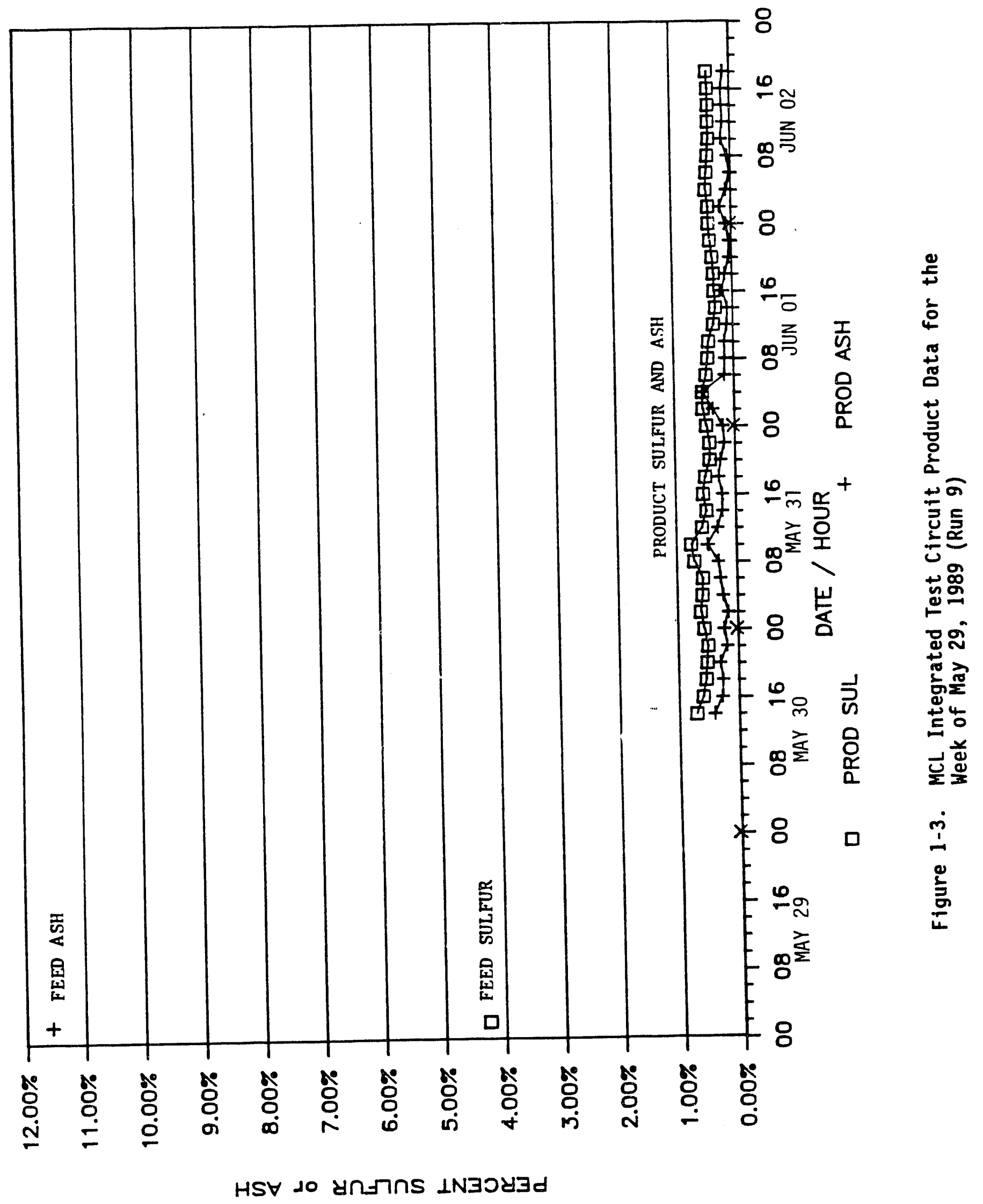




\subsection{RECOMMENDATIONS}

It is recommended that the U.S. Government review its position regarding further funding of the $\mathrm{MCL}$ technology in order to provide key data that are still required prior to full pilot/commercial demonstration. The further testing required is as follows:

- Evaluation of the coal-derived mineral matter reject from Section 400. The current program only funds simple precipitation of this mineral matter reject in order that the regenerated caustic can be successfully recycled and reused many times. This reject needs to be stabiiized and washed to recover absorbed caustic and to provide a landfill material consisting of cementitious silica, alumina and gypsum. The testing required includes:

- Operation of the complete molten caustic leaching integrated test circuit to produce quantities of mineral matter reject,

- Addition of washing stages or off-line use of the existing wash train to cleanup the reject and recover absorbed caustic,

- Heat treatment and roasting operations to stabilize the cementitious mineral matter, and

- Applications testing of the thus stabilized purified product.

- Product coal applications. The several tons of product coal produced during the mineral matter reject study described above need to be evaluated as pulverized fuel, as briquetted fuel and as coal water slurry, and for application as a toxic water cleanup medium.

Accomplishment of these tests will answer the major questions still associated with the MCL Process as to the environmental impact of the process mineral matter reject and applications of the MCL product coal. The process should then be ready for application both in the United States and on a world-wide export basis.

\subsection{REPORT OUTLINE}

The following sections of this report contain a compilation of run conditions and product coal data; an engineering assessment of the test results; a description of equipment modifications and Phase II off-line test results; and a computer model of the process. 


\section{TEST CIRCUIT DESCRIPTION}

One of the objectives of this project was to construct a continuous integrated MCL or Gravimelt test circuit which incorporated as much as possible the already existing Government-owned modular test circuit from the previous Gravimelt program (U.S. Department of Energy Contract No. $D E-A C 22-83 P(63032)$. That is, the current program involved the modification of the existing modular facility and the procurement and installation of additional equipment to upgrade the test circuit for continuous integrated operation. Major unit operations installed were a coal/caustic feed system for the existing rotary kiln reactor, coal washing and dewatering systerns, a caustic evaporation unit, and waste water treating systems.

In support of this integrated project, TRW spent its own funds to provide facilities for installation of equipment and to provide utilities to operate the plant. The major TRW facility improvements included:

- Design and construction of concrete foundations for equipment mounting.

- Design and installation of a major electrical substation supplying 480/208V 3-phase and 110V single phase for all motors, pumps, heaters and instrumentation (motor control center and electrical distribution system).

- Design aud installation of rain/weather covering for equipment and instrumentation.

- Design and installation of water and compressed air service to the facility.

- Refurbishment of the existing temperature controlled test area for use as a project control room to house the data acquisition and control system, and to provide desk facilities for the engineering staff during installation, shakedown and operation of the plant.

- Design and construction of a waste materials treatment system to interface with TRW waste treatment equipment allowing legal disposal of by-product solids and liquids generated during the project.

Following construction, the major objectives of the program were to operate the integrated plant on a long term steady-state basis in order to: 
1) demonstrate the production of coals meeting New Source Performance Standards (NSPS), 2) demonstrate process economic assumptions, 3) test an array of process conditions aimed at lowering processing costs, 4) obtain scaleup data, and 5) deliver product coal to DOE for subsequent combustion testing.

Although most of the above objectives were met and demonstrated during the Phase I operations, there were often equipment problems and failures that prevented operation in an integrated manner where all units were operating at optimum processing conditions. Budget constraints did not allow for the modification, replacement or repair of equipment during Phase $I$.

The purpose of Phase II of this program was to repair, refurbish, and modify the test circuit and to operate the plant in a continuous integrated manner at optimal conditions in order to obtain engineering, operational, and equipment performance data and to demonstrate process economic assumptions. The coal product obtained from the integrated MCL process met New Source Performance Standards (NSPS) for sulfur and contained very low levels of coal derived mineral matter.

The following six unit operations make up the MCL plant that was installed during Phase I of the program and modified during Phase II (Figure 2-1):

- Rotary Kiln Reactor System (Section 100)

- Water Washing System (Section 200)

- Acid Washing System (Section 300)

- Caustic Regeneration System (Section 400)

- Caustic Concentration System (Section 500)

- Water Treatment System (Section 600)

Al1 systems in the integrated test circuit were designed to operate continuously to support the production of 20 pounds of $M C L$ coal per hour. The material balance used to design the $20 \mathrm{lb} / \mathrm{hr}$ integrated test circuit is presented in Table 2-1 along with design assumptions. The design was based on TRW's knowledge and experience acquired from the previous modular Gravimelt program, as we 11 as information provided by equipment vendors. 


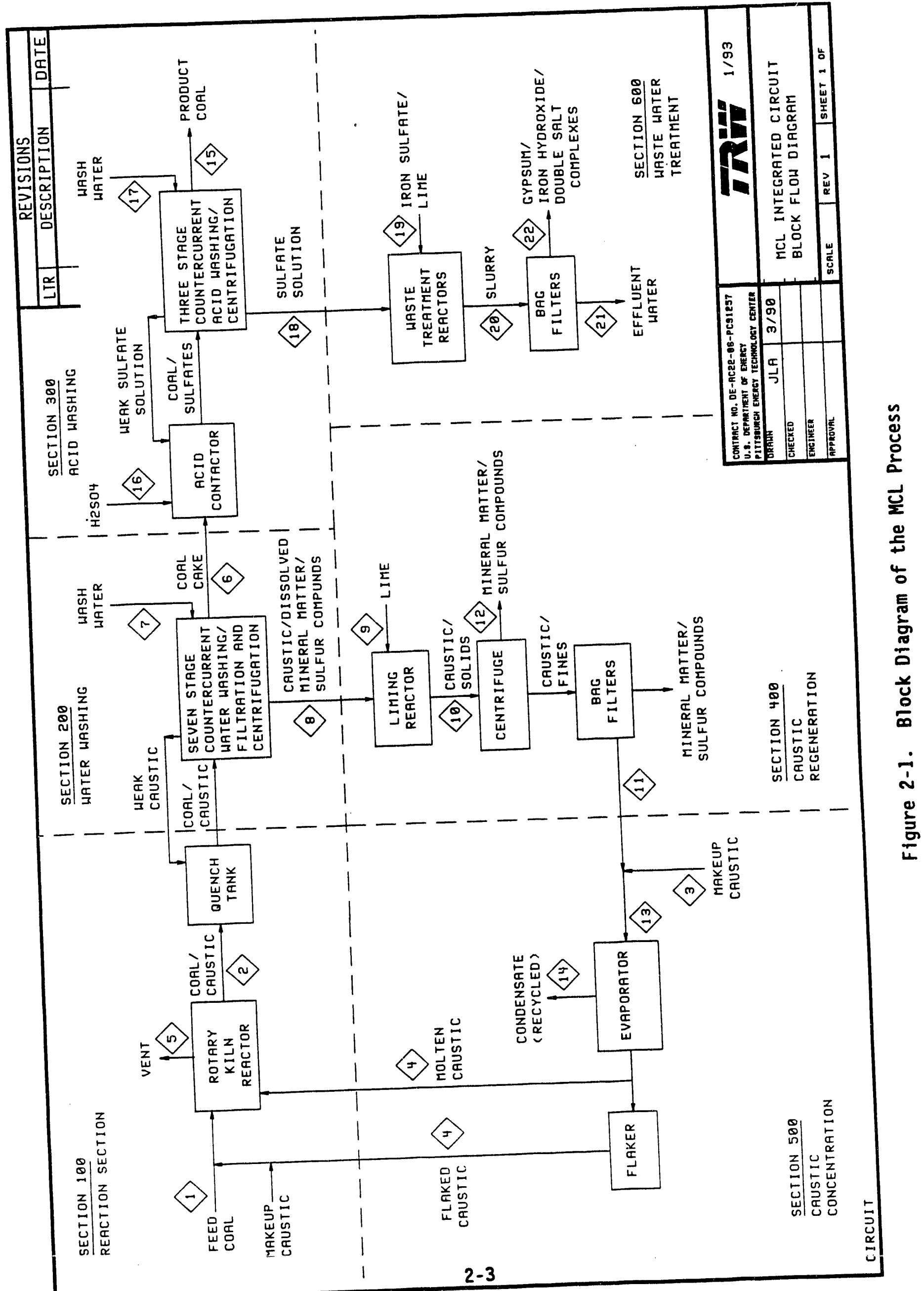




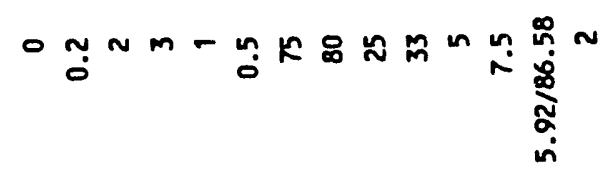

岂

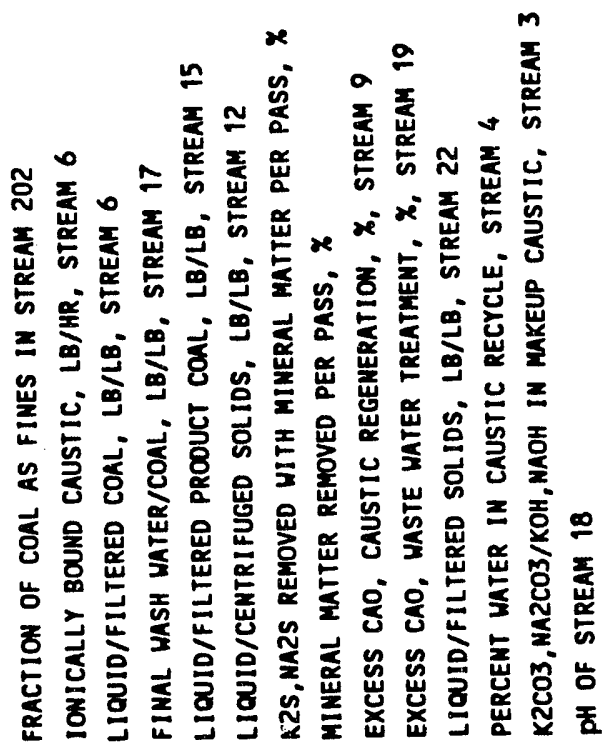

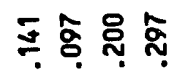

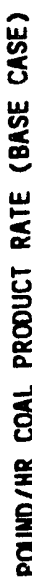

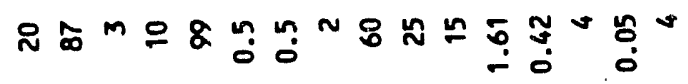

n

产

宕苔总

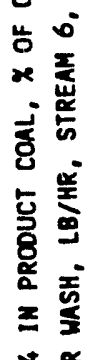




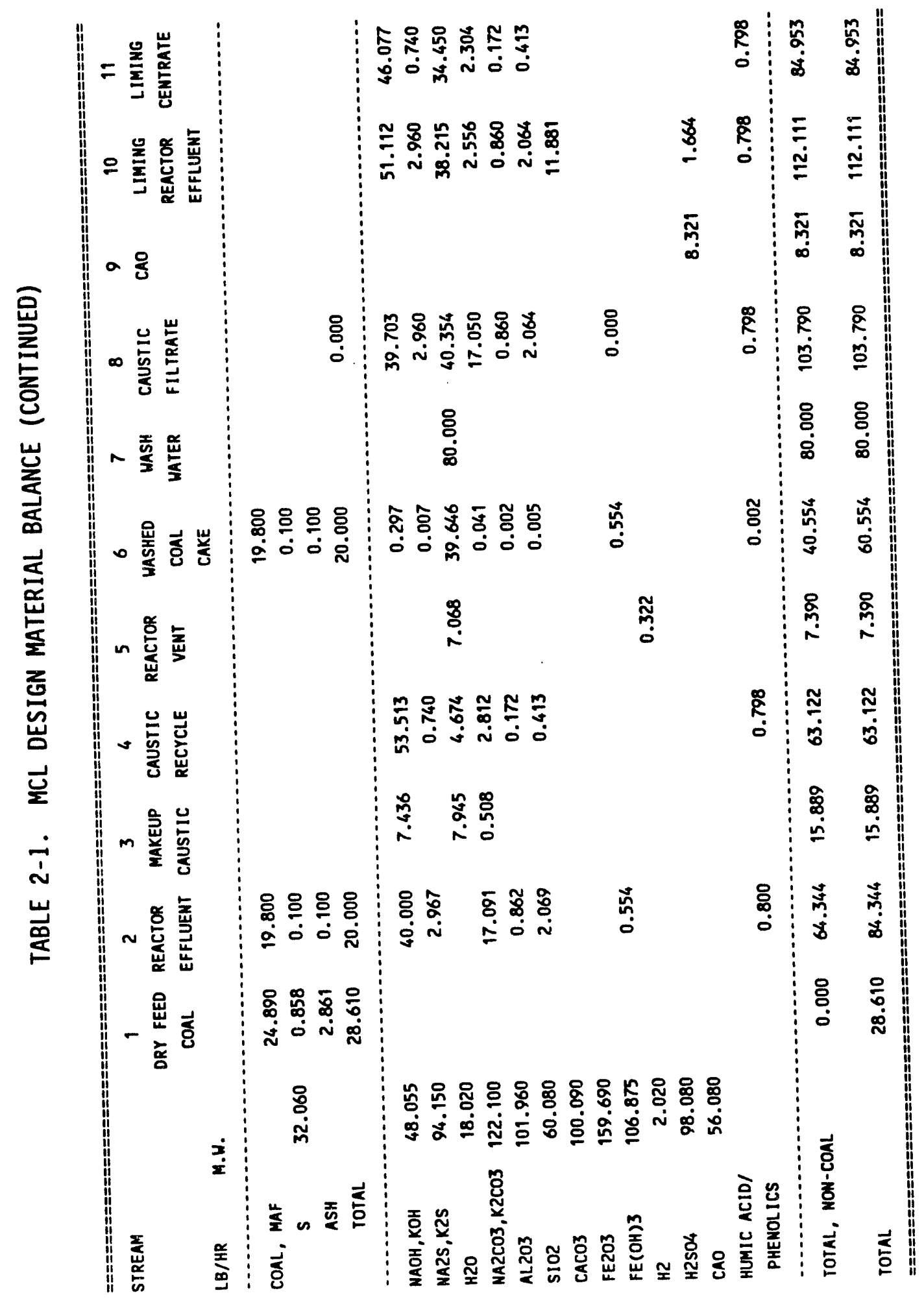




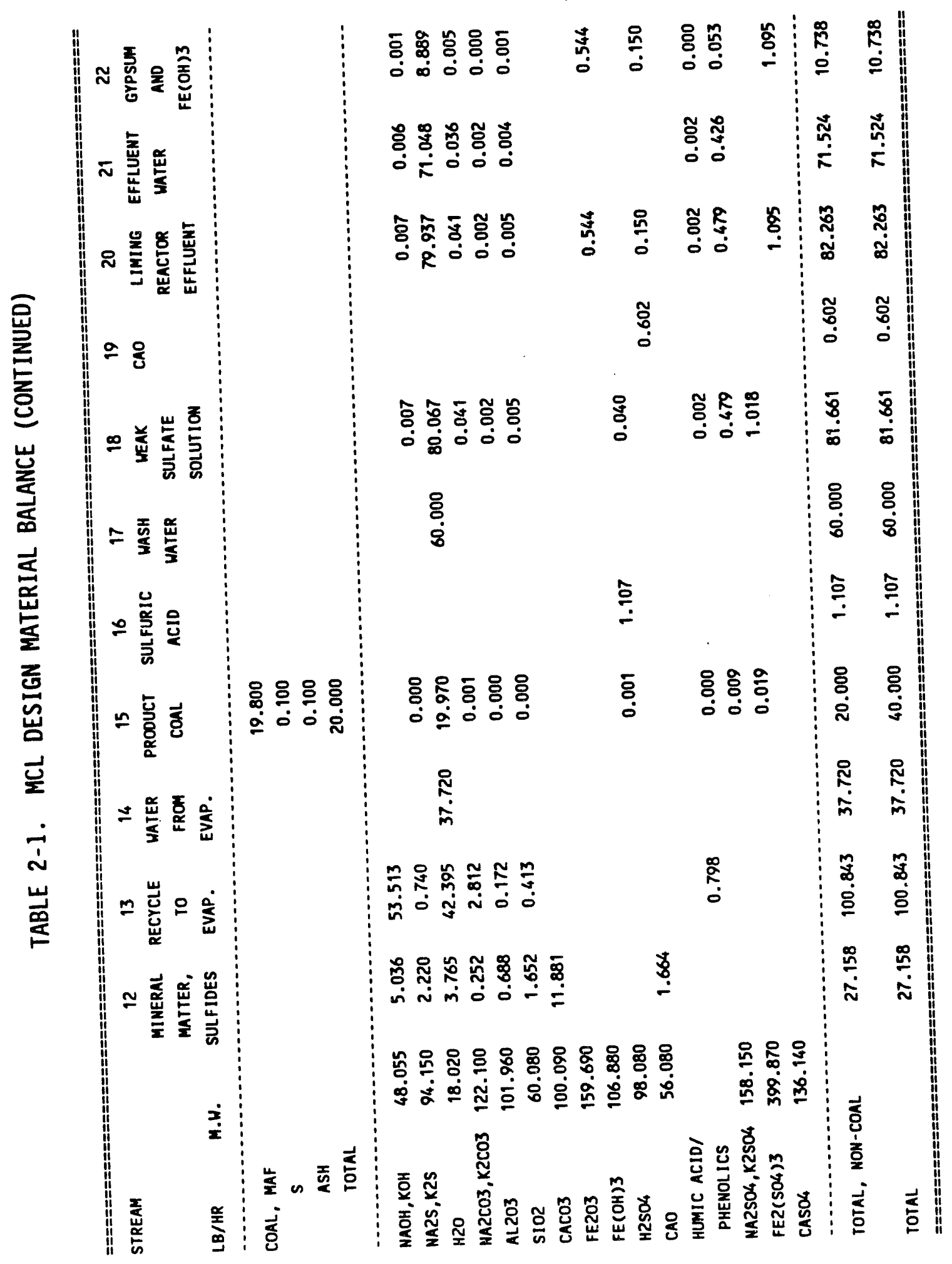


The final integrated test circuit, as built, contained about 150 major pieces of equipment including filters, centrifuges, tanks, reactors, feeders, a kiln, and a rising film evaporator. The major items of equipment are listed in Table 2-2. The materials of construction included stainless steel, nickel, Inconel, Alloy 20, carbon steel, polypropylene, and fiberglass. The test circuit occupies 3700 square feet and is fitted with more than 6000 feet of piping, 425 valves, 80 instruments and controls as well as a computer control room with a data acquisition and reduction system. The six unit operations including the modifications made during Phase II are described in detail in the following sections.

\subsection{ROTARY KILN REACTOR SYSTEM (SECTION 100)}

The rotary kiln reactor systen is used for the reaction of the coal/caustic mixture at a temperature of 325 to $415^{\circ} \mathrm{C}$ and a residence time of 1 to 2 hours. The kiln system was installed and successfully operated on the previous modular Gravimelt program but required some modifications to the kiln feed and exit to allow for continuous steady-state operation and integration with the other unit operations.

The kiln reactor unit accommodates a coal feed rate of $20 \mathrm{lbs} / \mathrm{hr}$ and a caustic (sodium hydroxide or a potassium/sodium hydroxide mixture) feed rate of 20-50 $\mathrm{lbs} / \mathrm{hr}$ (caustic to coal ratios of 1 to $21 / 2$ parts caustic to 1 part coal). The entire reactor system is operated at a slightly positive pressure and is swept with nitrogen to prevent oxidation of coal or contamination of the caustic by contact with the water vapor and $\mathrm{CO}_{2}$ present in ambient air. The nitrogen sweep also assures that the combustible gases evolved from the coal do not form a potentially explosive mixture. Schematics of the reactor system are shown in Figures 2-2 and 2-3. Figure 2-2 shows the reactor system as it was initially configured during Phase $I$ with cocurrent nitrogen flow. The system was reconfigured later in Phase I for countercurrent nitrogen flow. Figure 2-3 shows the reactor system after it was modified in Phase II. The nitrogen flow is still countercurrent and an acid scrubber has been added to the vent gas stream.

Flaked recycle caustic is mixed with fresh makeup caustic and coal in a stainless steel 55-galion drum. The drum is then attached to a tumbling 
TABLE 2-2. MAJOR EQUIPMENT

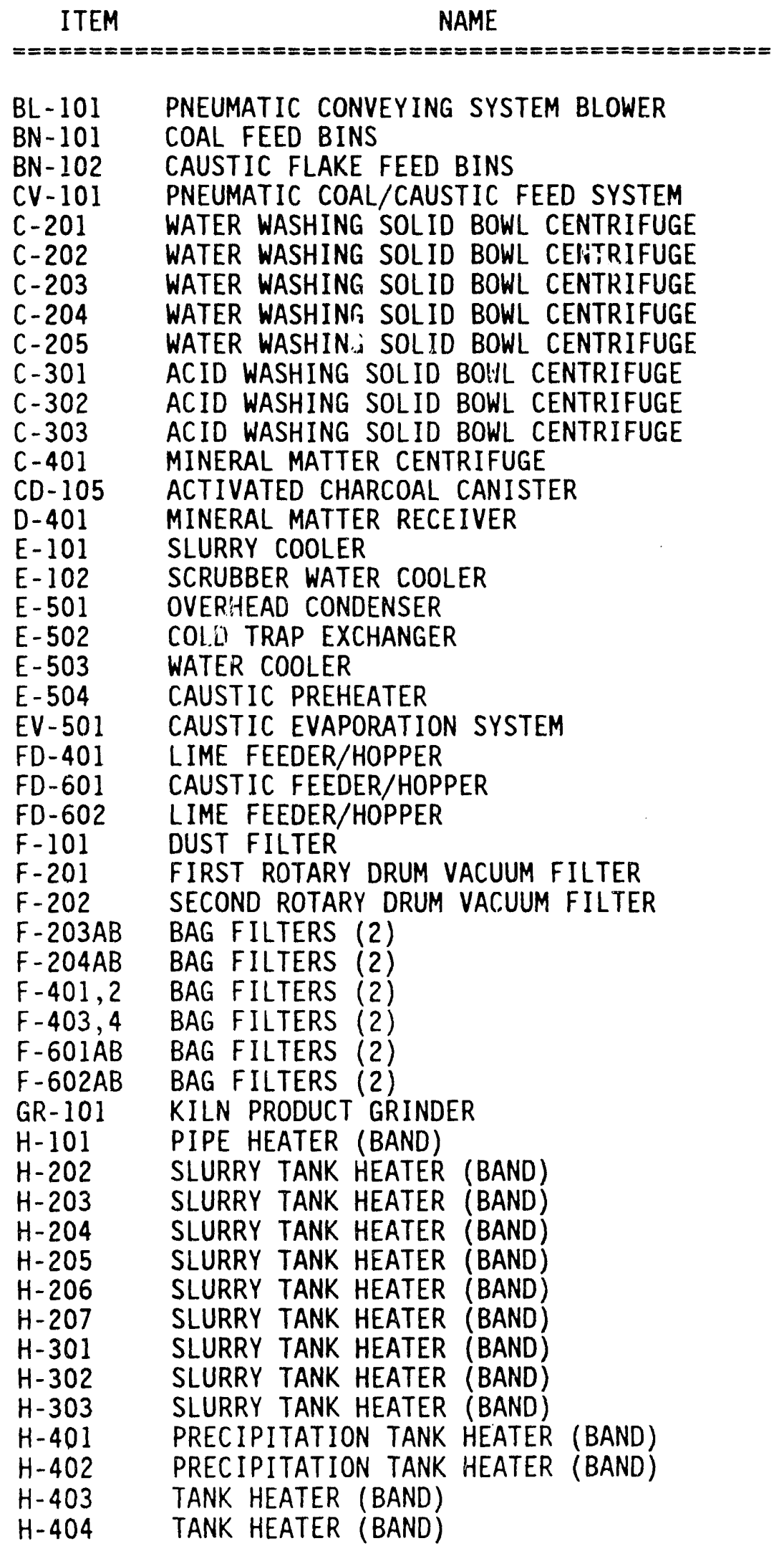


TABLE 2 2 . MAJOR EQUIPMENT (CONTINUED)

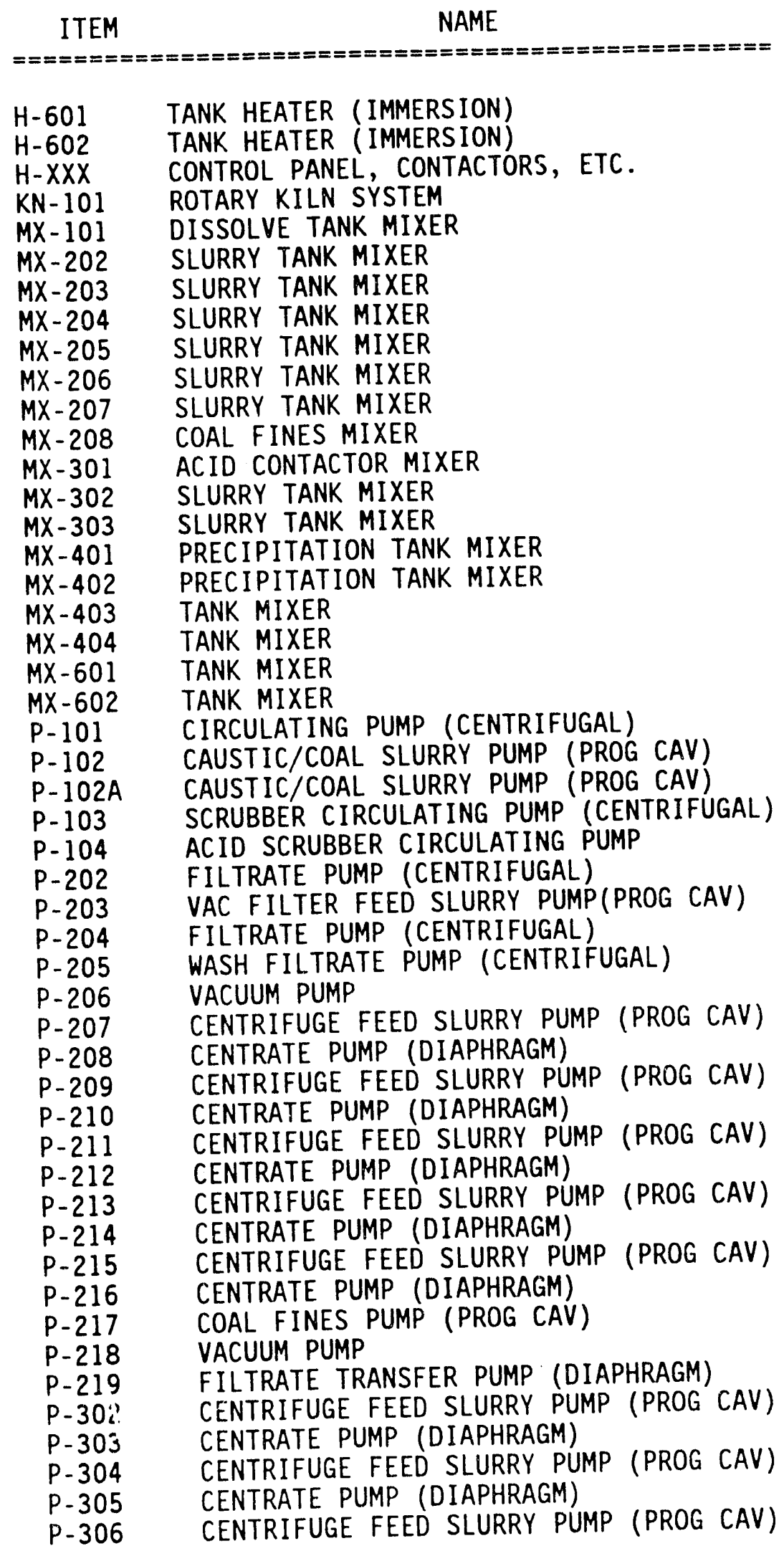


TABLE 2-2. MAJOR EQUIPMENT (CONTINUED)

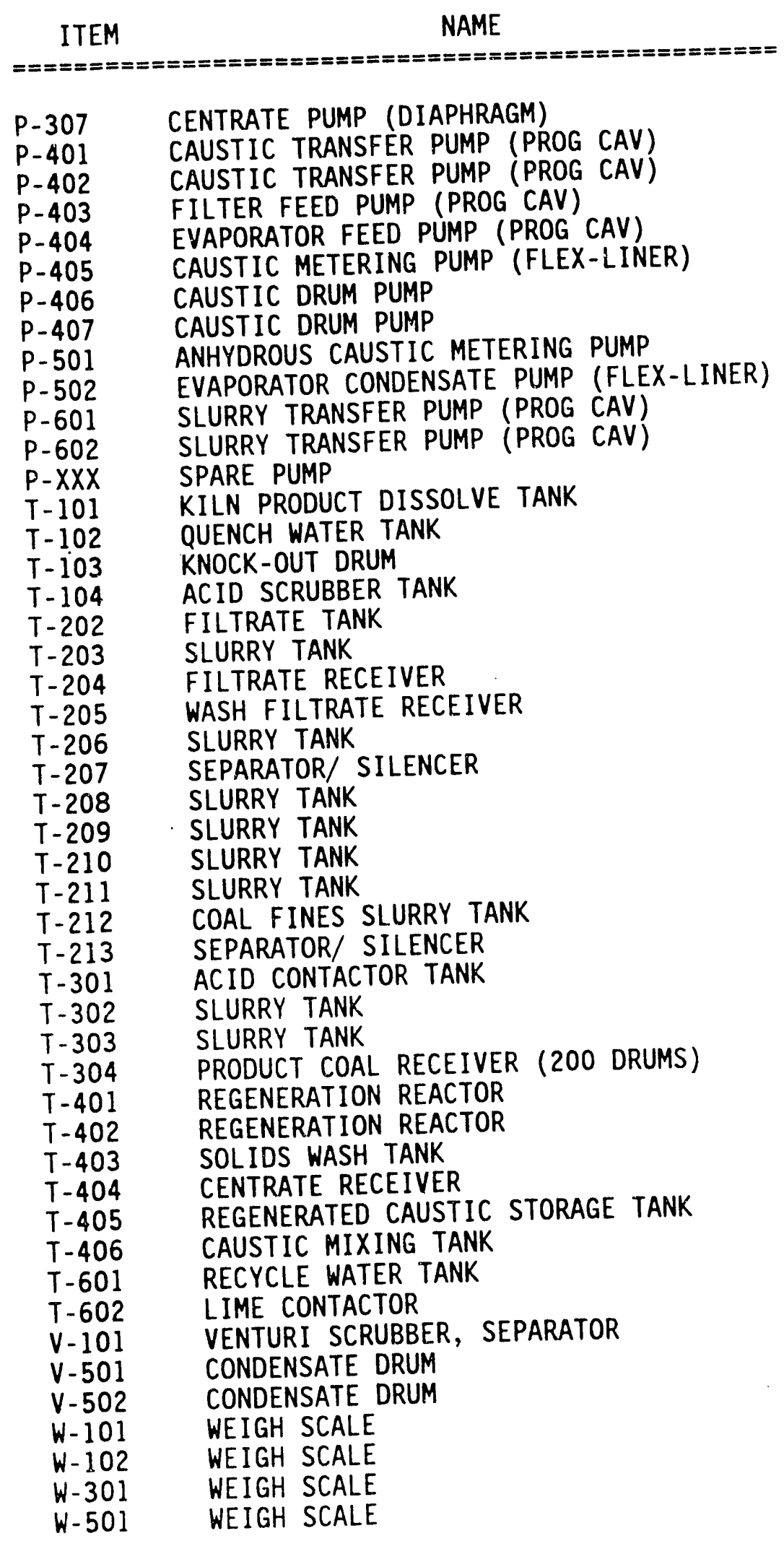




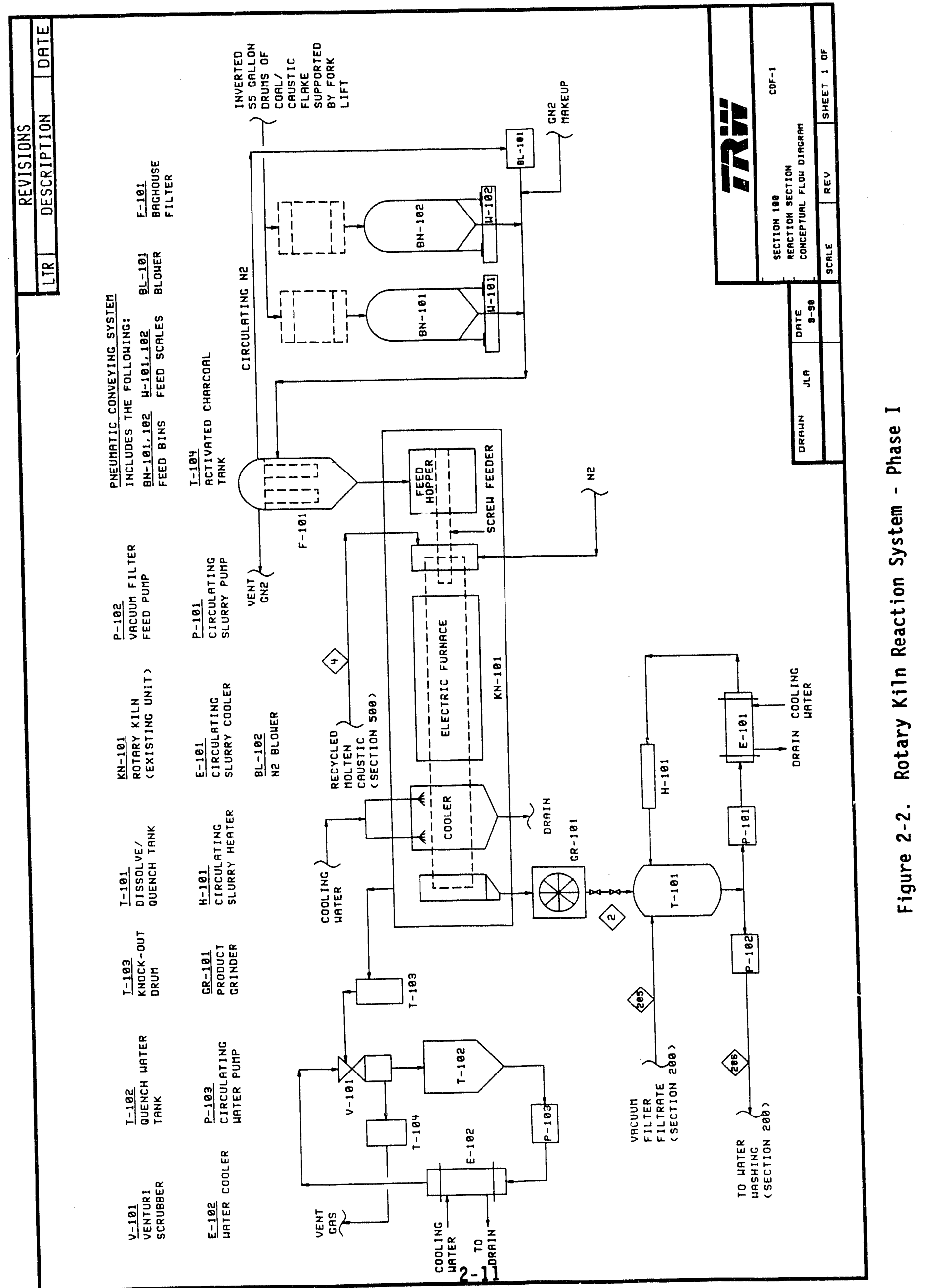




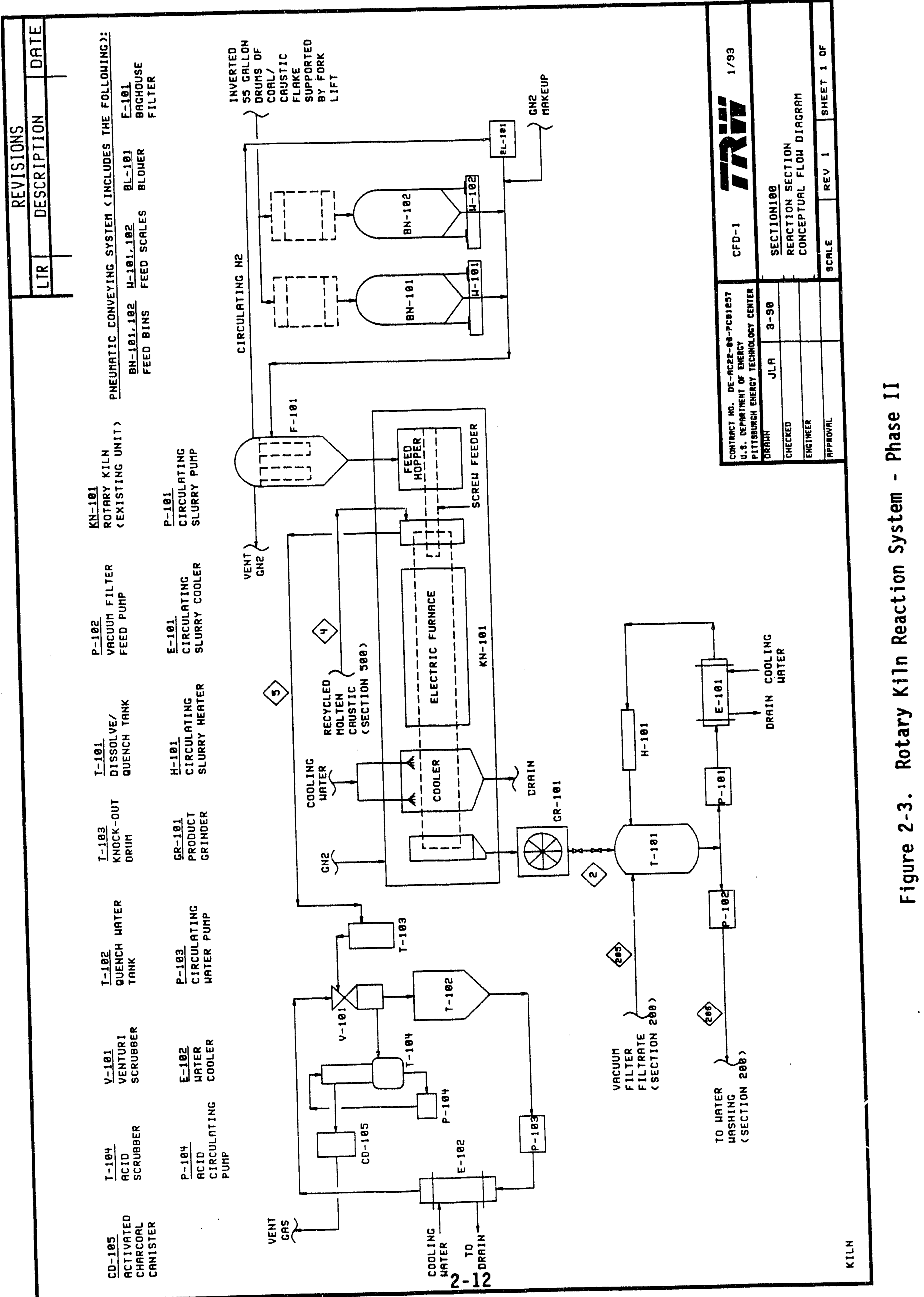


device to promote intimate mixing of caustic and coal. From the drum tumbling device, the coal/caustic feed is transferred to one of two coal/caustic feed bins (BN-101 and BN-102). The $10 \mathrm{ft}^{3}$ capacity feed bins were sized to store up to 12 hours of feed mixture at the nominal flow rate of $20 \mathrm{lbs} / \mathrm{hr}$ coal with 20 to $50 \mathrm{ibs} / \mathrm{hr}$ caustic. The pneumatic feed system for the kiln is depicted in Figure 2-4. Shown in the middle of this photo are the two feed bins $B N-101$ and $B N-102$. When fifty-five gallon drums containing a mixture of coal and caustic are attached to the top of these bins, the feed materials flow by gravity (assisted by compressed air vibration of the bins) into these bins. Shown at the bottom of this photo is the pneumatic nitrogen line. The feed mixture is pneumatically conveyed by nitrogen from the feed bins to a bag house filter, F-101. The dust free nitrogen from the filter is recirculated by blower, BL-101. The bag house filter is shown in Figure 2-5. The filter is located on a mezzanine above the feed end of the kiln. The kiln feed hopper can be seen in the lower left of this photo. The solids are periodically shaken off the filter bags and discharged into the coal/caustic feed hopper/screw feeder of the kiln. The screw feeder to the kiln has a variable speed driver which allows for varying the feed rate from $30 \mathrm{lbs} / \mathrm{hr}$ to $120 \mathrm{lbs} / \mathrm{hr}$.

The rotary kiln, supplied by C.E. Raymond, consists of a feed section, a 12-foot long furnace section, a 3-foot long cooling zone and a discharge area. The overall length of the kiln is about 32 feet. The kiln has an emergency hand crank for rotation of the kiln cylinder in the event of a power interruption or motor failure to prevent warpage of the kiln tube. The feed end of the rotary kiln is shown in Figure 2-6. The bag house filter can be seen in the upper right of this photo.

The kiln was modified for this program to have the capability of operating with either an all solid feed stream consisting of coal and caustic flake or with separate feed streams consisting of coal and recycled molten caustic. The coal or coal/caustic flake pneumatic solids feed system is described above. The heat traced molten caustic recycle line (described in Section 2.5) was not used during this program. 


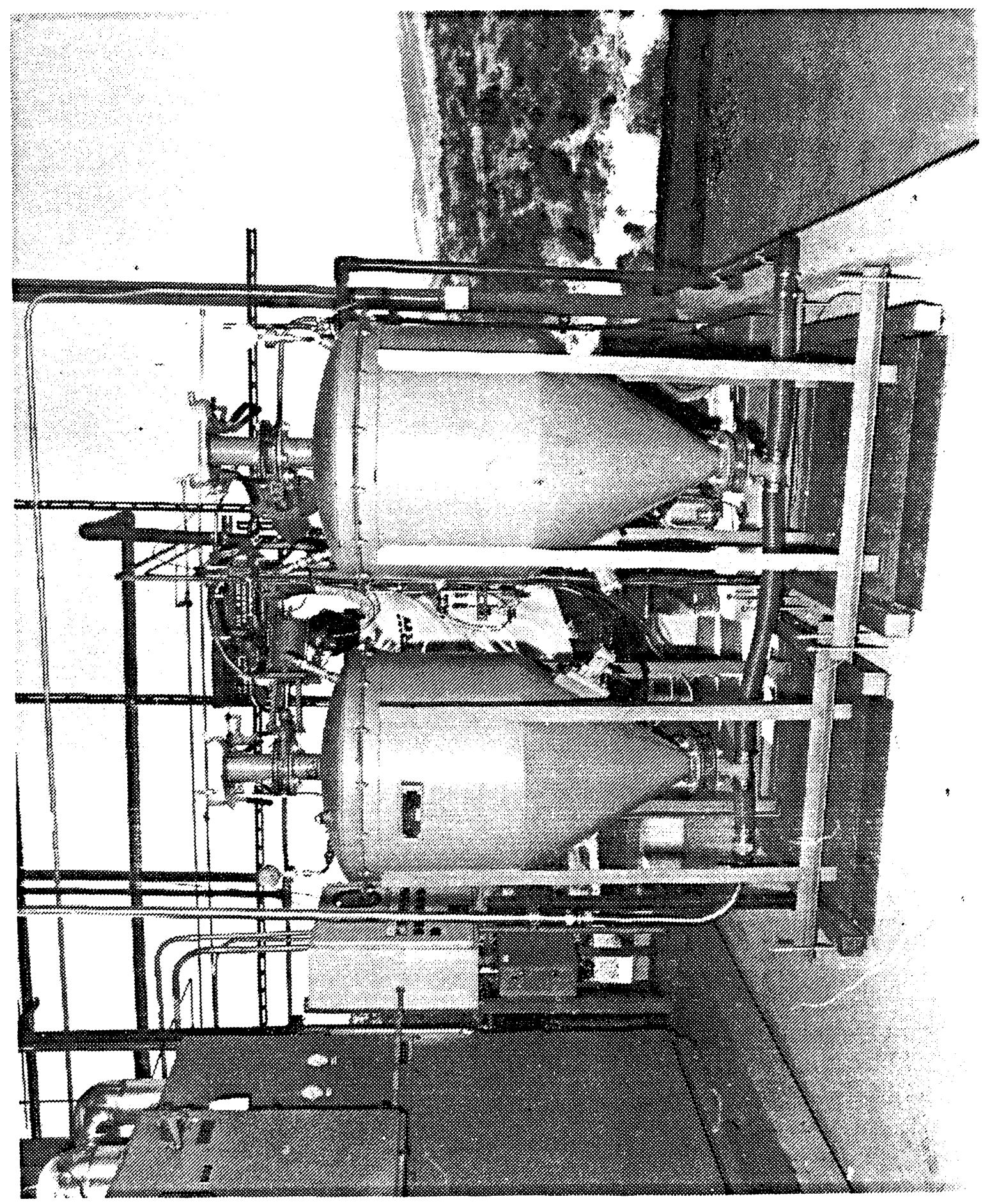

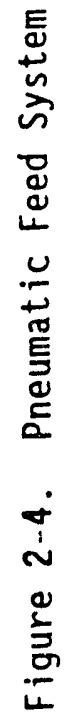




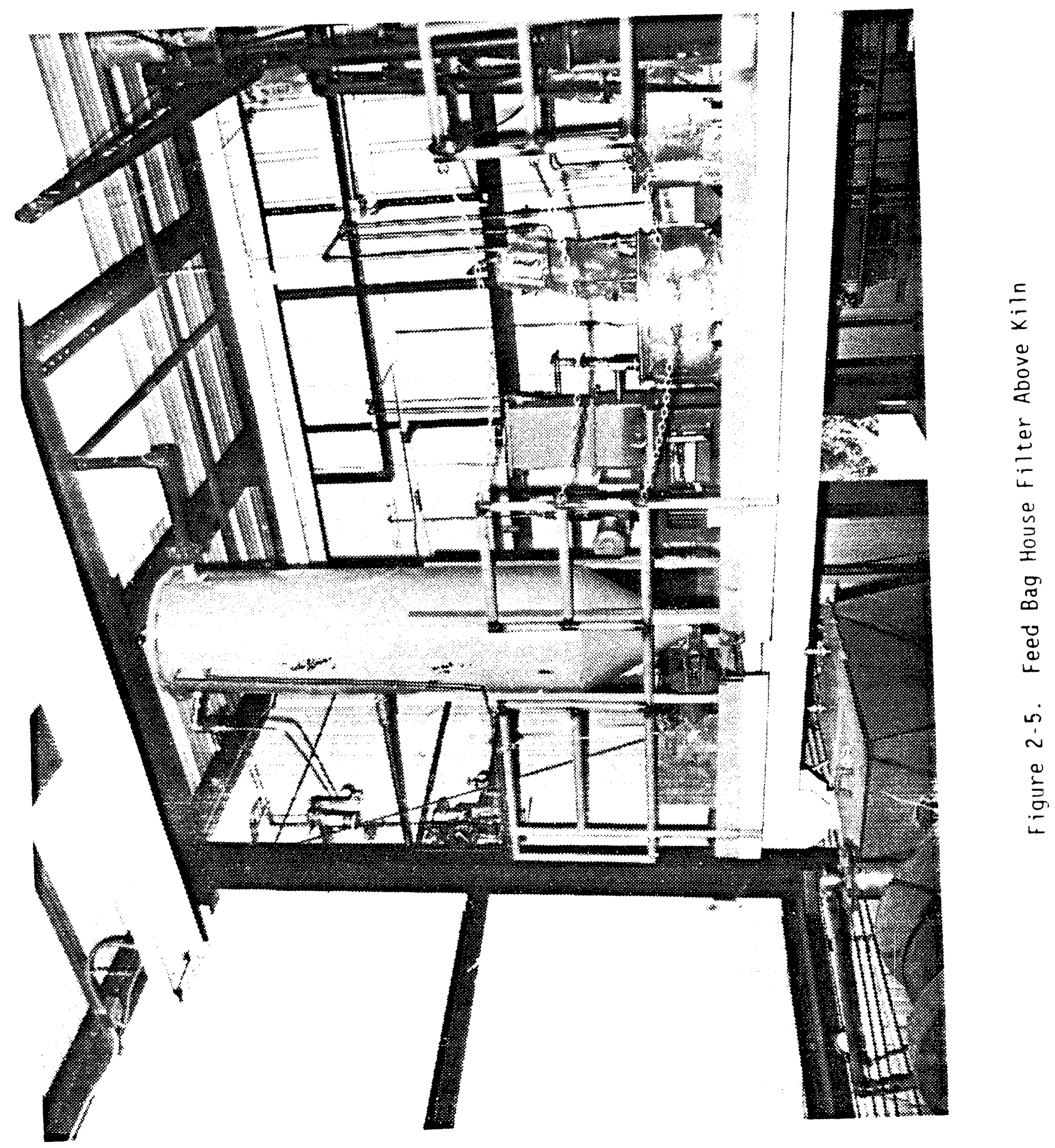




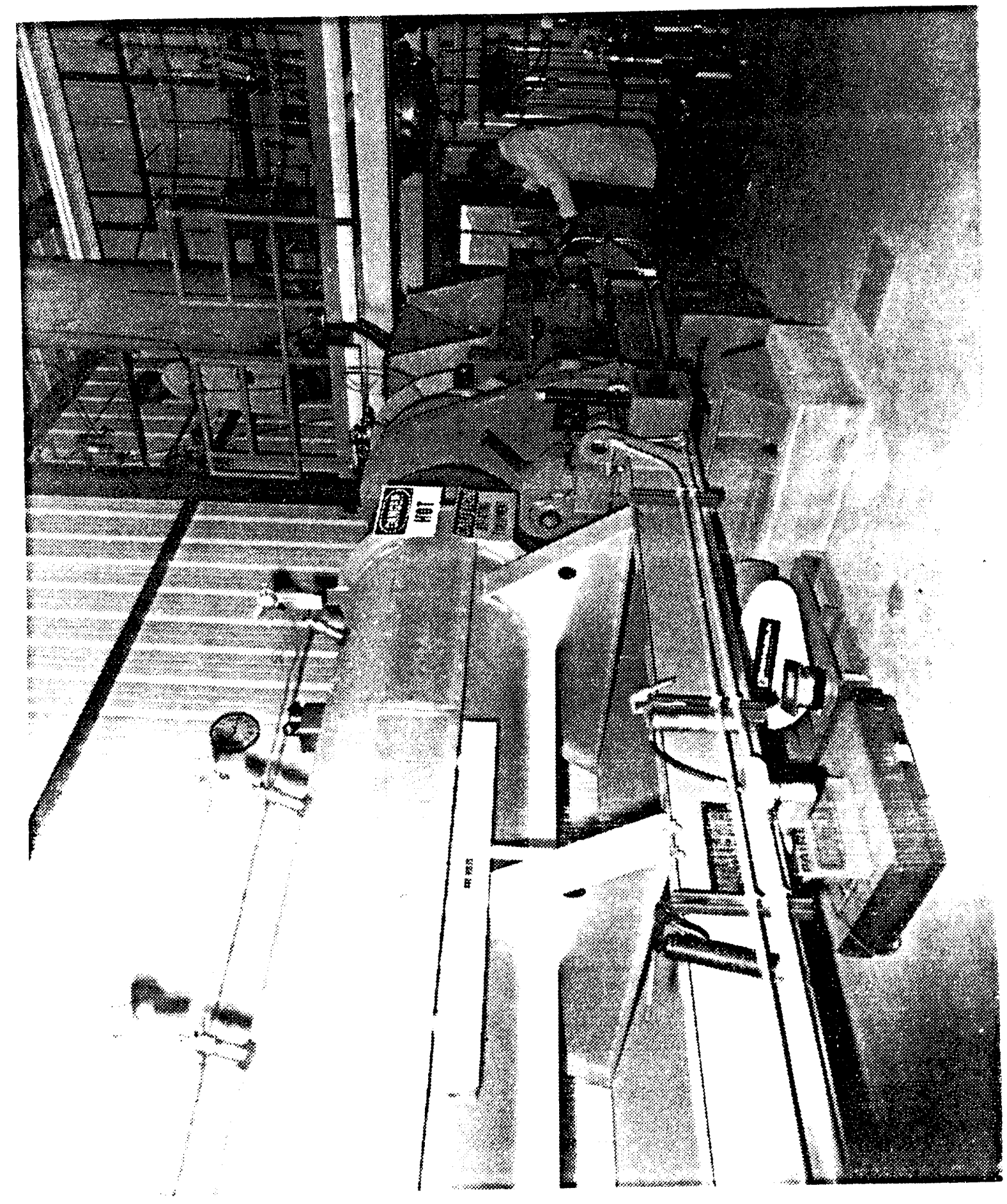


The main part of the kiln contains a 14 -inch diameter tube which is 26 feet long. The tube is constructed of Inconel 601 which had high corrosion resistance to caustic at elevated temperatures. Part of the tube is enclosed in a 12-foot long furnace section which is electrically heated in four separate zones. The wall temperature of each zone is measured and controlled separately. The kiln was normally operated with internal temperatures in the range of $325^{\circ}$ to $415^{\circ} \mathrm{C}$ (the skin temperatures were higher). The twelve-foot heated reaction section surrounded by a four-zone electric furnace can be seen in the foreground of Figure 2-6.

The solids are transported from the feeding zone into the furnace section and then into the cooling zone. The 3-foot cooling zone consists of the Inconel tube enclosed in a cooling jacket where cooling water is sprayed directly on the hot kiln tube. The jacket has the capability of cooling the coal-caustic mixture to $100^{\circ} \mathrm{F}$.

The kiln was equipped for this program with a rotating scraper to keep the internal walls of the kiln tube free of sticky solid deposits. The wall scrapper is an auger-like device, six inches in diameter, that runs the length of the kiln tube and is in contact with the tube wall 15 degrees offset from the kiln bottom. It has a variable rotary speed driver (up to 20 RPM) and is rotated in an automatically timed sequence in alternate clockwise and counterclockwise directions. The use of a variable kiln slope, kiln rotational speeds, and the rotation settings of the wall scraper provide for different volume loadings and retention times in the $\mathrm{kiln}$.

The exit end of the kiln is shown in Figure 2-7. The operator in the figure is looking into a window where he can see solids dropping out of the end of the kiln tube. Following the cooling zone, the solids exit the kiln through a bellows seal breaching arrangement. The bellows seal is capable of maintaining pressures up to \pm 2 inches W.G. in the kiln tube without leakage. The reacted solids leaving the kiln are broken up by the newly installed rotating breaker bar which is located in the conical discharge chute exiting the kiln (see figure 2-7). The breaker bar was installed during Phase II to prevent the large agglomerates seen in Phase I from hanging up in the chute and blocking the flow of solids from the kiln. The reacted solids from the 


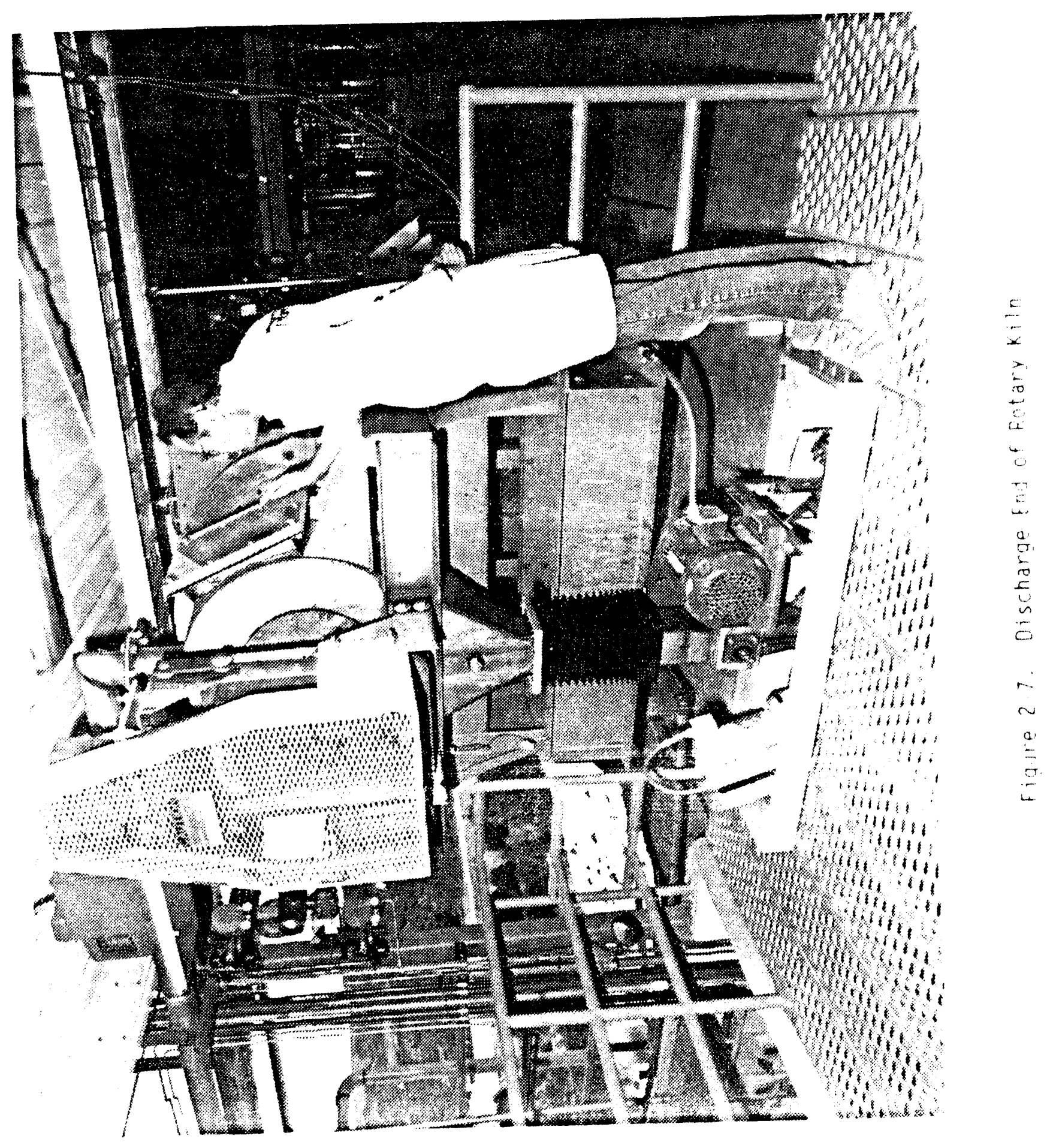


kiln then drop through a flexible connection into a crusher/delumper for further size reduction (to $<1 / 8$-inch). The crusher/delumper (with its motor) can be seen just above the floor grating in Figure 2-7. A constant, small particle size ensures a constant and fast rate of caustic dissolution in the reactor product dissolver/quench tank.

From the crusher/delumper, the solids flow through a series of two slide gate valves which provide an airlock and which are operated sequentially by a timer, such that one of the valves is always closed. This "lock hoppering" protects the kiln from any in-leakage of steam from the downstream quench subsystem. The slide valves are hidden from view in Figure 2-7 by the floor grating.

The kiln control panei can be seen in Figure 2-8. The unit includes a Honeywe11 10-point strip chart recorder (center) and a Barber-Coleman 560 series digital indicating-proportioning three-mode controller with deviation alarm and bar graph. The power supply to electric motors, the furnace and instrument pinel can be seen at the left.

Nitrogen is fed to the kiln to maintain a slightly positive pressure on the system to prevent in-leakage of air and to sweep away the small amount of hydrogen and methane gases and phenolic-type vapors produced as a byproduct. The gas exhaust is swept through a caustic venturi scrubber, a newly installed acid scrubber, and an activated carbon bed prior to venting (Figure 2-3).

The 40 to 70 pounds per hour of solids exiting the second gate valve drop into a stirred 12-gallon Inconel tank, T-101, where the caustic (as well as sulfur compounds and ash leached from the coal) dissolves in an aqueous caustic filtrate solution that flows into this tank from the second vacuum filter in the coal washing system (stream 205). The dissolver/quench tank, which is located in a trench at the outlet of the kiln, is shown in the bottom part of Figure 2-9. The trench was dug especially for this program in order that the solids discharged from the kiln can fall by gravity into the quench tank. The outlet of the kiln can be seen in the upper part of the photo behind the guard railing. The crusher/delumper and slide valves are hidden from view behind the platform on which the man is standing. 


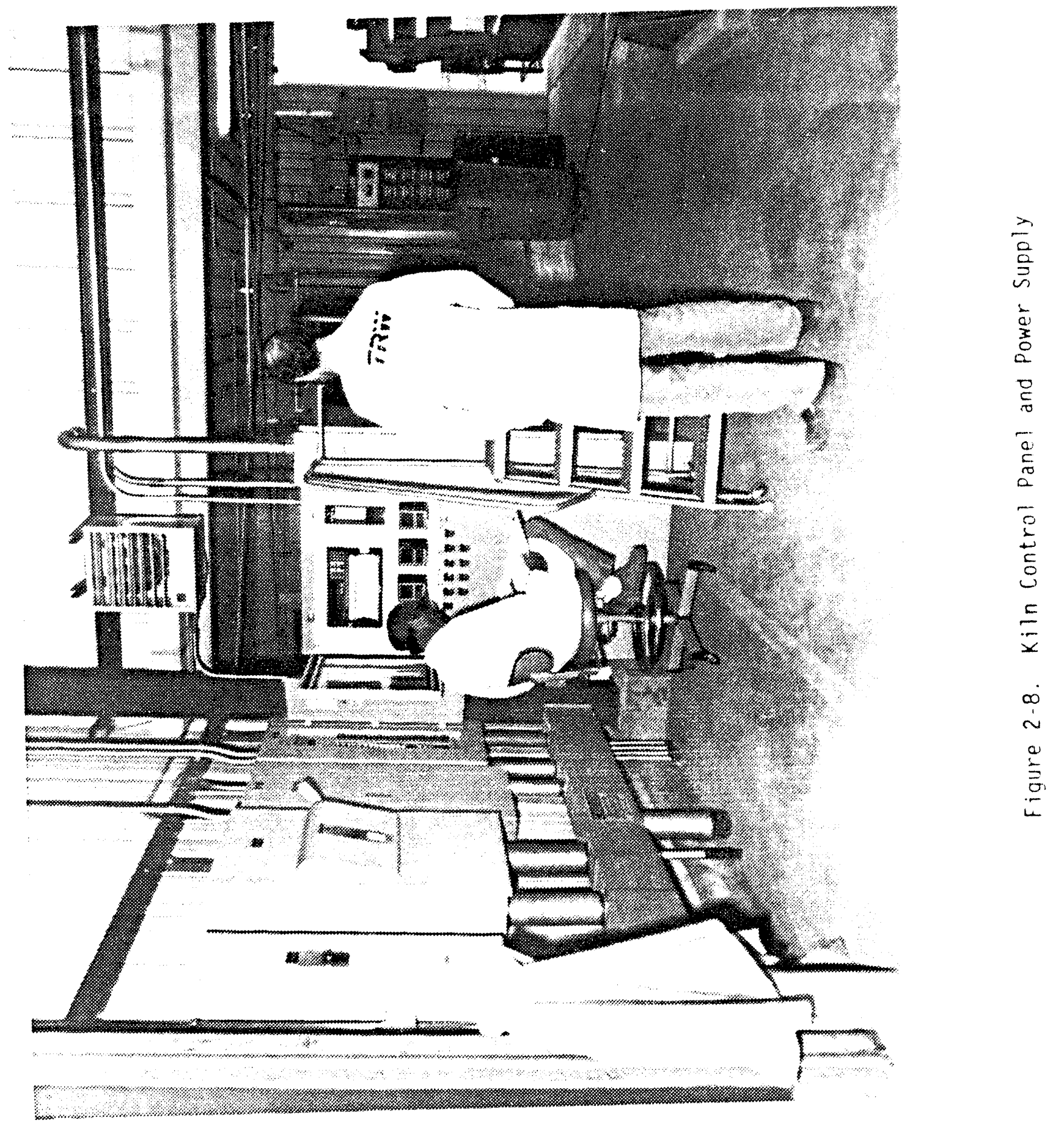




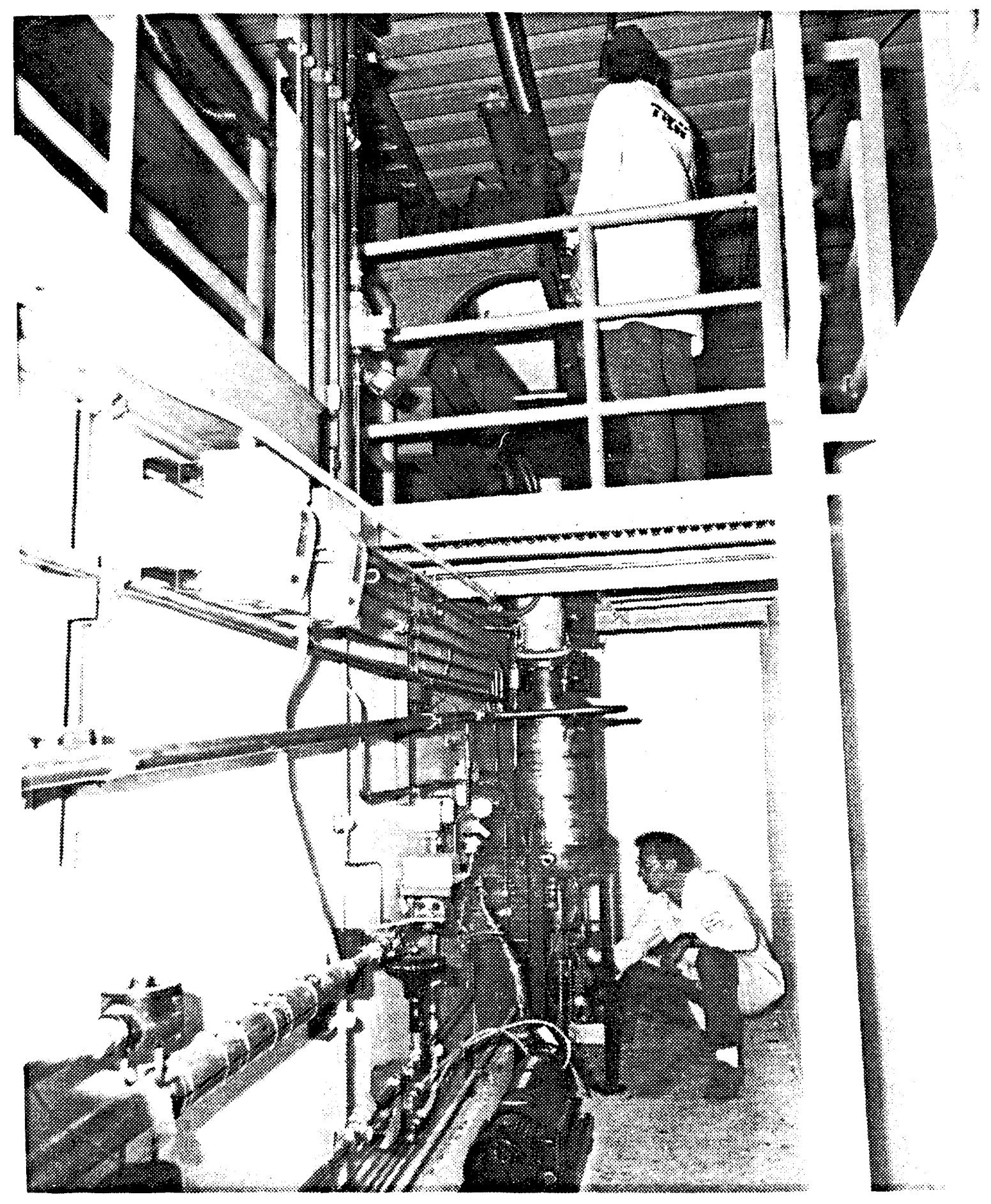

Figure 2-9. Dissolver/Quench Tank in Trench Below Kiln 
T-101 was sized for a nominal residence time of 30 minutes. However, the residence time can be varied from 15 to 45 minutes ( $1 / 4$ to $3 / 4$ ful1). Due to the propensity of the solution to precipitate dissolved mineral matter and sulfur compounds on standing for extended periods of time, the residence time in T-101 was kept at a minimum.

The temperature of the resulting slurry was controlled at the desired value $\left(180^{\circ} \mathrm{F}\right.$ maximum) by adding or removing heat using an electric slurry heater, H-101, or a water cooled slurry cooler, E-101, located in a pump-around loop external to dissolver/quench tank T-101 (Figure 2-3). The caustic solution produced in T-101 (stream 206) is pumped to the first vacuum filter in the water washing system by progressive cavity pump, P-102 (Inconel wetted parts). Excess filter feed in returned to $T-101$ via a spillback 1 ine.

\subsection{WATER WASHING SYSTEM (SECTION 200)}

In the water washing (or coal-caustic separation) system, the coal/caustic slurry from the reactor product dissolver/quench tank is water washed to remove the caustic, sulfur forms, and dissolved silica and alumina mineral matter. A block flow diagram of the multistage countercurrent water washing system is depicted in Figure 2-10 and a typical design material balance is given in Table 2-3. The coal/caustic slurry is sequentially washed and dewatered in two vacuum filters, F-201 and F-202, five centrifuges, C-201 through C-205, and six interstage slurry tanks, T-203, $T-206$, and $T-208$ through $T-211$, in a countercurrent manner producing a washed coal virtually free of dissolved caustic (stream 6) and a concentrated aqueous caustic filtrate stream requiring regeneration (stream 8 ). As the wash water streams move in a countercurrent direction with respect to the solids flow, they become more concentrated in caustic and salts while the residual liquid on the solids becomes more dilute in caustic and salts.

A11 new equipment was installed in this section during Phase I. TRW provided a 1400 square foot covered concrete process area adjacent to the kiln to house the water washing and acid washing system. This area, during groundbreaking, is depicted in Figure 2-11. The building housing the kiln is shown in the left rear of this photo. The same area is depicted in Figure 


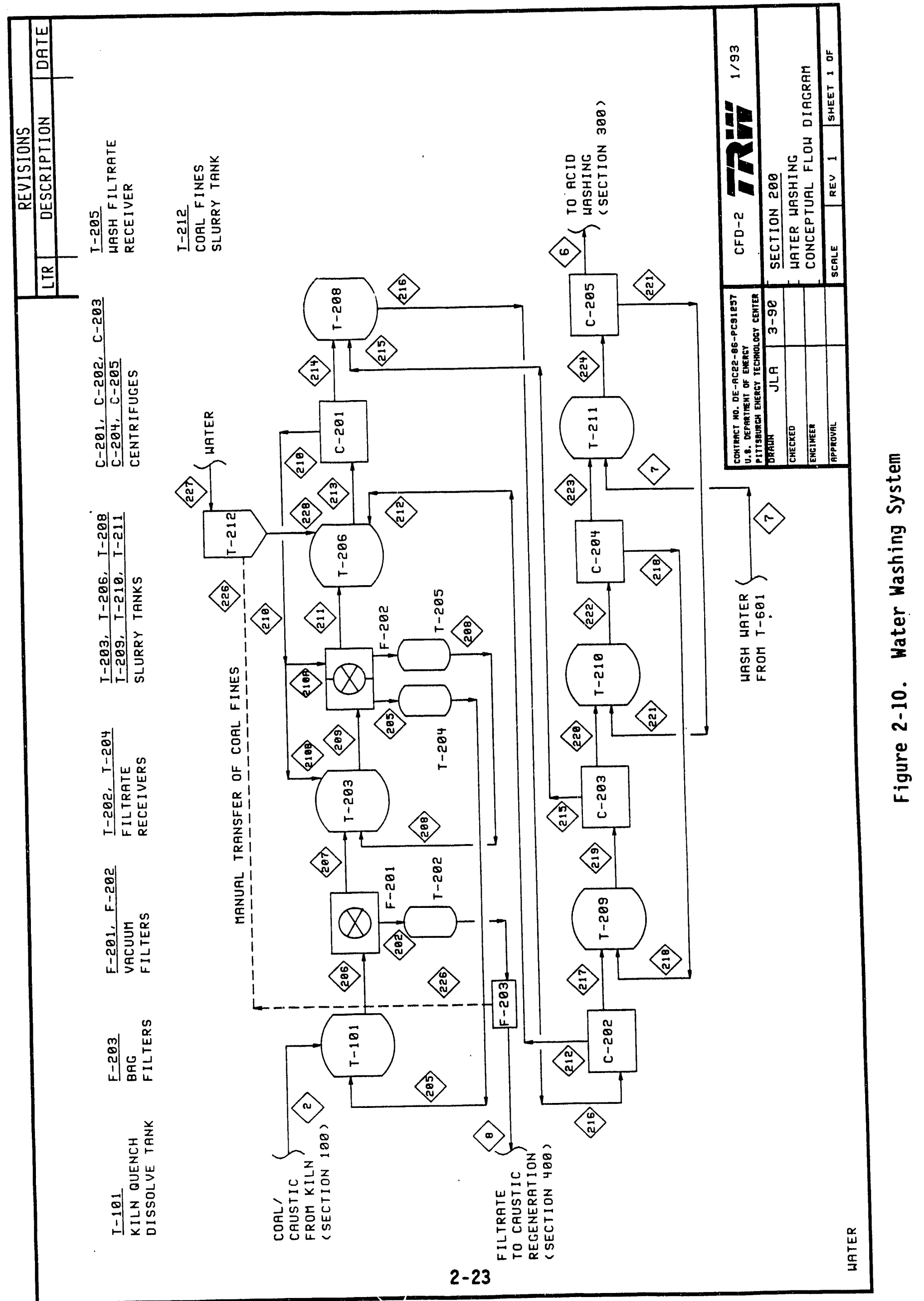




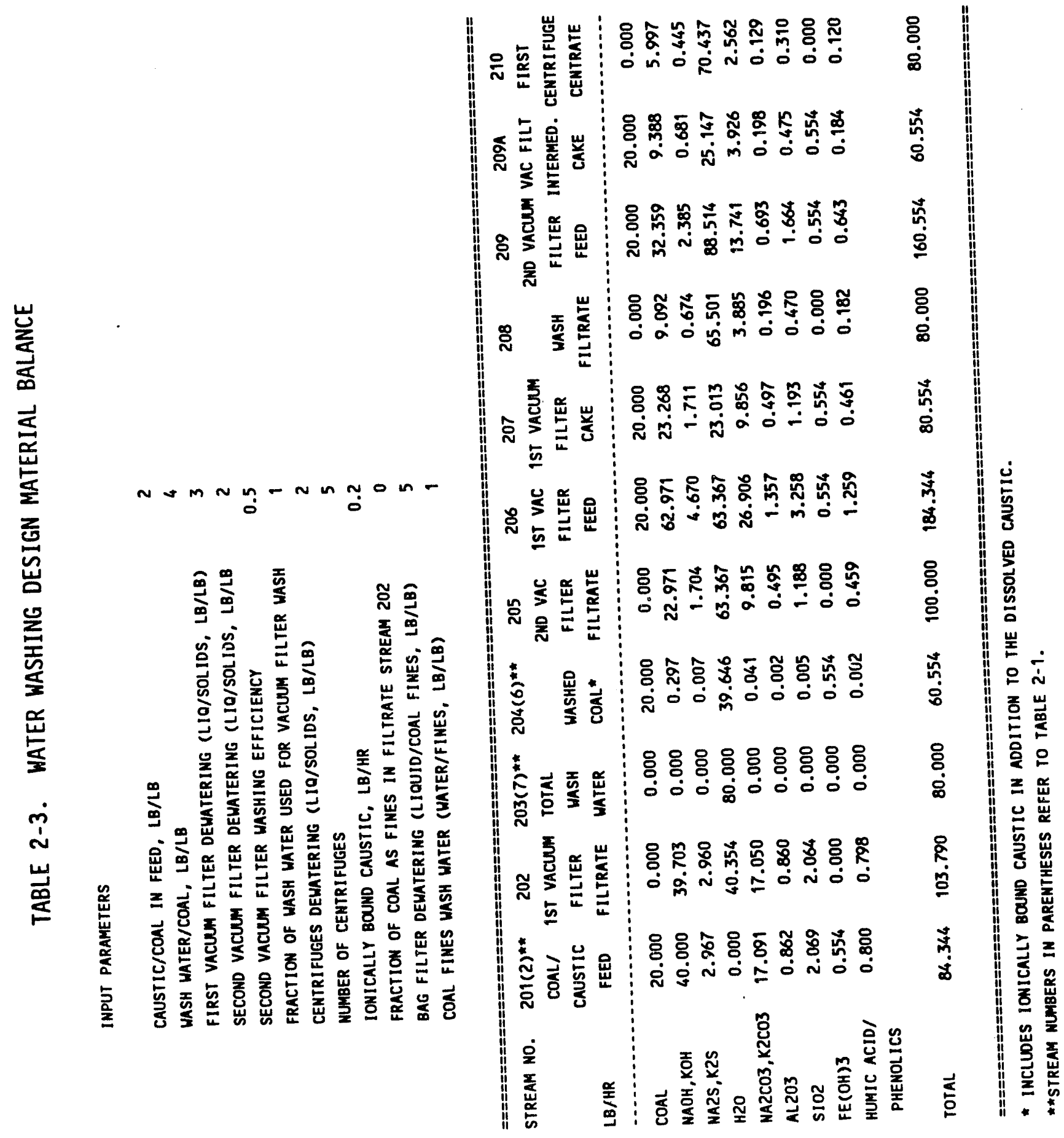




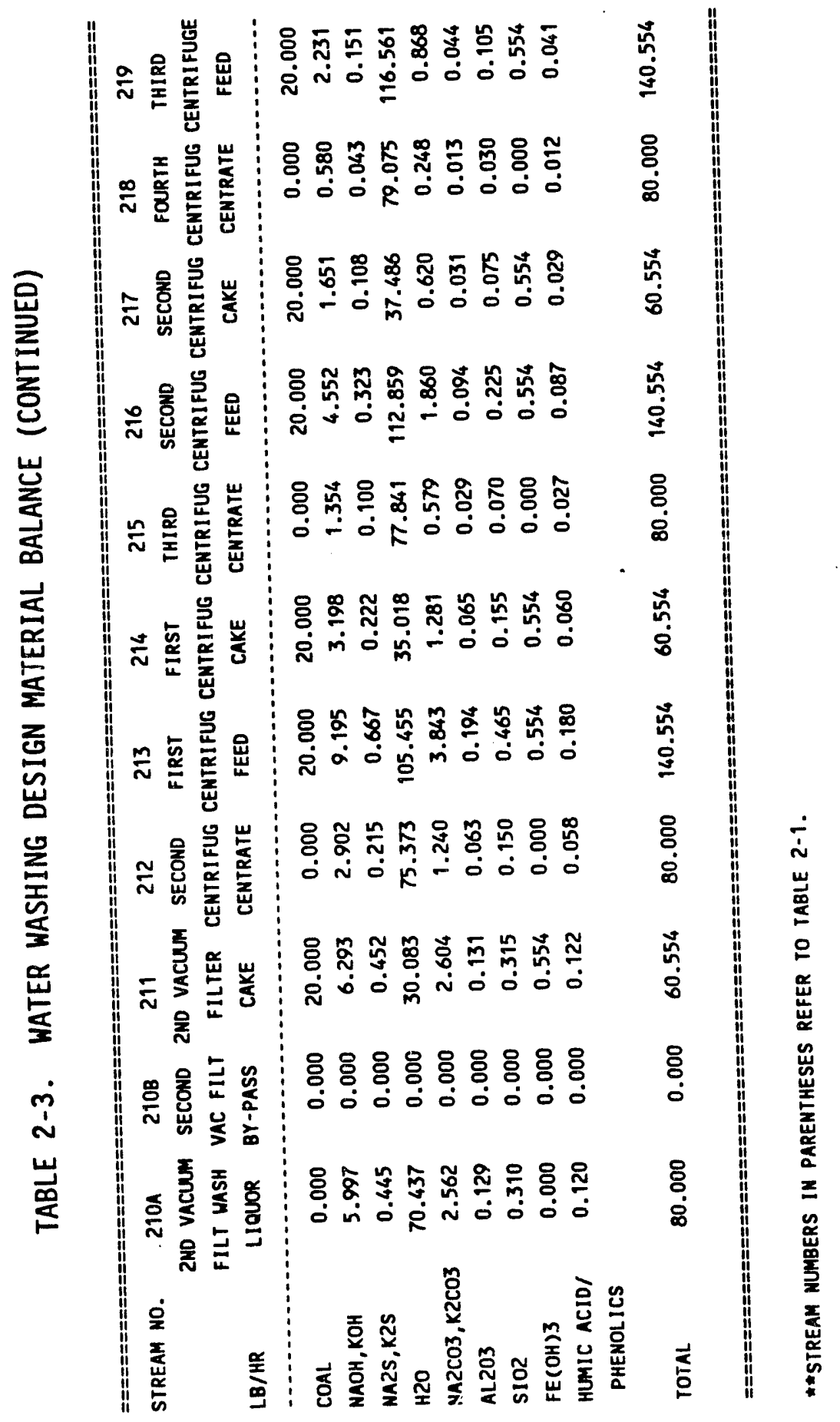




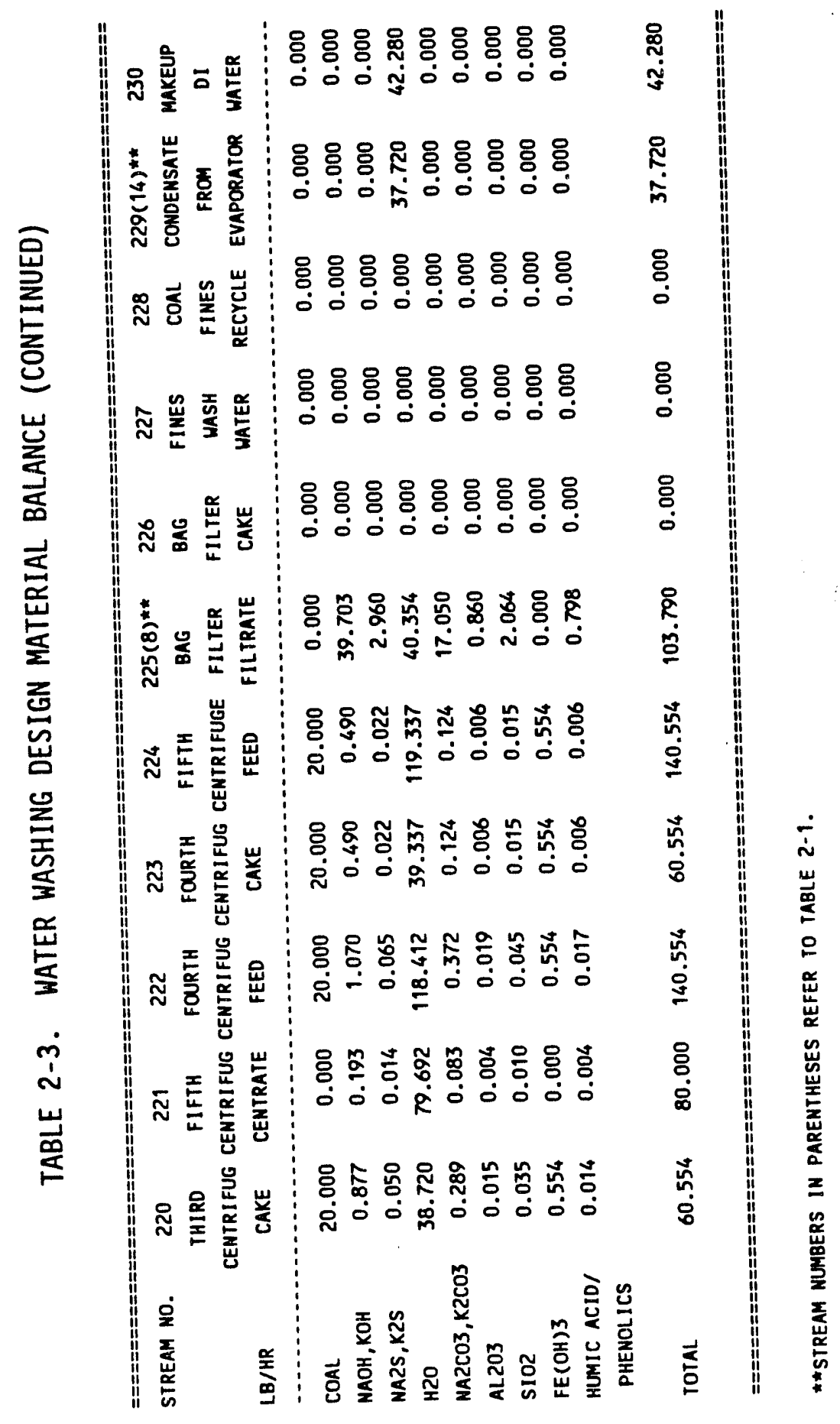




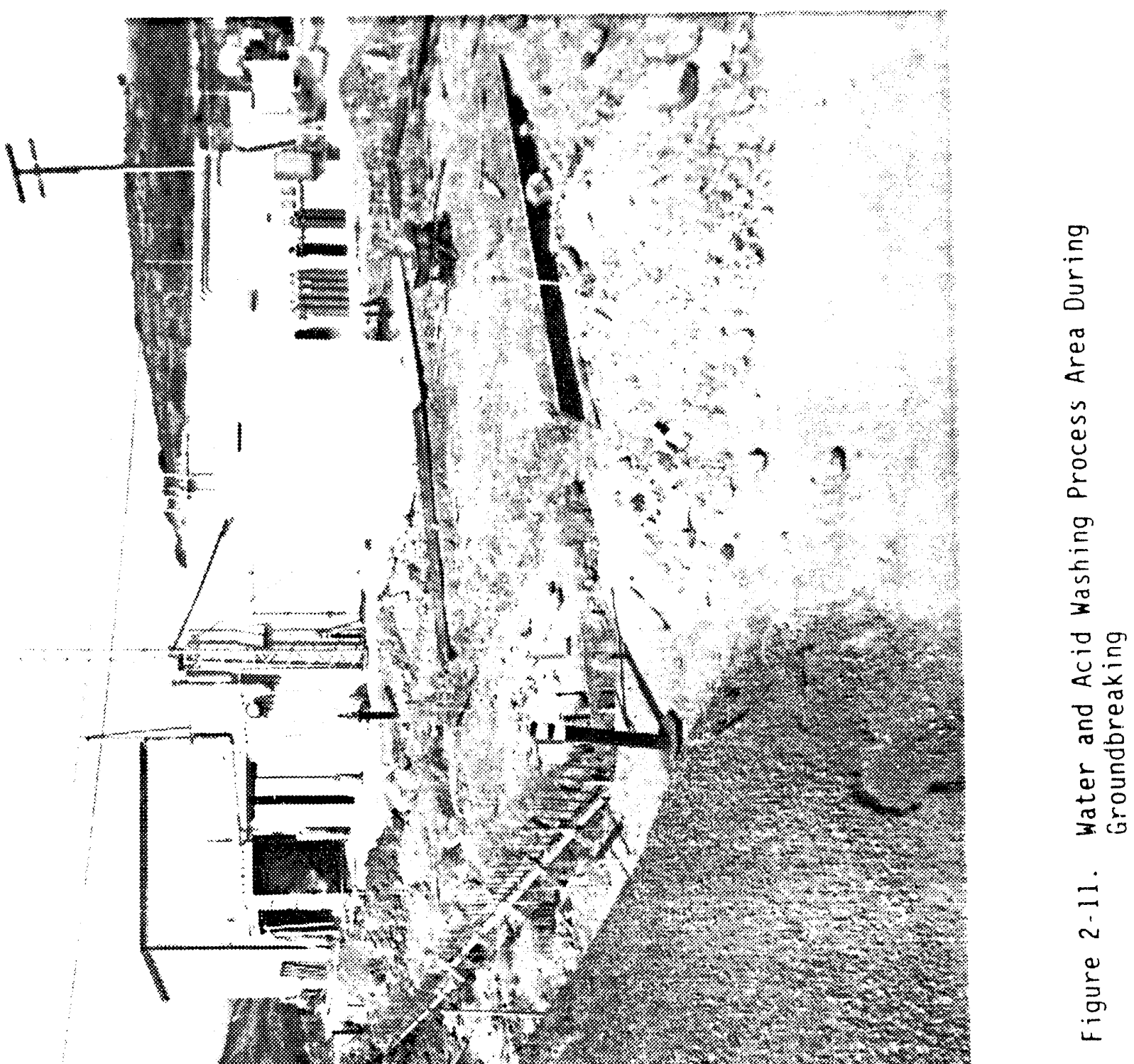


2-12 after the installation of the equipment. The water washing train of five centrifuges is shown on the left side of this photo and the acid washing train of three centrifuges is shown on the right side of the photo. One of the two vacuum filters can be seen in Figure 2-13. The filter is elevated above its 12-gallon reslurry tank which is shown on the right. To the left are the two filtrate receiving tanks. A centrifuge is depicted in Figure 2-14 with its 12-gallon reslurry tank with mixer to the right and a diaphragm pump and a progressive cavity pump on the ground underneath.

The system was designed with two vacuum filtration stages prior to the five centrifuge stages because at caustic concentrations of greaier than $20 \%$, the aqueous caustic solution has a greater specific gravity than the coal cake and thus the liquor cannot be separated from the cake using centrifuges. Only after two vacuum filtration stages is the liquor dilute enough for separation from the cake using centrifuges. The temperatures in the two vacuum filters is never higher than $180^{\circ} \mathrm{F}$ to avoid boiling in the filters when a vacuum is drawn. The temperature of the vacuum filters is established by the dissolver/quench tank pump-around loop containing a slurry heater and cooler (Figure 2-3).

There were a number of advantages in using centrifuges wherever possible in place of filters in the integrated $20 \mathrm{lb} / \mathrm{hr} \mathrm{plant}$. First and foremost, modular equipment testing during the previous Gravimeli program had shown that cake moisture levels for dewatering Gravimelt coal are considerably lower for centrifuge operation than for filters. Other advantages are the relative simplicity of operation and control in a multistage system and a lower power requirement for centrifuges due to elimination of the vacuum pump systems.

The design feed to the water washing system from the reactor system contained approximately two parts caustic per part coal (stream 2). The first vacuum filter, $F-201$, is of Inconel construction and is an enclosed system in an inert atmosphere. The design filtrate from this vacuum filter (based on a wash water to coal ratio of about four to one, stream 7) was an approximately $50 \%$ aqueous caustic solution containing most of the dissolved sulfur compounds and silica and alumina mineral matter leached from the coal (stream 202). This filtrate stream is filtered in bag filter $\mathrm{F}-203$ to remove 


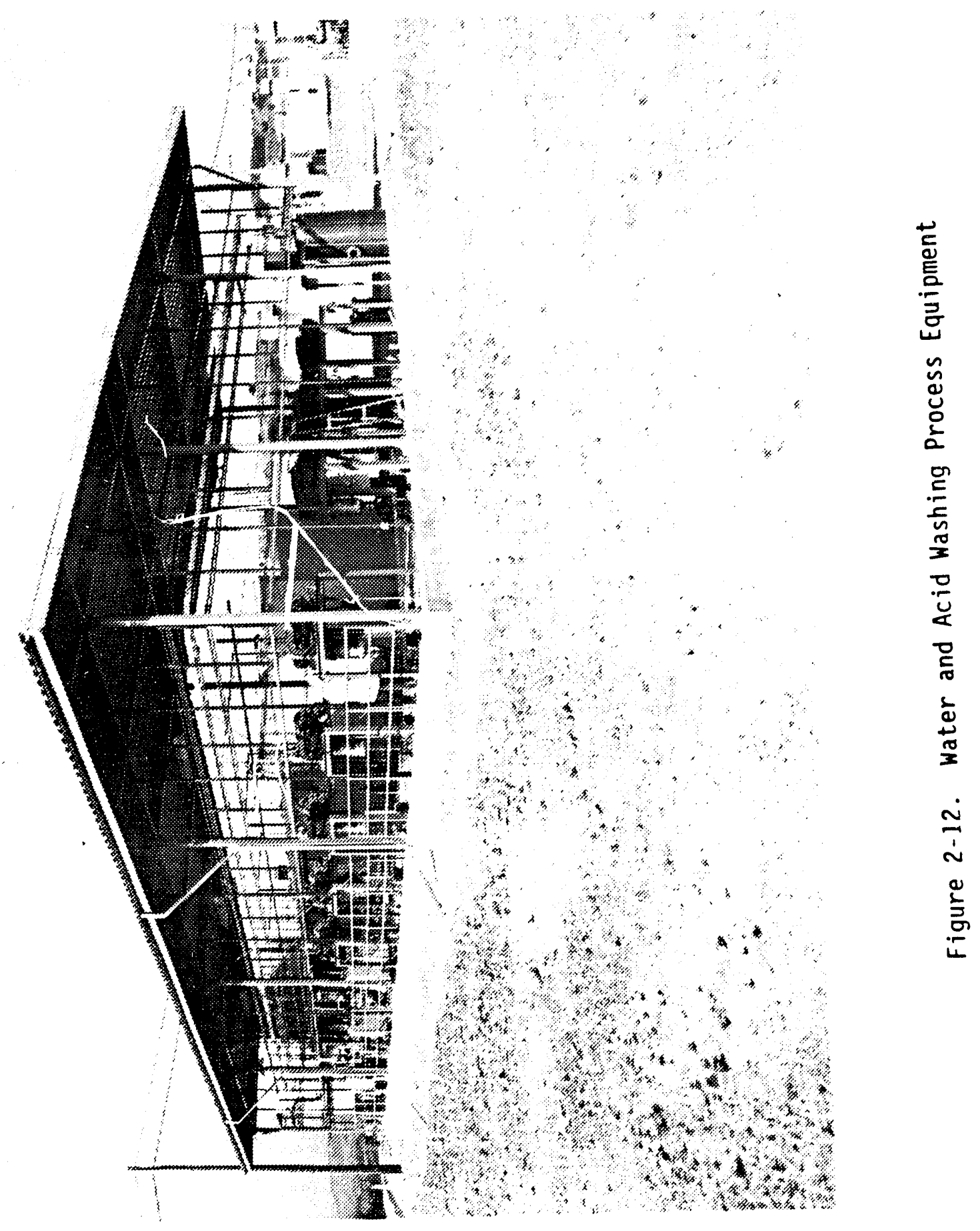




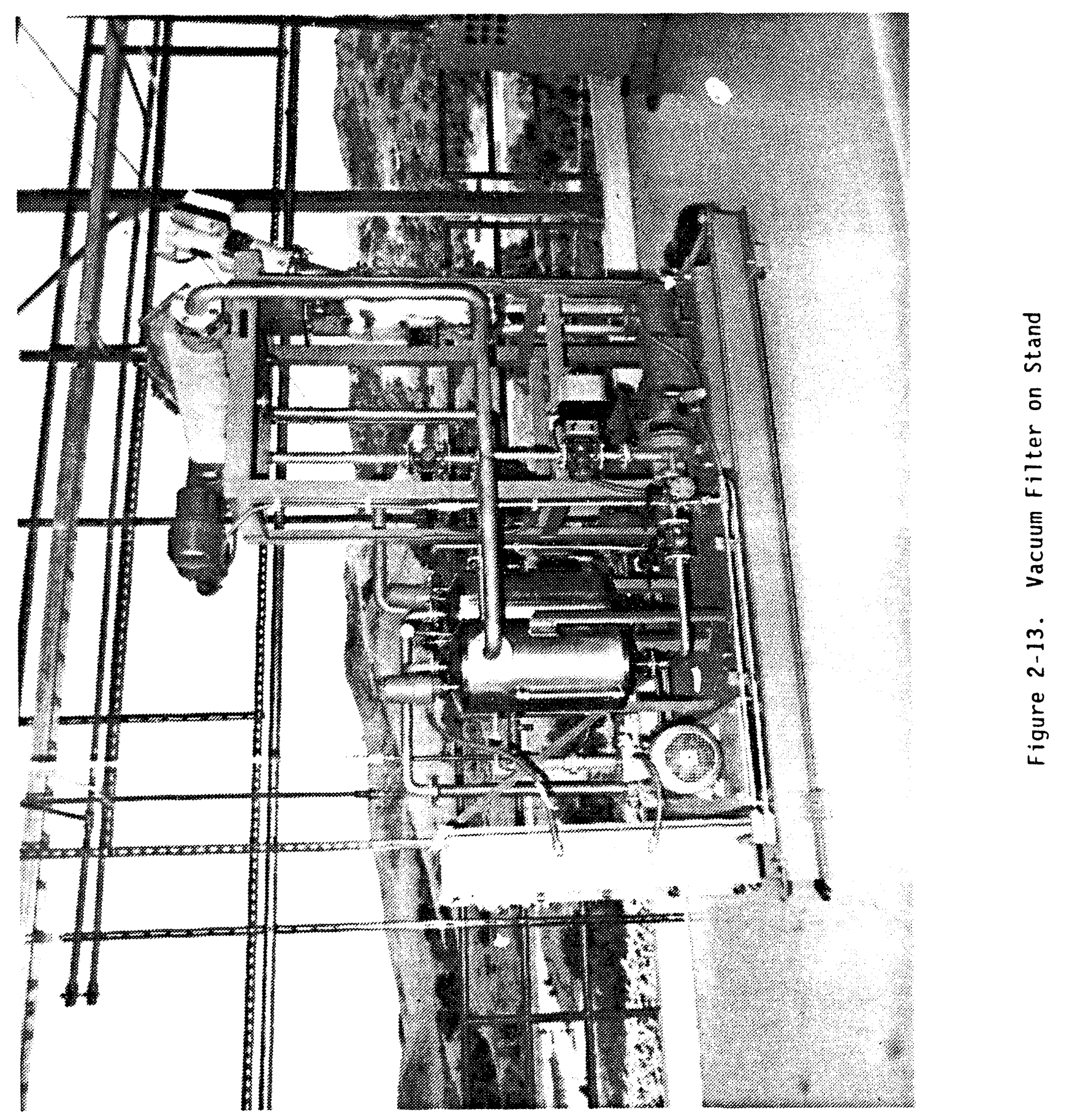




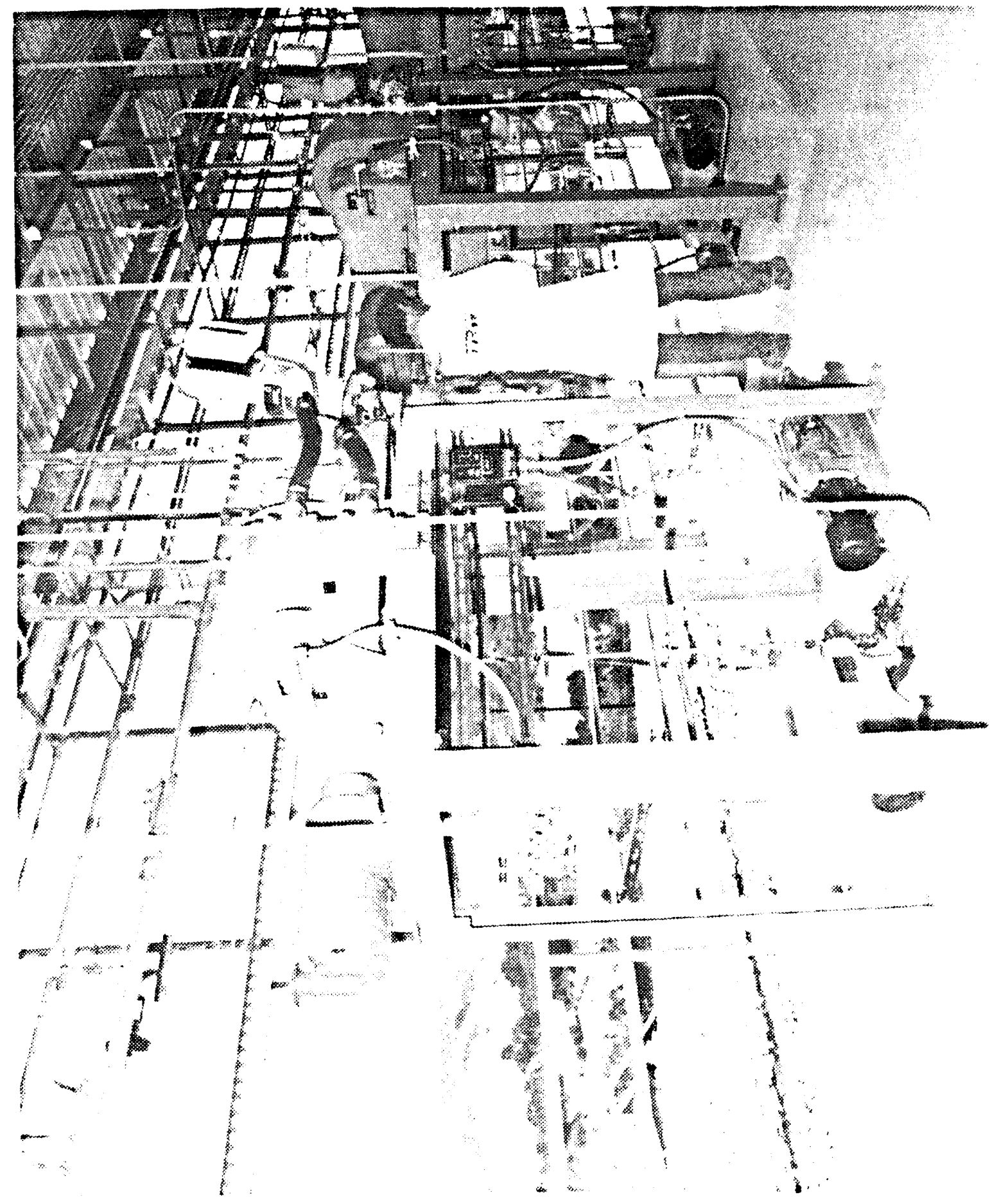


any coal fines before being sent to the caustic regeneration system (stream 8). The coal fines recovered in bag filter F-203 can be manually transferred to tank T-212, reslurried with water, and reintroduced into the water washing system ahead of the first centrifuge (Figure 2-10). This was not done during this program. Iron hydroxide for the most part does not dissolve in the aqueous caustic but remains on the coal cake until acid washing where it is dissolved as iron sulfate upon addition of acid and washed from the coal.

Due to problems with the vacuum filters (intermittent flows to and from the filters, solids buildup in the filter pan, blinding of the cloth on the filter drum), the vacuum filters were bypassed during most of Phase I testing and the caustic stream sent to caustic regeneration was the centrate from the first centrifuge, C-201, (stream 210) which was very dilute (containing only about $15 \%$ caustic) instead of the design $50 \%$ caustic (stream 202) from the first filter. For more information on the vacuum filter problems experienced during Phase I and the subsequent modifications made during Phase II see Section 5.2 of this Final Report.

The vacuum filter system was extensively modified during Phase II of this program. The system was replumbed so that the flow rates to the filters were greatly increased (spillback lines to the dissolver/feed tanks were provided along with filtrate recycle). Line sizes were incrsased and the feed pumps to the filters were replaced with higher capacity pumps. Level switches in the filtrate tanks were replaced with bubbler level indicators. The level indicator readings in the filtrate and dissolver/feed tanks are fed to the computer which automatically adjusts four newly installed control valves such that the levels in the tanks are maintained at preset levels by recycling some filtrate back to the dissolver/feed tanks. Figure 2-15 is a schematic of the replumbed, automated filtration system. A substantial number of hours of on-stream time were logged during Phase II for the first time with the modified vacuum filtration system operating in series with the rest of the integrated MCL facility and with filtrate sent to regeneration containing 20 to $25 \%$ caustic.

The coal solids are dewatered and reslurried in a series of five centrifuges ( $C-201$ through $C-205)$ and interstage slurry tanks to remove the caustic, sulfur forms, and dissolved silica and alumina mineral matter. The 


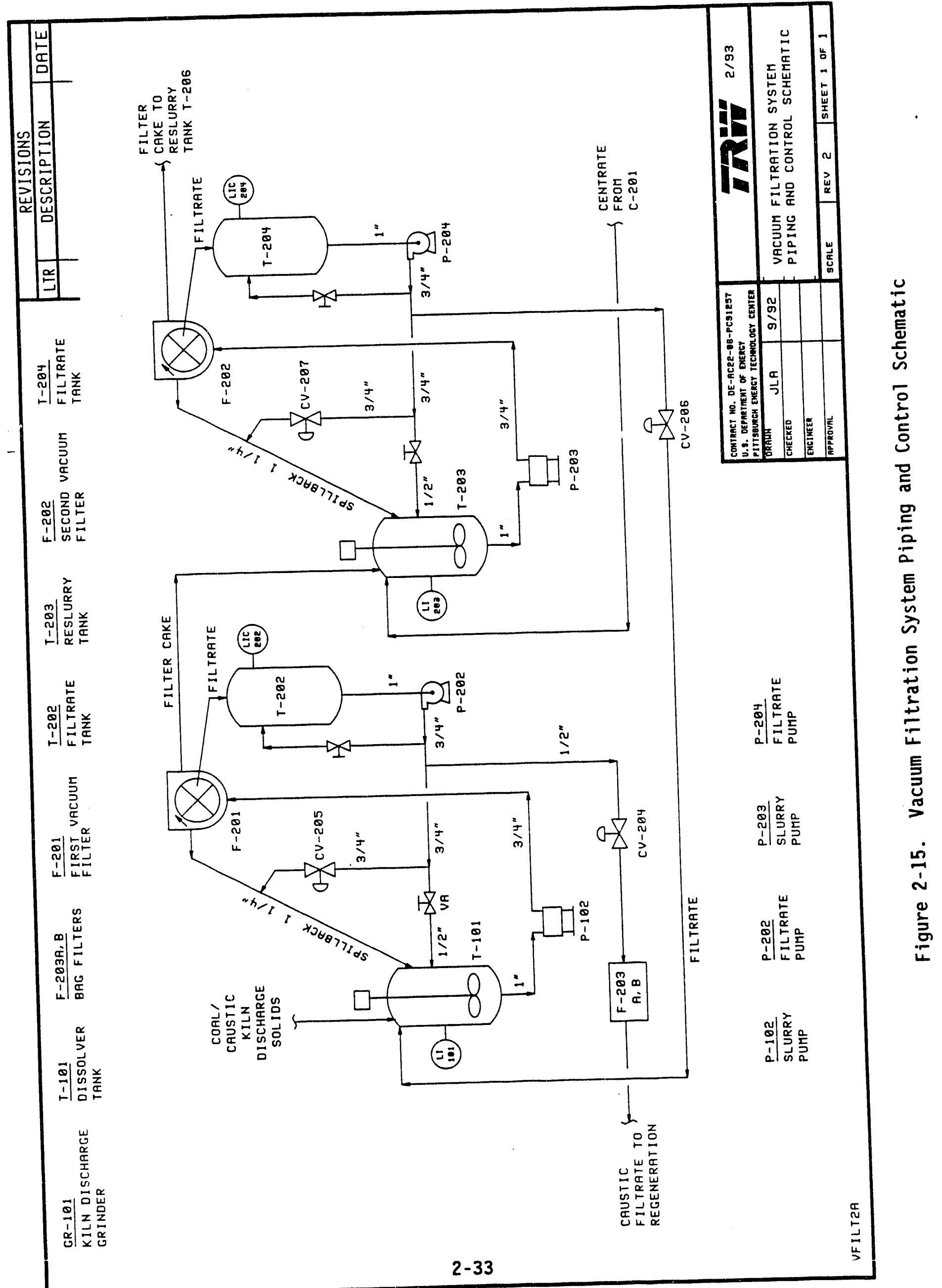


liquid content of the solids from each centrifuge had a design level of about 2 parts liquid per part of coal. The solid bowl centrifuges are six inches in diameter and of 316 stainless steel construction. The slurry tanks for all filters and centrifuges are located next to and slightly below the filters and centrifuges in order that the cake can drop by gravity directiy into the repulping tanks, eliminating the need for manualiy or mechanically transferring the cakes to the tanks.

Progressive cavity pumps were used throughout the water wash system to provide a steady feed stream to the filters and centrifuges. Diaphragm pumps were used to recirculate the filtrate and centrate streams to the repulping tanks.

A11 tanks in the water washing section are provided with mixers to aid the repulping operations. The mixer propellers on all slurry tanks were replaced with impellers during Phase II modifications for improved mixing to prevent solids buildup. Also, the electric band heaters and insulation on all tanks were replaced during Phase II in order to maintain high wash train temperatures (up to $180^{\circ} \mathrm{F}$ ) to facilitate the liquid-solids separation in the centrifuges. A pumped anti-foam injection system with manifolding to tanks T-101, T-202, T-205, T-209 and T-211 was installed during Phase II modifications since foaming in tanks caused problems with the level sensors in the Phase I program.

The coal cake from the final centrifuge, C-205 (stream 6), is further washed with acid and water in the acid washing system to remove the dissolved and ionically bound caustic along with the iron-containing mineral matter remaining on the coal cake.

The entire water washing system (filters, centrifuges, and tanks) is blanketed with nitrogen in order to prevent air (carbon dioxide) from entering the system.

\subsection{ACID WASHING SYSTEM (SECTION 300)}

In the acid washing system, the coal cake from the final water washing centrifuge is treated with sulfuric acid to neutralize and remove the iron 
hydroxides and the residual bound and dissolved caustic forming sulfate salts. The resulting slurry is then centrifuged and washed to remove the sulfate salts and excess acid, producing the final coal product. A block flow diagram of the acid washing system is depicted in Figure 2-16 and a typical design material balance is given in Table 2-4. All new equipment was installed in this system during Phase I. The acid washing train of three centrifuges is shown on the right side of Figure 2-12. The centrifuges, C-301 through $\mathrm{C}-303$, along with interstage slurry tanks are used to remove the sulfate salts and excess acid, producing the final coal product (stream 15). The coal product is low in sulfur, ash, and sulfate salts. The centrate from the first centrifuge, C-301, (stream 18) containing the excess acid and the sulfate salts is sent to the waste water treatment system. This centrate stream has a $\mathrm{pH}$ of about two.

The wash water is feed to the final slurry tank, T-303. As the wash water streams move in a countercurrent direction with respect to the solids flow, they become more concentrated in acid and sulfate salts while the residual liquid on the coal solids becomes more dilute in acid and salts. This countercurrent washing arrangement is very similar to that used in the water washing system. During Phase I testing, about ten parts wash water per part coal were used (instead of the design three parts wash water, stream 17) to increase the flow rates through the acid washing system. The design liquid content of the solids from each centrifuge is about one part liquid per part coal.

The coal cake is treated with sulfuric acid in a 12-galion Alloy 20 acid contacting vesse1, T-301. The sulfuric acid is metered into the acid contactor from a storage drum. The first two solid bowl centrifuges, C-301 and $C-302$, and slurry tank T-302 are also of Alloy 20 construction. The final solid bowl centrifuge, $C-303$, and the final tank, T-303, are of 316 stainless steel construction. All three centrifuges are six inches in diameter. The acid contactor and the two tanks are provided with mixers and with electric band heaters. The mixer propellers on all tanks were replaced with impellers during Phase II for improved mixing to prevent solids buildup. The electric band heaters and insulation on all tanks were also replaced in Phase II in order to maintain high wash train temperatures (up to $180^{\circ} \mathrm{F}$ ) to facilitate the liquid-solids separation in the centrifuges. 


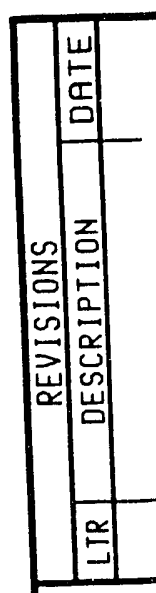

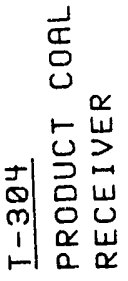

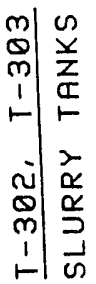
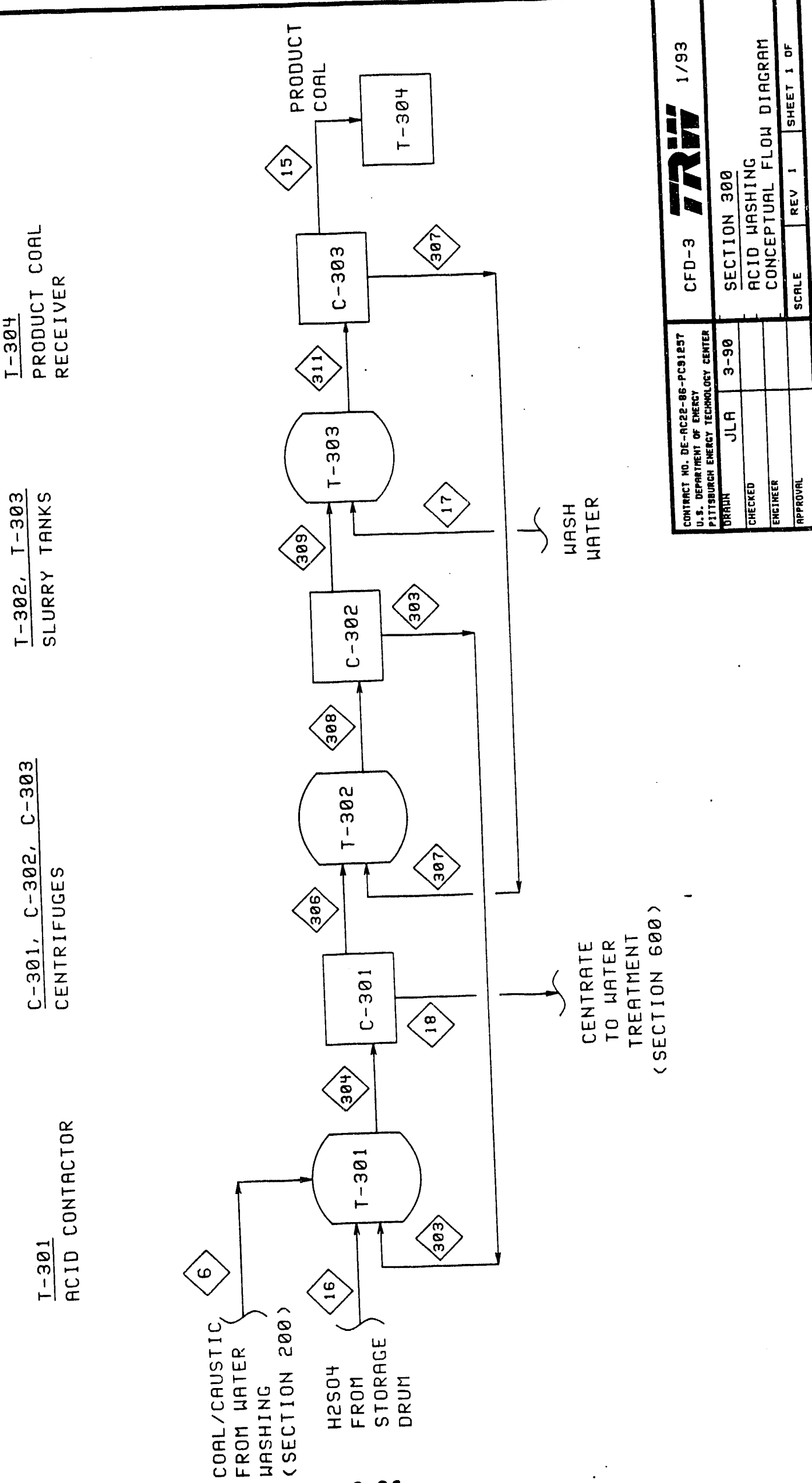

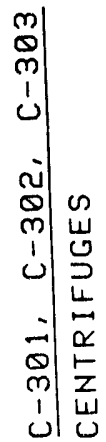

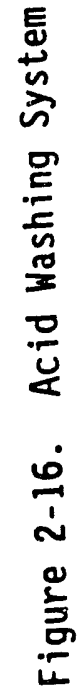

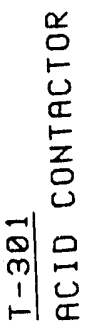




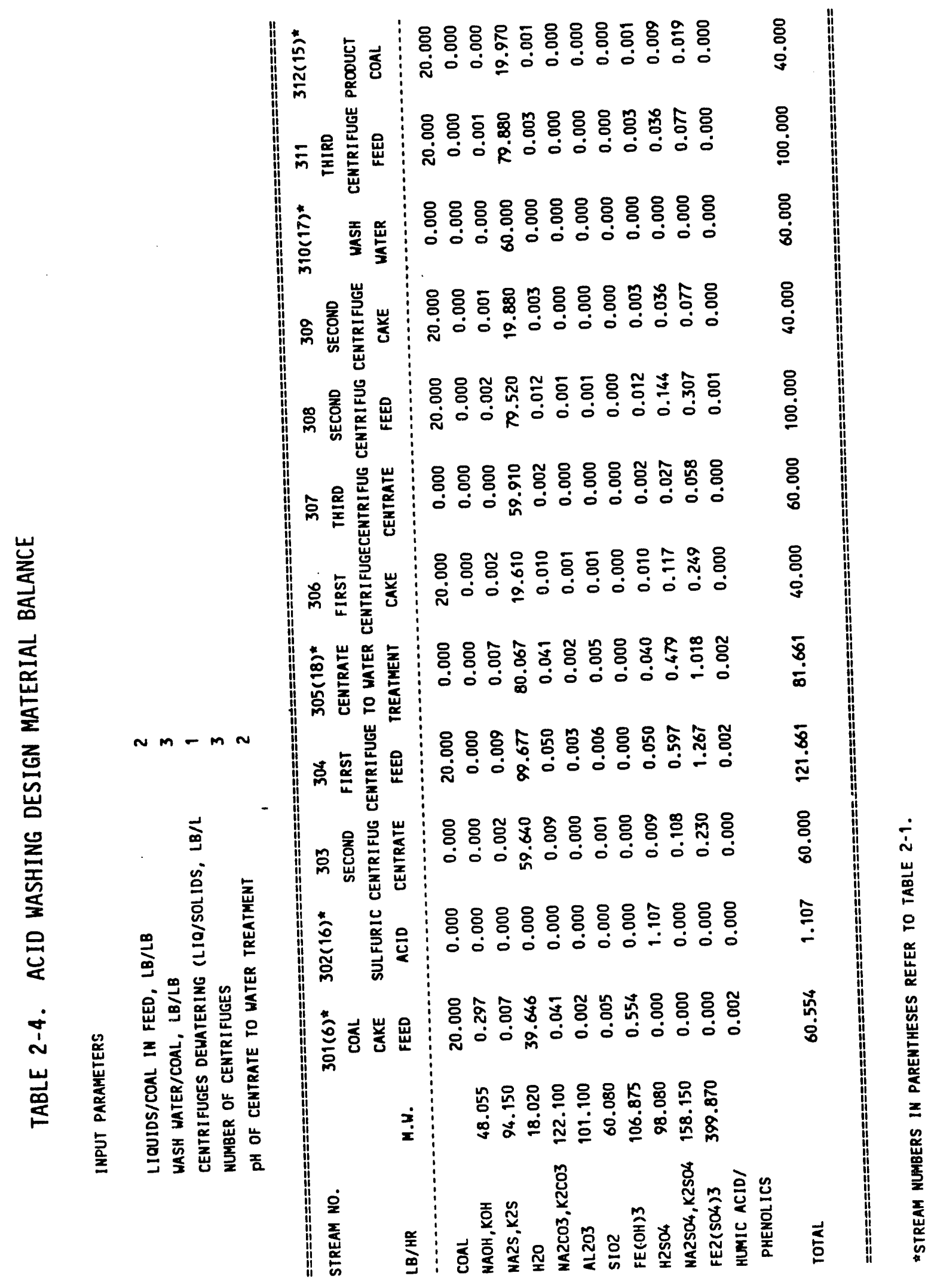


The acid contactor and the two slurry tanks are 12 gallons in size in order to provide a nominal 30 minutes of residence time each. In a full scale plant, the slurry tanks in both the water washing and acid washing systems will be sized for minimum residence time (about five minutes). In this integrated $20 \mathrm{lb} / \mathrm{hr} \mathrm{plant}$, a five minute residence time corresponds to only $0.75 \mathrm{gallions}$ or 0.1 cubic feet (based on a flow rate of $0.15 \mathrm{gpm}$ ). Tanks designed for only 5 minutes residence time at. this scale are simply too small to be practical. For this reason, the nominal slurry tank residence time in the water and acid washing systems was increased to 30 minutes ( 5 gallons).

Progressive cavity pumps were used to provide a steady slurry feed stream to the centrifuges. Diaphragm pumps were used to recirculate the centrate streams to the repulping tanks. Tanks T-302 and T-303 are located next to and slightly below centrifuges $c-301$ and $c-302$ in order that the cake produced by the centrifuges can drop by gravity directly into the repulping tanks. In a similar manner, the acid contactor, T-301, is located next to and slightly below the final centrifuge, $\mathrm{C}-205$, of the water washing system.

The relatively sulfur and ash-free coal product from centrifuge $\mathrm{C}-303$ is collected in plastic bags and then transferred to 55 gallon drums. Samples of the final coal cake were dried in ovens off-line. The centrate stream from the first centrifuge, $C-30 \overline{1}^{-}$(stream 18) containing the excess acid and most of the sodium sulfate and ferric sulfate salts formed by the neutralization reactions is sent to the waste water treatment system.

Late in Phase II, a new process configuration was tested in which an acid wash of the MCL coal product was not performed, although the acid wash train equipment was used for additional stages of water washing. The centrate from the acid wash train (without acid injection) was used as makeup wash water for the water wash train, thus eliminating the acid extract waste water stream. Eliminating acid washing had no effect on the sulfur levels of the product coal but increased the ash levels and has the potential of significantly reducing the cost of the process for those commercial applications that do not require low ash coal. 
The entire acid washing system (centrifuges and tanks) is blanketed with nitrogen in order to prevent air (carbon dioxide) from entering the system.

\subsection{CAUSTIC REGENERATION SYSTEM (SECTION 400)}

The caustic regeneration system was designed to remove the dissolved coal mineral matter and sulfur compounds from the caustic before the caustic is sent to the evaporator and recycled to the kiln. Caustic is recovered and recycled for economic and environmental reasons: caustic is expensive and difficult to dispose of. The feed to the caustic regeneration section is the filtrate from the first vacuum filter in the water washing section (stream 8, Figure 2-1). The design filtrate consisted of approximately $50 \%$ caustic (sodium hydroxide and potassium hydroxide) and 50\% water, with associated dissolved mineral matter and sulfur compounds. During Phase I testing, problems with the vacuum filters resulted in the filters being bypassed and the feed to the caustic regeneration system being very dilute, containing only about $15 \%$ caustic. These problems were corrected when the vacuum filter system was extensively modified during Phase II of this program. A substantial number of hours of on-stream time were subsequently logged during Phase II with the modified vacuum filtration system operating in series with the rest of the integrated $\mathrm{MCL}$ facility and with filtrate sent to regeneration containing 20 to $25 \%$ caustic.

The dissolved materials, together with water, must be removed in order to recover anhydrous caustic for recycle. The dissolved mineral matter and sulfur compounds are removed in the caustic regeneration system by the addition of lime and by providing sufficient residence time to permit precipitation. The precipitated solids are then mechanically separated from the caustic in a centrifuge and bag filters (Figure 2-17). The water is later removed from the caustic in the caustic concentration system (Section 500, Figure 2-1).

Most of the tanks and the centrifuge in the caustic regeneration system are existing equipment from the previous modular Gravimelt program that were repiped and refurbished for this program. Figure 2-18 shows the refurbished regeneration system. In the middle of the photo is the existing centrifuge with a small solids collection drum underneath. In the top of the photo can 


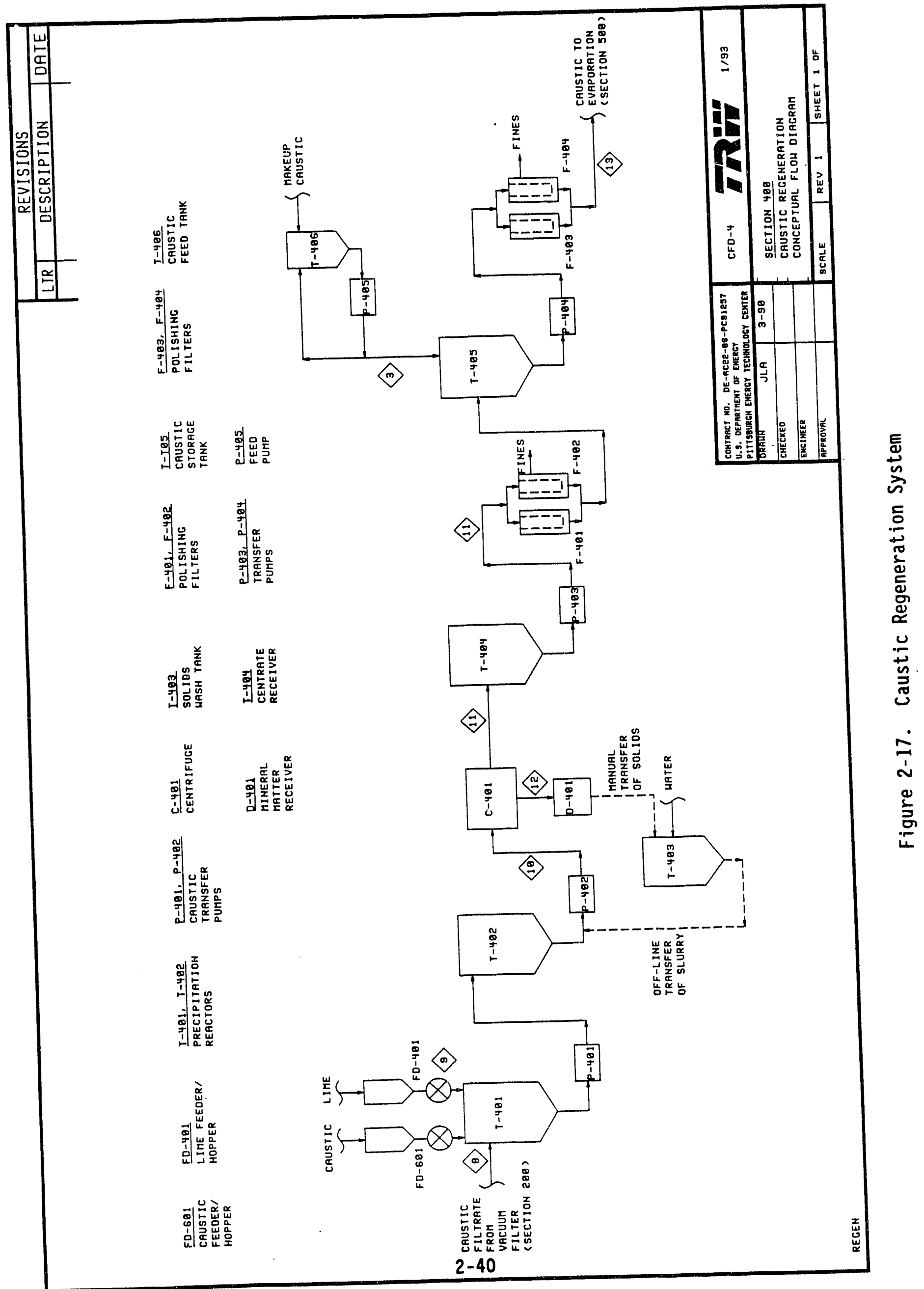




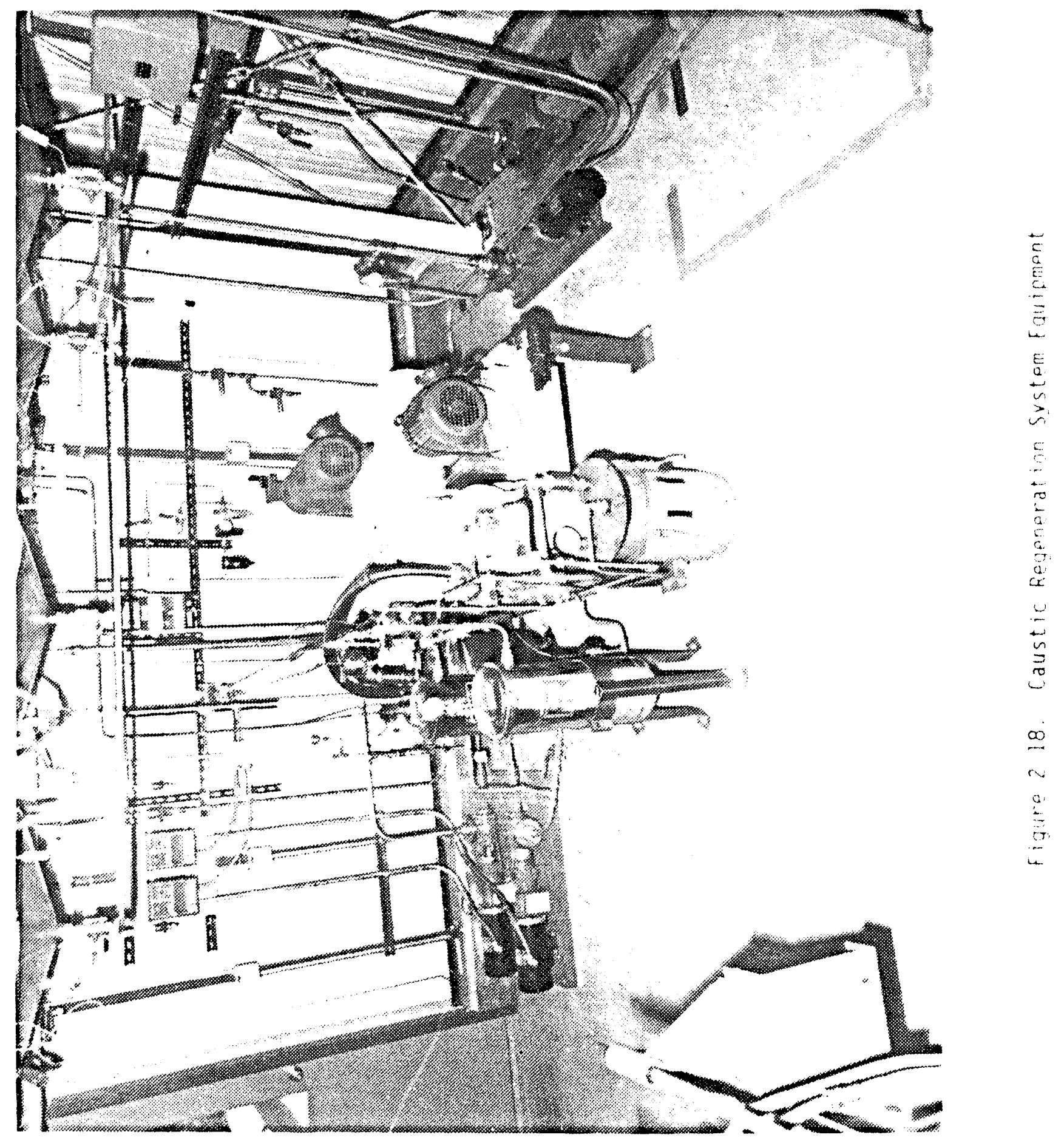


be seen the cone bottoms of the existing 100 and 65 gallon tanks which are located on a mezzanine. The tops of two of the tanks can be seen on the right in Figure $2-5$.

The feed to the caustic regeneration system is continuously pumped to an insulated 100 gallon precipitation tank, T-401, which provides a residence time range of four to eight hours (1/4 to $3 / 4$ ful1). The tank is provided with both immersion heaters and band heaters to heat and maintain the caustic solution at reaction temperatures up to $200^{\circ} \mathrm{F}$.

Lime, $\mathrm{CaO}$ or $\mathrm{Ca}(\mathrm{OH})_{2}$ (stream 9), is added by a solids feeder, FD-401, to tank T-401. The lime forms insoluble complexes with the mineral matter and also causes the precipitation of the sulfur compounds present. A second screw feeder, FD-601, was added to tank T-401 during the modifications made during Phase II and calibrated with caustic. A new, larger feed screw was installed in the feed hopper to allow for the feeding of flaked caustic. Since the caustic concentration from the newly modified vacuum filter system was expected to range from 25 to $35 \%$, the caustic concentration to the evaporator was increased during Phase II testing to $50 \%$ by metering anhydrous fresh caustic flake/beads into reactor T-401 to precipitate carbonate (and thus prevent precipitation of carbonate in the evaporator tubes). The equilibrium solubility of sodium carbonate in sodium hydroxide drops to a very low yalue $(<1 \%)$ at $50 \%$ caustic concentrations. During Phase II testing, 1 ime was not added to tank T-401.

The caustic solution with the precipitated solids (and excess lime) is continuously transferred to a second heated 100-gallon precipitation tank, $\mathrm{T}-402$, which can provide up to an additional eight hours of residence time to facilitate the further precipitation of mineral matter, carbonates, and sulfur-containing solids if needed. Such relatively long residence times were provided since the precipitation reactions involve potentially slow crystallizations. Tank T-402 may be bypassed.

The caustic along with the precipitated solids (and excess lime) is next transferred to centrifuge $\mathrm{C}-401$ where most of the precipitated solids and excess lime are removed from the caustic. The centrifuge is an existing unit 
from the previous Gravimelt program and is virtually identical to the centrifuges in the water washing and acid washing systems. The remaining precipitated solids are removed downstream in bag filters $F-401$ through F-404.

The centrifuge cake (siream 12) is collected in a small collection drum underneath the centrifuge and transferred to 55 gallon drums (D-401). These cake solids are wet with caustic solution and can be reslurried (washed with water) and recentrifuged off-1ine (Figure 2-17) at a later time to make the solids more environmentally suitable for disposal.

The mixer propellers in all tanks were replaced with impellers during the Phase II program for improved mixing and to prevent the solids from settling in the tanks and plugging the tank outlet lines. Tanks T-401, T-402 and T-405 are provided with both electrical band heaters and immersion heaters to maintain the caustic solutions at temperatures up to $200^{\circ} \mathrm{F}$ to facilitate the precipitation reactions and the liquid-solids separation in the centrifuge and bag filters.

The centrate (stream 11) is collected in a 10-gallon heated centrate receiver, T-404, before being pumped through polishing bag filters, F-401 and F-402, to remove the remaining precipitated solids and excess 1 ime not removed by the centrifuge. The filtrate from the bag filters is collected in a heated 100-galion storage tank, T-405, which also serves as the evaporator feed tank. Tank T-405 can hold up to 8 hours of caustic and provides a surge volume for :austic storage i.. zase of a temporary shutdown of the evaporator system.

The caustic is pumped from tank T-405 through polishing bag filters F-403 and F-404 and on to the evaporator where the remaining water is removed to produce an anhydrnus caustic for recycle to the kiln. Polishing filters, $F-403$ and F-404, are present to protect the evaporation system from plugs caused by additional solids precipitation in tank T-405. A new hot oil-heated caustic preheater was fabricated during Phase II and installed downstream of bag filters F-403 and F-404 and upstream of the evaporator. 
A11 equipment in the caustic regeneration system is of 316 stainless steel or fiberglass construction. The entire caustic regeneration system (bag filters, centrifuge, and tanks) is blanketed with nitrogen in order to prevent air. (carbon dioxide) from entering the system.

\subsection{CAUSTIC CONCENTRATION SYSTEM (SECTION 500)}

The feed to the caustic concentration or evaporation system is the caustic solution from the caustic regeneration system (Figure 2-1). Although the bulk of the mineral matter, carbonates and sulfur compounds present in the caustic solution was removed in the regeneration section, small amounts of these compounds are still present to the extent of their solubility limit in caustic solution. The existing batch evaporation unit from the previous Gravimelt program was inadequate for the integrated system both in terms of size and in terms of mode of operation (batch). A new continuous rising film evaporator was installed along with a caustic flaking unit and an overhead steam condensing system during Phase I (Figure 2-19). During Phase II, an oil-heated preheater was installed and the overhead steam condensing system was simplified to reduce back pressure (Figure 2-20).

The caustic evaporator purchased during Phase I for the integrated MCL process is a skid mounted package unit designed and built by the Buflovak Division of the Blaw Knox Food and Chemical Company. The aqueous caustic feed is stored and kept at about $200^{\circ} \mathrm{F}$ in a 100-gallon storage tank, T-405, in the caustic regeneration system. The hot caustic is pumped through bag filters, F-403 and F-404, and through the newly installed preheater, E-504, into the evaporator unit.

During Phase I testing, it appeared that a significant fraction of the evaporator tube heat iransfer surface was utilized to preheat the incoming caustic solution to its initial boiling point. This resulted in very low overall heat transfer coefficients for the evaporator system. A new hot oil-heated, double pipe caustic preheat exchanger, E-504, was fabricated and installed at the evaporator inlet during Phase II modifications (Figure $2-20)$. The purpose of the preheater is to remove the sensible heating requirement from the evaporator. The evaporator is now used strictiy for boiling. When the preheater was used during Phase ii lesting to heat the 


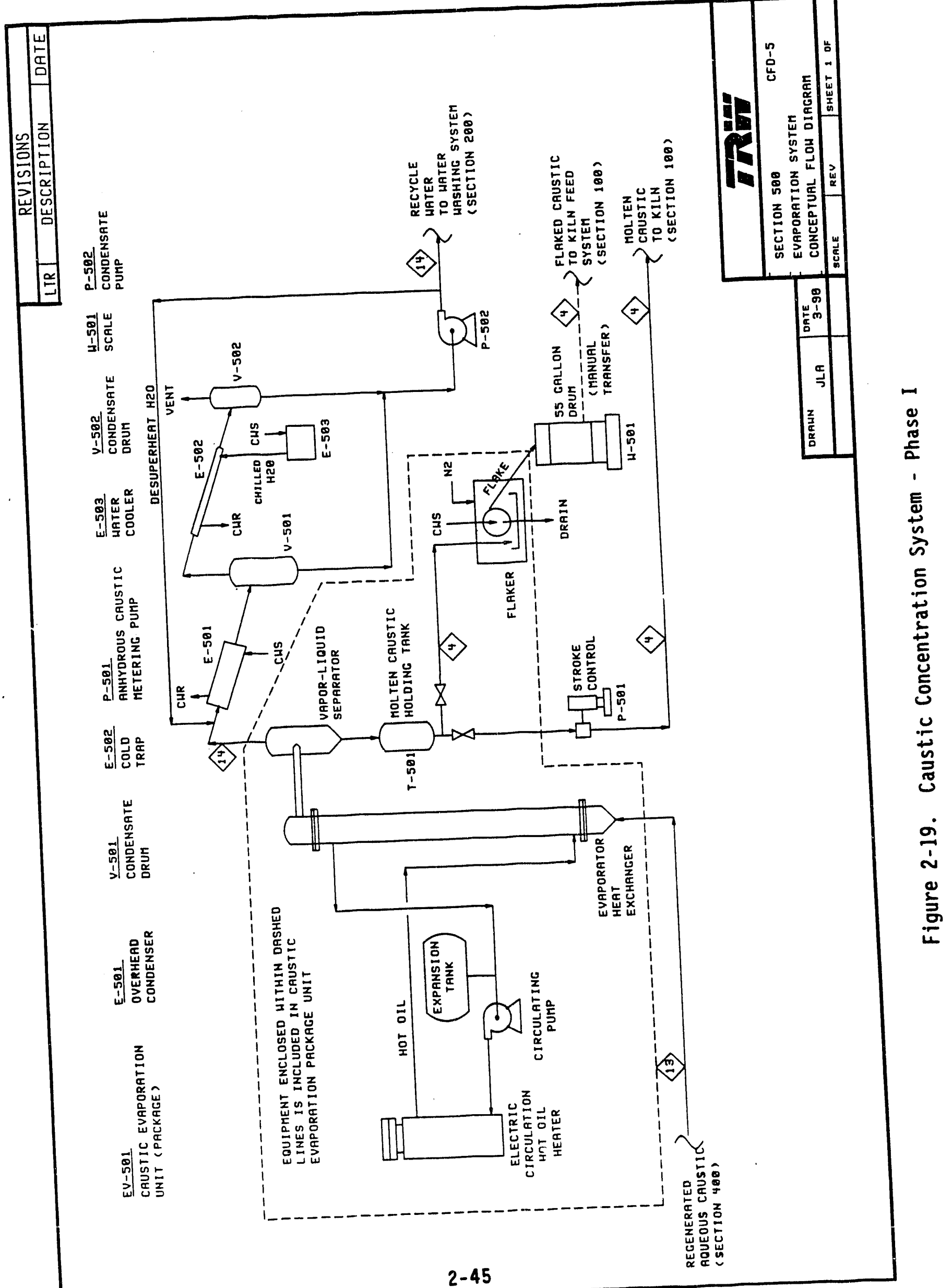




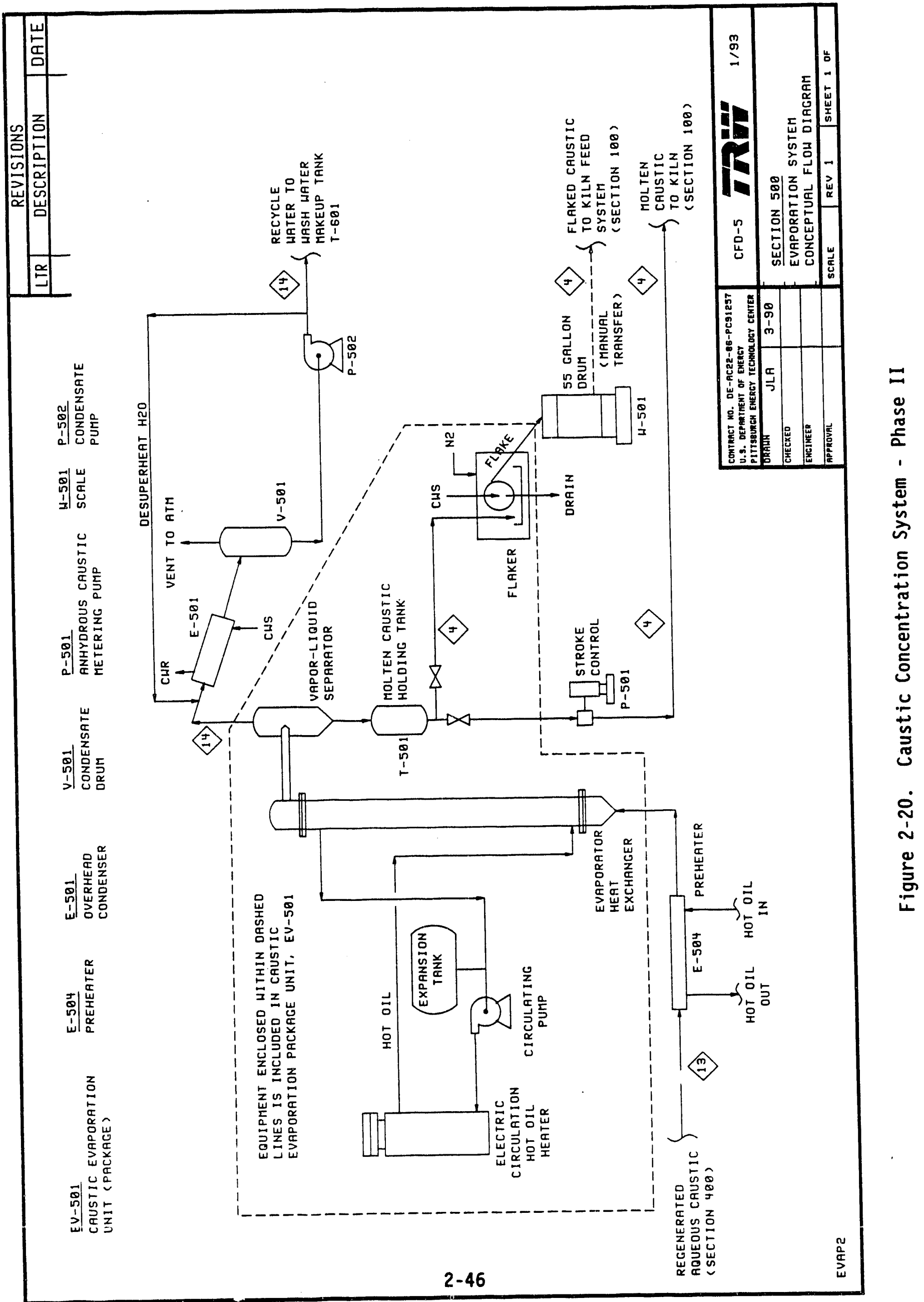


evaporator feed to its initial boiling point, a marked improvement in evaporator heat transfer coefficients was observed. The normal boiling point of 50 percent sodium hydroxide solution is $145^{\circ} \mathrm{F}\left(293^{\circ} \mathrm{F}\right)$. The double pipe preheater and the short 1 ine from it to the evaporator are insulated.

The evaporation unit consists of a shell and tube exchanger in which hot oil circulates through the shell and the caustic flows through the tubes. The exchanger is vertical and the caustic is carried up the walls as a thin film by the evaporated steam. The hot oil and caustic flows are cocurrent. The exchanger has a total heat transfer surface of $11.65 \mathrm{ft}^{2}$ (provided by three 15 feet long, one inch Inconel tubes).

Heat input to the evaporation unit is provided by electric resistance heaters in two $60 \mathrm{~kW}$ hot oil units (120 kW total). The initial single $60 \mathrm{~kW}$ unit proved to be inadequate and a second $60 \mathrm{~kW}$ unit was added. Syltherm 800 is heated in these units and is circulated by a centrifugal pump through the shell of the evaporating exchanger, the jacket on the molten caustic line from the evaporator to the flaker, the jacket of the flaker pan, and the jacket of the preheater. The oil exiting this equipment passes through an expansion tank, to allow for any necessary degassing, before returning to the heating units. Syltherm 800 is a silicone type $0 i 1$ which is extremely stable even at temperatures as high as $750^{\circ} \mathrm{F}$. All syltherm containing lines are insulated.

The caustic evaporator module is located on a specially constructed cement pad provided by TRW and equipped with electrical supply and cooling water utilities. The evaporator is shown in Figure 2-21 as it was delivered to TRW. The top of the evaporator unit is shown to the right. The horizontal Syltherm expansion tank is on the top in this photo with the vapor-liquid separator below. The evaporator after it was installed and insulated is shown in Figure 2-22. The 15-foot high rising film exchanger can be see in the middle of the photo. The vapor-liquid separator can be seen at the very top of the photo, extending above the roof. The horizontal Syltherm expansion tank which is also located at the top of the evaporator is $h$ idden from view by the roof. The evaporator control panel is depicted in Figure 2-23. The evaporator can be seen in the top part of this photo rising through the roof. 


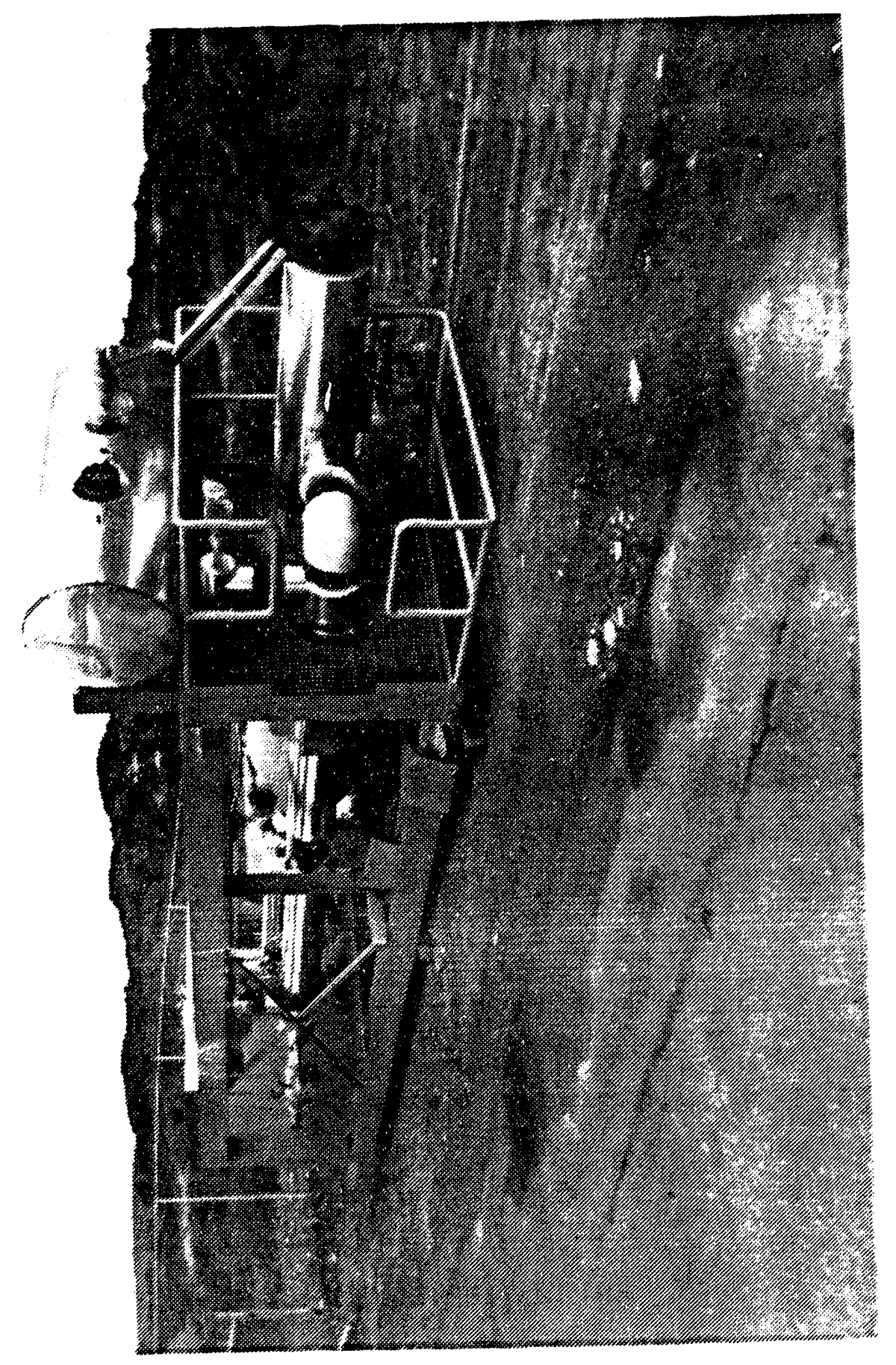

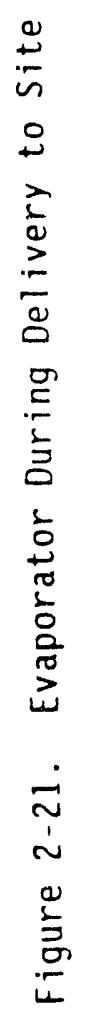




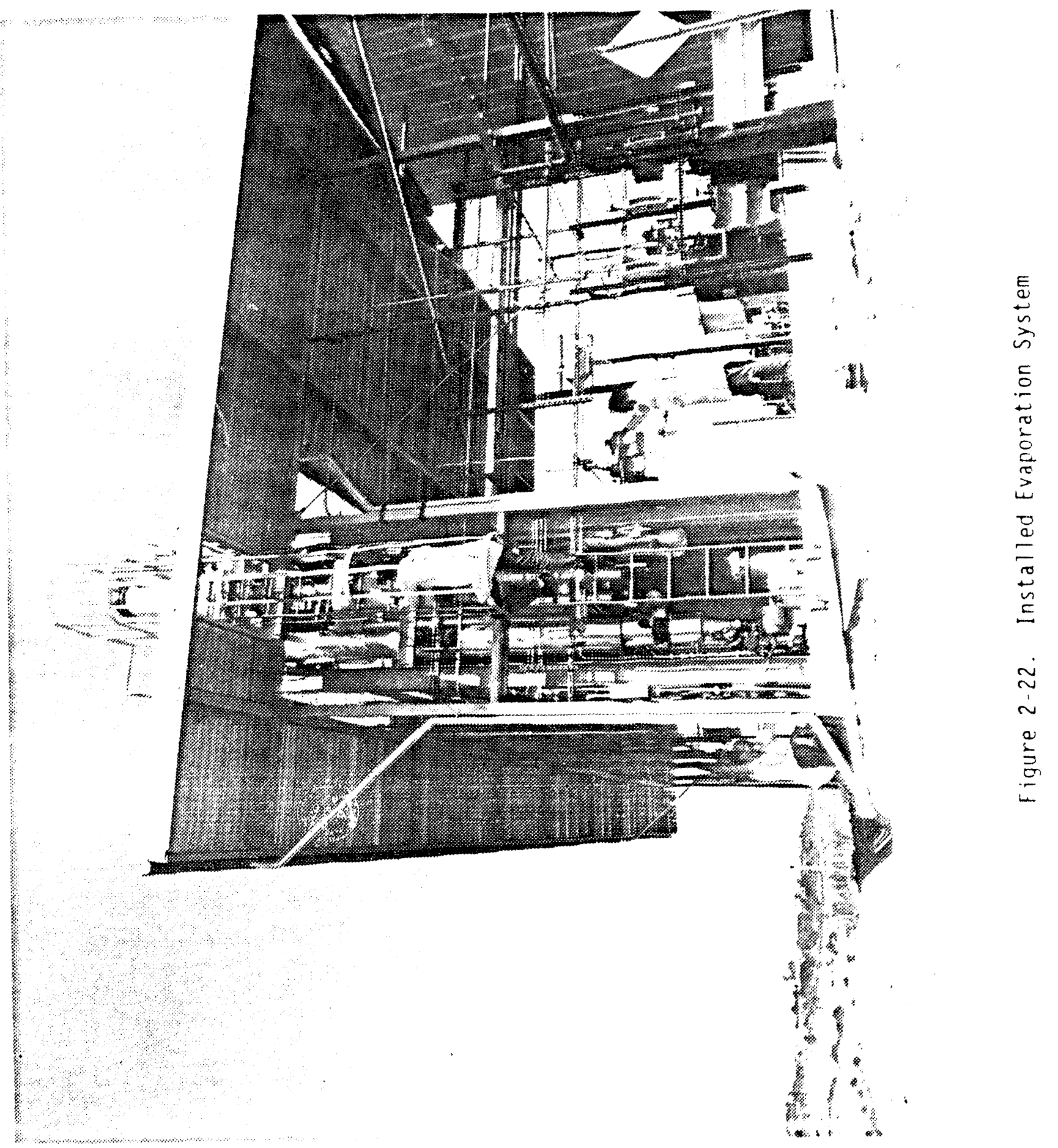




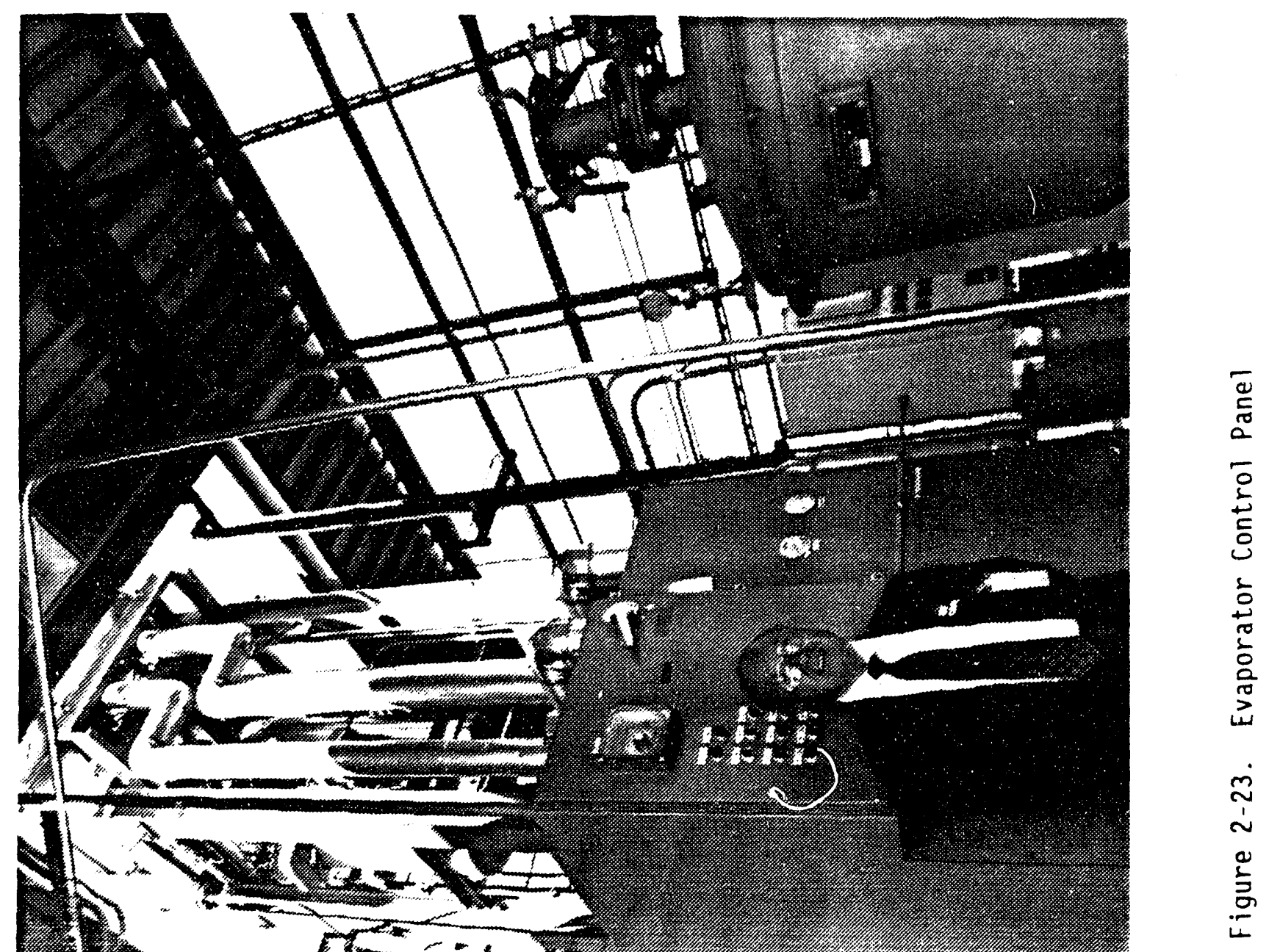


The evaporator unit was designed to process $180 \mathrm{lbs} / \mathrm{hr}$ of 30 percent aqueous caustic $(50: 50 \mathrm{NaOH}: \mathrm{KOH})$ or $130 \mathrm{lbs} / \mathrm{hr}$ of 50 percent caustic (50:50 $\mathrm{NaOH}: \mathrm{KOH}$ ) and dry these feeds to give a caustic product containing about eight percent moisture. The overhead product temperature from the evaporator at this product moisture level is about $600^{\circ} \mathrm{F}$.

Several tests were conducted in the caustic evaporation/flaking system during the Phase I program. Due to the problems with the vacuum filters which resulted in solutions of only $15 \%$ caustic being sent to the regeneration system, aqueous caustic evaporator feed solutions of $50 \%$ were specially prepared for these evaporator tests. The simultaneous operation of vacuum filters and evaporation using high caustic concentrations was not attempted during Phase I testing.

Because the off-line evaporator tests during Phase I experienced limited throughput, iow overall heat transfer coefficients, and periodic surges or upsets, the evaporation unit was modified during Phase II. The overhead vent system on the evaporator was modified to reduce the back pressure and prevent flow surges and uneven evaporation rates. The line sizes on the evaporator vent were increased from 1 inch to 2 inches. A preheater was installed on the evaporator feed to remove the sensible heating requirement from the evaporator. Hot oil jacketing and improved thermal insulation were installed on the molten caustic line from the evaporator to the flaker in order to allow for operation with $\mathrm{NaOH}$ only rather than the lower melting point $\mathrm{NaOH} / \mathrm{KOH}$ mixture used during the Phase I program. The evaporator performed well during Phase II testing with a fresh $50 \% \mathrm{NaOH}$ feed; with regenerated caustic, however, the evaporator continued to experience periodic upsets. Flaked caustic product with a moisture content of 5 to 7 percent was produced.

The vapor-liquid stream exiting the top of the heat exchanger tubes consists of steam and molten caustic. The steam is separated from the molten caustic in a vapor-liquid separating vessel. The steam flowing out the top of this vessel is desuperheated with a small stream of injected water and then condensed in a shell and tube water cooled condenser, E-501. The 
resulting condensate is collected in a separation drum, V-501, downstream of the condenser. The overhead steam condensing system as constructed during Phase $I$ is shown in Figure 2-19. In this configuration, the vapor from separation drum, V-501, flowed through a double pipe exchanger, E-502, that utilizes chilled water from a water cooler, E-503. Any resulting condensate was collected in a second separation drum, V-502. The uncondensed vapor was vented to the atmosphere. The condensate collected in the two separation drums was recycled to the water washing system (Section 200).

The steam condensing system shown in Figure 2-19 was undersized. Also, the condensate recycle pump was not adequate for the task. The intermittent and surging feed flow observed in the evaporator during Phase I testing was probably due in part to back pressure in the evaporator created by an undersized vapor line to the condenser. The size of the steam lines was increased from 1 -inch to 2 -inch tubing during the Phase II modifications to reduce the back pressure on the evaporator. The second condenser, E-502, the second condensate drum, V-502, and the water cooler, E-503, were disconnected from the system to further reduce the back pressure. The uncondensed vapor is now vented to the atmosphere from the first condensate drum, V-501. The condensate pump was replaced to allow recycle of the condensate to the wash train. The modified steam condensing system used during Phase II testing is shown in Figure 2-20.

The molten caustic product leaving the evaporator separator vessel drains by gravity into a heated holding tank, T-501, located directly below the vapor-liquid separator. The molten caustic can then be directed to either a metering pump for injection into the rotary kiln reactor as a liquid or to the pan of a caustic flaking unit for solidification. In the latter case, the caustic is fed to the kiln as a solid with the feed coal. Hot oil jacketing and insulation were installed during Phase II on the molten caustic lines from the vapor-liquid separator to the holding tank and from the holding tank to the flaker, replacing inadequate electrical tracing. The holding tank was already hot oil jacketed.

The flaker consists of a six-inch diameter rotating drum (nine inches long) cooled internally with a water spray. The flaker is shown in Figure 
2-24. The drum rotates through the pan collecting a thin film of molten caustic. Molten caustic picked up by the drum solidifies as it is cooled on the drum surface. The solid caustic is scraped off the drum as a thin flake by a knife and collected in 55 gallon drums. These 55 gallon drums are manually transferred to the rotary kiln reactor feed system where the recycled flaked caustic is mixed with fresh makeup caustic and feed coal. (The kiln feed system is described above in Section 2.1.)

In an alternative mode of operation, hot molten caustic from the evaporator can be fed directly into the kiln reactor, bypassing the flaking system entirely. When operated in this mode, holding tank T-501 acts as a molten caustic feed tank. Molten caustic is transferred from tank T-501 through a heated traced insulated line to a specially designed kiln feed inlet. The molten caustic is transferred by diaphragm pump, P-501, which has remotely mounted nickel diaphragms. The heat traced molten caustic recycle line was not used during the Phase I program since the evaporator was not tested on-line during Phase I due to the problems with the vacuum filters. The molten caustic recycle line was not tested during Phase II because it was decided early in Phase II to implement only those modifications that were critical to integrated operation of the plant (such as modification of the vacuum filter system and the evaporator overhead steam system) and defer other modifications that would have improsed plant operation. Modifications to the molten caustic feed system (hot oil jacketing and improved insulation) were not made since they were not critical to integrated operation.

The caustic concentration system including the molten caustic holding tank and the hot oil expansion tank is blanketed with nitrogen to prevent air from entering the system.

\subsection{WASTE WATER TREATMENT SYSTEM (SECTION 600)}

The waste treatment system was designed to treat the acid containing centrate stream from the acid washing system (stream 18, Figure 2-1) to make this stream suitable for disposal as waste water. This centrate stream contains the potassium sulfate, sodium sulfate, and ferric sulfate salts formed by the neutralization reactions between iron hydroxide, the residual 


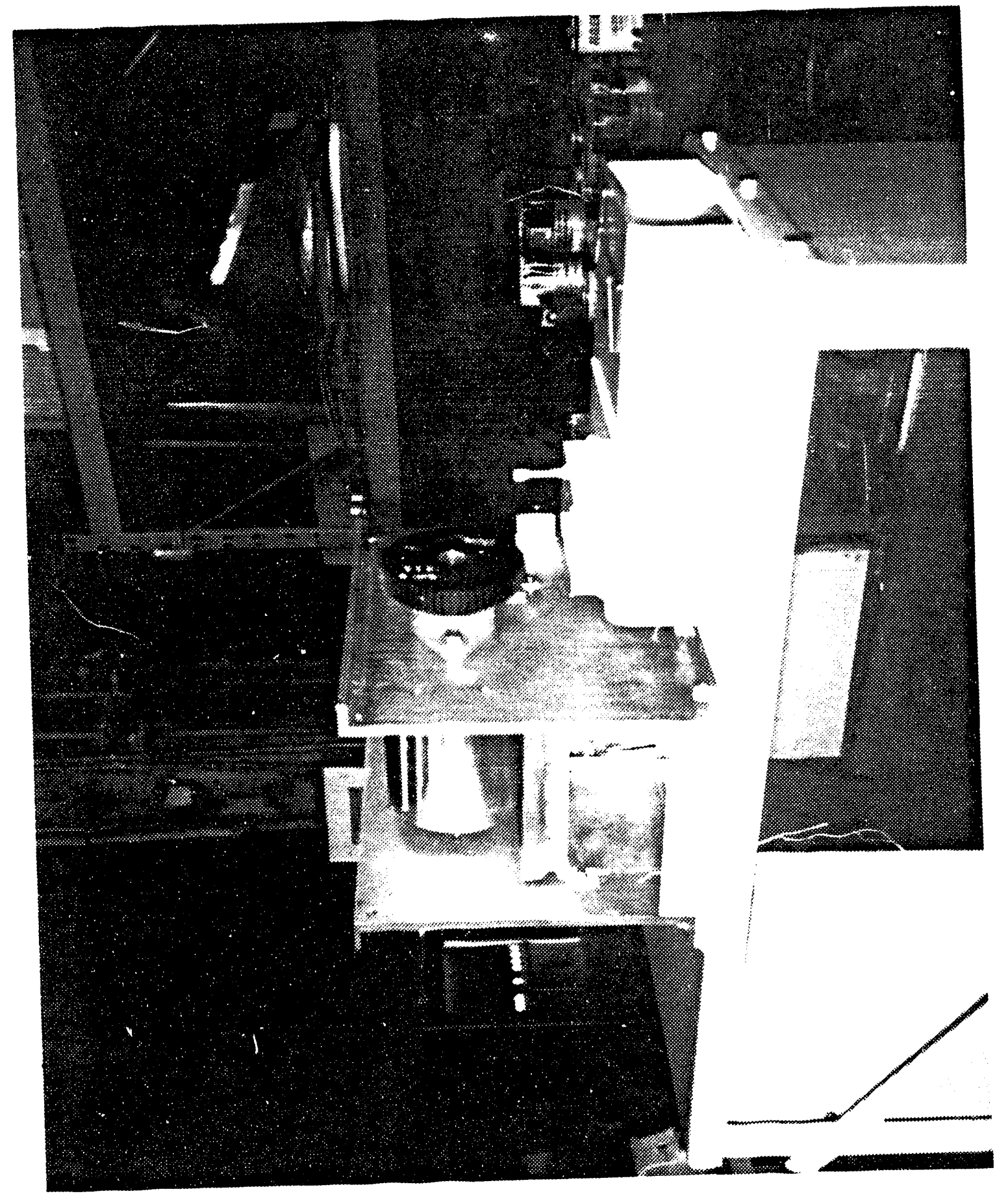

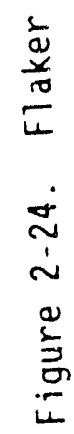


caustic, and sulfuric acid. The sulfate salts and any residual acid in this centrate stream when contacted with ferric sulfate, $\mathrm{Fe}_{2}\left(\mathrm{SO}_{4}\right)_{3}$, and lime, $\mathrm{CaO}$ or $\mathrm{Ca}(\mathrm{OH})_{2}$, in the waste water treatment system precipitate gypsum $\left(\mathrm{CaSO}_{4}\right)$, ferric hydroxide, $\mathrm{Fe}(\mathrm{OH})_{3}$, and double salts of sodium and iron such as sideronatrite $\left(\mathrm{NaSO}_{4}\right)_{2} \mathrm{FeOH} \cdot 3 \mathrm{H}_{2} \mathrm{O}$, ferrinatrite, $\left(\mathrm{NaSO}_{4}\right)_{3} \mathrm{Fe} \cdot 3 \mathrm{H}_{2} \mathrm{O}$, and natrojarosite, $\mathrm{NaFe}_{3}\left(\mathrm{SO}_{4}\right)_{2}(\mathrm{OH})_{6}$.

The water treatment system as originally installed during Phase I contained two fiberglass 300 gallon tanks each followed by a bag filter system to remove the precipitated solids. A flow diagram of the water treatment system installed during Phase $I$ is depicted in Figure 2-25. A11 new equipment was installed in this section. The tanks were sized to provide about 24 hours residence time each. Such relatively long residence times may be required as the reactions involve potentially slow crystallizations. The tanks were provided with immersion heaters, mixers, and screw feeders.

The system was originally designed to add ferric sulfate, $\mathrm{Fe}_{2}\left(\mathrm{SO}_{4}\right)_{3}$, to the first tank, T-601, in order to adjust the sodium to iron ratio to $1: 3$ to facilitate formation of complex, insoluble salts such as sideronatrite, ferrinatrite, and $n$. jarosite. The precipitated salts would be removed from the weak acid solution in a Alloy 20 bag filters, F-601, before the acid stream is sent to tank T-602.

However, the water washing system (Section 200) produced much drier coal cakes during Phase I testing than was expected (about one weight of water per weight of coal versus the design two weights of water per weight of coal), resulting in less caustic remaining on the coal sent to acid washing and a high iron to sodium ratio in the acid waste stream. As a result, there was no need to add iron sulfate to adjust the iron to sodium ratio in the waste treatment system. Thus tank T-601 was bypassed during Phase I testing and the acid centrate was sent directly to T-602 for lime addition. A pH monitor/controller was used to control the speed of the lime screw feeder.

In this configuration, the iron complex minerals are precipitated in tank $\mathrm{T}-602$ in the same step as the gypsum $\left(\mathrm{CaSO}_{4}\right)$ and other calcium salts. The liming of alkali metal sulfates sometimes results in the formation of highly 


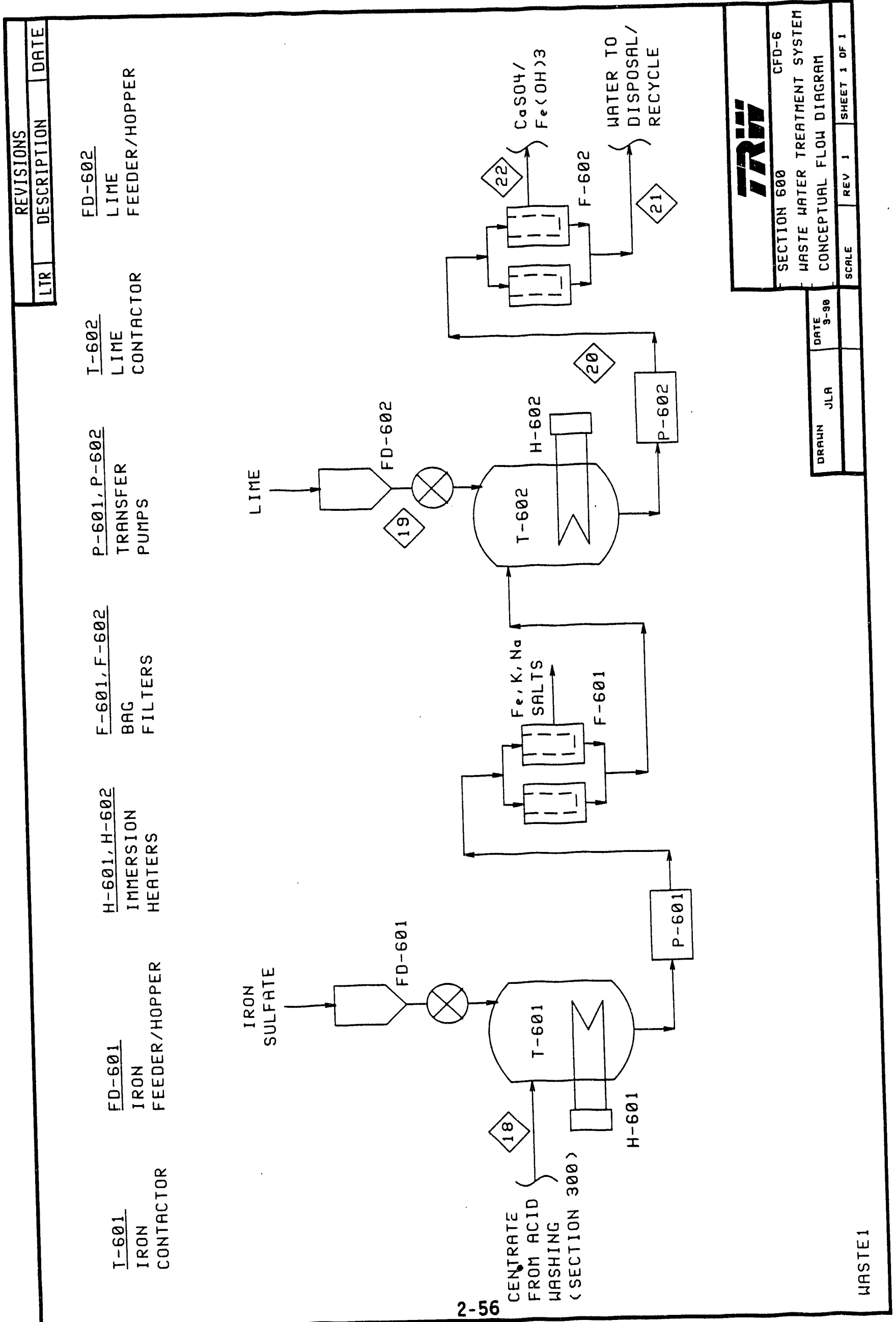


insolubie syngenite minerals which are intersalts of calcium and alkali metai sulfates. These salts are likely to be environmentally sound disposal products.

The precipitated solids (stream 22) are removed in bag filter F-602 before the waste water (stream 21) is sent to disposal. Since the scdium/potassium levels in the treated waste water were only $2000 \mathrm{ppm}$ versus the expected 5000 to $7000 \mathrm{ppm}$, the treated waste water was recycled as wash water to the water washing system (Section 200) during some of the Phase I tests. Otherwise, the waste water stream was further treated in the site waste treatment facility where it was subjected to the following: settling tank to remove solids, treatment to adjust the $\mathrm{pH}$ to ten, filtration, neutralization, and a second filtration. The final solids-free, neutralized filtrate was collected in a holding tank and hauled away by a disposal company.

Durirg the Phase II modifications, the wash water feed system to the water and acid wash sections was modified to maintain the wash water temperature at levels up to $180^{\circ} \mathrm{F}$. Tank T-601, which has an immersion heater, was converted for use as a wash water feed tank. The system was replumbed so that makeup DI water and recycled condensate from the evaporator are pumped to tank T-601. This tank is now used to preheat the wash water. A pump-around loop external to this tank was inctalled to circulate the water in the tank to maintain uniform temperature. A tank level switch was installed. A flow diagram of the modified Phase II waier treatment system is Jepicted in Figure 2-26. Tank T-602 is still used for lime addition.

The two 300-galion tanks, T-601 and T-602, are shown in Figur ?-27. Mixers and screw feeders with feed hoppers are shown above the tanks, and a pair of the bag filters is between the tanks. The immersion heaters are inscrted through the $f 1$ anges on the sides of the tanks. The tanks are lined fiberglass vessels. Bag filters F-602 are of 316 stainless stee 1 construction.

The waste water treatment system (taniks and bay filters) was not blanketed with nitrogen as were the other systems of the MCL plant since there ras no need to prevent air from entering this system. 


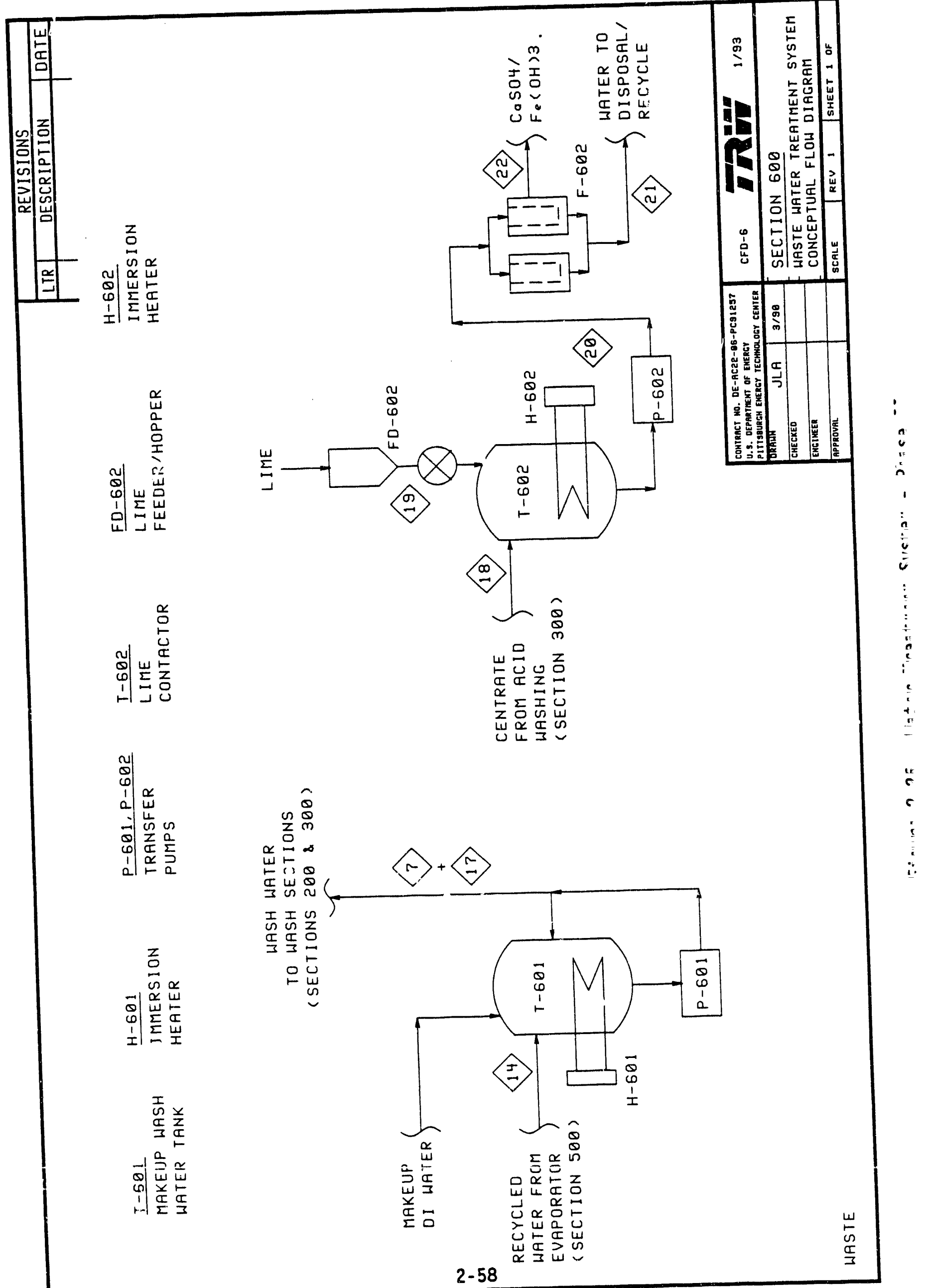




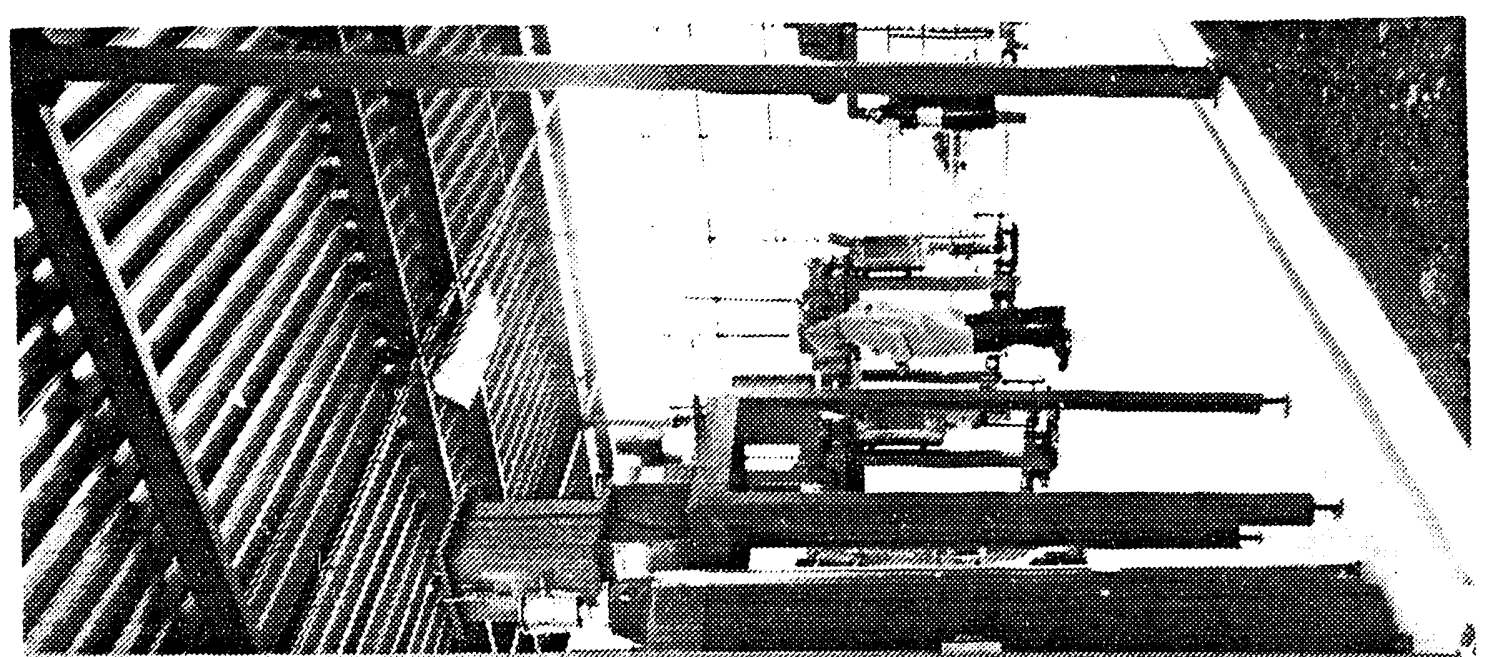

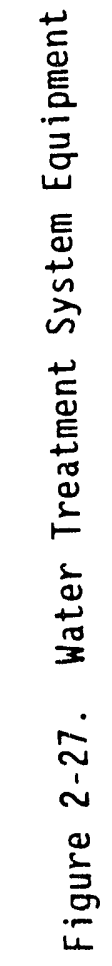


Samples were taken as needed from all gas, solid and liquid streams throughout the MCL plant in order to obtain sufficient data to assess the efficiency of the MCL system.

\subsection{DATA ACQUISITION AND CONTROL SYSTEM}

The operation of the integrated $\mathrm{MCL}$ test circuit resulted in the generation of an extensive data base. Included in this data base were pressure (psig), vacuum (inches $\mathrm{H}_{2} \mathrm{O}$ or inches $\mathrm{Hg}$ ), differential pressure (inches $\mathrm{H}_{2} \mathrm{O}$ ), liquid flow (gpm), air or nitrogen flow (SCFM), temperature $\left({ }^{\circ} \mathrm{F}\right)$, and tank level (percent). This information, being voluminous, was easily handled by a data acquisition and control system which collected and stored the process measurements in real time and utilized a portion of it for feedback control.

The microprocessor based data acquisition and control system installed at the test site consisted of modular components as much as possible including process interface equipment, process control units and operator's display stations. The two operator's display stations inccrporated CRT's displaying loop and alarm status and real time plant graphics. Keyboards allowed configuration and control function adjustments to be entered into the system. The system was designed so that the failure of any single component would not disable the entire system. The data acquisition and control system is shown during installation in Figure 2-28. One of the control monitors is shown at the left. There are approximately 80 instruments and con'rols in the integrated MCL plant.

The data acquisition and control system was designed to perform the following functions:

- To measure and collect data necessary to assess the technical performance of the process over the specified ranges of operating conditions.

- To control and monitor the operation of the continuous MCL test plant over a wide range of operating conditions.

- Tn evaluate the performance and reliability of selected instruments and contruis in MCL service. 

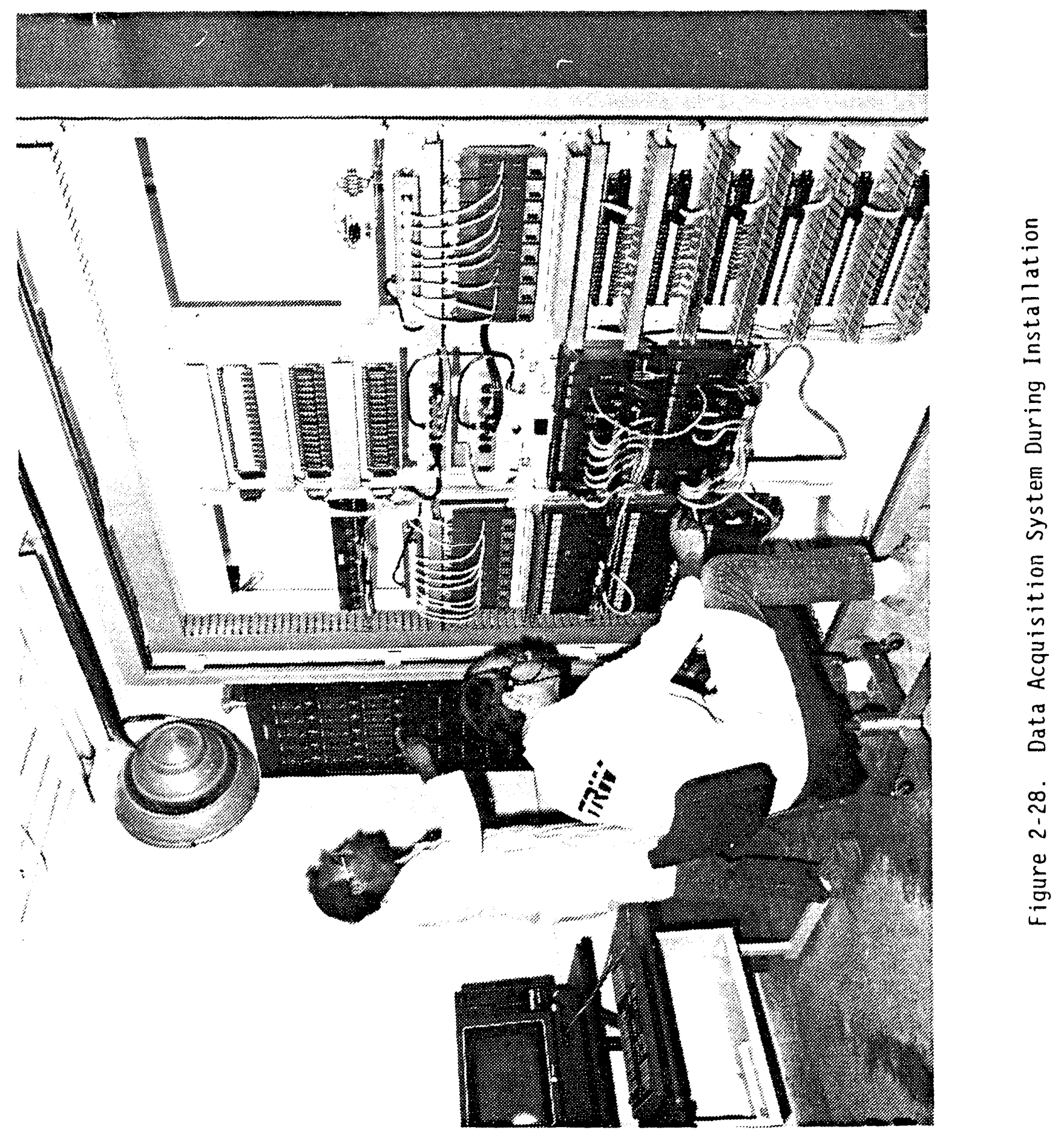


\section{SUMMARY OF DATA}

The MCL integrated test circuit was operated on an around-the-clock basis in a shakedown mode for approximately 100 hours in December 1988, following by nine operating weeks during Phase I over the period of time from January through June of 1989 and two additional operating weeks during Phase II for a total of about 1000 hours of matrix testing. The normal mode of operation during Phase I was to operate around-the-clock for a week (two 12-hour shifts for five days) followed uy a week of scheduled downtime for maintenance and refurbishment (repair and modify equipment as necessary), data analysis and future test planning. During Phase II, because of the large number of off-line vacuum filter and evaporator tests, only two weeks of around-the-clock operations (one for five days and one for seven days) were performed. Each week of operation was designated as a "run" for a total of eleven runs plus shakedown. Each run was then subdivided into smaller units of time ( $A, B, C, D)$ based on operational changes called for by the test matrix.

The following sections contain a compilation of the more critical test data including a test matrix summary, a run conditi uns summary, sulfur and ash results by run, voiatiles and heat content summaries by run, coal product data tabulation, and product coal shipping data. Analyses were obtained on process streams such as product coal, slurries, filtrates, centrates, kiln gas, and various effluent solids.

\subsection{TEST MATRIX AND RUN CONDITION SUMMARY}

In order to provide sufficient data for evaluation, a fractional factorial test matrix was formulated such that the three coals and the process variables were tested against each other in a statistically relevant manner. Process variables tested include potassium-to-sodium ratio, caustic-to-coal ratio, kiln temperature, kiln residence time (RPM, auger speed, auger direction - forward vs. backward), fresh vs. recycled caustic, feed coal mesh size ( 14 vs. 6 mesh $\times 0)$, and nitrogen flow direction in kiln (cocurrent vs. countercurrent). The test matrix was aimed at: 1) providing the highest grade compliance coal containing both ultra-low-sulfur and 
ultra-low-ash, 2) evaluation of the most economic processing conditions, and 3) production of coal with the minimum by-product formation.

The 63-test process matrix performed during integrated operations is presented in Table 3-1 on a variable-by-variable basis. A run-by-run summary of operating conditions is presented in Table 3-2. A much more detailed run-by-run summary of operating conditions including product coal analyses and heat content is presented in Appendix $A$.

\subsection{SULFUR AND ASH REMOVAL}

The sulfur and ash content of the MCL integrated test circuit product coal is presented on an hour-by-hour basis in Figure 3-1. These data are line-connected for ease of inspection. Analyses of intermediate samples taken at the kiln exit are also shown. Approximately four hours should be added to the abscissa of these samples to align with the time of exit of the product coal collected at the final wash station. This is to correct for the $\sim 4$ hours residence time in the water and acid washing sections.

As a point of reference it should be noted that the feed Pittsburgh No. 8 coal (Powhatan No. 6 mine) analyses are: $4.27 \%$ total sulfur; $2.54 \%$ organic sulfur; $11.57 \%$ ash; 13,016 MF Btu/1b, 14,719 MAF Btu/1b; the feed Kentucky No. 9 analyses are: $3.41 \%$ total sulfur; $1.85 \%$ organic sulfur; $9.25 \%$ ash; 13,259 MF Btu/1b; 14,610 MAF Btu/lb: and the feed Pittsburgh No. 8 coal (CONSOL's Blacksville No. 2 mine) for Run No. 11 are $2.26 \%$ total sulfur,

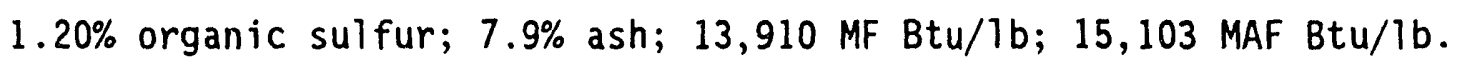

\subsection{VOLATILES AND HEAT CONTENT}

Volatiles, sulfur, and heat content by run of the product coal as a function of coal exit time are presented in Figure 3-2. A tabular listing of kiln exit coal and product coal sulfur and ash content along with product coal volatiles and heat content is included in Appendix B.

\subsection{PRODUCT COAL}

Table 3-3 contains a listing of all product coal samples fully processed in the integrated test circuit together with analyses to aid in the selection 
of samples for delivery to the DOE. Selected samples of MCL product coal were placed in a $\mathrm{GN}_{2}$-purged coal drying oven for six hours at $140^{\circ} \mathrm{F}$. This removed about 15\% of the moisture from the samples and provided a free-flowing granulated product. The dried samples were place in 55-gallon steel drums for shipment to the DOE. The total weight shipped to DOE at the end of Phase $I$ is summarized in Table 3-4. Additional coal from Run 11 in Phase II has been assigned to the DOE and is planned for shipment at the end of Phase II. 


\section{TABLE 3-1. TEST MATRIX: VARIABLE-BY-VARIABLE}

PITT 8 (Powhatan No. 6 mine)

$\mathrm{KOH} / \mathrm{NaOH}=1$

Caustic/Coal $=1$

$\begin{array}{lrrrrrr}\text { Run } & 1 A & 3 A 1 & 3 A 2 & 3 A 3 & 6 E 1 & 6 F 1 \\ \text { Temp, C } & 390 & 427 & 427 & 427 & 427 & 427 \\ \text { Res Time, hr } & 1.5 & 2.0 & 2.3 & 2.2 & 2.0 & 2.0 \\ \text { Kiln RPM } & 2 & 1 & 1 & 1 & 1 & 1 \\ \text { Auger Speed, X max } & 30 & 30 & 15 & 30 & 30 & 3 \\ \text { Auger sec F/sec B } & 20 / 10 & 20 / 10 & 20 / 10 & 17 / 13 & 20 / 10 & 20 / 10\end{array}$

PITT 8 (Powhatan No. 6 mine)

$\mathrm{KOH} / \mathrm{NaOH}=1$

Caustic/Coal $=1.5$

$\begin{array}{lrrrr}\text { Run } & 1 B & 1 C & 8 A & 8 B \\ \text { Temp, C } & 390 & 410 & 427 & 427 \\ \text { Res Time, hr } & 1.5 & 1.5 & 2.1 & 2.1 \\ \text { Kiln RPM } & 2 & 2 & 0.8 & 0.8 \\ \text { Auger Speed, X max } & 30 & 30 & 30 & 30 \\ \text { Auger sec F/sec B } & 20 / 10 & 20 / 10 & 20 / 10 & 20 / 10\end{array}$

* Nitrogen flow was countercurrent to coal feed in Runs $8 \mathrm{~A}$ and 88. Also, 6 mesh coal was used in Run 8B.

PITT 8 (Powhatan No. 6 mine)

$\mathrm{KOH} / \mathrm{NaOH}=1$

Caustic/Coal $=2$

\begin{tabular}{|c|c|c|c|c|c|c|c|c|c|c|}
\hline Run & 10 & $2 A$ & $2 B$ & $2 c$ & 383 & 382 & 3B1 & $5 A 1$ & To & TE \\
\hline Temp, C & 410 & 390 & 390 & 427 & 432 & 438 & 438 & 413 & 427 & 427 \\
\hline Res Time, hr & 1.5 & $1.5-2.0$ & 2.0 & 2.0 & 2.2 & 2.3 & 2.0 & 2.0 & 2.1 & 2.1 \\
\hline Kiln RPM & 2 & $2 / 1.5 / 1$ & 1 & 1 & 1 & 1 & 1 & 1 & 0.8 & 0.8 \\
\hline Auger speed, $\% \max$ & 30 & 30 & 30 & 30 & 30 & 15 & 30 & 30 & 30 & \\
\hline Auger $\sec \mathrm{F} / \mathrm{sec} B$ & $20 / 10$ & $20 / 10$ & $20 / 10$ & $20 / 10$ & $17 / 13$ & $20 / 10$ & $20 / 10$ & $20 / 10$ & $20 / 10$ & $0 / 1$ \\
\hline
\end{tabular}

*Nitrogen flow was countercurrent to coal feed in Runs 70 and $T E$. Also, recycled caustic was fed with coal in Run 70 .

PITT 8 (Powhatan No. 6 mine)

$\mathrm{KOH} / \mathrm{NaOH}=1$

Caustic/Coal $=2.5$

$\begin{array}{lrrr}\text { Run } & 9 A & 9 A 1 & 9 B . \\ \text { Temp, C } & 427 & 438 & 438 \\ \text { Res Time, hr } & 2.1 & 2.1 & 2.1 \\ \text { Kiln RPM } & 0.8 & 0.8 & 0.8 \\ \text { Auger Speed, X max } & 30 & 30 & 30 \\ \text { Auger sec F/sec B } & 20 / 10 & 20 / 10 & 20 / 10\end{array}$

* Nitrogen flow was countercureent to coal feed in Runs $9 A, 9 A 1$ and $9 B$. Also, recycled caustic was fed with coal in Run $9 B$. 
TABLE 3-1. TEST MATRIX: VARIABLE-BY-VARIABLE (CC:YTINUED)

PITT 8 (Powhatan No. 6 mine)

$\mathrm{KOH} / \mathrm{NaOH}=1$

Caust $\mathrm{ic} / \mathrm{COB}=3$

$\begin{array}{lrr}\text { Run } & 3 C 1 & 7 C \\ \text { Temp, C } & 438 & 427 \\ \text { Res Time, hr } & 2.0 & 2.1 \\ \text { Kiln RPM } & 1 & 0.8 \\ \text { Auger Speed, X max } & 30 & 30 \\ \text { Auger sec F/sec B } & 20 / 10 & 20 / 10\end{array}$

* Nitrogen flow was countercurrent to 6 mesh coal feed in Run $7 C$.

PITT 8 (Powhatan No. 6 mine)

$\mathrm{KOH} / \mathrm{MaOH}=0$

Caustic/Coal $=0$

$\begin{array}{lr}\text { Run } & 4 A \\ \text { Temp, C } & 410 \\ \text { Res Time, hr } & 2.0 \\ \text { Kiln RPM } & 1 \\ \text { Auger Speed, X max } & 30 \\ \text { Auger sec F/sec B } & 20 / 10\end{array}$

PITT 8 (Powhatan No. 6 mine)

$\mathrm{KOH} / \mathrm{MaOH}=0$

Caustic/Coal $=1$

$\begin{array}{lrr}\text { Run } & 6 A 1 & 6 C 1 \\ \text { Temp, C } & 427 & 410 \\ \text { Res Time, hr } & 2.0 & 2.0 \\ \text { Kiln RPM } & 1 & 1 \\ \text { Auger Speed, X max } & 30 & 30 \\ \text { Auger sec F/sec B } & 20 / 10 & 20 / 10\end{array}$

PITT 8 (Powhatan No. 6 mine)

$\mathrm{KOH} / \mathrm{MaOH}=0$

Caustic/Coal $=1.5$

$\begin{array}{lrrrrr}\text { Run } & 681 & 601 & 8 C & 80 & 8 E \\ \text { Temp, C } & 427 & 410 & 427 & 442 & 412 \\ \text { Res Time, hr } & 2.0 & 2.0 & 2.1 & 2.1 & 2.1 \\ \text { Kiln RPM } & 1 & 1 & 0.8 & 0.8 & 0.8 \\ \text { Auger Speed, X max } & 30 & 30 & 30 & 30 & 30 \\ \text { Auger sec F/sec B } & 20 / 10 & 20 / 10 & 20 / 10 & 20 / 10 & 20 / 10\end{array}$

*Nitrogen flow was countercurrent to 6 mesh coal feed in Runs $8 C, 80$ and $8 E$. 


\section{TABLE 3-1. TEST MATRIX: VARIABLE-BY-VARIABLE (CONTINUED)}

PITT 8 (Powhatan No. 6 mine)

$\mathrm{KOH} / \mathrm{NaOH}=\mathrm{O}$

Caustic/COal=2

$\begin{array}{lrrrrrrr}\text { Run } & 4 C 1 & 4 C 2 & 4 C 3 & 4 C 4 & 4 C 5 & 4 C 6 & 4 C 7 \\ \text { Temp, C } & 413 & 416 & 420 & 423 & 430 & 435 & 441 \\ \text { Res Time, hr } & 2.0 & 2.0 & 2.0 & 1.5^{\star} & 1.0^{\star} & 1.0^{\star} & 1.0^{\star} \\ \text { Kiln RPM } & 1 & 1 & 1 & 1 & 1 & 1 & 1 \\ \text { Auger Speed, X max } & 30 & 30 & 30 & 30 & 30 & 30 & 30 \\ \text { Auger sec F/sec B } & 20 / 10 & 20 / 10 & 20 / 10 & 20 / 10 & 20 / 10 & 20 / 10 & 20 / 10\end{array}$

* Residence time based on heated zones only.

PITT 8 (Powhatan No. 6 mine)

$\mathrm{KOH} / \mathrm{NaOH}=0$

Caustic/Coal $=2$

\begin{tabular}{|c|c|c|c|c|c|c|c|c|c|c|}
\hline Run & 581 & 582 & 583 & 584 & $5 B 5$ & $8 F$ & $10 A^{\star \star}$ & $108 * *$ & $10 c^{\star \star}$ & $100 * \star$ \\
\hline Temp, C & 413 & 420 & 423 & 430 & 427 & 412 & 380 & 380 & 360 & 400 \\
\hline Res Time, hr & 2.0 & 2.0 & $1.5^{\star}$ & $1.0 *$ & 2.0 & 2.1 & 2.0 & 2.0 & 2.0 & 2.0 \\
\hline$K i \ln R P M$ & 1 & 1 & 1 & 1 & 1 & 0.8 & 1 & 1 & 1 & 1 \\
\hline Auger speed, $x$ max & 30 & 30 & 30 & 30 & 30 & 30 & 30 & 30 & 30 & 30 \\
\hline Auger sec $F / \sec B$ & $20 / 10$ & $20 / 10$ & $20 / 10$ & $20 / 10$ & $20 / 10$ & $20 / 10$ & $20 / 10$ & $20 / 10$ & $20 / 10$ & $20 / 10$ \\
\hline
\end{tabular}

* Residence time based on heated zones only.

* Weathered (3-year old) coal was used in Runs 10A, 10B, 10C and 100.

Nitrogen flow was countercurrent to coal feed in Runs 8F, 10A, 108, $10 \mathrm{C}$ and 100.

PITT 8 (Powhatan No. 6 mine)

$\mathrm{KOH} / \mathrm{NaOH}$ :

Caustic/Coal $=2.5$

$\begin{array}{lr}\text { Run } & 9 C \\ \text { Temp, C } & 438 \\ \text { Res Time, hr } & 2.1 \\ \text { Kiln RPM } & 0.8 \\ \text { Auger Speed, \% max } & 30 \\ \text { Auger sec F/sec B } & 20 / 10\end{array}$

- Nitrogen flow was countercurrent to coal feed in Run $9 C$.

KENT 9

$\mathrm{KOH} / \mathrm{NaOH}=1$

Caustic/Coal $=2$

$\begin{array}{lrr}\text { Run } & 2 D & 2 E \\ \text { Temp, C } & 427 & 438 \\ \text { Res Time, hr } & 2.0 & 2.0 \\ \text { Kiln RPM } & 1 & 1 \\ \text { Auger Speed, \% max } & 30 & 30 \\ \text { Auger sec F/sec B } & 20 / 10 & 20 / 10\end{array}$




\section{TABLE 3-1. TEST MATRIX: VARIABLE-BY-VARIABLE (CONTINUED)}

KENT 9

$\mathrm{KOH} / \mathrm{NaOH}=0$

Caust ic/Coal $=2$

$\begin{array}{lrrrrr}\text { Run } & 5 C 5 & 5 C 4 & 5 D 1 & 502 & 503 \\ \text { Temp, C } & 427 & 427 & 427 & 399 & 371 \\ \text { Res Time, hr } & 2.0 & 1.5^{\star} & 1.0^{\star} & 2.0 & 2.0 \\ \text { Kiln RPM } & 1 & 1 & 1 & 1 & 1 \\ \text { Auger Speed, X max } & 30 & 30 & 30 & 30 & 30 \\ \text { Auger sec F/sec B } & 20 / 10 & 20 / 10 & 20 / 10 & 20 / 10 & 20 / 10\end{array}$

- Residence time based on heated zones only.

KENT 9

$\mathrm{KOH} / \mathrm{NaOH}=1$

Caustic/Coal $=2.5$

$\begin{array}{lr}\text { Run } & \text { TA } \\ \text { Temp, C } & 427 \\ \text { Res Time, hr } & 2.1 \\ \text { Kiln RPM } & 0.8 \\ \text { Auger Speed, X max } & 30 \\ \text { Auger sec F/sec B } & 20 / 10\end{array}$

*Nitrogen flow was countercurrent to coal feed in Run 7A.

KENT 9

$\mathrm{KOH} / \mathrm{NaOH}=1$

Caustic/Coal $=3$

$\begin{array}{lr}\text { Run } & 78 \\ \text { Temp, C } & 427 \\ \text { Res Time, hr } & 2.1 \\ \text { Kiln RPM } & 0.8 \\ \text { Auger Speed, X max } & 30 \\ \text { Auger sec F/sec B } & 20 / 10\end{array}$

*Nitrogen flow was countercurrent to coal feed in Run $7 B$.

PITT 8 (CONSOL's Blacksville No. 2 mine)

$\mathrm{KOH} / \mathrm{NaOH}=0$

Caustic/Coal $=2.5$

$\begin{array}{lrrrrr}\text { Run } & 11 A & 11 B & 11 C & 110 & 11 E \\ \text { Temp, C } & 424 & 434 & 434 & 434 & 434 \\ \text { Res Time, hr } & 2.0 & 2.0 & 2.0 & 2.0 & 2.0 \\ \text { Kiln RPM } & 1 & 1 & 1 & 1 & 1 \\ \text { Auger Speed, X max } & 30 & 30 & 30 & 30 & 30 \\ \text { Auger sec F/sec B } & 20 / 10 & 20 / 10 & 20 / 10 & 20 / 10 & 20 / 10\end{array}$

"Nitrogen flow was countercurrent to coal feed in Runs 11A, 118, 11C, 110 and $11 E$. 


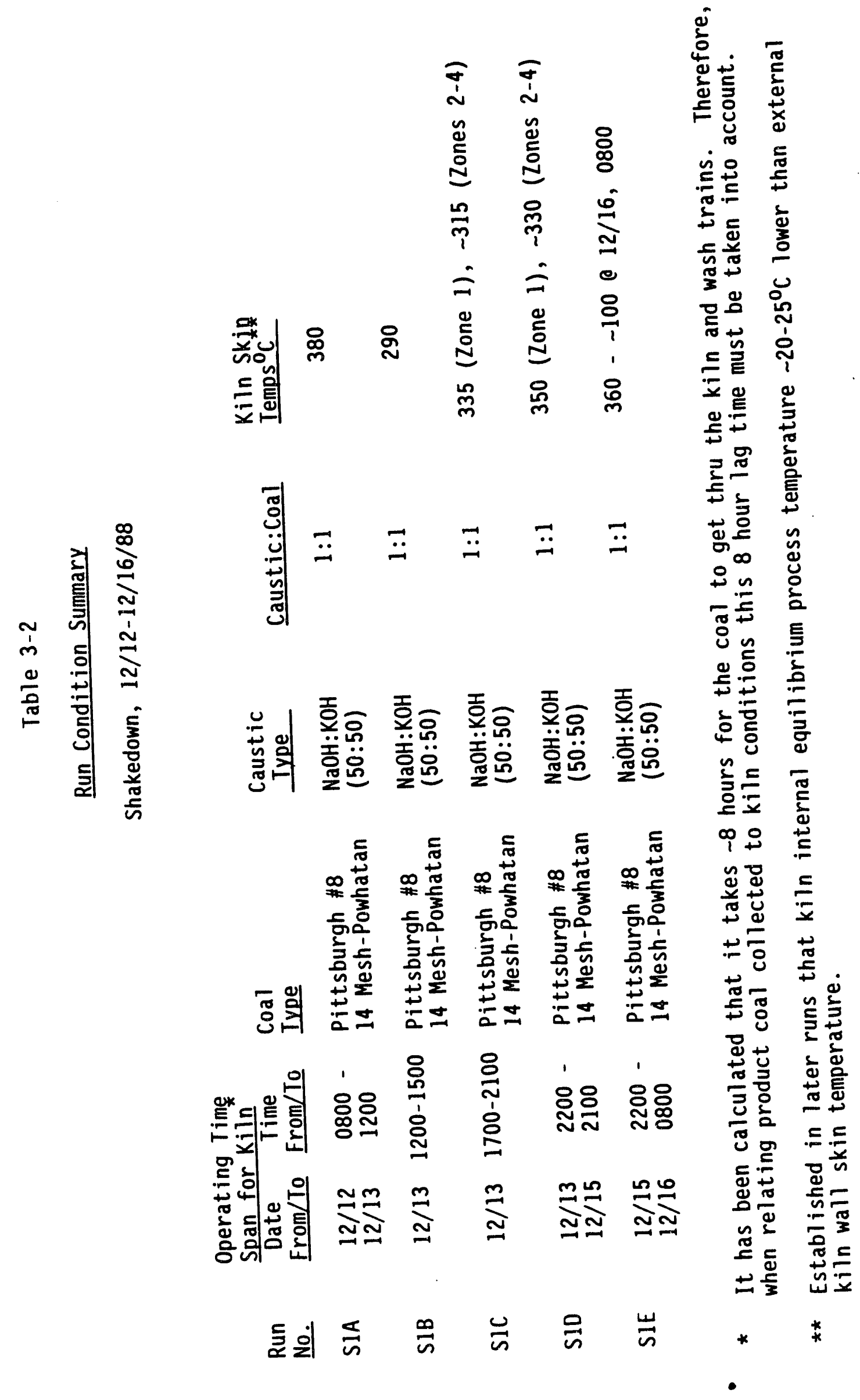




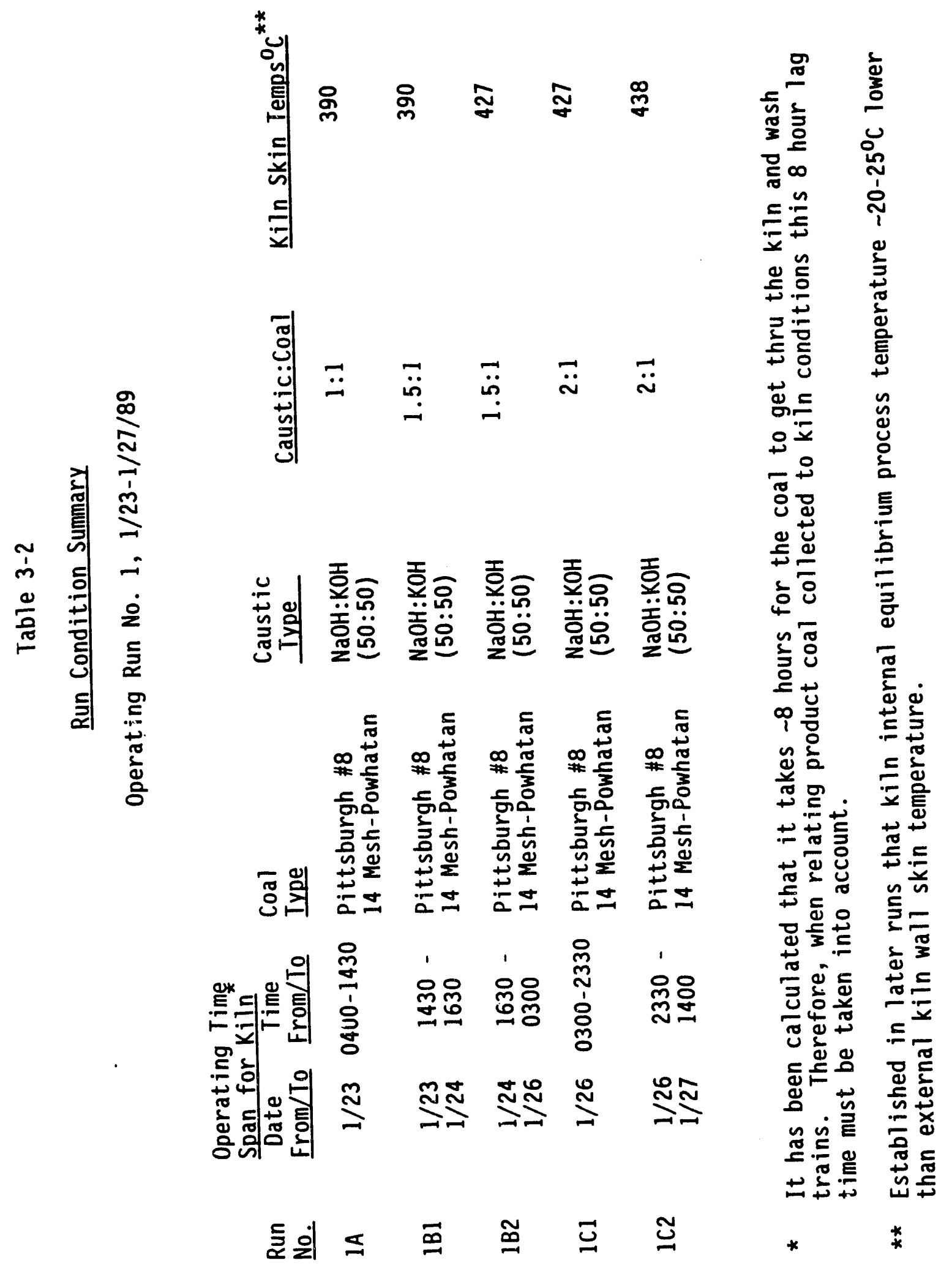




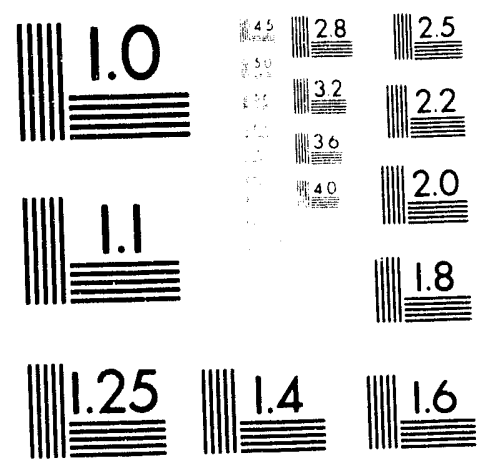



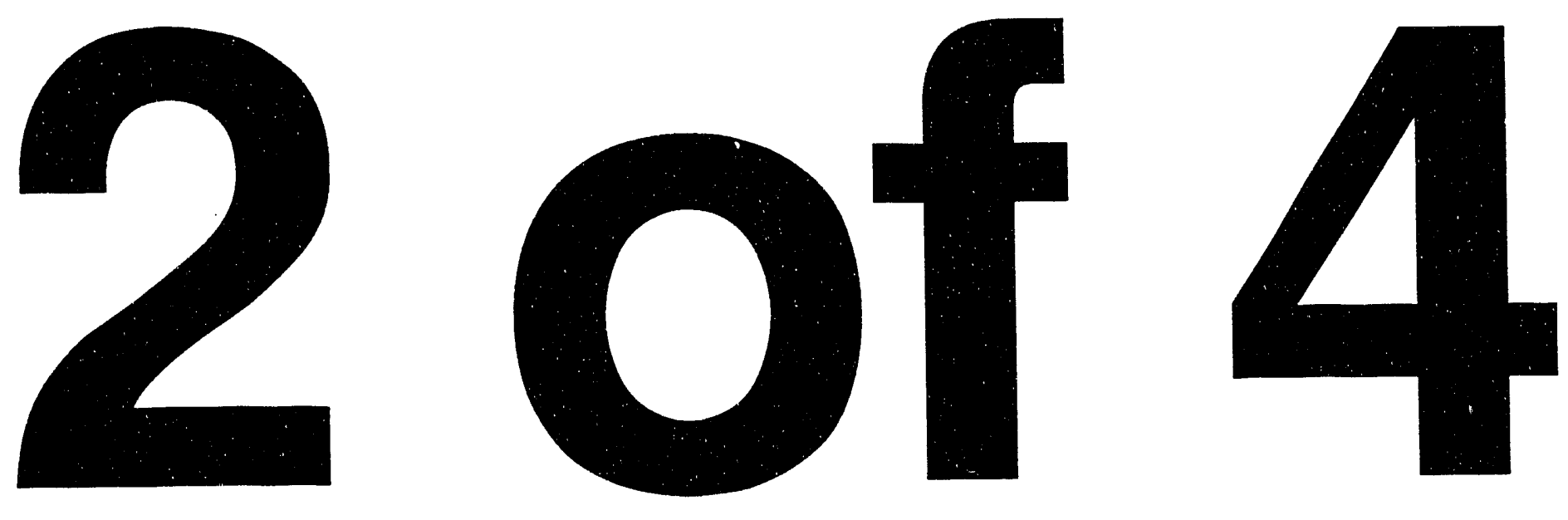


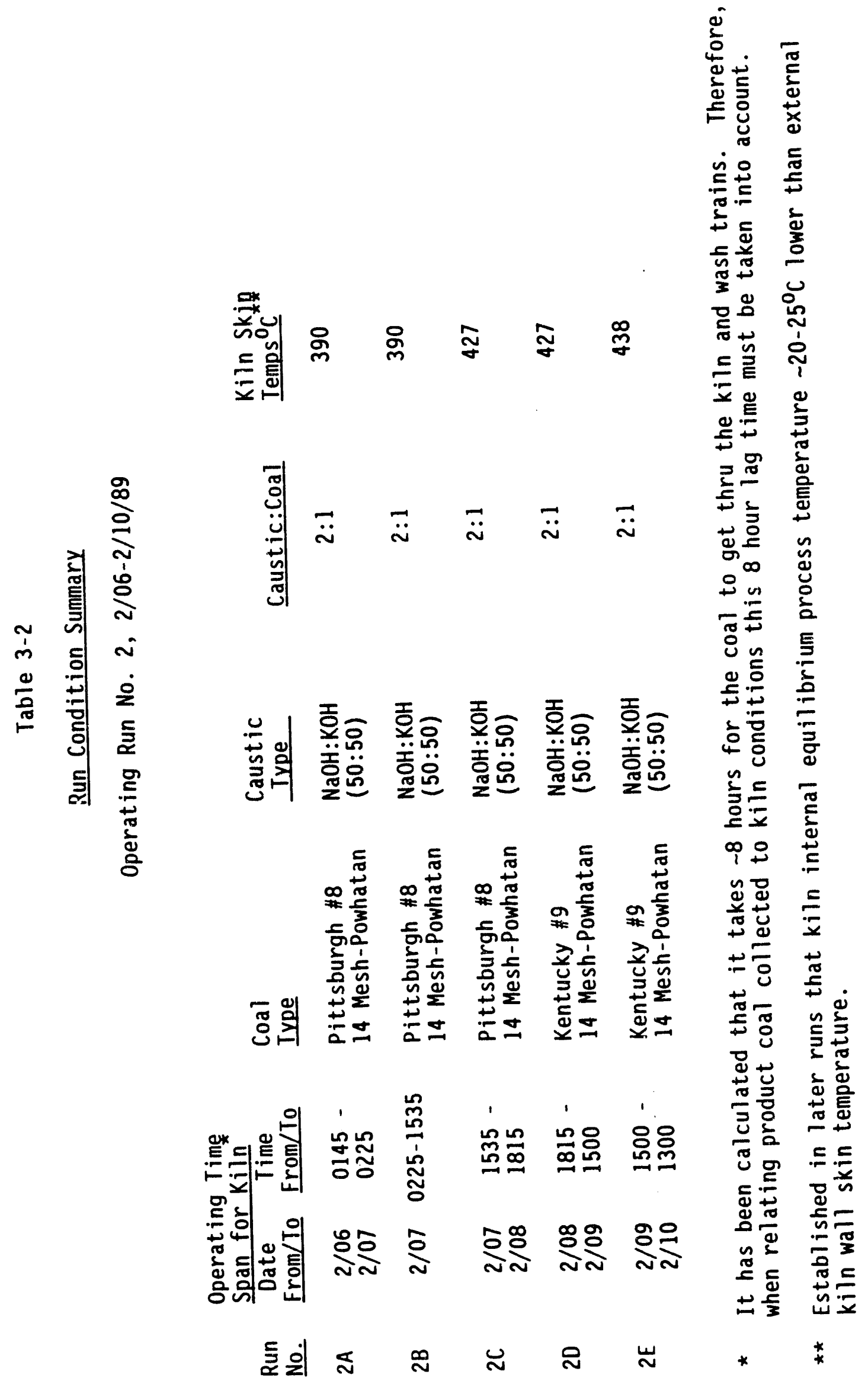



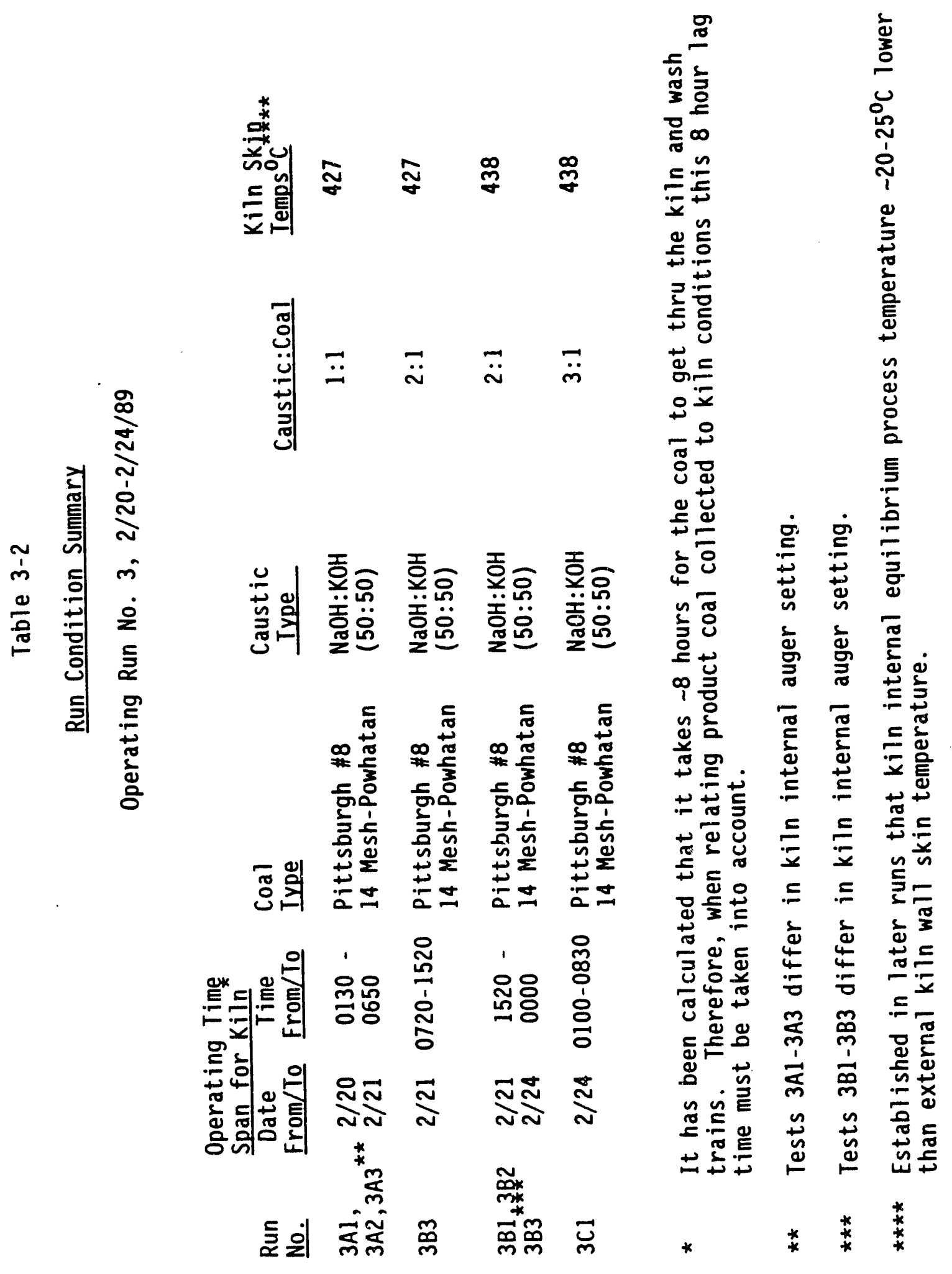


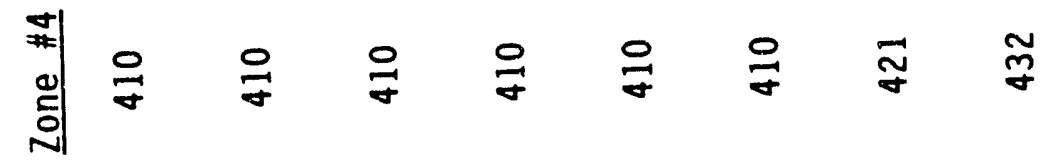

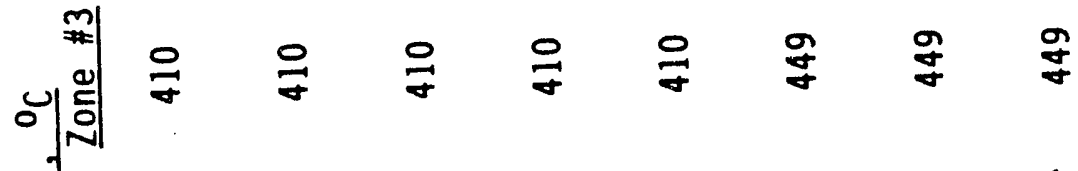

$$
\begin{aligned}
& \text { : }
\end{aligned}
$$

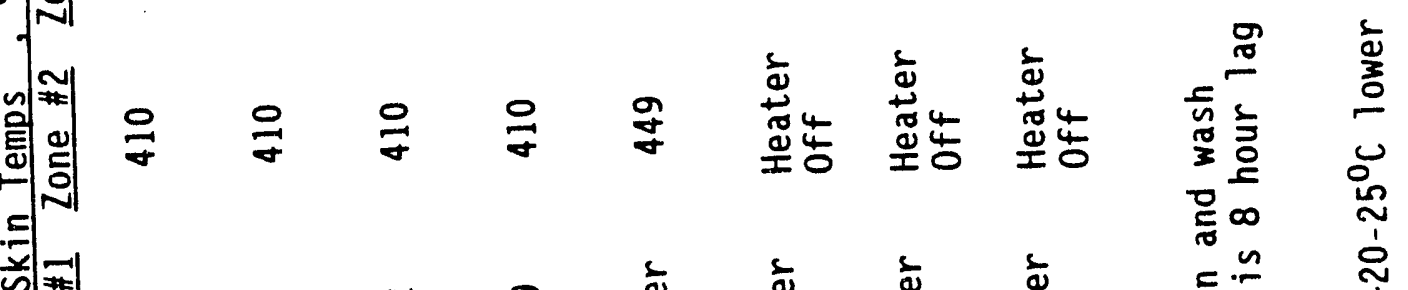

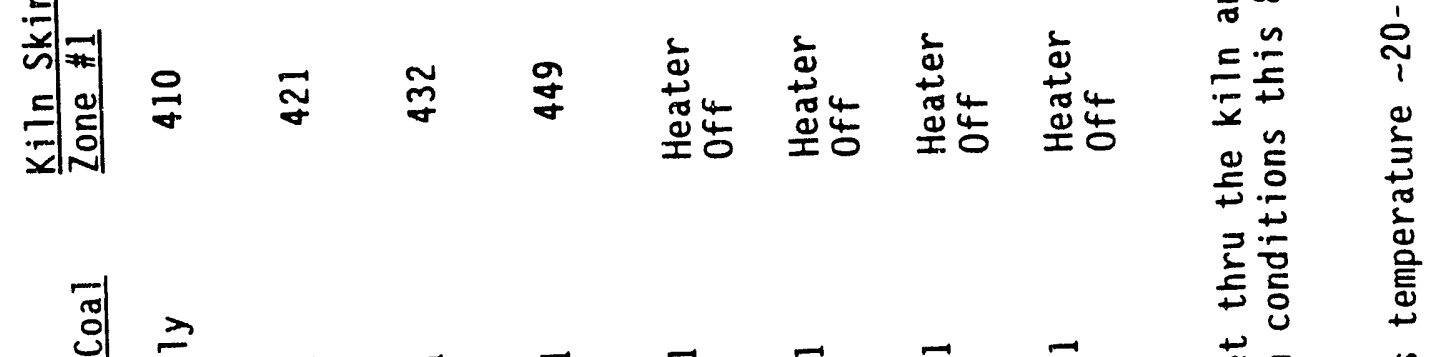

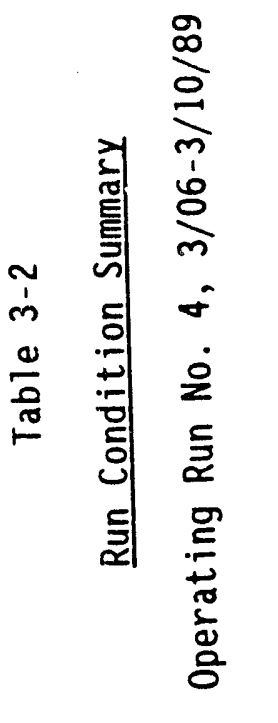

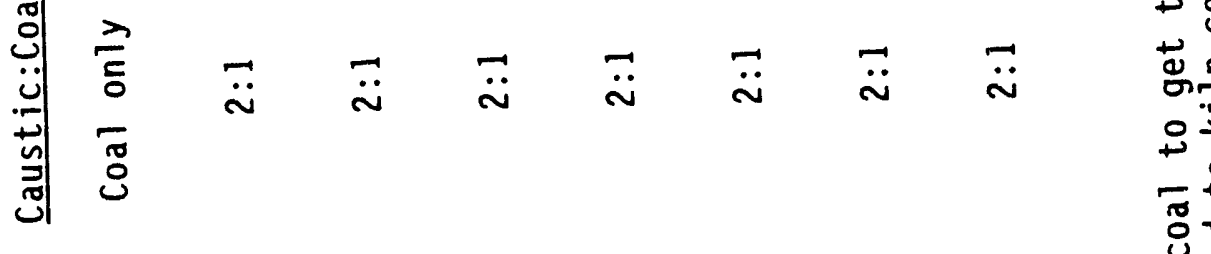

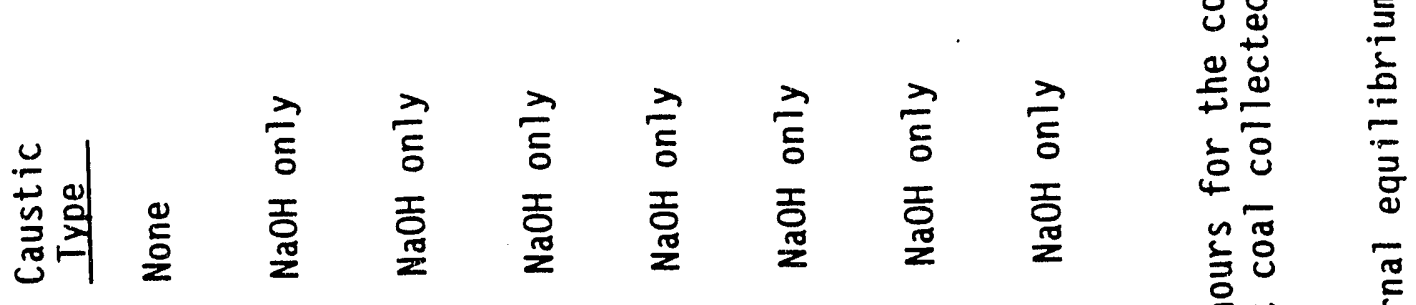

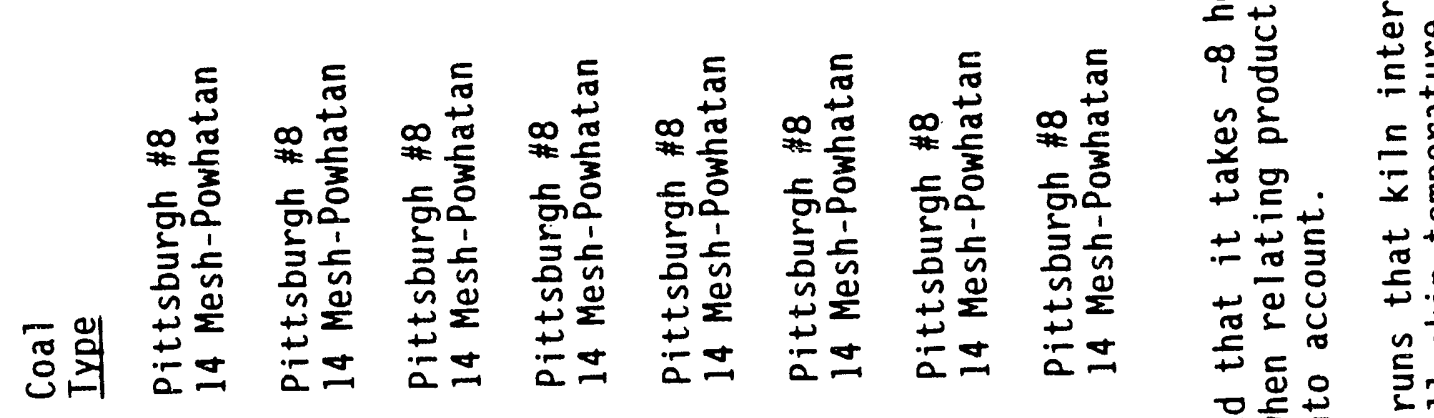

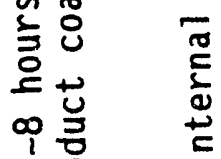

$$
\begin{aligned}
& \text { 兘市 } \\
& \text { 造宁 }
\end{aligned}
$$

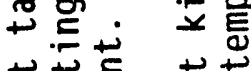

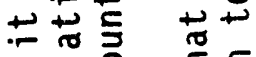

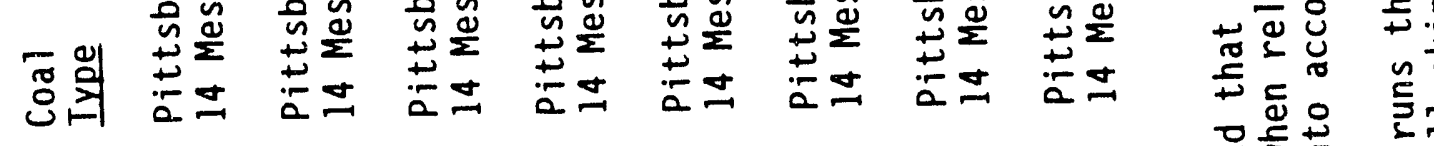

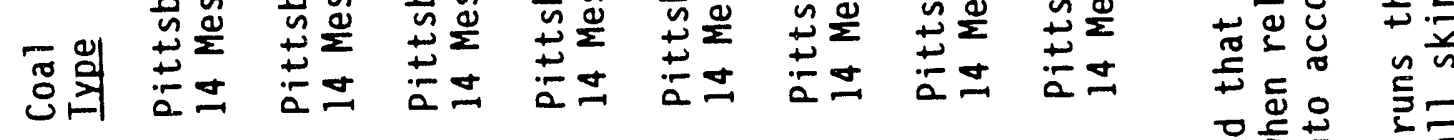

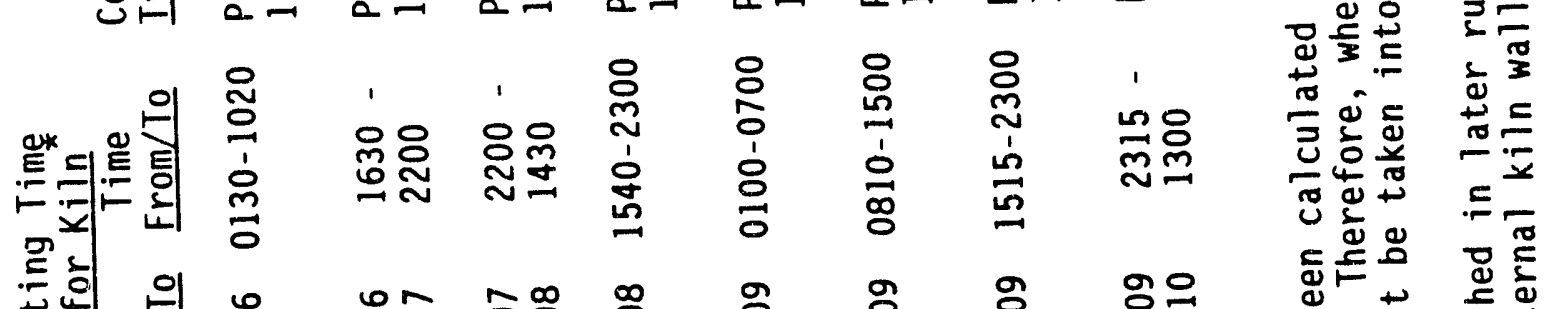

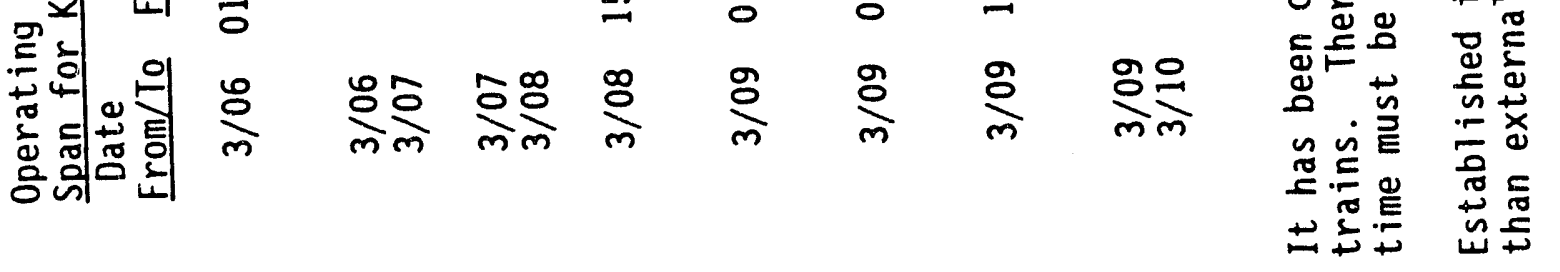

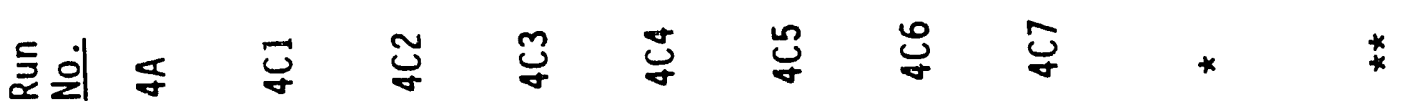




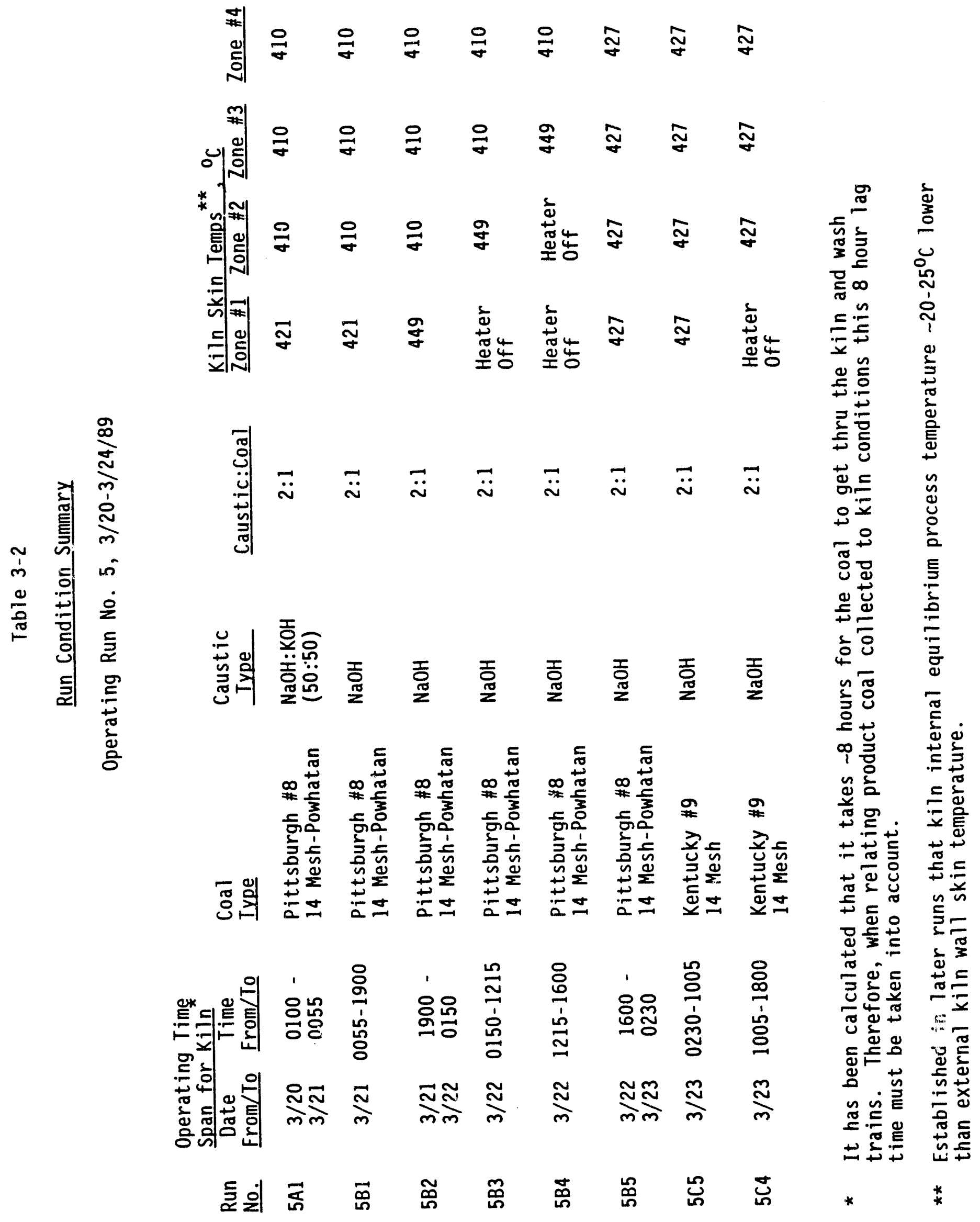




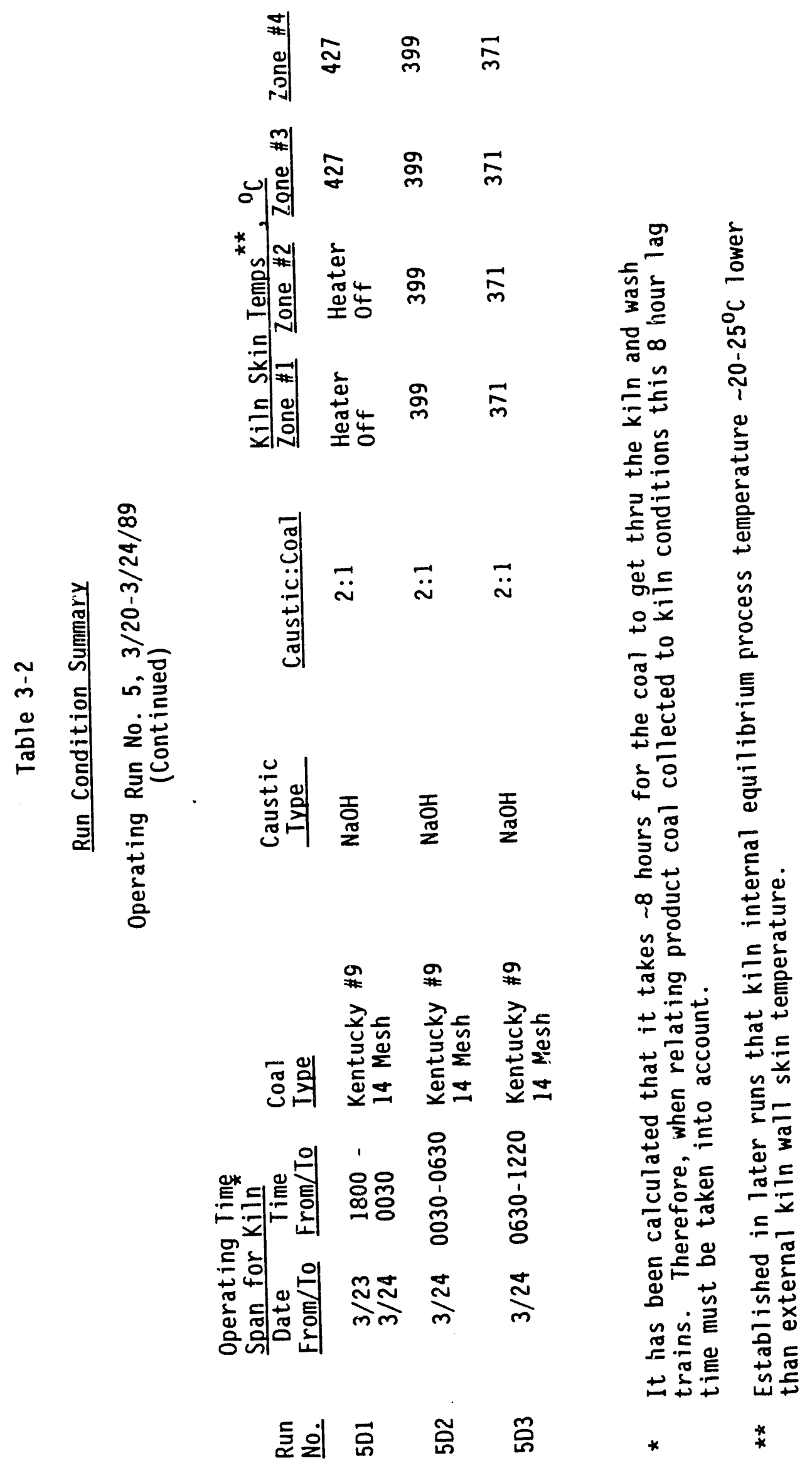




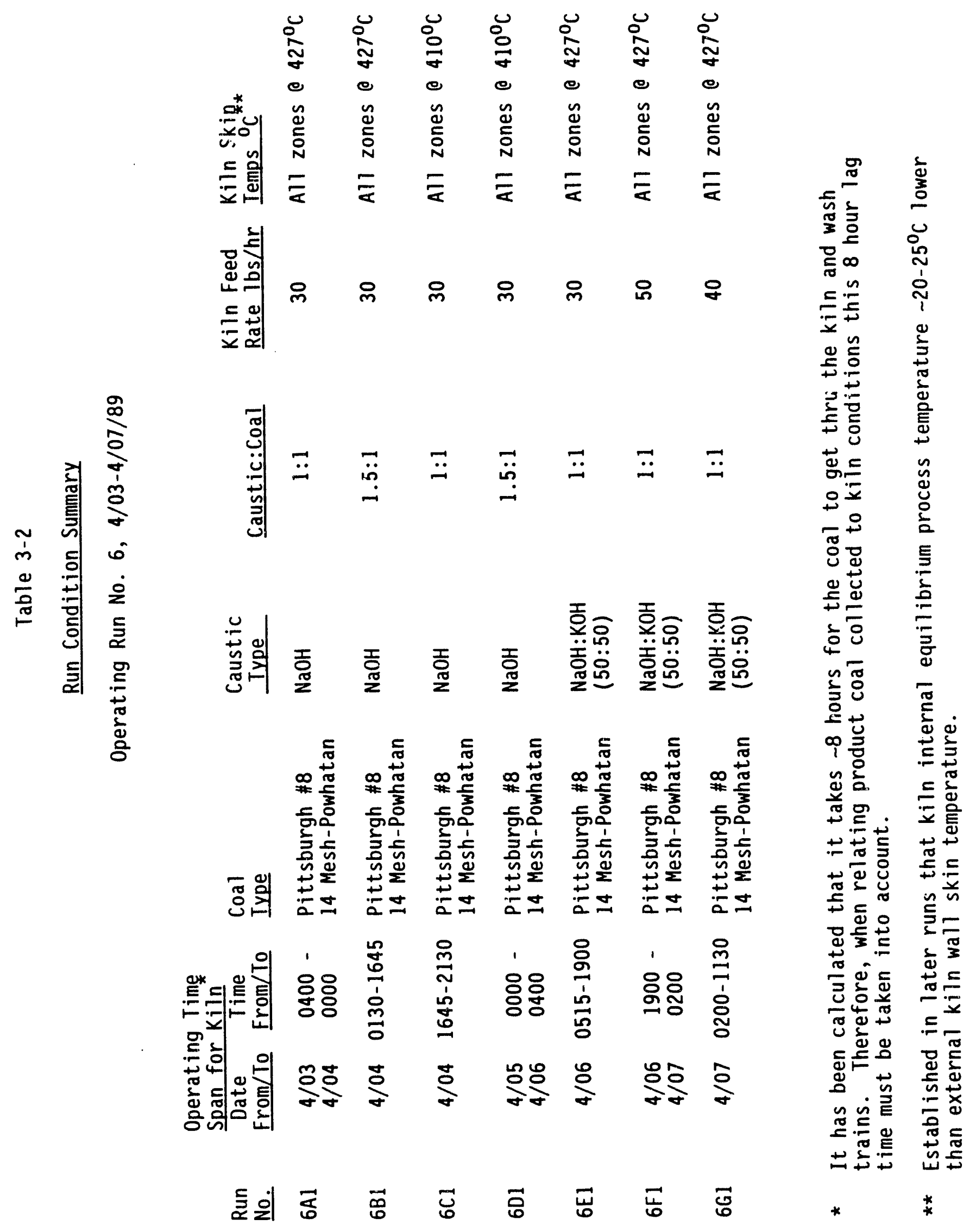




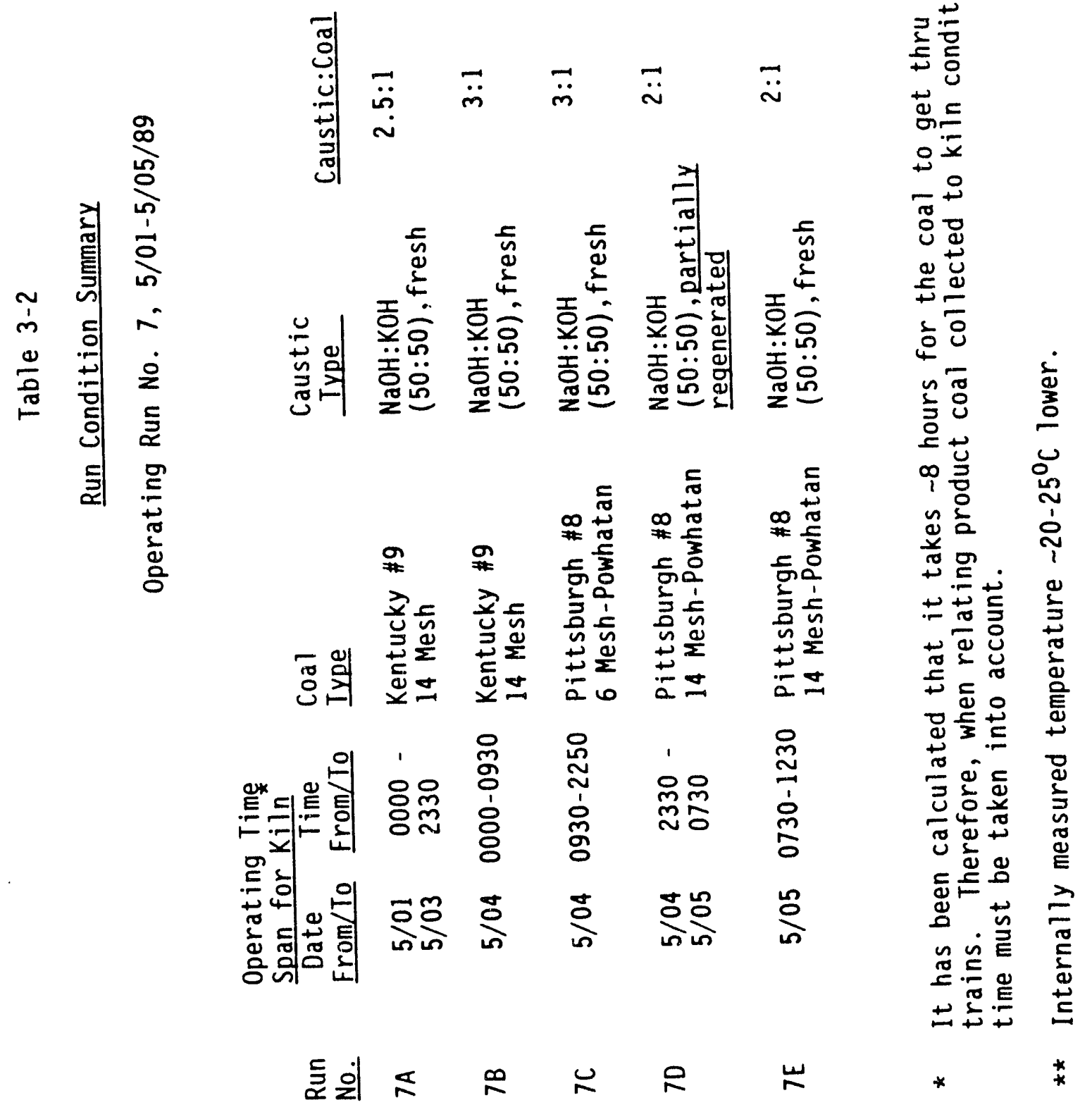

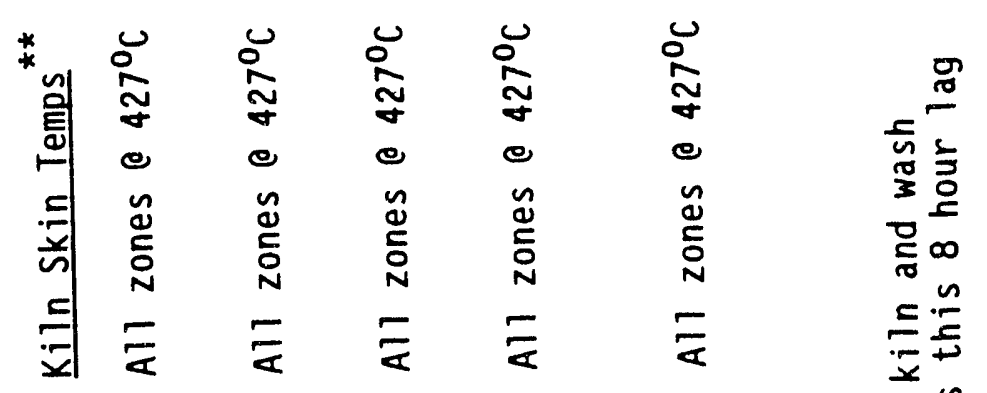

통 

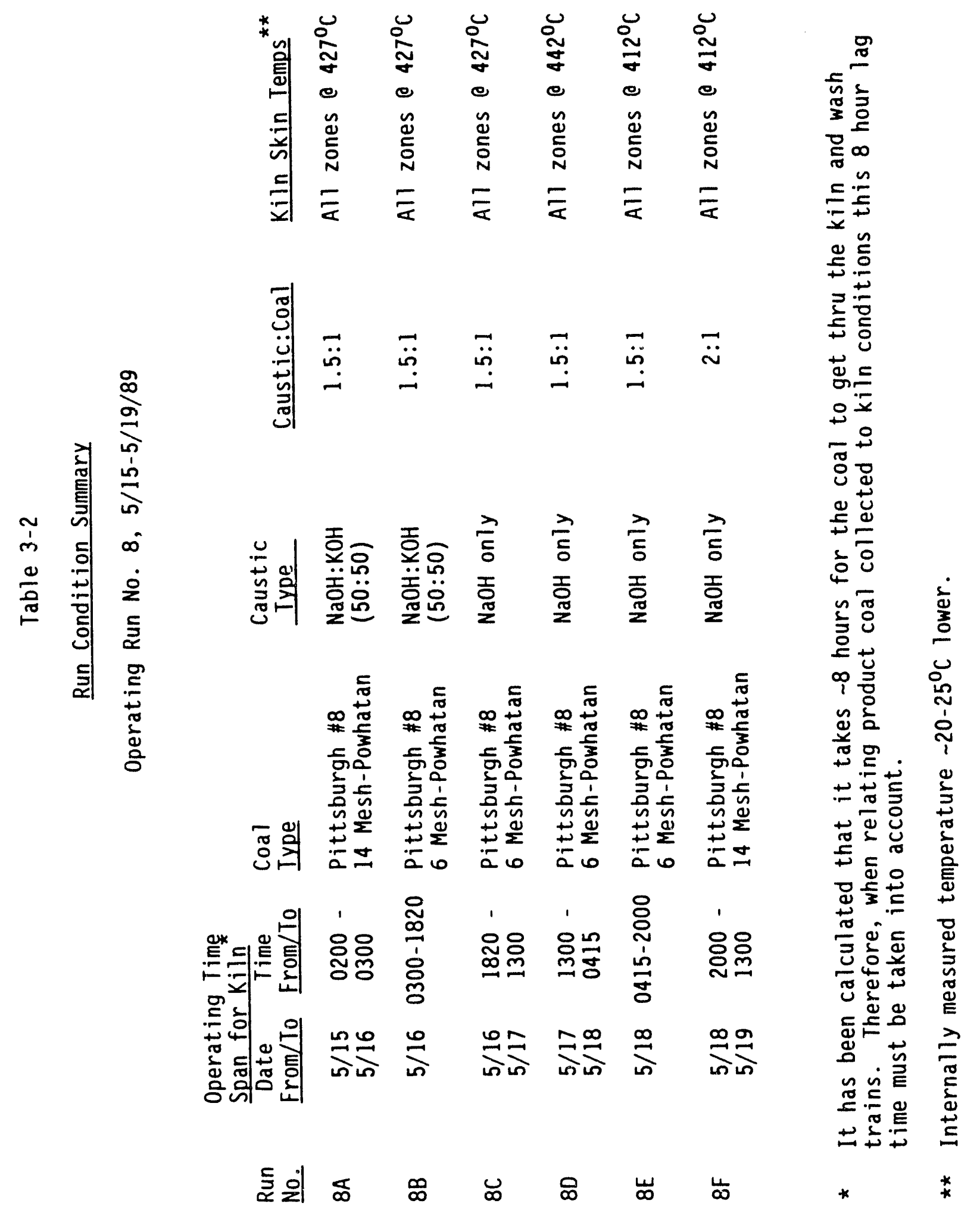

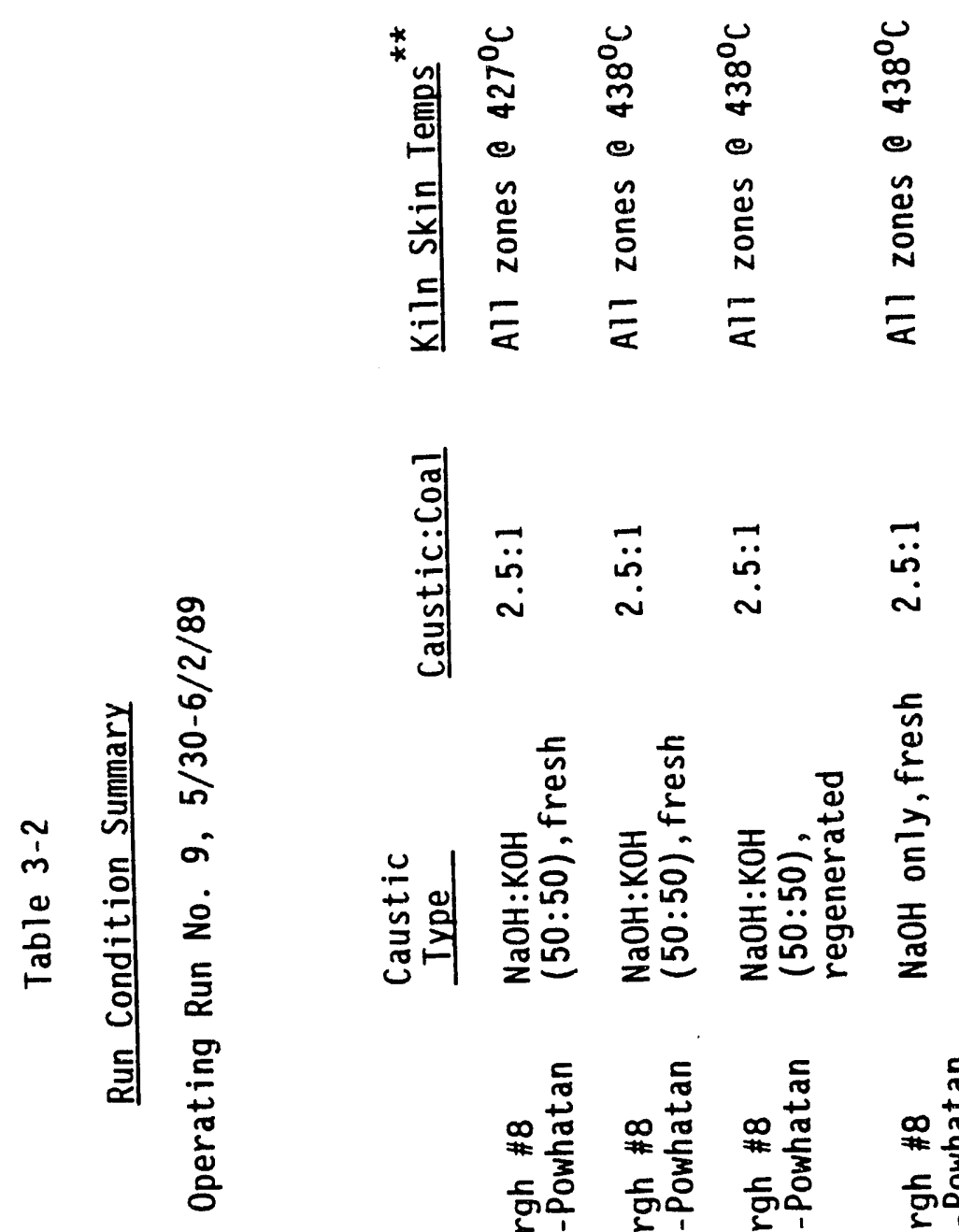

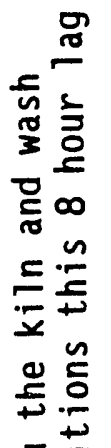

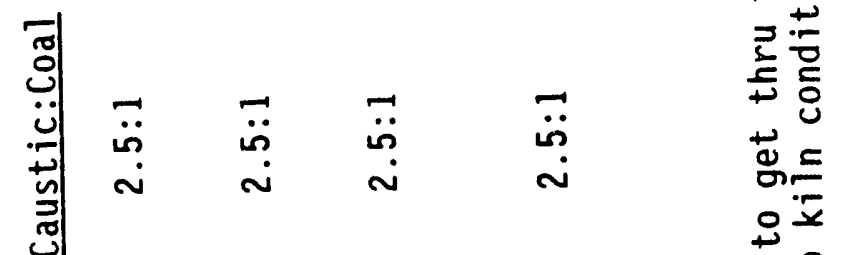

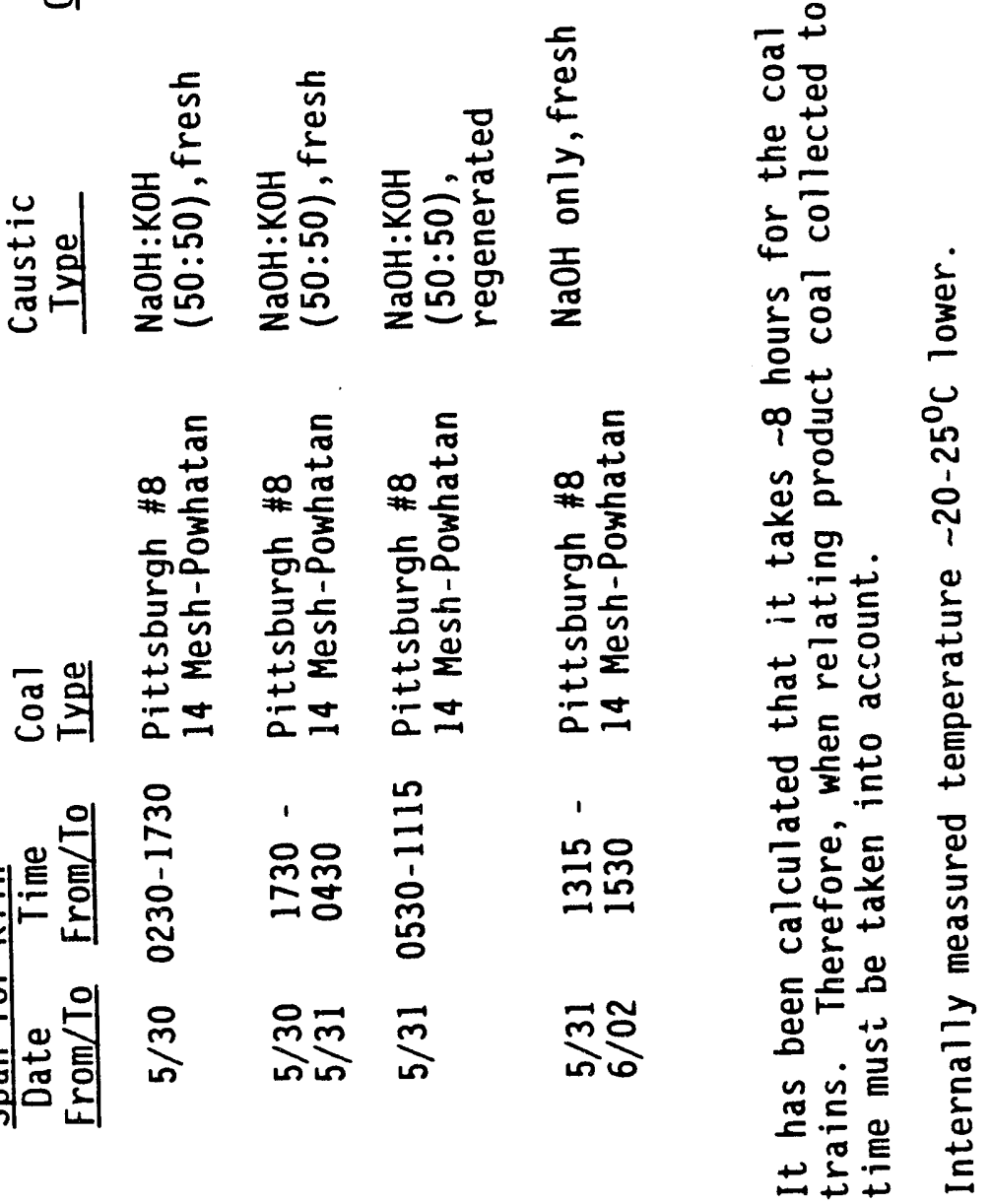

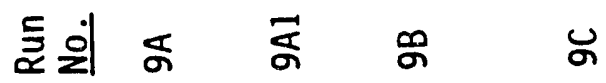




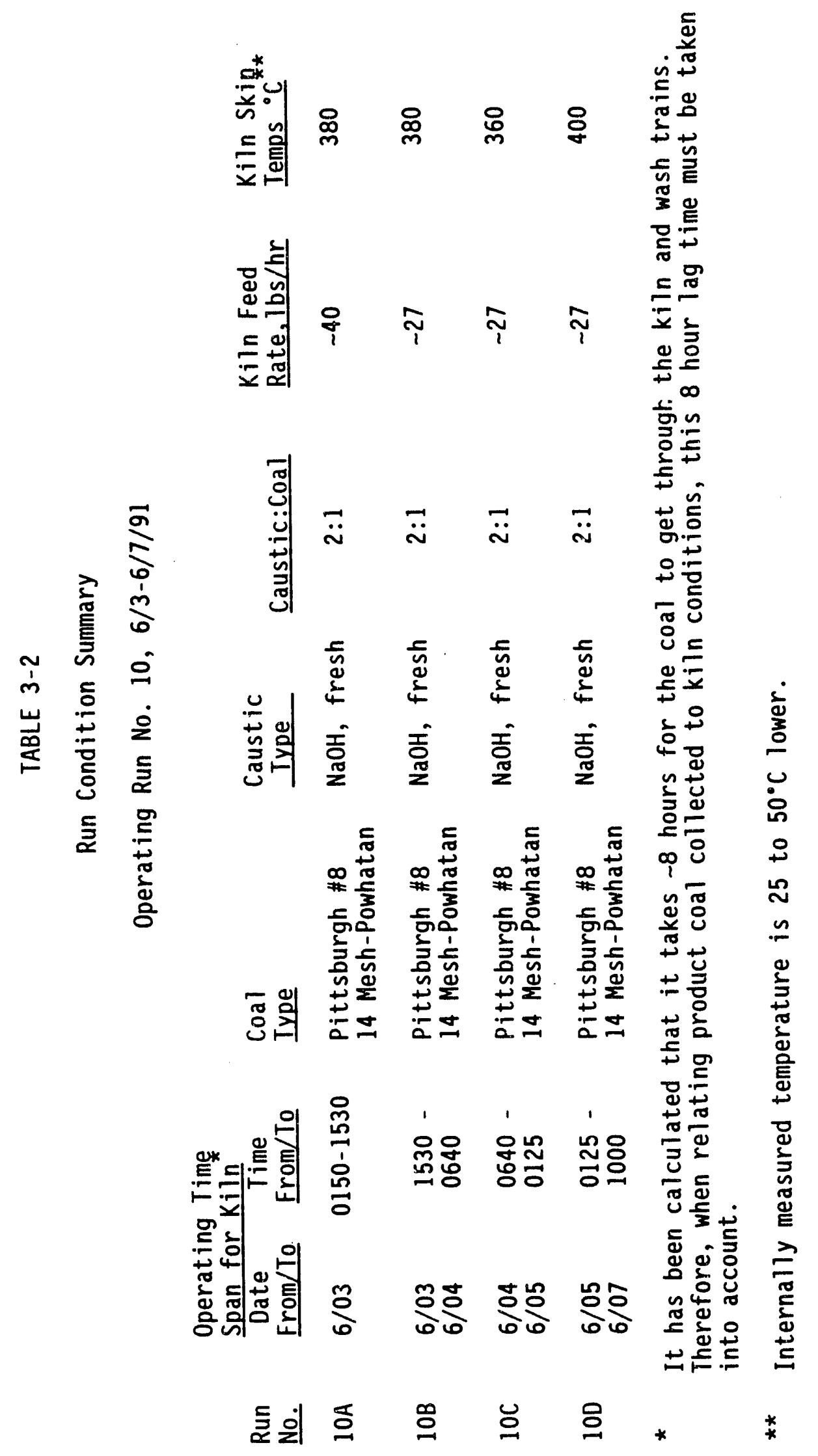




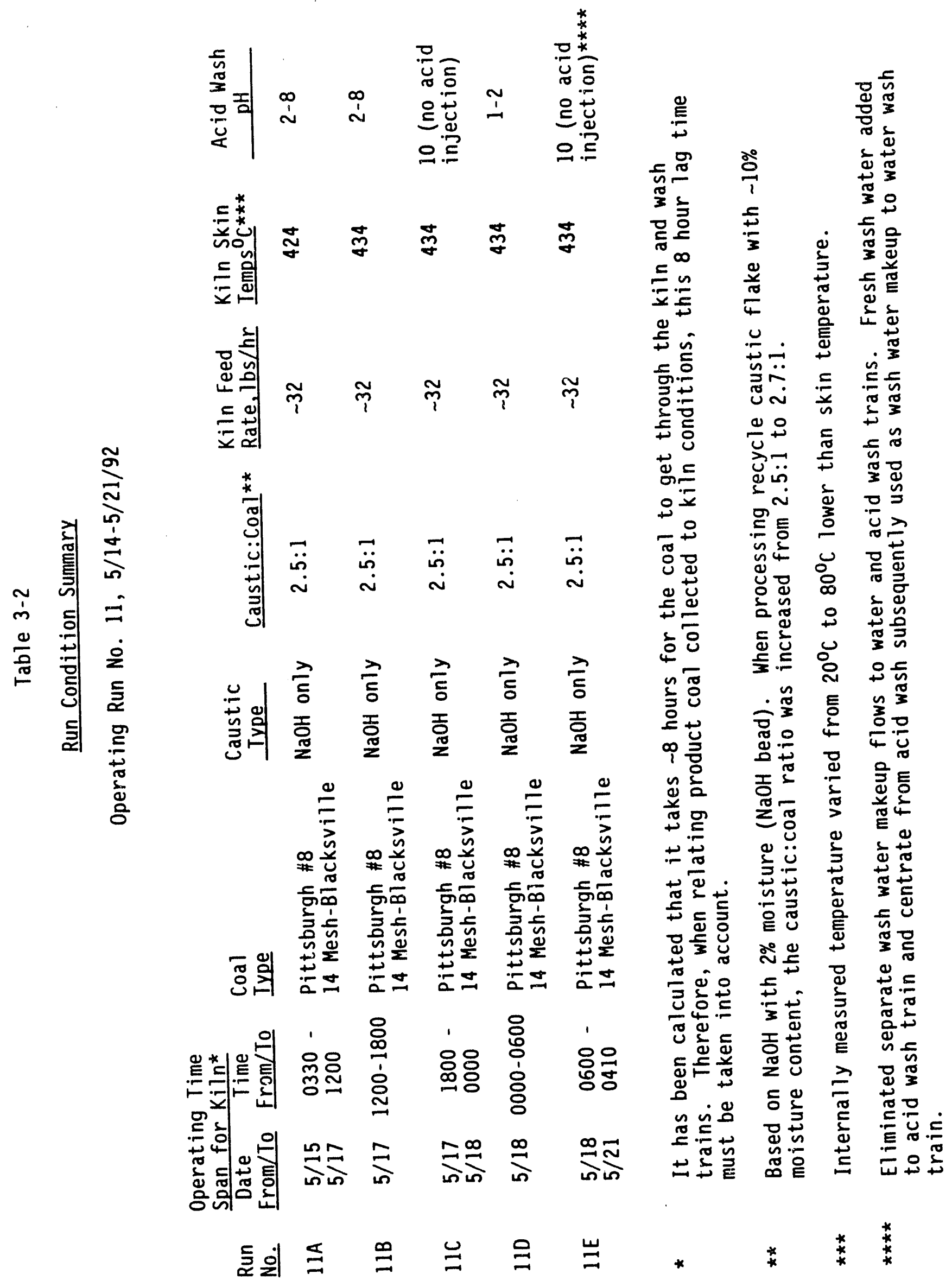


Figure 3-1. Sulfur and Ash Data for Kiln and Product Coal as a Function of Coal Exit Time 


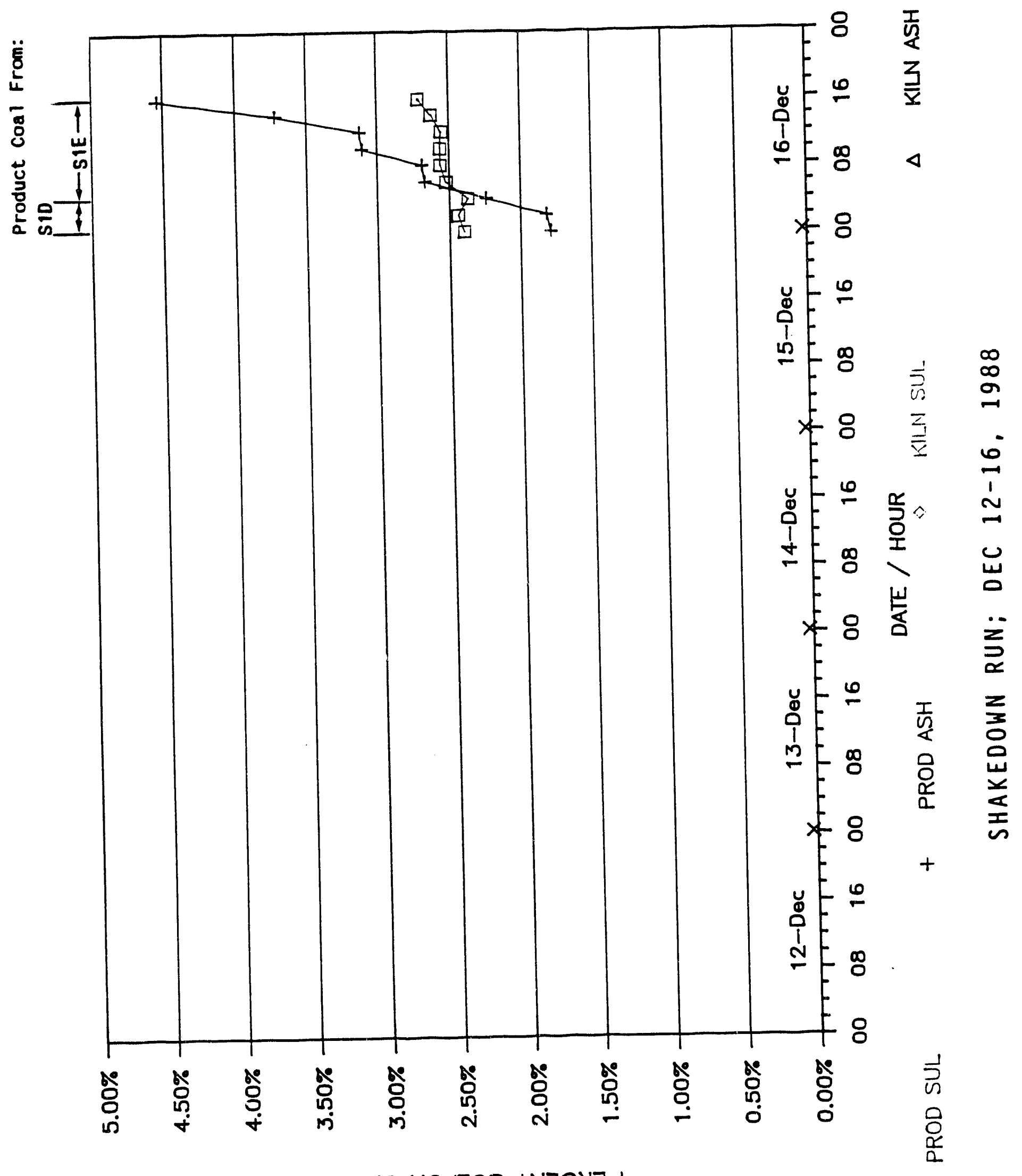

HS $\forall$ do $\forall$ ชก 


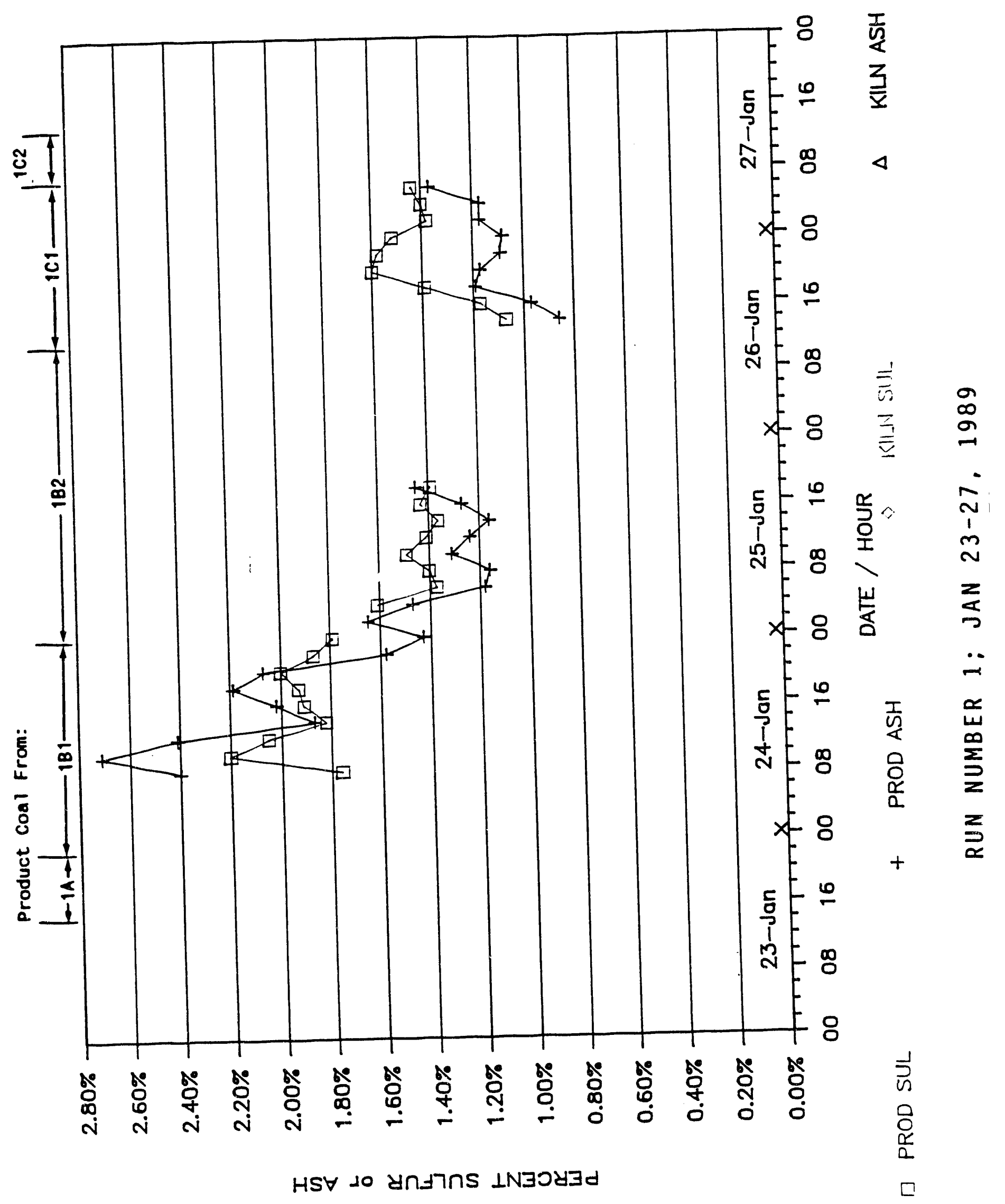




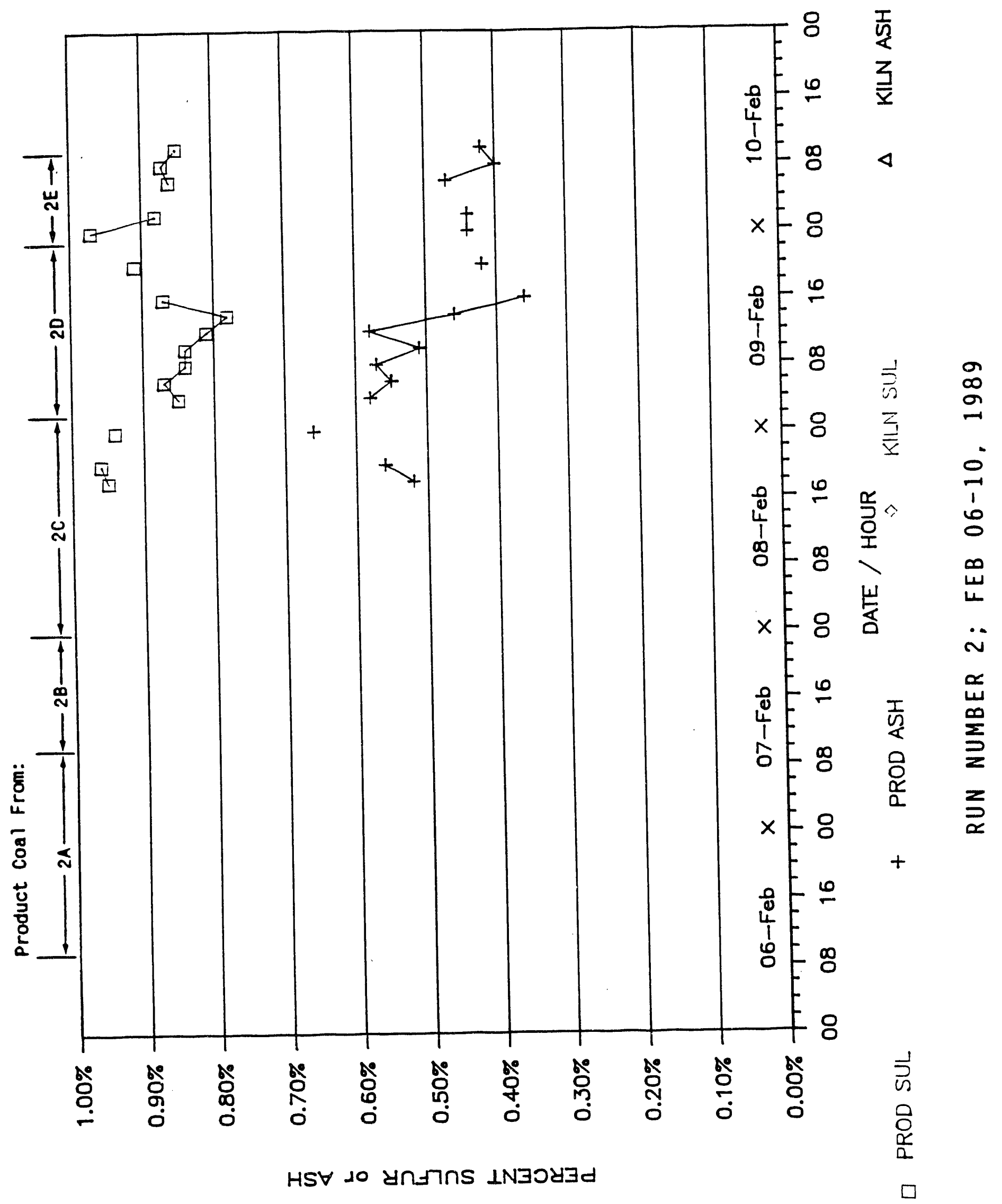




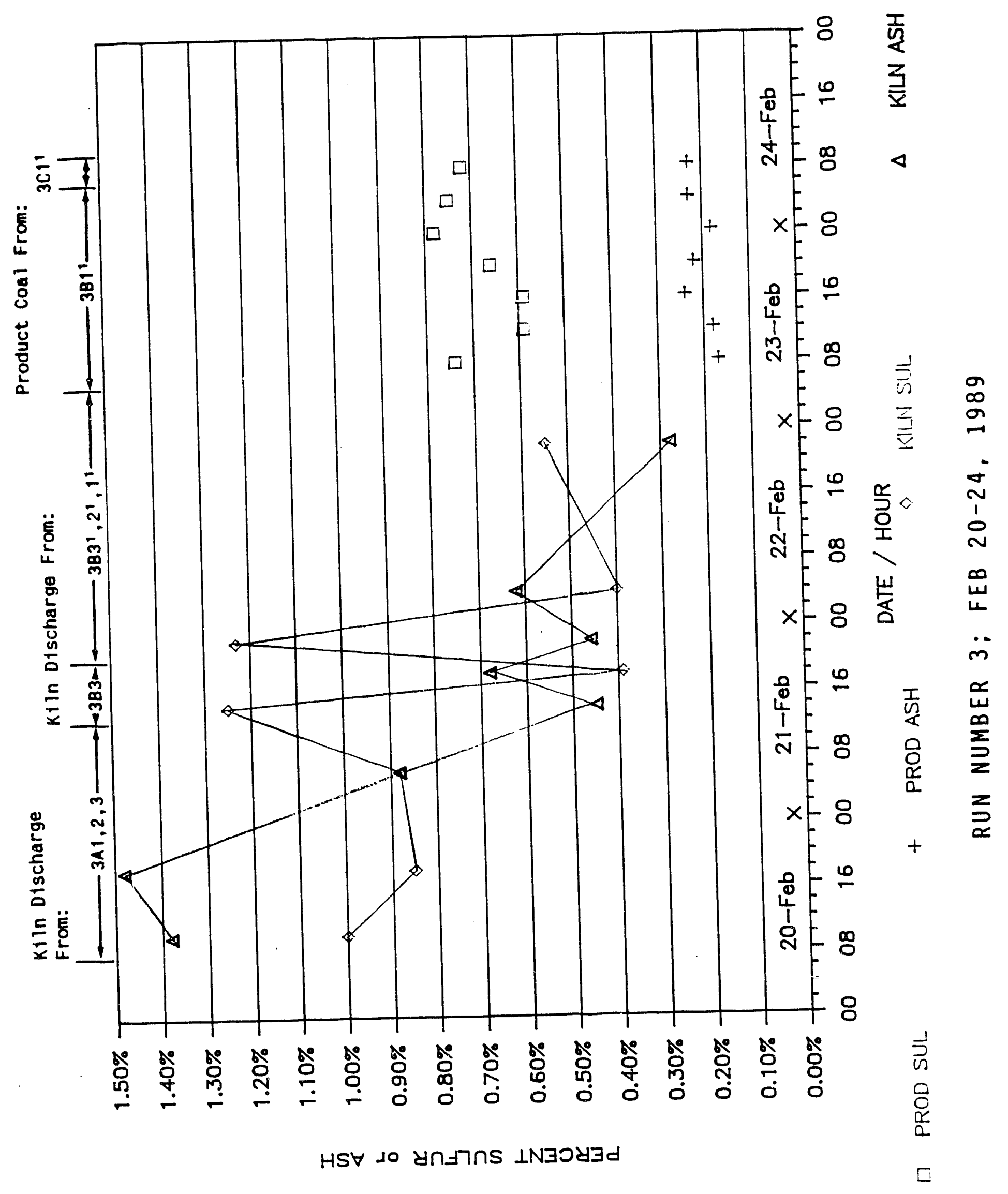




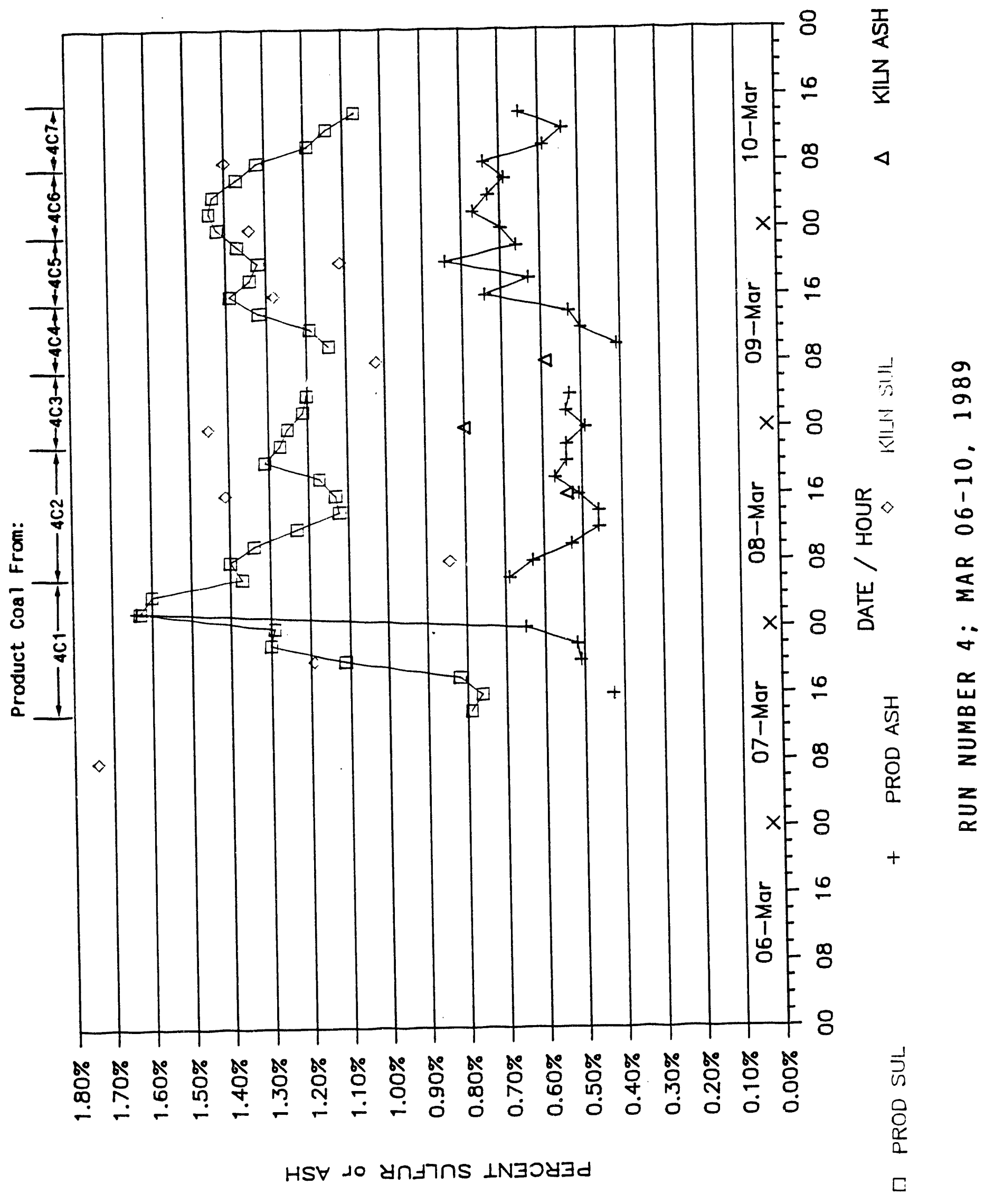




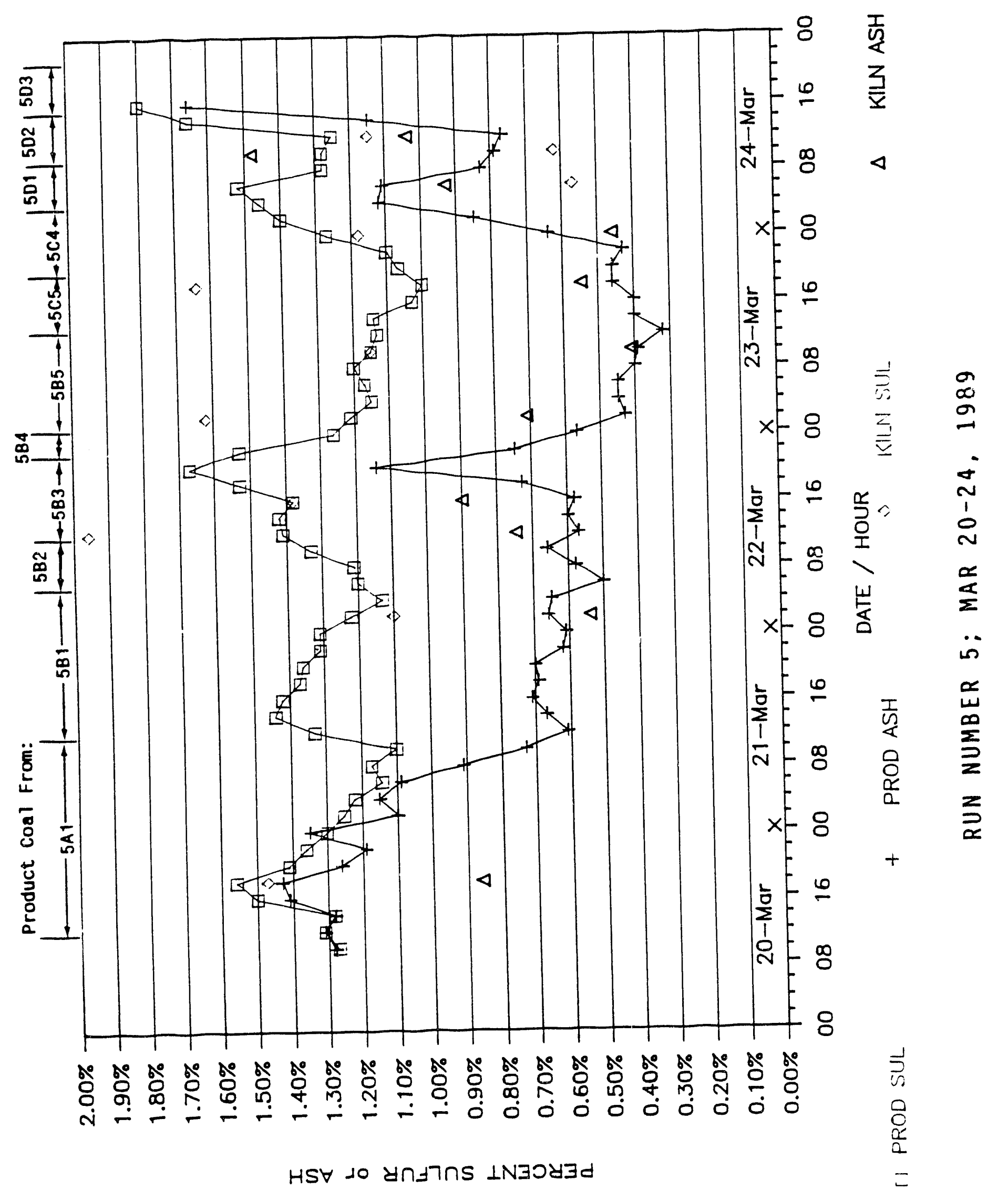




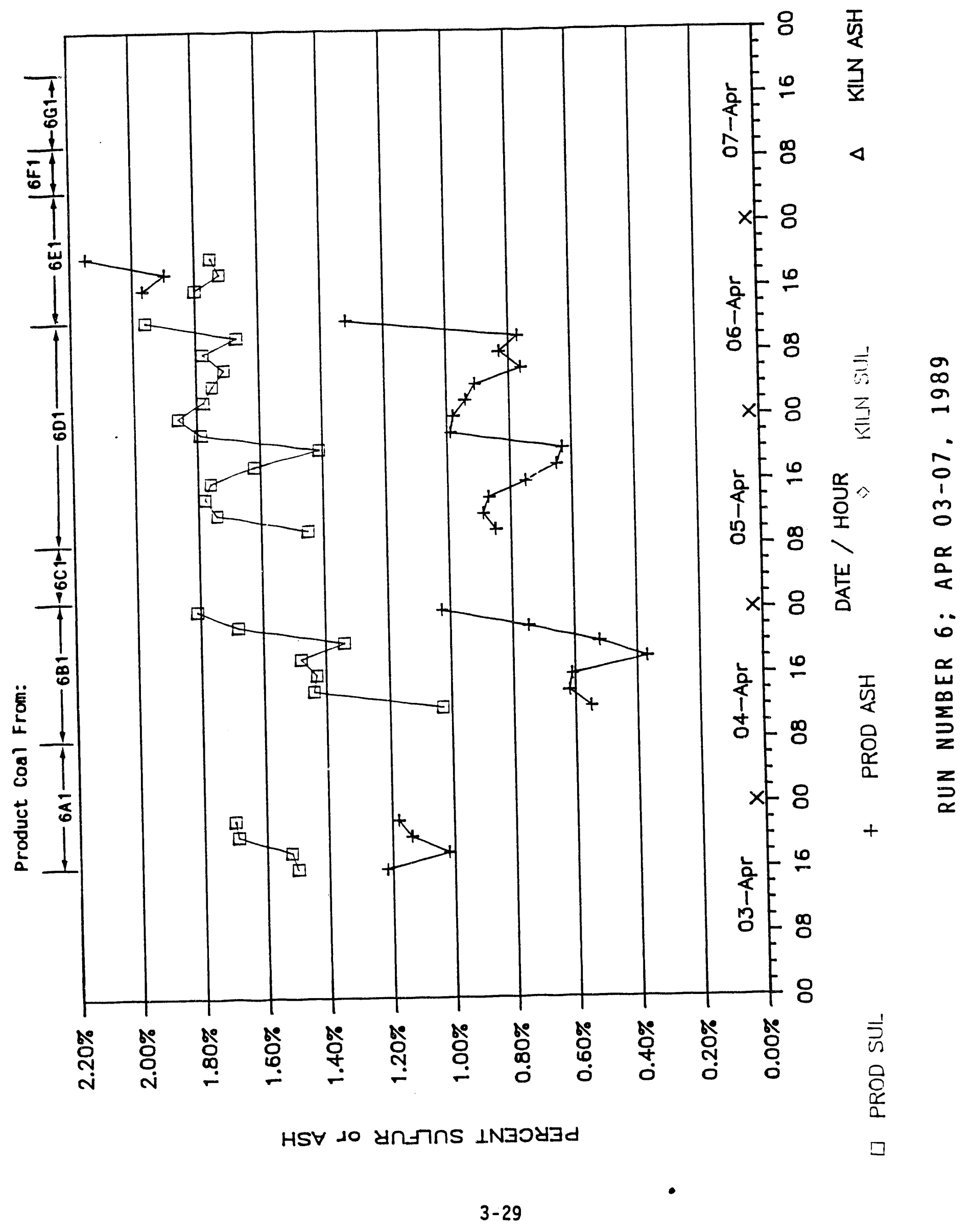




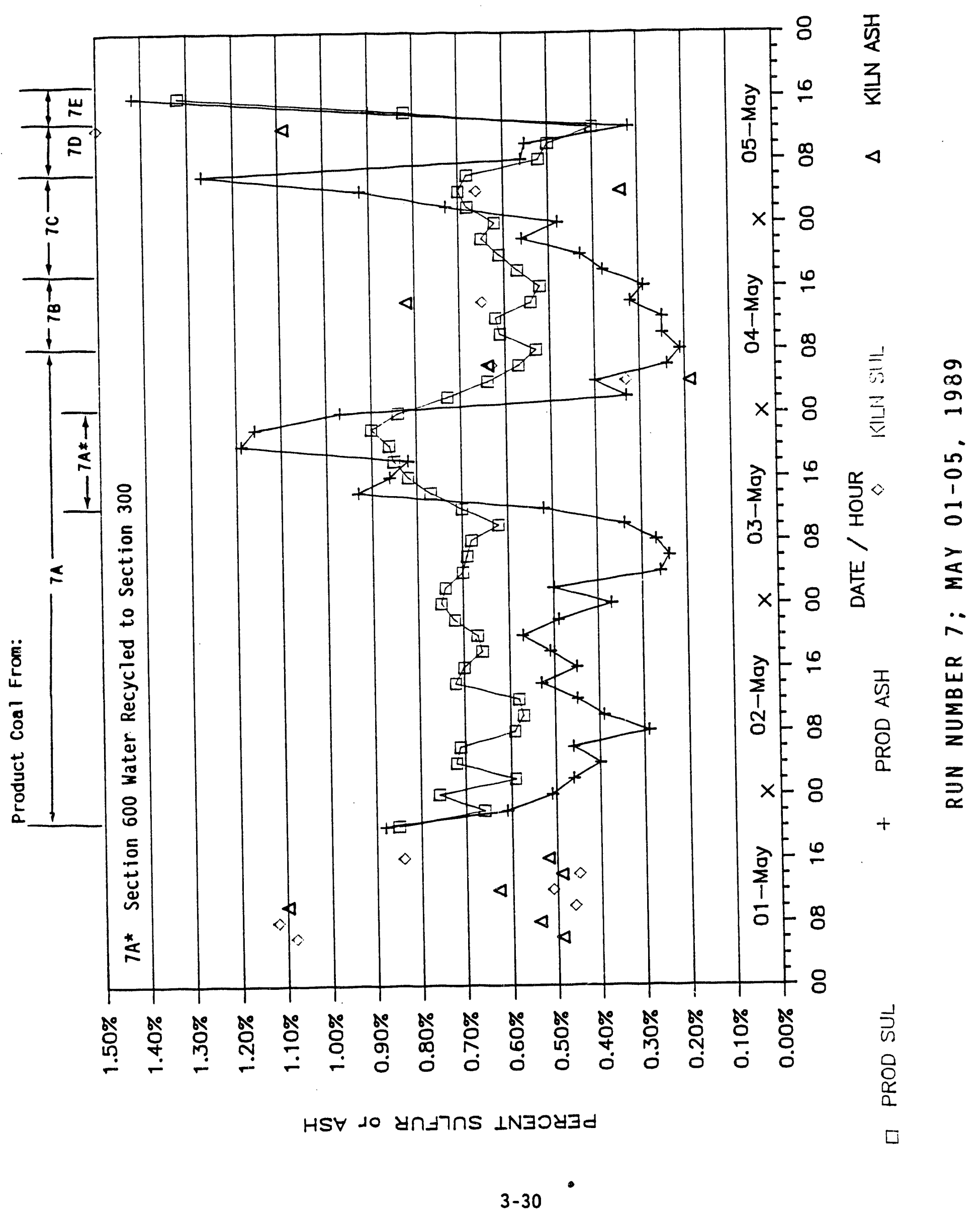




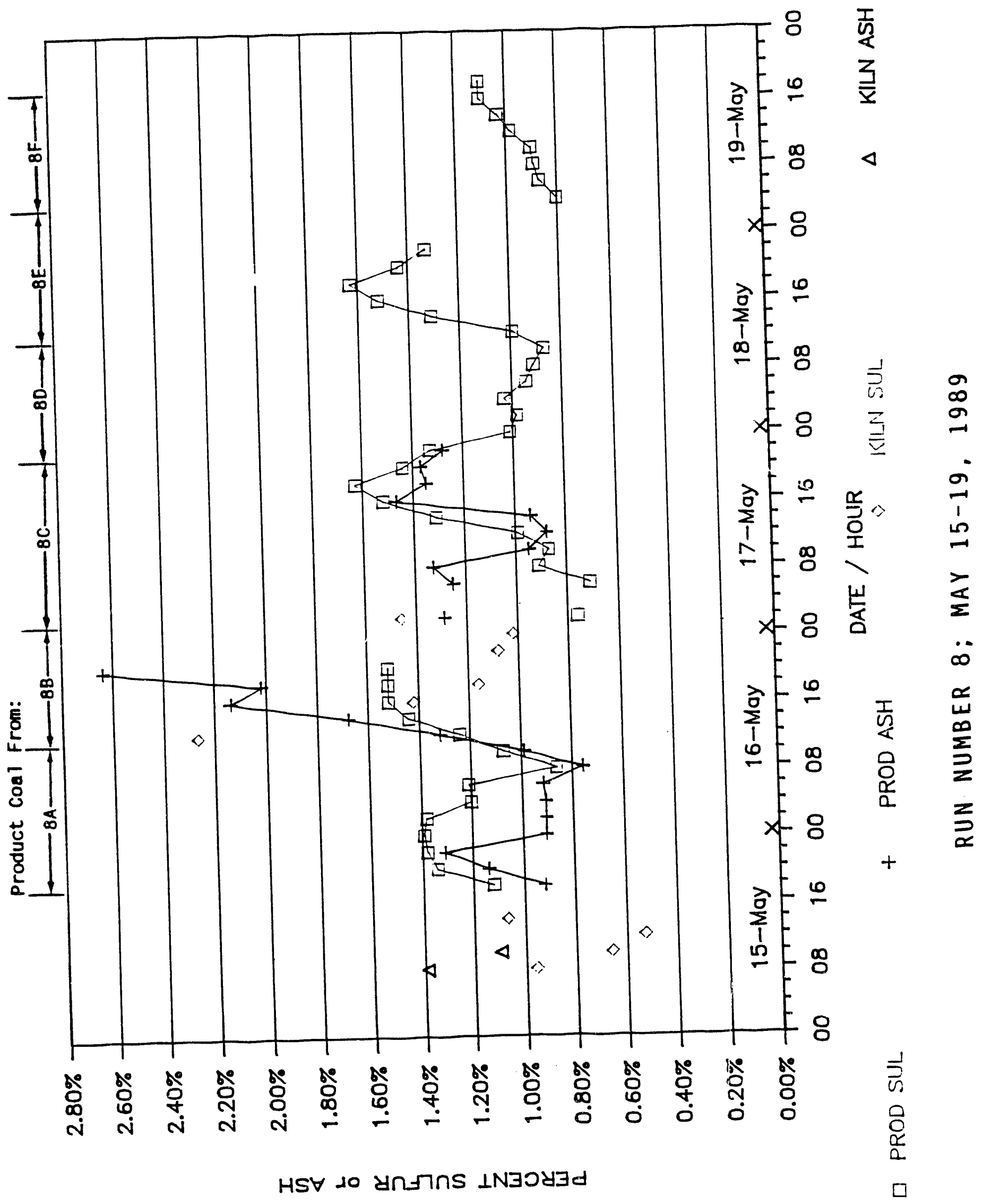




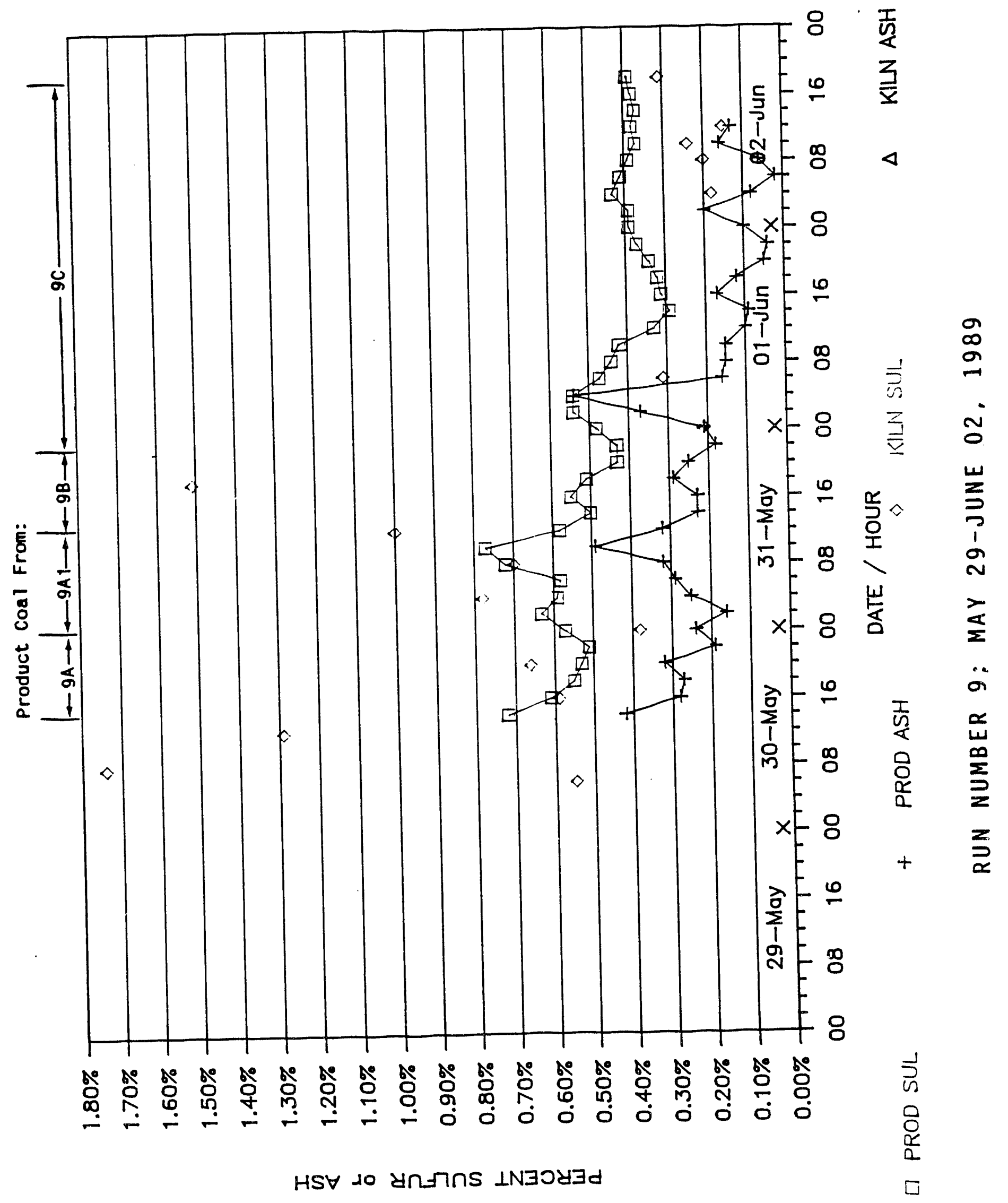




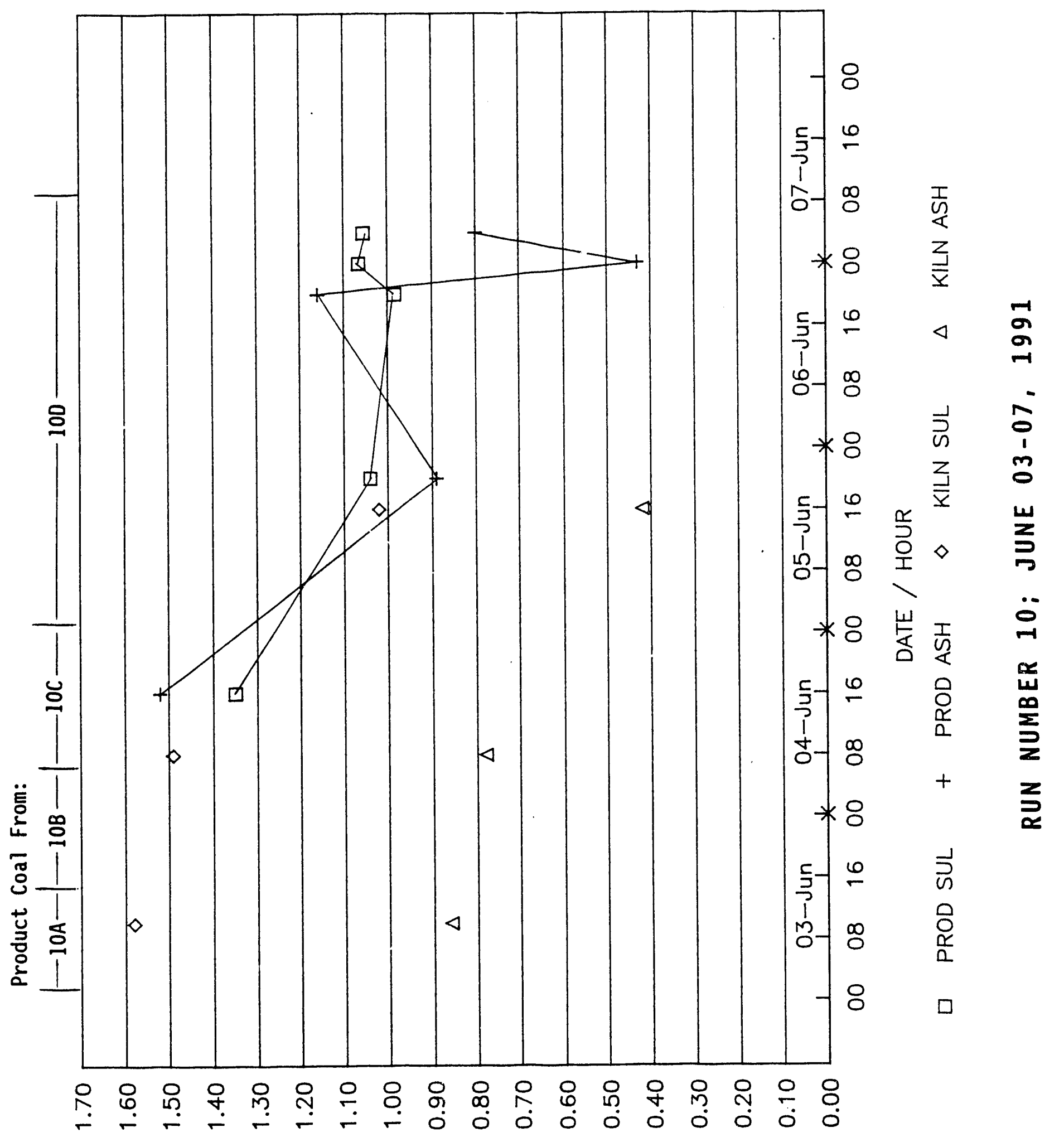

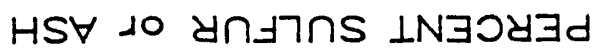




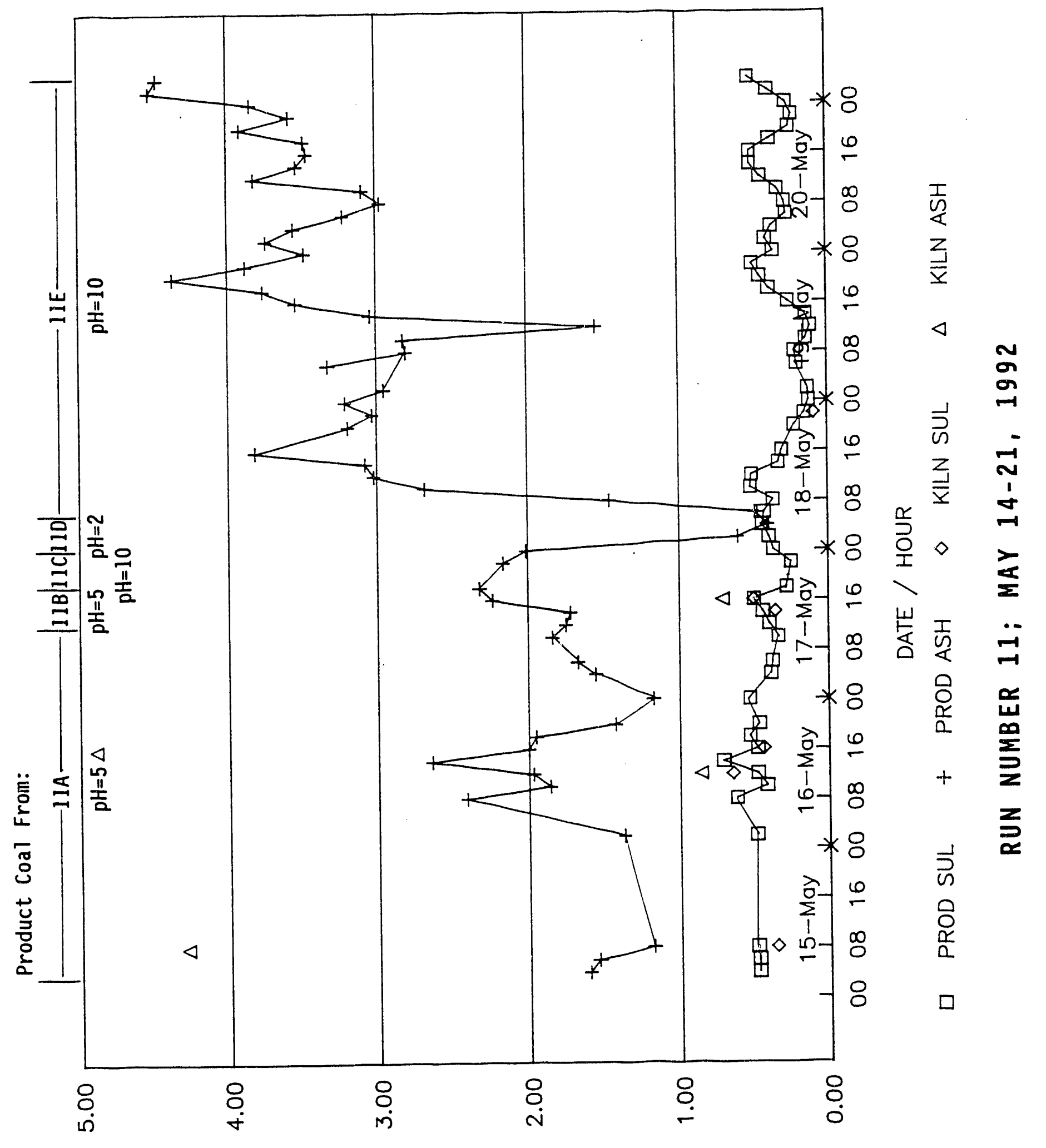

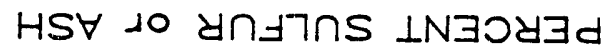


Figure 3-2. Volatiles, Sulfur and Heat Content Data for Product Coal as a Function of Coal Exit Time 
3-36 


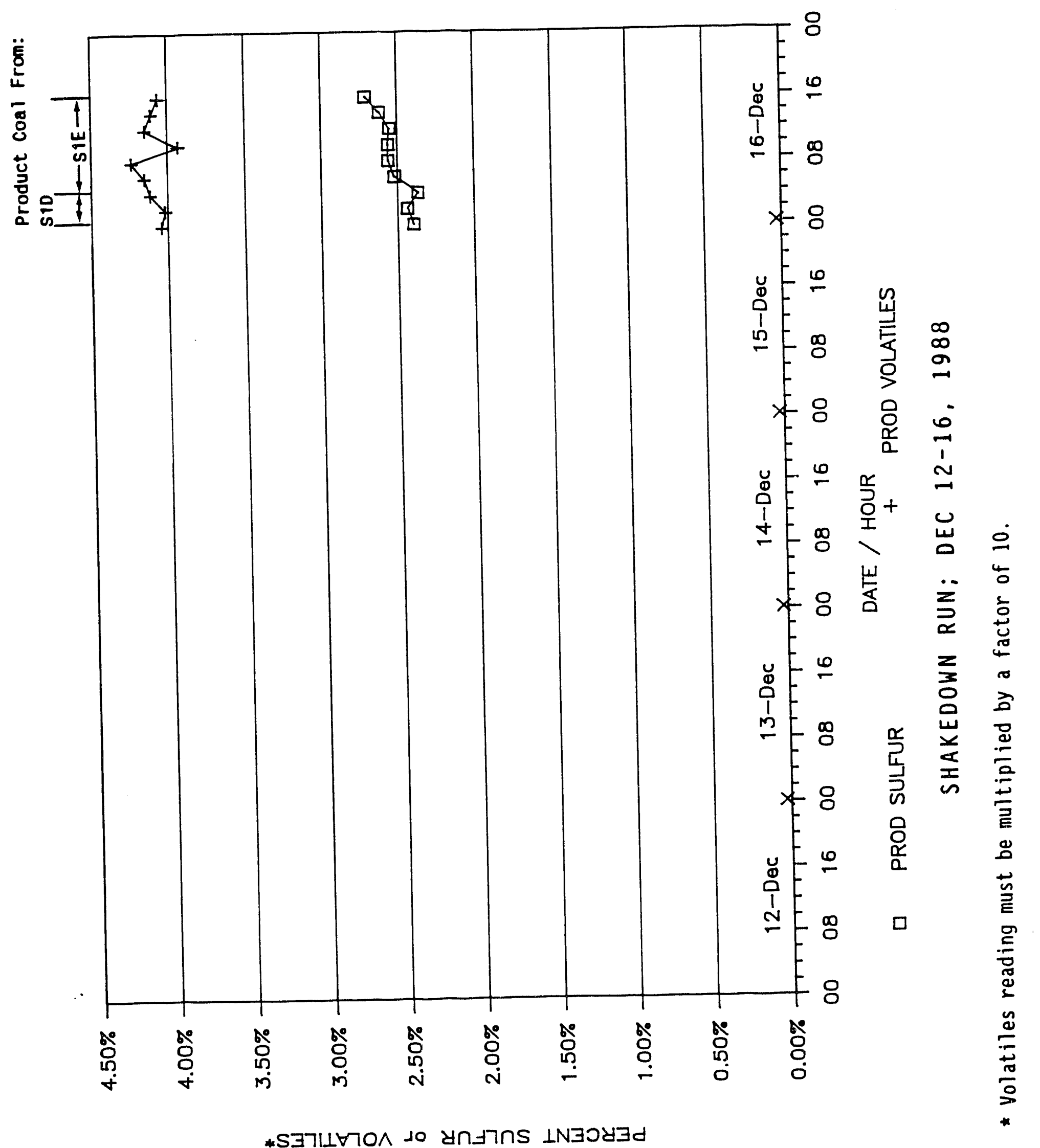




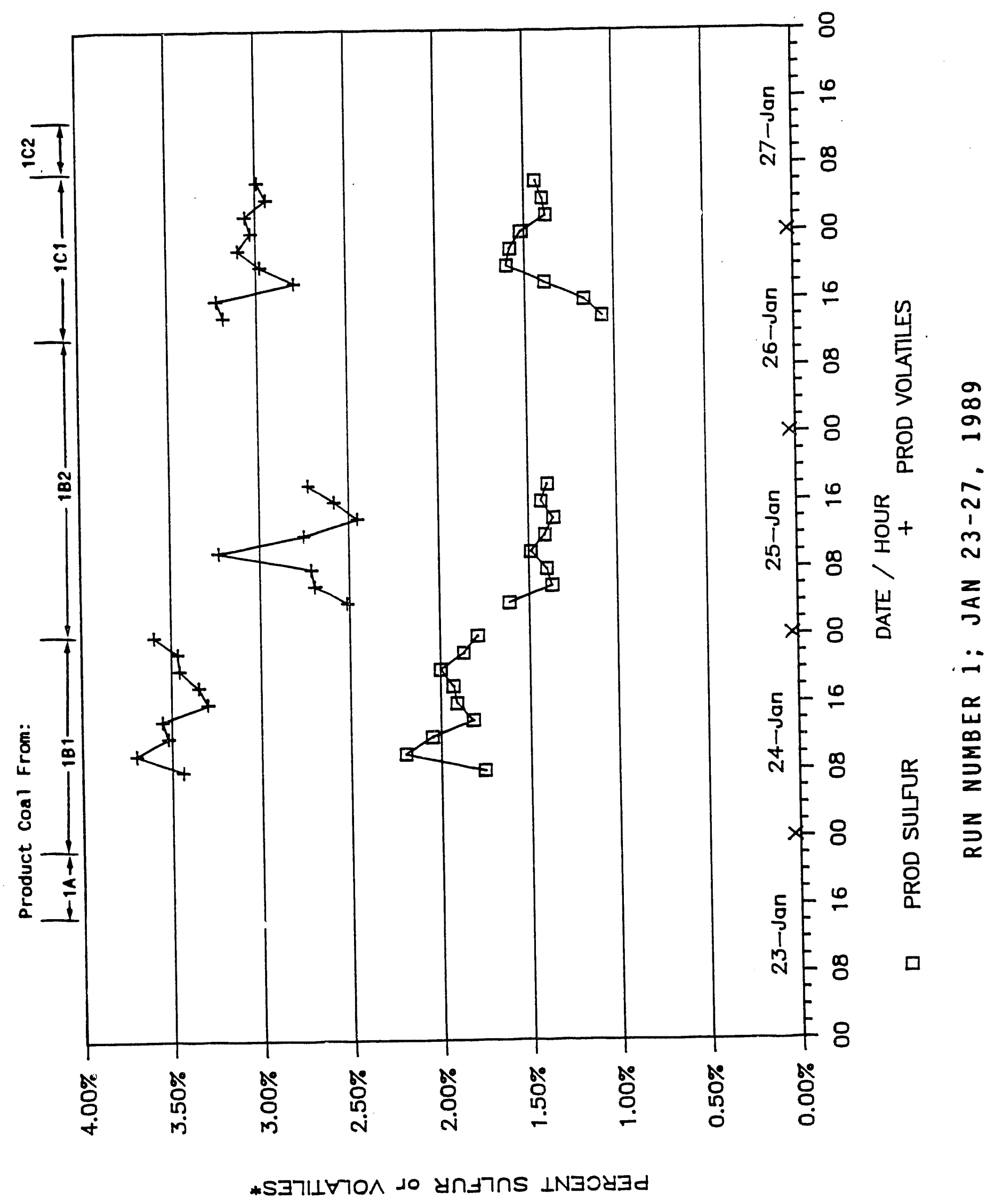




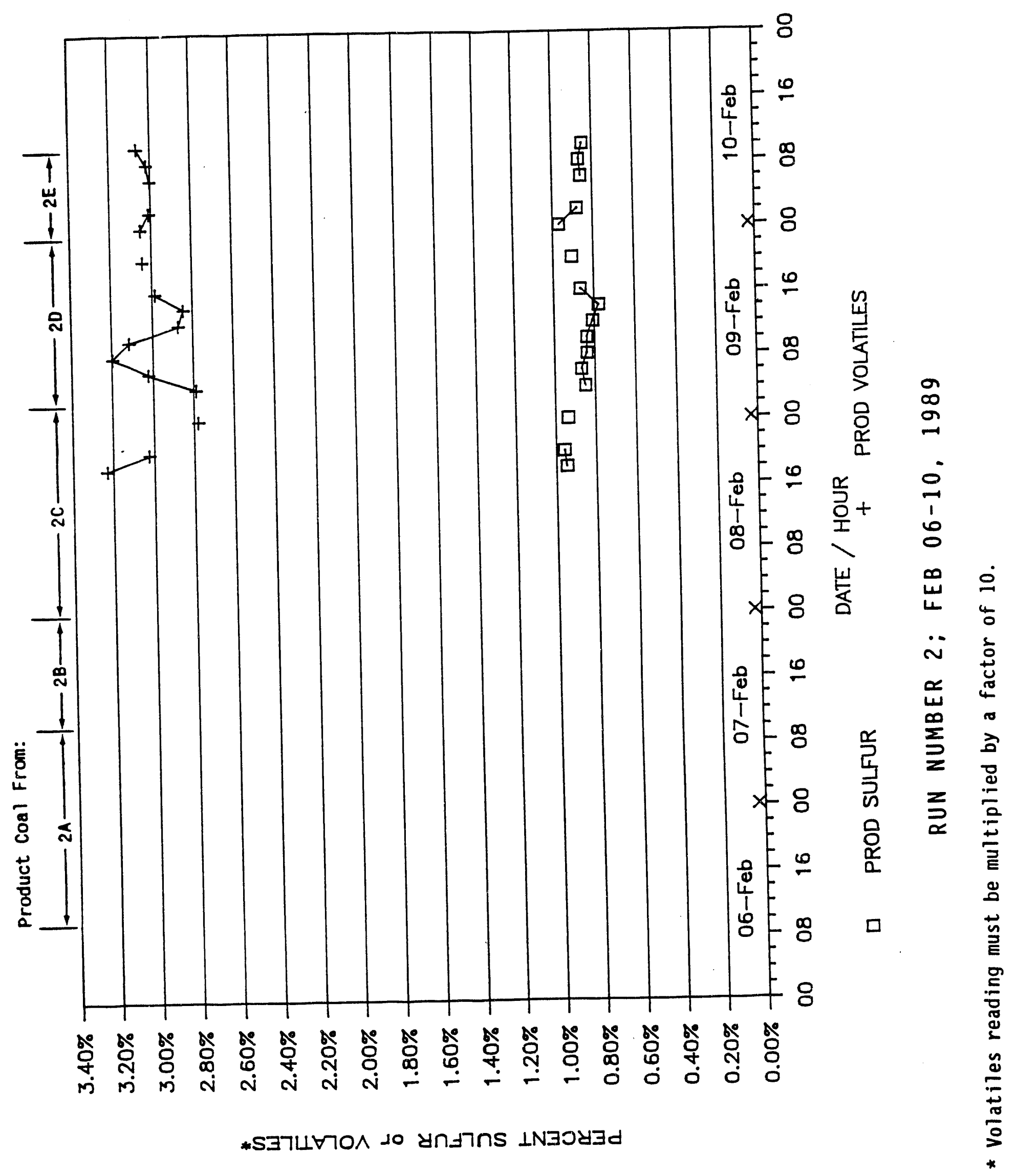




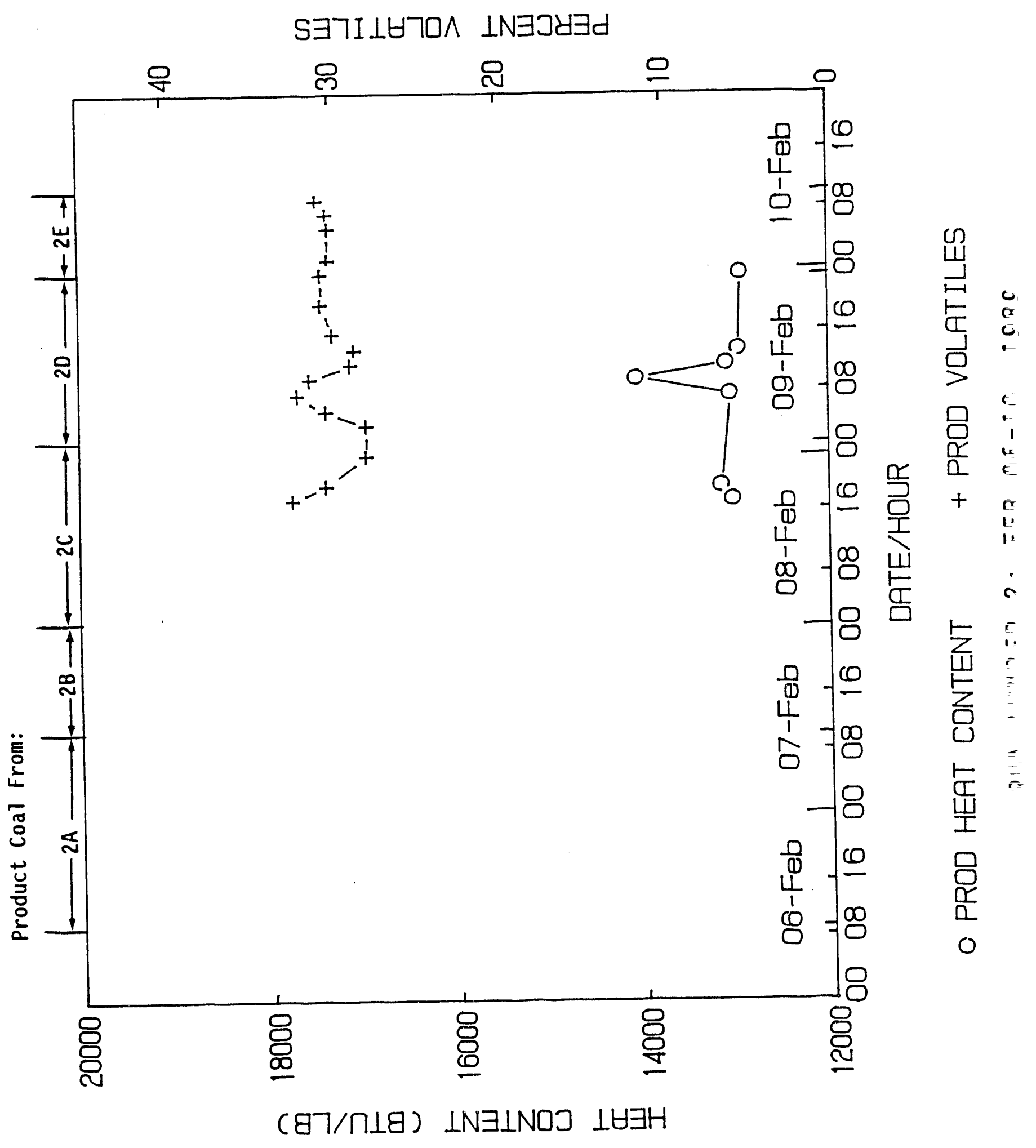




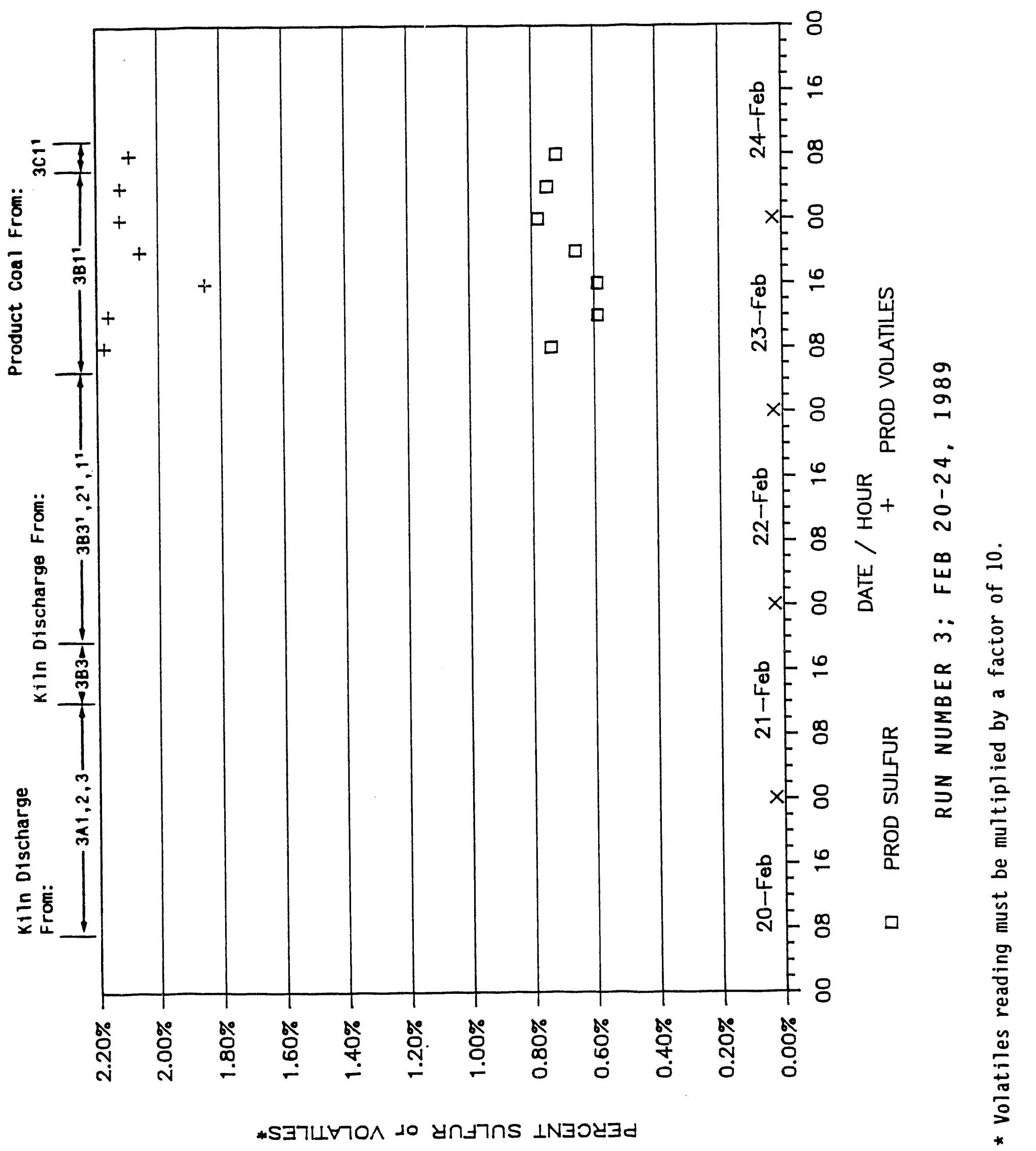




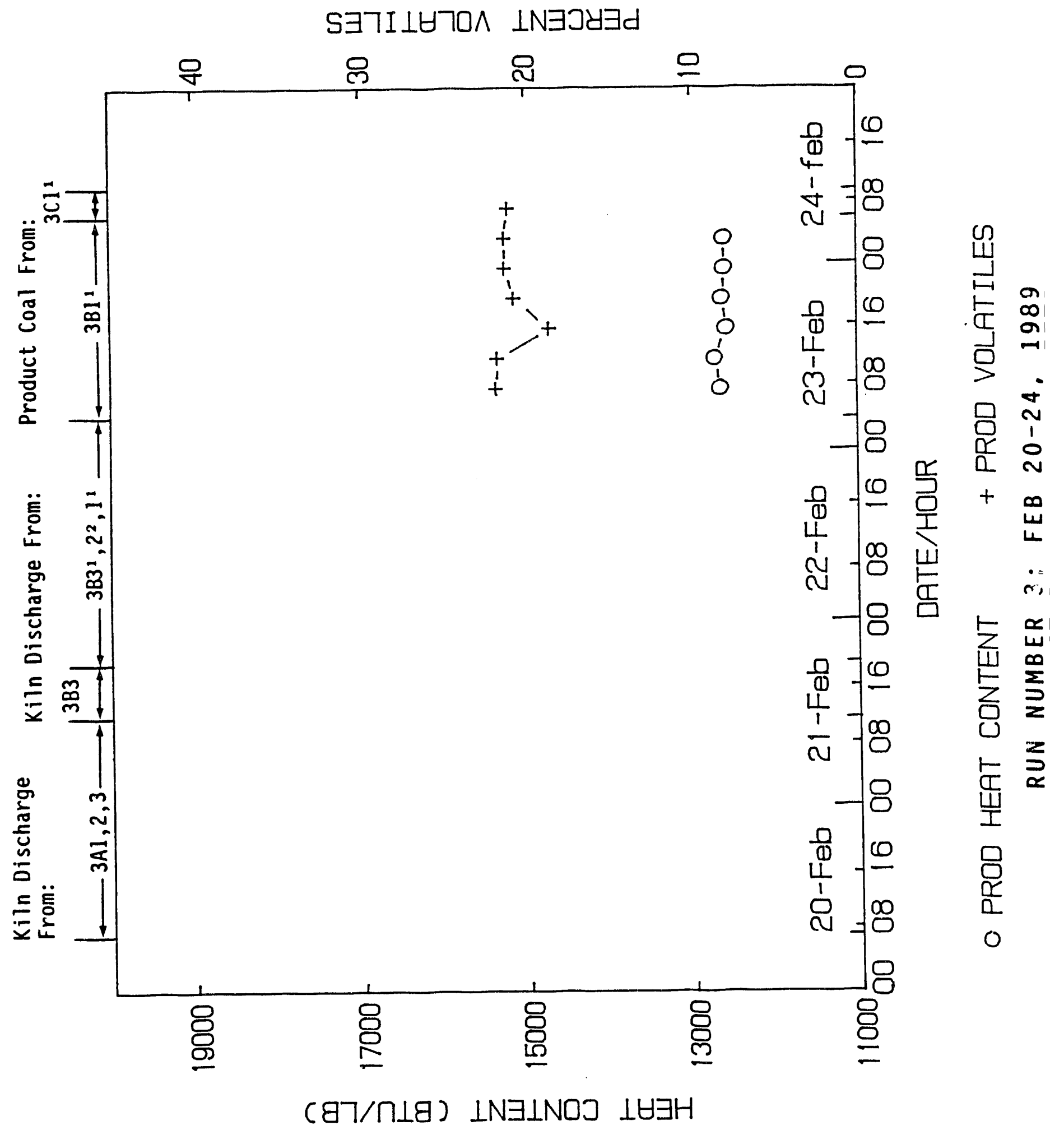




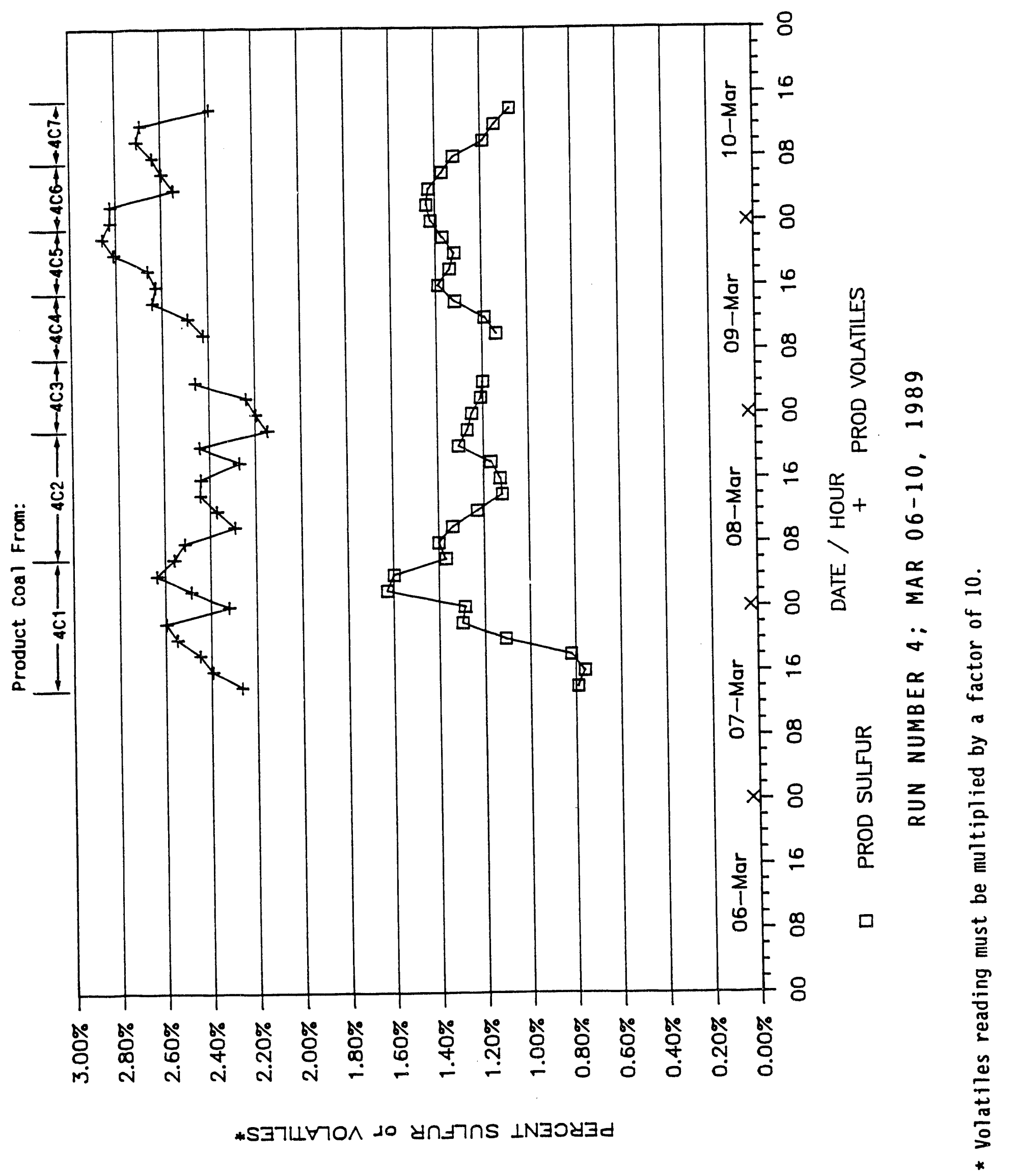




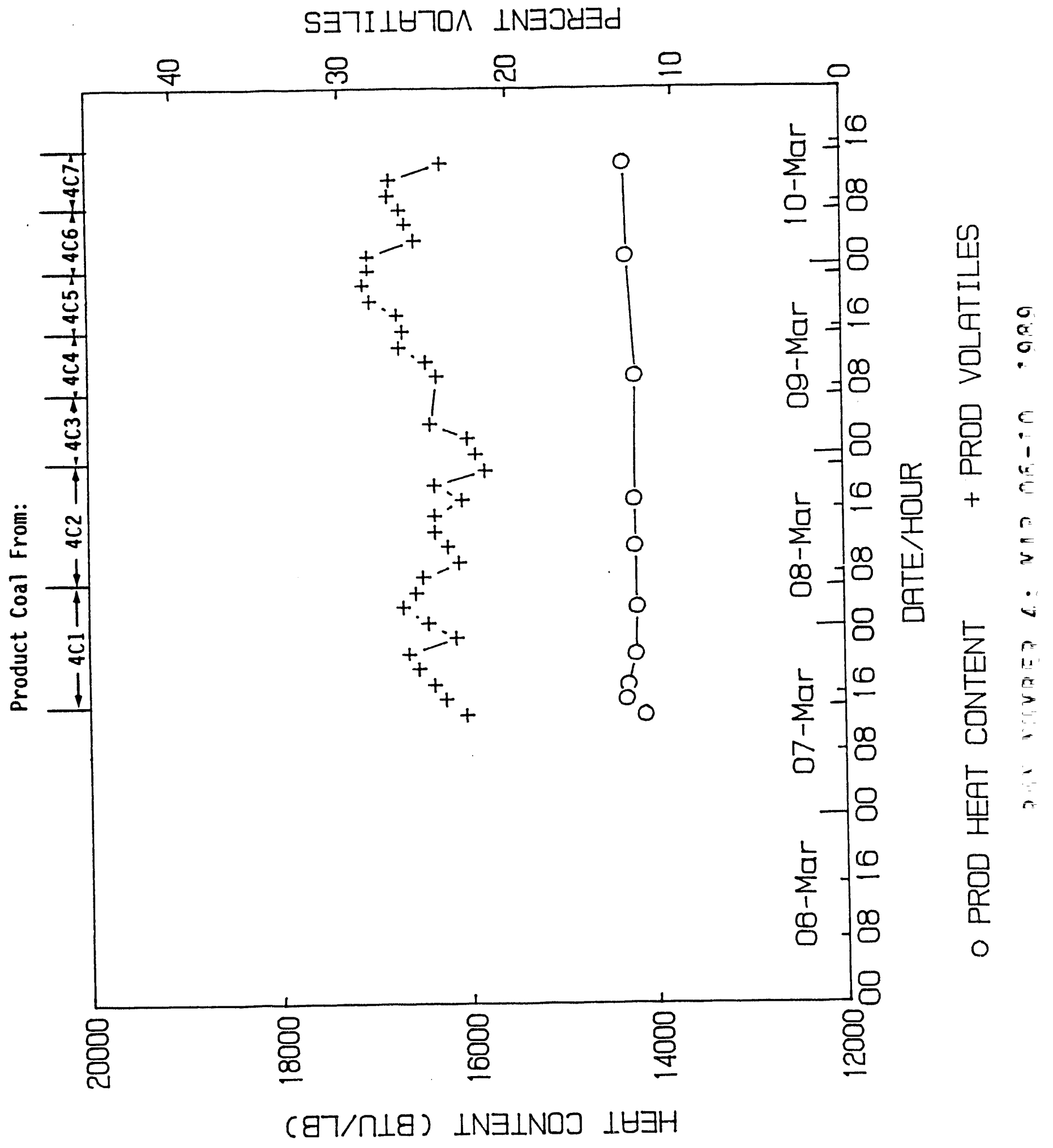




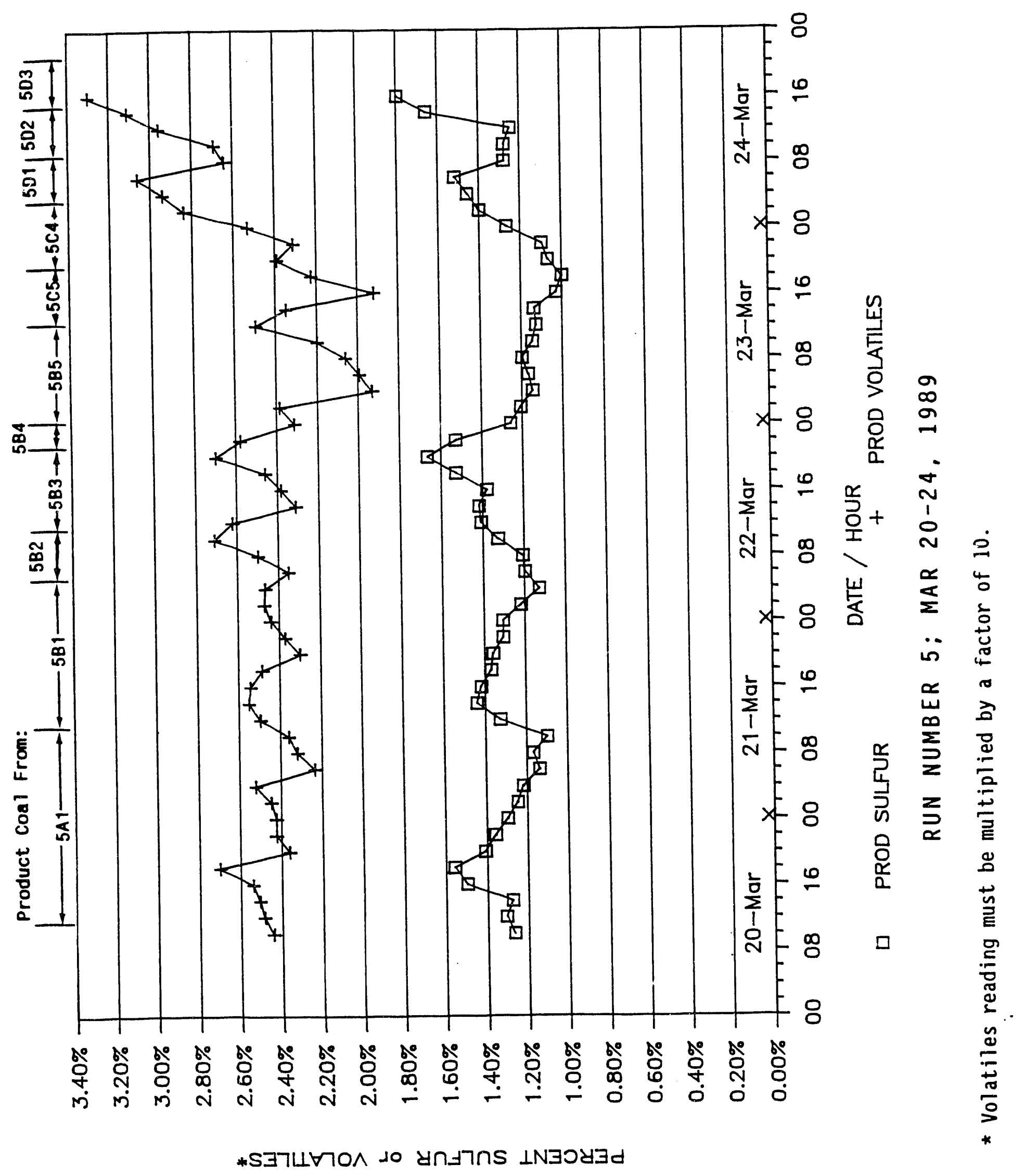




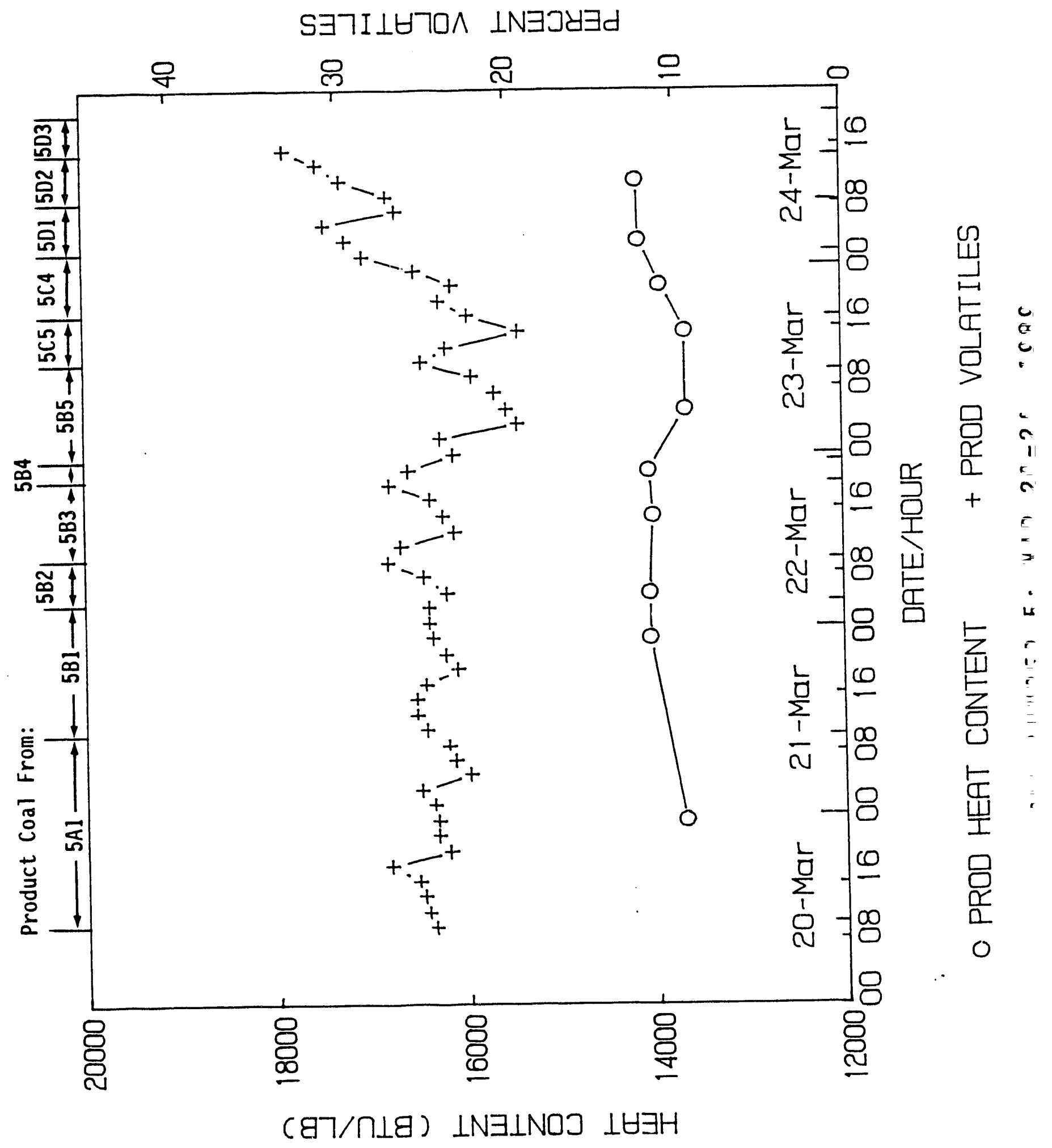




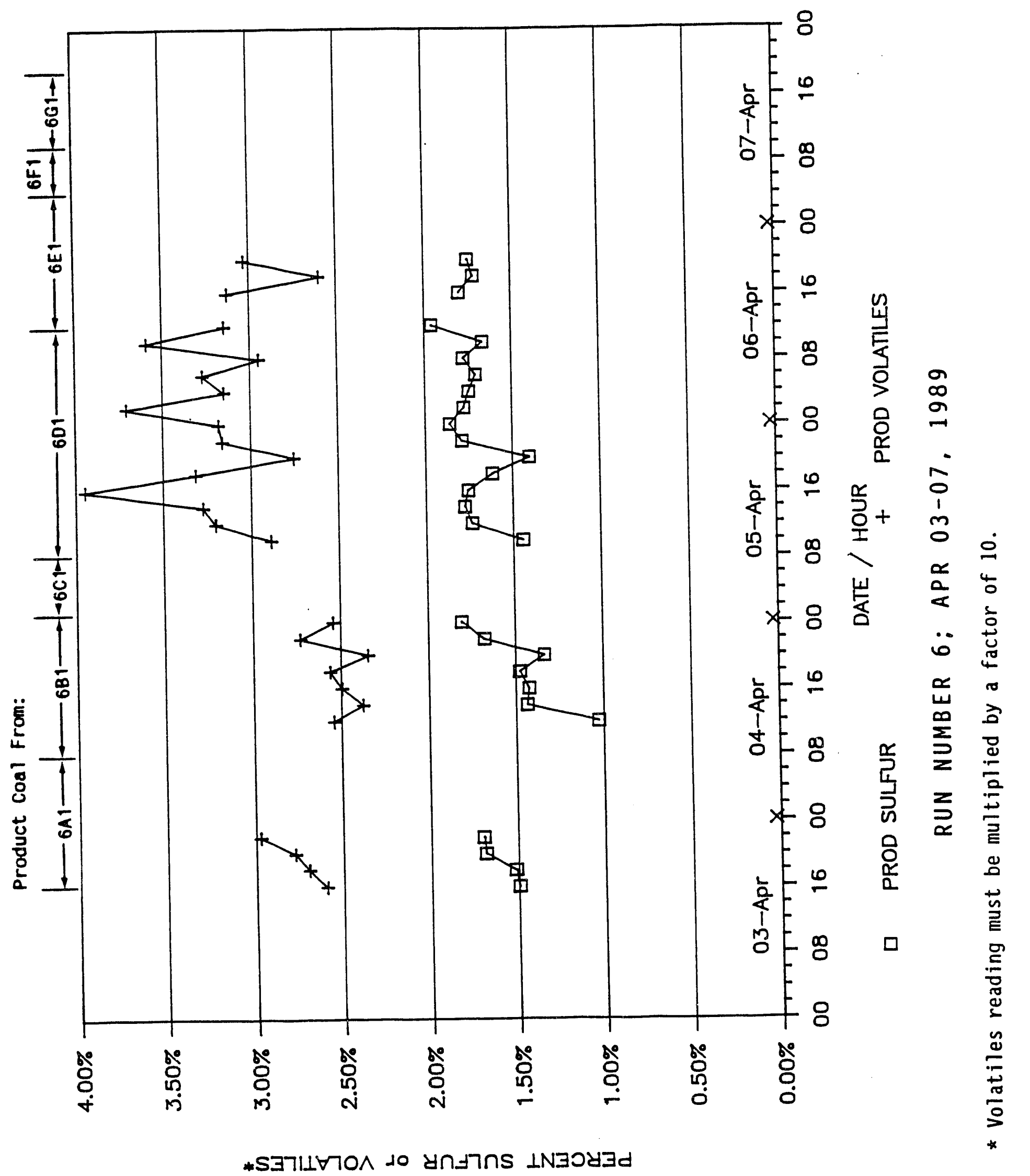




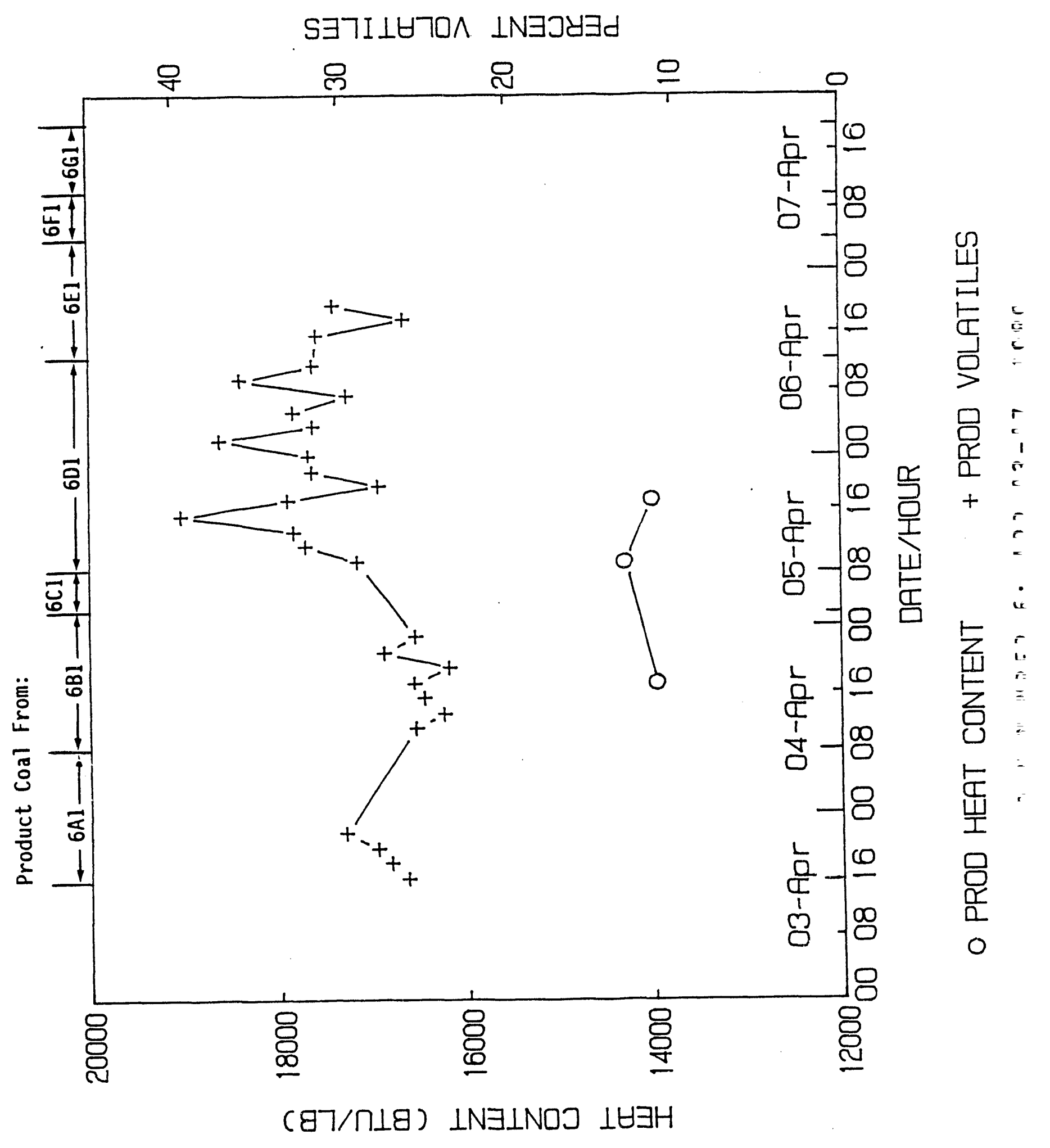




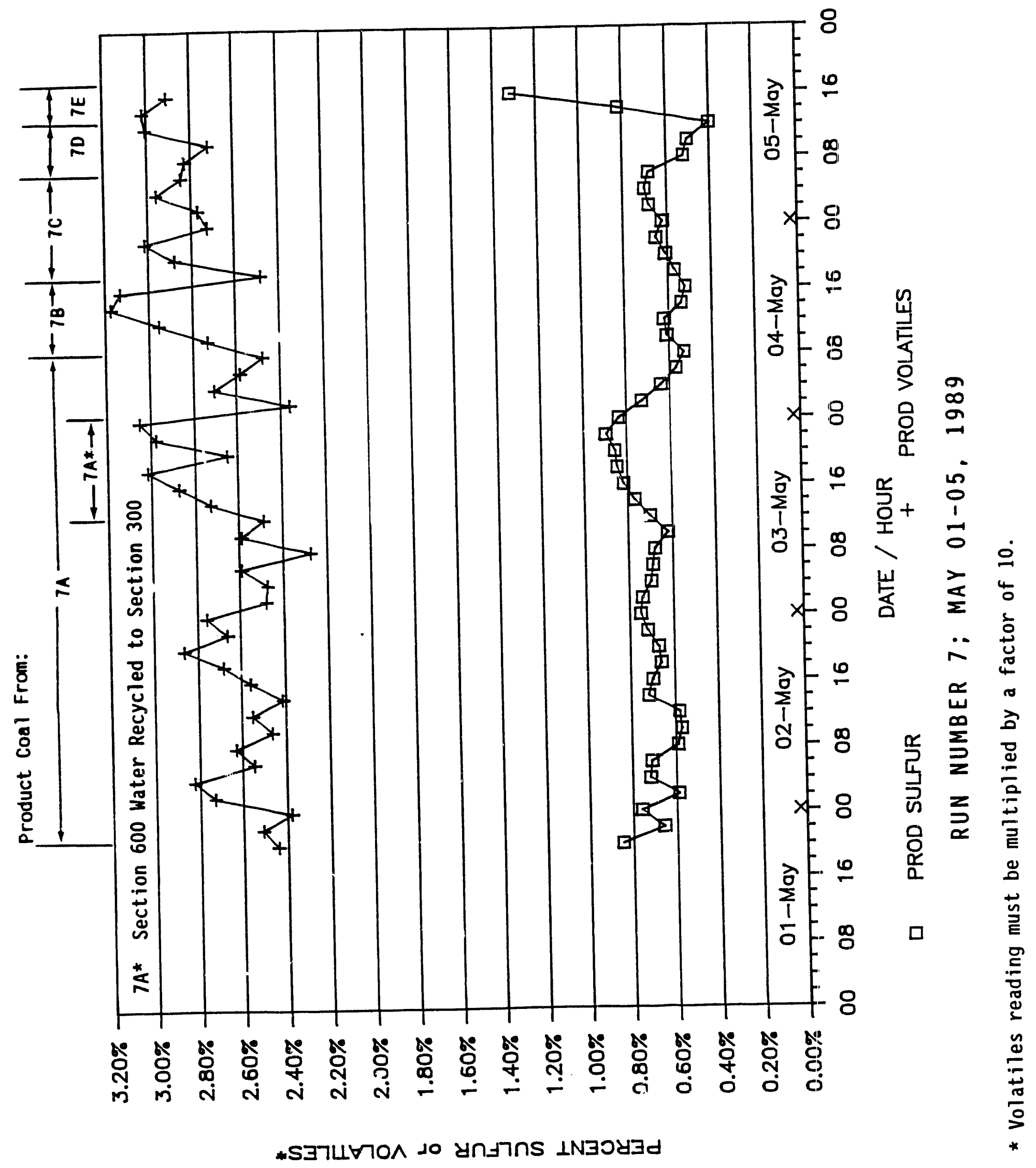




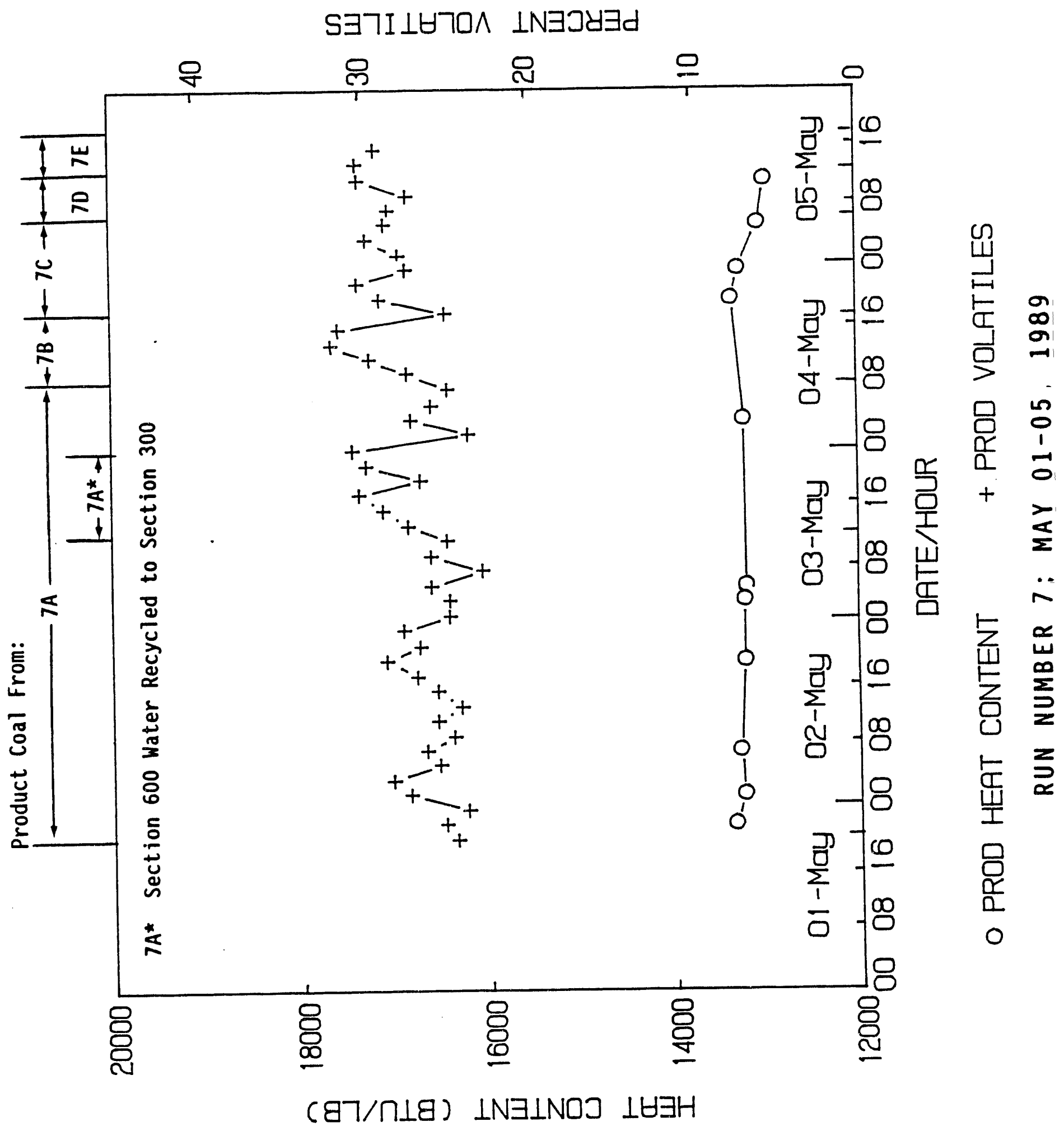




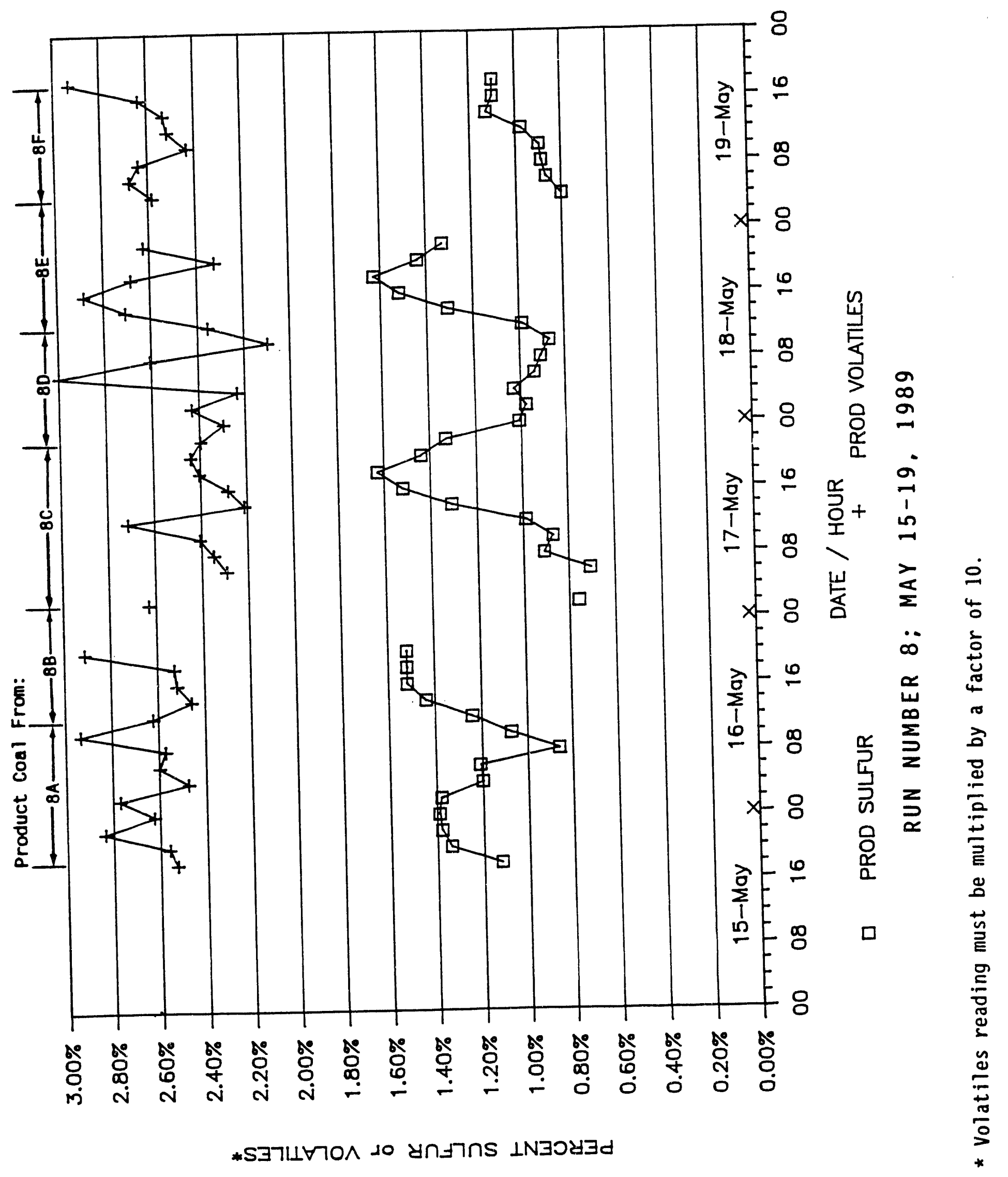




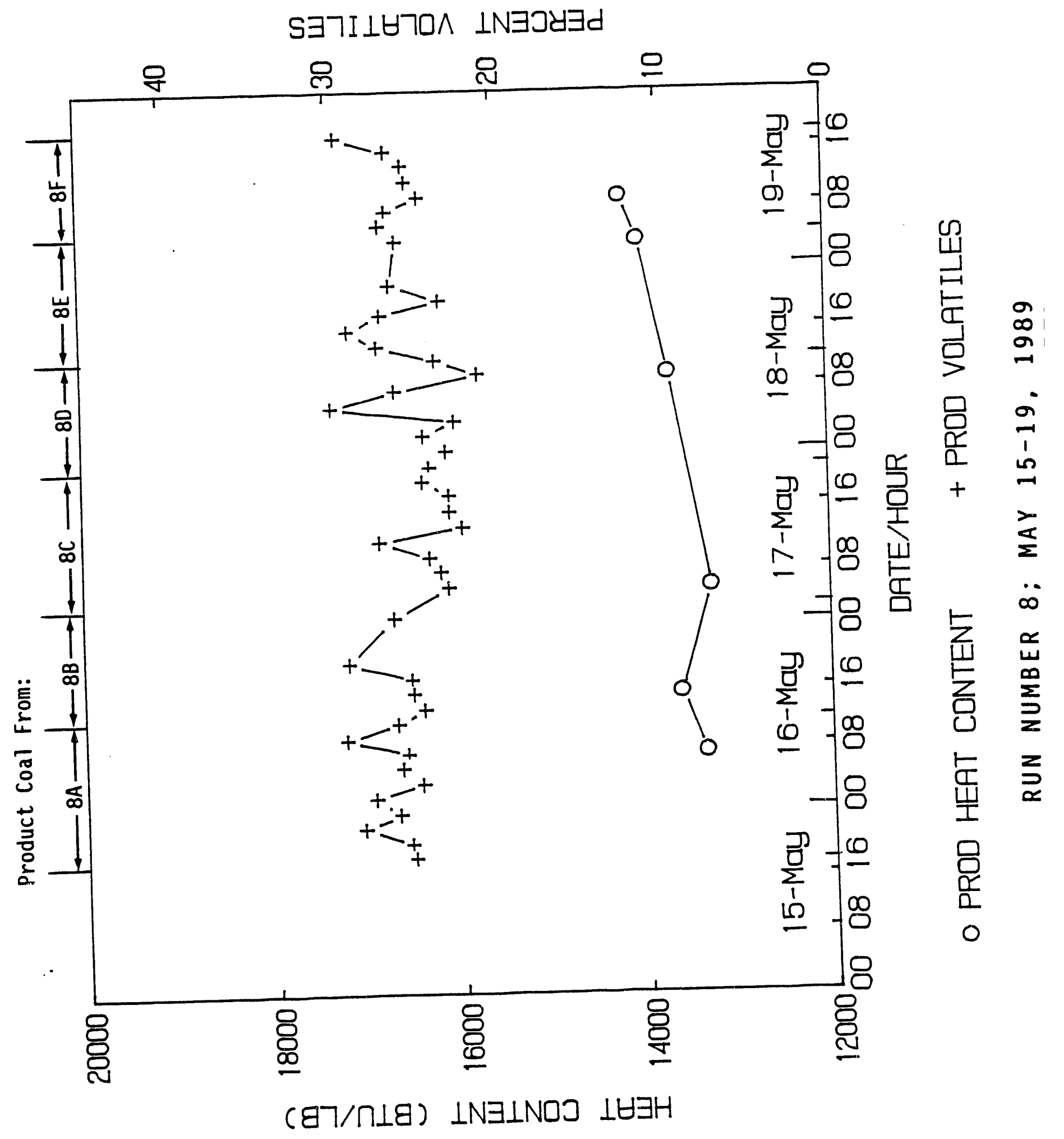




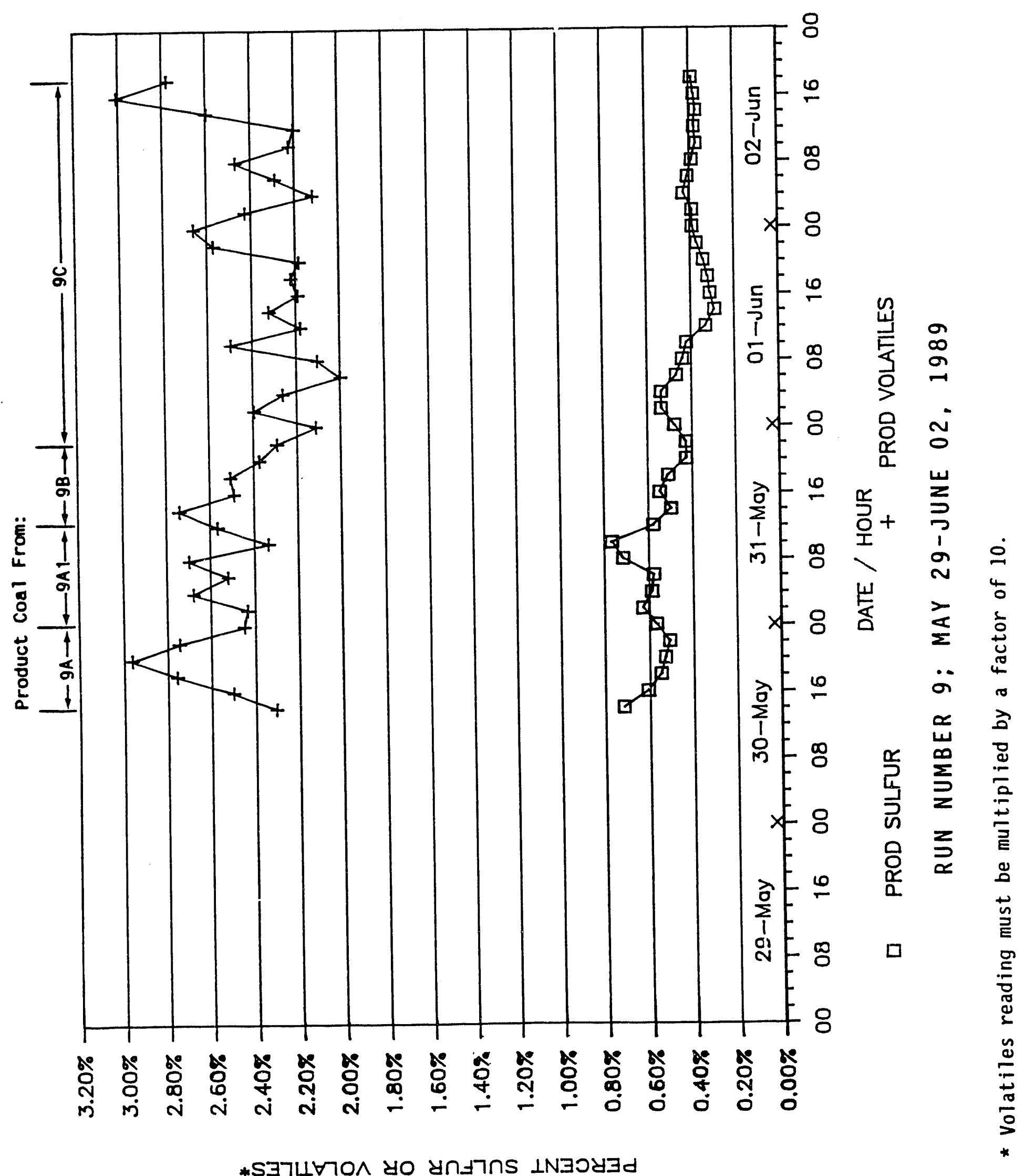




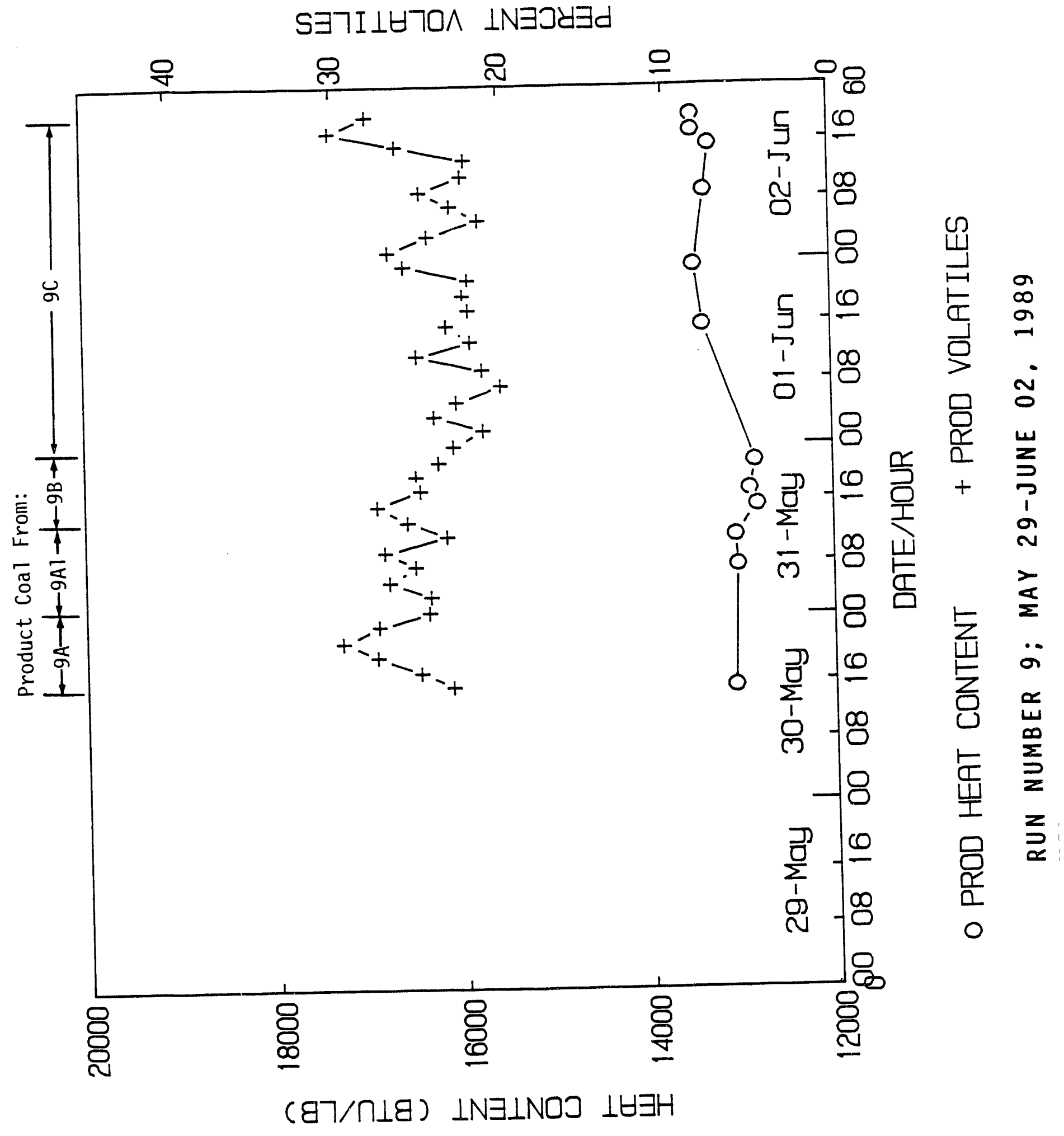




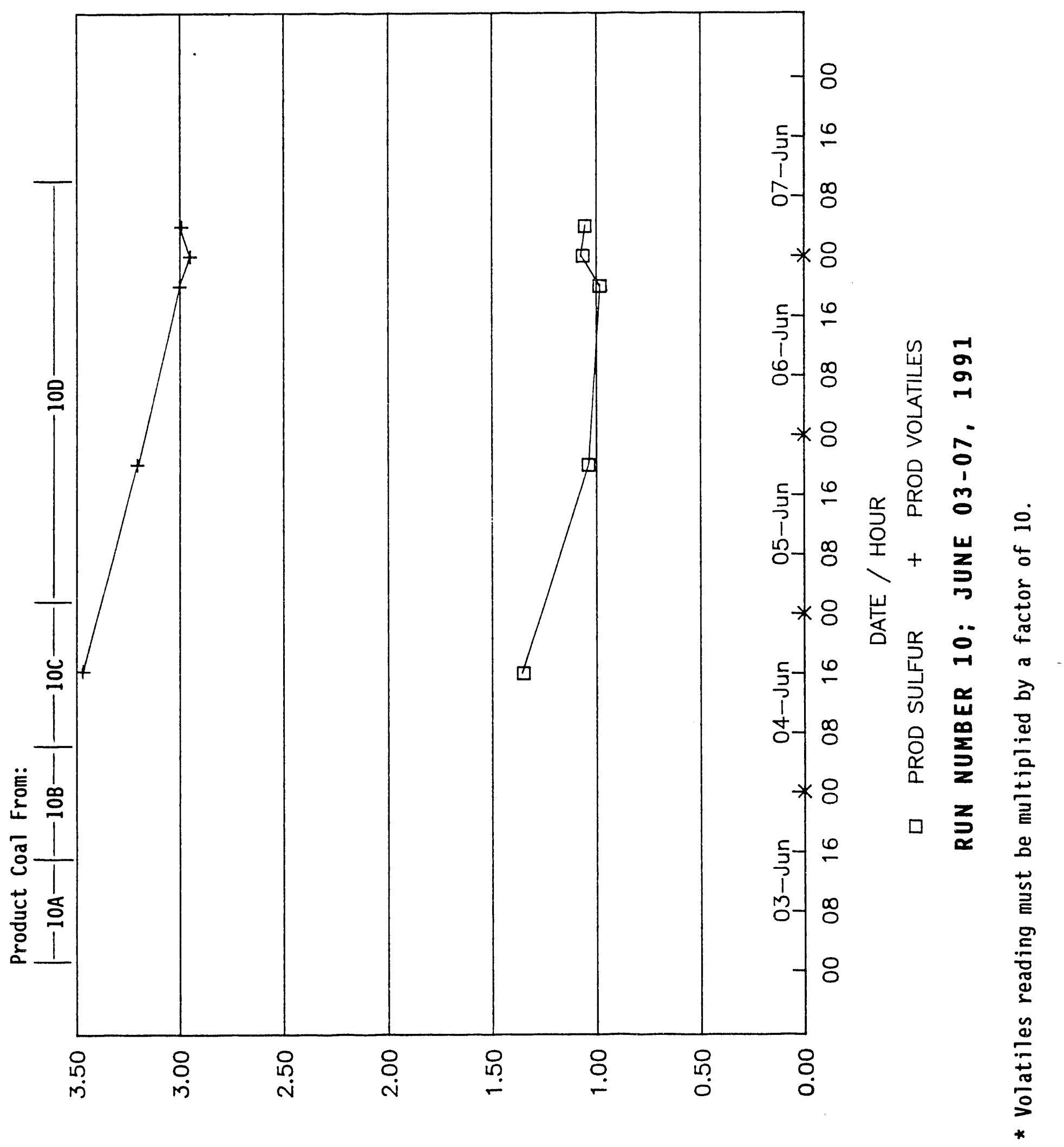

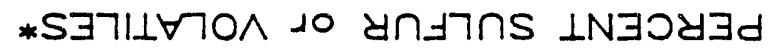




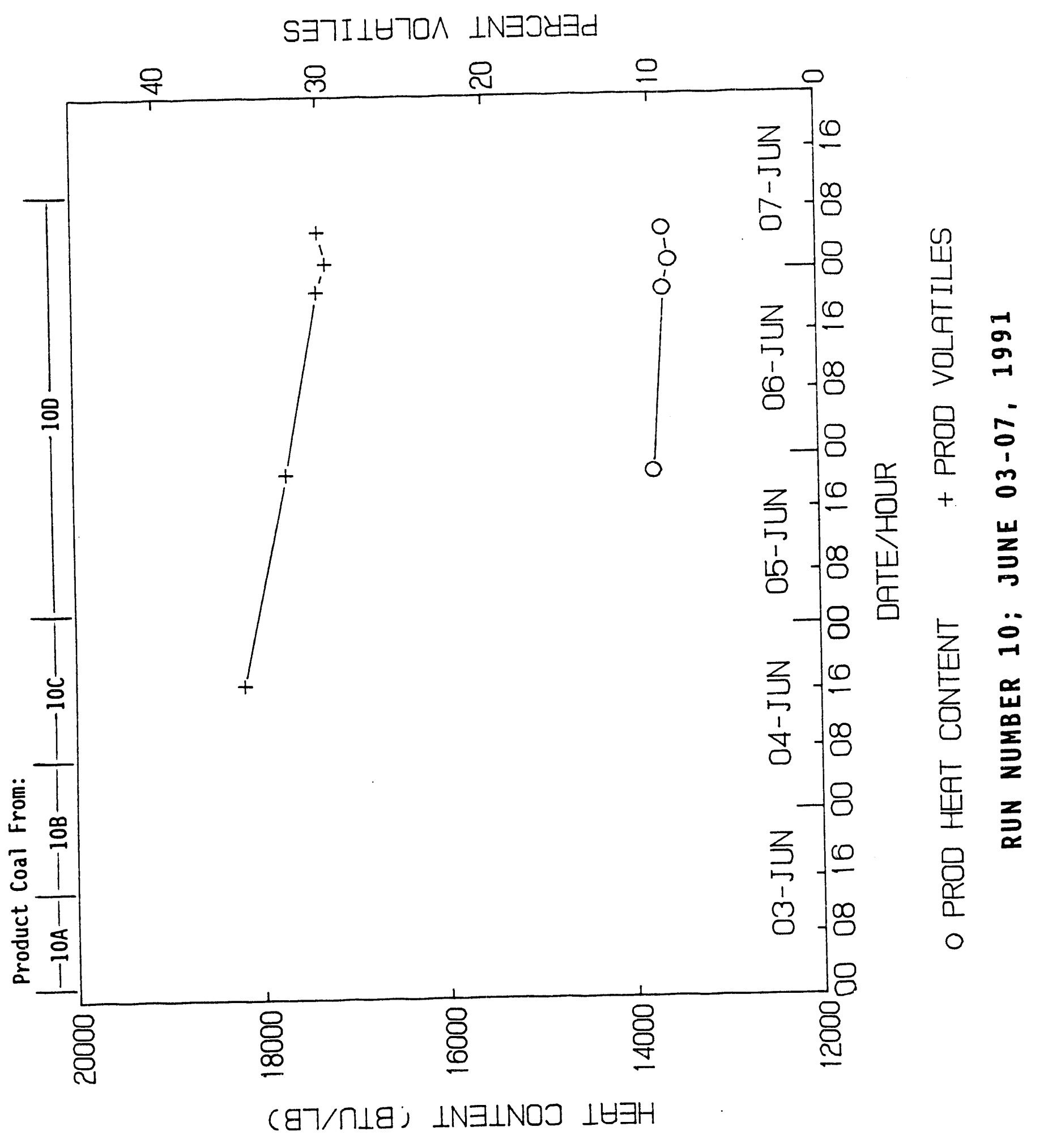




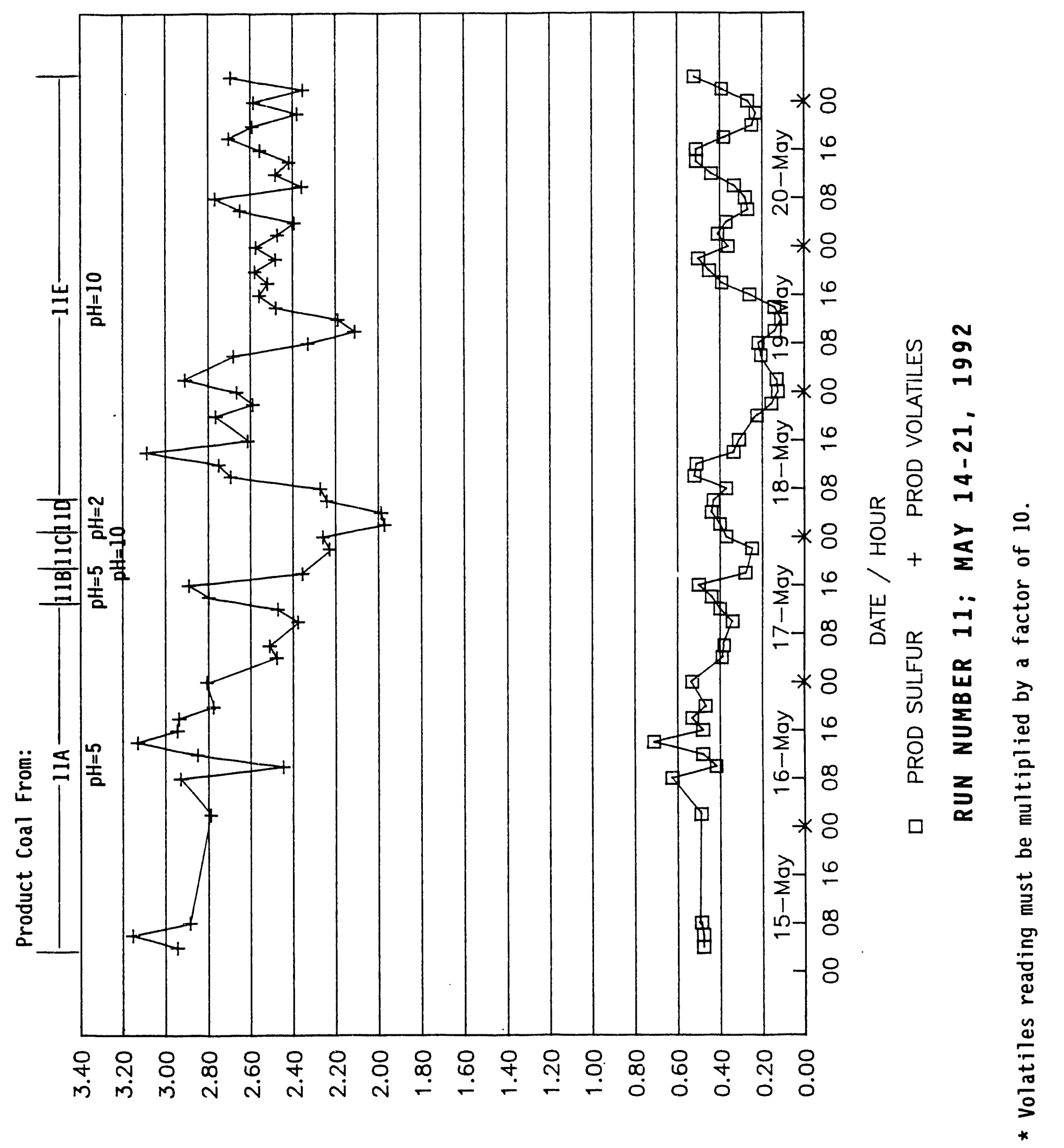

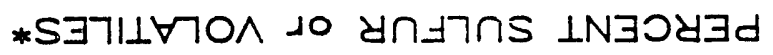




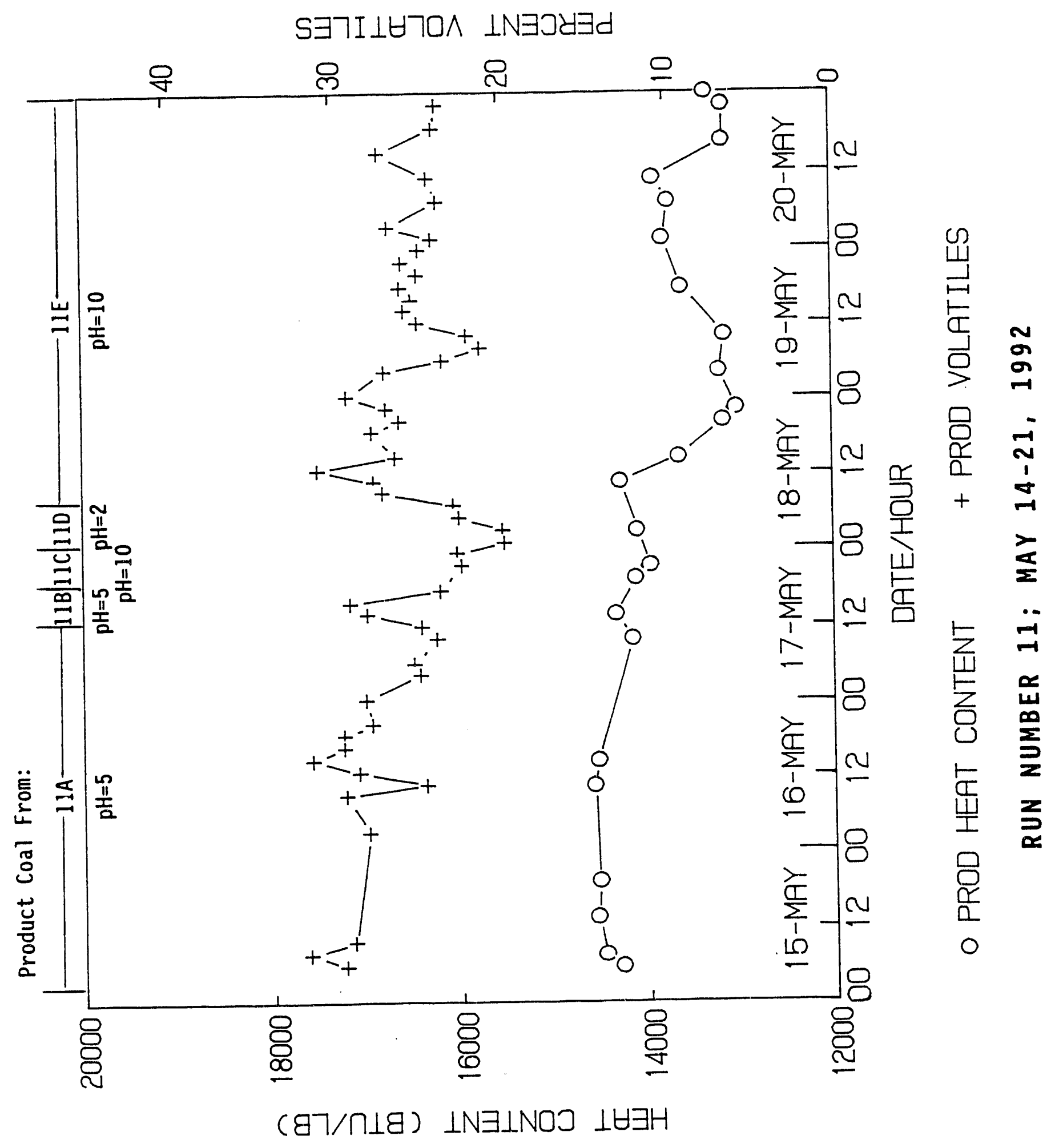


TABLE 3-3. QUANTITIES AND CHARACTERISTICS OF COAL PRODUCTS COLLECTED FROM MCL OPERATIONS

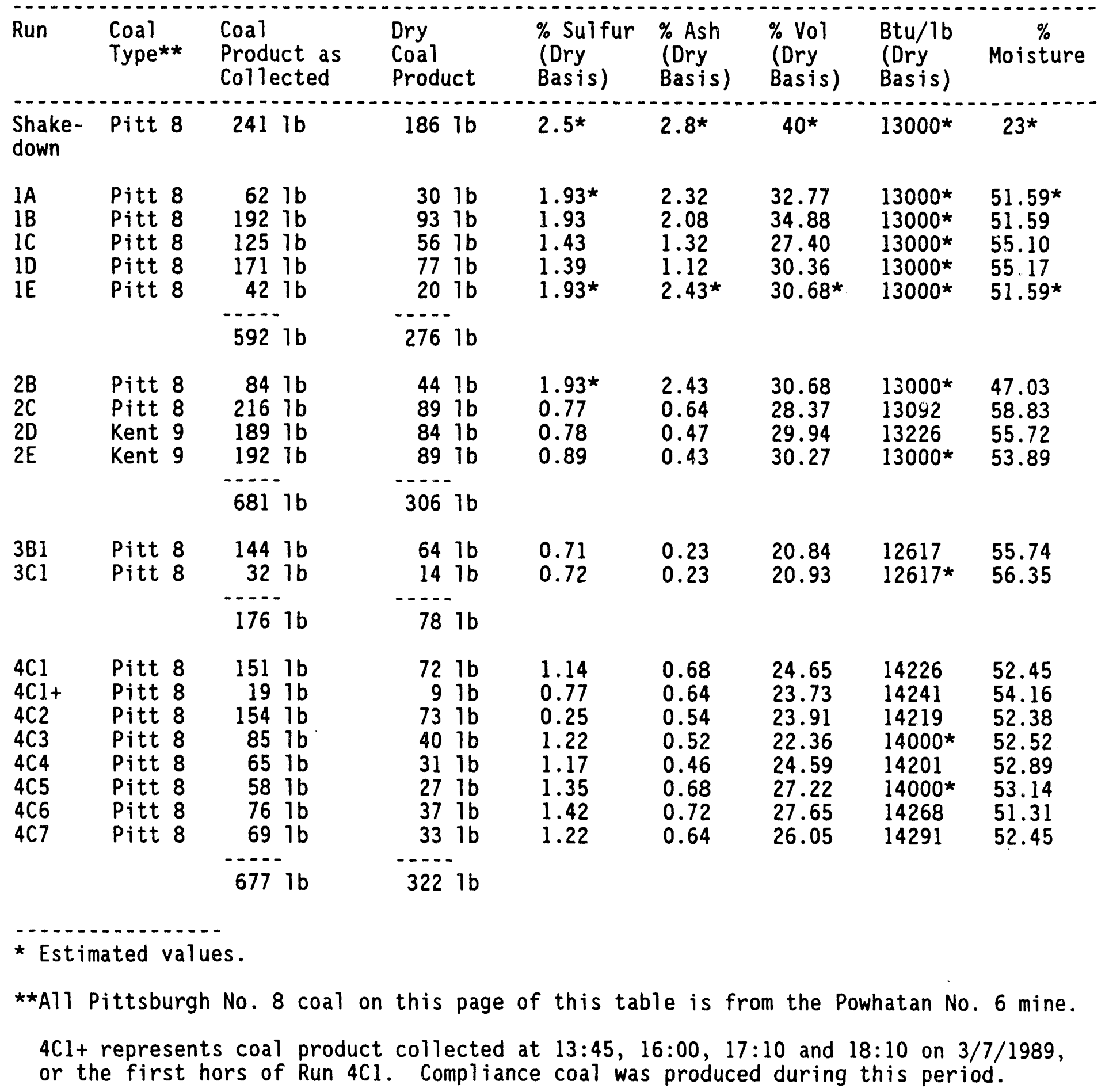


TABLE 3-3. QUANTITIES AND CHARACTERISTICS OF COAL PRODUCTS

COLLECTED FROM MCL OPERATIONS (CONTINUED)

\begin{tabular}{|c|c|c|c|c|c|c|c|c|}
\hline Run & $\begin{array}{l}\text { Coal } \\
\text { Type** }\end{array}$ & $\begin{array}{l}\text { Coal } \\
\text { Product as } \\
\text { Collected }\end{array}$ & $\begin{array}{l}\text { Dry } \\
\text { Coal } \\
\text { Product }\end{array}$ & $\begin{array}{l}\% \text { Sulfur } \\
\text { (Dry } \\
\text { Basis) }\end{array}$ & $\begin{array}{l}\% \text { Ash } \\
\text { (Dry } \\
\text { Basis) }\end{array}$ & $\begin{array}{l}\% \text { Vol } \\
\text { (Dry } \\
\text { Basis) }\end{array}$ & $\begin{array}{l}\text { Btu/1b } \\
\text { (Dry } \\
\text { Basis) }\end{array}$ & $\begin{array}{c}\% \\
\text { Moisture }\end{array}$ \\
\hline $\begin{array}{l}5 A 1 \\
5 B 1 \\
5 B 2 \\
5 B 3 \\
5 B 4 \\
5 B 5 \\
5 C 5 \\
5 C 4 \\
5 D 1 \\
5 D 2\end{array}$ & $\begin{array}{l}\text { Pitt } 8 \\
\text { Pitt } 8 \\
\text { Pitt } 8 \\
\text { Pitt } 8 \\
\text { Pitt } 8 \\
\text { Pitt } 8 \\
\text { Kent } 9 \\
\text { Kent } 9 \\
\text { Kent } 9 \\
\text { Kent } 9\end{array}$ & $\begin{array}{rl}227 & 1 \mathrm{~b} \\
181 & 1 \mathrm{~b} \\
70 & 1 \mathrm{~b} \\
91 & 1 \mathrm{~b} \\
42 & 1 \mathrm{~b} \\
106 & 1 \mathrm{~b} \\
73 & 1 \mathrm{~b} \\
78 & 1 \mathrm{~b} \\
40 & 1 \mathrm{~b} \\
431 \mathrm{~b}\end{array}$ & \begin{tabular}{rl}
124 & $1 \mathrm{~b}$ \\
81 & $1 \mathrm{~b}$ \\
30 & $1 \mathrm{~b}$ \\
41 & $1 \mathrm{~b}$ \\
19 & $1 \mathrm{~b}$ \\
48 & $1 \mathrm{~b}$ \\
35 & $1 \mathrm{~b}$ \\
35 & $1 \mathrm{~b}$ \\
19 & $1 \mathrm{~b}$ \\
21 & $1 \mathrm{~b}$ \\
\hdashline
\end{tabular} & $\begin{array}{l}1.31 \\
1.32 \\
1.20 \\
1.46 \\
1.60 \\
1.19 \\
1.10 \\
1.11 \\
1.46 \\
1.27\end{array}$ & $\begin{array}{l}1.23 \\
0.67 \\
0.58 \\
0.63 \\
0.95 \\
0.45 \\
0.37 \\
0.50 \\
1.03 \\
0.80\end{array}$ & $\begin{array}{l}24.52 \\
24.47 \\
24.65 \\
24.69 \\
26.39 \\
21.49 \\
22.57 \\
23.66 \\
29.48 \\
27.64\end{array}$ & $\begin{array}{l}13686 \\
14052 \\
14059 \\
14023 \\
14047 \\
13656 \\
13651 \\
13918 \\
14130 \\
14152\end{array}$ & $\begin{array}{l}55.28 \\
55.16 \\
56.61 \\
54.40 \\
55.23 \\
54.81 \\
51.69 \\
55.73 \\
53.65 \\
52.05\end{array}$ \\
\hline $\begin{array}{l}6 \mathrm{Al} \\
6 \mathrm{Bl} \\
6 \mathrm{Cl} \\
6 \mathrm{Dl} \\
6 \mathrm{El}\end{array}$ & $\begin{array}{l}\text { Pitt } 8 \\
\text { Pitt } 8 \\
\text { Pitt } 8 \\
\text { Pitt } 8 \\
\text { Pitt } 8\end{array}$ & $\begin{array}{rl}91 & 1 \mathrm{~b} \\
160 & 1 \mathrm{~b} \\
11 & 1 \mathrm{~b} \\
419 & 1 \mathrm{~b} \\
140 & 1 \mathrm{~b} \\
821 & 1 \mathrm{~b}\end{array}$ & $\begin{array}{rl}43 & 1 b \\
74 & 1 b \\
5 & 1 b \\
190 & 1 b \\
73 & 1 b \\
385 & 1 b\end{array}$ & $\begin{array}{l}1.60 \\
1.55 \\
1.74^{\star} \\
1.74 \\
1.72\end{array}$ & $\begin{array}{l}1.14 \\
0.64 \\
1.14^{\star} \\
0.87 \\
2.02\end{array}$ & $\begin{array}{l}27.65 \\
25.15 \\
32.56^{*} \\
32.56 \\
30.84\end{array}$ & $\begin{array}{l}13948^{\star} \\
13948 \\
14000^{\star} \\
14150 \\
13000^{\star}\end{array}$ & $\begin{array}{l}52.24 \\
53.93 \\
52.24 \\
54.74 \\
47.73\end{array}$ \\
\hline $\begin{array}{l}7 \mathrm{AA} \\
7 \mathrm{~A}+ \\
7 \mathrm{~B} \\
7 \mathrm{C} \\
7 \mathrm{D} \\
7 \mathrm{E}\end{array}$ & $\begin{array}{l}\text { Kent } 9 \\
\text { Kent } 9 \\
\text { Kent } 9 \\
\text { Pitt } 8 \\
\text { Pitt } 8 \\
\text { Pitt } 8\end{array}$ & $\begin{array}{rl}571 & \mathrm{lb} \\
30 & \mathrm{lb} \\
85 & \mathrm{lb} \\
80 & \mathrm{lb} \\
29 & \mathrm{lb} \\
13 & \mathrm{lb} \\
808 \mathrm{lb}\end{array}$ & $\begin{array}{rr}238 & 1 \mathrm{~b} \\
12 & 1 \mathrm{~b} \\
35 & 1 \mathrm{~b} \\
33 & 1 \mathrm{~b} \\
13 & 1 \mathrm{~b} \\
6 & 1 \mathrm{~b} \\
337 & 1 \mathrm{~b}\end{array}$ & $\begin{array}{l}0.71 \\
0.58 \\
0.56 \\
0.64 \\
0.56 \\
1.32\end{array}$ & $\begin{array}{l}0.55 \\
0.38 \\
0.27 \\
0.68 \\
0.58 \\
1.42\end{array}$ & $\begin{array}{l}26.36 \\
25.49 \\
29.07 \\
28.13 \\
28.94 \\
29.15\end{array}$ & $\begin{array}{l}13262 \\
13262 \\
13285 \\
13349 \\
12976 \\
13349 \star\end{array}$ & $\begin{array}{l}58.26 \\
59.29 \\
59.37 \\
58.35 \\
55.86 \\
51.35\end{array}$ \\
\hline $\begin{array}{l}8 A \\
8 B \\
8 C \\
8 D \\
8 E \\
8 F\end{array}$ & $\begin{array}{l}\text { Pitt } 8 \\
\text { Pitt } 8 \\
\text { Pitt } 8 \\
\text { Pitt } 8 \\
\text { Pitt } 8 \\
\text { Pitt } 8\end{array}$ & $\begin{array}{r}2531 \mathrm{lb} \\
1061 \mathrm{lb} \\
1691 \mathrm{lb} \\
126 \mathrm{lb} \\
991 \mathrm{lb} \\
112 \mathrm{lb} \\
865 \mathrm{lb}\end{array}$ & $\begin{array}{rr}113 & 1 \mathrm{~b} \\
50 & 1 \mathrm{~b} \\
82 & 1 \mathrm{~b} \\
60 & 1 \mathrm{~b} \\
48 & 1 \mathrm{~b} \\
52 & 1 \mathrm{~b} \\
4051 \mathrm{~b}\end{array}$ & $\begin{array}{l}1.22 \\
1.35 \\
1.18 \\
0.96 \\
1.46 \\
0.97\end{array}$ & $\begin{array}{l}1.01 \\
1.95 \\
1.20 \\
1.03 \\
1.18 \\
0.54\end{array}$ & $\begin{array}{l}26.54 \\
26.12 \\
23.91 \\
24.41 \\
26.45 \\
26.19\end{array}$ & $\begin{array}{l}13359 \\
13616 \\
13278 \\
13694 \\
14001 \\
14177\end{array}$ & $\begin{array}{l}55.14 \\
52.66 \\
51.58 \\
52.12 \\
51.34 \\
54.03\end{array}$ \\
\hline
\end{tabular}

* Estimated values.

${ }^{\star \star A}$ All Pittsburgh No. $8 \mathrm{coal}$ on this page of this table is from the Powhatan No. 6 min:

7 At represents coal product collected at $8: 15,9: 15,10: 15$ and $12: 15$ on 5/2/1989. Compliance coal was produced during this period of $7 \mathrm{~A}$. 
TABLE 3-4. MCL PRODUCT COAL SHIPPED AT END OF PHASE I

$\begin{array}{lrr}\begin{array}{l}\text { Ship } \\ \text { Drum } \\ \text { No. }\end{array} & \begin{array}{c}\text { Coal } \\ \text { Type }\end{array} & \begin{array}{c}\text { Net } \\ \text { Ship } \\ \text { Wt. Lbs }\end{array} \\ 096 & \text { Ky } 9 & 176.5 \\ 098 & \text { Ky } & 200.8 \\ 108 & \text { Ky } 9 & 218.1 \\ 109 & \text { Ky } 9 & 247.0 \\ \text { Total Ship Wt. Ky } 9 & \\ \text { Total Dry Wt. Ky } 9 & 842.4 \\ & & 486.0 \\ 107 & & \\ 112 & & 173.9 \\ 116 & & 173.4 \\ 119 & \text { Pitt } 8 & 214.7 \\ 120 & \text { Pitt } 8 & 173.8 \\ \text { Total Ship Wt. Pitt } 8 & 244.9 \\ \text { Total Dry Wt. Pitt } 8 & 980.7 \\ \end{array}$

All Pittsburgh No. 8 coal shipped was from Powhatan No. 6 mine. 


\section{TEST RESULTS}

The objectives of shakedown and operational testing of the 20 pounds of coal per hour integrated MCL test circuit were: 1) to demonstrate the technical capability of the MCL process for producing a demineralized and desulfurized coal that meets New Source Performance Standards (NSPS), 2) to determine the range of effective process operation, 3) to test process conditions aimed at significantly lower costs, 4) to obtain scaleup data, and 5) to deliver product coal. The integrated test circuit produced coal with sulfur content well below the New Source Performance Standards and with equipment performance consistent with commercial design requirements. An assessment of the results indicated that the MCL circuit (as modified for Phase II) is capable of integrated, continuous, long-duration operational testing.

The MCL integrated test circuit was operated in a shakedown mode for approximately 100 hours in Decemier 1988, following by nine series of operational tests (Runs 1 thiough i) during Phase I between January and June 1989, and two additional operational tests (Runs 10 and 11) during Phase II for a total of approximately 1000 hours of operational time during which a 63-test matrix was covered. About 3,700 pounds of treated coal suitable for further testing and evaluation were produced. Either a 50/50 potassium hydroxide/sodium hydroxide mixture or pure sodium hydroxide was used as the leaching agent. The three feed coals processed were representative of the Eastern United States coal production: Pittsburgh No. 8 (Powhatan No. 6 mine), Pittsburgh No. 8 (Blacksville No. 2 mine), and Kentucky No. 9. Pittsburgh No. 8 (Powhatan No. 6 mine) coal was used for most of the testing. The feed coal size was 14 mesh in all but 5 of the tests, during which 6 mesh coal was fed to the kiln. The rotary kiln was operated with average wall temperatures of 340 to $438^{\circ} \mathrm{C}$. The residence tinie of the coal/caustic mixture in the heated zones of the rotary kiln was approximately 2 hours for a majurity of the runs. However, the heaters in the first, or the first and second, zones of the rotary kiln were shut-off during selected runs to simulate the conditions of shorter residence time. Nitrogen gas was passed through the kiln to suppress carbonate formation and to assure that 
the hydrogen and hydrocarbons present in the kiln gas would be below the flammability limit. The flow of nitrogen inside the kiln was cocurrent with the coal/caustic flow during shakedown and the first six series of tests, and countercurrent to the coal/caustic flow in the last five series of tests.

The wash train during shakedown and the operational tests included five centrifuge stages in the caustic washing section and three centrifuge stages in the acid washing section. Due to problems with the two rotary drum vacuum filters in the caustic wash train, the vacuum filters were bypassed during most of Phase I testing. When the filters were bypassed, the caustic wash train utilized only the five centrifuges and was operated with elevated wash water to caustic ratio to provide a first stage liquid specific gravity under 1.2 as is required for centrifuge coal separation. The vacuum filtration system was redesigned as part of Phase II and was operated as part of the wash train during Phase II testing (Runs 10 and 11 ).

During Phase I, the spent caustic solution sent to the regenerator contained less than $15 \%$ caustic (instead of $50 \%$ caustic) as a result of the vacuum filters not being on-line, and regeneration of the caustic was conducted by the addition of 1 ime to the spent caustic solution, typically at a temperature of $43^{\circ} \mathrm{C}\left(109^{\circ} \mathrm{F}\right)$. Several tests were conducted in the caustic evaporation/flaking system during Phase I system operation. Due to the problems with the vacuum filters, aqueous $50 \%$ caustic evaporator feed solutions were specially prepared for these tests. However, once the vacuum filtration system was modified during Phase II, the vacuum filters were operated on-7ine with the rest of plant (Runs 10 and 11 ).

The week of shakedown testins was conducted at a kiln wall temperature of $340^{\circ} \mathrm{C}$ which was $50^{\circ} \mathrm{C}$ less than the next lowest Phase I operating temperature of $390^{\circ} \mathrm{C}$ (Run $1 \mathrm{~A}$, Run $1 \mathrm{~B}$, Run $2 \mathrm{~A}$ and Run $2 \mathrm{~B}$ ). Run 10 in Phase II was operated at low kiln wall temperatures of 360 to $400^{\circ} \mathrm{C}$. Since shakedown was the first time the refurbished kiln was operated at temperature with its new auger/scraper and also the first time in conjunction with the integrated MCL system, shakedown testing was essentiall / part of the final checkout of the system. For this reason, a mild kiln cperating temperature was used. Since such a low kiln temperature is not remresentative of full scaie kiin operating temperatures, the shakedown test results are not 
incorporated in some of the tests results presented in this section. Whenever, shakedown data were excluded, it is noted in the text. For a more detailed discussion of shakedown test results, see the report entitled "Topical Report for Test Circuit Procurement, Fabrication, Installation, Shakedown and Test Plan Formulation" dated November 1990.

Run 10 was the first integrated test conducted in Phase II after the MCL facility had undergone extensive refurbishments and design modifications. Also the plant had been dormant for two years (Run No. 9 was conducted in June 1989 and Run No. 10 in June 1991). Since Run 10 was in many ways a shakedown of the modified system, mild kiln operating temperatures were used in order to checkout the system rather than produce low sulfur coal. Also, problems with filtration (high levels of coal fines, blinding of the filter cloth) indicated that the three-year old Pittsburgh No. 8 (Powhatan) coal used for Run 10 test might be weathered. Subsequent analysis of coal samples indicated weathering as noted by the increase in sulfate sulfur and the decrease in heating value when compared to the original analysis provided when the coal was purchased in June 1988. Weathered coal is highly friable. Since low kiln temperatures and weathered coal are not representative of full scale operations, test results from Run 10 are not incorporated in some of the test results presented in this section. Whenever, Run 10 data were excluded, it is noted in the text.

To avoid the filter problems encountered in Run 10, fresh Pittsburgh No. 8 coal (Blacksville) was acquired for Run 11. Since the Blacksville coal was much lower in both sulfur $(2.26 \%$ vs. $4.27 \%)$ and ash $(7.90 \%$ vs. $11.57 \%)$ than the Powhatan coal, the results from Run 11 are not incorporated in some of the correlations presented in this section but are discussed separately. For a more detailed discussion of Run 10 and Run 11 test results, see the Phase II report entitled "Topical Report for Test Circuit Operation" dated February 1993.

\subsection{COAL PRODUCT QUALITY}

Coal product samples were obtained either from the end of the wash train (CP samples) or from the kiln discharge (KD samples) for analysis. The KD samples were acquired for several reasons: to provide an early indication 
whether the kiln operating conditions were conducive to production of compliance coal; to determine the residence time of the coal/caustic mixture inside the kiln by collection of all discharge products; to perform material balances; and to evaluate the formation of carbonates and sulfates inside the rotary $\mathrm{kiln}$. The $C P$ samples, on the other hand, were more representative of the product obtained from an integrated MCL plant. There was also less variability in the $C P$ samples from the same run because of the mixing and reslurrying processes in the wash train. For most of the runs, analysis results for both $C P$ and $K D$ samples are available. In these cases, the $C P$ results were used as the basis for assessing the effects of operating parameters on coal product quality. The KD results were used whenever $C P$ results were unavailable.

The complete analysis results for the sulfur content, ash content, volatile content, moisture content and heating value of the coal product samples are presented in Appendix $A$ of this report. These analysis results are summarized in Tables 4-1 through 4-4 for Pittsburgh No. 8 (Powhatan) coal processed with mixed caustic (sodium and potassium hydroxides), Pittsburgh No. 8 coal (Powhatan) processed with only sodium hydroxide, Kentucky No. 9 coal processed with either mixed caustic or sodium hydroxide, and Pittsburgh No. 8 coal (Blacksville) processed with only sodium hydroxide, respectively.

\subsubsection{Sulfur Removal}

The sulfur content of the MCL coal product generally decreases with increasing caustic/coal ratio and increasing reaction temperature, but also depends on the type of coal and the type of caustic used, as well as the residence time of the coal/caustic mixture in the heated zones of the rotary kiln.

\subsubsection{Pittsburgh No. 8 (Powhatan); $50: 50 \mathrm{KOH} / \mathrm{NaOH}$}

The analysis results presented in Table 4-1 show that the combination of high kiln wall temperature, long residence time and high caustic/coal ratio (R) will likely produce compliance coal. Approximately $90 \%$ sulfur dioxide removal (based on $1 \mathrm{bs} / \mathrm{mill}$ ion Btu removed from run-of-mine coal) was achieved with kiln wall temperature $\geq 427^{\circ} \mathrm{C}$, residence time of 2.0 hours, and caustic/coal $=2$, as indicated by the Run $2 C$, Run $3 B 1$ and Run $7 D$ test results 


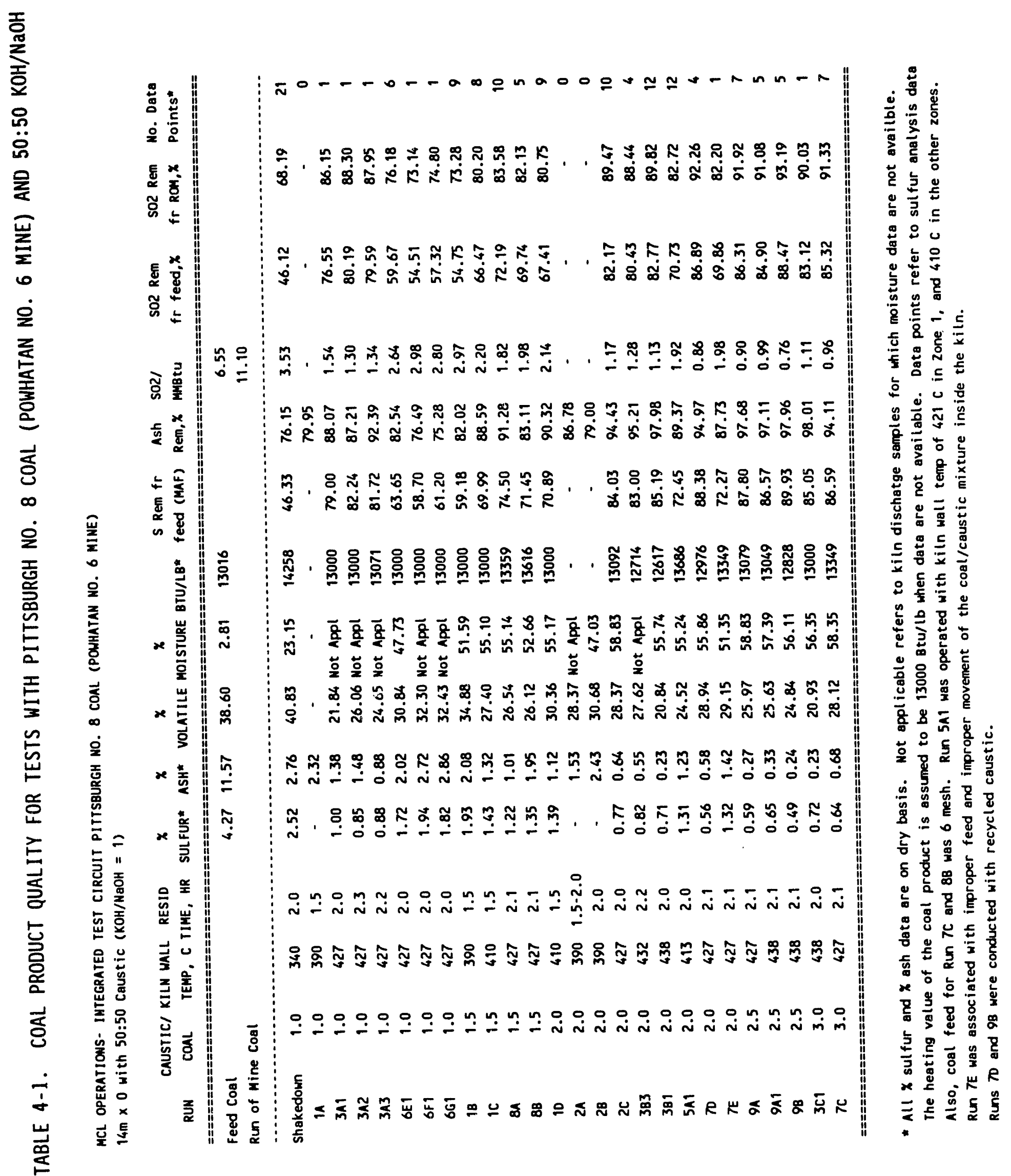



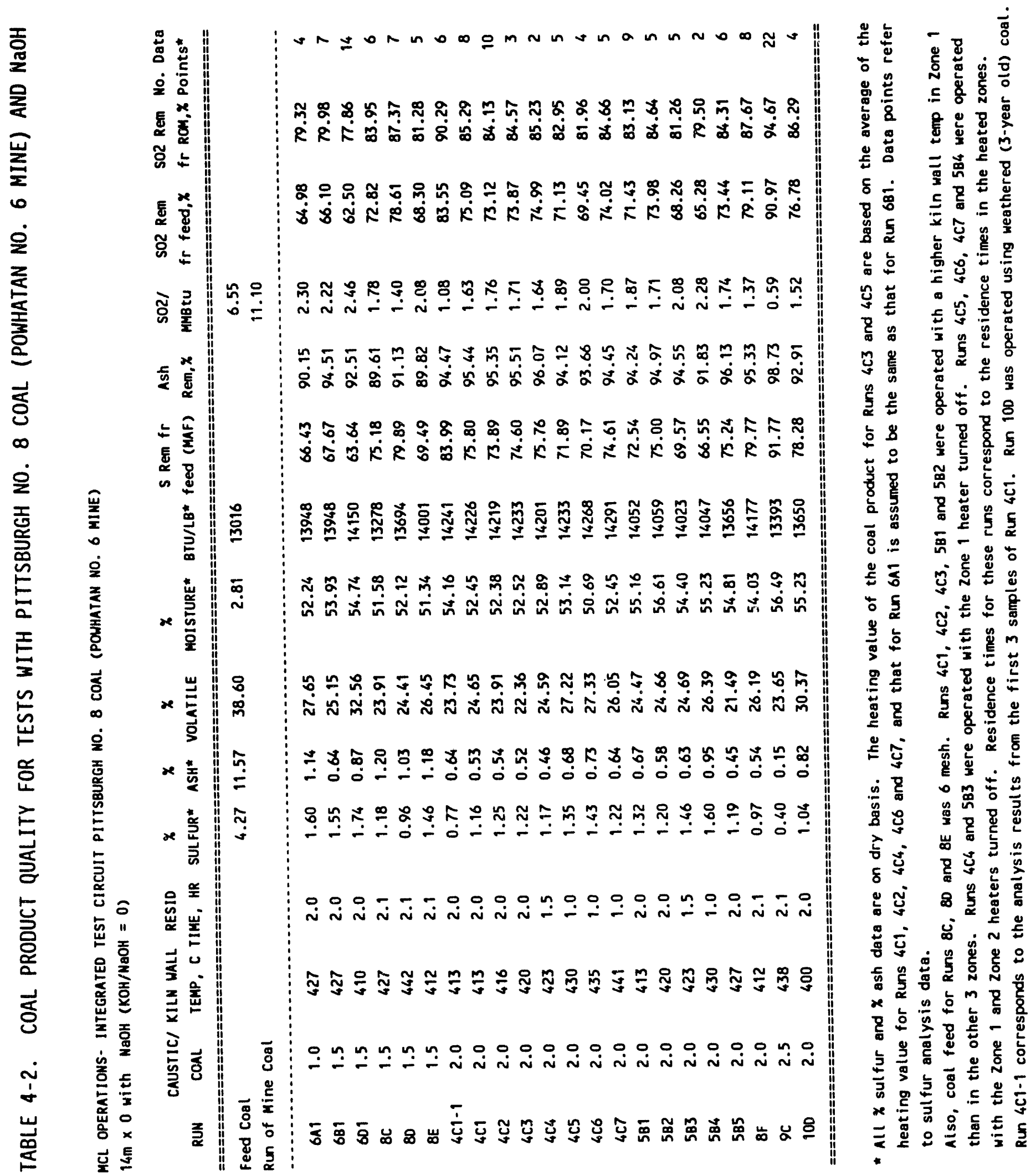


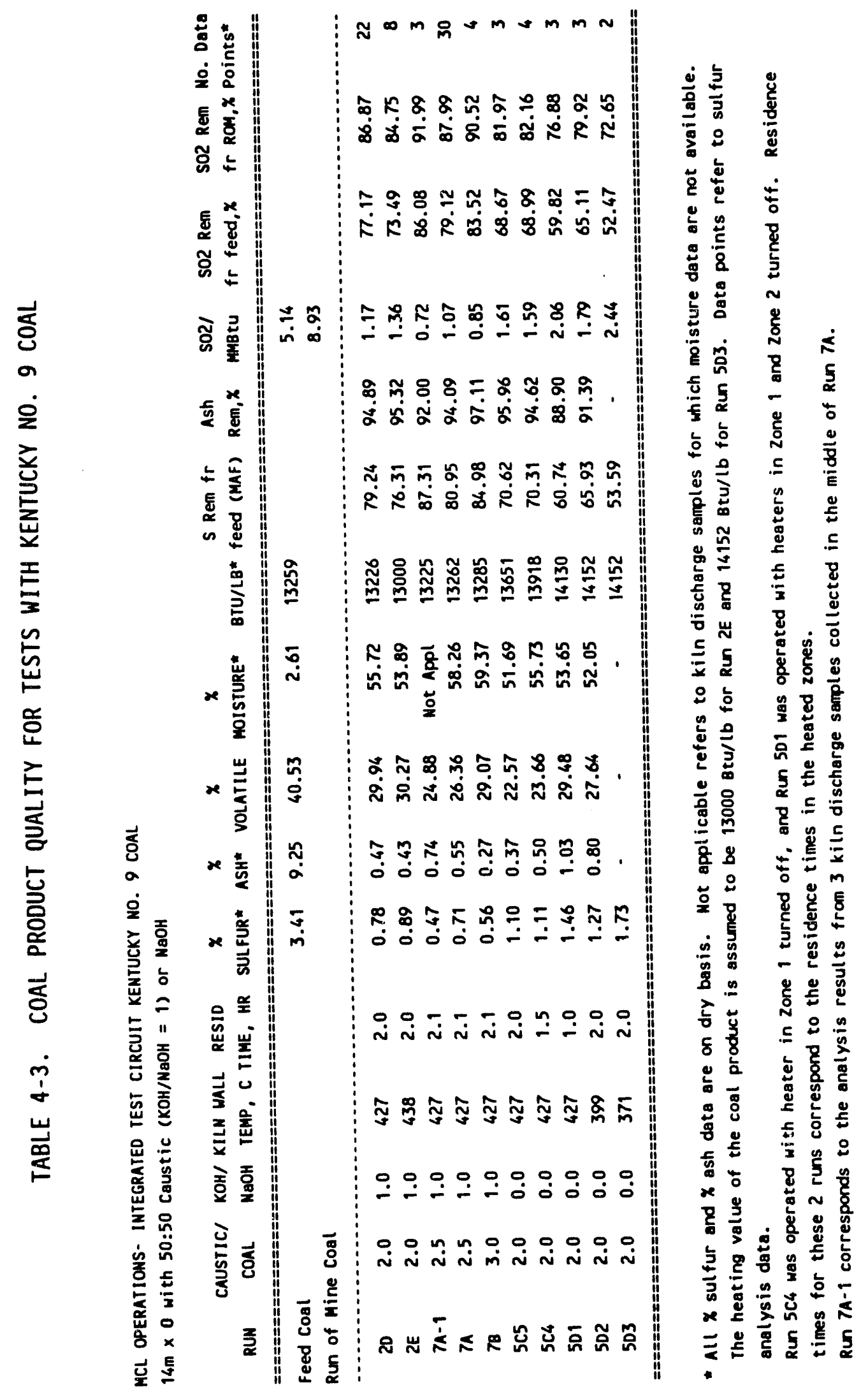




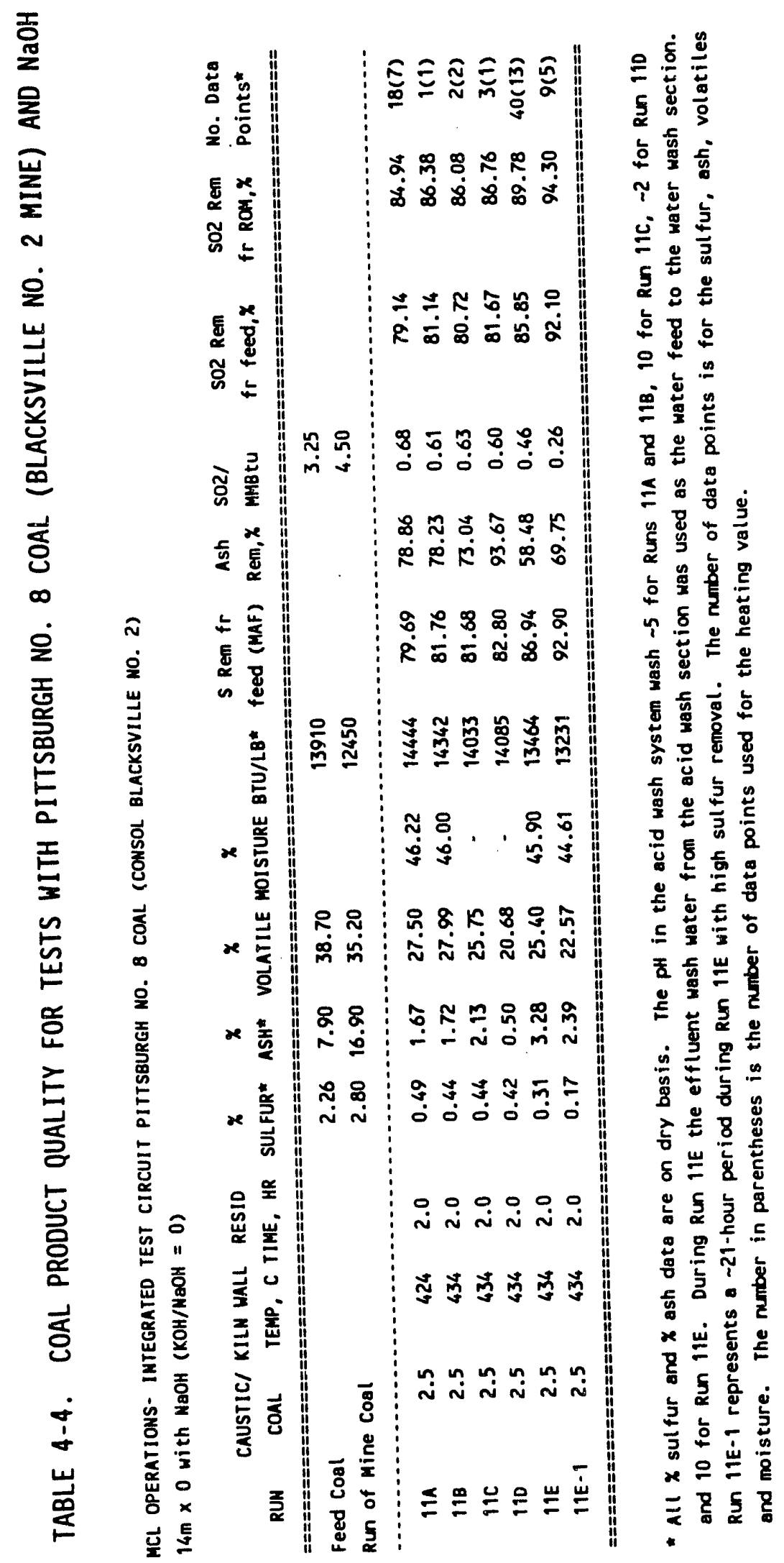


at $89.5 \%, 89.8 \%$ and $92.3 \%$ sulfur dioxide removal, respectively. Further, sulfur dioxide removal of $90 \%$ or above was achieved with kiln wall temperature $\geq 427^{\circ} \mathrm{C}$, residence time of 2.0 hours, and caustic/coal $\geq 2.5$, as indicated by the Run $3 C 1$, Run $7 C$, Run $9 A$, Run $9 A 1$ and Run $9 B$ test results at $90.0 \%, 91.3 \%, 91.9 \%, 91.1 \%$ and $93.3 \%$ sulfur dioxide removal, respectively.

The effects of caustic/coal ratio and reaction temperature on sulfur removal was also evaluated by regression analysis. The correlation between the sulfur content of the coal product and the caustic/coal ratio (R) and the kiln wall temperature $\left(T\right.$ in $\left.{ }^{\circ} \mathrm{C}\right)$ is as follows:

$$
\% \text { sulfur }=10.143-0.3697 R-0.01975 T
$$

with a correlation coefficient of 0.7848 (excluding shakedown data). The correlation between the sulfur content of the coal product and the kiln wall temperature, if only runs with caustic/coal ratio $\geq 2$ are included in the regression analysis, is as follows:

$$
\% \text { sulfur }=11.393-0.02478 \mathrm{~T}
$$

with a correlation coefficient of -0.8209 . The negative regression coefficients in these correlations indicate that the sulfur content of the MCL coal product decreases with increasing caustic/coal ratio and increasing kiln wall temperature. These same effects are illustrated by plotting the sulfur analysis results in Figure 4-1. However, Figure 4-1 and Table 4-1 also show that based on the limited test results from Runs $3 A 1,3 A 2$ and $3 A 3$, it may be possible to achieve 86 to $88 \%$ sulfur dioxide removal with caustic/coal ratio of 1 and kiln wall temperature of $427^{\circ} \mathrm{C}$. At higher reaction temperatures, it is conceivable that $90 \%$ sulfur dioxide removal (corresponding to $0.7 \%$ sulfur in $\mathrm{MCL}$ COal) may be achieved even at a caustic/coal ratio of 1 . On the other hand, Figure 4-1 and Table 4-1 indicate that at kiln wall temperatures below or near $410^{\circ} \mathrm{C}$ and with nitrogen flow and coal feed in cocurrent direction, less than $81 \%$ sulfur removal can be obtained, as shown by the test results from Runs 1B, 1C, 10 and 5Al. Shakedown which was operated at a mild kiln wall temperature of $340^{\circ} \mathrm{C}$ achieved a sulfur dioxide removal of only $68 \%$. 


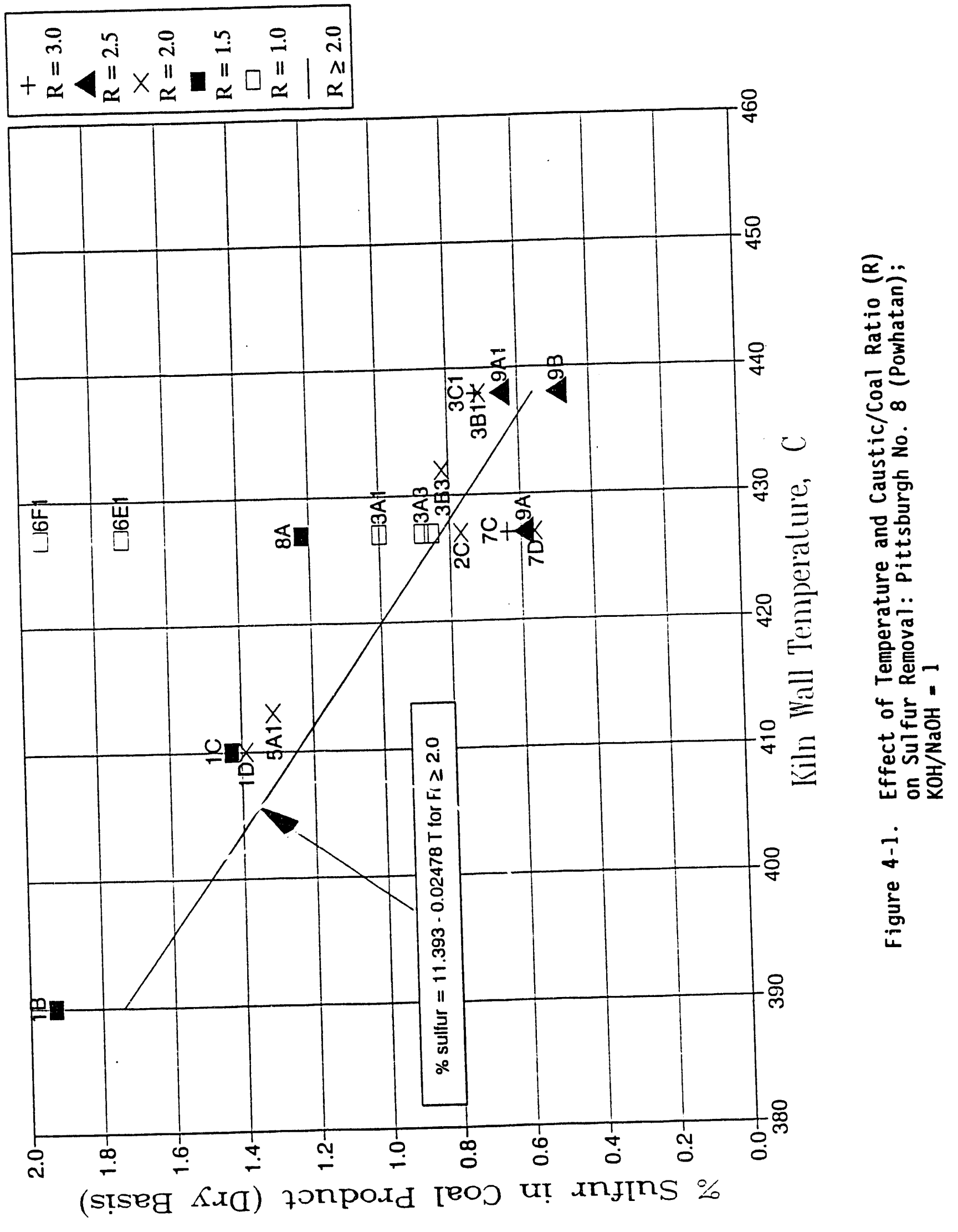


In Figure 4-2, the sulfur content of the MCL coal product from analysis of the samples is compared with the predicted values according to the correlation represented by equation [1]. For 12 of the 21 runs, the predicted and experimental sulfur levels are in very good agreement. For Runs $2 C, 3 A 1,3 A 2,3 A 3$ and $7 D$, the predicted sulfur levels are higher than the experimental values. For Runs $3 C 1,6 E l, 6 F 1$ and $6 \mathrm{Gl}$, the predicted sulfur levels are lower than the experimental values. Only one sample each from Runs $3 A 1,3 A 2,3 A 3,3 C 1,6 F 1$ and $6 \mathrm{Gl}$, however, was acquired and analyzed. Therefore, some of the disagreement between predicted and experimentally determined sulfur levels may be simply due to the small number of samples analyzed in these runs to provide a good estimate of the actual sulfur level.

The effects of countercurrent versus cocurrent nitrogen flow was evaluated by comparing sulfur removal from runs with otherwise similar operating conditions. Runs $7 C$ and $7 D$, with nitrogen flow in countercurrent direction to the coal/caustic flow, resulted in sulfur dioxide removal of 91.3\% and 92.3\%. By comparison, the corresponding Runs $2 \mathrm{C}$ and $3 \mathrm{Cl}$ with nitrogen and coal/caustic flow in cocurrent direction, resulted in lower sulfur dioxide removal of $89.5 \%$ and $90.0 \%$. In runs with cocurrent nitrogen and coal/caustic flow, the temperature of the coal/caustic mixture in the first heated zone was typically about $130^{\circ} \mathrm{C}$ below the kiln wall temperature. (Shakedown testing was conducted with cocurrent flows). In runs with countercurrent nitrogen and coal/caustic flow, the temperature sensor for the coal/caustic mixture was moved downstream near the second heated zone. However, the sensor temperature in these runs was typically only $30^{\circ} \mathrm{C}$ below the kiln wall temperature. Therefore, the higher sulfur removal for the countercurrent nitrogen flow runs was due to longer residence time of the coal/caustic mixture at temperatures of near $400^{\circ} \mathrm{C}$. For Pitisburgh No. 8 (Powhatan) coal processed with 50:50 $\mathrm{KOH} / \mathrm{NaOH}$, no tests were conducted with some of the kiln heaters shut off. Thus, no data are available to assess the effects of shorter residence time on sulfur removal due to shut off of the heated zones.

Runs $7 D$ and $9 B$ were conducted using caustic mixtures that contained regenerated caustic. In Run 70 , this caustic mixture was the evaporator 


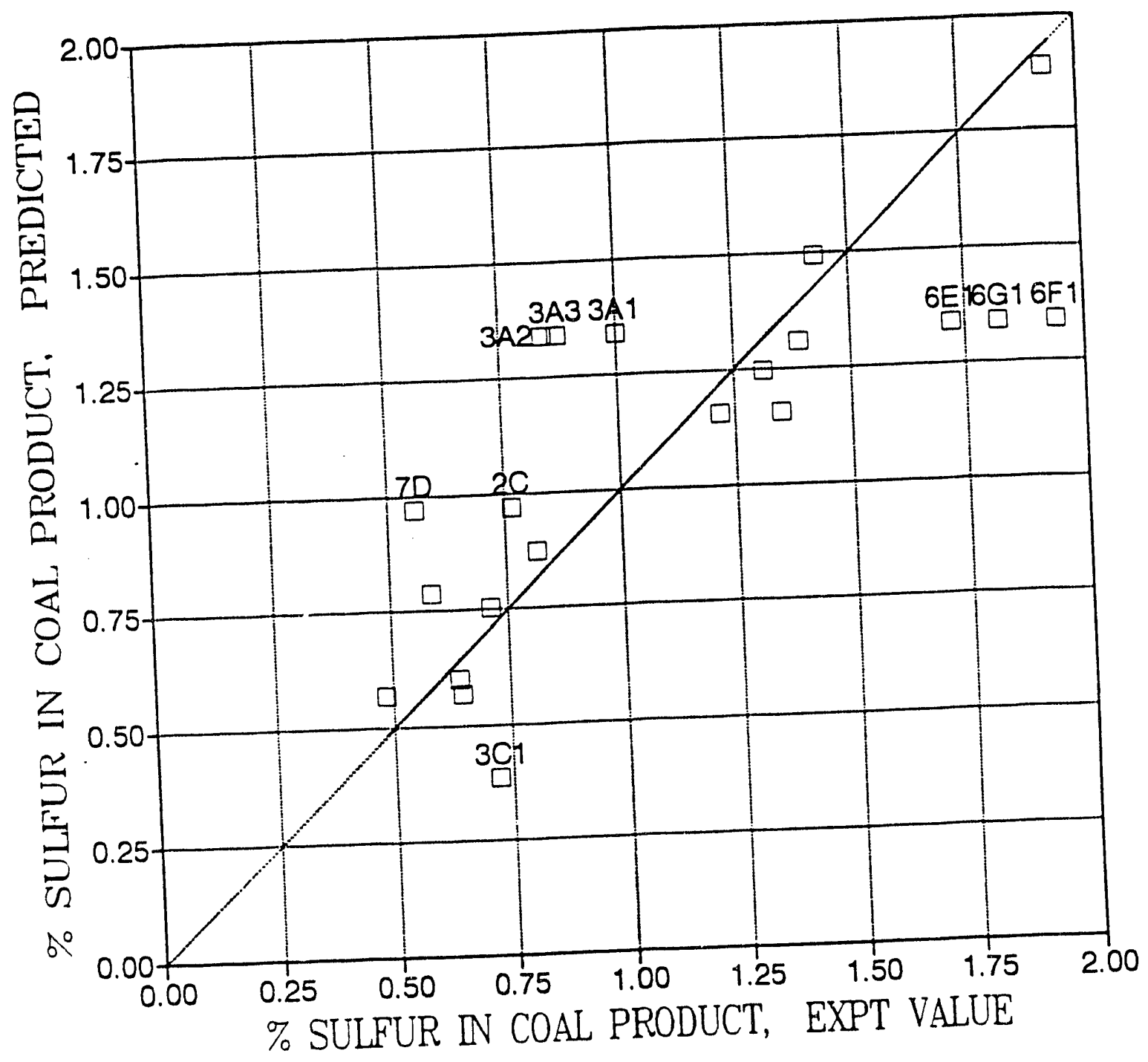

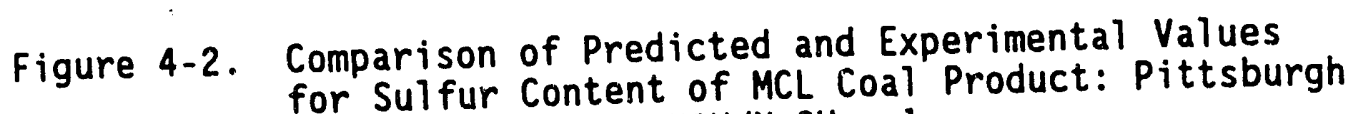
No. 8 (Powhatan); $\mathrm{KOH} / \mathrm{NaOH}=1$ 
flake product obtained from an evaporator feed consisting of $51 \%$ caustic (50:50 $\mathrm{KOH} / \mathrm{NaOH})$, prepared by mixing regenerated $13 \%$ caustic $(50: 50 \mathrm{KOH} / \mathrm{NaOH})$ with purchased caustic solids $(50: 50 \mathrm{KOH} / \mathrm{NaOH})$. The concentration of regenerated caustic in the caustic feed to the kiln in Run 70 was $14 \%$. In Run $9 B$, the caustic feed was the evaporator flake product obtained totally from regenerated caustic. This evaporator product contained $80.6 \%$ potassium and sodium hydroxide, $5.6 \%$ potassium and sodium carbonate, $0.4 \%$ potassium and sodium sulfide, and approximately $13.4 \%$ moisture. The Run $7 D$ and Run 98 test results show sulfur dioxide removal of $92.3 \%$ and $93.2 \%$, respectively. These test results demonstrate that high sulfur removal can be achieved using regenerated caustic.

Runs $8 A$ and $8 B$ were conducted under similar conditions except that 14 mesh coal feed was used in Run $8 A$ and 6 mesh coal feed in Run 8B. The average sulfur content of the MCL coal product was $1.22 \%$ for Run $8 A$ and $1.35 \%$ for Run $8 \mathrm{~B}$. Therefore, it appears that processing with a finer coal feed would result in higher sulfur removal.

\subsubsection{Pittsburgh No. 8 (Powhatan): NaOH Only}

In the analysis results presented in Table 4-2, $94.7 \%$ sulfur dioxide removal (based on lbs/million Btu removed from run-of-mine coal) was obtained for Run $9 \mathrm{C}$, with $\mathrm{kiln}$ wall temperature of $438^{\circ} \mathrm{C}$, residence time of 2.1 hours, caustic/coal ratio of 2.5 , and nitrogen and coal/caustic flow in countercurrent direction. In addition, over $90 \%$ sulfur dioxide removal was obtained during the first 14 hours of operation for Run $4 C 1$ (shown as Run 4C1-1 in Table 4-2). However, the same level of sulfur removal was not achieved in latter parts of the same run. Sulfur dioxide removal for all other runs was less than $88 \%$. At caustic/coal of 1.5 or 1.0 , and with nitrogen and coal/caustic flow in cocurrent direction, sulfur dioxide removal was less than $80 \%$, as shown by the test results from Runs $6 \mathrm{Al}, 6 \mathrm{Bl}$ and 601 .

The correlation between the sulfur content of the coal product and caustic/coal ratio $(R)$ and the kiln wall temperature $\left(T\right.$ in $\left.{ }^{\circ} \mathrm{C}\right)$ is:

$$
\% \text { sulfur }=9.785-0.6440 R-0.01756 T
$$


with a correlation coefficient of 0.8971 , if the regression analysis exclude data points for runs with shut-off of one or two kiln heaters (Runs $4 C 4,4 C 5$, $4 C 6,4 C 7,5 B 3$ and 5B4). These excluded data points represent runs operated with shorter residence time in the heated zones, which is an additional parameter not addressed in the above correlation. In addition, Run 100 data were excluded from this correlation due to the fact that weathered coal was used for that run. The correlation between the sulfur content of the coal product and the kiln wall temperature, if in addition only runs with caustic/coal ratio $\geq 2$ are included in the regression analysis, is as follows:

$$
\% \text { sulfur }=11.443-0.02466 \mathrm{~T}
$$

with a correlation coefficient of -0.7366 (excluding Run 10D). The correlation represented by equation [4] is very similar to the correlation represented by equation [2], and gives only slightly higher sulfur content for the coal product. This implies that with caustic/coal $\geq 2, \mathrm{NaOH}$ is almost as effective as $50: 50 \mathrm{KOH} / \mathrm{NaOH}$ in removing sulfur from Pittsburgh No. 8 coal. By comparing the correlations represented by equations [1] and [3], it also appears that sulfur removal is more sensitive to the caustic/coal ratio for $\mathrm{NaOH}$ than for $50: 50 \mathrm{KOH} / \mathrm{NaOH}$. At caustic/coal ratio of 1.0 or 1.5 , these correlations indicate that $50: 50 \mathrm{KOH} / \mathrm{NaOH}$ would be more effective than $\mathrm{NaOH}$ in removing sulfur from Pittsburgh No. 8 coal. The difference in sulfur removal could be partly attributed to the lower melting point of the 50:50 $\mathrm{KOH} / \mathrm{NaOH}$ (approximately $220^{\circ} \mathrm{C}$ ) versus $\mathrm{NaOH}\left(318^{\circ} \mathrm{C}\right.$ ), so that there is effectively longer residence time for the mixed caustic than for $\mathrm{NaOH}$ in molten form to penetrate the coal matrix. The intimate contact between molten caustic and the coal matrix is also apparently more important for low caustic/coal ratios, where less molten caustic is accessible to the coal matrix. However, these comparisons may no longer be applicable if molten caustic feed were to be used in the MCL process.

The effects of reaction temperature and caustic/coal ratio are also illustrated in Figure 4-3. (Run 100 is shown in this figure although Run 100 was excluded from the above correlations due to weathered coal.) It is shown that operation with high kiln wall temperature and caustic/coal ratio $\geq 2$ 


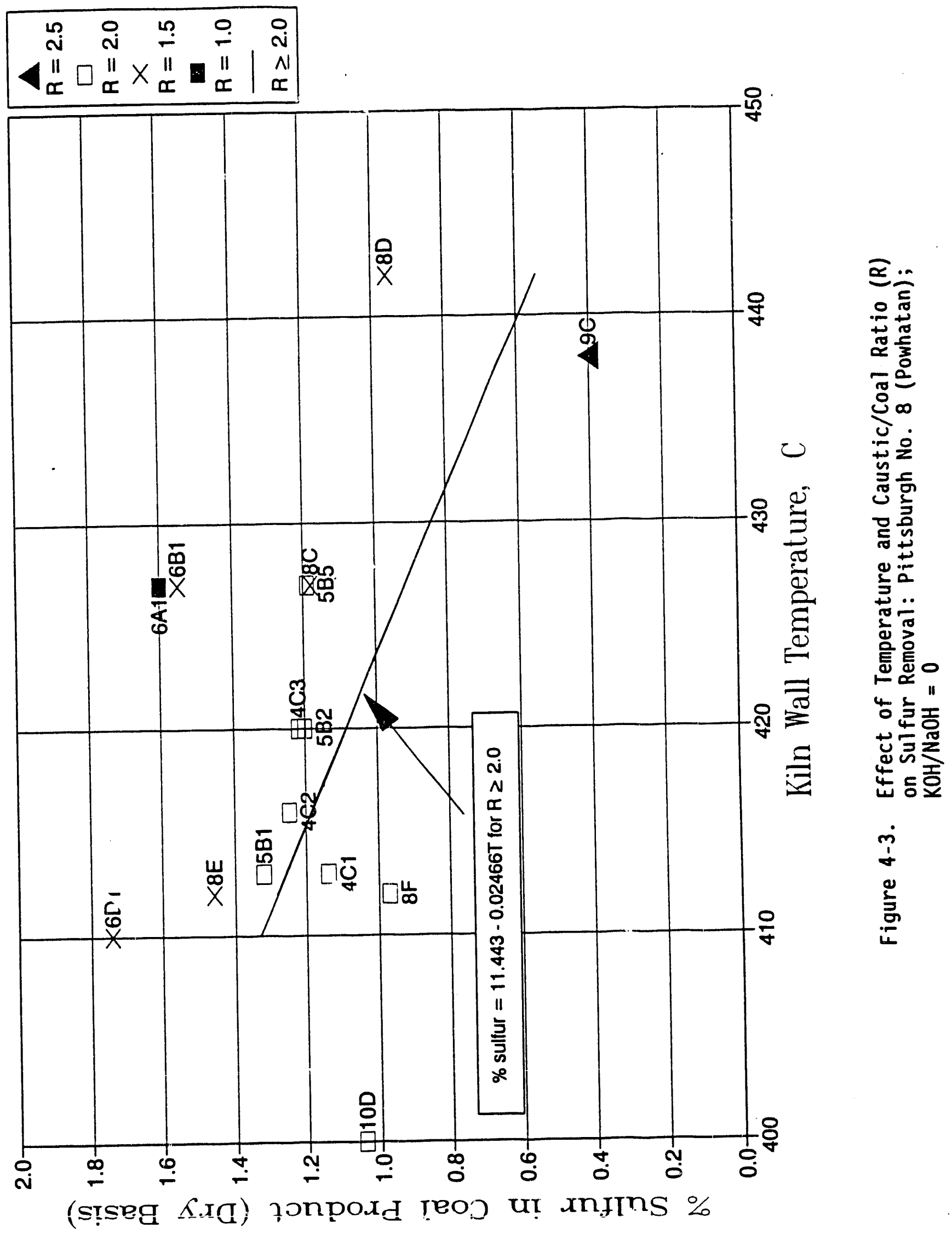


would result in low sulfur content in the coa? product. Again, it is conceivable that with molten caustic feed, high sulfur removal can be achieved even at low caustic/coal ratios.

In Figure 4-4, the sulfur content of the $M C L$ coal product from analysis of the samples is compared with the predicted values according to the correlation represented by equation [3]. For all 14 runs with residence time of 2 hours, the predicted and experimental sulfur levels are in good agreement. (Run 100 is shown in this figure although Run 100 was excluded from the above correlation due to weathered coal.) The runs with shorter residence times are not represented in this figure. Examination of the data points, however, indicated that for all six runs (Runs $8 \mathrm{C}, 8 \mathrm{D}, 8 \mathrm{E}, 8 \mathrm{~F}, 9 \mathrm{C}$ and 10D) conducted with nitrogen flow in countercurrent direction to the flow of the coal/caustic mixture, the predicted sulfur levels are higher than the experimental values. This suggests that the correlation equation [3] over predicts the sulfur levels in the case of countercurrent nitrogen flow. which is more effective. than cocurrent nitrogen flow in sulfur removal.

There is only one pair of runs to directly compare the effects of countercurrent versus cocurrent nitrogen flow on sulfur removal. Runs $6 \mathrm{Bl}$ and $8 \mathrm{C}$ were conducted under similar conditions except that nitrogen flow was in countercurrent direction to coal/caustic flow in the latter run, and that 6 mesh coal was fed to the kiln. Even with the coarser coal fed, the sulfur dioxide removal for Run $8 \mathrm{C}$, at $84.0 \%$, was higher than the $80.0 \%$ obtained for Run $6 \mathrm{Bl}$. This again demonstrates that countercurrent nitrogen flow is more effective than cocurrent nitrogen flow in sulfur removal.

The effect of coal feed size on sulfur removal is less clear when only sodium hydroxide was used as the leaching agent. Runs $8 \mathrm{C}, 8 \mathrm{D}$ and $8 \mathrm{E}$ were conducted with 6 mesh coal feed. However, these runs were also all conducted with a caustic/coal ratio of 1.5 and nitrogen and coal/caustic flow in countercurrent direction. There were no other runs conducted at similar operating conditions and with 14 mesh coal feed to provide direct comparison. Runs $8 E$ and $8 F$ ( 14 mesh coal feed) were both conducted with kiln wall temperature of $412^{\circ} \mathrm{C}$, residence time of 2.1 hours and countercurrent nitrogen flow. With a caustic/coal ratio of 2.0 , the sulfur content of the 


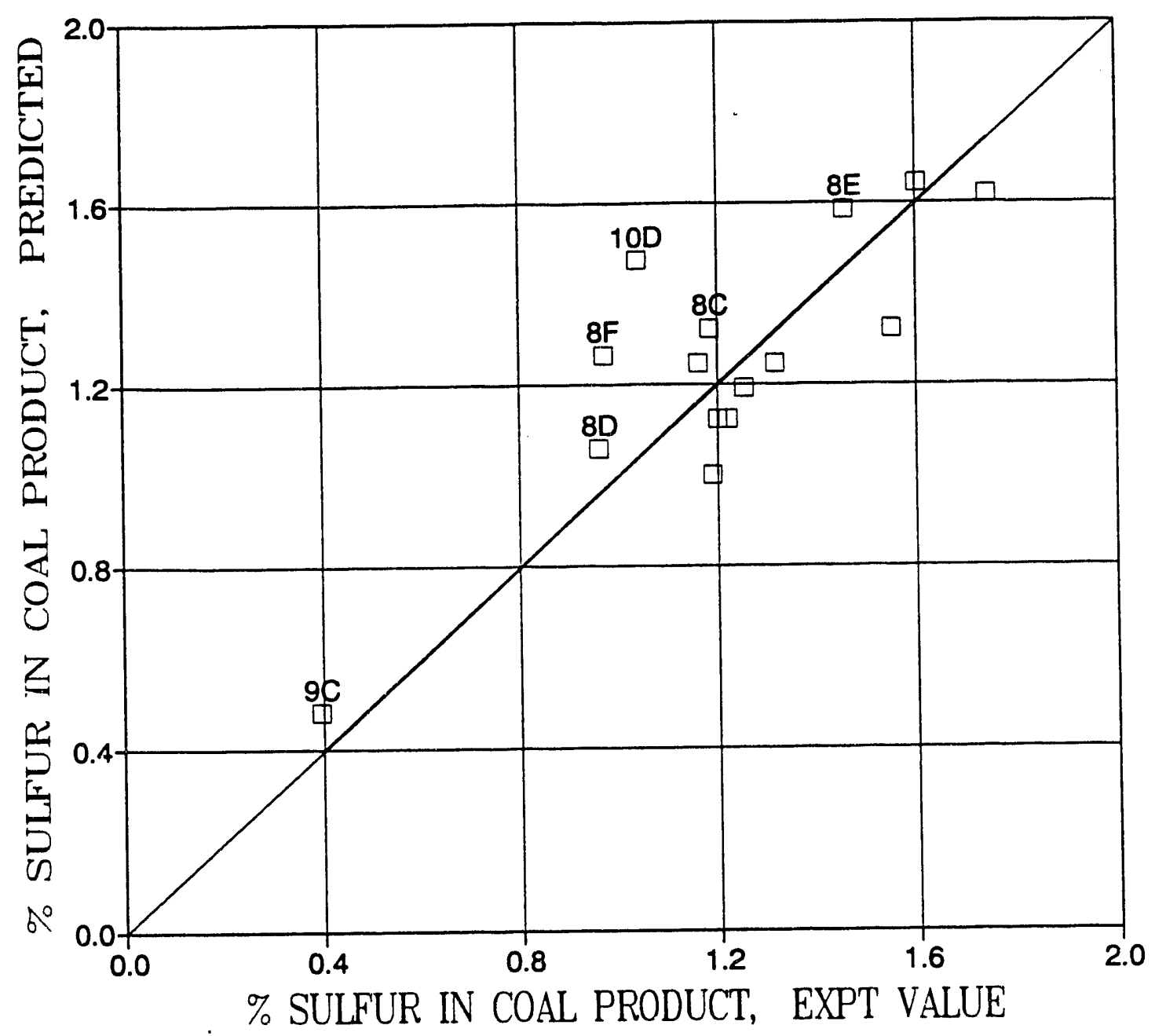

Figure 4-4. Comparison of Predicted and Experimental Values for Sulfur Content of MCL Coal Product: Pittsburgh No. 8 (Powhatan); $\mathrm{KOH} / \mathrm{NaOH}=0$ 
coal product from Run $8 F$ was $0.97 \%$. Using the dependence of sulfur content on caustic/coal ratio as represented by equation [3], the projected sulfur content of the coal product would be $1.29 \%$ at a caustic/coal ratio of 1.5 with 14 mesh coal feed. The sulfur content of the coal product from Run $8 E$ was $1.46 \%$. Thus, this indirect comparison shows that sulfur removal would be higher for 14 mesh than for 6 mesh coal feed.

Runs $4 C 4$ and $5 B 3$ were operated with the Zone 1 heater turned off. In addition, Runs 4C5,4C6, 4C7 and 5B4 were operated with Zone 1 and Zone 2 heaters turned off. Turning the heaters off effectively reduced the residence times of the coal/caustic mixture in the heated zones to 1.5 hours (one heater turned off) or 1.0 hour ( 2 heaters turned off). The suifur removal for these runs are compared with the sulfur removal for runs with all four kiln heaters operating (residence time of 2.0 hours):

Run

\begin{tabular}{llllllll}
$4 C 3$ & $4 C 4$ & $4 C 5$ & $4 C 6$ & $4 C 7$ & $5 B 2$ & $5 B 3$ & $5 B 4$ \\
\hline 420 & 423 & 430 & 435 & 441 & 420 & 423 & 430 \\
2.0 & 1.5 & 1.0 & 1.0 & 1.0 & 2.0 & 1.5 & 1.0 \\
1.22 & 1.17 & 1.35 & 1.43 & 1.22 & 1.20 & 1.46 & 1.60
\end{tabular}

The effects of residence time on sulfur removal are difficult to assess by only comparing the test results from any two runs. However, it is clear from the Runs $4 C 5,4 C 6$ and $4 C 7$ test results that at a residence time of 1.0 hour, less sulfur was removed from the coal than in Runs $4 \mathrm{C} 3$ and $4 \mathrm{C} 4$, which were operated for longer residence times at lower kiln wall temperatures. The Runs 5B2, 5B3 and 5B4 test results also show that as the residence time decreased from 2.0 hours to 1.5 hours and then to 1.0 hour, the sulfur content of the coal product increased from $1.20 \%$ to $1.46 \%$ and then to $1.60 \%$, notwithstanding the higher kiln wall temperatures for runs with the shorter residence times.

\subsubsection{Kentucky No. 9}

There were fewer number of tests conducted with Kentucky No. 9 than with Pittsburgh No. 8 (Powhatan) coal feed. In the analysis results presented in Table 4-3, four tests were conducted using $50: 50 \mathrm{KOH} / \mathrm{NaOH}$ and five tests were 
conducted using only $\mathrm{NaOH}$. Specifically, the test results from Run $7 \mathrm{~B}$ show that with nitrogen flow in countercurrent direction to coal feed, $90.5 \%$ sulfur dioxide removal (from run-of-mine coal on lbs/million Btu basis) was achieved using 50:50 KOH/NaOH, caustic/coal $=3$, $\mathrm{kiln}$ wall temperature of $427^{\circ} \mathrm{C}$, and residence time of 2.1 hours. For Run $7 \mathrm{~A}$ with caustic/coal $=2.5$ and the same operating conditions as Run 7B, an average sulfur dioxide removal of $88.0 \%$ was obtained. There was a period in the middle of Run $7 \mathrm{~A}$ (designated as Run $7 A-1$ ), however, during which $92.0 \%$ sulfur dioxide removal was obtained. These test results demonstrate that under the appropriate operating conditions, compliance coal can be obtained using the MCL process and Kentucky No. 9 coal feed.

The sulfur dioxide removal for the Kentucky No. 9 tests conducted using $\mathrm{NaOH}$ was all under $83 \%$, but all these tests were at mild temperature conditions and caustic/coal ratio of 2.0. It may be possible to attain much higher sulfur dioxide removal with nitrogen flow in countercurrent direction to flow of the coal/caustic mixture, kiln wall temperature $\geq 427^{\circ} \mathrm{C}$, and using either a caustic/coal ratio $>2.0$ or caustic feed in molten form. (If caustic is fed to the kiln at temperature in molten form instead of as flakes at room temperature, the coal/caustic mixture would be at reaction temperature for a longer time.)

As shown in Figure 4-5, high kiln wall temperature and the use of 50:50 $\mathrm{KOH} / \mathrm{NaOH}$ instead of $\mathrm{NaOH}$ generally lead to high sulfur removal. There is an insufficient number of data points to obtain a two-parameter correlation between the sulfur content of the coal product and the caustic/coal ratio (R) and the kiln wall temperature $\left(T\right.$ in $\left.{ }^{\circ} \mathrm{C}\right)$. However, for processing with $50: 50 \mathrm{KOH} / \mathrm{NaOH}$, there is good correlation between the sulfur content of the coal product and caustic/coal ratio, at a kiln wall temperature of $427^{\circ} \mathrm{C}$ :

$$
\% \text { sulfur }=1.217-0.2174 R
$$

with a correlation coefficient of -0.9748 . Also, for processing with $\mathrm{NaOH}$, there is good correlation between the sulfur content of the coal product and kiln wall temperature, at a caustic/coal ratio of 2.0 :

$$
\% \text { suifur }=5.855-0.01125 \mathrm{~T}
$$




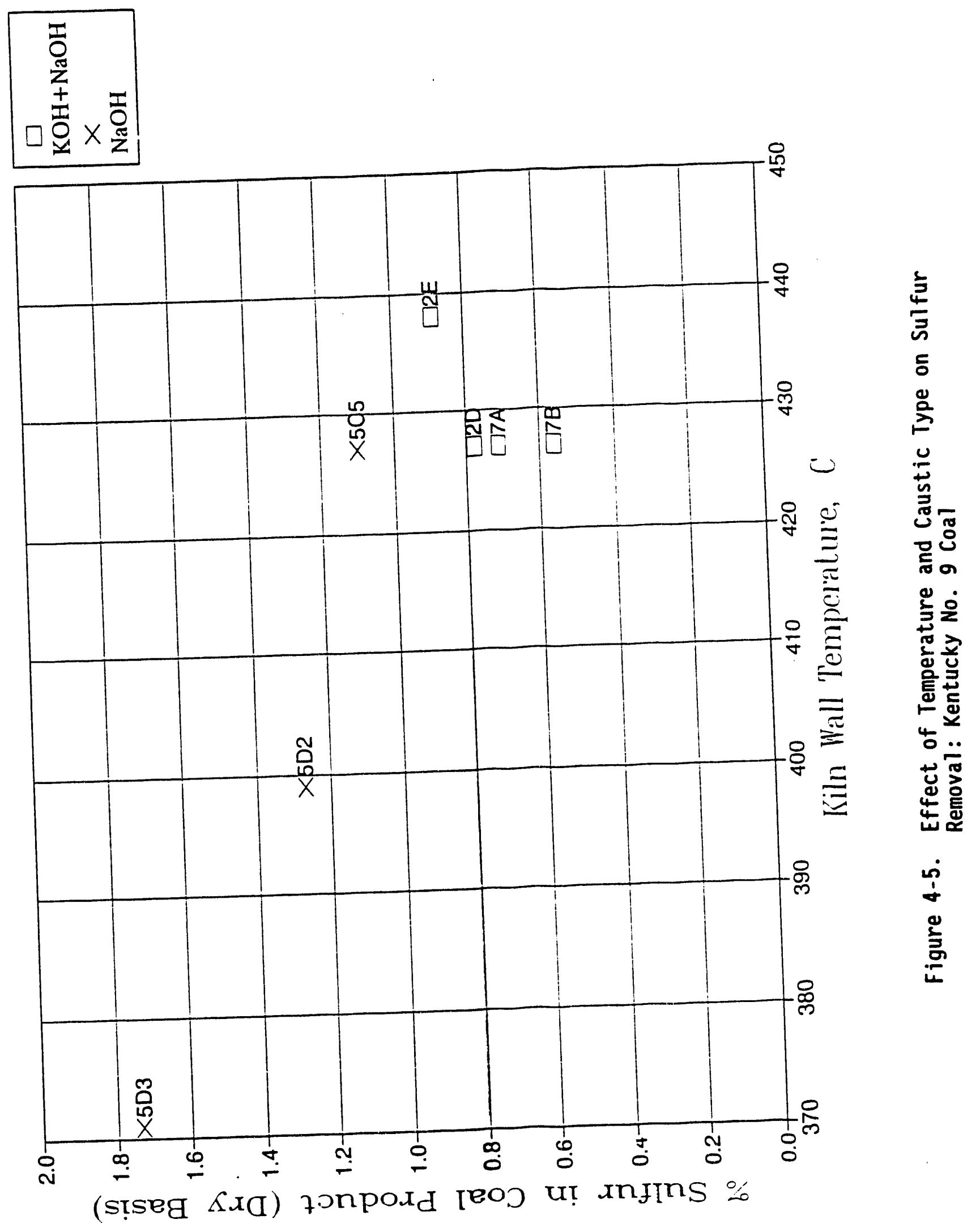


with a correlation coefficient of -0.9665 . Compared to the correlations [1] to [4] for Pittsburgh No. 8 (Powhatan) coal, the smaller regression coefficients in the above correlations appear to indicate lesser dependence of sulfur removal on caustic/coal ratio and kiln wall temperature in the processing of Kentucky No. 9 coal.

There are four pairs of runs that can be used to compare sulfur removal for Kentucky No. 9 coal versus Pittsburgh No. 8 (Powhatan) coal. Each pair represents operation at the same conditions except with a different coal feed. The sulfur contents of the Kentucky No. 9 and Pittsburgh No. 8 (Powhatan) coal product for these runs were:

$\begin{array}{llllll}\text { Coal } & \frac{\text { lst Pair }}{\text { KENT 9 }} & 0.56 \% \text { (Run 7B) } & 0.78 \% \text { (Run 2D) } & 0.47 \% \text { (Run 7A-1) } & \text { 1.10\% (Run 5C5) } \\ \text { PITT 8 } & 0.64 \% \text { (Run 7C) } & 0.77 \% \text { (Run 2C) } & 0.59 \% \text { (Run 9A) } & 1.19 \% \text { (Run 5B5) }\end{array}$

Overal1, the sulfur content of the coal product was approximately the same for the two coals under similar operating conditions. This also implies that more sulfur was removed from the Pittsburgh No. 8 (Powhatan) coal, because the average sulfur content of this feed coal is $4.27 \%$ as compared to the $3.41 \%$ for Kentucky No. 9 coal.

\subsubsection{Pittsburgh No. 8 (Blacksville); $\mathrm{NaOH}$ Only}

In the analysis results presented in Table 4-4, sulfur dioxide removal (based on lbs/million Btu removed from run-of-mine coal) averaged about $87 \%$ for Run 11 , with a kiln wall temperature of $434^{\circ} \mathrm{C}$, residence time of 2.0 hours, caustic/coal ratio of 2.5 , and nitrogen and coal/caustic flows in countercurrent direction. In addition, a $94.3 \%$ sulfur dioxide removal was obtained during a -21-hour period of operation during for Run $11 E$ (designated Run $11 \mathrm{E}-1$ ) when the kiln internal temperature most closely approached the kiln wall temperatures, indicating a relatively clean kiln wall (see Figure 4-6).

Since Pittsburgh No. 8 (Blacksville) coal was used for only one run and since on?y two temperatures were used that were only $10^{\circ} \mathrm{C}$ apart $(424$ and 


\section{女กาไกร \%}

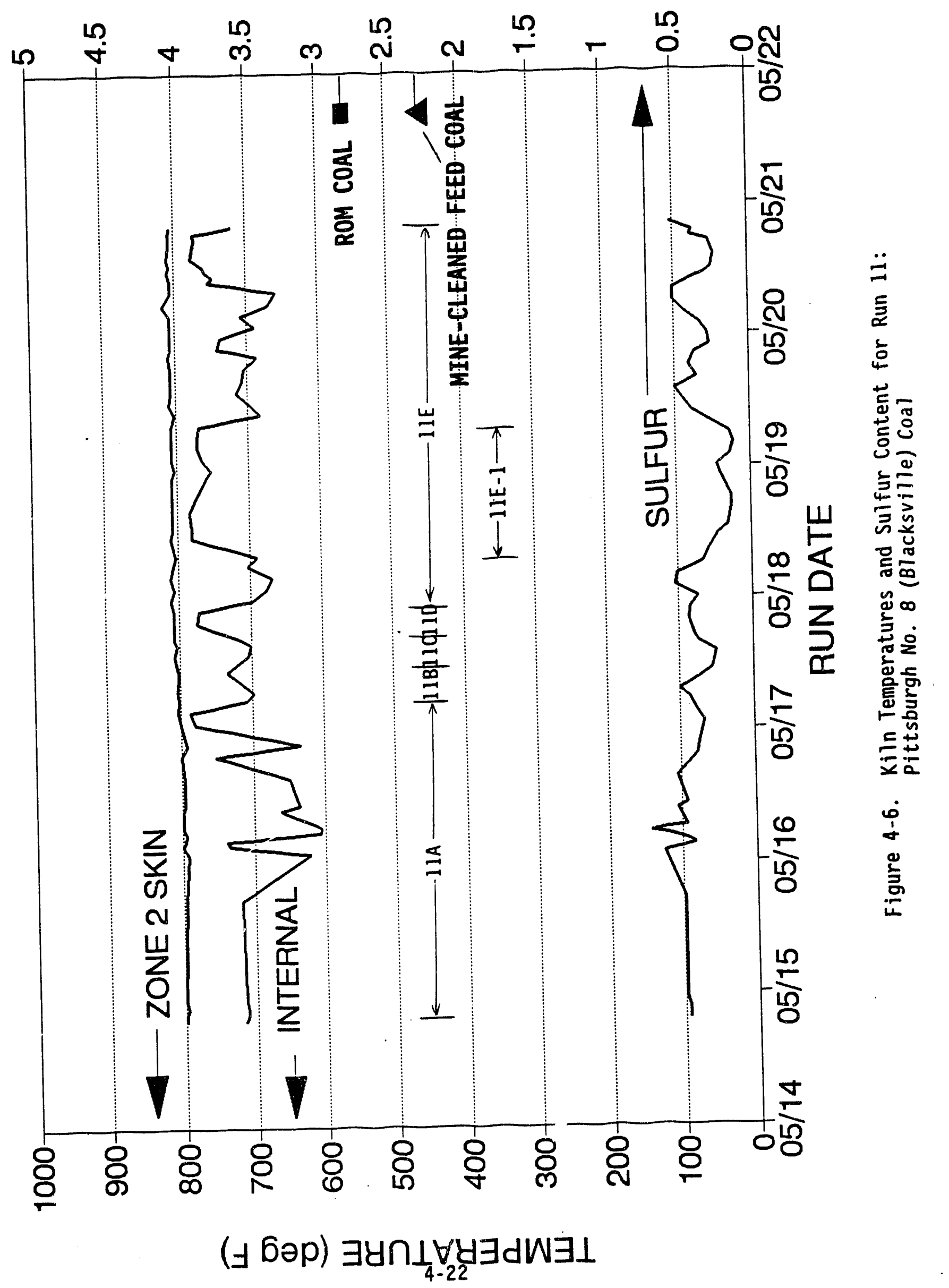


$434^{\circ} \mathrm{C}$ ) and since only one caustic:coal ratio $(2.5: 1)$ was used, there is an insufficient number of data points to obtain a correlation boticen the sulfur content of the coal product and the caustic/coal ratio or the kiln wall temperature.

\subsubsection{Ash Removal}

The ash content of the MCL coal product also decreases with increasing caustic/coal ratio and increasing reaction temperature, and depends on the type of coal and caustic used in processing, and the residence time of the coal/caustic mixture in the heated zones of the rotary kiln.

\subsubsection{Pittsburgh No. 8 (Powhatan); $50: 50 \mathrm{KOH} / \mathrm{NaOH}$}

As shown in Table 4-1, ash removal for Pittsburgh No. 8 coal (Powhatan) is strongly dependent on reaction temperature. The ash content of the coal product was above $1.1 \%$ for all runs with kiln wall temperature $\leq 410^{\circ} \mathrm{C}$. $\mathrm{Kiln}$ wall temperatures $\geq 427^{\circ} \mathrm{C}$ were generally needed to reduce the ash content of the coal product to below $0.5 \%$, as indicated by the test results from Runs $3 \mathrm{~B} 1,3 \mathrm{Cl}, 9 \mathrm{~A}, 9 \mathrm{Al}$ and $9 \mathrm{~B}$.

The effect of caustic/coal ratio on ash removal appears to be more pronounced at low caustic/coal ratio levels. For example, the ash content of the coal product increased from $0.64 \%$ for Run $2 \mathrm{C}$ (caustic/coal $=2$ ) to $1.48 \%$ for Run $3 A 2$ (caustic/coal = 1 ), at the same kiln wall temperature of $427^{\circ} \mathrm{C}$ and approximately the same residence time. On the other hand, the ash content of the coal product was $0.23 \%$ for both Run $3 B 1$ (caustic/coal $=2$ ) and Run $3 \mathrm{Cl}$ (caustic/coal $=3$ ). These two runs were both operated at the kiln wall temperature of $438^{\circ} \mathrm{C}$ and residence time of 2.0 hours. Thus, it appears that a minimum caustic/coal ratio may be needed for efficient ash removal. It is possible, however, that a lower caustic/coal ratio would be effective if molten caustic were to be fed to the kiln, because of the greater accessibility of the caustic to the coal matrix.

Regression analysis of the test data shows that the correlation between the ash content of the coal product and the caustic/coal ratio $(R)$ and the kiln wall temperature $\left(T\right.$ in ${ }^{\circ} \mathrm{C}$ ) is: 


$$
\% \text { ash }=11.870-0.7869 R-0.02191 \mathrm{~T}
$$

with a correlation coefficient of 0.8109 (excluding shakedown data). There is better correlation between the ash content of the coal product and the kiln wall temperature, when only runs with caustic/coal ratio $\geq 2.0$ are included in the regression analysis:

$$
\% \text { ash }=15.941-0.03588 T
$$

with a correlation coefficient of -0.9407 . The effects of high caustic/coal ratio and high kiln wall temperature in reducing the ash content of the coal product are also illustrated in Figure 4-7. Shakedown which was operated at a mild kiln wall temperature of only $340^{\circ} \mathrm{C}$ achieved an ash removal of only $68 \%$.

In Figure 4-8, the ash content of the MCL coal product from analysis of the samples is plotted against the predicted values represented by the correlation equation [7]. For 15 of the 24 runs, the predicted and experimental ash levels are in fairly good agreement. For Runs $1 C, 3 A 3,3 B 1$ and $8 A$, the predicted ash levels are higher than the experimental values. For Runs $2 \mathrm{~B}, 6 \mathrm{Fl}, 6 \mathrm{Gl}, 7 \mathrm{C}$ and $8 \mathrm{~B}$, the predicted ash levels are lower than the experimental values. The largest discrepancies between the predicted and experimental ash levels are associated with fiuns $2 B, 3 A 3,6 F 1$ and $6 G 1$. For these four runs, however, only one sample from each run was acquired and analyzed, and the disagreement may be due to the insufficient sample size to provide a good estimate of the actual ash level. The coal feed in Run $8 B$ was 6 mesh, which led to higher than predicted ash level in the coal product. In addition, Run $3 B 1$ was operated with a caustic/coal ratio of 2.0 and kiln wall temperature of $438^{\circ} \mathrm{C}$, whereas Run $7 \mathrm{C}$ was operated with a caustic/coal ratio of 3.0 and $k i l n$ wall temperature of $427^{\circ} \mathrm{C}$. The lower than predicted ash level for Run $3 B 1$, along with the higher than predicted ash level for Run $7 C$, is an indication that the dominating factor for ash removal is kiln temperature when the caustic/coal ratio $\geq 2.0$. In fact, the predicted ash levels using correlation equation [8] are $0.23 \%$ for Run $3 \mathrm{~B} 1$ and $0.62 \%$ for Run $7 C$, which agree very well with the corresponding experimental ash levels of $0.23 \%$ and $0.68 \%$. 


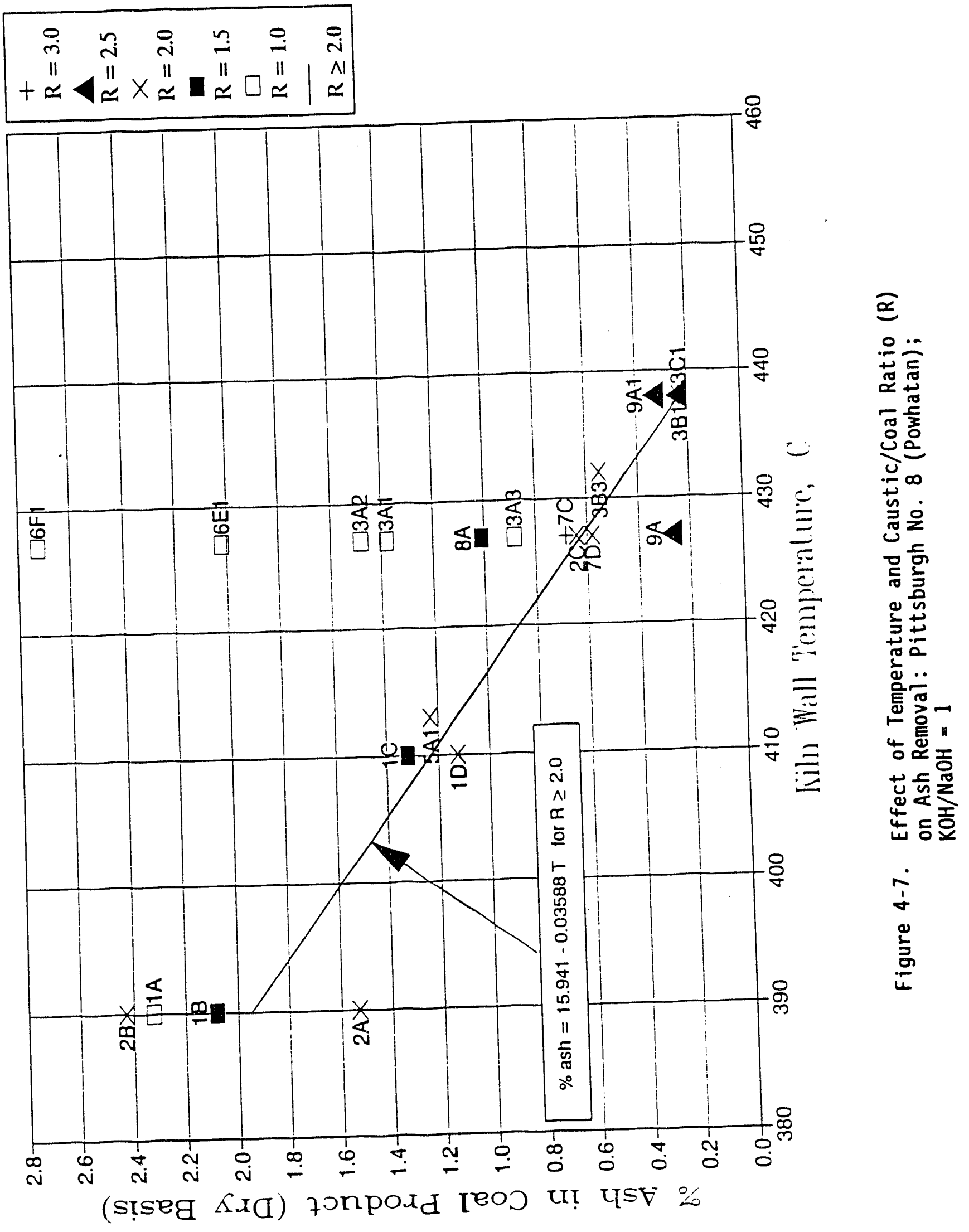




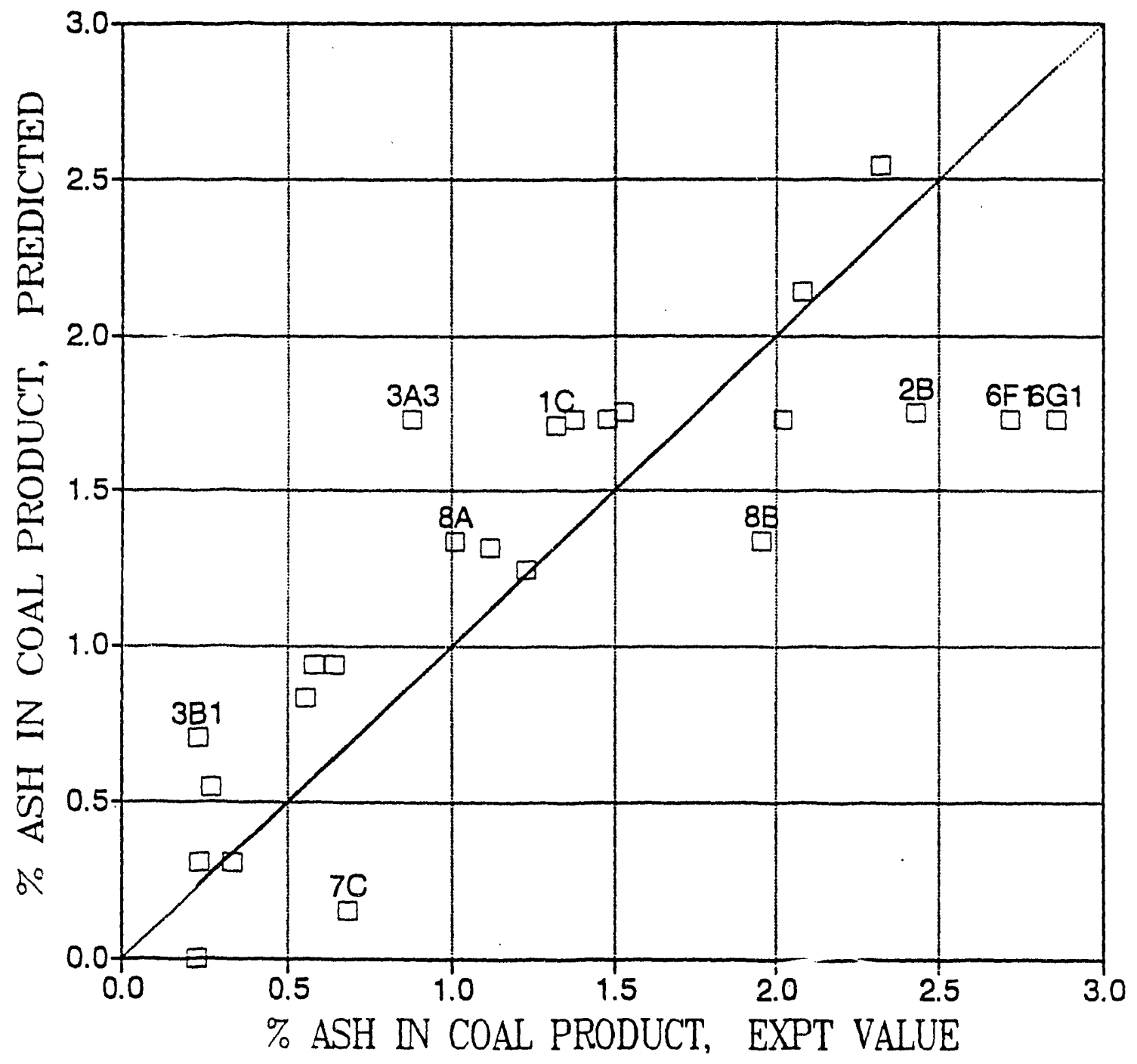

Figure 4-8. Comparison of Predicted and Experimental Values for Ash Content of MCL Coal Product: Pittsburgh No. 8 (Powhatan); $\mathrm{KOH} / \mathrm{NaOH}=1$ 
The ash content of the coal product from Runs $7 D$ and $9 B$ was $0.58 \%$ and $0.24 \%$, respectively. Both runs were conducted using caustic mixtures containing regenerated caustic. These test results confirm that high ash removal can be readily achieved using regenerated caustic.

The effect of feed coal size on ash removal can be assessed by comparing the test results from Runs $8 A$ and $8 B$, which were conducted under otherwise similar conditions but with 14 mesh and 6 mesh coal feed, respectively. The average ash content of the coal product from Run $8 \mathrm{~A}$ was $1.01 \%$. By comparison, the average ash content of the coal product from Run $8 B$ was $1.95 \%$. Therefore, it appears that the use of a finer coal feed in the MCL process could effect higher ash removal, at least at a low caustic/coal ratio of 1.5 .

As shown in Figure 4-9, there is reasonably good correlation between the sulfur content and ash content of the coal product:

$$
\% \text { sulfur }=0.478+0.5383 \% \text { ash }
$$

with a correlation coefficient of 0.9075 (excluding shakedown data), despite some scattering of data points on both sides of the correlation line. This implies that in general, the operating conditions that favor sulfur removal also facilitate ash removal.

\subsubsection{Pittsburgh No. 8 (Powhatan): $\mathrm{NaOH}$ Only}

The analysis results presented in Table 4-2 show that $\mathrm{NaOH}$ is apparently more effective than $50: 50 \mathrm{KOH} / \mathrm{NaOH}$ in removing ash from coal. This is demonstrated by comparing the test results from three sets of runs, all conducted at a residence time of approximately 2.0 hours and under similar operating conditions within each set:

\begin{tabular}{|c|c|c|c|c|}
\hline Run & Caustic/Coal & Wall Temp, ${ }^{\circ} \mathrm{C}$ & $\mathrm{KOH} / \mathrm{NaOH}$ & Ash Content of Coal Product \\
\hline $\begin{array}{l}2 \mathrm{C} \\
5 \mathrm{~B} 5\end{array}$ & $\begin{array}{l}2.0 \\
2.0\end{array}$ & $\begin{array}{l}427 \\
427\end{array}$ & $\begin{array}{l}1 \\
0\end{array}$ & $\begin{array}{l}0.64 \% \\
0.45 \%\end{array}$ \\
\hline $\begin{array}{l}5 A 1 \\
8 F\end{array}$ & $\begin{array}{l}2.0 \\
2.0\end{array}$ & $\begin{array}{l}413 \\
412\end{array}$ & $\begin{array}{l}1 \\
0\end{array}$ & $\begin{array}{l}1.23 \% \\
0.54 \%\end{array}$ \\
\hline $\begin{array}{l}9 B \\
9 C\end{array}$ & $\begin{array}{l}2.5 \\
2.5\end{array}$ & $\begin{array}{l}438 \\
438\end{array}$ & $\begin{array}{l}1 \\
0\end{array}$ & $\begin{array}{l}0.24 \% \\
0.15 \%\end{array}$ \\
\hline
\end{tabular}



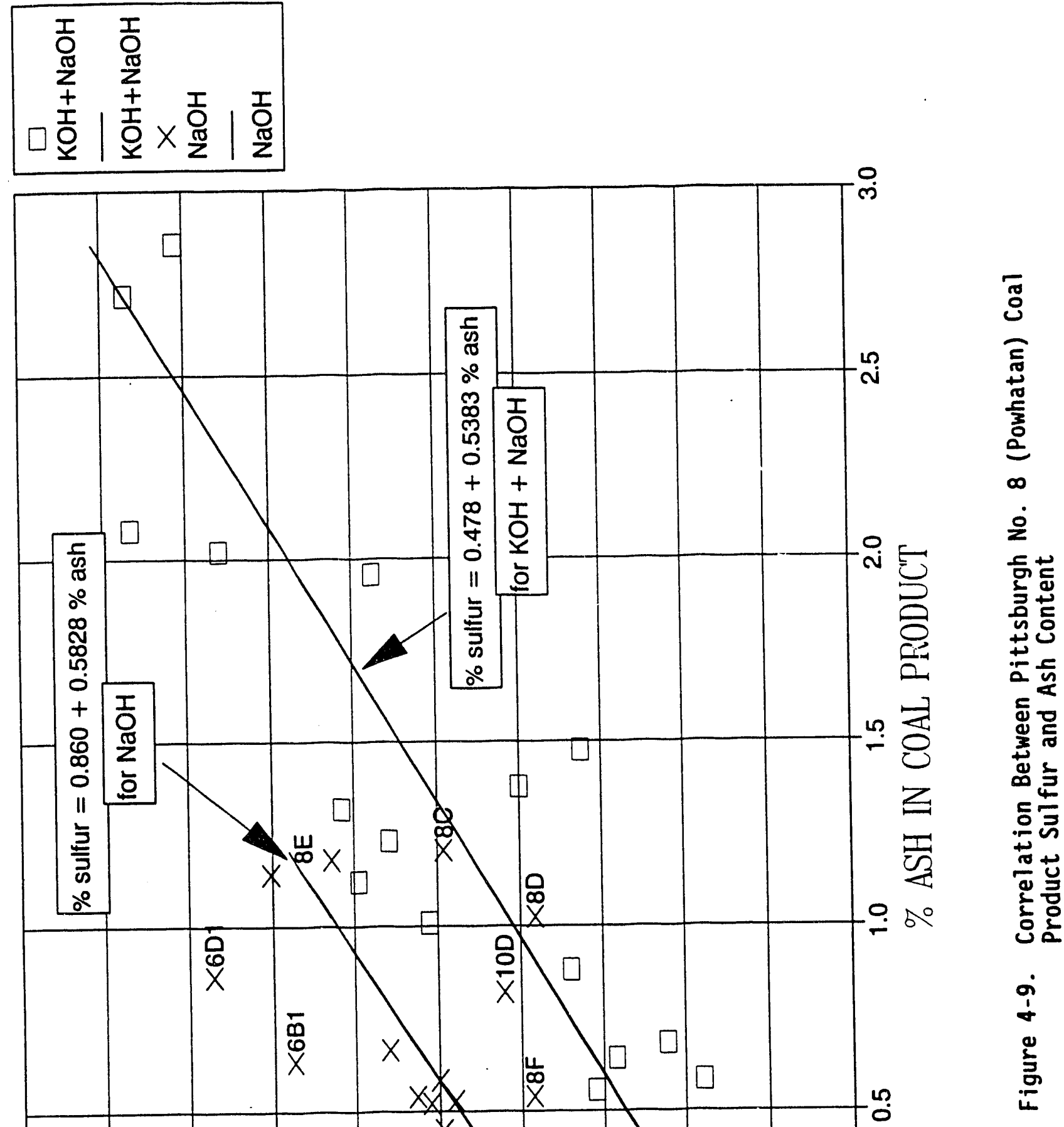
In each of the three sets, the run using $\mathrm{NaOH}$ resulted in a coal product containing less ash than the coal product from the run using 50:50 $\mathrm{KOH} / \mathrm{NaOH}$. In particular, the average ash content of the coal product from Run $9 C$ was only $0.15 \%$. This suggests that $\mathrm{NaOH}$ may be used for the production of ultra low-ash coal under appropriate operating conditions.

The ash content of the coal product can be correlated with the caustic/coal ratio $(R)$ and the kiln wall temperature $\left(T\right.$ in ${ }^{\circ} \mathrm{C}$ ), by regression analysis of the test data for runs with residence time of approximately 2.0 hours:

$$
\% a s h=2.774-0.7416 R-0.00174 T
$$

with a correlation coefficient of 0.8836 (excluding Run 10D). In addition, if only runs with caustic/coal ratio $\geq 2.0$ are considered, the correlation between the ash content of the coal product and $k i$ in wall temperature is:

$$
\% \text { ash }=7.010-0.01551 \mathrm{~T}
$$

with a correlation coefficient of -0.8945 (excluding Run 100). In comparing the correlation equations [7] for $50: 50 \mathrm{KOH} / \mathrm{NaOH}$ and [10], the predicted ash content of the coal product would always be lower for processing with $\mathrm{NaOH}$ than for 50:50 KOH/NaOH, at any caustic/coal ratio and up to a kiln wall temperature of $444^{\circ} \mathrm{C}$.

In Figure 4-10, it is shown that high caustic/coal ratios and high kiln wall temperatures are necessary to reduce the ash content of the coal product to very low levels. A data point from Run 100 is shown in Figure $2-10$ even though Run 10D was excluded from the above correlation. Run 100 which was operated with weathered coal achieved an average ash removal of $93 \%$ at a kiln wall temperature of $400^{\circ} \mathrm{C}$.

In Figure 4-11, the ash contents of the coal product as predicted from the correlatior equation [10] are compared with the corresponding experimentai vaiues. For ii of the 14 ruins, the predicted and experimenta? 


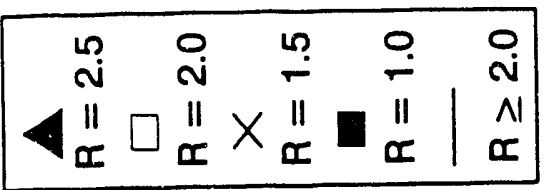

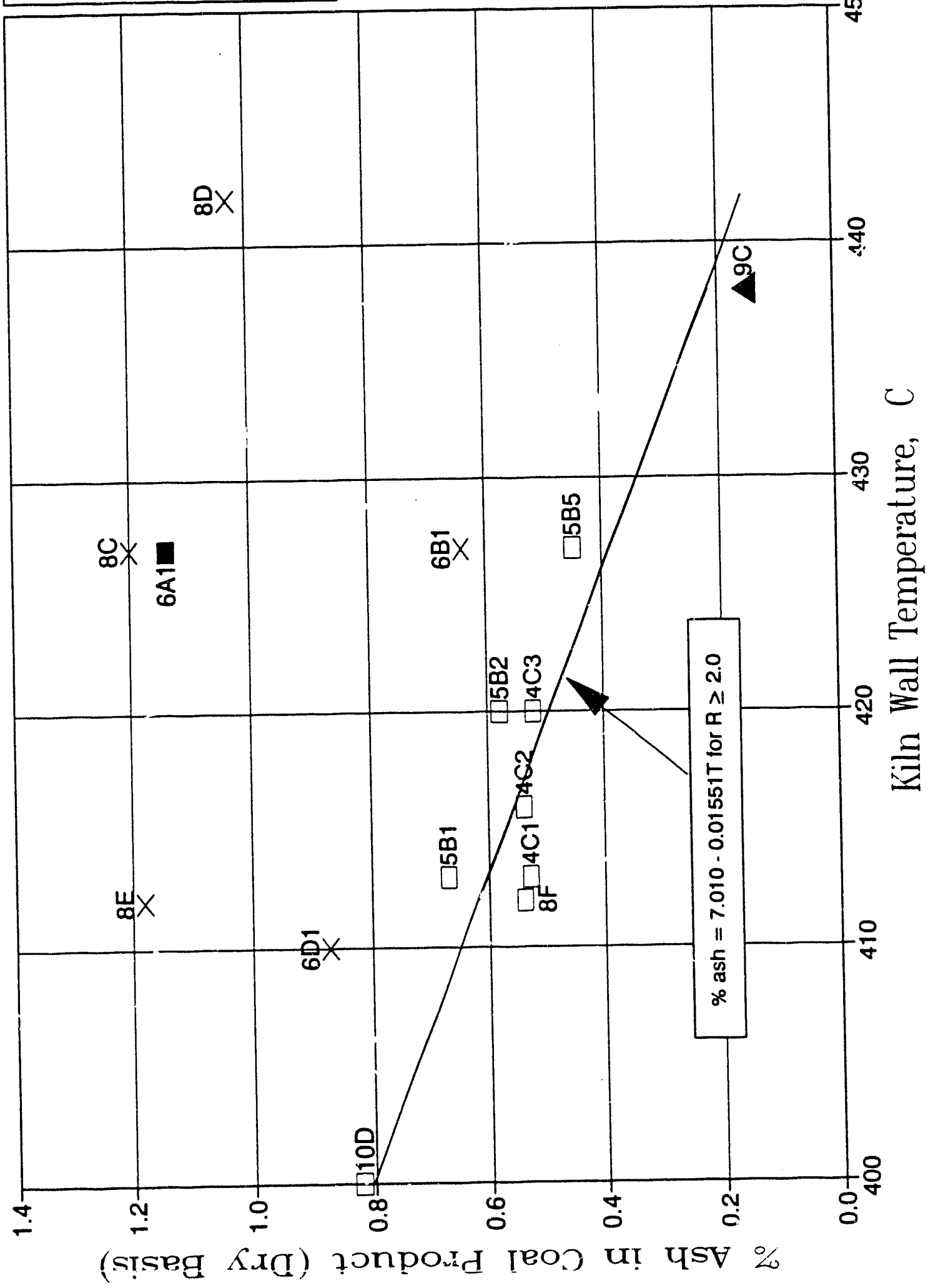

$\underline{\simeq}$

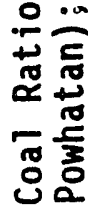

눙 


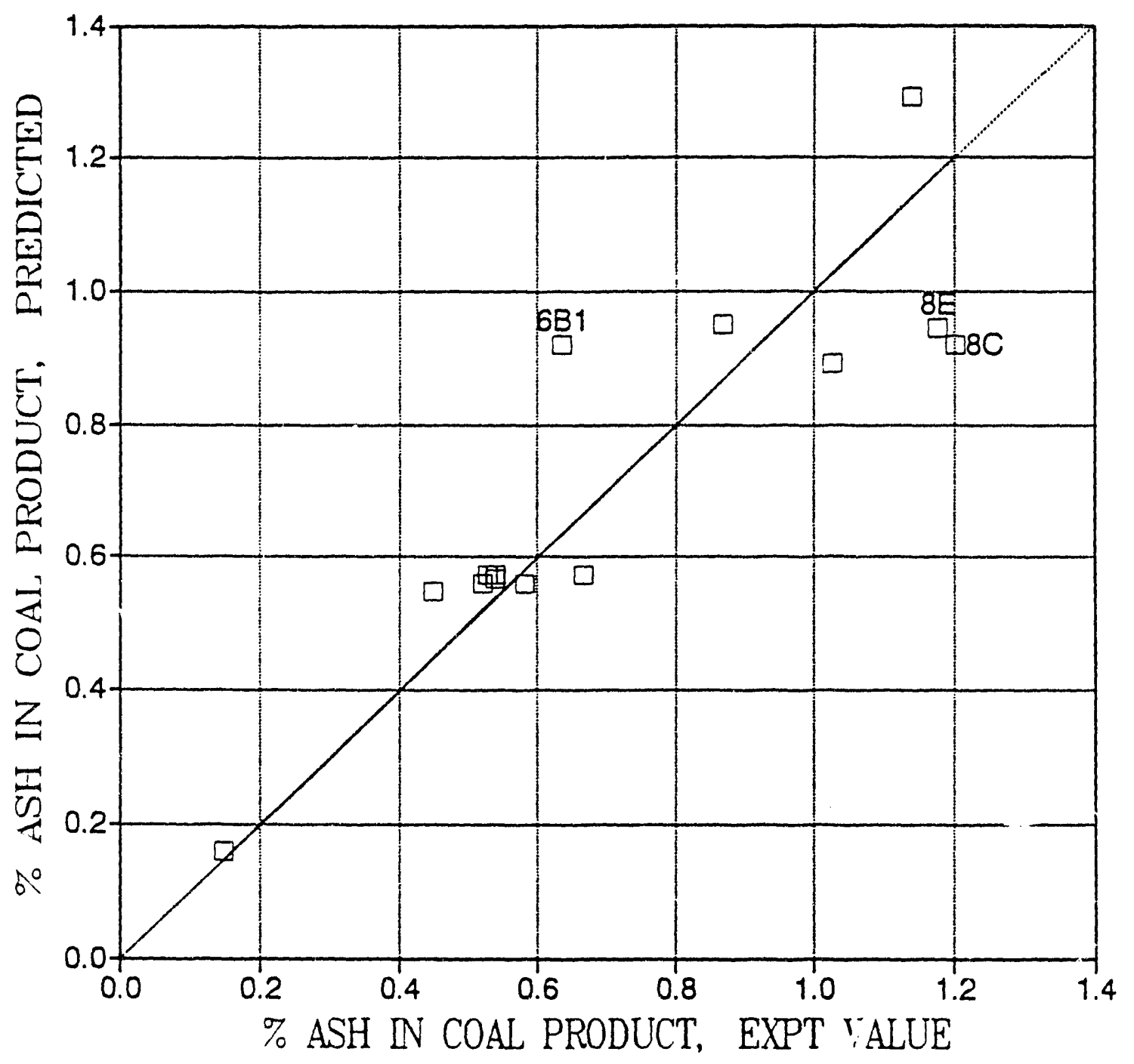

Figure 4-11. Comparison of Predicted and Experimental Values for Ash Content of MCL Coal Product: Pittsburgh No. 8 . (Powhatan); $\mathrm{KOH} / \mathrm{NaOH}=0$ 
ash levels are in good agreement. The exceptions are Runs $6 \mathrm{~B} 1,8 \mathrm{C}$ and $8 \mathrm{E}$. However, both Runs $8 i$ and $8 E$ were conducted using 6 mesh coal feed, which resulted in higher ash levels than predicted by the correlation. In addition to these runs, Run 80 was also conducted with 6 mesh coal feed. The average ash content of the coal product was $1.03 \%$, as compared to the predicted value of $0.89 \%$. Thus, for all three runs with 6 mesh coal feed, the ash contents of the coal product are higher than the predicted values. Because the predicted ash levels are based on a correlation based primarily on test data from runs with 14 mesh coal feed, the implication is that a finer coal feed would produce a MCL coal of lower ash content.

As in the case of sulfur removal, the effects of residence time on ash removal can be assessed by examining the test results from the runs with one or two kiln heaters turned off, thereby reducing the residence time of the coal/caustic mixture in the heated zones:

Run

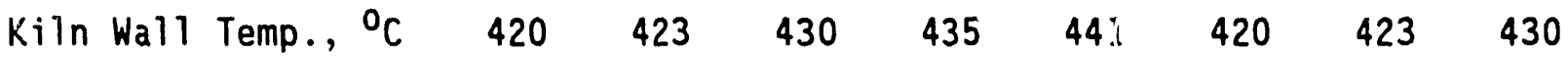

$\begin{array}{lllllllll}\text { Residence Time, } \mathrm{hr} & 2.0 & 1.5 & 1.0 & 1.0 & 1.0 & 2.0 & 1.5 & 1.0\end{array}$

$\%$ Ash in Coal

$\begin{array}{llll}0.52 & 0.46 & 0.68 & 0.73\end{array}$

0.64

0.58

0.63

0.95

Comparison of the test results from Runs $4 C 3$ versus $4 C 4$, and $5 B 2$ versus 5B3 suggest that there is no significant impact on ash removal in reduins the residence time from 2.0 to 1.5 hours. At a residence time of 1.0 hour, however, it is evident from the Runs $4 C 5,4 C 6$ and $4 C 7$ test results that less ash was removed from the coal than in Runs $4 C 3$ and $4 C 4$. Similarly, less ash was removed from the coal in Run 5B4 than in Runs 582 and 583 . For Pittsburgh No. 8 (Powhatan) coal processed with $\mathrm{NaOH}$, it appears that a residence time of about 1.5 hours is needed for effective ash removal.

For processing with $\mathrm{NaOH}$, there is little correlation between the sulfur content and the ash content of the coal product:

$$
\% \text { sulfur }=0.860+0.5828 \% \text { ash }
$$


with a correlation coefficient of 0.5521 (excluding Run 10D). As shown in Figure 4-9, there is considerable data scatter on both sides of the correlation line. Examination of the data scatter indicates that Runs 6BI and $6 \mathrm{Dl}$, both operated with a caustic/coal ratio of 1.5 , resulted in coal products with higher sulfur content than predicted by the correlation. This is attributed to the higher than usual ash removal under the operating conditions for these two runs. On the other hand, Runs $8 \mathrm{C}, 8 \mathrm{D}, 8 \mathrm{E}, 8 \mathrm{~F}, 9 \mathrm{C}$ and 100, all operated with nitrogen flow in countercurrent direction to flow of the coal/caustic mixture, resulted in coal products with lower sulfur content than predicted by the correlation. (A data point from Run 100 (weathered coal) is shown in Figure 4-9 even though it was not used in calculating the correlations.) Therefore, it appears that in processing with $\mathrm{NaOH}$, countercurrent nitrogen flow is needed more for sulfur removal than for ash removal. This is probably because in countercurrent nitrogen flow, the temperature of the coal/caustic mixture in the first zone is considerably higher than that in concurrent nitrogen flow, and a longer residence time in the high temperature zones is needed for sulfur removal than for ash removal.

\subsubsection{Kentucky No. 9}

As indicated in Table 4-3, high ash removal can be achieved with Kentucky No. 9 coal feed. Specifically, the test results from Runs 2D, 2E, 5C5 and 7B demonstrate that coal products containing less than $0.5 \%$ ash can be obtained using either $50: 50 \mathrm{KOH} / \mathrm{NaOH}$ or $\mathrm{NaOH}$. Figure 4-12 shows that kiln wall temperatures $\geq 427^{\circ} \mathrm{C}$ are generally needed to produce MCL coal containing less than $0.5 \%$ ash.

There is an insufficient number of data points to obtain a correlation between the ash content of the coal product and the caustic/coal ratio or the kiln wall temperature. The effect of residence time on ash removal, however, can be assessed by comparing the test results from Runs 5C5, 5C4 and 5D1, all operated with $\mathrm{NaOH}$, caustic/coal ratio $=2.0$, and kiln wall temperature of $427^{\circ} \mathrm{C}$ for the heated zones, except that the Zone 1 heater was turned off in Run 5C4 and both the Zone 1 and Zone 2 heaters were turned off in Run 5D1. The ash content of the coal product was $0.37 \%$ for Run 5C5 (residence time of 2.0 hours), $0.50 \%$ for Run $5 C 4$ (residence time of 1.5 hours), and increased to $1.03 \%$ for Run $50 \mathrm{I}$ (residence time of 1.0 hour). As in the case of Pittsburgh 

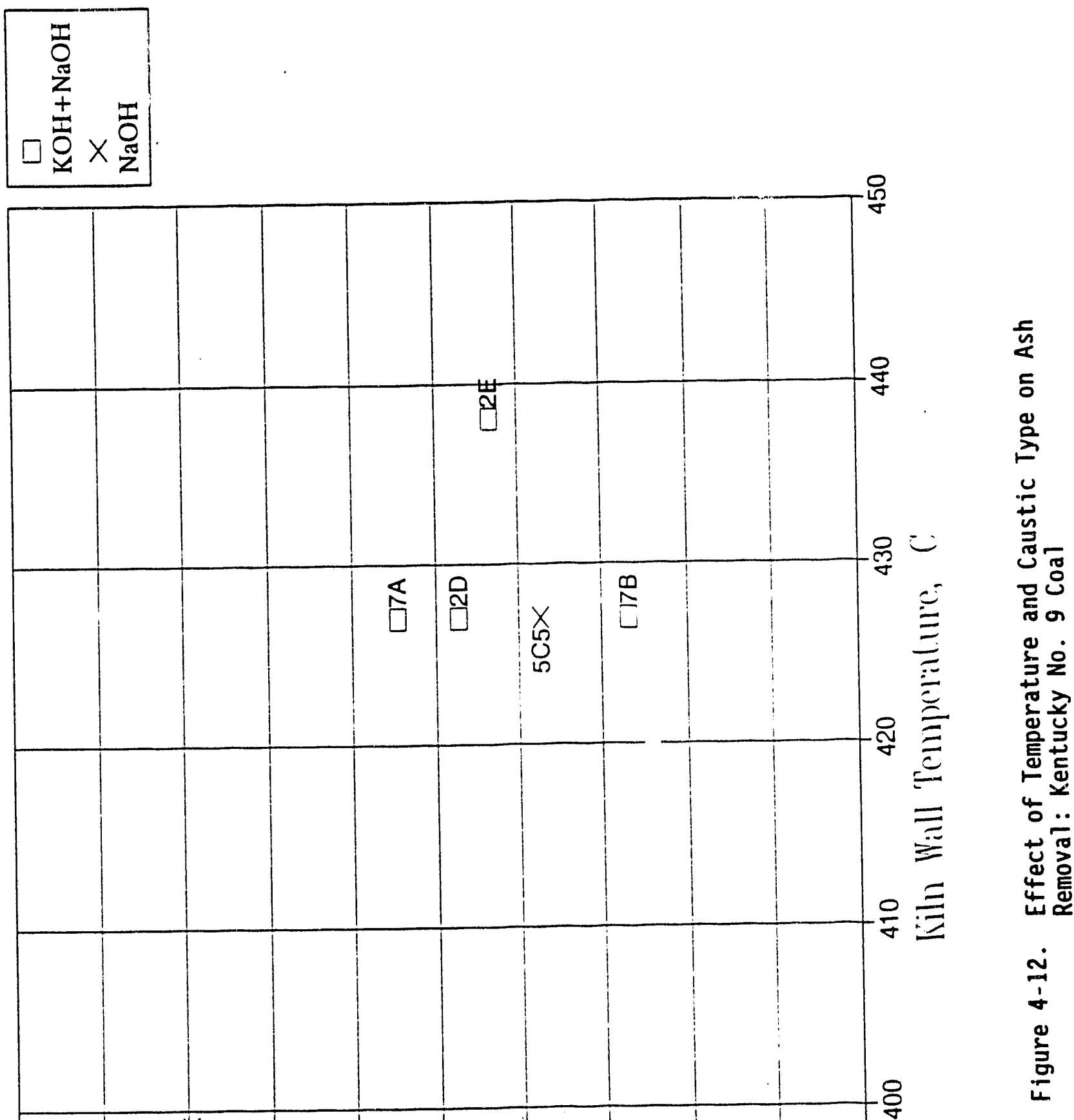
(Powhatan) coal, it appears that a residence time of 1.5 hours is needed to produce low ash MCL coal.

Both Runs $2 \mathrm{D}$ and $5 \mathrm{C} 5$ were conducted at a kiln wall temperature of $427^{\circ} \mathrm{C}$, residence time of 2.0 hours, and caustic/coal ratio $=2.0$. The average ash content of the coal product from Run 2D, processed with 50:50 $\mathrm{KOH} / \mathrm{NaOH}$, was $0.47 \%$. By comparison, Run $5 \mathrm{C5}$ was operated with $\mathrm{NaOH}$ and resulted in a coal product containing an average of $0.37 \%$ ash. This again demonstrates that $\mathrm{NaOH}$ may be more effective than $50: 50 \mathrm{KOH} / \mathrm{NaOH}$ in removing ash from coal.

There are four pairs of runs that can be used to compare ash removal for Kentucky No. 9 coal versus Pittsburgh No. 8 (Powhatan) coal. Each pair represents operation at the same conditions except with a different coal feed. The ash contents of the Kentucky No. 9 and Pittsburgh No. 8 (Powhatan) coal product for these runs were:

$\begin{array}{lllllll}\text { Coal } & \frac{1 \text { 1st Pair }}{\text { KENT 9 }} & 0.27 \% \text { (Run 7B) } & 0.47 \% \text { (Run 2D) } & 0.55 \% \text { (Run 7A) } & 0.37 \% \text { (Run 5C5) } \\ \text { PITT } 8 & 0.68 \% \text { (Run 7C) } & 0.64 \% \text { (Run 2C) } & 0.27 \% \text { (Run 9A) } & 0.45 \% \text { (Run 5B5) }\end{array}$

Even for Run 7A, the ash content for 5 of the 30 coal product samples collected and analyzed was between 0.24 to $0.29 \%$, or the same as that for Run $9 A$. Therefore, it appears that in most cases, a lower ash coal product could be obtained with Kentucky No. 9 than with Pittsburgh No. 8 (Powhatan) coal feed. This could be partly due to the fact that the average ash content of the feed Kentucky No. 9 coal is $9.25 \%$ as compared to $11.57 \%$ for Pittsburgh No. 8 (Powhatan) coal. Additional tests are needed to determine the specific processing conditions and mechanism that result in higher ash removal for Kentucky than for Pittsburgh (Powhatan) coal.

\subsubsection{Pittsburgh No. 8 (Blacksville); $\mathrm{NaOH}$ Only}

In the analysis results presented in Table 4-4, the product coal ash content for Run 11 ranged from $0.50 \%$ to $3.28 \%$ corresponding to a $97 \%$ to $81 \%$ reduction of ash from the $16.9 \%$ ash content of the raw, run-of-mine coal or a 
$94 \%$ to $58 \%$ reduction of ash from the $7.9 \%$ ash content of the feed coal. Run 11 was conducted at kiln wall temperatures of $424^{\circ} \mathrm{C}$ (Run $11 \mathrm{~A}$ ) to $434^{\circ} \mathrm{C}$ (Runs 11B through 11E), residence time of 2.0 hours, caustic/coal ratio of 2.5, and nitrogen and coal/caustic flows in countercurrent direction. The product coal ash values are plotted as a function of run time in Figure 4-13. During Run 11, the $\mathrm{pH}$ in the acid wash train was varied from 2 to 10. The $\mathrm{pH}$ level employed in the acid wash train had a very significant effect on the product coal ash content. When the average acid wash $\mathrm{pH}$ was $\sim 5$, the average ash in the product coal collected was $-1.7 \%$ (Runs $11 \mathrm{~A}$ and $11 \mathrm{~B}$ ). When no acid was being injected and the $\mathrm{pH}$ was allowed to increase to $\sim 10$, the average ash content of the product coal was $-2.1 \%$ (Run $11 \mathrm{C}$ ). When acid was injected to give a $\mathrm{pH}$ of about 2 , the average ash content of the product coal fel? to $-0.5 \%$ (Run 110 ).

Finally in Run $11 \mathrm{E}$ during which no acid was being injected ( $\mathrm{pH}$ increased to -10$)$ and the effluent wash water from the acid wash section was used as the water feed to the water wash section, the average ash content of the product coal was $3.28 \%$, representing only a $58 \%$ reduction of ash from the feed coal. The ash value for Run $11 \mathrm{E}$ is artificially high because unfortunately caustic-contaminated recycled wash wash (due to an evaporator upset) was used. During a 24-hour wash train test conducted after Run 11 under the same conditions as Run 11E, the ash content of the product coal was $2.7 \%$.

Eliminating acid washing (and the acid waste water treatment step) has no effect on the sulfur levels of the coal product but has the potential of significantly reducing the cost of the process for those commercial applications that do not require low ash coal.

Since Pittsburgh No. 8 (Blacksville) coal was used for only one run and since only two temperatures were used that were oiriy $10^{\circ} \mathrm{C}$ apart $(424$ and $434^{\circ} \mathrm{C}$ ) and since only one caustic:coal ratio $(2.5: 1)$ was used, there is an insufficient number of data points to obtain a correlation between the ash content of the coal product and the caustic/coal ratio or the kiln wall temperature. In addition, since all of the other runs (Runs 1 through 10) plus shakedown were conducted with acid injection resulting in an acid wash 


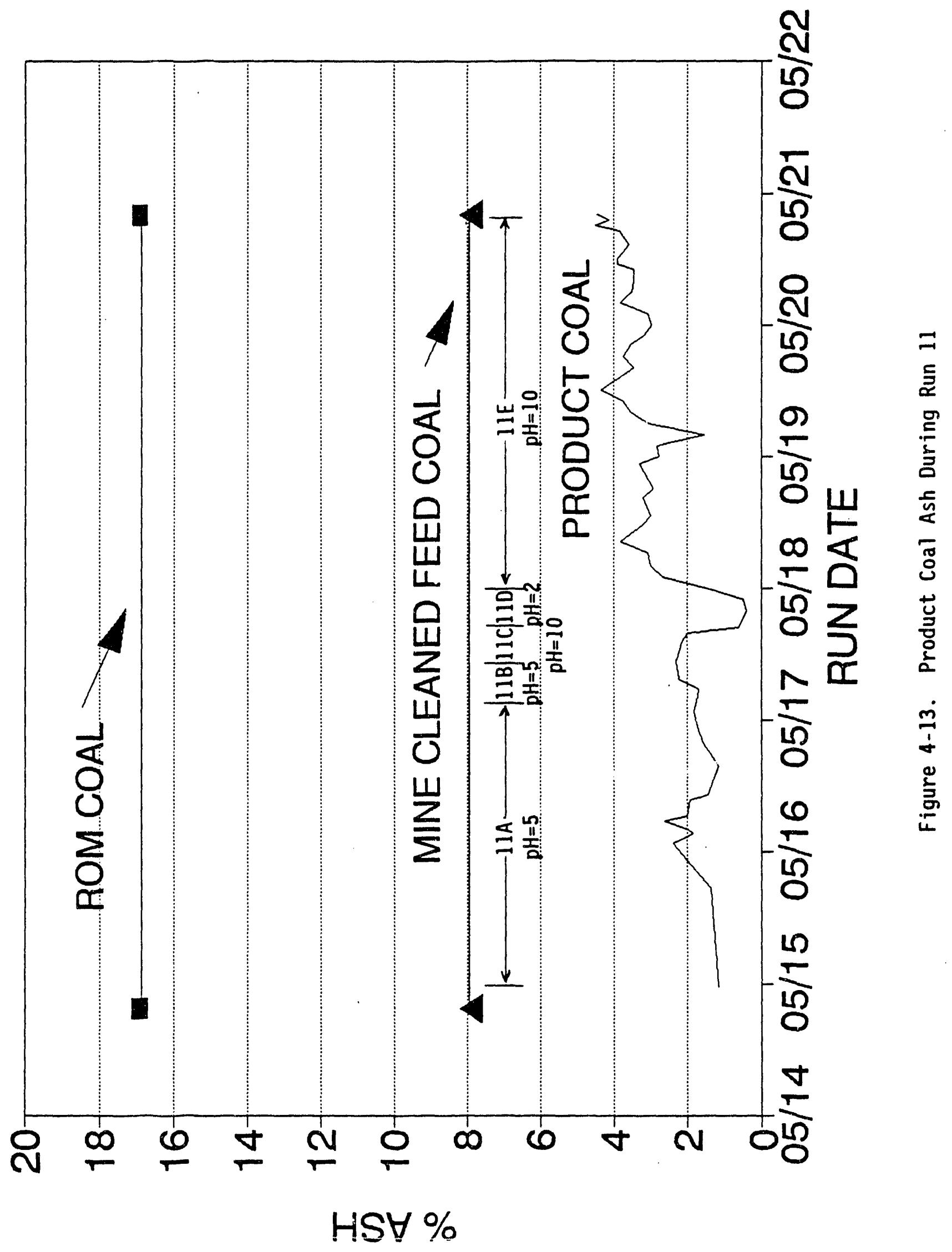


$\mathrm{pH}$ of about 2, only the data from Run 110 would have been of use for a correlation that could be used for comparison with the other runs.

\subsubsection{Heating Value}

As shown by the test results presented in Tables 4-1 through 4-4, the heating value of the coal product depends on the type of caustic used for processing. With 50:50 $\mathrm{KOH} / \mathrm{NaOH}$, the average heating value of the coal product was approximately $13000 \mathrm{Btu} / \mathrm{lb}$ (dry basis) (excluding shakedown data). With $\mathrm{NaOH}$, the average heating value of the coal product increased to about $14000 \mathrm{Btu} / \mathrm{lb}$ (dry basis). This was true for both Pittsburgh No. 8 coal feeds (Powhatan and Blacksville) and for Kentucky No. 9 coal feed.

The heating value of the coal product also depends on the severity of the processing conditions. For runs conducted with Pittsburgh No. 8 (Powhatan) coal feed and caustic/coal ratio $\geq 2.0$, the correlation between the heating value and the kiln wall temperature $\left(T\right.$ in $\left.{ }^{\circ} \mathrm{C}\right)$ for processing with 50:50 $\mathrm{KOH} / \mathrm{NaOH}$ is:

$$
\text { Btu/lb }=27483-33.603 T
$$

with a correlation coefficient of -0.8346 . The correlation for processing with $\mathrm{NaOH}$ is:

$$
\mathrm{Btu} / \mathrm{Tb}=27394-31.895 \mathrm{~T}
$$

with a correlation coefficient of -0.9094 (excluding Run 10D). For both Kentucky No. 9 and Pittsburgh No. 8 (Blacksville) coal feeds, there were insufficient data to use regression analysis to obtain any meaningful correlation with the process parameters. Nevertheless, it is expected that high kiln wall temperature would also decrease the heating value of the coal product. Shakedown which was operated at mild conditions (kiln wall temperature of $340^{\circ} \mathrm{C}$ ) resulted in a coal product heating value of 14258 Btu/lb. Run 11 which used Pittsburgh No. 8 ( $B$ lacksville) coal resulted in a coal product heating value of about $14,000 \mathrm{Btu} / 1 \mathrm{~b}$ at $434^{\circ} \mathrm{C}$. 


\subsubsection{Volatiles Content}

The volatiles content of the coal product ranged from $20.93 \%$ (run $3 C 1$ ) to $34.88 \%$ (Run 1B) for Pittsburgh No. 8 (Powhatan) coal feed (excluding shakedown data), from $22.57 \%$ (Run $5 C 5$ ) to $30.27 \%$ (Run $2 E$ ) for Kentucky No. 9 coal feed, and from $20.68 \%$ (Run 11D) to $28.0 \%$ (Run 11B) for Pittsburgh No. 8 (Blacksville) coal feed. In general, the volatiles content decreases with increasing caustic/coal ratio $(R)$ and increasing $k i l n$ wall temperature ( $T$ in ${ }^{0} \mathrm{C}$ ). There is little correlation between the volatiles content of the coal product and these two process parameters. For Pittsburgh No. 8 (Powhatan) coal processed with $50: 50 \mathrm{KOH} / \mathrm{NaOH}$ :

$$
\% \text { volatile }=82.362-1.3507 R-0.1246 \mathrm{~T}
$$

with a correlation coefficient of 0.5912 (excluding shakedown data). Shakedown which was operated at a very mild kiln wall temperature $\left(340^{\circ} \mathrm{C}\right)$ and a low caustic/coal ratio (1.0) produced a product coal with a high volatiles content $(40.83 \%)$ which is consistent with the above correlation. For Pittsburgh No. 8 (Powhatan) $\mathrm{COal}$ processed with $\mathrm{NaOH}$ :

$$
\% \text { volatile }=78.854-3.7421 R-0.1116 \mathrm{~T}
$$

with a correlation coefficient of 0.6657 (excluding Run 10D). There is no clear indication whether processing with $50: 50 \mathrm{KOH} / \mathrm{NaOH}$ or $\mathrm{NaOH}$ would produce a coal product of higher volatiles content. For both Kentucky No. 9 and Pittsburgh No. 8 (Blacksville) coal feeds, there were again insufficient data to perform meaningful regression analysis.

\subsubsection{Moisture Content}

The moisture content of the coal product ranged from $43.73 \%$ (Run 6E1) to 58.35\% (Run 7C) for Pittsburgh No. 8 (Powhatan) coal feed (excluding shakedown data), from $51.69 \%$ (Run 5 C5) to $59.37 \%$ (Run 78 ) for Kentucky No. 9 coal feed, and from $44.61 \%$ (Run $11 E-1$ ) to $46.22 \%$ (Run $11 A$ ) for Pittsburgh No. 8 (Blacksville) coal feed. Both the kiln processing conditions and the wash train performance affect the moisture content. However, it appears that wash train performance was fairly uniform from run to run, and correlations could 
be obtained between the moisture content and the ash content of the coal product. For Pittsburgh No. 8 (Powhatan) coal processed with 50:50 KOH/NaOH:

$$
\% \text { moisture }=58.951-4.0373 \% \text { ash }
$$

with a correlation coefficient of -0.8668 (excluding shakedown data). For Pittsburgh No. 8 (Powhatan) coal processed with $\mathrm{NaOH}$ :

$$
\% \text { moisture }=56.223-3.6669 \% \text { ash }
$$

with a correlation coefficient of -0.6620 (excluding Run 10D). These correlations are depicted in Figure 4-14. (A data point from Run 100 (weathered coal) is shown in Figure 4-14 even though it was not used in calculating the correlations.) These correlations indicate that the kiln operating conditions which lead to lower ash removal would result in lower coal porosity and surface area, and therefore also lower moisture content for the coal product. For example, shakedown which was operated at a very mild $\mathrm{kiln}$ wall temperature $\left(340^{\circ} \mathrm{C}\right)$ and which had low ash removal resulted in a product coal with a very low moisture content ranging from $10.69 \%$ to $36.04 \%$. The average shakedown coal product moisture content was $23.15 \%$.

No equivalent correlations were obtained for either Kentucky No. 9 or for Pittsburgh No. 8 (Blacksville) coal feeds because of an insufficient number of data points.

\subsubsection{Trace Element Analysis}

Assessment of trace elements in the feed and product coals for two runs (Run 7A with Kentucky No. 9 coal and Run $9 C$ with Pittsburgh No. 8, Powhatan coal) was performed. These two coals were subjected to a series of analyses as follows:

- Quantitative analysis for $\mathrm{Na}, \mathrm{K}, \mathrm{Cl}$ and $\mathrm{F}$

- Semi-quantitative analysis for any remaining cations.

The above analyses required different sample preparation techniques which were as follows: 


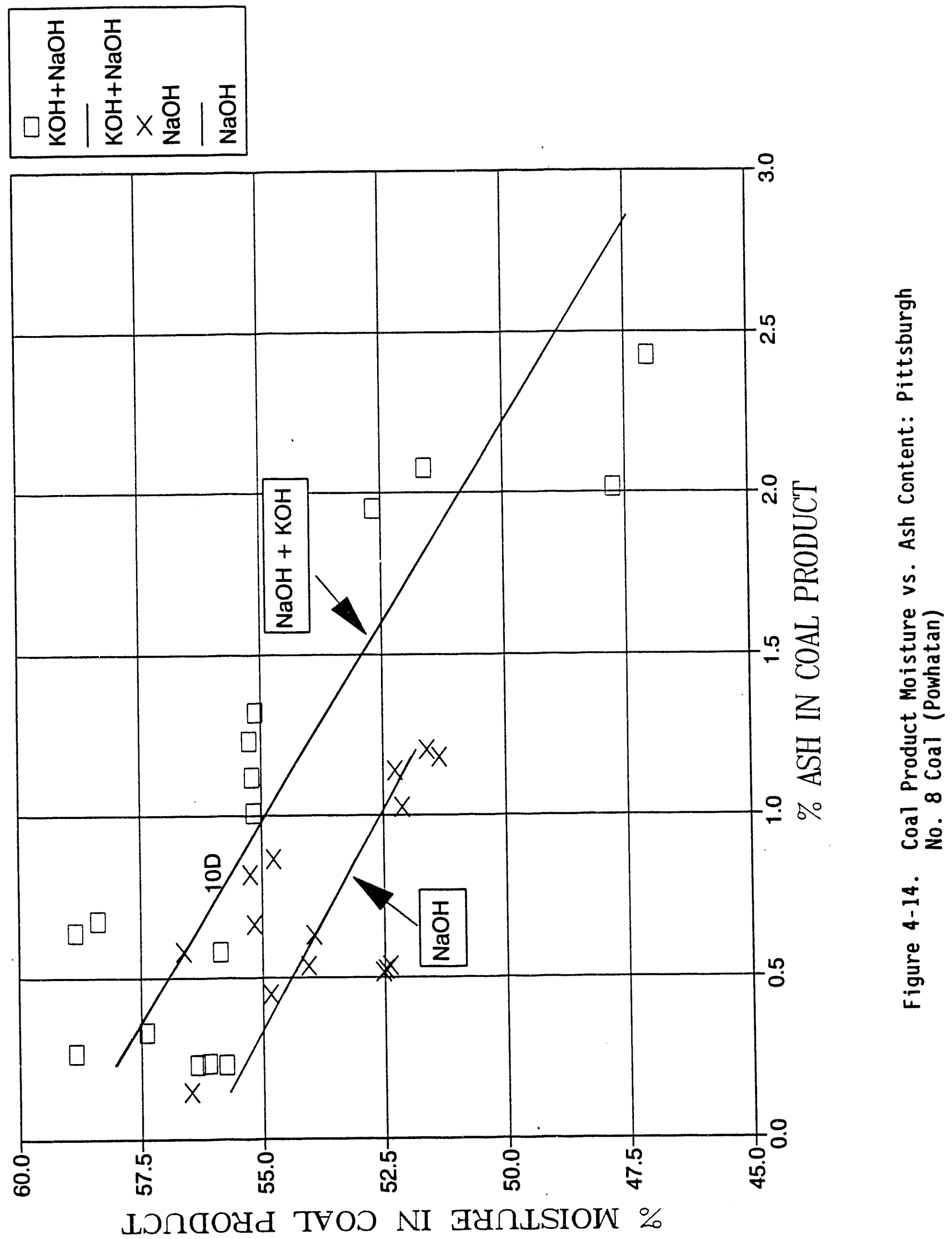


- Quantitative $\mathrm{Na}$ and $\mathrm{K}$ analysis - used ASTM D2795 which is a muffle ash at $750^{\circ} \mathrm{C}$ followed by $\mathrm{H}_{2} \mathrm{SO}_{4}$ - HF digestion.

- Quantitative $\mathrm{Cl}$ analysis - used a Parr oxygen bomb combustion over standard anion eluent. Solutions were analyzed directly by ion chromatography.

- Semi-quantitative Emission Spectrographic Analysis (ES) - the coal ash sample, which was ultimately analyzed, was obtained from the parent coal using a low temperature $\left(<130^{\circ} \mathrm{C}\right)$ oxygen plasma procedure. The ash, after plasma ashing, was removed and submitted to Pacific Spectrochemical for ES analysis. (Note: low temperature ashing is used to retain elements normally lost using high temperature muffle ashing techniques).

The quantitative results obtained for $\mathrm{Na}, \mathrm{K}, \mathrm{Cl}$ and $\mathrm{F}$ are presented in Table 4-5. The ES results reduced to a $\mathrm{mg} / \mathrm{g}$ basis are summarized in Table 4-6 for 21 elements. No equivalent analytical data was taken for Pittsburgh No. 8 (Blacksville) coal.

\subsection{SULFUR FORMS ANALYSIS}

Samples of kiln discharge coal and washed coal product from selected $\mathrm{MCL}$ runs were analyzed to determine the forms of residual sulfur present in $\mathrm{MCL}$ coal. A secondary objective for this analysis was to resolve whether sulfate sulfur was formed in kiln processing, or as a result of subsequent washing by dilute sliffuric acid. Therefore, some of the samples were only analyzed to determine sulfate sulfur and total sulfur. If sulfates were formed in kiln processing, it may be possible to delineate the operating conditions that suppress sulfate formation. If sulfates were formed ly dilute sulfuric acid wash, then it may be recessary to consider alternate washing schemes in order to further reduce residual sulfur levels in $\mathrm{MCL}$ coal.

The sulfur form analysis results are presented in Table 4-7. As shown in this table, the amount of sulfate sulfur in $M C L$ coal appears to remain invariant of coal type or processing conditions (if shakedown and Run 11 data are excluded). The mean sulfate sulfur level for the $\mathrm{MCL}$ coal samples is $0.28 \%$, with a standard deviation of only $0.06 \%$. However, shakedown which was operated at a very mild kiln wall temperature $\left(340^{\circ} \mathrm{C}\right)$ had a coal product sulfate content of only $0.02 \%$. Since the product coal during shakedown was 


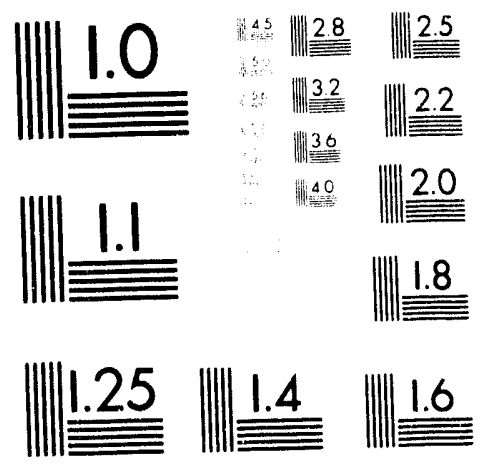



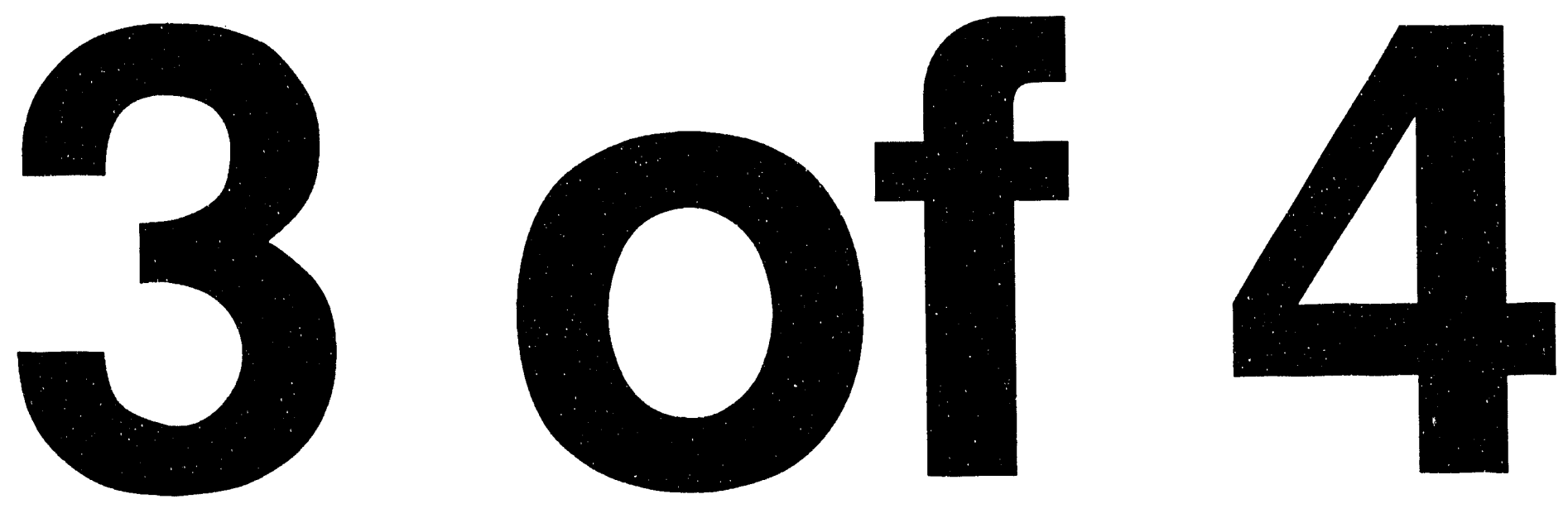
TABLE 4-5. QUANTITATIVE CATION AND ANION ANALYSIS

\begin{tabular}{|c|c|c|c|c|}
\hline Element & $\begin{array}{l}\text { Feed } \\
\text { Ky } 9 \\
\end{array}$ & $\begin{array}{l}\text { Feed } \\
\text { Pitt } 8^{*}\end{array}$ & $\begin{array}{l}\text { Prod } \\
\text { Ky } 9 \\
\end{array}$ & $\begin{array}{c}\text { Prod } \\
\text { Pitt } 8^{\star}\end{array}$ \\
\hline $\mathrm{Na}$ & 0.034 & 0.060 & C. 012 & 0.022 \\
\hline$k$ & 0.15 & 0.20 & 0.025 & 0.013 \\
\hline $\mathrm{Cl}$ & 0.16 & 0.11 & 0.0090 & 0.0105 \\
\hline $\mathrm{F}$ & 0.0027 & 0.0050 & 0.0017 & 0.0044 \\
\hline
\end{tabular}

* Pittsburgh No. 8 coal from Powhatan No. 6 mine. 
TABLE 4-6. REMOVAL OF MINOR AND TRACE ELEMENTS FROM COAL SEMI - QUANTITATIVE EMISSION SPECTROGRAPHIC ANALYSIS, $\mathrm{mg} / \mathrm{g} \mathrm{dmf}$

\begin{tabular}{|c|c|c|c|c|c|c|}
\hline ELEMENT & $\begin{array}{l}\text { FEED } \\
\text { KYN } 9 \\
\end{array}$ & $\begin{array}{l}\text { FEED } \\
\text { PITT 8* }\end{array}$ & $\begin{array}{l}\text { PROD } \\
\text { KYN } 9 \\
\end{array}$ & $\begin{array}{l}\text { PROD } \\
\text { PITT 8* }\end{array}$ & $\begin{array}{l}\text { \%REM } \\
\text { KYN } 9 \\
\end{array}$ & $\begin{array}{l}\text { \%REM } \\
\text { PITT 8* }\end{array}$ \\
\hline $\begin{array}{l}\mathrm{Cu} \\
\mathrm{Na} \\
\mathrm{Zr} \\
\mathrm{Co} \\
\mathrm{Sr}\end{array}$ & $\begin{array}{r}40 \\
8.8 \\
9.7 \\
0.5 \\
0.045 \\
0.57 \\
2 \\
0.63 \\
0.045 \\
0.2 \\
0.026 \\
0.02 \\
0.004 \\
0.004 \\
0.008 \\
0.007 \\
0.44 \\
0.023 \\
0.002 \\
0.025\end{array}$ & $\begin{array}{r}55 \\
16 \\
13 \\
0.63 \\
0.1 \\
3.4 \\
2 \\
0.43 \\
0.03 \\
0.2 \\
0.05 \\
0.02 \\
0.004 \\
0.004 \\
0.027 \\
0.023 \\
0.37 \\
0.055 \\
0.002 \\
0.069\end{array}$ & $\begin{array}{r}1.6 \\
0.24 \\
0.26 \\
0.024 \\
0.0037 \\
0.013 \\
0.24 \\
0.26 \\
0.014 \\
0.003 \\
0.0018 \\
0.0054 \\
0.0044 \\
5 E-05 \\
0.0018 \\
0.003 \\
0.1 \\
0.015 \\
0.0015 \\
0.0008\end{array}$ & $\begin{array}{r}1.1 \\
0.12 \\
0.09 \\
0.009 \\
0.003 \\
0.046 \\
0.0035 \\
0.19 \\
0.017 \\
0.0035 \\
0.001 \\
0.024 \\
0.003 \\
5 E-05 \\
0.002 \\
0.006 \\
0.15 \\
0.018 \\
0.001 \\
0.001\end{array}$ & $\begin{array}{l}96 \\
97 \\
97 \\
95 \\
92 \\
98 \\
88 \\
59 \\
69 \\
99 \\
93 \\
73 \\
-10 \\
99 \\
78 \\
57 \\
77 \\
35 \\
25 \\
97\end{array}$ & $\begin{array}{r}98 \\
99 \\
99 \\
99 \\
97 \\
99 \\
100 \\
56 \\
43 \\
98 \\
98 \\
-20 \\
25 \\
99 \\
93 \\
74 \\
59 \\
67 \\
50 \\
99\end{array}$ \\
\hline$\star * *$ & 0.16 & 0.11 & 0.01 & 0.01 & 94 & 90 \\
\hline
\end{tabular}

* Pittsburgh No. 8 coal from Powhatan No. 6 mine. ** Chloride analysis by Parr bomb ion chromatography. 
TABLE 4-7. SULFUR FORMS ANALYSIS RESULTS

\begin{tabular}{|c|c|c|c|c|c|c|c|c|}
\hline & Coal & Sample & $\%$ Su & 11 fate su & Ifur & $\%$ Pyritic & $\%$ Organic & $\%$ Total \\
\hline Run & Iype & Type & Mean & Std Dev. & Data Pts & Sulfur & $\underline{\text { Sulfur }}$ & \\
\hline SHDW & PITT 8* & $C P$ & 0.02 & 0.00 & 2 & 0.52 & 1.79 & 2.33 \\
\hline $4 C 1$ & PITT 8* & $C P$ & 0.24 & 0.08 & 2 & 0.04 & 0.47 & 0.74 \\
\hline $\begin{array}{l}6 \mathrm{~B} 1 \\
6 \mathrm{DI}\end{array}$ & $\begin{array}{l}\text { PITT 8* } \\
\text { PITT 8* }\end{array}$ & $\begin{array}{l}C P \\
C P\end{array}$ & $\begin{array}{l}0.29 \\
0.28\end{array}$ & $0 . \overline{01}$ & $\begin{array}{l}1 \\
2\end{array}$ & $\begin{array}{l}0.01 \\
0.02\end{array}$ & $\begin{array}{l}1.05 \\
1.09\end{array}$ & $\begin{array}{l}1.35 \\
1.38\end{array}$ \\
\hline $\begin{array}{l}7 A \\
7 A \\
7 B \\
7 B \\
7 C \\
7 C \\
7 D \\
7 D \\
7 E\end{array}$ & $\begin{array}{l}\text { KENT } 9 \\
\text { KENT } 9 \\
\text { KENT } 9 \\
\text { XENT } 9 \\
\text { PITT } 8^{\star} \\
\text { PITT } 8^{\star} \\
\text { PITT } 8^{\star} \\
\text { PITT } \\
\text { PITT } \\
8^{*}\end{array}$ & $\begin{array}{l}C P \\
K D \\
C P \\
K D \\
C P \\
K D \\
C P \\
K D \\
K D\end{array}$ & $\begin{array}{l}0.35 \\
0.29 \\
0.27 \\
0.35 \\
0.26 \\
0.26 \\
0.21 \\
0.36 \\
0.29\end{array}$ & $\begin{array}{c}0.08 \\
0.09 \\
- \\
- \\
- \\
- \\
- \\
0.04\end{array}$ & $\begin{array}{l}3 \\
3 \\
1 \\
1 \\
1 \\
1 \\
1 \\
1 \\
3\end{array}$ & $\begin{array}{c}0.01 \\
- \\
- \\
- \\
- \\
- \\
- \\
-\end{array}$ & $\begin{array}{c}0.22 \\
- \\
- \\
- \\
- \\
- \\
- \\
- \\
-\end{array}$ & $\begin{array}{l}0.59 \\
0.73 \\
0.56 \\
0.63 \\
0.54 \\
- \\
0.38 \\
0.66 \\
1.50\end{array}$ \\
\hline $\begin{array}{l}8 A \\
8 A \\
8 B \\
8 C \\
8 D \\
8 E \\
8 F\end{array}$ & $\begin{array}{l}\text { PITT } 8^{\star} \\
\text { PITT } 8^{\star} \\
\text { PITT } 8^{*} \\
\text { PITT } 8^{\star} \\
\text { PITT } 8^{*} \\
\text { PITT } 8^{\star} \\
\text { PITT } \\
\text { PITT } \\
8^{*}\end{array}$ & $\begin{array}{l}C P \\
K D \\
C P \\
C P \\
C P \\
C P \\
C P \\
K D\end{array}$ & $\begin{array}{l}0.28 \\
0.34 \\
0.21 \\
0.13 \\
0.25 \\
0.23 \\
0.36 \\
0.35\end{array}$ & $\begin{array}{c}-\overline{0} \\
0.03 \\
- \\
0.07 \\
- \\
0.10 \\
-\end{array}$ & $\begin{array}{l}1 \\
4 \\
1 \\
1 \\
2 \\
1 \\
3 \\
1\end{array}$ & $\begin{array}{c}0.02 \\
- \\
0.07 \\
0.03 \\
0.03 \\
0.02 \\
0.01 \\
-\end{array}$ & $\begin{array}{l}0.55 \\
1.00 \\
0.61 \\
0.56 \\
0.52 \\
0.63 \\
-\end{array}$ & $\begin{array}{l}0.85 \\
0.80 \\
1.37 \\
0.77 \\
0.80 \\
0.76 \\
0.92 \\
1.01\end{array}$ \\
\hline $\begin{array}{l}9 A \\
9 A \\
9 A 1 \\
9 A 1 \\
9 B \\
9 B \\
9 C \\
9 C\end{array}$ & 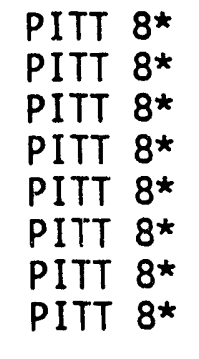 & $\begin{array}{l}C P \\
K D \\
C P \\
K D \\
C P \\
K D \\
C P \\
K D\end{array}$ & $\begin{array}{l}0.27 \\
0.33 \\
0.30 \\
0.41 \\
0.26 \\
0.28 \\
0.30 \\
0.19\end{array}$ & $\begin{array}{c}- \\
0.03 \\
0.03 \\
- \\
0.03 \\
0.08 \\
0.02 \\
-\end{array}$ & $\begin{array}{l}1 \\
3 \\
2 \\
1 \\
3 \\
1 \\
6 \\
1\end{array}$ & $\begin{array}{c}0.01 \\
-\overline{0} \\
0.01 \\
- \\
0.003 \\
-\overline{0} \\
0.002 \\
-\end{array}$ & $\begin{array}{c}0.60 \\
0 . \overline{41} \\
0 . \overline{35} \\
0 . \overline{19}\end{array}$ & $\begin{array}{l}0.88 \\
0.97 \\
0.72 \\
0.79 \\
0.62 \\
1.25 \\
0.49 \\
0.23\end{array}$ \\
\hline $\begin{array}{l}11 \mathrm{~A} \\
11 \mathrm{C} \\
110 \\
11 \mathrm{E}\end{array}$ & $\begin{array}{ll}\text { PITT } & 8 * \star \\
\text { PITT } & 8 * \star \\
\text { PITT } & 8 * \star \\
\text { PITT } & 8 * \star\end{array}$ & $\begin{array}{l}C P \\
C P \\
C P \\
C P\end{array}$ & $\begin{array}{l}0.02^{\star \star \star x} \\
0.02^{\star \star *} \\
0.23^{\star \star *} \\
0.06^{\star \star *}\end{array}$ & $\begin{array}{cc}* & 0.01 \\
* & - \\
* & - \\
* & 0.03\end{array}$ & $\begin{array}{l}4 \\
1 \\
1 \\
4\end{array}$ & $\begin{array}{l}0.08 \\
0.07 \\
0.01 \\
0.15\end{array}$ & $\begin{array}{l}0.46 \\
0.17 \\
0.18 \\
0.28\end{array}$ & $\begin{array}{l}0.56 \\
0.26 \\
0.42 \\
0.49\end{array}$ \\
\hline
\end{tabular}

For pyritic and organic sulfur, a "-" indicates no data.

* Pittsburgh No. 8 coal from Powhatan No. 6 mine.

** Pittsburgh No. 8 coal from Blacksville No. 2 mine.

$\star \star *$ pH was -5 during Run $11 \mathrm{~A},-10$ during Run 11C, $\sim 2$ during Run 110 , and $\cdot 10$ during Run IIE. Also during Run IIE the acid wash effluent was used as makeup water to the water wash train. 
subjected to the same wash train processing steps as the product coal for the other runs, this seems to indicate that under mild operating temperatures, insoluble sulfate is not formed by kiln processing in the MCL process. That is, the mild operating temperatures may have resulted in lower coal porosity and surface area, and therefore lower sulfate pick-up and/or more efficient washing in the acid wash train. Shakedown feed coal (Pittsburgh No. 8, Powhatan) contained only $0.04 \%$ sulfate sulfur.

Ten sets of sulfate suifur data are compared in Figure 4-15, with each set representing kiln discharge (KD) coal versus washed coal product (CP) data from the same run. The comparisons show higher KD than CP sulfate sulfur levels in 6 data sets, same KD and CP sulfate sulfur level in 1 data set, and lower KD than CP sulfate levels in 3 data sets. In addition, the Pittsburgh No. 8 (Powhatan) and the Kentucky No. 9 coal feeds only contain $0.04 \%$ and $0.02-0.06 \%$ sulfate sulfur, respectively. This indicates that sulfate sulfur in MCL coal is formed in kiln processing, and not due to washing by dilute sulfuric acid. It also appears that in at least some of the runs, downstream washing removes part of the sulfate sulfur initially formed. Thus, this sulfate level comparison provides evidence of the effectiveness of the countercurrent washing scheme in the integrated $\mathrm{MCL}$ plant.

The sulfate in MCL coal is probably present as iron sulfate, formed from the thiosulfate derived from the reaction of sodium or potassium hydroxide with coal pyritic sulfur:

$$
8 \mathrm{FeS}_{2}+30 \mathrm{NaOH}=14 \mathrm{Na}_{2} \mathrm{~S}+\mathrm{Na}_{2} \mathrm{~S}_{2} \mathrm{O}_{3}+4 \mathrm{Fe}_{2} \mathrm{O}_{3}+15 \mathrm{H}_{2} \mathrm{O}
$$

The above reaction indicates that $1 / 8$ of the pyritic sulfur could be converted to thiosulfate and subsequently to iron sulfate, which apparentiy cannot be readily removed from coal by washing with water or dilute sulfuric acid. For Pittsburgh No. 8 (Powhatan) coal, the $1.69 \%$ pyritic sulfur arid $0.04 \%$ sulfate sulfur in the coal feed could result in $0.25 \%$ sulfate sulfur in MCL coal. Similarly, the $1.84 \%$ pyritic sulfur and 0.02 to $0.06 \%$ sulfate sulfur in Kentucky No. 9 coal feed could result in $0.27 \%$ sulfate sulfur in $M C L$ coal. These sulfate sulfur leveis are approximately the same as the 


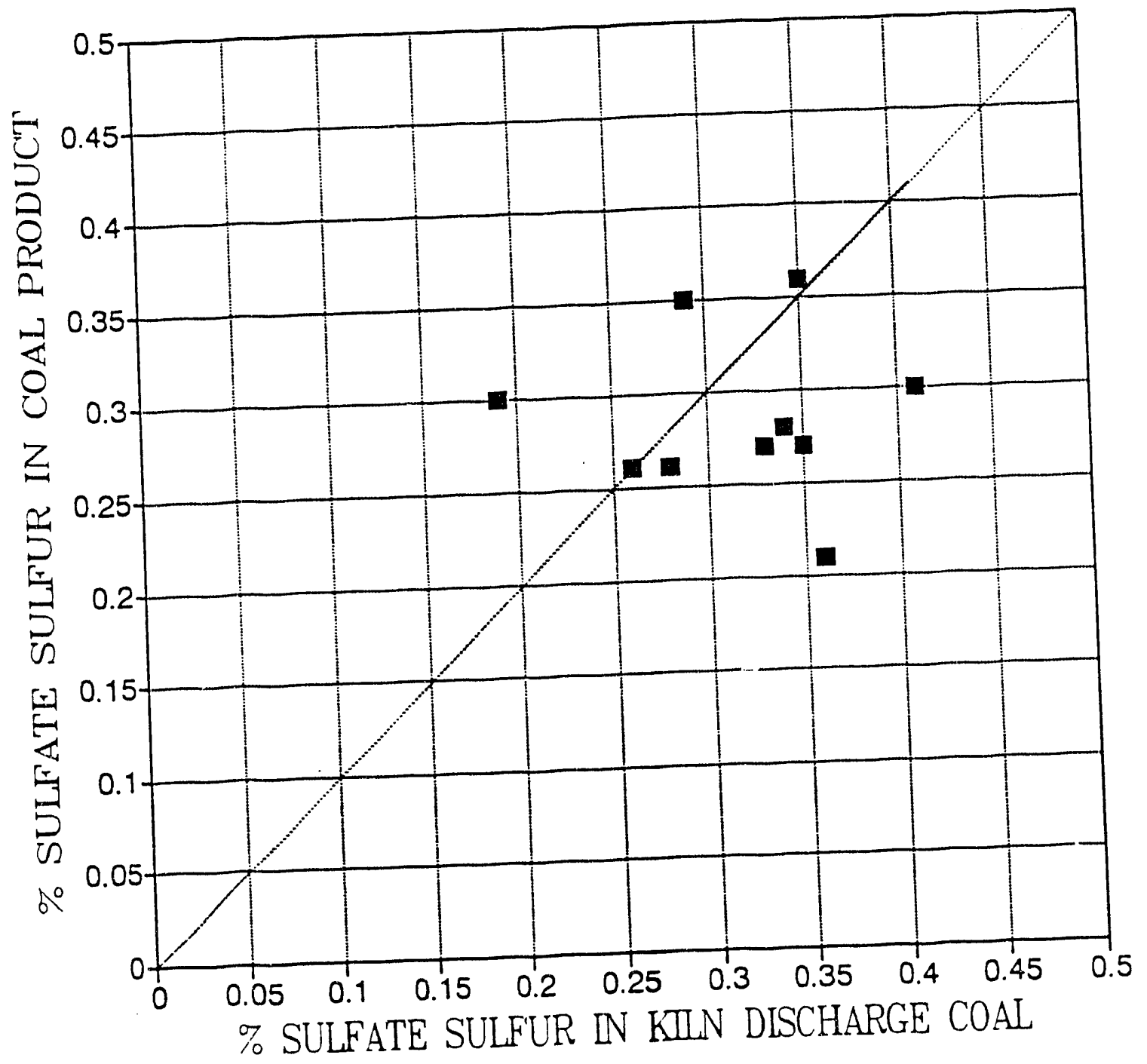

Figure 4-15. Comparison of Sulfate Sulfur in Kiln Discharge
Coal and Washed Coal Product 
average $0.28 \%$ sulfate sulfate level determined from the $\mathrm{MCL}$ coal samples (excluding shakedown and Run 11 data). The fact that the shakedown product coal had such a high percentage of pyritic sulfur remaining (Table 4-7) indicates that at mild kiln operating conditions, the above reaction does not take place.

The retention of sulfate sulfur can probably be reduced by utilizing low pyrite (highly cleaned) coals in the MCL process. For example, coal deeply cleaned to $0.5 \%$ pyritic sulfur, but containing any amount of organic sulfur should be reducible to $0.1 \%$ organic sulfur (one of the samples from Run 9C), $0.02 \%$ pyritic sulfur (Table $4-7$ ), and $0.07 \%$ sulfate sulfur (Table $4-7$ ) for a total sulfur of $0.19 \%$.

The data presented in Table 4-7 also show that in almost every case, the pyritic sulfur in MCL coal has been reduced to extremely low levels. The mean residual pyritic sulfur level is $0.022 \%$ for Pittsburgh (Powhatan) coal (excluding shakedown data), $0.01 \%$ for Kentucky coal. These represent $98.7 \%$ and $99.5 \%$ removal of the pyritic sulfur present in the Pittsburgh (Powhatan) and Kentucky coals fed to the rotary kiln.

Pittsburgh No. 8 (Blacksville) coal was used in only one run (Run 11). However, Run 11 was also the only run during which the $\mathrm{pH}$ in the acid wash train was varied from 2 to 10 by adjusting the amount of sulfuric acid injected. The $\mathrm{pH}$ level employed in the acid wash train had a very significant effect on the product coal sulfate sulfur content. When the $\mathrm{pH}$ was allowed to increase to 5 or 10 by cutting back on or eliminating sulfuric acid injection, the sulfate sulfur in the coal product samples decreased to $0.02-0.06 \%$ (Runs $11 \mathrm{~A}, 11 \mathrm{C}$ and $11 \mathrm{E}$ ). During Run 110 when the $\mathrm{pH}$ was 2 (as it was during Runs 1 through 10), the sulfate sulfur in the coal product was $0.23 \%$, indicating that the bulk of the sulfate sulfur in the product coal is from the sulfuric acid and not from the feed coal. No kiln discharge samples were analyzed for Run 11. The Pittsburgh No. 8 (Blacksville) feed coal contained only $0.01 \%$ sulfate sulfur.

For Pittsburgh No. 8 (Blacksville) coal, the $0.65 \%$ pyritic sulfur and $0.01 \%$ sulfate sulfur in the coal feed could result in $0.09 \%$ sulfate sulfur in 
MCL coal versus the 0.02 to $0.06 \%$ sulfate sulfur in actual MCL coal samples without acid wash (the pyritic sulfur ranged from 0.07 to $0.15 \%$ during the same period). When an acid wash was used and the $\mathrm{pH}$ was about 2 (Run 11D), the sulfate sulfur rose to $0.23 \%$ while the pyritic sulfur dropped to $0.01 \%$.

The amount of residual organic sulfur in MCL coal is strongly dependent on processing conditions. The lowest organic sulfur level found is $0.08 \%$ from a Run $9 \mathrm{C}$ coal product sample and the highest is $1.79 \%$ from the shakedown run. The effects of processing conditions on sulfur removal are discussed in Section 4.1 .1 of this report.

\subsection{KILN GAS}

Eight $\mathrm{ki}$ ln gas samples obtained from $7 \mathrm{MCL}$ runs were analyzed to determine the gas composition, as well as the percentage of coal feed converted to kiln gas. The results of the gas composition analysis and coal conversion calculations are presented in Table 4-8.

Except for Runs $5 \mathrm{D} 3$ and $11 \mathrm{E}$, data from the other five runs provide consistent results. Run $5 \mathrm{D} 3$ indicates much higher methane and $\mathrm{C}_{2}^{+}$ hydrocarbon content in the kiln gas and higher conversion of coal feed to kiln gas (as compared with the other runs), despite mild kiln operating temperatures. The Run $11 \mathrm{E}$ results indicate much lower $\mathrm{H}_{2}$ content in the $k i l n$ gas, despite operating temperatures similar to most of the other runs.

Comparison of the analytical results from Runs 1C, 2E, 5C4, 6G1, 100 and $11 \mathrm{E}$ shows that an average of $2.59 \%$ of the coal feed was converted to hydrogen and hydrocarbon gases. Operation at higher kiln temperatures (indicated by the Zone 1 or 2 sensor temperature) generally led to conversion of a higher percentage of the cDal feed to kiln gas, when analysis results from Runs $2 E$ and 5C4 (Kentucky No. 9) or Runs 1C and 6G1 (Pittsburgh No. 8, Powhatan) are compared. Results from these runs also suggest conversion of a higher percentage of the Kentucky No. 9 than Pittsburgh No. 8 (both Powhatan and Blacksville) coal feed to kiln gis.

Analyses show the volatiles content of the feed coals to be $38.60 \%$ for Pittsburgh No. 8 (Powhatan), $40.53 \%$ for Kentucky No. 9, and $38.70 \%$ for 


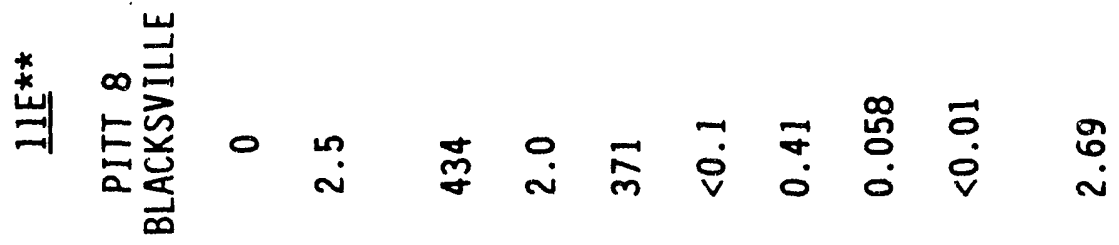

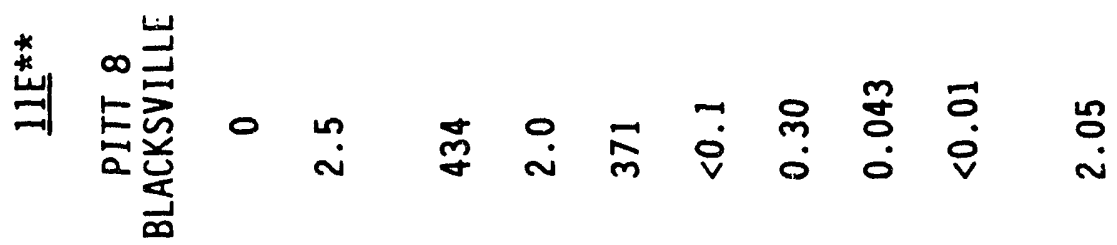

$\ddot{8}$

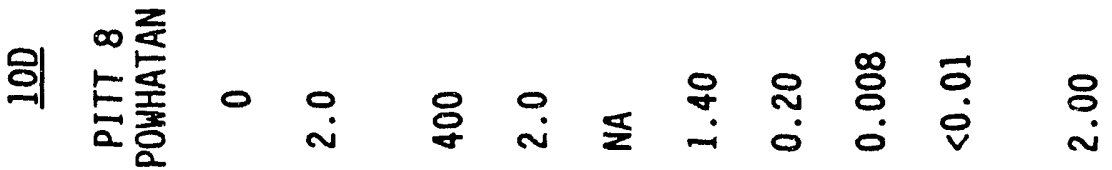

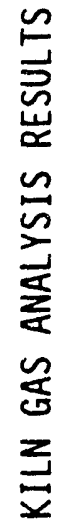

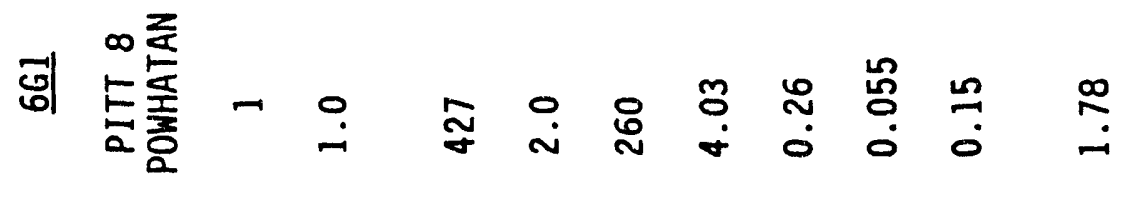

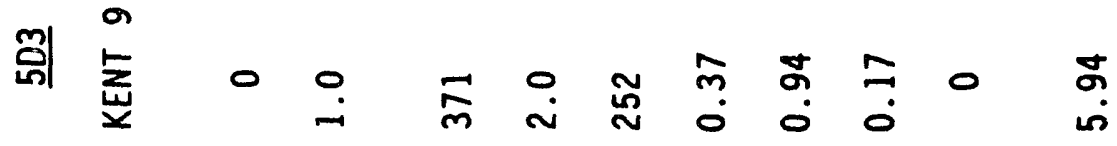

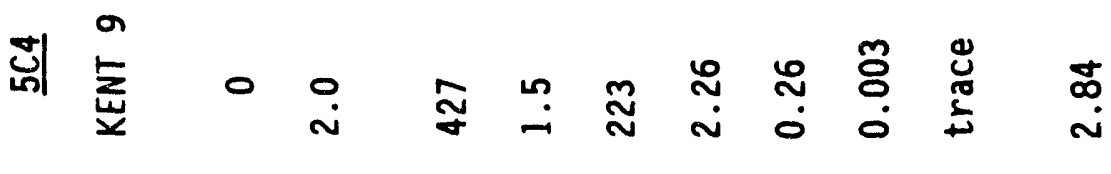

5

$\frac{\infty}{1}$

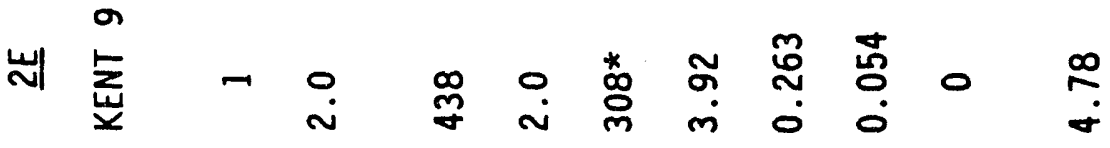

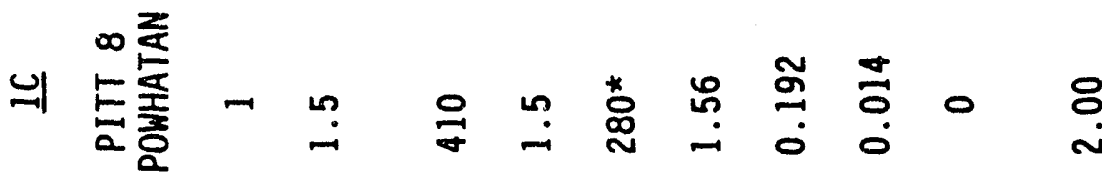

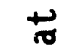

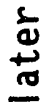

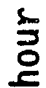

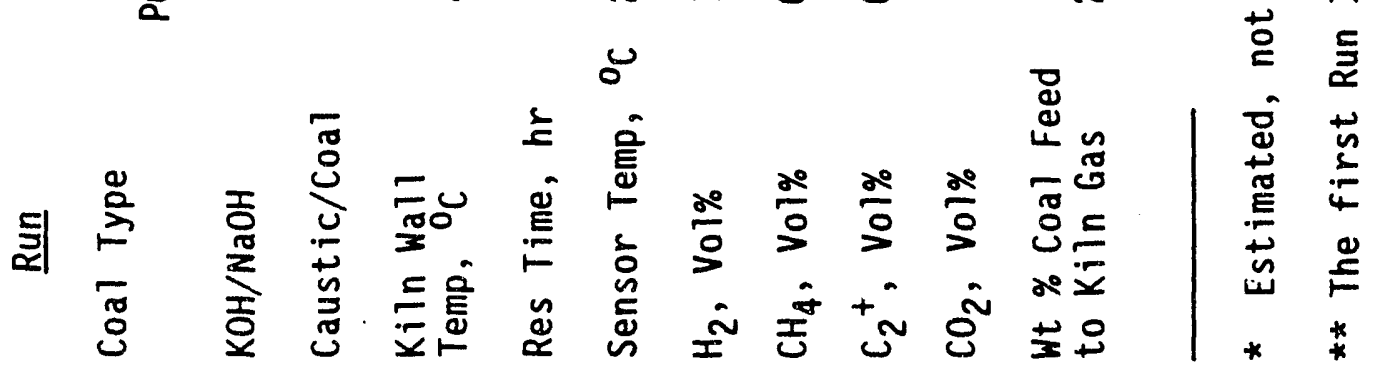


Pittsburgh No. 8 (Blacksville) (see Table 1-1). The volatiles content of the product coal averages about 27\% (see Appendix A), for an overall decrease in volatiles of approximately $13 \%$. It is 1 ikely chat 7 to $8 \%$ by weight of the volatiles loss is due to the desiccating conditions of dry molten caustic and temperatures around $390^{\circ} \mathrm{C}$ which would most likely remove the decomposition moisture of the organic constituents of coal as well as the water of hydration of the coal mineral matter. For example, Friedman, et al, " have shown that there is an $8 \%$ water component in the volatile matter of Pittsburgh seam coal when the coal is heated to $600^{\circ} \mathrm{C}$. It is possible that the MCL process conditions, while at a lower temperature, may be even more severe in desiccating action due to the presence of caustic. If this $8 \%$ moisture loss is added to the $2 \%$ loss to phenolics and the $3 \%$ loss to hydrogen/hydrocarbon gases, the $13 \%$ overall decrease in volatiles content is accounted for.

Two samples of kiln off-gas were collected during Run $11 \mathrm{E}$ ( 1 hour apart) at the same kiln conditions. At a $\mathrm{GN}_{2}$ feed rate to the kiln of 17 SCFM during the sampling period and a coal feed rate to the kiln of $\sim 91 \mathrm{bs} / \mathrm{hour}$, the average weight percent coal converted to $\mathrm{CH}_{4}$ is $1.83 \%$ and to $\mathrm{C}_{2}{ }^{+}$is $0.48 \%$. Less than $0.06 \%$ of the weight of the coal was converted to hydrogen. While an average total weight of coal converted to hydrogen and volatile hydrocarbon gases of $-2.36 \%$ is not out of 1 ine with the average coal weight loss to volatiles with other coals processed during the other six tests for which there is data, the conversion of coal to hydrogen is about an order of magnitude lower than that seen previously (hydrogen content of the kiln off-gas averaged 2.26 mole percent during the other six runs versus $<0.1$ mole percent for Run IIE). The very small amount of hydrogen observed in the kiln off-gas raises questions about the mechanism of carbonate formation in the kiln. One reaction mechanism theorized to explain carbonate formation is:

$$
2 \mathrm{NaOH}+\mathrm{C}+\mathrm{H}_{2} \mathrm{O}-->\mathrm{Na}_{2} \mathrm{CO}_{3}+2 \mathrm{H}_{2}{ }^{\dagger}
$$

* Friedman, L. D., et al, Fuel, 47, 149 (1968). 
If this mechanism is correct, hydrogen will be formed. At the carbonate make seen in Run No. $11 \mathrm{E}$ ( $-9 \%$ of caustic converted to carbonate, see Section 4.5.1), the hydrogen level in the kiln off-gas (exclusive of any hydrogen driven from the coal itself as it is heated) would calculate to 2.0 mole percent versus the $<0.1$ mole percent observed. Another possible mechanism for carbonate formation is:

$$
2 \mathrm{NaOH}+\mathrm{CO}_{2} \rightarrow \mathrm{Na}_{2} \mathrm{CO}_{3}+\mathrm{H}_{2} \mathrm{O}
$$

The source of the $\mathrm{CO}_{2}$ could be: 1) $\mathrm{CO}_{2}$ evolved from the coal during heatup, or 2) a small amount of air leakage into the kiln to form $\mathrm{CO}_{2}$ by combustion of a small amount of the coal. Since the coal tested is of relatively high rank (bituminous), it is doubtful that the coal would have evolved (during heatup) more than a small fraction of the $\mathrm{CO}_{2}$ needed to account for the observed carbonate make. The air leakage into the kiln needed to account for the observed carbonate make would be -0.8 SCFM (or $~-5 \%$ of the $\mathrm{GN}_{2}$ volume used to sweep the $\left.k i l n\right)$. It should be noted that the kiln is normally operated under a slightly positive pressure $\left(<1\right.$ inch $\left.\mathrm{H}_{2} \mathrm{O}\right)$ to avoid the in-leakage of air.

The -25 gallon inventory of dilute (10\%) caustic solution that was used in the venturi scrubber for the scrubbing of the kiln off-gas was analyzed at the end of Run 11 for total organic carbon (TOC) content. The solution has a TOC of 550 micrograms/gram.

\subsection{HUMIC ACID AND PHENOLICS FORMATION}

Soluble organic compounds present in the spent caustic solutions are classified as humic materials if they precipitate upon acidification, and as phenolic materials if they remain soluble upon acidification. Data on the conversion of coal feed to humics are presented in Table 4-9. Data on the conversion to phenolics are not reported because of analytical and sampling problems in the test circuit. Also, experience has showed only very small amounts of phenolic formation in laboratory studies.

Data on the conversion of coal feed to humics are also plotted in Figure 4-16 as a function of the caustic/cnal temperature in Zone 1 (Runs 1 to 5 ) or 
TABLE 4-9. FORMATION OF HUMIC ACID FOR SHAKEDOWN THROUGH RUN 9

\begin{tabular}{|c|c|c|c|c|c|c|c|}
\hline \multirow[b]{2}{*}{ Run } & \multirow{2}{*}{$\begin{array}{l}\text { Coal } \\
\text { Iype }\end{array}$} & \multirow{2}{*}{$\begin{array}{l}\mathrm{KOH} / \\
\mathrm{NaOH} \\
\end{array}$} & \multirow{2}{*}{$\begin{array}{l}\text { Leach } \\
\text { Temp, }{ }^{0}{ }^{C * *}\end{array}$} & \multicolumn{3}{|c|}{$\%$ Coal Converted to Humics } & \multirow{2}{*}{ Range } \\
\hline & & & & Mean & Std Dev. & Data Pts & \\
\hline DW & PITT 8 & 1 & 209 & 4.5 & - & 1 & 4.5 \\
\hline $\begin{array}{l}1 B \\
1 C\end{array}$ & $\begin{array}{l}\text { PITT } 8 \\
\text { PITT } 8 \\
\text { PITT } 8\end{array}$ & $\begin{array}{l}1 \\
1 \\
1\end{array}$ & $\begin{array}{l}259 \\
279 \\
279\end{array}$ & $\begin{array}{l}0.533 \\
0.794 \\
1.231\end{array}$ & $\begin{array}{l}- \\
1.041 \\
0.676\end{array}$ & $\begin{array}{l}1 \\
3 \\
4\end{array}$ & $\begin{array}{c}0.533 \\
0.105-1.992 \\
0.278-1.872\end{array}$ \\
\hline $\begin{array}{l}2 B \\
2 C \\
2 D \\
2 E\end{array}$ & $\begin{array}{ll}\text { PITT } & 8 \\
\text { PITT } & 8 \\
\text { KENT } & 9 \\
\text { KENT } & 9\end{array}$ & $\begin{array}{l}1 \\
1 \\
1 \\
1\end{array}$ & $\begin{array}{l}259 \\
296 \\
296 \\
307\end{array}$ & $\begin{array}{l}0.249 \\
0.067 \\
0.236 \\
0.039\end{array}$ & $\begin{array}{c}0.148 \\
0.060 \\
- \\
-\end{array}$ & $\begin{array}{l}2 \\
3 \\
1 \\
1\end{array}$ & $\begin{array}{c}0.144-0.354 \\
0.027-0.136 \\
0.236 \\
0.039\end{array}$ \\
\hline $\begin{array}{l}B 1 \\
\text { C1 }\end{array}$ & $\begin{array}{l}\text { PITT } 8 \\
\text { PITT } 8\end{array}$ & $\begin{array}{l}1 \\
1\end{array}$ & $\begin{array}{l}307 \\
307\end{array}$ & $\begin{array}{l}0.507 \\
0.217\end{array}$ & $\begin{array}{c}0.392 \\
-\end{array}$ & $\begin{array}{l}6 \\
1\end{array}$ & $\begin{array}{c}0.066-1.02 \\
0.217\end{array}$ \\
\hline $\begin{array}{l}C 1 \\
C 2 \\
C 23\end{array}$ & $\begin{array}{l}\text { PITT } 8 \\
\text { PITT } 8 \\
\text { PITT } 8\end{array}$ & $\begin{array}{l}0 \\
0 \\
0\end{array}$ & $\begin{array}{l}290 \\
302 \\
323\end{array}$ & $\begin{array}{l}3.97 \\
5.38 \\
1.74\end{array}$ & $0 . \overline{445}$ & $\begin{array}{l}1 \\
2 \\
1\end{array}$ & $\begin{array}{l}3.97 \\
5.06-5.69 \\
1.74\end{array}$ \\
\hline $\begin{array}{l}5 B 1 \\
5 B 3 \\
5 C 5 \\
5 C 4 \\
5 D 3\end{array}$ & $\begin{array}{ll}\text { PITT } & 8 \\
\text { PITT } & 8 \\
\text { KENT } & 9 \\
\text { KENT } & 9 \\
\text { KENT } & 9\end{array}$ & $\begin{array}{l}0 \\
0 \\
0 \\
0 \\
0\end{array}$ & $\begin{array}{l}302 \\
237 \\
298 \\
223 \\
252\end{array}$ & $\begin{array}{l}3.17 \\
10.4 \\
1.67 \\
5.95 \\
2.49\end{array}$ & $\begin{array}{l}- \\
- \\
- \\
-\end{array}$ & $\begin{array}{l}1 \\
1 \\
1 \\
1 \\
1\end{array}$ & $\begin{array}{c}3.17 \\
10.4 \\
1.67 \\
5.95 \\
2.49\end{array}$ \\
\hline $\begin{array}{l}8 \mathrm{~A} \\
8 \mathrm{~B} \\
8 \mathrm{C} \\
8 \mathrm{D} \\
8 \mathrm{E}\end{array}$ & $\begin{array}{l}\text { PITT } 8 \\
\text { PITT } 8 \\
\text { PITT } 8 \\
\text { PITT } 8 \\
\text { PITT } 8 \\
\text { PITT } 8\end{array}$ & $\begin{array}{l}1 \\
1 \\
0 \\
0 \\
0 \\
0\end{array}$ & $\begin{array}{l}395 \\
395 \\
403 \\
417 \\
389 \\
384\end{array}$ & $\begin{array}{l}1.94 \\
1.20 \\
0.744 \\
1.14 \\
1.27 \\
1.24\end{array}$ & $\begin{array}{c}- \\
- \\
0.276 \\
- \\
- \\
-\end{array}$ & $\begin{array}{l}1 \\
1 \\
2 \\
1 \\
1 \\
1\end{array}$ & $\begin{array}{c}1.94 \\
1.20 \\
0.548-0.939 \\
1.14 \\
1.27 \\
1.24\end{array}$ \\
\hline & $\begin{array}{l}\text { PITT } 8 \\
\text { PITT } 8 \\
\text { PITT } 8\end{array}$ & $\begin{array}{l}1 \\
1 \\
0\end{array}$ & $\begin{array}{l}407 \\
415 \\
419\end{array}$ & $\begin{array}{l}1.17 \\
0.209 \\
0.318\end{array}$ & $\begin{array}{c}- \\
0 . \overline{3} 19\end{array}$ & $\begin{array}{l}1 \\
1 \\
2\end{array}$ & $\begin{array}{c}1.17 \\
0.209 \\
0.093-0.524\end{array}$ \\
\hline
\end{tabular}

* Pittsburgh No. 8 coal from Powhatan No. 6 mine (Powhatan Point, Ohio).

** Leaching temperature is based on temperatures measured by the thermocouple probe placed in either Zone 1 (Runs 4 and 5) or Zone 2 (Runs 8 and 9) of the rotary kiln. For shakedown and Runs 1 to 3 , the leaching temperature is estimated by assuming a $131^{\circ} \mathrm{C}$ temperature difference between the Zone $1 \mathrm{kiln}$ wall and the caustic/coal mixture. 


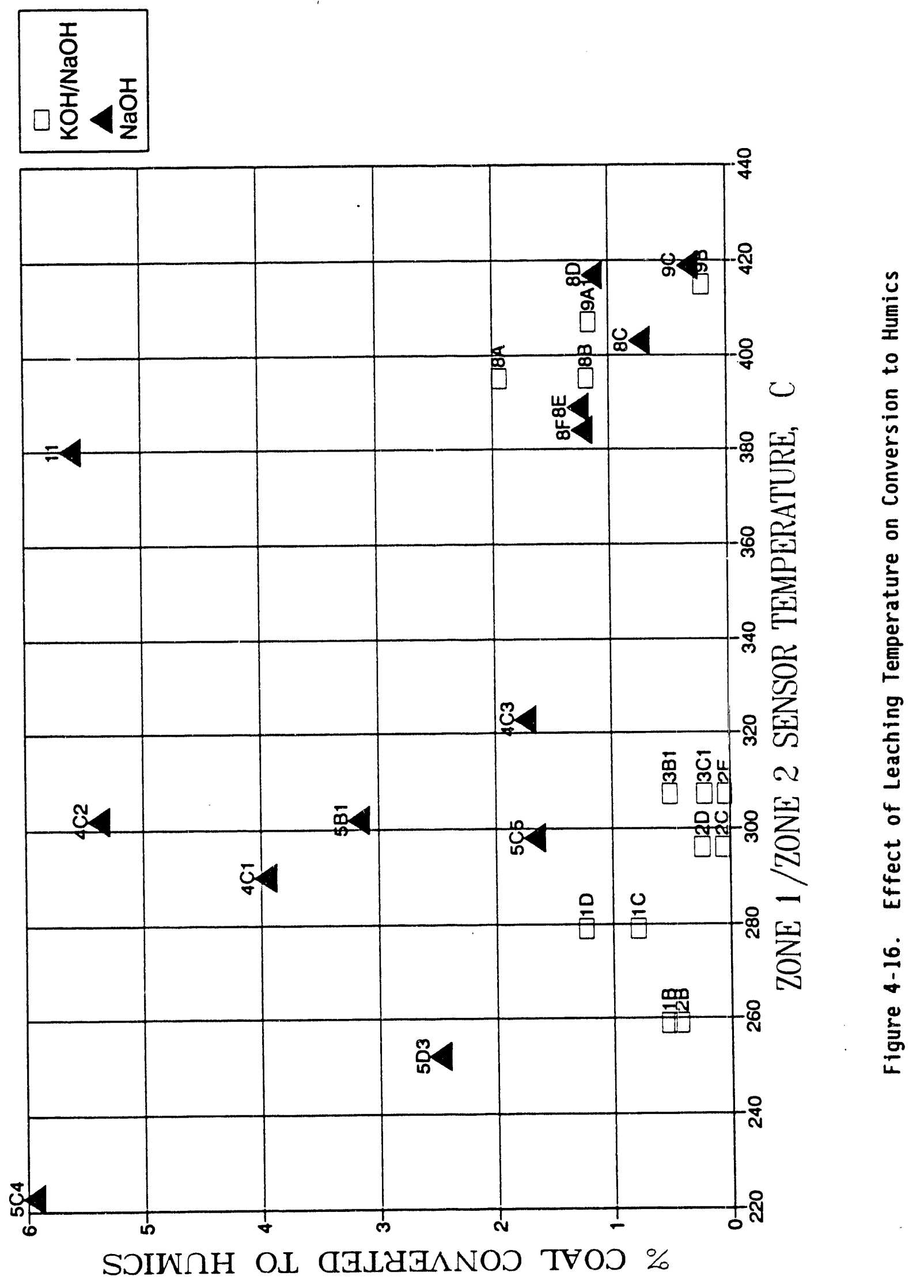


Zone 2 (Runs 8 and 9) of the rotary kiln. Shakedown data are not included in this plot. The nitrogen flow was concurrent with the coal/caustic fiow during shakedown and Runs 1 to 5 , and countercurrent to the coal/caustic flow in Runs 8,9 and 11 . The net result was that during shakedown and Runs 1 to 5 , the temperature difference between the kiln wall and the coal/caustic mixture in Zone 1 was typically $131^{\circ} \mathrm{C}$. By comparison, the temperature difference between the kiln wall and the coal/caustic mixture in Zone 2 was only in the $19-32^{\circ} \mathrm{C}$ range in Runs 8 and 9 . Run 11 , the only fully integrated test, is the subject of a separate discussion later in this section. During shakedown and Runs 1 to 5 , the leaching temperature would increase in the subsequent zones of the rotary kiln and would be higher than the temperature indicated by the Zone 1 probe sensor. For Runs 8 and 9 , the leaching temperature in all four zones of the rotary kiln would be approximately the same as indicated by the Zone 2 probe sensor.

Based on the data from Runs $4 C 1,4 C 2,5 B 1,5 B 3$ (not shown in Figure 4-16, off the scale), $5 \mathrm{C} 4$ and 5D3, formation of humics appears to be maximum when only sodium hydroxide was used and when Zone 1 leaching temperatures were near or below $300^{\circ} \mathrm{C}$. When mixed caustic was used for leaching, the maximum conversion of coal feed to humics was less than $2 \%$ (excluding shakedown data). However, at leaching temperatures higher than $384^{\circ} \mathrm{C}$, the conversion to humics was about the same whether sodium hydroxide or mixed caustic was used, and an average of $0.96 \%$ of the coal feed was converted to humics. At these temperatures, humics formation appears to decrease with leaching temperature. At leaching temperatures in excess of $415^{\circ} \mathrm{C}$ (Runs $9 \mathrm{~B}$ and $9 \mathrm{C}$ ), the data also show that low conversions of only 0.2 to $0.3 \%$ of the coal feed to humics are achievable.

At Zone 1 leaching temperatures near or below $300^{\circ} \mathrm{C}$, humics formation was also low when mixed caustic was used (Runs 1 to 3 ). Even though the average leaching temperature was higher than that for Zone 1 , these data suggest that for mixed caustic, it is possible to achieve low humics formation under Zone 1 leaching temperatures as $10 \mathrm{w}$ as $260^{\circ} \mathrm{C}$.

The total $4.5 \%$ conversion of feed coal to humic acid during shakedown is indicative of the mild $\mathrm{kiln}$ operating temperatures (approximately $209^{\circ} \mathrm{C}$ in 
Zone 1) during the shakedown run. Humics formation is known to decrease with increasing temperature. It is thought that the humics, which are polycarboxy organics, dissolve in the caustic even under the mild conditions used during shakedown. Of the total $4.5 \%$ humic acid formed during shakedown, $0.35 \%$ was recovered in the first centrate sample in the acid wash, and $4.1 \%$ recovered in the caustic regenerator effluent sample. These data show that most of the humic acid would be found in the spent caustic and not in the acid wash. Under more severe operating conditions, the humics would decarboxylate to insoluble coal-like particles and exit the plant with the coal product.

Run 11 was the only run in which a continuous recycle of regenerated caustic to the kiln was maintained for most of the test (Run 9B had a total of -8 hours of operation with regenerated caustic recycled to the kiln). Fresh caustic was fed to the kiln during the first 24 hours of Run 11 to build caustic inventory in the system, and thereafter, fresh caustic was fed only sporadically and for short periods of time when the inventory of regenerated caustic flake available for recycle to the kiln ran low. The method of calculating humics make in all the runs conducted up to Run 11 was to compute the humics in the exit material from the kiln based on either kiln discharge solids or water wash train filtrate sample analysis (with the assumption that the humics inventory within the kiln is relatively constant). This calculation is valid in a "once-through" (no recycle of caustic to the kiln) system. A different approach to the calculation of humics formation needs to be taken for a system in which regenerated caustic (with a significant humics content) is recycled to the kiln.

Run 11 was conducted at a fairly constant kiln condition (Pittsburgh No. 8, Blacksville coal, 2.5:1 caustic ( $\mathrm{NaOH}$ only):coal, constant feed rate) for the entire week of operations. The kiln skin temperature was varied only slightly, from $424^{\circ} \mathrm{C}$ for the first 56 hours of the run to $434^{\circ} \mathrm{C}$ for the final 88 hours of the run. Since the kiln condition remained "constant" during the run, the approach to determining humics formation was to calculate the total humics make for the week based on "average" stream analyses and known stream flows. This approach, as opposed to the calculation of humics production at points of time within the test based on single sample stream 
analysis, practically eliminates the effect of changing plant humics inventory on the humics formation calculation.

Table 4-10 summarizes the humics analysis for water wash train filtrate (regeneration feed) and regenerated alkali (evaporator feed) samples taken during Run 11. The humics material balance calculation is shown schematically in Figure 4-17. The water wash train aqueous caustic effluent (which is assumed to contain all of the humics in the kiln discharge solids entering the wash train) had an average humic content of $0.41 \%$. This filtrate flow was calculated to average $-110 \mathrm{lbs} / \mathrm{hr}$ for a period of 137 hours. Total humics in this filtrate stream was calculated to be 62 pounds. Due to occasional kiln upsets, approximately $21 \%$ of the time the kiln discharge solids were collected in plastic bags rather than allowing the solids to enter the water wash train. (However, during this time, the wash water filtrate continued to flow to regeneration.) The amount of humics in the kiln discharge material bagged, which was assumed to have the same humics content as the solids entering the wash train, was calculated to be 17 pounds $[62$ lbs $\times 0.21+(1-0.21)]$. Therefore, the total humics exiting the kiln during Run 11 was 79 pounds (62 1b: + 17 1bs).

The average regenerated alkali fed to the caustic evaporator contained $0.17 \%$ humics and $27.2 \% \mathrm{NaOH}$ (Table 4-10). During evaporation there was no evidence of humics precipitation. The average $\mathrm{NaOH}$ content of the evaporated regenerated flake produced was $-85 \%$. Since the humics did not separate from the caustic during evaporation, the humics concentration was assumed to increase from an average value of $0.17 \%$ in the evaporator feed to $0.53 \%$ in the caustic flake. A total of 2,265 pounds of regenerated caustic flake (humics content $0.53 \%=12$ lbs) was fed to the kiln during Run 11 . The net production of humics during Run 11 therefore was 67 pounds [humics from kiln (79 lbs) minus humics to kiln (12 lbs)].

Based on a total of 1,193 lbs of MAF coal fed to the kiln during Run 11 , coal conversion to humics was $5.6 \%(67 \mathrm{lbs}+1193$ lbs $\times 100)$. This is a relatively high humics make compared to Runs 8 and 9 (see Figure 4-16) which utilized a different Pittsburgh No. 8 (Powhatan) coal. The skin temperature during Run 11 was $-430^{\circ} \mathrm{C}$, approximately the same as in Runs 8 and 9 . 


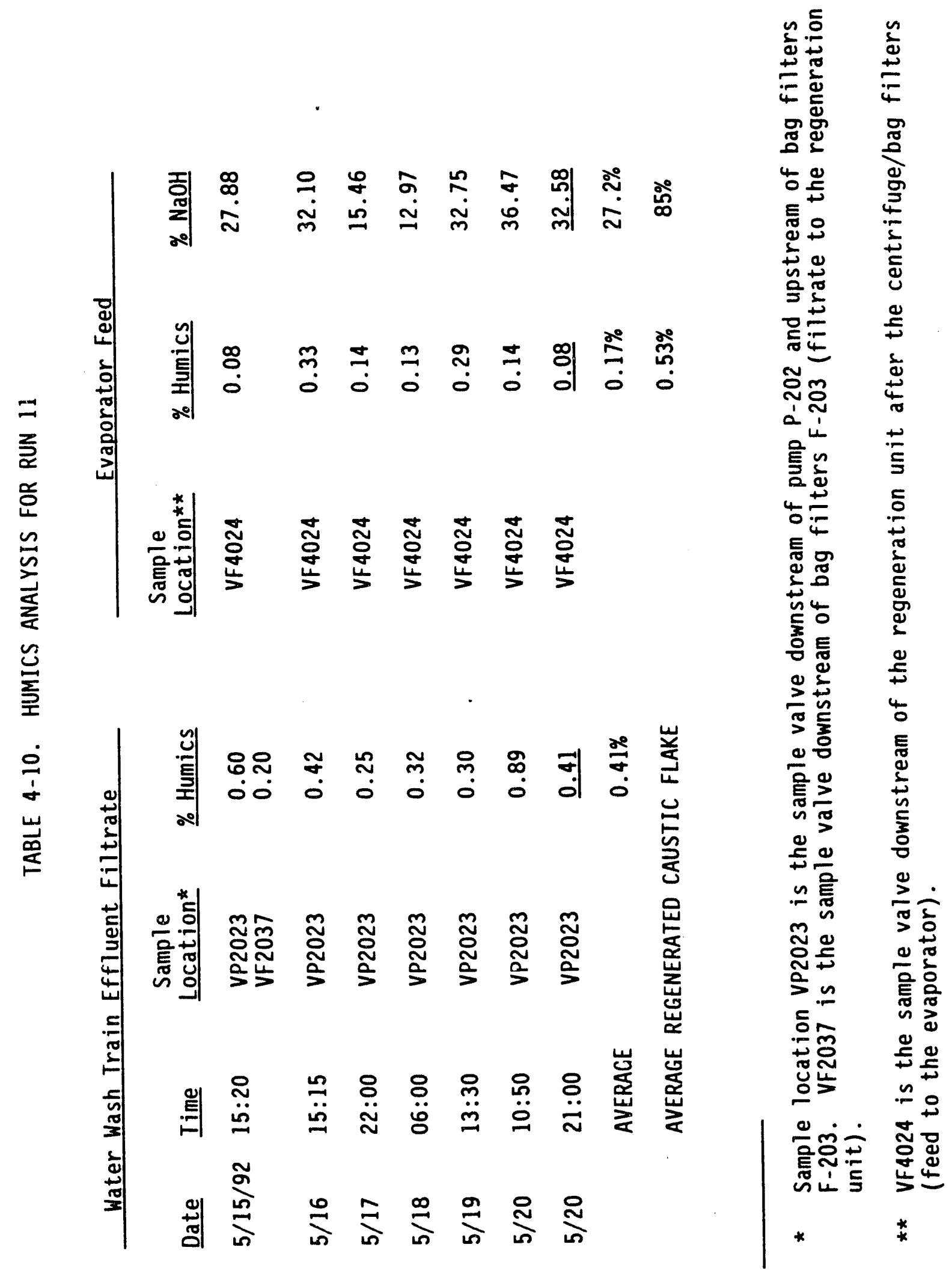




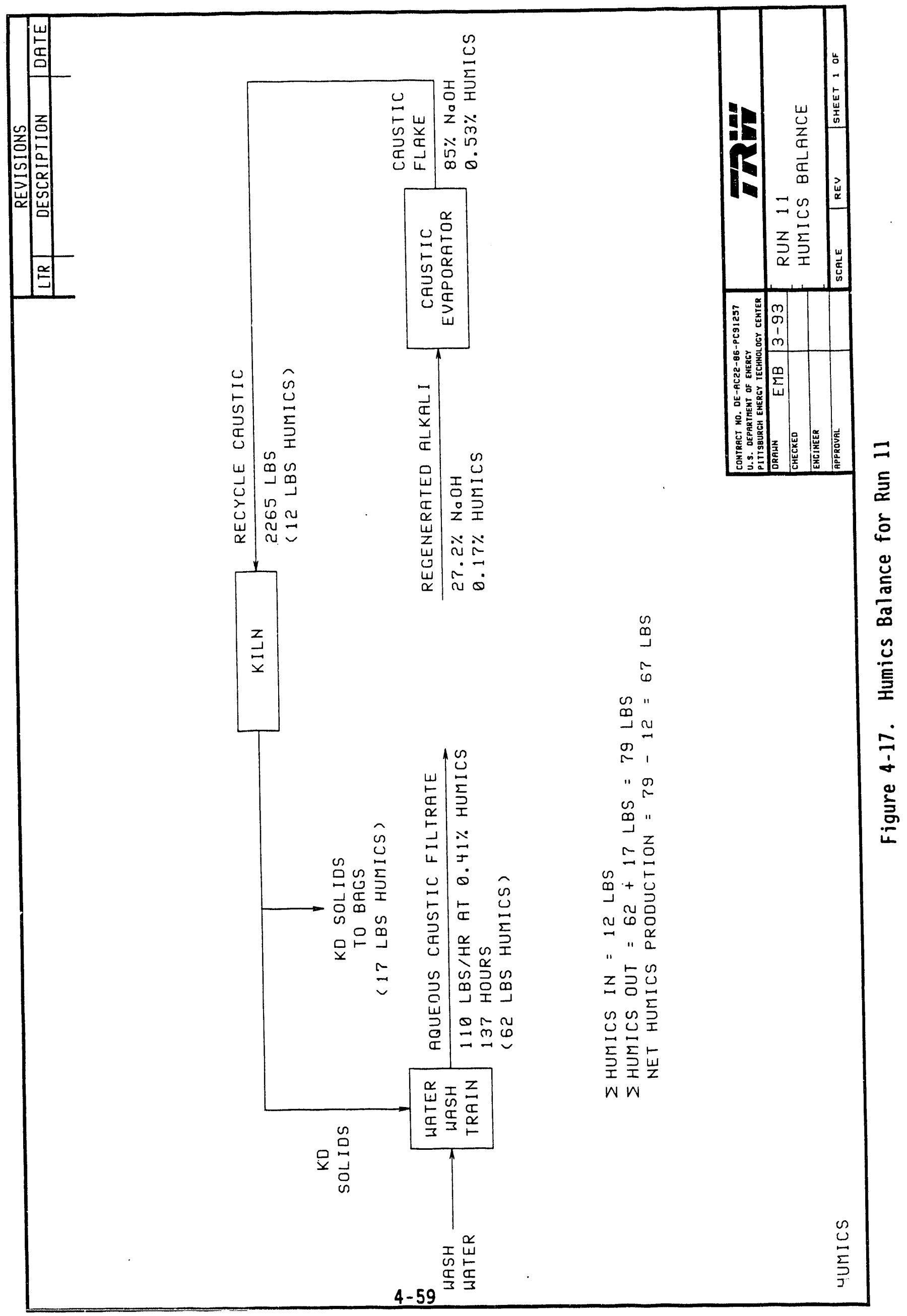


During Runs 8 and 9, the Zone 2 internal temperature was observed to be $19-32^{\circ} \mathrm{C}$ below the kiln skin temperature. However, during Run 11 the Zone 2 temperature was observed to vary between $15-110^{\circ} \mathrm{C}$ below the kiln skin temperature. (Note: inspection of the kiln's internal auger after Run 11 revealed that a number of the auger flights had sheared off. Thus the auger may not have been effective in scraping the wall and maintaining a low temperature differential between the wall and the kiln contents.)

The average Zone 2 temperature was estimated to be $-380^{\circ} \mathrm{C}$. The Zone 1 temperature undoubtedly was even lower than the Zone 2 temperature. These low temperatures in Zones 1 and 2 of the kiln during Run 11 may explain the relatively high conversion of coal to humics. A data point representative of Run 11 conversion to humics has been plotted in Figure 4-16.

The humics formation experienced during these test runs is highly specific to this plant and this scale of operation. Humics formation in a large scale plant should neariy be eliminated by the following considerations:

1) Kiln residence time of 0.5 hours versus the -2 hours used during most of this testing. Humics formation is a function of time. Laboratory studies have shown that high sulfur removal can be achieved in only 30 minutes. In a full scale plant, the caustic would be feed as molten rather than as flake, thus speeding up thermal transfer (by eliminating heating and melting of the caustic) and making a 0.5 hour residence time feasible.

2) Smooth, uninterrupted operation versus the start-stop operation in the test plant. The smooth, uninterrupted operation of a full scale plant would eliminate sources of air in-leakage that were present in the test plant.

3) Molten versus flake caustic feed to kiln. The flaked caustic used during testing brought adsorbed $\mathrm{CO}_{2}$ and $\mathrm{O}_{2}$ into the kiln. This would not be the case in a full scale plant with molten caustic feed.

4) Fresh coal versus weathered coal. A full scale plant would be built next to a coal mine, thus only hours or a few days would elapse between mining and processing in the $M C L$ plant. Even the "unweathered" coal used in Runs 1 through 9 and Run 11 was several months old (from the time it was mined to grinding by Tra-Det to transportation to TRW until the time of testing). There is information in the 1 iterature indicating that weathered coal forms more humics in the presence of caustic than fresh coal. 
5) Enclosed filters versus open filters. The vacuum filters in the test plant were operated with their cases open to the atmosphere (even though they had vapor enclosures) in order that the operators could observe cake thickness, etc. Thus, some air was undoubtedly drawn through the hot filter cakes. There is data in the literature indicating that coal dissolves in caustic and air $\left(0_{2}\right)$ to form humics and carbon dioxide. (Also, the dissolver tank in the test circuit was open to the atmosphere via the opening for the mixer.)

\subsection{CARBONATE FORMATION}

Carbonate is formed by the reaction of molten caustic with coal carbon in the presence of carbon dioxide (air). The formation of carbonate results in both loss of carbon from coal as well as loss of caustic. Data from the $\mathrm{MCL}$ integrated plant were evaluated to determine the effects of process parameters on carbonate formation, and to delineate the operating conditions that will minimize its formation.

During the operational runs, selected kiln discharge (KD) samples and spent caustic solution (VP2023 or VF2037)* samples were collected and analyzed for carbonate and caustic compositions. These composition data were used to compute the fraction of caustic converted to carbonate, as well as the fraction of coal or coal carbon converted to carbonate. The computations were based on the following equations:

$\mathrm{KOH}+\mathrm{NaOH}$ feed $=\mathrm{KOH}+\mathrm{NaOH}$ in product $+\mathrm{KOH}+\mathrm{NaOH}$ used for sulfur and ash removal $+\mathrm{KOH}+\mathrm{NaOH}$ converted to carbonate

Carbonate formed $=\mathrm{K}_{2} \mathrm{CO}_{3}+\mathrm{Na}_{2} \mathrm{CO}_{3}$ in product $-\mathrm{K}_{2} \mathrm{CO}_{3}+\mathrm{Na}_{2} \mathrm{CO}_{3}$ in caustic feed ${ }^{\star \star}$

Similar equations were used for $\mathrm{NaOH}$ feed. For $50: 50 \mathrm{KOH} / \mathrm{NaOH}$ feed, one pound of $\mathrm{KOH}+\mathrm{NaOH}$ converted to carbonate would result in 1.2783 pounds of potassium and sodium carbonate. Therefore:

\footnotetext{
VP2023 and VF2037 refer to the valve locations where the samples were taken. VP2023 is the sample valve downstream of pump P-202 (and upstream of bag filters F-203). VF2037 is the sample valve downstream of bag filters F-203 (on the filtrate to the regeneration section).

Fresh caustic feed + recycled regenerated caustic feed
} 
$\%$ of caustic converted to carbonate $=\frac{100 \times \text { carbonate formed }}{1.2783 \times \mathrm{KOH}+\mathrm{NaOH} \text { feed }}$

Similarly, for $100 \% \mathrm{NaOH}$ feed, one pound of $\mathrm{NaOH}$ converted to carbonate would result in 1.3250 pounds of sodium carbonate. In this case:

$$
\% \text { of caustic converted to carbonate }=\frac{100 \times \text { carbonate formed }}{1.3250 \times \text { NaOH feed }}
$$

In reducing the data, the following composition for the commercial solid caustic mixture was used: $92.42 \%$ sodium and potassium hydroxide, $3.37 \%$ sodium and potassium carbonate, and $4.21 \%$ water. This is the average composition of the mixed fresh caustic fed to the rotary kiln, as determined from the analysis of nine samples. The same composition was used in reducing data for runs with sodium hydroxide feed, except substituting sodium for sodium/potassium. For Run 9B, recycled caustic from the evaporator was used as the feed, with the following composition: $80.61 \%$ sodium and potassium hydroxide, $5.59 \%$ sodium and potassium carbonate, $0.37 \%$ sodium and potassium sulfide, and $13.43 \%$ water. This is the composition used for reducing Run $9 B$ data. Run 11 which was the only run in which a continuous recycle of regenerated caustic to the kiln was maintained for most of the test. (Run $9 B$ had a total of $\sim 8$ hours of operation with regenerated caustic recycled to the $k i 7 n$ ) is the subject of a separate discussion (see Section 4.5.1).

It was also assumed that the carbon in the carbonate formed was derived from the carbon in the coal feed. For $50: 50 \mathrm{KOH} / \mathrm{NaOH}$ feed, one pound of $\mathrm{KOH}+\mathrm{NaOH}$ reacts with 0.12859 pounds of coal carbon (or 100 pounds of caustic with 12.859 lbs of coal carbon) to form carbonate. Since the caustic feed contains only $92.42 \%$ sodium and potassium hydroxide and only part of this hydroxide is converted to carbonate, the percentage of coal feed converted to carbonate was calculated as follows:

$$
\% \text { coal converted to carbonate }=\frac{0.12859 \times 92.42 \times \text { carbonate formed } \times r}{1.2783 \times \mathrm{KOH}+\mathrm{NaOH} \text { feed }}
$$

where $r$ is the caustic/coal feed ratio. For $100 \% \mathrm{NaOH}$ feed, one pound of $\mathrm{NaOH}$ reacts with $0.15015 \mathrm{lb}$ of coal (or 100 pounds of $\mathrm{NaOH}$ with $15.015 \mathrm{lbs}$ of coal), and the percentage of coal feed converted to carbonate was calculated as follows: 
$\%$ coal converted to carbonate $=\frac{0.15015 \times 92.42 \times \text { carbonate formed } \times r}{1.3250 \times \mathrm{NaOH} \text { feed }}$

In Table 4-11, data on carbonate formation are presented on a run-by-run basis, in terms of fraction of caustic converted to carbonate. These data can also be readily translated to fraction of coal or coal carbon converted to carbonate, since the caustic/coal ratio for each run is known. No carbonate formation data was collected for Run 10 . Run 11, the only fully integrated test, is discussed separately in Section 4.5.1.

Based on the carbonate formation data for the 46 runs through Run 9 (excluding shakedown data) presented in Table 4-11, the average conversion of caustic to carbonate is $17.57 \%$. This corresponds to an average conversion of $4.18 \%$ of the feed coal to carbonate. (For those runs that had more than one carbonate formation data point, the data points were averaged for the run and this average run value was used in the calculation for the average conversion of caustic to carbonate for the 46 runs.) For shakedown, the average conversion of caustic to carbonate is $5.03 \%$ and of the feed coal to carbonate is $1.20 \%$. In analyzing the carbonate formation data, the effects of four process parameters were investigated: caustic/coal ratio, kiln temperature, coal type, and caustic type. The effect of residence time on carbonate formation was not assessed because almost all the data were collected at a kiln residence time of two hours. Carbonates in the MCL product were measured either using the kiln discharge (KD) product, or by analysis of the carbonates and caustic in the spent caustic solution (VF2037 or VP2023). The kiln discharge carbonate data were compared with the spent caustic carbonate data to determine whether the two data sets are similar, or whether additional carbonate is generated in the wash section.

\subsubsection{Carbonate Formation During Run 11}

Run 11 was the only run in which a continuous recycle of regenerated caustic to the kiln was maintained for most of the test (Run 9B had a total of -8 hours of operation with regenerated caustic recycled to the kiln). Fresh caustic was fed to the kiln during the first 24 hours of Run 11 to build caustic inventory in the system, and thereafter, fresh caustic was fed only sporadically and for short periods of time when the inventory of 
TABLE 4-11. CARBONATE FORMATION FOR S1:AKEDOWN THROUGH RUN 9

Coal KOH/ Caustic/ Wall Sample Frac of Caustic to Carbonate

Run Iype ${ }^{\star \star} \underline{\mathrm{NaOH}} \underline{\mathrm{Coal}}$ Temp. ${ }^{\circ} \mathrm{C}$ Iype Mean Std Dev. Data Pts

\begin{tabular}{|c|c|c|c|c|c|c|c|}
\hline SHDW & PITT 8 & 1 & 1.0 & 340 & $\begin{array}{c}\text { KD } \\
\text { VP2086 }\end{array}$ & $\begin{array}{l}0.0501 \\
0.0505\end{array}$ & $\begin{array}{l}0.0402 \\
0.0154\end{array}$ \\
\hline $1 B$ & PITT 8 & 1 & 1.0 & 390 & $\begin{array}{c}K D \\
\text { VP2023 } \\
K D+V P\end{array}$ & $\begin{array}{l}0.0416 \\
0.197 \\
0.119\end{array}$ & $\begin{array}{l}0.0264 \\
0.0271 \\
0.0885\end{array}$ \\
\hline $1 C$ & PITT 8 & 1 & 1.5 & 410 & $\begin{array}{c}\text { KD } \\
\text { VP2023 }\end{array}$ & $\begin{array}{l}0.224 \\
0.216\end{array}$ & - \\
\hline $1 D$ & PITT 8 & 1 & 2.0 & 410 & $\begin{array}{c}\mathrm{KD}+\mathrm{VP} \\
\mathrm{KD}\end{array}$ & $\begin{array}{l}0.220 \\
0.0943\end{array}$ & $\begin{array}{l}0.0059 \\
0.0699\end{array}$ \\
\hline $\begin{array}{l}2 \mathrm{~A} \\
2 \mathrm{~B} \\
2 \mathrm{C} \\
2 \mathrm{D} \\
2 \mathrm{E}\end{array}$ & $\begin{array}{l}\text { PITT } 8 \\
\text { PITT } 8 \\
\text { PITT } 8 \\
\text { KENT } 9 \\
\text { KENT } 9\end{array}$ & $\begin{array}{l}1 \\
1 \\
1 \\
1 \\
1\end{array}$ & $\begin{array}{l}2.0 \\
2.0 \\
2.0 \\
2.0 \\
2.0\end{array}$ & $\begin{array}{l}390 \\
390 \\
427 \\
427 \\
438\end{array}$ & $\begin{array}{c}\text { KD } \\
\text { VF2037 } \\
\text { VF2037 } \\
\text { VF2037 } \\
\text { VF2037 }\end{array}$ & \begin{tabular}{l}
\multicolumn{1}{c}{0} \\
0.0844 \\
0.181 \\
0.203 \\
0.228
\end{tabular} & $\begin{array}{l}0 . \\
0.0257 \\
0.0274 \\
0.0183 \\
0.0146\end{array}$ \\
\hline $\begin{array}{l}3 A 1 \\
3 A 2 \\
3 A 3 \\
3 B 3 \\
3 B 2 \\
3 B 1\end{array}$ & $\begin{array}{l}\text { PITT } 8 \\
\text { PITT } 8 \\
\text { PITT } 8 \\
\text { PITT } 8 \\
\text { PITT } 8 \\
\text { PITT } 8\end{array}$ & $\begin{array}{l}1 \\
1 \\
1 \\
1 \\
1 \\
1\end{array}$ & $\begin{array}{l}1.0 \\
1.0 \\
1.0 \\
2.0 \\
2.0 \\
2.0\end{array}$ & $\begin{array}{l}427 \\
427 \\
427 \\
432 \\
438 \\
438\end{array}$ & $\begin{array}{l}K D \\
K D \\
K D \\
K D \\
K D \\
K D \\
K D \\
\text { VF2037 } \\
\text { KD+VF }\end{array}$ & $\begin{array}{l}0.314 \\
0.279 \\
0.227 \\
0.267 \\
0.253 \\
0.342 \\
0.330 \\
0.332\end{array}$ & $\begin{array}{c}- \\
- \\
-\overline{0} \\
0 . \\
- \\
0.0361 \\
0.0337\end{array}$ \\
\hline $3 \mathrm{Cl}$ & PITT 8 & 1 & 3.0 & 438 & VF2037 & 0.387 & - \\
\hline $4 \mathrm{Cl}$ & PITT 8 & 0 & 2.0 & $421^{\star}$ & $\begin{array}{c}K D \\
V F 2037 \\
\text { KD+VF }\end{array}$ & $\begin{array}{l}0.139 \\
0.206 \\
0.161\end{array}$ & $\begin{array}{c}0.0396 \\
- \\
0.0475\end{array}$ \\
\hline $4 C 2$ & PITT 8 & 0 & 2.0 & $432^{\star}$ & $\begin{array}{c}K D \\
V F 2037 \\
K D+V F\end{array}$ & $\begin{array}{l}0.136 \\
0.265 \\
0.201\end{array}$ & $\begin{array}{l}0.0384 \\
0.0316 \\
0.0802\end{array}$ \\
\hline $\begin{array}{l}4 C 3 \\
4 C 4\end{array}$ & $\begin{array}{l}\text { PITT } 8 \\
\text { PITT } 8\end{array}$ & $\begin{array}{l}0 \\
0\end{array}$ & $\begin{array}{l}2.0 \\
2.0\end{array}$ & $\begin{array}{l}449 * \\
336^{*}\end{array}$ & $\begin{array}{c}\text { KD } \\
\text { KD } \\
\text { VF2037 }\end{array}$ & $\begin{array}{l}0.167 \\
0.105 \\
0.419\end{array}$ & - \\
\hline & & 0 & & & $\begin{array}{c}\mathrm{KD}+\mathrm{VF} \\
\mathrm{KD}\end{array}$ & 0.262 & 0.222 \\
\hline $\begin{array}{l}4 C 6 \\
4 C 7\end{array}$ & $\begin{array}{l}\text { PITT } 8 \\
\text { PITT } 8\end{array}$ & $\begin{array}{l}0 \\
0\end{array}$ & $\begin{array}{l}2.0 \\
2.0\end{array}$ & $\begin{array}{l}293^{\star} \\
293^{\star}\end{array}$ & $\begin{array}{l}\mathrm{KD} \\
\mathrm{KD} \\
\mathrm{KD}\end{array}$ & $\begin{array}{l}0.162 \\
0.102\end{array}$ & 5 \\
\hline
\end{tabular}

* These represent the wall temperatures in Zone 1 of the rotary kiln. For Runs $4 C 1,4 C 2$ and $4 C 3$, the $k i l n$ wall temperatures in Zones 2 to 4 were all $410^{\circ} \mathrm{C}$. For Runs $4 C 4,4 C 5,4 C 6$ and $4 C 7$, the $k j l n$ wall temperatures in the other 3 zones were: Zone $2-449^{\circ} \mathrm{C}(4 \mathrm{C} 4), 341^{\circ} \mathrm{C}(4 \mathrm{C} 5,4 \mathrm{C} 6,4 \mathrm{C} 7)$; Zone 3 $-410^{\circ} \mathrm{C}(4 \mathrm{C} 4), 449^{\circ} \mathrm{C}(4 \mathrm{C} 5,4 \mathrm{C} 6,4 \mathrm{C} 7)$; Zone $4-410^{\circ} \mathrm{C}(4 \mathrm{C} 4), 410^{\circ} \mathrm{C}$ $(4 \mathrm{C} 5), 420^{\circ} \mathrm{C}(4 \mathrm{C} 6), 432^{\circ} \mathrm{C}(4 \mathrm{C} 7)$.

** Pittsburgh No. coal from Powhatan No. 6 mine (Powhatan Point, Ohio). 
TABLE 4-11. CARBONATE FORMATION FOR SHAKEDOWN THROUGH RUN 9 (CONTINUED)

\begin{tabular}{|c|c|c|c|c|c|c|c|c|}
\hline Run & $\begin{array}{l}\text { Coal } \\
\text { Iype }\end{array}$ & $\begin{array}{l}\mathrm{KOH} / \\
\mathrm{NaOH} \\
\end{array}$ & $\begin{array}{l}\text { Caustic/ } \\
\text { Coal }\end{array}$ & $\begin{array}{r}\text { Wall } \\
\text { Temp }{ }^{\circ} \mathrm{C}\end{array}$ & $\begin{array}{l}\text { Sample } \\
\text { Iype }\end{array}$ & $\frac{\text { Frac of }}{\text { Mean }}$ & $\begin{array}{l}\text { Caustic to } \\
\text { Std Dev. }\end{array}$ & $\frac{\text { Carbonat }}{\text { Data Pt }}$ \\
\hline B1 & PITT 8 & 0 & 2.0 & $421^{\star}$ & $\begin{array}{c}K D \\
\text { VP2023 } \\
\text { KD+ VP }\end{array}$ & $\begin{array}{l}0.151 \\
0.149 \\
0.150\end{array}$ & $\begin{array}{c}- \\
\overline{0} \overline{0}\end{array}$ & $\begin{array}{l}1 \\
1 \\
2\end{array}$ \\
\hline 5B3 & PITT 8 & 0 & 2.0 & $339 *$ & $\begin{array}{l}K D \\
K F 2037 \\
K D+V F\end{array}$ & $\begin{array}{l}0.125 \\
0.191 \\
0.151\end{array}$ & $\begin{array}{l}0.0298 \\
0.0621 \\
0.0519\end{array}$ & $\begin{array}{l}3 \\
3 \\
2 \\
5\end{array}$ \\
\hline $5 \mathrm{~B} 5$ & PITT 8 & 0 & 2.0 & 427 & $\begin{array}{c}K D \\
V F 2037 \\
K D+V F\end{array}$ & $\begin{array}{l}0.0993 \\
0.169 \\
0.122\end{array}$ & $\begin{array}{l}0.0139 \\
0.0411\end{array}$ & $\begin{array}{l}2 \\
1 \\
3\end{array}$ \\
\hline $5 C 4$ & KENT 9 & 0 & 2.0 & $333^{*}$ & $\begin{array}{l}K D \\
K F 2037 \\
V D+V F\end{array}$ & $\begin{array}{l}0.164 \\
0.208 \\
0.187\end{array}$ & $\begin{array}{l}0.0495 \\
0.141 \\
0.0900\end{array}$ & $\begin{array}{l}2 \\
2 \\
4\end{array}$ \\
\hline $\begin{array}{l}501 \\
5 D 2\end{array}$ & $\begin{array}{l}\text { KENT } 9 \\
\text { KENT } 9\end{array}$ & $\begin{array}{l}0 \\
0\end{array}$ & $\begin{array}{l}2.0 \\
2.0\end{array}$ & $\begin{array}{l}281^{*} \\
399\end{array}$ & $\begin{array}{l}\text { KD } \\
K D \\
V F 2037 \\
K D+V F\end{array}$ & $\begin{array}{l}0.146 \\
0.0918 \\
0.113 \\
0.102\end{array}$ & $\begin{array}{c}- \\
- \\
0.0149\end{array}$ & $\begin{array}{l}1 \\
1 \\
1 \\
2\end{array}$ \\
\hline $\begin{array}{l}6 \mathrm{~B} 1 \\
6 \mathrm{D} 1 \\
6 \mathrm{E} 1 \\
6 \mathrm{~F} 1\end{array}$ & $\begin{array}{l}\text { PITT } 8 \\
\text { PITT } 8 \\
\text { PITT } 8 \\
\text { PITT } 8\end{array}$ & $\begin{array}{l}0 \\
0 \\
1 \\
1\end{array}$ & $\begin{array}{l}1.5 \\
1.5 \\
1.0 \\
1.0\end{array}$ & $\begin{array}{l}427 \\
410 \\
427 \\
427\end{array}$ & $\begin{array}{l}\text { VP2023 } \\
K D \\
K D \\
K D\end{array}$ & $\begin{array}{l}0.179 \\
0.0835 \\
0.139 \\
0.192\end{array}$ & $\begin{array}{l}0.0526 \\
0.0575\end{array}$ & $\begin{array}{l}2 \\
1 \\
2 \\
1\end{array}$ \\
\hline $7 A$ & KENT 9 & 1 & 2.5 & 427 & $\begin{array}{c}K D \\
\text { VF2037 } \\
\text { KD+VF }\end{array}$ & $\begin{array}{l}0.144 \\
0.0166 \\
0.126\end{array}$ & $\begin{array}{l}0.0907 \\
0 . \overline{102}\end{array}$ & $\begin{array}{l}6 \\
1 \\
7\end{array}$ \\
\hline $\begin{array}{l}7 C \\
7 D \\
7 E\end{array}$ & $\begin{array}{l}\text { PITT } 8 \\
\text { PITT } 8 \\
\text { PITT } 8\end{array}$ & $\begin{array}{l}1 \\
1 \\
1\end{array}$ & $\begin{array}{l}3.0 \\
2.0 \\
2.0\end{array}$ & $\begin{array}{l}427 \\
427 \\
427\end{array}$ & $\begin{array}{l}K D \\
K D \\
K D\end{array}$ & $\begin{array}{l}0.112 \\
0.289 \\
0.0067\end{array}$ & - & $\begin{array}{l}1 \\
1 \\
1\end{array}$ \\
\hline
\end{tabular}

* These represent the wall temperatures in Zone 1 of the rotary kiln. For Run $5 \mathrm{~B} 1$, the $\mathrm{ki}$ in wall temperatures in Zones 2 to 4 were all $410^{\circ} \mathrm{C}$. For Run $5 \mathrm{~B} 3$, the $\mathrm{kiln}$ wall temperatures were: $449^{\circ} \mathrm{C}$ (Zone 2), $410^{\circ} \mathrm{C}$ (Zones 3 and 4 ). For Run $5 \mathrm{C} 4$, the kiln wall temperatures in Zones 2 to 4 were al1 $427^{\circ} \mathrm{C}$. For Run $5 \mathrm{Dl}$, the kiln wall temperatures were: $331^{\circ} \mathrm{C}$ (Zone 2), $427^{\circ} \mathrm{C}$ (Zones 3 and 4 ).

** Pittsburgh No. 8 coal from Powhatan No. 6 mine (Powhatan Point, Ohio). 
TABLE 4-11. CARBONATE FORMATION FOR SHAKEDOWN THROUGH RUN 9 (CONTINUED)

\begin{tabular}{|c|c|c|c|c|c|c|c|c|}
\hline & $\begin{array}{l}\text { Coal } \\
\text { Iype }\end{array}$ & $\begin{array}{l}\mathrm{KOH} / \\
\mathrm{NaOH}\end{array}$ & $\begin{array}{c}\text { Caustic/ } \\
\text { Coal }\end{array}$ & $\begin{array}{c}\text { Wall } \\
\text { Iemp }{ }^{\circ} \mathrm{C}\end{array}$ & $\begin{array}{l}\text { Sample } \\
\text { Iype }\end{array}$ & $\frac{\text { Frac of }}{\text { Mean }}$ & $\begin{array}{l}\text { Caustic to } \\
\text { Std Dev. }\end{array}$ & $\frac{\text { Carbonate }}{\text { Data Pts }}$ \\
\hline$B A$ & PITT 8 & 1 & 1.5 & 427 & $\begin{array}{c}\text { KD } \\
\text { VF2037 }\end{array}$ & $\begin{array}{l}0.141 \\
0.127\end{array}$ & $\begin{array}{l}0.0942 \\
0.0114\end{array}$ & $\begin{array}{l}3 \\
2 \\
5\end{array}$ \\
\hline $8 B$ & PITT 8* & 1 & 1.5 & 427 & $\begin{array}{l}K D+V P \\
K D \\
V F 2037 \\
K D+V F\end{array}$ & $\begin{array}{l}0.136 \\
0.154 \\
0.205 \\
0.163\end{array}$ & $\begin{array}{l}0.00 / 4 \\
0.0451 \\
0.0453\end{array}$ & $\begin{array}{l}5 \\
5 \\
1 \\
6\end{array}$ \\
\hline $8 C$ & PITT 8* & 0 & 1.5 & 427 & $\begin{array}{l}K U+V F \\
K D \\
V F 2037 \\
K D+V F\end{array}$ & $\begin{array}{l}0.161 \\
0.208 \\
0.180\end{array}$ & $\begin{array}{l}0.0180 \\
0.0788 \\
0.0486\end{array}$ & $\begin{array}{l}6 \\
3 \\
2 \\
5\end{array}$ \\
\hline $\begin{array}{l}8 D \\
8 E\end{array}$ & $\begin{array}{l}\text { PITT 8* } \\
\text { PITT 8* }\end{array}$ & $\begin{array}{l}0 \\
0\end{array}$ & $\begin{array}{l}1.5 \\
1.5\end{array}$ & $\begin{array}{l}442 \\
412\end{array}$ & $\begin{array}{c}\text { VF2037 } \\
\text { KD } \\
\text { VF2037 }\end{array}$ & $\begin{array}{l}0.218 \\
0.155 \\
0.151\end{array}$ & $0 . \overline{0} 167$ & $\begin{array}{l}1 \\
2 \\
1\end{array}$ \\
\hline $8 \mathrm{~F}$ & PITT 8 & 0 & 2.0 & 412 & $\begin{array}{l}K D+V F \\
K D \\
V F 2037 \\
K D+V F\end{array}$ & $\begin{array}{l}0.154 \\
0.126 \\
0.171 \\
0.156\end{array}$ & $\begin{array}{l}0.0121 \\
- \\
0.0157 \\
0.0279\end{array}$ & $\begin{array}{l}3 \\
1 \\
2 \\
3\end{array}$ \\
\hline $9 A$ & PITT 8 & 1 & 2.5 & 427 & $\begin{array}{c}\text { KD } \\
\text { VF2037 } \\
\text { KD+VF }\end{array}$ & $\begin{array}{l}0.221 \\
0.174 \\
0.212\end{array}$ & $\begin{array}{l}0.141 \\
- \\
0.124\end{array}$ & $\begin{array}{l}4 \\
1 \\
5\end{array}$ \\
\hline $\begin{array}{l}9 A 1 \\
9 B\end{array}$ & $\begin{array}{l}\text { PITT } 8 \\
\text { PITT } 8\end{array}$ & $\begin{array}{l}1 \\
1\end{array}$ & $\begin{array}{l}2.5 \\
2.5\end{array}$ & $\begin{array}{l}438 \\
438\end{array}$ & $\begin{array}{l}\text { KD } \\
\text { KD } \\
\text { VF2037 }\end{array}$ & $\begin{array}{l}0.167 \\
0.170 \\
0.299\end{array}$ & $\begin{array}{l}0.0679 \\
0.0790 \\
-\end{array}$ & $\begin{array}{l}2 \\
2\end{array}$ \\
\hline $9 C$ & PITT 8 & 0 & 2.5 & 438 & $\begin{array}{c}K D+V F \\
K D \\
V F 2037 \\
K D+V F\end{array}$ & $\begin{array}{l}0.213 \\
0.124 \\
0.251 \\
0.166\end{array}$ & $\begin{array}{l}0.0931 \\
0.0731 \\
0.174 \\
0.116\end{array}$ & $\begin{array}{l}4 \\
2 \\
6\end{array}$ \\
\hline
\end{tabular}

* For Runs $8 \mathrm{~B}, 8 \mathrm{C}, 8 \mathrm{D}$ and $8 \mathrm{E}, 6$ mesh coal was used in the coal feed to the rotary kiln. The coal feed for all the other runs was 14 mesh coal. Also, recycled caustic was used in Runs 70 and $9 B$.

** Pittsburgh No. 8 coal from Powhatan No. 6 mine (Powhatan Point, Ohio). 
regenerated caustic flake available for recycle to the kiln ran low. The method of calculating carbonate make in all the runs conducted up to Run 11 was to compute the carbonate in the exit material from the kiln based on either kiln discharge solids or water wash train filtrate (VP2023 or VF2037) sample analysis and subtract from this the amount of carbonate being fed to the kiln with the fresh caustic bead. This method of calculation (which assumes that the carbonate inventory within the kiln is relatively constant) is applicable in a "once-through" (no recycle of caustic to the kiln) system. A different approach to the calculation of carbonate formation needs to be taken for a system in which regenerated caustic (with a significant carbonate content) is recycled to the kiln.

Run 11 was conducted at a fairly constant kiln condition (Pittsburgh No. 8, Blacksville coà1, 2.5:1 caustic ( $\mathrm{NaOH}$ only):coal, constant feed rate) for the entire week of operations. The kiln skin temperature was varied only slightly, from $424^{\circ} \mathrm{C}$ for the first 56 hours of the run to $434^{\circ} \mathrm{C}$ for the final 88 hours of the run. Since the kiln condition remained "constant" during the run, the approach to determining carbonate formation was to calculate the total carbonate make for the week based on "average" stream analyses and known stream flows. This approach, as opposed to the calculation of carbonate production at points of time within the test based on single sample stream analysis, practically eliminates the effect of changing plant carbonate inventory on the carbonate formation calculation. This approach is very similar to that used to calculate humics production during this run (see Section 4.4).

Table 4-12 summarizes the carbonate analysis for water wash train filtrate (regeneration feed) and regenerated alkali (evaporator feed) samples taken during Run 11. The carbonate material balance calcuiation is shown schematically in Figure 4-18. The water wash train aqueous caustic effluent (which is assumed to contain all of the carbonate in the kiln discharge solids entering the wash train) had an average carbonate content of $2.8 \%$. This filtrate flow was calculated to average -110 ibs/hr for a period of 137 hours. Total carbonate in this filtrate stream was calculated to be 422 pounds. Due to occasional kiln upsets, approximately $21 \%$ of the time the 


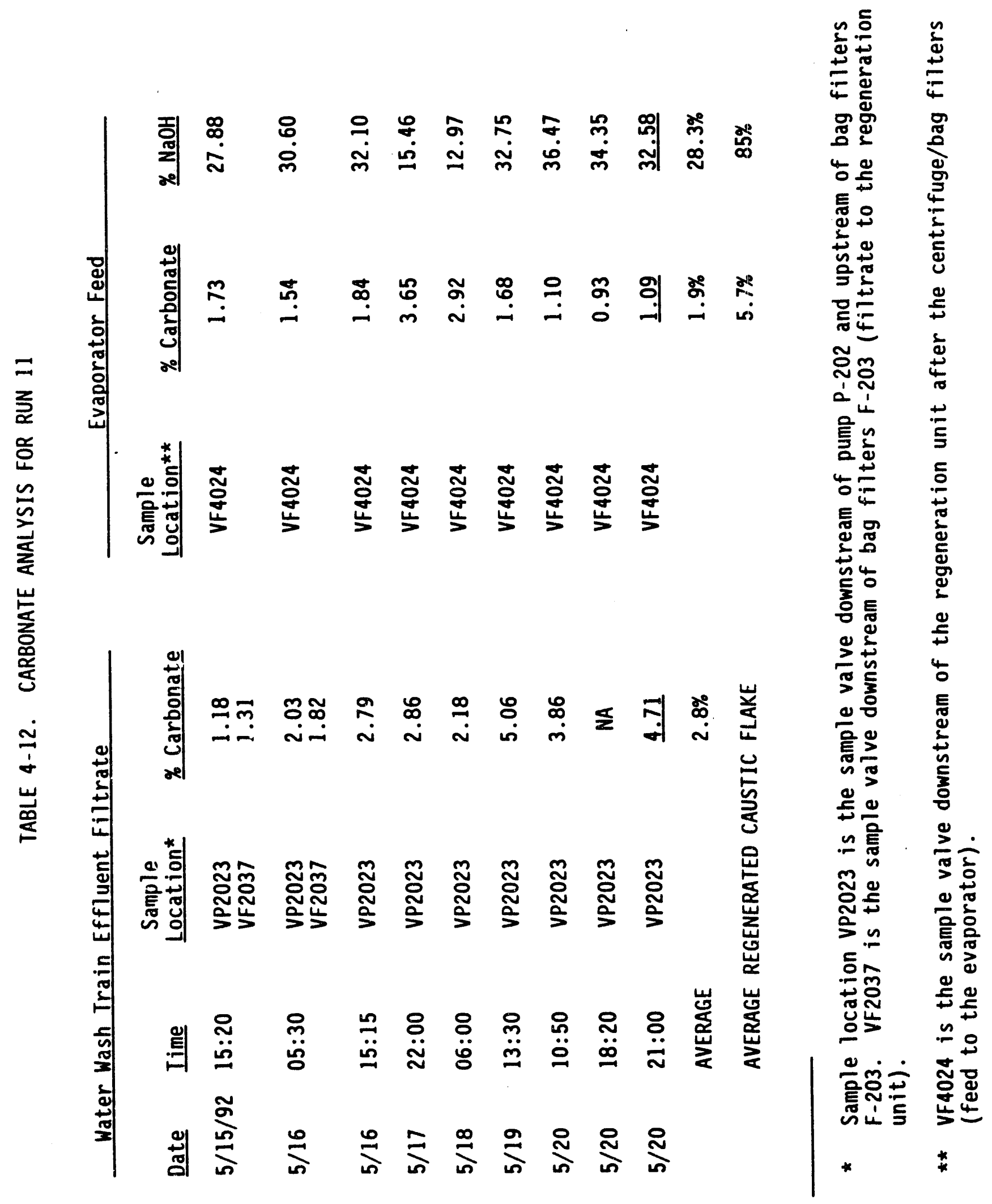




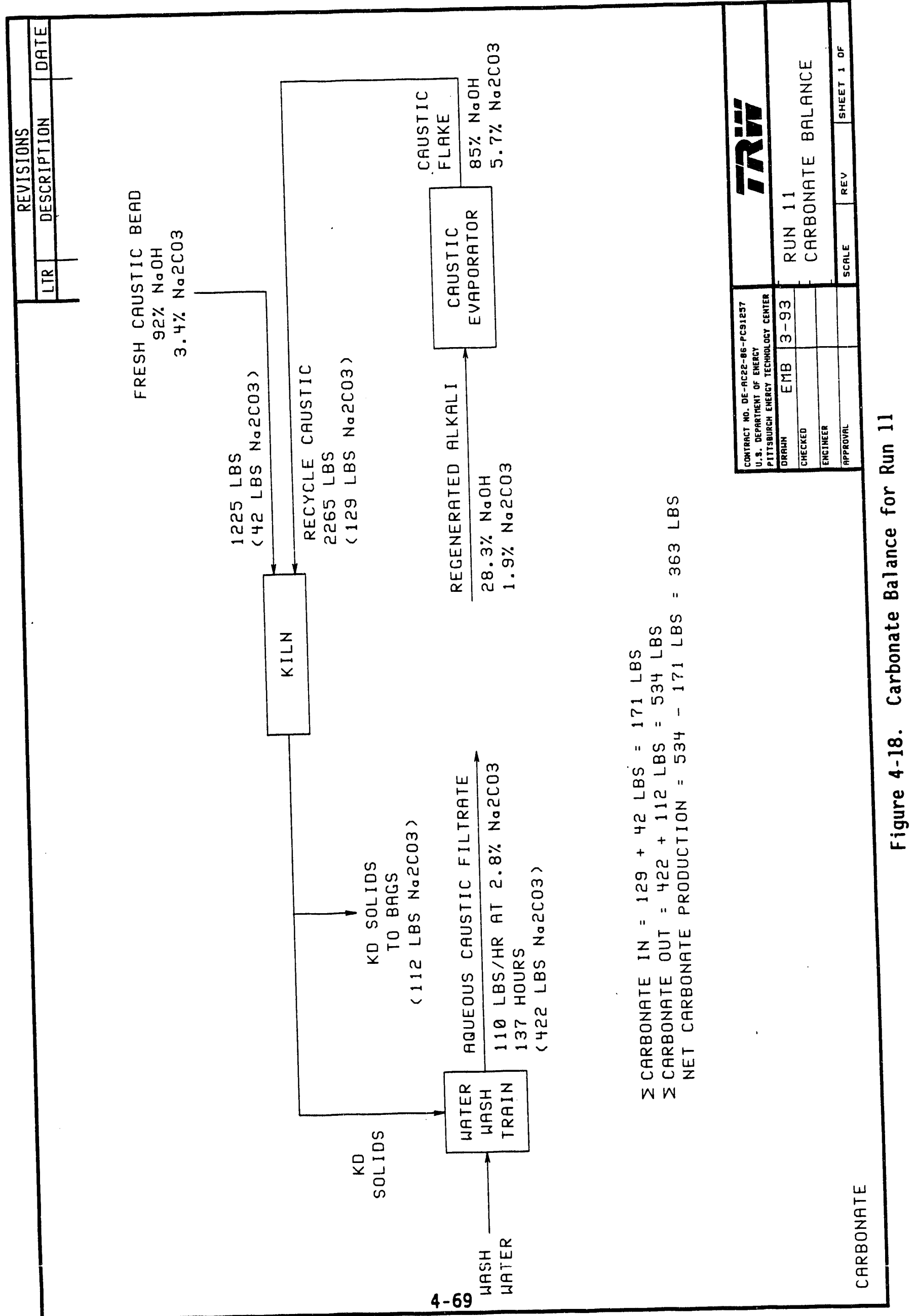


kiln discharge solids were collected in plastic bags rather than allowing the solids to enter the water wash train. (However, during this time, the wash water filtrate continued to flow to regeneration.) The amount of carbonate in the kiln discharge material bagged, which was assumed to have the same carbonate content as the solids entering the wash train, was calculated to be 112 pounds [422 lbs $\times 0.21 \div(1-0.21)]$. Therefore, the total carbonate exiting the kiln during Run 11 was 534 pounds (422 lbs +112 lbs).

The average regenerated alkali fed to the caustic evaporator contained $1.9 \%$ carbonate and $28.3 \% \mathrm{NaOH}$ (Table $4-12$ ). During evaporation there was no evidence of carbonate precipitation (carbonate precipitate would have rapidly scaled the evaporator heat transfer surface). The average $\mathrm{NaOH}$ content of the evaporated regenerated flake produced was $-85 \%$. Since the carbonate did not separate from the caustic during evaporation, the carbonate concentration was assumed to increase from an average value of $1.9 \%$ in the evaporator feed to $5.7 \%$ in the caustic flake. A total of 2,265 pounds of regenerated caustic flake (carbonate content $05.7 \%=129$ lbs) was fed to the kiln during Run 11. In addition, a total of 1,225 pounds of fresh caustic bead (carbonate content $3.4 \%=42 \mathrm{lbs}$ ) was fed to the kiln. Therefore, the total carbonate entering the kiln during Run 11 was 171 pounds. The net production of carbonate during Run 11 therefore was 363 pounds [carbonate from kiln (534 lbs) minus carbonate to kiln (171 lbs)].

Therefore, the total caustic converted to carbonate during Run 11 was 274 pounds $(80+106 \times 363 \mathrm{lbs})$, and coal carbon converted to carbonate was 41 pounds $(12+106 \times 363 \mathrm{lbs})$. Based on a fresh caustic bead $\mathrm{NaOH}$ content of $92 \%$ and a recycled regenerated caustic flake $\mathrm{NaOH}$ content of $85 \%$, the caustic conversion to carbonate during Run 11 was $9.0 \%$ calculated as follows:

$$
\frac{274 \text { lbs }}{(2295 \text { Tbs } \times 0.85)+(1225 \text { bs } \times 0.92)} \times 100=9.0 \%
$$

Based on a total of 1,193 pounds of MAF coal fed to the kiln during Run 11, coal conversion to carbonate was $3.4 \%$ (41 lbs +1193 lbs $\times 100)$. 


\subsubsection{Effect of Caustic/Coal Ratio on Carbonate Formation}

The effect of caustic/coal ratio on carbonate formation is evaluated by plotting the percentage of coal converted to carbonate at different caustic/coal ratios, at a kiln wall temperature of $427^{\circ} \mathrm{C}$. The fraction of coal feed converted to carbonate was computed as the ratio of the weight of carbonate carbon to the weight of coal feed. As shown in Figure 4-19, the general trend is that a higher percentage of the coal feed is converted to carbonate as the caustic/coal ratio increases. At a caustic/coal ratio of 1.0, the conversion of coal feed to carbonate averages $2.73 \%$ and ranges from $1.65 \%$ to $3.73 \%$. By comparison, at a caustic/coal ratio of 2.0 or greater, the conversion of coal feed to carbonate averages $3.86 \%$ (including Run 11) and exceeds $3.70 \%$ in 5 of the 7 runs. The slightly higher conversion of coal carbon to carbonates at the higher ratio of caustic/coal is probably due to the increased accessibility of coal surface to molten caustic.

\subsubsection{Effect of Kiln Temperature on Carbonate Formation}

The effect of operating temperature on carbonate formation was examined by plotting the percentage of coal and the percentage of caustic converted to carbonate versus the kiln wall temperature, at a caustic/coal ratio of 2.0 and greater. These plots, as presented in Figures 4-20 and 4-21, indicate a definite trend of increasing carbonate formation with increasing kiln operating temperature. Run 11 had a relatively low conversion of coal and caustic to carbonate despite a rather high kiln wall temperature. The actual bulk temperature in the kiln varied from 15 to $110^{\circ} \mathrm{C}$ below the wall temperature during this run. Thus the effective process temperature during Run 11 may have been much lower than during Run 9 in which the average delta temperature between the kiln wall and the internal temperature only varied between 19 and $32^{\wedge} \mathrm{C}$. This may explain the relatively low carbonate formation during Run 11.

At kiln wall temperatures of $410^{\circ} \mathrm{C}$ or below, carbonate formation is relatively low. For the four runs (Runs $1 D, 2 A, 2 B$ and 5D2) at these temperatures, the average conversion of caustic to carbonate is $7.03 \%$. The corresponding average conversion of coal to carbonate is $1.78 \%$. 


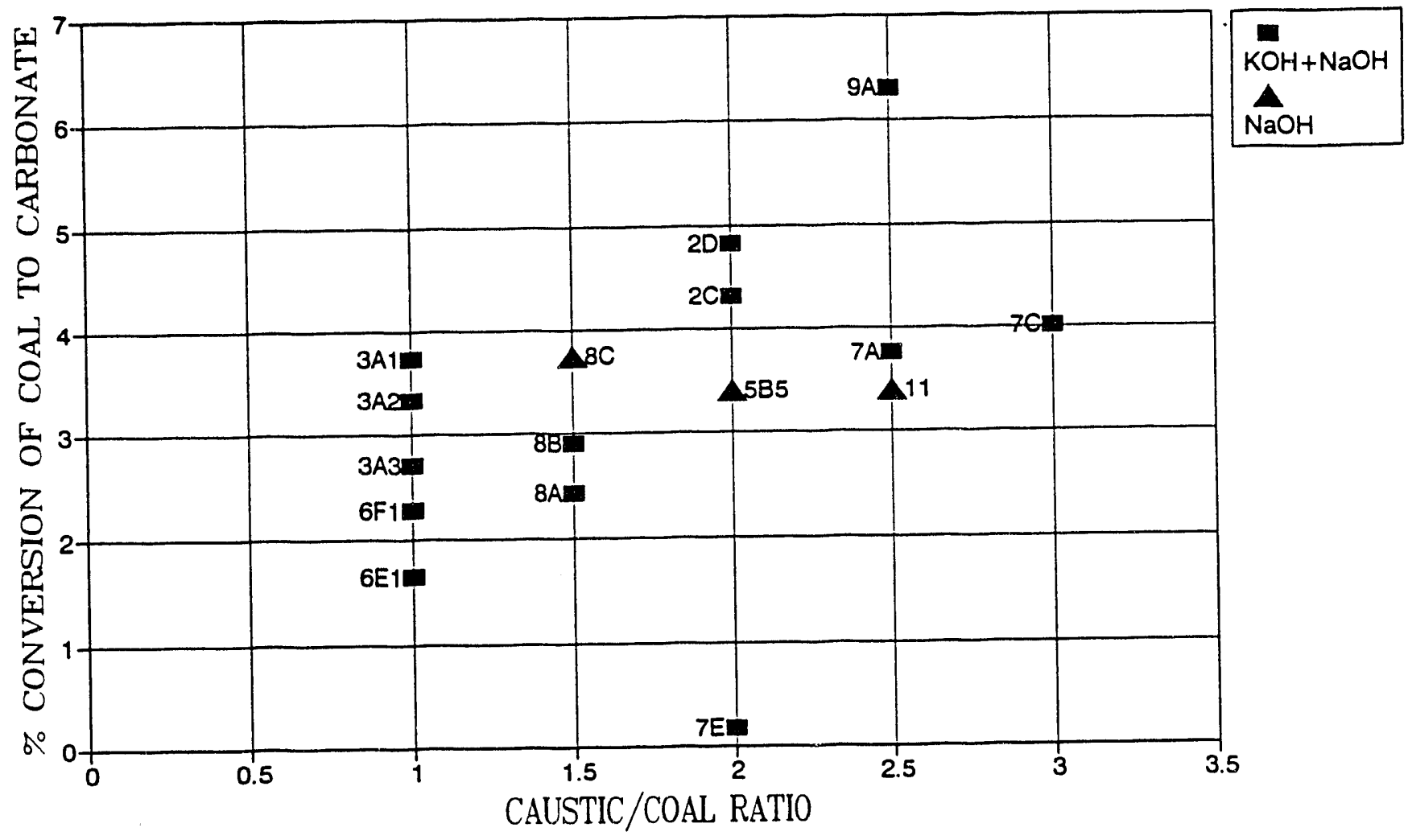

Figure 4-19. Effect of Caustic/Coal Ratio on Carbonate
Formation at $427^{\circ} \mathrm{C} \mathrm{Kiln} \mathrm{Wall} \mathrm{Temperature}$ 


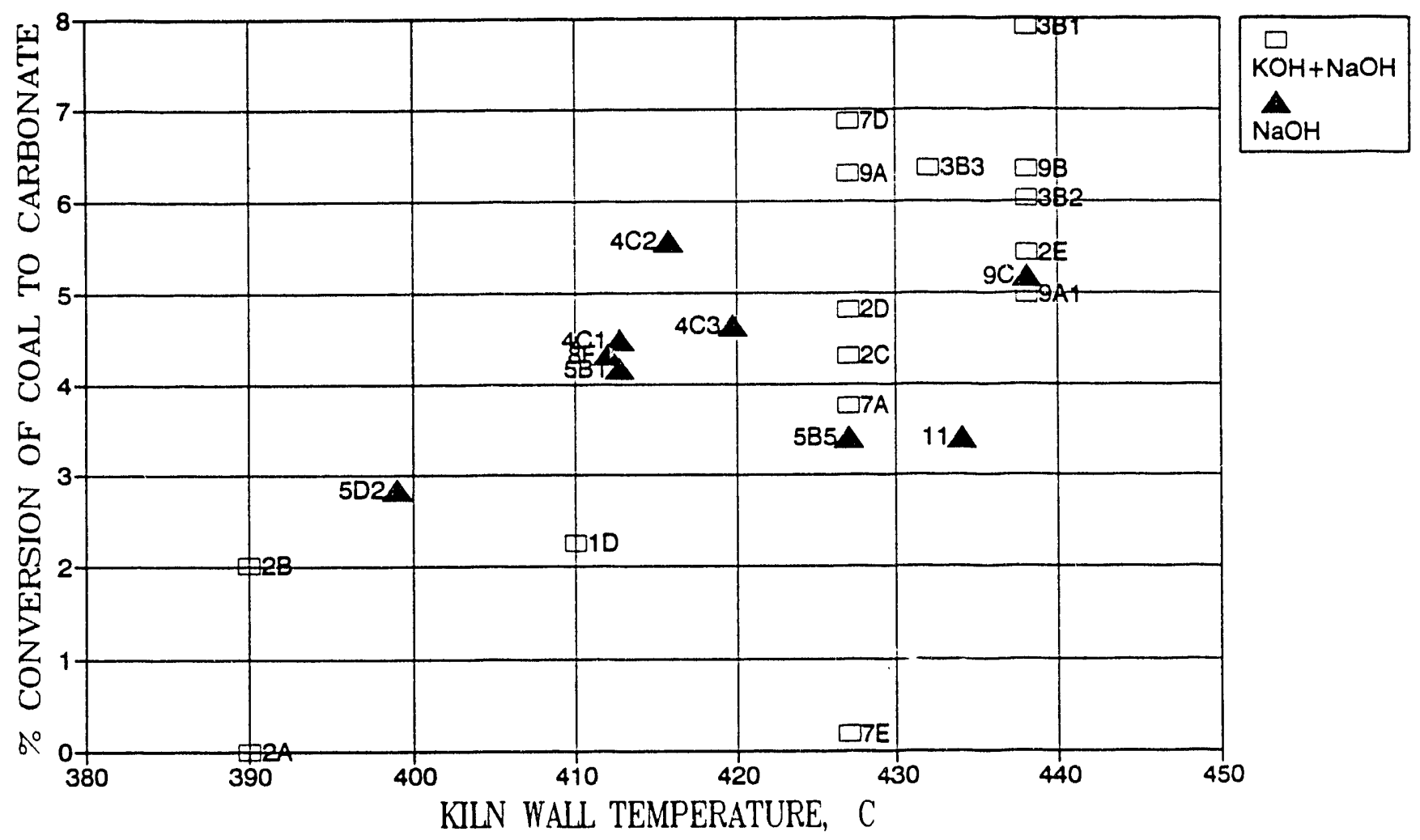

Figure 4-20 Effect of Kiln Temperature on Conversion of Coal to Carbonate at Caustic/Coal $\geq 2.0$ 


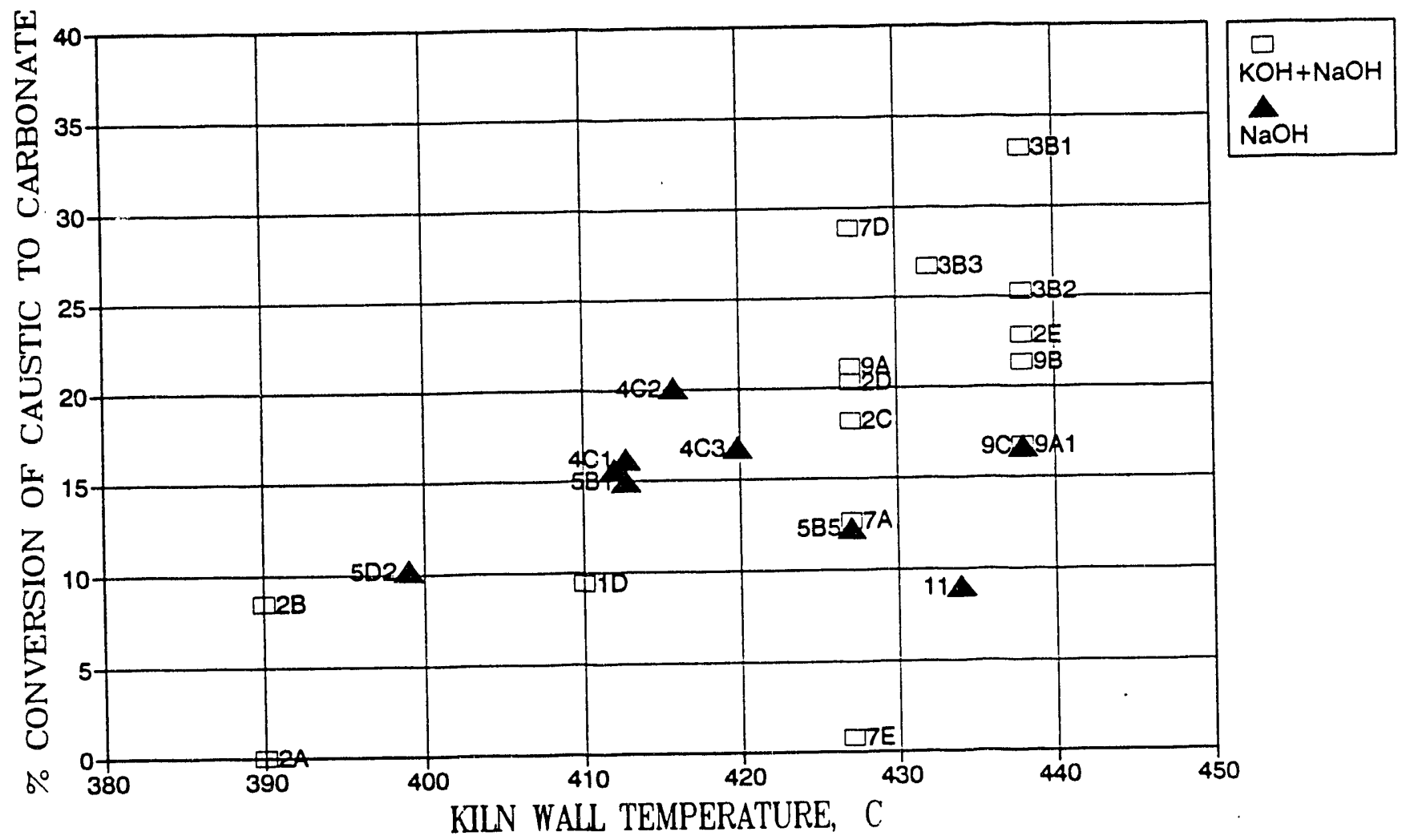

Figure 4-21. Effect of Kiln Temperature on Conversion of Caustic to Carbonate at Caustic/Coal $\geq 2.0$ 
An important observation is that the temperature dependence of carbonate formation is much less pronounced when sodium hydroxide instead of mixed caustic was used. At kiln wall temperatures between $412^{\circ} \mathrm{C}$ and $438^{\circ} \mathrm{C}$, Figures 4-20 and 4-21 indicate that carbonate formation is not dependent on kiln wall temperature for sodium hydroxide.

For shakedown, the average conversion of caustic to carbonate is $5.03 \%$ which corresponds to a feed coal to carbonate conversion of $1.20 \%$. The low conversions of caustic and coal carbon to carbonate are due to the relatively low kiln wall operating temperature $\left(340^{\circ} \mathrm{C}\right)$ during shakedown. In addition, a low caustic/coal ratio of 1.0 was used during shakedown which would also lead to low conversion to carbonates.

\subsubsection{Effect of Coal Type on Carbonate Formation}

The data presented in Table 4-11 include those from 6 Kentucky No. 9 coal runs and 41 high sulfur Pittsburgh No. 8 (Powhatan) coal runs. Data for Run 11 which utilized a low sulfur Pittsburgh No. 8 (Blacksville) coal were presented in Table 4-12 and Figure 4-18. The average conversion of Kentucky coal to carbonate is $4.35 \%$, with a standard deviation of $0.98 \%$. By comparison, the average conversion of high sulfur Pittsburgh (Powhatan) coal to carbonate is $4.16 \%$, with a standard deviation of $2.41 \%$ (excluding shakedown data). The difference between these average carbonate conversions for the two coal types is statistically insignificant. The average conversion of low sulfur Pittsburgh (Blacksville) coal to carbonate is $3.4 \%$.

The effect of coal type can also be evaluated by comparing carbonate formation in runs with similar operating conditions except coal type: Run $2 C$ versus Run 2D, and Run $3 B 1$ versus Run $2 E$. Data from these runs are summarized in the following table:

\begin{tabular}{|c|c|c|c|c|c|c|c|}
\hline Run & $\begin{array}{l}\text { Coal } \\
\text { Iype }\end{array}$ & $\begin{array}{l}\mathrm{KOH} / \\
\mathrm{NaOH}\end{array}$ & $\begin{array}{c}\text { Caustic/ } \\
\text { Coal }\end{array}$ & $\begin{array}{l}\text { Wall } \\
\text { Temp, }{ }^{\circ} \mathrm{C} \\
\end{array}$ & $\begin{array}{l}\text { Percent } \\
\text { Mean }\end{array}$ & $\begin{array}{l}\text { of Coal to } \\
\text { Std Dev. }\end{array}$ & $\begin{array}{c}\text { Carbonate } \\
\text { Data Pts }\end{array}$ \\
\hline $\begin{array}{l}2 C \\
2 D\end{array}$ & $\begin{array}{l}\text { PITT } 8 \\
\text { KENT } 9\end{array}$ & $\begin{array}{l}1 \\
1\end{array}$ & $\begin{array}{l}2.0 \\
2.0\end{array}$ & $\begin{array}{l}427 \\
427\end{array}$ & $\begin{array}{l}4.31 \\
4.82\end{array}$ & $\begin{array}{l}0.652 \\
0.435\end{array}$ & $\begin{array}{l}3 \\
2\end{array}$ \\
\hline $\begin{array}{l}3 \mathrm{Bl} \\
2 \mathrm{E}\end{array}$ & $\begin{array}{l}\text { PITT } 8 \\
\text { KENT } 9\end{array}$ & $\begin{array}{l}1 \\
1\end{array}$ & $\begin{array}{l}2.0 \\
2.0\end{array}$ & $\begin{array}{l}438 \\
438\end{array}$ & $\begin{array}{l}7.90 \\
5.43\end{array}$ & $\begin{array}{l}0.802 \\
0.347\end{array}$ & $\begin{array}{l}6 \\
2\end{array}$ \\
\hline
\end{tabular}


These data indicate that at a $427^{\circ} \mathrm{C} \mathrm{ki} / \mathrm{n}$ wall temperature, the average conversion to carbonate is essentially the same for Pittsburgh (Powhatan) and Kentucky coals. At a $438^{\circ} \mathrm{C}$ kiln wall temperature, data for Runs $3 B 1$ and $2 E$ show that the average conversion to carbonate may be lower for Kentucky coal than for Pittsburgh (Powhatan) coal. However, there is only one data set to support this observation, and the overall data base still indicates small differences in carbonate formation between the Pittsburgh (Powhatan) and Kentucky coals.

\subsubsection{Effect of Caustic Type on Carbonate Formation}

The average coal conversion to carbonate is $4.22 \%$ for the 26 mixed caustic runs, with a standard deviation of $2.84 \%$ (excluding shakedown data). For the 21 sodium hydroxide runs, the average coal conversion to carbonate is $4.06 \%$ (including Run 11 ), with a standard deviation of $1.22 \%$. The difference between these average conversions for the two caustic types is statistically insignificant. A more detailed analysis indicates that the effect of caustic type on carbonate formation appears to be temperature dependent. Examination of Figures 4-20 and 4-21 shows that at kiln wall temperatures of $420^{\circ} \mathrm{C}$ and below, the use of sodium hydroxide results in higher conversion to carbonate than the use of mixed caustic. However, at temperatures above $420^{\circ} \mathrm{C}$, lower conversion to carbonate is obtained with sodium hydroxide as compared to mixed caustic. This is due to the increased carbonate formation at high kiln temperatures for mixed caustic, and the relative insensitivity of carbonate formation to kiln temperatures for sodium hydroxide. Therefore, the use of sodium hydroxide instead of mixed caustic may result in lower carbonate formation at high kiln temperatures.

The effect of caustic type can also be assessed by comparing carbonate formation data from 4 pairs of runs: $1 C / 6 \mathrm{D} 1,2 \mathrm{C} / 5 \mathrm{~B} 5,8 \mathrm{~B} / 8 \mathrm{C}$, and $9 \mathrm{Al} / 9 \mathrm{C}$. Each pair contains two runs with Pittsburgh No. 8 (Powhatan) coal at the same caustic/coal ratio and kiln conditions, except one run used mixed caustic and the other run used sodium hydroxide. Data from these runs are summarized in the following table: 


\begin{tabular}{|c|c|c|c|c|c|c|}
\hline Run & $\begin{array}{l}\mathrm{KOH} / \\
\mathrm{NaOH}\end{array}$ & $\begin{array}{c}\text { Caustic/ } \\
\text { Coal }\end{array}$ & $\begin{array}{l}\text { Wal1 } \\
\text { Temp } \\
\end{array}$ & $\begin{array}{l}\text { Percent } \\
\text { Mean }\end{array}$ & $\begin{array}{l}\text { of Coal to } \\
\text { Sid Dev. }\end{array}$ & $\begin{array}{c}\text { Carbonate } \\
\text { Data Pts }\end{array}$ \\
\hline $\begin{array}{l}1 C \\
6 D 1\end{array}$ & $\begin{array}{l}1 \\
0\end{array}$ & $\begin{array}{l}1.5 \\
1.5\end{array}$ & $\begin{array}{l}410 \\
410\end{array}$ & $\begin{array}{l}3.93 \\
1.74\end{array}$ & $\begin{array}{c}0.105 \\
-\end{array}$ & $\begin{array}{l}2 \\
1\end{array}$ \\
\hline $\begin{array}{l}2 C \\
5 B 5\end{array}$ & $\begin{array}{l}1 \\
0\end{array}$ & $\begin{array}{l}2.0 \\
2.0\end{array}$ & $\begin{array}{l}427 \\
427\end{array}$ & $\begin{array}{l}4.31 \\
3.40\end{array}$ & $\begin{array}{l}0.652 \\
1.15\end{array}$ & $\begin{array}{l}3 \\
3\end{array}$ \\
\hline $\begin{array}{l}8 B \\
8 C\end{array}$ & $\begin{array}{l}1 \\
0\end{array}$ & $\begin{array}{l}1.5 \\
1.5\end{array}$ & $\begin{array}{l}427 \\
427\end{array}$ & $\begin{array}{l}2.90 \\
3.74\end{array}$ & $\begin{array}{l}0.806 \\
1.10\end{array}$ & $\begin{array}{l}6 \\
5\end{array}$ \\
\hline $\begin{array}{l}9 A 1 \\
9 C\end{array}$ & $\begin{array}{l}1 \\
0\end{array}$ & $\begin{array}{l}2.5 \\
2.5\end{array}$ & $\begin{array}{l}438 \\
438\end{array}$ & $\begin{array}{l}4.97 \\
5.18\end{array}$ & $\begin{array}{l}2.02 \\
3.62\end{array}$ & $\begin{array}{l}2 \\
6\end{array}$ \\
\hline
\end{tabular}

The data for Runs $2 \mathrm{C} / 5 \mathrm{~B} 5,8 \mathrm{~B} / 8 \mathrm{C}$ and $9 \mathrm{Al} / 9 \mathrm{C}$ show statistically the same coal conversions to carbonate for either type of caustic. Run $1 C / 6 D 1$ data show lower coal conversions to carbonate for sodium hydroxide than for mixed caustic. Comparison of the Run $1 C$ and Run $6 D 1$ data, however, must take into account the limited number of data points. Run 1D, operated at the same conditions as Run $1 C$ except with a caustic/coal ratio of 2.0 , resulted in an average conversion of $2.24 \%$ coal to carbonate, based on the analysis of 4 kiln discharge samples. This is essentially the same average coal conversion to carbonate as in 6D1. Therefore, these comparisons show no significant difference in carbonate formation between the use of mixed caustic and sodium hydroxide.

\subsubsection{Kiln Discharge Versus Spent Caustic Data}

Twenty-one sets of carbonate conversion data are compared in Figure 4-22, with each set representing kiln discharge (KD) coal versus spent caustic data from the same run. The comparisons show that in 7 of the 21 runs, carbonate conversions are found to be approximately the same in the KD and spent caustic samples. In 12 of the 21 runs, higher carbonate conversions are obtained in the spent caustic than in the KD samples. There are only 2 runs for which carbonate conversions are higher in the KD samples than in the spent caustic samples. Therefore, it appears that at least in some of the runs, additional conversion of caustic to carbonate had taken place in the wash section of the integrated MCL circuit. 


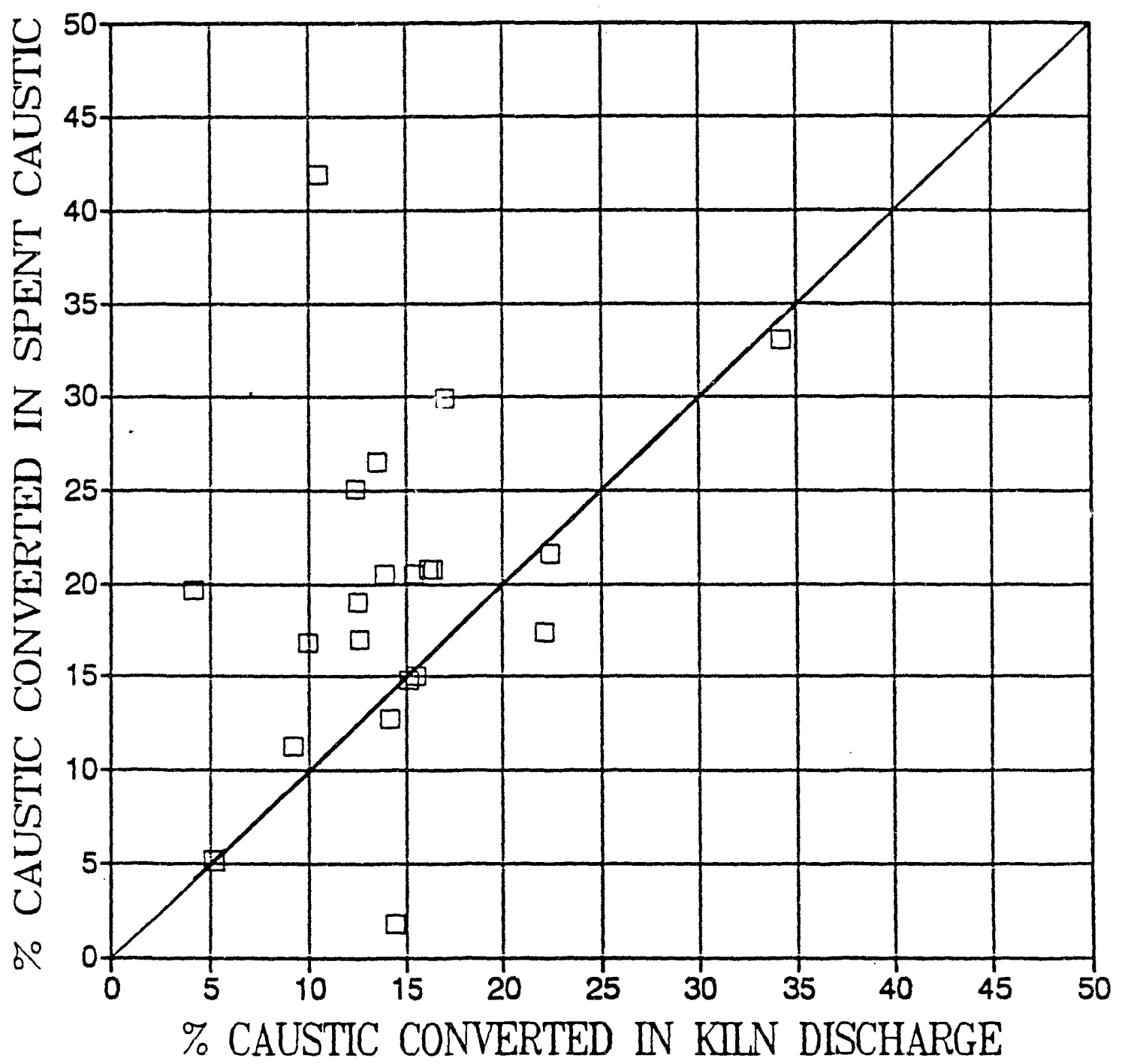

Figure 4-22. Comparison of Carbonate Formation in Kilii Discharge and Spent Caustic Samples 


\subsubsection{Suppression of Carbonate Formation}

The discussion in the above sections show that carbonate formation can generally be suppressed by operating with low caustic/coal ratio, low kiln temperature, and possibly using sodium hydroxide instead of mixed caustic. Operation with low caustic/coal may result in unacceptably high residual sulfur and ash in the MCL coal due to poor mixing and poor penetration into coal pores. However, if molten caustic is injected onto the coal inside the kiln, it may be possible to achieve high sulfur and ash removal with a relatively low caustic/coal ratio. In this approach, the molten caustic will be sprayed onto the coal as the kiln continually exposes fresh coal surface. This is also the best mechanical and thermal approach for a larger plant where a large part of the kiln heat is provided by molten caustic from the evaporator. Also, use of sodium hydroxide may result in fairly low carbonate formation even at high kiln temperatures.

Review of the carbonate formation data indicates in seven of the samples analyzed, the conversion of caustic to carbonate is at or below $3.3 \%$. These seven samples include one sample each from Runs $1 B, 1 D, 2 A, 7 E$ and $8 A$, and two samples from Run 7A. In general, these runs are not associated with operating conditions that will specifically minimize carbonate formation. A possible explanation is that for these samples, the coal had been partially devolatilized in the heating process prior to reaction with caustic, such as in the case of poor mixing in the first heating zone.

As discussed previously, the average conversion of coal to carbonate is $4.22 \%$ for the 26 mixed caustic runs (excluding shakedown data), and $4.06 \%$ for the 21 sodium hydroxide runs (including Run 11). The corresponding average caustic conversion to carbonate is $19.0 \%$ for mixed caustic (excluding shakedown data), and $15.4 \%$ for sodium hydroxide (including Run 11). These results were achieved by exclusion of air in the rotary kiln with nitrogen flow. Research conducted at the Ames laboratory has demonstrated that carbonate formation can be further suppressed by the pretreatment devolatilization of coal prior to MCL. Thus, the optimum mode of operation for low carbonate formation may include using the first heated zone of the rotary kiln for coal devolatilization, followed by injection of molten caustic onto the partially devolatilized coal in the second heated zone. 
Also, "it is clear that carbonate formation is a reaction separate from desulfurization, and since laboratory tests show that desulfurization is complete within 30 minutes, limiting residence time in the reactor will result in a large reduction in both carbonate and humics formation.

The same factors that reduce humics formation al so reduce carbonate formation. Those factors were discussed above for humics at the end of Section 4.4 and can be summarized as follows:

1) Reduce kiln residence time to 0.5 hours

2) Smooth, uninterrupted operation

3) Molten caustic feed to kiln

4) Fresh coal feed

5) Enclosure (for air exclusion) of all equipment, including the filters

The above factors would be present in a continuously operated full scale plant and thus carbonate formation in such a plant would be minimized.

\subsection{PARTICLE SIZE DISTRIBUTION}

Particle size distribution data for the feed coal and processed coal are presented in Table 4-13. Representative data from Table 4-13 are plotted in Figures 4-23 through 4-26 to provide better illustration of the effects of $\mathrm{MCL}$ processing on coal particle size. The data plotted are derived from the experimental data, assuming that the coal particles follow a logarithmic-probability distribution of the form:

Cumulative $\%$ by Weight Undersize $=50\left[1+\operatorname{erf}\left(\log \left(X / X^{\prime}\right) / s\right)\right]$

where $X=$ particle size, $X^{\prime}=$ parameter with dimension of size, $s=$ standard deviation parameter, and erf = normal probability function or error function. The parameters $X^{\prime}$ and $s$ were determined from 1 inear regression of the experimental data. In all cases, it was found that the experimental data can be well represented by the logarithmic-probability function. This indicates that the logarithm of the coal particle size is normally distributed. 
TABLE 4-13. COAL PARTICLE SIZE DISTRIBUTION

Run/

Cumulative \% Retained on Screen

Sample Date/Time

\begin{tabular}{lllll}
\hline 0 mesh & $60 \mathrm{mesh}$ & $80 \mathrm{mesh}$ & $100 \mathrm{mesh}$ & 200 \\
& &
\end{tabular}

Feed* $\quad 1212$

47.0

64.0

71.4

75.2

85.3100

SHDW/KD $\quad 1214 / 0130$

37.9

56.0

63.8

68.1

$80.3 \quad 100$

SHDW/KD $\quad 1214 / 0830$

39.7

56.7

63.8

68.1

$79.7 \quad 100$

SHDW/T101 1214/0805

$8.5 \quad 33.0$

47.1

55.7

$77.1 \quad 100$

SHDW/C201 1214/0550

19.5

13.9

41.4

35.3

SHDW/C2O1 1214/0815

28.9

53.6

53.3

50.3

60.0

75.9

100

65.9

58.8

80.4

100

SHDW/CP $\quad 1216 / 0115$

SHDW/CP $\quad 1216 / 0200$

1.5

1.0

SHDW/CP

$1216 / 0400$

1.3

1.8

$\begin{array}{ll}\text { SHDW/CP } & 1216 / 0700 \\ \text { SHDW/CP } & 1216 / 1130\end{array}$

1.3

3.7

\section{3}

3.3

72.5

87.6

100

SHDW/CP $1216 / 1600$

\begin{tabular}{|c|c|c|c|c|c|c|c|}
\hline $\begin{array}{l}1 B / C P \\
1 B / C P \\
1 B / C P \\
1 C / C P \\
1 C / C P\end{array}$ & $\begin{array}{l}0123 / 2230 \\
0124 / 0600 \\
0124 / 0830 \\
0124 / 2230 \\
0125 / 0630\end{array}$ & $\begin{array}{l}0.83 \\
0.79 \\
0.33 \\
0.24 \\
0.18\end{array}$ & $\begin{array}{l}3.03 \\
4.72 \\
1.42 \\
1.29 \\
0.74\end{array}$ & $\begin{array}{l}6.22 \\
9.57 \\
3.14 \\
3.65 \\
2.42\end{array}$ & $\begin{array}{c}9.1 \\
13.17 \\
4.96 \\
7.4 \\
4.93\end{array}$ & $\begin{array}{l}28.6 \\
32.29 \\
19.3 \\
35.58 \\
22.72\end{array}$ & $\begin{array}{l}100 \\
100 \\
100 \\
100 \\
100\end{array}$ \\
\hline $\begin{array}{l}2 B / K D \\
2 C / K D \\
2 C / K D\end{array}$ & $\begin{array}{l}0207 / 1430 \\
0208 / 0330 \\
0208 / 1100\end{array}$ & $\begin{array}{r}3.9 \\
46.2 \\
8.5\end{array}$ & $\begin{array}{l}16.1 \\
60.2 \\
31.0\end{array}$ & $\begin{array}{l}27.3 \\
66.2 \\
44.1\end{array}$ & $\begin{array}{l}34.6 \\
69.4 \\
50.9\end{array}$ & $\begin{array}{l}56.2 \\
78.2 \\
68.4\end{array}$ & $\begin{array}{l}100 \\
100 \\
100\end{array}$ \\
\hline $\begin{array}{l}3 \mathrm{~B} 1 / \mathrm{CP} \\
3 \mathrm{~B} 1 / \mathrm{CP} \\
3 \mathrm{C} 1 / \mathrm{CP} \\
3 \mathrm{C} 1 / \mathrm{CP}\end{array}$ & $\begin{array}{l}0223 / 0815 \\
0224 / 0415 \\
0224 / 0815 \\
0224 / 1215\end{array}$ & $\begin{array}{l}0.6 \\
0.3 \\
0.5 \\
0.5\end{array}$ & $\begin{array}{l}3.2 \\
1.7 \\
2.9 \\
2.8\end{array}$ & $\begin{array}{r}10.4 \\
6.4 \\
10.3 \\
9.5\end{array}$ & $\begin{array}{l}16.7 \\
11.4 \\
17.1 \\
15.7\end{array}$ & $\begin{array}{l}37.5 \\
35.0 \\
41.0 \\
38.4\end{array}$ & $\begin{array}{l}100 \\
100 \\
100 \\
100\end{array}$ \\
\hline $\begin{array}{l}6 D 1 / K D \\
6 D 1 / K D \\
6 G 1 / K D\end{array}$ & $\begin{array}{l}0406 / 1435 \\
0406 / 0130 \\
0407 / 0655\end{array}$ & $\begin{array}{l}41.1 \\
28.3 \\
16.8\end{array}$ & $\begin{array}{l}59.0 \\
46.9 \\
39.4\end{array}$ & $\begin{array}{l}67.9 \\
58.0 \\
53.1\end{array}$ & $\begin{array}{l}72.6 \\
64.9 \\
60.8\end{array}$ & $\begin{array}{l}86.5 \\
83.8 \\
82.0\end{array}$ & $\begin{array}{l}100 \\
100 \\
100\end{array}$ \\
\hline $\begin{array}{l}100 / K D \\
10 D / K D \text {-chur } \\
100 / K D \text {-pows }\end{array}$ & $\begin{array}{c}0605 / 1515 \\
\text { unk } 0605 / 1340 \\
\text { vder } 0605 / 1340\end{array}$ & $\begin{array}{l}0.21 \\
0.22 \\
3.99\end{array}$ & $\begin{array}{r}1.88 \\
1.78 \\
22.94\end{array}$ & $\begin{array}{r}8.14 \\
8.25 \\
35.66\end{array}$ & $\begin{array}{l}15.24 \\
15.62 \\
42.64\end{array}$ & $\begin{array}{l}52.61 \\
52.90 \\
65.33\end{array}$ & $\begin{array}{l}100 \\
100 \\
100\end{array}$ \\
\hline $\begin{array}{c}\text { Run/ } \\
\text { Sample }\end{array}$ & Date/Time & & $\begin{array}{l}100 \text { mesh } \\
0.150 \mathrm{~mm}\end{array}$ & $\begin{array}{l}150 \text { mesh } \\
0.105 \mathrm{~mm}\end{array}$ & $\begin{array}{l}200 \text { mesh } \\
0.075 \mathrm{~mm}\end{array}$ & $\begin{array}{l}325 \text { mesh } \\
0.045 \mathrm{~mm} \\
\end{array}$ & $\begin{array}{l}\text { Pan } \\
0 \\
\end{array}$ \\
\hline $\begin{array}{l}100 / C P \\
100 / C P\end{array}$ & $\begin{array}{l}0605 / 2000 \\
0607 / 0400\end{array}$ & & $\begin{array}{l}3.42 \\
4.47\end{array}$ & $\begin{array}{l}10.95 \\
12.29\end{array}$ & $\begin{array}{l}19.17 \\
22.90\end{array}$ & $\begin{array}{l}33.55 \\
45.81\end{array}$ & $\begin{array}{l}100 \\
100\end{array}$ \\
\hline
\end{tabular}

* All coal on this page of this table is Pittsburgh No. 8, Powhatan No. 6 mine. 
TABLE 4-13. COAL PARTICLE SIZE DISTRIBUTION (CONTINUED)

Cumulative \% Retained on Screen**

Run/ Sample

Feed*

Run/

Sample

$11 \mathrm{~A} / \mathrm{CP}$

$11 \mathrm{~A} / \mathrm{CP}$

$11 A / C P$

$11 A / C P$

$11 A / C P$

$11 A / C P$

$11 A / C, P$

$110 / C P$

$11 E / C P$

$11 E / C P$

$11 E / C P$

$11 E / C P$

$\vdots 1 E / C P$

$11 E / C P$

$11 / C P$

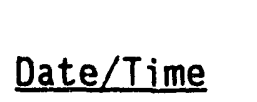

By Tra-Det

Date/Time

$0515 / 0330$

$0515 / 1430$

$0516 / 0935$

$0516 / 1330$

$0516 / 1730$

$0517 / 0335$

$0517 / 1530$

$0518 / 0400$

$0518 / 1615$

$0519 / 0635$

$0519 / 1600$

$0520 / 0422$

$0520 / 1605$

$0521 / 0410$

Average
14 mesh

$1.19 \mathrm{~mm}$

1.72

40 mesh

$\underline{0.425 \mathrm{~mm}}$

0.2

0.2

0.2

0.6

0.5

1.6

0.3

0.1

0.3

0.3

0.1

0.1

0.2

0.4

0.4
100 mesh 200 mesh

$0.150 \mathrm{~mm}$

78.42

36.73

60 mesh

$\underline{0.250 \mathrm{~mm}}$

0.4

1.0

1.3

3.0

1.2

3.5

1.1

4.4

1.2

1.3

1.8

0.6

1.2

1.7

1.7
80 mesh

$0.180 \mathrm{~mm}$

0.6

2.1

3.4

6.5

2.8

6.8

2.6

17.3

3.6

4.3

5.5

2.4

3.4

3.8

4.6
$0.075 \mathrm{~mm}$

88.05

100 mesh

$0.150 \mathrm{~mm}$

0.8

3.9

5.7

9.9

5.1

10.8

4.8

28.0

6.7

7.7

9.6

4.8

6.7

6.4

7.9
325 mesh Pan

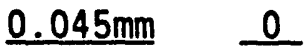

$91.01 \quad 100$

200 mesh Pan

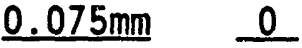

8.5100

$28.1 \quad 100$

$29.9 \quad 100$

$29.4 \quad 100$

$27.9 \quad 100$

$32.9 \quad 100$

$23.1 \quad 100$

$59.0 \quad 100$

$33.9 \quad 100$

$33.1 \quad 100$

$38.2 \quad 100$

$30.5 \quad 100$

$36.3 \quad 100$

$30.5 \quad 100$

$31.5 \quad 100$

* All coal on this page of this iable is Pittsburgh No. 8 coal from CONSOL's Blacksville No. 2 mine.

** The screen sizes are given in both mesh size (14 mesh to pan) and in the size of the corresponding screen opening (1.19 $\mathrm{mm}$ to zero). CP is washed coal product and $K D$ is coal obtained from kiln discharge. 


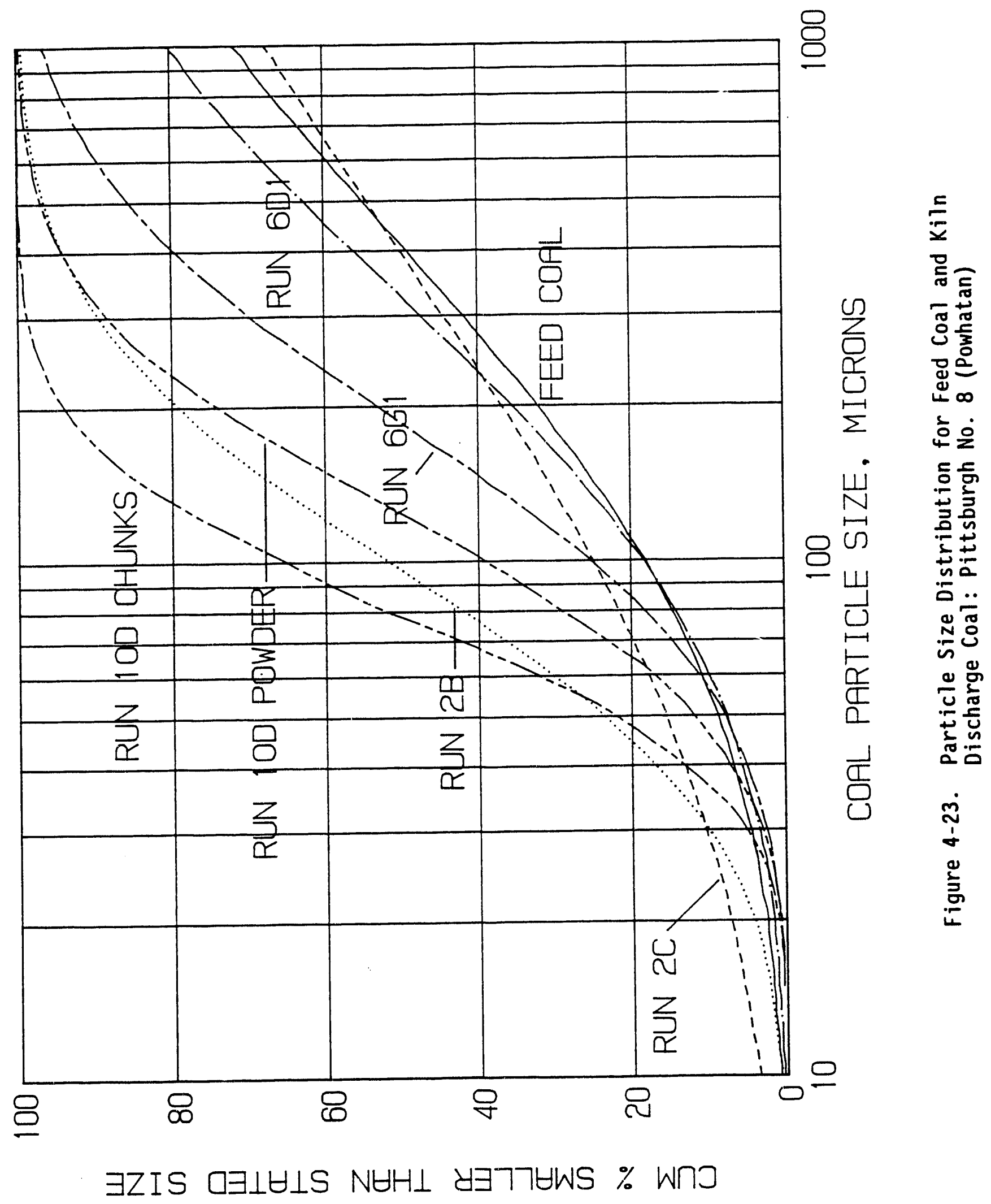




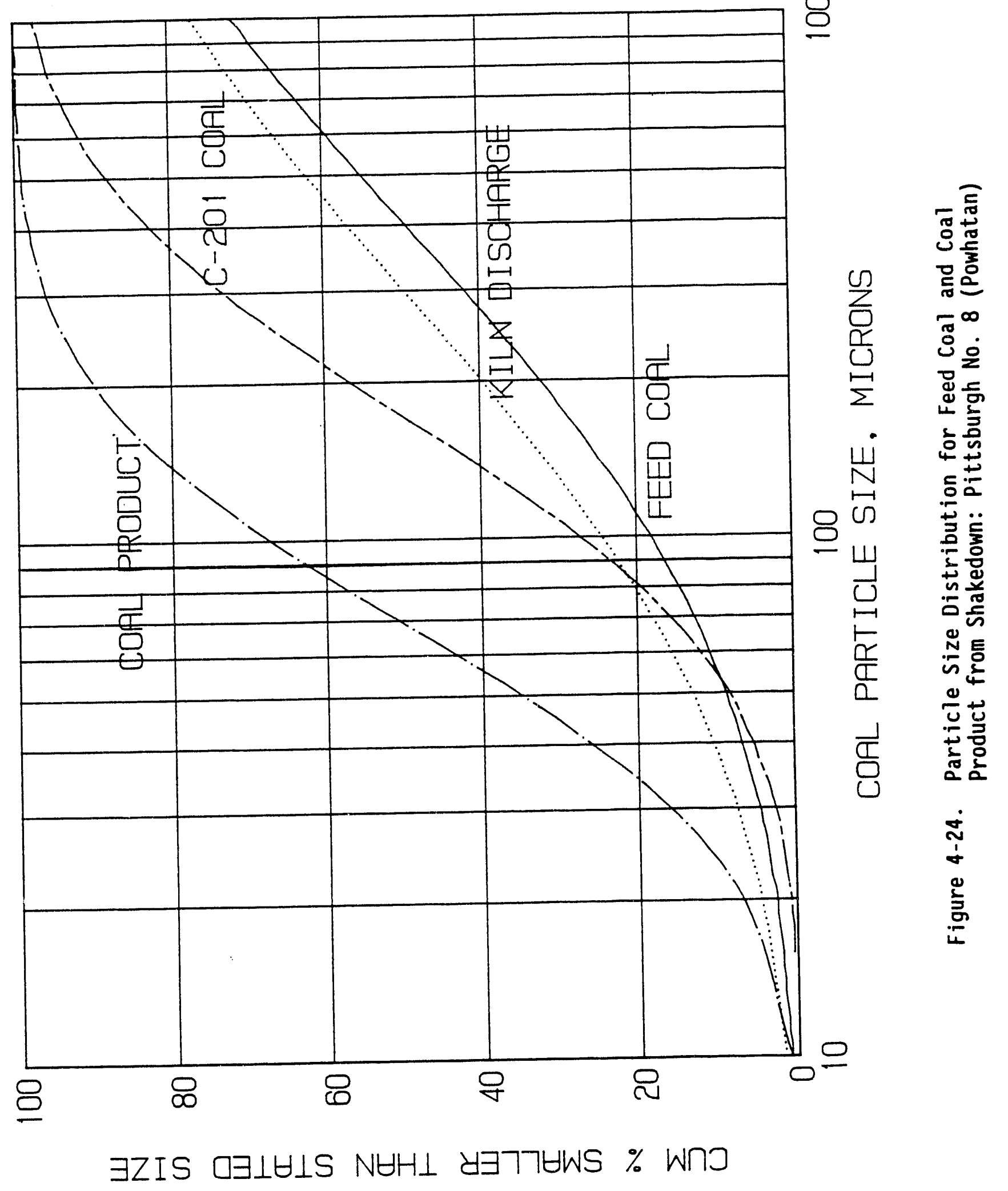




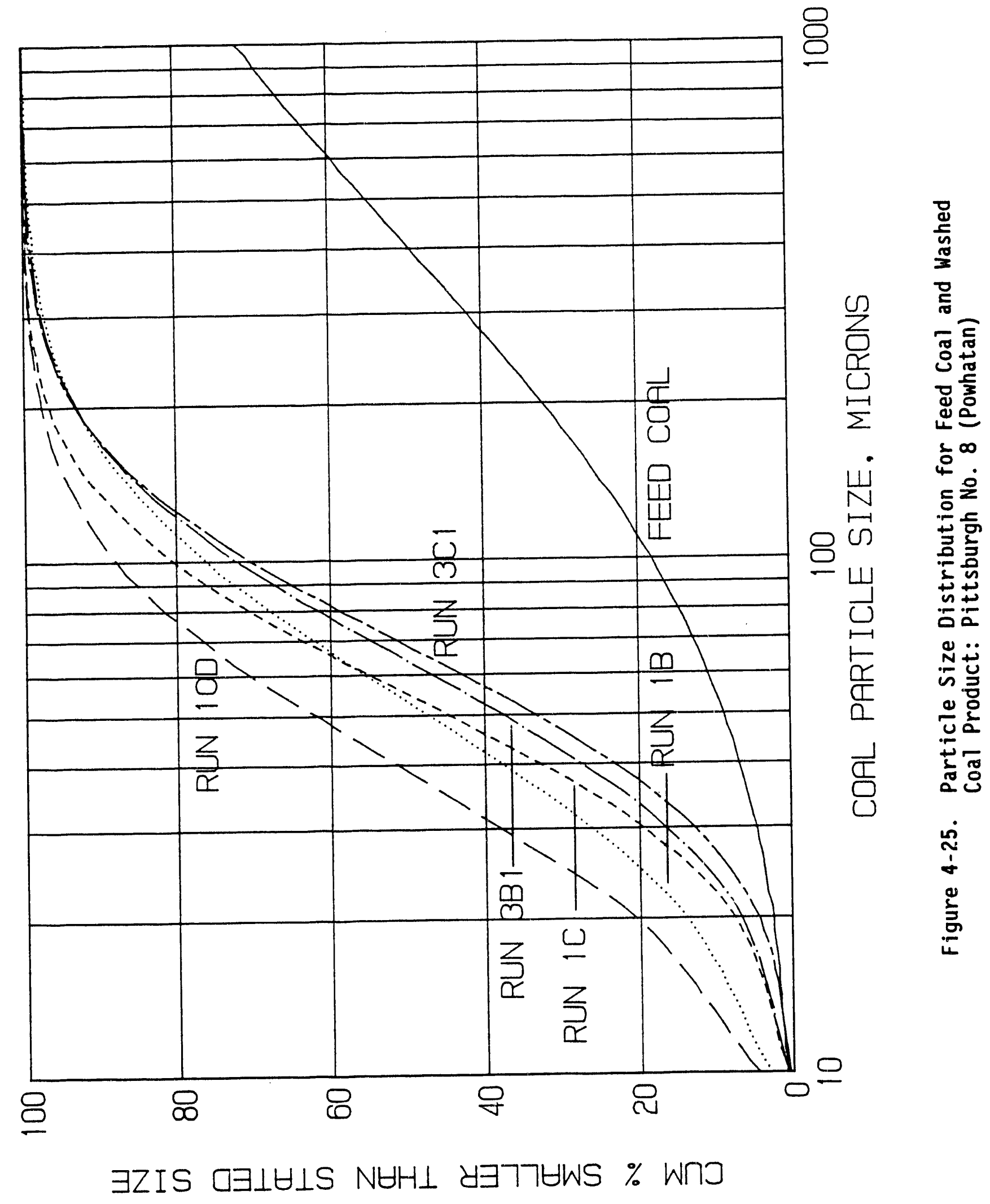




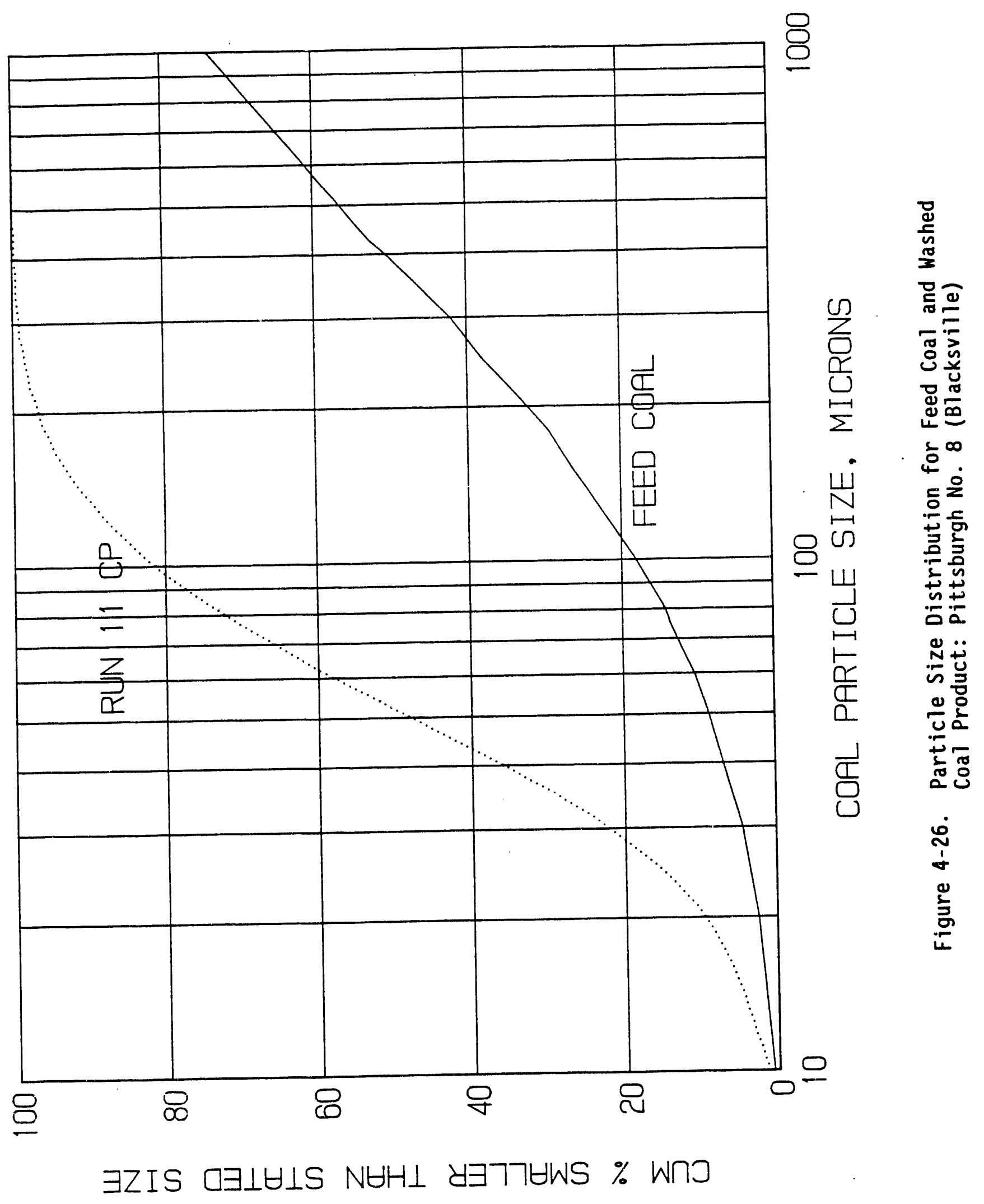


As shown in Figures 4-23 and 4-24, the effect of rotary kiln operation on coal particle size reduction is not totally clear. Data from Run $2 \mathrm{C}$ (0208/0330), Run 6DI (0406/1435), and shakedown (1214/0130 and 1214/0830) show that there was little or no size reduction for the coal particles after processing in the rotary kiln, based on examination of the kiln discharge (KD) coal particle size data illustrated in Figures 4-23 and 4-24. Data from Run $2 B$ and Run $6 \mathrm{Gl}$, however, indicate that there was size reduction for at least the large coal particles (Figure 4-23). For example, approximately $48 \%$ of the coal particles in the feed coal were greater than 400 microns ( 40 mesh) in size. In Run 2B, only $7 \%$ of the KD coal particles were greater than this size. In Run $6 G 1,20 \%$ of the $K D$ coal particles were greater than 400 microns (40 mesh). Thus, there appears to be some coal particle size reduction due to kiln processing, although the effects were not as significant as dissolver pump around and downstream proressing by washing/centrifuge.

Initial data from Run 100 showed a much higher minus 100 mesh cut ( $-85 \%)$ than was seen for any of the kiln discharge coals analyzed in the earlier Phase I runs which averaged about 39\% minus 100 mesh (including shakedown). A screening of a second kiln discharge sample, collected about an hour earlier in the test run, was performed to confirm the initial findings. The second kiln discharge sample was segregated into its 'powdery' and 'chunk' forms to determine the importance of selecting a 'representative' sample for the screening analysis.

As can be seen from Table 4-13, the 'chunk' portion of the second kiln discharge coal sample had a size consist almost identical to the kiln discharge material analyzed earlier. Most likely, the operator selectively chose the 'chunk' form when sending the first kiln discharge sample for analysis. The 'powder' form of the kiln discharge sample actually has a much lower (-57\%) minus 100 mesh content than the chunk form. This is probably related to the greater surface adhesion of the small particles that make up the chunks. The powder and chunk kiln discharge samples from Run 100 are included in Figure 4-23.

The problems with filtration during Run 10 (high coal fines losses, blinding of the filter cloth) indicated that the three-year old Pittsburgh 
(Powhatan) coal in inventory at TRW used for this run might be weathered. Coal samples were analyzed for comparison to the original analysis provided when the coal was purchased in June 1988. The analysis indicated weathering as noted by the increase in sulfate sulfur (from $-0.04 \%$ to $-0.6 \%$ ) and the decrease in heating value (from 13,016 to $-12,700 \mathrm{Btu} / \mathrm{lb}$ ). The plant had been dormant for two years (Run No. 9 was conducted in June 1989 and Run No. 10 in June 1991). It is believed that the weathering of the coal is responsible for the high level of coal fines in the kiln discharge material and for the filter problems during Run 10. Weathered coal is highly friable. Based on this information, it was concluded that it was risky to attempt further integrated tests using the coal feedstocks in inventory since control of fines losses and fines content of the feed slurries to the dewatering equipment was critical to successful operation of the plant. As a result, fresh coal was purchased for the remaining tests (Pittsburgh No. 8 , Blacksville) and the coal in inventory at TRW (both Pittsburgh No. 8 (Powhatan) and Kentucky No. 9) was disposed of (it was sent to a coal-fired power plant where it was burned).

The data presented in Table 4-13 and the plots in Figures 4-24, 4-25 and 4-26 show that there is significant reduction in coal particle size due to dissolver pump around and subsequent washing/centrifuging, as illustrated by the first water washing centrifuge cake $(C-201)$ and coal product data from shakedown (Figure 4-24), shakedown dissolver tank data (T-101, Table 4-13), and by the coal product $(C P)$ data from Runs $1 B, 1 C, 3 B 1,3 C 1$ and $10 D$ (Figure 4-25). In the feed coal (Pittsburgh No. 8, Powhatan), approximately 69\% of the coal particles were greater than 200 microns (65 mesh) in size. By comparison, only 2.8 to $8 \%$ of the coal particles are greater than 200 microns in size in the washed coal product (excluding the weathered coal used in Run 100 which has $-1 \%$ of the coal particles greater than 200 microns in size, see Figure 2-25).

Figure 4-26 is a similar plot of feed coal and product coal for Run 11 which utilized a different coal (Pittsburgh No. 8, Blacksville). For this coal, approximately $78 \%$ of the coal particles in the feed coal were greater than 150 microns (100 mesh) in size. By comparison, only about $7.9 \%$ of the coal particles are greater than 150 microns in size in the washed coal product. 
The shakedown C-201 data (Figure 4-24) show that in the dissolver loop pump around and in the first centrifuge, only the larger coal particles have been reduced in size, and there is no significant size reduction for coal particles less than 75 microns (200 mesh). About 47 percent of the feed coal particles were greater than 425 microns (40 mesh). By comparison, only 13.9 to 28.9 percent of the $C-201$ coal particles were greater than this size (Table 4-13).

The shakedown data indicate that the washing, centrifuge and pump around operations downstream of $\mathrm{C}-201$ appear to further reduce the size of coal particles in all size ranges, as illustrated in Figure 4-24. In the c-201 coal cake, only about 15 percent of the coal particles were smaller than 75 microns (200 mesh). In the final coal product, an average of 61 percent of the coal particles were smaller than this size, and less than 1.0 percent of the coal particles were greater than 425 microns (40 mesh) (Figures 4-24 and 4-25).

With the additional operation of the two vacuum filters to remove most of the spent caustic from MCL coal before centrifuge separation, it may be possible to lessen coal particle size reduction by reducing the number of centrifuge stages, or by reducing centrifuge speed and minimizing shear generated in pump around loops and stirring of the coal slurry.

\subsection{PROCESS LIQUID ANALYSIS}

Selected liquid samples were collected during shakedown and the operational runs and analyzed to determine the partition of aluminum, silicon and iron in the wash sections, the effectiveness of caustic regeneration, and the effectiveness of acid wash water treatment. The analysis results are discussed in the following sections.

\subsubsection{Partition of Aluminum, Silicon and Iron for Sh.kedown Through Run 9}

The liquid samples collected for analysis included VP2023 (spent caustic solution upstream of the bag filter), VF2037 (caustic regenerator inlet downstream of the bag filter), and VP304l (centrate from the first centrifuge 
in the acid wash section, C-301, to water treatment). The concentration levels of aluminum, silicon and iron in these samples are presented in Table 4-14 for shakedown through Run 9. Also presented in this table are the computed concentration levels of aluminum, silicon and iron for a liquid stream entering the wash sections, based on the coal product generation rate and assuming that all mineral matter from coal is in solution. These computed concentration levels can be compared with the sum of the concentration levels of aluminum, silicon and iron in the VP2023+VP3041 samples, because these samples represent the only exit streams for mineral matter from the wash sections. In cases where data for the VP2023 samples are unavailable, the computed concentration levels can be compared with the sum of the concentration levels in the VF2037 and VP3041 samples. This latter comparison does not take into account the amount of aluminum, silicon and iron that could be trapped by the wash section bag filters. For shakedown, neither VF2037 nor VP2023 data are available. Run 11, the only fully integrated test with caustic recycle, is the subject of a separate discussion (see Section 4.7.2).

The data presented in Table 4-14 show that essentially all the aluminum and silicon in the coal feed were found in the caustic regeneration feed (VP2023 and VF2037), whereas most of the iron in the coal feed was found in the acid wash section (VP3041). The expected aluminum and silicon concentrations are in fairly good agreement with the measured values. On the average, approximately $107 \%$ of the aluminum and $125 \%$ of the silicon in the coal feed could be found in the exit streams from the wash sections. These are good mass balance closures considering the fluctuations in concentration values during the course of each run. However, only about an average of $64 \%$ of the iron present in the coal feed could be found in the same exit streams (the iron mass balance was not as good as the aluminum and silicon mass bal ances).

For Runs $7 A, 8 A$, and $8 E$, some silicon and aluminum in the caustic regeneration feed were precipitated in the bag filter, as indicated by comparing the VP2023 and VF2037 concentration levels for these elements. Also, for Runs $7 A, 8 A$ and $9 A$, some of the iron present in the caustic 
TABLE 4-14. PARTITION OF ALUMINUM, SILICON AND IRON FOR SHAKEDOWN THROUGH RUN 9

\begin{tabular}{|c|c|c|c|c|}
\hline Run & Sample & Al $(\mathrm{mg} / \mathrm{L})$ & $\underline{\mathrm{si}}(\mathrm{mg} / \mathrm{L})$ & $\mathrm{Fe}(\mathrm{mg} / \mathrm{L})$ \\
\hline SHDW & VP3041 & 14 & $<20$ & 160 \\
\hline $\begin{array}{l}2 B \\
2 B \\
2 B \\
2 B \\
2 B\end{array}$ & $\begin{array}{c}\text { VP2023 } \\
\text { VF2037 } \\
\text { VP3041 } \\
\text { VP2023+VP3041 } \\
\text { Computed }\end{array}$ & $\begin{array}{r}470 \\
500 \\
7 \\
477 \\
557\end{array}$ & $\begin{array}{r}950 \\
1100 \\
7 \\
957 \\
993\end{array}$ & $\begin{array}{l}3.9 \\
11 \\
220 \\
224 \\
971\end{array}$ \\
\hline $\begin{array}{l}2 C \\
2 C \\
2 C \\
2 C\end{array}$ & $\begin{array}{c}\text { VF2037 } \\
\text { VP3041 } \\
\text { VF2037+VP3041 } \\
\text { Computed }\end{array}$ & $\begin{array}{r}540 \\
10 \\
550 \\
451\end{array}$ & $\begin{array}{r}1120 \\
8 \\
1128 \\
805\end{array}$ & $\begin{array}{r}36 \\
355 \\
391 \\
787\end{array}$ \\
\hline $\begin{array}{l}2 D \\
2 D \\
2 D \\
2 D \\
2 D\end{array}$ & $\begin{array}{c}\text { VP2023 } \\
\text { VF2037 } \\
\text { VP3041 } \\
\text { VP2023+VP3041 } \\
\text { Computed }\end{array}$ & $\begin{array}{r}630 \\
568 \\
10 \\
640 \\
534\end{array}$ & $\begin{array}{r}1500 \\
1250 \\
8 \\
1508 \\
802\end{array}$ & $\begin{array}{r}26 \\
6 \\
370 \\
396 \\
729\end{array}$ \\
\hline $\begin{array}{l}3 \mathrm{BB} 1 \\
3 \mathrm{~B} 1 \\
3 \mathrm{~B} 1 \\
3 \mathrm{~B} 1 \\
3 \mathrm{~B} 1\end{array}$ & $\begin{array}{c}\text { VP2023 } \\
\text { VF2037 } \\
\text { VP3041 } \\
\text { VP2023+VP3041 } \\
\text { Computed }\end{array}$ & $\begin{array}{r}385 \\
453 \\
.47 \\
432 \\
380\end{array}$ & $\begin{array}{l}748 \\
888 \\
147 \\
895 \\
678\end{array}$ & $\begin{array}{l}140 \\
105 \\
363 \\
503 \\
663\end{array}$ \\
\hline $\begin{array}{l}4 C 2 \\
4 C 2 \\
4 C 2 \\
4 C 2\end{array}$ & $\begin{array}{c}\text { VF2037 } \\
\text { VP3041 } \\
\text { VF2037+VP3041 } \\
\text { Computed }\end{array}$ & $\begin{array}{r}737 \\
12 \\
745 \\
551\end{array}$ & $\begin{array}{c}1482 \\
7.7 \\
1490 \\
983\end{array}$ & $\begin{array}{r}29 \\
500 \\
529 \\
961\end{array}$ \\
\hline $\begin{array}{l}4 C 3 \\
4 C 3 \\
4 C 3 \\
4 C 3\end{array}$ & $\begin{array}{c}\text { VF2037 } \\
\text { VP3041 } \\
\text { VF2037+VP3041 } \\
\text { Computed }\end{array}$ & $\begin{array}{l}349 \\
7.6 \\
357 \\
519\end{array}$ & $\begin{array}{l}806 \\
3.8 \\
810 \\
926\end{array}$ & $\begin{array}{r}13 \\
340 \\
353 \\
906\end{array}$ \\
\hline $\begin{array}{l}4 C 6 \\
4 C 6 \\
4 C 6 \\
4 C 6\end{array}$ & $\begin{array}{c}\text { VF2037 } \\
\text { VP3041 } \\
\text { VF2037+VP3041 } \\
\text { Computed }\end{array}$ & $\begin{array}{c}454 \\
8.1 \\
462 \\
529\end{array}$ & $\begin{array}{c}1040 \\
5.0 \\
1045 \\
943\end{array}$ & $\begin{array}{r}11 \\
400 \\
411 \\
922\end{array}$ \\
\hline $\begin{array}{l}5 B \\
5 B \\
5 B \\
5 B\end{array}$ & $\begin{array}{c}\text { VF2037 } \\
\text { VP3041 } \\
\text { VF2037+VP3041 } \\
\text { Computed }\end{array}$ & $\begin{array}{r}482 \\
47 \\
529 \\
574\end{array}$ & $\begin{array}{r}989 \\
44 \\
1033 \\
1024\end{array}$ & $\begin{array}{r}65 \\
550 \\
615 \\
1002\end{array}$ \\
\hline
\end{tabular}


TABLE 4-14. PARTITION OF ALUMINUM, SILICON AND IRON FOR SHAKEDOWN THROUGH RUN 9 (CONTINUED)

\begin{tabular}{|c|c|c|c|c|}
\hline Run & Sample & Al $(\mathrm{mg} / \mathrm{L})$ & $\underline{S i}(\mathrm{mg} / \mathrm{L})$ & $\mathrm{Fe}(\mathrm{mg} / \mathrm{L})$ \\
\hline $\begin{array}{l}5 C / 5 D \\
5 C / 5 D \\
5 C / 5 D \\
5 C / 5 D\end{array}$ & $\begin{array}{c}\text { VF2037 } \\
\text { VP3041 } \\
\text { VF2037+VP3041 } \\
\text { Computed }\end{array}$ & $\begin{array}{r}404 \\
12 \\
416 \\
435\end{array}$ & $\begin{array}{r}913 \\
9 \\
922 \\
654\end{array}$ & $\begin{array}{l}243 \\
495 \\
738 \\
594\end{array}$ \\
\hline $\begin{array}{l}7 A \\
7 A \\
7 A \\
7 A \\
7 A\end{array}$ & $\begin{array}{c}\text { VP2023 } \\
\text { VF2037 } \\
\text { VP3041 } \\
\text { VP2023+VP3041 } \\
\text { Computed }\end{array}$ & $\begin{array}{l}378 \\
302 \\
<0.5 \\
378 \\
521\end{array}$ & $\begin{array}{l}864 \\
756 \\
<0.5 \\
864 \\
783\end{array}$ & $\begin{array}{r}378 \\
30 \\
420 \\
798 \\
711\end{array}$ \\
\hline $\begin{array}{l}8 A \\
8 A \\
8 A \\
8 A \\
8 A\end{array}$ & $\begin{array}{c}\text { VP2023 } \\
\text { VF2037 } \\
\text { VP3041 } \\
\text { VP2023+VP3041 } \\
\text { Computed }\end{array}$ & $\begin{array}{r}1049 \\
860 \\
29 \\
1078 \\
918\end{array}$ & $\begin{array}{r}2105 \\
1873 \\
62 \\
2167 \\
1637\end{array}$ & $\begin{array}{r}525 \\
483 \\
540 \\
965 \\
1601\end{array}$ \\
\hline $\begin{array}{l}8 C \\
8 C \\
8 C \\
8 C \\
8 C\end{array}$ & $\begin{array}{c}\text { VP2023 } \\
\text { VF2037 } \\
\text { VP3041 } \\
\text { VP2023+VP3041 } \\
\text { Computed }\end{array}$ & $\begin{array}{r}526 \\
482 \\
13 \\
539 \\
518\end{array}$ & $\begin{array}{r}972 \\
959 \\
13 \\
985 \\
924\end{array}$ & $\begin{array}{l}137 \\
120 \\
299 \\
436 \\
903\end{array}$ \\
\hline $\begin{array}{l}8 \mathrm{E} \\
8 \mathrm{E} \\
8 \mathrm{E} \\
8 \mathrm{E} \\
8 \mathrm{E}\end{array}$ & $\begin{array}{c}\text { VP2023 } \\
\text { VF2037 } \\
\text { VP3041 } \\
\text { VP2023+VP3041 } \\
\text { Computed }\end{array}$ & $\begin{array}{l}311 \\
250 \\
10 \\
321 \\
427\end{array}$ & $\begin{array}{r}642 \\
531 \\
11 \\
653 \\
761\end{array}$ & $\begin{array}{r}88 \\
89 \\
313 \\
401 \\
744\end{array}$ \\
\hline $\begin{array}{l}9 A \\
9 A \\
9 A \\
9 A \\
9 A\end{array}$ & $\begin{array}{c}\text { VP2023 } \\
\text { VF2037 } \\
\text { VP3041 } \\
\text { VP2023+VP3041 } \\
\text { Computed }\end{array}$ & $\begin{array}{l}666 \\
710 \\
5.6 \\
722 \\
443\end{array}$ & $\begin{array}{r}1332 \\
1332 \\
<2 \\
1332 \\
789\end{array}$ & $\begin{array}{l}233 \\
178 \\
230 \\
463 \\
772\end{array}$ \\
\hline $\begin{array}{l}9 C \\
9 C \\
9 C \\
9 C \\
9 C\end{array}$ & $\begin{array}{c}\text { VP2023 } \\
\text { VF2037 } \\
\text { VP3041 } \\
\text { VP2023+VP3041 } \\
\text { Computed }\end{array}$ & $\begin{array}{r}400 \\
475 \\
4 \\
404 \\
419\end{array}$ & $\begin{array}{r}950 \\
1147 \\
<2 \\
950 \\
747\end{array}$ & $\begin{array}{l}140 \\
300 \\
195 \\
335 \\
730\end{array}$ \\
\hline
\end{tabular}


regeneration feed were precipitated in the bag filter. The amount precipitated was likely a function of the residence time of the solution in the filter tank ( $T-202)$ prior to being pumped to the caustic regenerator. Precipitation may also depend on the temperature and $\mathrm{pH}$ of the solution.

For Runs $7 A, 8 A, 9 A$ and $9 C$, the iron removed from the coal feed was almost equaliy partitioned between the caustic regeneration feed (before filtration) and the acid wash water. All four runs were conducted with caustic/coal feed ratios of 2.5 or 3.0 , which resulted in relatively high caustic concentrations. Thus, the amount of iron in the caustic regenerator feed appears to increase with high caustic concentrations.

\subsubsection{Partition of Aluminum, Silicon and Iron for Run 11}

The partition of aluminum, silicon and iron during Run 11, the only run in which caustic was continuously recycled to the kiln, is shown in Table 4-15. Run 110 was conducted with fresh water makeup flows to both the water and acid wash trains and with the $\mathrm{pH}$ in the acid train controlled at 1 to 2 . Approximately 25\% (3 GPH) of the filtrate to regeneration (VP2023) was diverted to waste disposal because of limitations on the capacity of the caustic evaporator. It should be noted that the aluminum and silicon concentrations in the water wash train effluent to regeneration have built to levels considerably in excess of the "computed" value corresponding to complete removal of these minerals from the coal fed to the kiln. Aluminum and silicon actually exit the plant in only the $25 \%$ of this stream ( $3 \mathrm{GPH}$ ) sent to waste disposal. The elevated aluminum and silicon levels in the regeneration unit feed (and the precipitation of an insignificant amount of these components from solution in the regeneration unit during this part of Run 11) probably indicates that the "solubility limits" for these components in caustic have not been reached.

During Run 110 , approximately $63 \%$ of the aluminum, $143 \%$ of the silicon and $114 \%$ of the iron in the coal feed can be accounted for in the wash train effluents exiting the plant. Of the total iron exiting the plant, fully $90 \%$ appears in the acid wash train effluent. This is consistent with the iron partition noted in the earlier runs (see Section 4.7.1). 
TABLE 4-15. PARTITION OF ALUMINUM, SILICON AND IRON FOR RUN 11

\begin{tabular}{|c|c|c|c|c|}
\hline Run & Sample & Al $(\mathrm{mg} / \mathrm{L})$ & $\mathrm{Si}(\mathrm{mg} / \mathrm{L})$ & $\mathrm{Fe} / \mathrm{mg} / \mathrm{L}$ \\
\hline $\begin{array}{l}110 \\
110 \\
110 \\
110 \\
110\end{array}$ & $\begin{array}{l}\text { VP2023(1) } \\
\text { VF2037 } \\
\text { VP3041(2) } \\
\text { VP2023+VP3041(3) } \\
\text { Computed }\end{array}$ & $\begin{array}{l}875 \\
N A \\
246 \\
420 \\
665\end{array}$ & $\begin{array}{l}2820 \\
N A \\
1100 \\
1665 \\
1160\end{array}$ & $\begin{array}{l}525 \\
N A \\
924 \\
1030 \\
900\end{array}$ \\
\hline $\begin{array}{l}11 \mathrm{E} \\
11 \mathrm{E} \\
11 \mathrm{E} \\
11 \mathrm{E} \\
11 \mathrm{E}\end{array}$ & $\begin{array}{l}\text { VP2023(1) } \\
\text { VF2037 } \\
\text { VP3041(4) } \\
\text { VP2023+VP3041(3) } \\
\text { Computed }\end{array}$ & $\begin{array}{c}2210 \\
N A \\
- \\
2210 \\
3250\end{array}$ & $\begin{array}{c}4670 \\
N A \\
- \\
4670 \\
5600\end{array}$ & $\begin{array}{c}3160 \\
- \\
- \\
3160 \\
4320\end{array}$ \\
\hline
\end{tabular}

(1) $-25 \%(-3 \mathrm{GPH})$ of F-201 filtrate exits the plant as a waste stream. Balance of filtrate sent to regeneration/evaporation for treatment and concentration prior to recycle to kiln.

(2) This run conducted with $\mathrm{pH}$ in first stage of acid wash (T-301) controlled at a value of 1 to 2 . Approximately $15 \mathrm{GPH}$ of acid wash water from this stage exits the plant as a disposal product.

(3) Computed by adding aluminum, silicon, and iron in $3 \mathrm{GPH}$ of VP2023 filtrate sent to waste disposal to amount of aluminum, silicon and iron in acid wash waste stream (VP3041).

(4) This run was conducted with no acid addition to first stage of acid wash ( $\mathrm{pH}$ in T-301-10). Also, the effluent from the acid wash train was used as makeup water to the water wash train, eliminating the waste stream from the acid wash. 
During Run 11E, no acid was injected into the acid wash train (with a resulting $\mathrm{pH}$ of -10 being recorded in $\mathrm{T}-301$ ), and the aqueous effluent from the acid wash train was used as water makeup to the water wash train. Therefore, the only exit stream for the aluminum, silicon and iron removed from the coal is in the $3 \mathrm{GPH}$ of water wash train filtrate sent to waste disposal and in the mineral matter precipitated from solution in the regeneration reactor. The elimination of the acid wash effluent stream resulted in a significant buildup (relative to Run 110) of aluminum, silicon and iron in the filtrate from the water wash train. At these elevated component concentrations, there is analytical evidence suggesting that some mineral precipitation from solution was occurring in the regeneration reactors.

\subsubsection{Regeneration of Caustic Solution}

Although the caustic solution obtained from the MCL operation contained less than $15 \%$ caustic through Run 9 (due to problems with the vacuum filters, see Section 2.2), analysis results of the regeneration inlet (VF2037) and outlet (VF4024) samples indicate significant removal of silicon and aluminum compounds. Regeneration was conducted by the addition of lime to the caustic solution during shakedown through Run 9, typically at a temperature of $110^{\circ} \mathrm{F}\left(43^{\circ} \mathrm{C}\right)$. The VF4024 samples were acquired after the regenerated solution was centrifuged for solids removal, followed by passing the centrate through a bag filter. The spent caustic solution was also passed through a bag filter prior to entering the caustic regeneration unit. During selected runs, samples (VP2023) of the spent caustic solution were collected upstream of the bag filter. As discussed in the previous sections, comparison of the VF2037 and VP2023 data would provide an indication whether some of the mineral matter were removed in the bag filter even before entering the regenerator unit. For shakedown, neither VF2037 nor VP2023 data are available.

The concentration levels of aluminum, silicon and iron in the VP2023, VF2037 and VF4024 samples through Run 8 are presented in Table 4-16, including some of the data already contained in Table 4-14. Again presented in this table are the computed concentration levels for a liquid stream entering the wash sections, based on the coal product generation rate and 
TABLE 4-16. CONCENTRATION LEVELS OF ALUMINUM, SILICON AND IRON IN S'PENT. AND REGENERATED CAUSTIC FOR RUNS 2 THROUGH 8

\begin{tabular}{|c|c|c|c|c|}
\hline Run & Sample & A) $(m g / L)$ & $\underline{\mathrm{Si}(\mathrm{mg} / L)}$ & $\mathrm{Fe}(\mathrm{mg} / \mathrm{L})$ \\
\hline $\begin{array}{l}2 C \\
2 C \\
2 C\end{array}$ & $\begin{array}{l}\text { VF2037 } \\
\text { VF4024 } \\
\text { Computed }\end{array}$ & $\begin{array}{l}540 \\
374 \\
451\end{array}$ & $\begin{array}{r}1120 \\
142 \\
805\end{array}$ & $\begin{array}{r}36 \\
44 \\
787\end{array}$ \\
\hline $\begin{array}{l}3 \mathrm{~B} 1 \\
3 \mathrm{~B} ! \\
3 \mathrm{LI} \\
3 \mathrm{~B} 1\end{array}$ & $\begin{array}{l}\text { VP2023 } \\
\text { VF2037 } \\
\text { VF4024 } \\
\text { Computed }\end{array}$ & $\begin{array}{l}385 \\
453 \\
353 \\
380\end{array}$ & $\begin{array}{l}748 \\
888 \\
612 \\
678\end{array}$ & $\begin{array}{r}140 \\
105 \\
31 \\
663\end{array}$ \\
\hline $\begin{array}{l}3 C 1 \\
3 C 1 \\
3 C 1 \\
3 C 1\end{array}$ & $\begin{array}{l}\text { VP2023 } \\
\text { VF2037 } \\
\text { VF4024 } \\
\text { Computed }\end{array}$ & $\begin{array}{l}540 \\
515 \\
400 \\
418\end{array}$ & $\begin{array}{r}1300 \\
1170 \\
650 \\
746\end{array}$ & $\begin{array}{r}140 \\
140 \\
30 \\
729\end{array}$ \\
\hline $\begin{array}{l}5 B \\
5 B \\
5 B\end{array}$ & $\begin{array}{l}\text { VF2037 } \\
\text { VF4024 } \\
\text { Computed }\end{array}$ & $\begin{array}{l}482 \\
664 \\
574\end{array}$ & $\begin{array}{r}989 \\
411 \\
1024\end{array}$ & $\begin{array}{r}65 \\
31 \\
1002\end{array}$ \\
\hline $\begin{array}{l}5 C / 5 D \\
5 C / 5 D \\
5 C / 5 D\end{array}$ & $\begin{array}{l}\text { VF } 2037 \\
\text { VF } 4024 \\
\text { Comput ed }\end{array}$ & $\begin{array}{l}404 \\
702 \\
435\end{array}$ & $\begin{array}{r}913 \\
1253 \\
654\end{array}$ & $\begin{array}{r}243 \\
46 \\
594\end{array}$ \\
\hline $\begin{array}{l}7 A \\
7 A \\
7 A \\
7 A\end{array}$ & $\begin{array}{l}\text { VP2023 } \\
\text { VF2037 } \\
\text { VF } 4024 \\
\text { Computed }\end{array}$ & $\begin{array}{l}378 \\
302 \\
352 \\
521\end{array}$ & $\begin{array}{l}864 \\
756 \\
264 \\
783\end{array}$ & $\begin{array}{r}378 \\
30 \\
44 \\
711\end{array}$ \\
\hline $\begin{array}{l}8 A \\
8 A \\
8 A \\
8 A\end{array}$ & $\begin{array}{l}\text { VP2C23 } \\
\text { VF2O37 } \\
\text { VF4024 } \\
\text { Computed }\end{array}$ & $\begin{array}{r}1049 \\
860 \\
659 \\
918\end{array}$ & $\begin{array}{r}2105 \\
1873 \\
395 \\
1637\end{array}$ & $\begin{array}{r}525 \\
483 \\
28 \\
1601\end{array}$ \\
\hline $\begin{array}{l}8 C \\
8 C \\
8 C \\
8 C\end{array}$ & $\begin{array}{l}\text { VP2023 } \\
\text { VF2037 } \\
\text { VF4024 } \\
\text { Computed }\end{array}$ & $\begin{array}{l}526 \\
482 \\
163 \\
518\end{array}$ & $\begin{array}{l}972 \\
959 \\
178 \\
924\end{array}$ & $\begin{array}{r}137 \\
120 \\
5 \\
903\end{array}$ \\
\hline $\begin{array}{l}8 \mathrm{E} \\
8 \mathrm{E} \\
8 \mathrm{E} \\
8 \mathrm{E}\end{array}$ & $\begin{array}{l}\text { VP2023 } \\
\text { VF2037 } \\
\text { VF4024 } \\
\text { Computed }\end{array}$ & $\begin{array}{l}311 \\
250 \\
379 \\
427\end{array}$ & $\begin{array}{l}642 \\
531 \\
139 \\
761\end{array}$ & $\begin{array}{r}88 \\
89 \\
11 \\
744\end{array}$ \\
\hline $\begin{array}{l}8 F \\
8 F \\
8 F \\
8 F\end{array}$ & $\begin{array}{l}\text { VP2023 } \\
\text { VF2037 } \\
\text { VF } 4024 \\
\text { Computed }\end{array}$ & $\begin{array}{l}774 \\
512 \\
348 \\
542\end{array}$ & $\begin{array}{r}1473 \\
1050 \\
165 \\
966\end{array}$ & $\begin{array}{r}225 \\
152 \\
11 \\
945\end{array}$ \\
\hline
\end{tabular}


assuning that all mineral matter from the coal is in solution. These computed concentration levels can be compared with the concentration levels of aluminum, silicon and iron in the VF2037 and VP2023 samples.

The data presented in Table 4-16 show that essentially all calculated aluminum and silicon in the coal feed were found in the caustic regenerator feed (VP2023 and VF2037), as pointed out in the previous discussion on partition of these elements.

The aluminum concentration levels in VF2037 were sometimes lower than those in the corresponding VF4024 samples. This is because the sample concentration levels represent instantaneous values which fluctuated during the course of the run. In the regeneration process through Run 9 , the calculated removal efficiency for aluminum ranged from 0 to $66.2 \%$ and averaged $21.9 \%$. Removal efficiency for silicon ranged from 31.1 to $87.3 \%$ and averaged $67.2 \%$. The iron concentration in the spent caustic solution was typically fairly low, as most of the iron in the coal feed is partitioned to the acid wash water. Regardless of the the iron concentration in the spent caustic solution, the regenerated caustic contained 5 to $46 \mathrm{mg} / \mathrm{L}$ iron. In a concentrated caustic solution (i.e. $50 \%$ caustic), the removal efficiencies for both silicon and aluminum compounds are expected to be even higher.

Runs 10 and 11 differed from the earlier runs in that no lime was added to the regeneration reactor. The concentrations of aluminum, silicon, iron and sulfur in the feed (VF2023) and effluent (VF4024) from regeneration are presented in Table 4-17 for these two runs.

Regeneration was conducted in a batch mode during Run 10 . Water wash train filtrate flowed into the regeneration reactor for -3.5 hours $(6 / 5$, $22: 40$ to $6 / 6,02: 15)$ late in the run. Caustic bead was metered into the regeneration reactor to increase the solutiol's caustic concentration thereby precipitating sodium carbonate from solution. (The presence of carbonate can cause tube scaling in the evaporator.) The reactor was agitated and maintained at $\sim 150^{\circ} \mathrm{F}$ for -35 hours. At the end of this time, the contents of the regeneration reactor were processed through a centrifuge and bag filter and the resulting solids-free solution collected in the evaporator feed tank. 


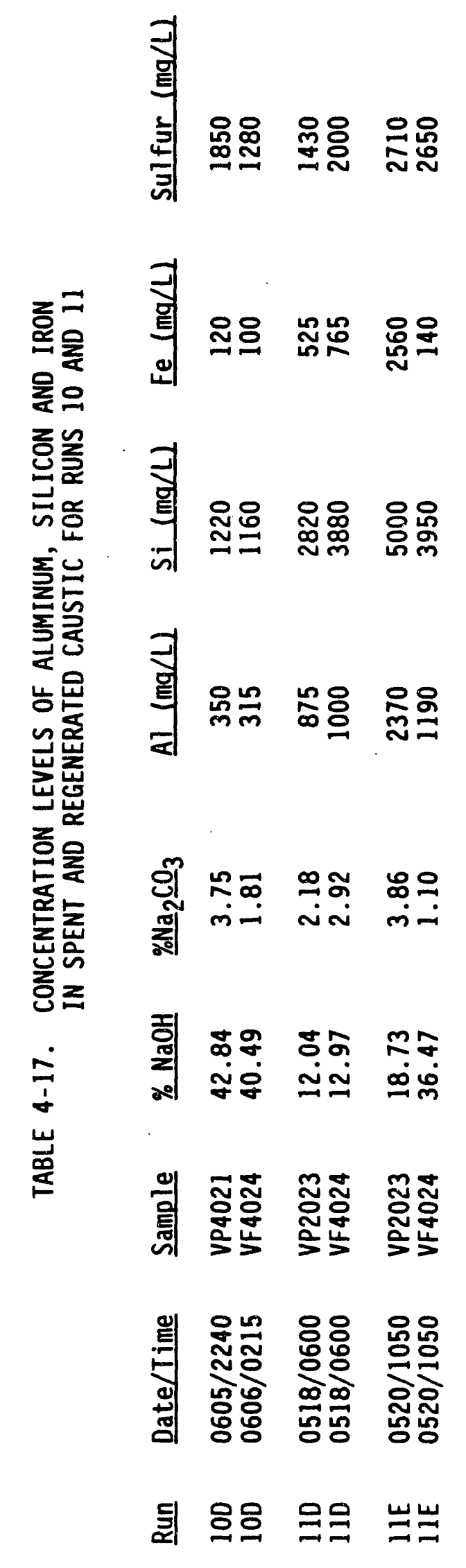


The wash train filtrate that was fed into the regeneration reactor during Run 10 was not analyzed for its aluminum, silicon, iron or sulfur content. However, samples were taken both upstream (VP4021) and downstream (VP4024) of the regeneration effluent centrifuge/bag filter. The upstream sample contains all of the aluminum, silicon, iron and sulfur fed to regeneration, whether in solution or as precipitate. The downstream sample does not contain any of the aluminum, silicon, iron or sulfur which precipitated in the regeneration reactor.

As can be seen in Table 4-17, very little, if any, aluminum, silicon or iron were removed from solution in the regeneration reactor during Run 100 . A significant amount of carbonate precipitation resulted from the addition of the caustic bead. Some sulfur rejection (-30\%) from solution al so occurred.

Run 11 was the only run in which wash water train caustic filtrate was continuously regenerated and recycled to the kiln. Although no lime was added to the regeneration reactor, caustic bead was added to increase the caustic content of the regenerating solution thereby precipitating carbonate from solution. The regeneration results for Runs 110 and $11 E$ are summarized in Table 4-17.

During Run 110 the flow of caustic bead into the regeneration reactor was inadvertently interrupted. This is reflected in the approximately constant caustic content of the solution in and out of the regeneration reactor. It should be remembered when examining the results of Run 110 that although the feed to the regeneration reactor (VP2023) is for a sample at a single point in time, the regenerator effluent (VF4024) is taken downstream of a continuously stirred tank with approximately 8 hours of liquid inventory. This sample therefore reflects the processing of feed streams (of possibly varying compositions) over a period of several hours. Thus it is possible, as in Run 11D, at a given point in time, for the regenerator effluent to contain higher concentrations of aluminum, silicon, iron and sulfur than does the regenerator feed of the moment. No significant regeneration of caustic appears to have occurred during Run 110. 
During Run $11 E$ the caustic concentration of the regenerator feed was increased from $-19 \%$ to $-37 \%$ by the addition of caustic bead to the regeneration reactor. The concentrations of aluminum, silicon, iron and sulfur in the regenerator feed for Run $11 \mathrm{E}$ were significantly higher than they were for Run 110 (Table 4-17). This was a result of the utilization of the acid wash train effluent as makeup water for the water wash train, thus eliminating the acid wash effluent as an exit point for aluminum, silicon, iron or sulfur from the process. At the elevated aluminum, silicon, iron and sulfur regenerator feed concentrations and an elevated caustic concentration $(-37 \%)$, some rejection of aluminum $(50 \%)$, silicon $(20 \%)$, and iron (95\%) from solution appears to be taking place. No change in solution sulfur content was noted in the regeneration unit.

Approximately $70 \%$ of the carbonate in the feed solution was precipitated in the regeneration reactor by the addition of caustic bead to increase the solution caustic concentration from $-19 \%$ to $-37 \%$.

\subsubsection{Treatment of Acid Wash Water}

The liquid samples collected to determine the effectiveness of acid wash water treatment included VP304l (centrate from the first centrifuge in the acid wash section, C-301, which is sent to water treatment), and VF6027 (the effluent from the lime contactor T-602). The concentration levels of aluminum, silicon, iron, sodium and potassium in these samples are presented in Table 4-18.

In almost all cases, the aluminum and iron levels in the treated water was less than $1 \mathrm{mg} / \mathrm{L}$, and the silicon level was less than $5 \mathrm{mg} / \mathrm{L}$. The single exception is the Run $8 E$ data, which indicate higher levels of aluminum and silicon in the treated water as compared to the untreated acid wash water, and a relatively high iron concentration of $142 \mathrm{mg} / \mathrm{L}$ in the treated water. It appears that $8 E / V F 6027$ was not a representative sample of the treated acid wash water. Overall, the test results show that lime addition is effective for removing iron from acid wash water.

For Runs $1 \mathrm{~B}, 2 \mathrm{C}, 4 \mathrm{Cl}$ and $8 \mathrm{C}$, sodium removal ranged from 38 to $61 \%$ and averaged $47 \%$. For Runs $1 B$ and $2 C$, potassium removal was $48 \%$ and $36 \%$, 
TABLE 4-18. CONCENTRATION LEVELS OF ALUMINUM, SILICON, IRON, SODIUM AND POTASSIUM IN ACID WASH WATER

\begin{tabular}{|c|c|c|c|c|c|c|}
\hline Run & Sample & $\mathrm{Al}(\mathrm{mg} / \mathrm{L})$ & $\underline{S i(m g / L)}$ & $\mathrm{Fe}(\mathrm{mg} / \mathrm{L})$ & $\mathrm{Na}(\mathrm{mg} / \mathrm{L})$ & $\underline{K(m g / L)}$ \\
\hline $\begin{array}{l}\text { SHDW } \\
\text { SHDW }\end{array}$ & $\begin{array}{l}\text { VP3041 } \\
\text { VP6027 }\end{array}$ & $\begin{array}{l}14 \\
<3\end{array}$ & $\begin{array}{r}<20 \\
<3\end{array}$ & $\begin{array}{l}160 \\
<0.2\end{array}$ & $\begin{array}{l}21.1 \\
17.8\end{array}$ & $\begin{array}{l}4.2 \\
3.3\end{array}$ \\
\hline $\begin{array}{l}1 \mathrm{~B} \\
1 \mathrm{~B}\end{array}$ & $\begin{array}{l}\text { VP3041 } \\
\text { VF6027 }\end{array}$ & $\begin{array}{l}83 \\
<1\end{array}$ & $\begin{array}{l}87 \\
<1\end{array}$ & $\begin{array}{r}506 \\
<1\end{array}$ & $\begin{array}{l}200 \\
118\end{array}$ & $\begin{array}{r}187 \\
97\end{array}$ \\
\hline $\begin{array}{l}1 \mathrm{C} \\
1 \mathrm{C}\end{array}$ & $\begin{array}{l}\text { VP3041 } \\
\text { VF6027 }\end{array}$ & $\begin{array}{l}41 \\
<1\end{array}$ & $\begin{array}{r}27 \\
3\end{array}$ & $\begin{array}{r}260 \\
<1\end{array}$ & 425 & 505 \\
\hline $\begin{array}{l}10 \\
10\end{array}$ & $\begin{array}{l}\text { VP3041 } \\
\text { VF6027 }\end{array}$ & $\begin{array}{r}5 \\
<1\end{array}$ & $\begin{array}{l}6 \\
2\end{array}$ & $\begin{array}{r}578 \\
<1\end{array}$ & 231 & 315 \\
\hline $\begin{array}{l}2 C \\
2 C\end{array}$ & $\begin{array}{l}\text { VP3041 } \\
\text { VF6027 }\end{array}$ & $\begin{array}{l}10 \\
<1\end{array}$ & $\begin{array}{l}8 \\
2\end{array}$ & $\begin{array}{l}355 \\
0.1\end{array}$ & $\begin{array}{l}631 \\
388\end{array}$ & $\begin{array}{l}985 \\
632\end{array}$ \\
\hline $\begin{array}{l}2 E \\
2 E\end{array}$ & $\begin{array}{l}\text { VP3041 } \\
\text { VF6027 }\end{array}$ & $\begin{array}{r}9 \\
<1\end{array}$ & $\begin{array}{r}8 \\
<5\end{array}$ & $\begin{array}{l}390 \\
<0.5\end{array}$ & $\begin{array}{l}1538 \\
1400\end{array}$ & $\begin{array}{l}2188 \\
2092\end{array}$ \\
\hline $\begin{array}{l}3 \mathrm{~B} 1 \\
3 \mathrm{~B} 1\end{array}$ & $\begin{array}{l}\text { VP3041 } \\
\text { VF6027 }\end{array}$ & $\begin{array}{l}47 \\
<1\end{array}$ & $\begin{array}{r}147 \\
<5\end{array}$ & $\begin{array}{l}363 \\
<0.5\end{array}$ & - & - \\
\hline $\begin{array}{l}4 C 1 \\
4 C 1\end{array}$ & $\begin{array}{l}\text { VP3041 } \\
\text { VF6027 }\end{array}$ & $\begin{array}{l}15 \\
<1\end{array}$ & $\begin{array}{r}11 \\
2\end{array}$ & $\begin{array}{l}500 \\
<0.5\end{array}$ & $\begin{array}{r}225 \\
88\end{array}$ & - \\
\hline $\begin{array}{l}4 C 2 \\
4 C 2\end{array}$ & $\begin{array}{l}\text { VP3041 } \\
\text { VF6027 }\end{array}$ & $\begin{array}{l}12 \\
<1\end{array}$ & $<1$ & $\begin{array}{l}500 \\
<0.5\end{array}$ & $\begin{array}{l}769 \\
950\end{array}$ & - \\
\hline $\begin{array}{l}4 C 5 \\
4 C 5\end{array}$ & $\begin{array}{l}\text { VP3041 } \\
\text { VF6027 }\end{array}$ & $<1.3$ & $\begin{array}{l}4.8 \\
<1\end{array}$ & $\begin{array}{r}320 \\
<1\end{array}$ & $\begin{array}{l}353 \\
370\end{array}$ & - \\
\hline $\begin{array}{l}5 B \\
5 B\end{array}$ & $\begin{array}{l}\text { VP3041 } \\
\text { VF6027 }\end{array}$ & $\begin{array}{l}47 \\
2.5\end{array}$ & $\begin{array}{l}44 \\
<1\end{array}$ & $\begin{array}{l}550 \\
<0.5\end{array}$ & $\begin{array}{r}1248 \\
993\end{array}$ & - \\
\hline $\begin{array}{l}5 C \\
5 C\end{array}$ & $\begin{array}{l}\text { VP3041 } \\
\text { VF6027 }\end{array}$ & $\begin{array}{r}11 \\
4\end{array}$ & $\begin{array}{l}14 \\
<1\end{array}$ & $\begin{array}{l}470 \\
<0.5\end{array}$ & $\begin{array}{r}950 \\
1085\end{array}$ & - \\
\hline $\begin{array}{l}5 D \\
5 D\end{array}$ & $\begin{array}{l}\text { VP3041 } \\
\text { VF6027 }\end{array}$ & $\begin{array}{r}7 \\
24\end{array}$ & $\begin{array}{r}10 \\
7\end{array}$ & $\begin{array}{l}520 \\
<0.5\end{array}$ & 1085 & - \\
\hline
\end{tabular}


TABLE 4-18. CONCENTRATION LEVELS OF ALUMINUM, SILICON, IRON, SODIUM AND POTASSIUM IN ACID WASH WATER (CONTINUED)

\begin{tabular}{|c|c|c|c|c|c|c|}
\hline Run & Sample & $\operatorname{Al}(\mathrm{mg} / \mathrm{L})$ & $\underline{S i(m g / L)}$ & $\mathrm{Fe}(\mathrm{mg} / \mathrm{L})$ & $\mathrm{Na}(\mathrm{mg} / \mathrm{L})$ & $K(m g / L)$ \\
\hline $\begin{array}{l}7 \mathrm{~A} \\
7 \mathrm{~A}\end{array}$ & $\begin{array}{l}\text { VP3041 } \\
\text { VF6027 }\end{array}$ & $\begin{array}{l}<0.5 \\
<0.5\end{array}$ & $\begin{array}{l}<0.5 \\
<0.5\end{array}$ & $\begin{array}{l}420 \\
<0.1\end{array}$ & : & - \\
\hline $\begin{array}{l}8 A \\
8 A\end{array}$ & $\begin{array}{l}\text { VP3041 } \\
\text { VF6027 }\end{array}$ & $\begin{array}{l}29 \\
1.4\end{array}$ & $\begin{array}{r}62 \\
3\end{array}$ & $\begin{array}{r}540 \\
<1\end{array}$ & - & - \\
\hline $\begin{array}{l}8 B \\
8 B\end{array}$ & $\begin{array}{l}\text { VP3041 } \\
\text { VF6027 }\end{array}$ & $<1$ & $\begin{array}{r}110 \\
3\end{array}$ & $\begin{array}{r}885 \\
2\end{array}$ & - & - \\
\hline $\begin{array}{l}8 C \\
8 C\end{array}$ & $\begin{array}{l}\text { VP3041 } \\
\text { VF6027 }\end{array}$ & $\begin{array}{r}13 \\
2\end{array}$ & $\begin{array}{r}13 \\
5\end{array}$ & $\begin{array}{r}299 \\
6\end{array}$ & $\begin{array}{r}1075 \\
550\end{array}$ & - \\
\hline $\begin{array}{l}8 \mathrm{E} \\
8 \mathrm{E}\end{array}$ & $\begin{array}{l}\text { VP3041 } \\
\text { VF6027 }\end{array}$ & $\begin{array}{l}10 \\
20\end{array}$ & $\begin{array}{l}11 \\
84\end{array}$ & $\begin{array}{l}313 \\
142\end{array}$ & 580 & - \\
\hline $\begin{array}{l}9 A \\
9 A\end{array}$ & $\begin{array}{l}\text { VP3041 } \\
\text { VF6027 }\end{array}$ & $<1$ & $\begin{array}{l}<2 \\
<2\end{array}$ & $\begin{array}{l}230 \\
<0.2\end{array}$ & - & - \\
\hline $\begin{array}{l}9 C \\
9 C\end{array}$ & $\begin{array}{l}\text { VP3041 } \\
\text { VF6027 }\end{array}$ & $<1$ & $\begin{array}{l}<2 \\
<2\end{array}$ & $\begin{array}{l}195 \\
1.3\end{array}$ & - & - \\
\hline 10 & $\begin{array}{l}\text { For Run } \\
\text { to wast } \\
\text { wash tr }\end{array}$ & $\begin{array}{l}\text { and } 11, \\
\text { posal }\end{array}$ & cid wa & $\begin{array}{l}\text { er was } \\
\text { IIE as }\end{array}$ & $\begin{array}{l}\text { treated } \\
r \text { makeu }\end{array}$ & $\begin{array}{l}\text { sent as } \\
\text { the wat }\end{array}$ \\
\hline
\end{tabular}

"-" indicates either no data or not applicable. 
respectively. For the other runs for which data are available, sodium or potassium removal was insignificant. The reason for the low rates of sodium and potassium removal was that ferric sulfate was not added to precipitate the double salts of sodium and potassium.

The sodium and potassium concentrations in the C-301 centrate (VP3041) totaled $25.3 \mathrm{mg} / \mathrm{L}$ for shakedown indicating that most of the caustic was effectively removed from the coal product in the caustic wash section. During shakedown, very dry coal cakes were obtained from the centrifuges in both the water and acid washing systems. This was probably due to the mild operating temperature $\left(340^{\circ} \mathrm{C}\right.$ at $\mathrm{kiln}$ wall) during shakedown which led to low sulfur and ash removal, low coal porosity and surface area, and thus to low cake moisture contents.

The T-602 effluent data (VP6027) show that lime treatment of the waste water from acid wash would effect almost total removal of the aluminum, silicon, and iron, but not necessary any additional removal of sodium and potassium salts. Because the treated water contains very low levels of aluminum, silicon and iron, it may be suitable for recycle as wash water.

During Run 11, the $\mathrm{pH}$ in the acid wash system was varied from 2 to 10 by curtailing acid injection. The iron content of the acid wash effluent stream increased (from $101 \mathrm{ppm}$ to $924 \mathrm{ppm}$ ) when the pH was decreased (from 10 to 2), confirming that acidification of the coal from the water wash train is effective in removing iron (probably present as iron hydroxide) from the coal.

\subsection{WASH TRAIN PERFORMANCE}

The wash train during shakedown and Runs 1 through 9 included five centrifuge stages in the water washing section and three centrifuge stages in the acid washing section, except for short periods during which one of the vacuum filters was on-line. At least one vacuum filter was on-line at all times during Runs 10 and 11 . The performance of the centrifuges can be evaluated from measurements of coal particles in the centrates and cake moisture in the coal product. The effectiveness of the wash train can be determined from $\mathrm{pH}$ measurements as the washing progressed downstream. 
The $\mathrm{pH}$ of the centrifuge centrates from selected runs was measured and is presented in Table 4-19. As expected, the $\mathrm{pH}$ in the centrate for all the runs decreased from $\mathrm{C}-202$ to $\mathrm{C}-205$, as washing progressed in the water washing section. In the acid washing section, the $\mathrm{pH}$ in the centrate increased from C-301 (where sulfuric acid solution was separated to be sent to waste water treatment) to $\mathrm{C}-303$ (where wash water was added), again as expected. These $\mathrm{pH}$ values suggest the effectiveness of the wash stations in removing caustic from coal in the water washing section, and in removing acid from coal in the acid washing section.

The percent of coal fines in the centrate from $C-201$ is presented in Table 4-20. The concentrations of these coal fines ranged from $0.01 \%$ to as much as $2.37 \%$. The average coal fines concentration in the $\mathrm{C}-201$ centrate was $0.26 \%$ (excluding shakedown data), with a standard deviation of $0.46 \%$. The differences in coal fines concentrations were probably caused by the differences in kiln coal product size distributions, as well as fluctuations in the caustic concentrations, both from run-to-run and within the same run. Also, separation in the first stage was not as good as in the later stages because of the smaller density difference between the concentrated caustic solution and the coal product in the first stage.

During shakedown, the coal fines concentrations in the centrates from the other centrifuges were also measured (Table 4-21). Except in the C-201 centrate, the concentrations of coal particles in the centrates were very low. The average concentration of coal particles in the $\mathrm{C}-202$ to $\mathrm{C}-205$ centrates was 0.038 percent, as compared to the average coal particle concentration of 1.42 percent in the $\mathrm{C}-202$ to $\mathrm{C}-205$ feed slurry. For the acid wash section, the average concentration of coal particles in the centrates was 0.035 percent, as compared to the average coal particle concentration of 1.80 percent in the feed slurry. Thus, the centrifuges performed well and showed good solids separation.

As shown in Table 4-22, the moisture content of the centrifuge cake generally decreased from the $60-75 \%$ range in the water wash section (excluding shakedown data) to $51-57 \%$ in the acid wash section. Good liquid 
TABLE 4-19. CENTRIFUGE CENTRATE PH MEASUREMENTS

$\begin{array}{lrrrrrrr}\text { Sample } & \text { SHDW } & \text { Run 2B } & \underline{\text { Run 2C }} & \text { Run 2C } & \text { Run 2D } & \text { Run 2D } & \text { Run 2E } \\ \text { C-202 } & 11.5 & 13.5 & 13.8 & 13.9 & 13.9 & 13.9 & 14.0 \\ \text { C-203 } & 11.0 & 12.7 & 13.0 & 13.1 & 13.2 & 13.2 & 13.4 \\ \text { C-204 } & 10.8 & 12.0 & 12.4 & 11.2 & 12.7 & 12.7 & 12.9 \\ \text { C-205 } & 10.0 & 11.5 & 11.9 & 10.8 & 12.1 & 12.1 & 12.4 \\ \text { C-301 } & 1.7 & 1.2 & 1.0 & 0.6 & 1.0 & \text { ND } & \text { ND } \\ \text { C-302 } & 4.9 & 1.8 & 1.7 & 1.3 & 1.5 & 1.6 & 1.6 \\ \text { C-303 } & 6.7 & 2.4 & 2.2 & 1.9 & 2.0 & 2.1 & 2.0\end{array}$
* ND indicates no data. For Runs $2 C$ and $2 D$, the two samples from each run
were obtained 12 hours apart. 
TABLE 4-20. COAL FINES IN C-201 CENTRATE

$\begin{array}{ccc}\text { Run } & \text { Sample Date/Time } & \text { \% Coal Fines } \\ \text { SHDW } & \text { - } & 0.38 \\ \text { IA } & 0123 / 2000 & 2.37 \\ \text { 1B } & 0124 / 0900 & 0.05 \\ \text { 1B } & 0124 / 2100 & 0.36 \\ \text { IC } & 0125 / 0300 & 0.02 \\ \text { IC } & 0125 / 0900 & 0.04 \\ \text { IC } & 0125 / 1500 & 0.01 \\ \text { IC } & 0125 / 2030 & 0.10 \\ \text { 1D } & 0126 / 1200 & 0.02 \\ \text { 1D } & 0126 / 1800 & 0.58 \\ \text { 1D } & 0127 / 0600 & 0.47 \\ \text { 1D } & 0127 / 1200 & 0.77 \\ \text { 2B } & 0207 / 1130 & 0.04 \\ \text { 2C } & 0207 / 2130 & 0.42 \\ \text { 2C } & 0208 / 0330 & 0.02 \\ \text { 2C } & 0208 / 0930 & 0.02 \\ \text { 2C } & 0208 / 1530 & 0.04 \\ \text { 2D } & 0209 / 0330 & 0.19 \\ \text { 3B1 } & 0223 / 0044 & 0.18 \\ \text { 3B1 } & 0223 / 1215 & 0.03 \\ \text { 3B1 } & 0223 / 1815 & 0.03 \\ \text { 3B1 } & 0224 / 0015 & 0.02 \\ \text { 3B1 } & 0224 / 0615 & 0.02 \\ \text { 3C1 } & 0224 / 1215 & 0.06 \\ \text { 9C } & 0601 / 1200 & 0.28 \\ \text { 10D } & 0605 / 0820 & 0.41 \\ \text { 10D } & 0605 / 1515 & 0.03 \\ \text { 10D } & 0605 / 1650 & 0.62 \\ \text { 10D } & 0605 / 2000 & \end{array}$


TABLE 4-21. CENTRIFUGE CENTRATE AND SLURRY CHARACTERISTICS

\begin{tabular}{llll} 
Sample & Iype & pH & \% Coal \\
\hline C-201 & Centrate & 14.0 & 0.38 \\
C-202 & Centrate & 11.5 & 0.01 \\
C-203 & Centrate & 11.0 & 0.081 \\
C-204 & Centrate & 10.8 & 0.022 \\
C-205 & Centrate & 10.0 & 0.038 \\
C-201 & Feed Slurry & 13.8 & 1.93 \\
C-202 & Feed Slurry & 12.3 & 2.62 \\
C-203 & Feed Slurry & 11.0 & 1.45 \\
C-204 & Feed Slurry & 10.6 & 0.33 \\
C-205 & Feed Slurry & 9.7 & 1.26 \\
& & & \\
C-301 & Centrate & 1.7 & 0.04 \\
C-302 & Centrate & 4.9 & 0.05 \\
C-303 & Centrate & 6.7 & 0.015 \\
C-301 & & & \\
C-302 & Feed Slurry & 2.1 & 1.78 \\
C-303 & Feed Slurry & 3.4 & 2.09 \\
& Feed Slurry & 6.4 & 1.52
\end{tabular}


TABLE 4-22. MOISTURE CONTENT OF CENTRIFUGE CAKE

\begin{tabular}{|c|c|c|c|}
\hline Run & Sample Date/Time & Sample Location & \% Cake Moisture \\
\hline $\begin{array}{l}\text { SHDW } \\
\text { SHDW }\end{array}$ & $\begin{array}{l}1214 / 0805 \\
1214 / 0550\end{array}$ & $\begin{array}{l}C-201 \\
C-201\end{array}$ & $\begin{array}{l}33.77 \\
48.98\end{array}$ \\
\hline $\begin{array}{l}2 \mathrm{E} \\
2 \mathrm{E}\end{array}$ & $\begin{array}{l}0210 / 1450 \\
0210 / 1505\end{array}$ & $\begin{array}{l}C-205 \\
C-205\end{array}$ & $\begin{array}{l}65.79 \\
66.05\end{array}$ \\
\hline $\begin{array}{l}4 C 2 \\
4 C 2 \\
4 C 2 \\
4 C 2 \\
4 C 2 \\
4 C 2\end{array}$ & $\begin{array}{l}0308 / 1700 \\
0308 / 1700 \\
0308 / 1700 \\
0308 / 1700 \\
0308 / 1700 \\
0308 / 1700\end{array}$ & $\begin{array}{l}C-201 \\
C-202 \\
C-203 \\
C-204 \\
C-205 \\
C-302\end{array}$ & $\begin{array}{l}65.81 \\
61.03 \\
57.13 \\
53.19 \\
54.08 \\
51.53\end{array}$ \\
\hline $\begin{array}{l}9 C \\
9 C \\
9 C \\
9 C \\
9 C \\
9 C \\
9 C\end{array}$ & $\begin{array}{l}0601 / 1030 \\
0601 / 1030 \\
0601 / 1030 \\
0601 / 1030 \\
0601 / 1030 \\
0601 / 1030 \\
0601 / 1030\end{array}$ & $\begin{array}{l}C-201 \\
C-202 \\
C-203 \\
C-204 \\
C-205 \\
C-301 \\
C-302\end{array}$ & $\begin{array}{l}65.02 \\
76.72 \\
72.96 \\
75.89 \\
56.47 \\
55.78 \\
57.39\end{array}$ \\
\hline
\end{tabular}


removal was attained even for $\mathrm{C}-201$, where the four data points indicated cake moisture contents of $33.8 \%$ to $66.0 \%$. In general, cake moisture should decrease as washing progresses downstream, as the density difference between the coal particles and the wash solution becomes greater. This is illustrated by the Run $4 \mathrm{C} 2$ data, but not totally supported by the Run $9 \mathrm{C}$ data. The C-201 cake moisture was low for shakedown because the mild kiln operating temperature led to low sulfur and ash removal, low coal porosity and surface area, and thus to lower cake moisture contents.

As discussed in Section 4.1 .5 of this report, the moisture content of the final coal product ranged from $10.69 \%$ (shakedown) to $58.4 \%$ (Run $7 C$ ) for Pittsburgh No. 8 (Powhatan) coal feed (excluding shakedown data), from $51.7 \%$ (Run 5C5) to 59.4\% (Run 7B) for Kentucky No. 9 coal feed, and from $44.6 \%$ (Run $11 E-1$ ) to $46.2 \%$ (Run $11 \mathrm{~A}$ ) for Pittsburgh No. 8 (Blacksvilie) feed coal. This is an indication of the good performance of the centrifuges in removing liquid from the coal product. The moisture content is effected by both the centrifuge train performance and by kiln processing conditions, which determine the porosity of the coal product. It is shown in Section 4.1.5 that fairly good correlations could be obtained between the moisture content and the ash content of the coal product and that the centrifuge train performance was fairly uniform from run to run. The lowest coal product moisture contents $(10.69 \%$ to $36.04 \%)$ were obtained during shakedown (average $23.15 \%$ ) as a result of the mild shakedown kiir operating conditions.

\subsection{MATERIAL BALANCE}

Three types of material balances were performed using the analysis results obtained for the operational runs. The overall material balances for coal on MAF basis and for sulfur are discussed in this section. The material balances for aluminum, silicon and iron have been discussed in Sections 4.7.1 and 4.7 .2 , by comparing concentration levels of these elements in the exit streams with calculated concentration levels in the coal feed and the caustic recycle stream.

\subsubsection{Coal Material Balance for Runs 1 through 9}

The coal material balances for Runs 1 through 9 (excluding shakedown) are shown in Table 4-23. These material balances are based on the coal feed to 


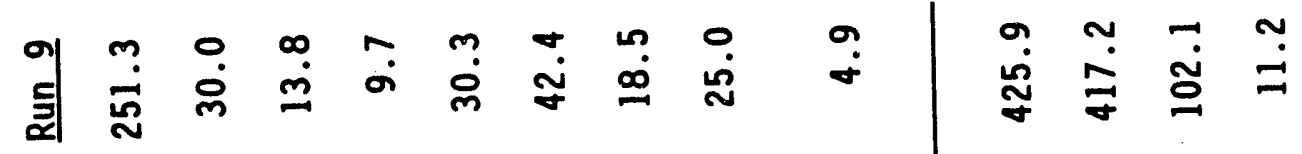

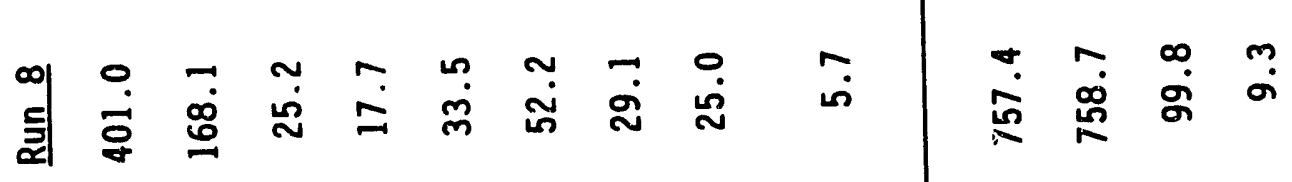

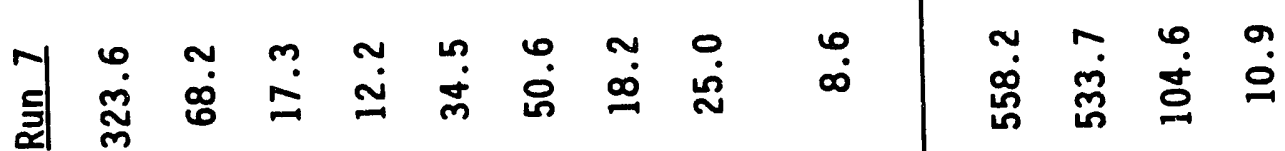

$$
\begin{aligned}
& \text { 旁 }
\end{aligned}
$$

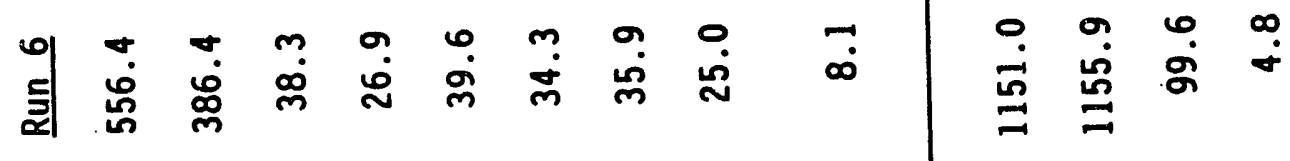

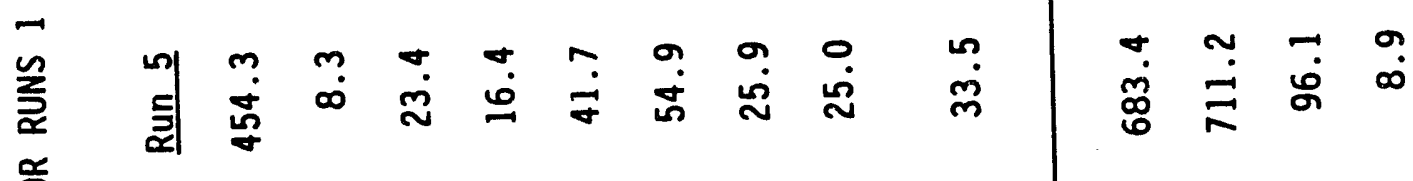

$$
\begin{aligned}
& \text { 운 } \\
& \text { 蓄 }
\end{aligned}
$$

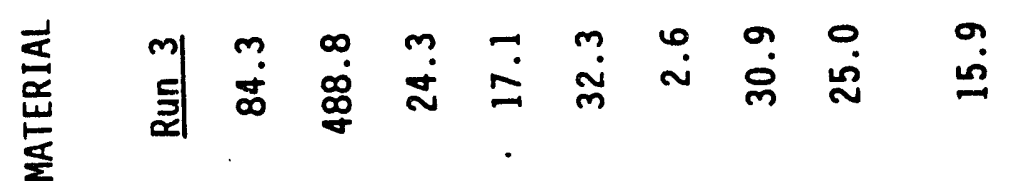

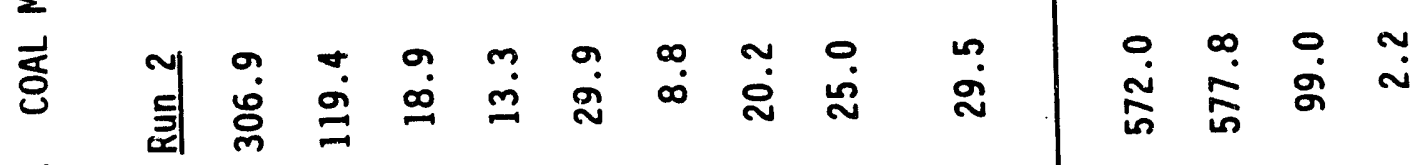

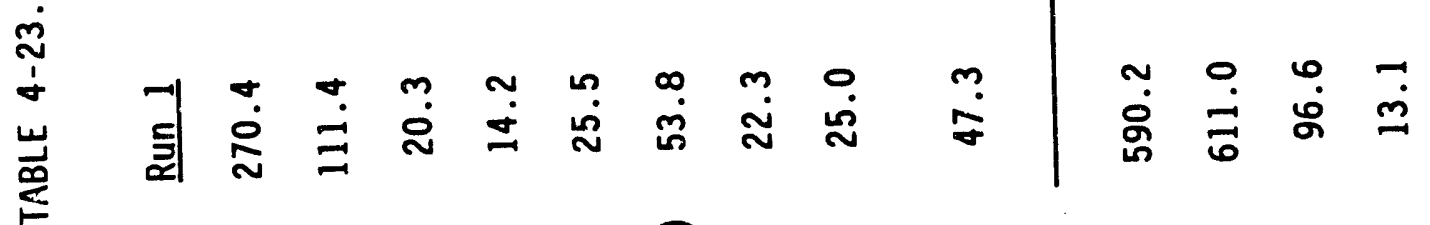

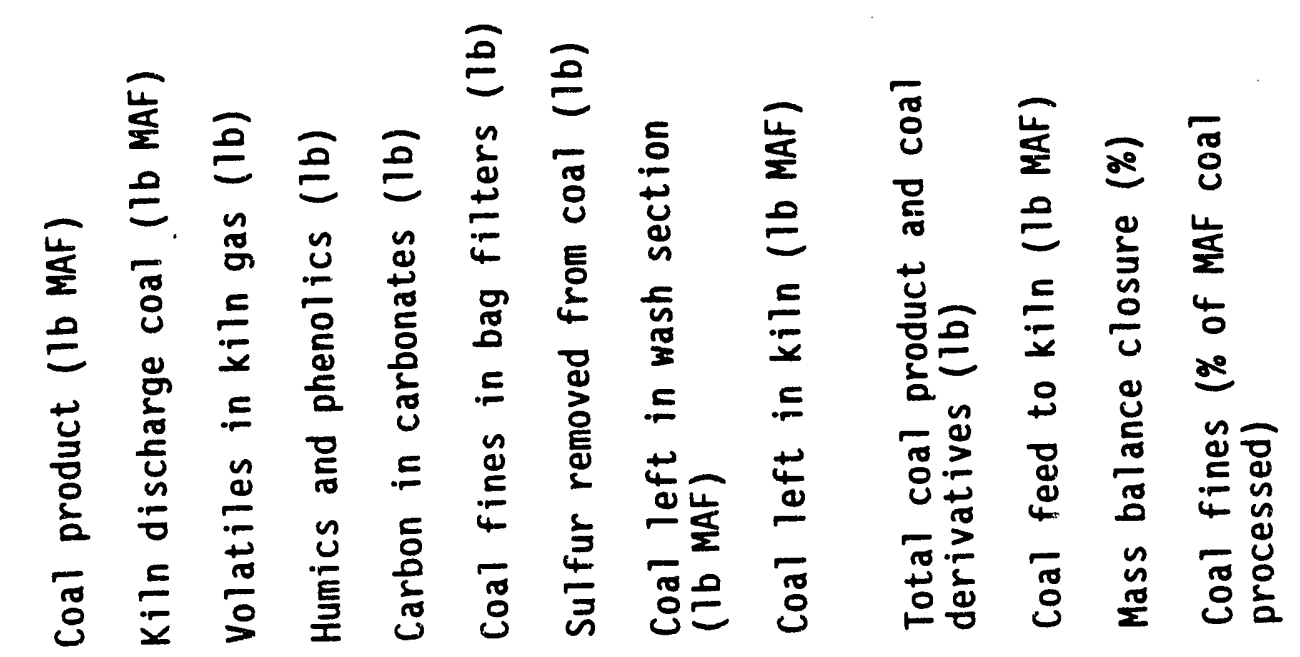


the rotary kiln, and the washed coal product and kiln discharge coal product collected, all obtained from the operation log book and corrected to MAF coal, using feed coal and product coal analysis data. In addition, data from the MCL operations show that an average of $2.85 \%$ of the feed coal was converted to volatiles (mainly methane and hydrogen) in the kiln gas, and approximately $2 \%$ to humics and phenolics. These were used as the basis for estimating the amount of volatiles and humics and phenolics in each run. No material balance was attempted for Run 10 which experienced wash train problems for most of the run, resulting in only a small percentage of the coal being collected as product coal. The material balance for Run 11, the only fully integrated test with caustic recycle, is the subject of a separate discussion (see Section 4.9.2).

As discussed in Section 4.5, analysis of the kiln discharge product and the spent caustic shows that an average of $17.57 \%$ of the caustic feed was converted to carbonates during Runs 1 through Run 9 (excluding shakedown data). The amount of coal carbon in carbonates was computed using the $17.57 \%$ caustic feed conversion rate, the caustic/coal feed ratio, and the percentage of carbon in carbonates. The sulfur removed from coal was computed from the? difference of sulfur content of the feed coal and the sulfur contents of the washed coal product and kiln discharge coal product.

The amount of coal fines collected was estimated using the 120 pound per hour caustic solution flowrate, the total hours of operation of the caustic wash section in each run, and the concentration of coal fines in the caustic solution sent to the bag filters (where coal fines were collected). Except for Runs 2, 3 and 4 , the amount of coal fines was based on a $0.435 \%$ coal fines concentration in the caustic wash solution. This $0.435 \%$ coal fines concentration in caustic solution was obtained from the average of Run 1 data. For Runs 2 and 3, coal fines concentrations of $0.122 \%$ and $0.057 \%$ from the analysis of the caustic wash solution were used. For Run 4, the amount of coal fines was the sum of the coal fines collected on the bag filters (46.60 lbs on dry basis) and the residual coal fines that passed through these bag filters $(85.43$ lbs). The amount of residual coal fines was exceptionally high for Run 4 , because an average of $0.85 \%$ coal fines concentration was found in the filtered caustic wash solution, probably due 
to the use of filter media of incorrect mesh size during the operation of the vacuum filter. The amount of coal fines in caustic solution fluctuated during the operation of each run, and would be significantly reduced if the two vacuum filters were in operation and with the proper filter media.

The amount of coal left in the wash section at the end of each run was estimated to be 25 pounds. This was derived by first estimating that the amount of process solutions left in the wash tanks at the end of the run would be 514 lb ( 8 tanks $\times 14$ gallons per tank $\times 50 \%$ full $\times 8.34$ lb/gallon $\times$ 1.1 specific gravity), and then using an average $3.8 \%$ coal concentration in the solution (obtained from Run 1 data). The amount of coal left in the wash tanks is, therefore, 19.5 pounds. The 25 -pound estimate includes allowance for some additional coal left in centrifuges and process piping. The amount of coal left in the rotary kiln at the end of each run was estimated from review of the operation $\mathrm{log}$ book data. For five of the nine runs, the equivalent of one hour of feed material was left inside the rotary kiln at the end of each run. For Runs 1, 2, 3 and 5, however, it appears that large quantities of feed material were still left inside the kiln, because the kiln was shut off shortly after the transfer of additional coal/caustic to the feed hopper.

\subsubsection{Coal Material Balance for Run 11}

Run 10 (June 1991) was the first test in the plant following extensive equipment modifications. Plugging problems were experienced in the water wash train during most of the run resulting in the production of only small quantities of product coal. Most of the coal fed to the kiln during Run 10 was sent to waste disposal with the numerous aqueous purge streams from the wash train. Therefore no attempt was made to mass balance Run 10 .

Run 11 (May 1992) was the final test in the program and the only one to be conducted under fully integrated (caustic recycle) conditions. The regenerated caustic recycled to the kiln contained carbonates as well as humics. These "recycled" components were accounted for in calculating the "net" conversion of coal fed to the kiln to carbonate and humics. Approximately $3.4 \%$ of the MAF coal fud to the kiln was converted to carbonate. Coa? conversion to humics (plus phenols) was 5.6\%. Approximately 
$2.7 \%$ of the coal was converted to volatiles in the kiln gas. Due to capacity limitations in the caustic evaporation unit, approximately 250 gallons of the wash train filtrate (regeneration feed) was diverted to waste disposal. The average coal fines content of this stream was 2.3\%. The coal material balance for Run 11 is given in Table 4-24.

As shown in Tables 4-23 and 4-24, excellent mass balance closure is obtained for each of the ten runs, ranging from $96.1 \%$ for Run 5 to $105.0 \%$ for Run 9. For the ten runs combined, the total coal product and coal derivatives amounted to 7,395 pounds, and the total coal feed to the rotary kiln was 7,436 pounds. The resulting combined mass balance closure is $99.4 \%$.

\subsection{3 sulfur Material Balance}

Oniy limited amount of analyses was performed to determine the sulfur levels in process liquids. The sulfur analysis data presented in Table 4-25 include those for the caustic regenerator inlet (VP2023 or VF2037) and the caustic regenerator outlet (VF4024). The spent caustic solution was passed through a bag filter prior to entering the regenerator, and the VP2023 and VF2037 samples represent samples collected upstream and downstream of the bag filter, respectively. Since sulfur in the form of sodium or potassium sulfide is not expected to be collected in the bag filter, the VP2023 and VF2037 samples should both provide information on the amourit of sulfur extracted from processed coal.

Also presented in Table 4-25 are the computed sulfur levels in the spent caustic, based on the coal product generation rate and the difference in sulfur levels between the coal feed and the coal product. These computed sulfur levels can be compared with the corresponding sulfur levels in VP2023/VF2037 samples to determine the closeness of the sulfur balance.

As shown in Table 4-25, the sulfur levels in the VP2023/VF2037 samples for Runs 2D, $3 \mathrm{~B} 1$ and $3 \mathrm{Cl}$ exceed the computed values, whereas those for Runs $5 \mathrm{~B}, 5 \mathrm{C} / 5 \mathrm{D}, 8 \mathrm{~A}, 8 \mathrm{C}, 8 \mathrm{D}, 8 \mathrm{E}$ and $8 \mathrm{~F}$ are well below the computed values. The experimentally determined sulfur levels range from $22 \%$ to $170 \%$ of the computed values. This relatively poor sulfur balance closure could be due to fluctuations in sulfur levels during the course of the run. For example, the 
TABLE 4-24. COAL MATERIAL BALANCE FOR RUN 11

$\begin{array}{lc} & \text { Run } 11 \\ \text { Coal Product (1b MAF) } & 634 \\ \text { Kiln Discharge Coal (1b MAF) } & 172 \\ \text { Volatiles in Kiln Gas (1b) } & 32 \\ \text { Humics and Phenolics (1b) } & 67 \\ \text { Carbon in Carbonates (1b) } & 41 \\ \text { Coal Fines in Bag Filters (1b) } & 34 \\ \text { Sulfur Removed from Coal (1b) } & 19 \\ \text { Coal Left in Wash Section (1b MAF) } & 25 \\ \text { Coal Left in Kiln (1b MAF) } & 10 \\ \text { Coal Fines in Filtrate to Waste Disposal (1b) } & 58 \\ \text { Coal Fines in Regeneration Unit Centrifuge Cake (1b) } & 45 \\ \text { Coal Fines Left in Regeneration Unit Tanks (1b) } & 19 \\ \text { Coal Fines in Evaporator Flake at End-of-Run (1b) } & 9 \\ \text { Total Coal Product and Coal Derivatives (1b) } & 1165 \\ \text { Coal Feed to Kiln (1b MAF) } & 1193 \\ \text { Material Balance Closure (\%) } & 97.7 \% \\ \text { Coal Fines (\% of MAF Coal Processed) } & 13.8 \% \\ \end{array}$


TABLE 4-25. CONCENTRATION LEVELS OF SULFUR IN SPENT AND REGENERATED CAUSTIC

\begin{tabular}{|c|c|c|c|c|c|}
\hline \multirow[b]{2}{*}{ Run } & \multicolumn{2}{|c|}{$\begin{array}{l}\text { Sulfur Conc. in } \\
\text { VP2023/VF2037 (mg/L) }\end{array}$} & \multicolumn{2}{|c|}{$\begin{array}{l}\text { Sulfur Conc. in } \\
\text { VF4024 (mg/L) }\end{array}$} & \multirow{2}{*}{$\begin{array}{l}\text { Computed Sulfur } \\
\text { Conc. (mg/L) }\end{array}$} \\
\hline & Mean & Range & Mean & Range & \\
\hline $2 D$ & 1840 & 1840 & No Data & - & 1145 \\
\hline $3 B 1$ & 1430 & $880-1980$ & No Data & - & 838 \\
\hline $3 \mathrm{Cl}$ & 1980 & 1980 & No Data & - & 1240 \\
\hline $5 B$ & 1003 & $695-1350$ & 1403 & $1275-1500$ & 1465 \\
\hline $5 C / 5 D$ & 746 & $550-860$ & 1355 & $1320-1390$ & 865 \\
\hline $8 A$ & 1100 & $790-1300$ & 2307 & $2200-2480$ & 2407 \\
\hline $8 B$ & No Data & - & 1780 & 1780 & 1370 \\
\hline $8 C$ & 630 & $570-690$ & 1355 & $1280-1430$ & 1828 \\
\hline 80 & 670 & 670 & 1730 & $1330-2130$ & 1049 \\
\hline $8 E$ & 340 & 340 & 1700 & 1700 & 1515 \\
\hline $8 F$ & 680 & $590-770$ & 1825 & $1680-1970$ & 2163 \\
\hline $11 E$ & $2600 *$ & & $2700 *$ & & 1500 \\
\hline
\end{tabular}

* End of run values. 
sulfur levels in the six VF2037 samples for Run 5B range from 695 to 1350 $\mathrm{mg} / \mathrm{L}$. Other possibilities are non-representative sampling or analysis error. For Runs $8 \mathrm{C}, 8 \mathrm{D}, 8 \mathrm{E}$ and $8 \mathrm{~F}$, only sodium hydroxide was present in the caustic feed. The sodium levels in the VF2037 samples for these runs range from 11600 to $24500 \mathrm{mg} / \mathrm{L}$, whereas those in the corresponding VP2023 samples range from 33400 to $75200 \mathrm{mg} / \mathrm{L}$. The sodium levels in the VP2023 and VF2037 samples should be approximately the same, unless a significant fraction of the sodium compounds (sodium hydroxide, sodium carbonate and sodium sulfide) was collected in the bag filter as a result of precipitation. Since the sulfur levels for Run 8 were determined using the VF2037 samples, it is possible that these sulfur levels should be corrected by a factor of 2 to 4 , or the ratio of the sodium levels in the corresponding VP2023 and VF2037 samples.

The sulfur levels in the VF4024 samples, however, are in fairly good agreement with the computed sulfur levels in the spent caustic. This good agreement is probably due to the mixing of caustic solution in the regenerator (residence time of 8 hours), so that fluctuations in sulfur levels in the VF4024 samples are less pronounced, as noted in the range of VF4024 sulfur values presented in Table 4-25. The good agreement between the VF4024 and computed sulfur levels also suggests that: 1) the Run 8 VF2037 sulfur values are in error as a result of non-representative sampling or incorrect analysis, because low sulfur levels in the caustic regenerator inlet cannot lead to high and predicted sulfur levels in the caustic regenerator outlet; and 2) sulfur was not removed from the spent caustic solution in the caustic regenerator.

Run 11 was the only run in which operations were conducted under fully integrated (caustic recycle) conditions. The sulfur values presented in Table 4-25 for VP2023/VF2037 and VF4024 for Run IIE are for samples taken towards the end of the week of operations. It can be seen that at the sulfur levels in the regeneration unit feed (VP2023/VF2037), sulfur is not being removed to any measurable extent in the regeneration reactor (VF4024). Sulfur in the regenerated caustic is consequently being recycled to the kiln. This is evident in that the sulfur level in the wash train filtrate $(2600 \mathrm{mg} / \mathrm{L}$ ) has built to about two times the computed sulfur concentration 
$\left(1500^{\circ} \mathrm{mg} / \mathrm{L}\right)$ expected for "once-through" (no recycle) operation with the known sulfur removal from the coal. In fact, some of the sulfur removed from the coal is exiting the plant with the $25 \%(-3 \mathrm{GPH})$ of wash train filtrate purged to waste disposal. At $2600 \mathrm{mg} / \mathrm{L}$ sulfur in the filtrate, approximately $50 \%$ of the known sulfur removed from the coal leaves the plant in this stream. The conclusion to be reached is that at the end of the week of operations, the plant had not reached steady-state with regard to sulfur removal. If operations had been allowed to continue, the sulfur level in VP2023/VF2037 would have continued to increase until the sulfur in the wash train filtrate purge to waste disposal (plus any sulfur that may precipitate in the regeneration reactor at the higher sulfur concentrations) becomes equal to the sulfur removed from the coal.

\subsection{CORROSION/EROSION DATA}

Two types of techniques were used to evaluate corrosion/erosion in the MCL integrated test facility: 1) placement of erosion coupons at selected locations; and 2) placement of electronic monitoring devices (Rohrback corrosometer probes) at selected locations. For the test coupons, the rates of corrosion/erosion were determined by weight and dimensional losses. The corrosometer probes measure corrosion/erosion by monitoring an electric potential across electrodes.

A total of 17 erosion coupons were installed in the MCL test loop. At the conclusion of Run 9, these coupons were removed from the system. A visual inspection of each coupon was performed, and the coupons were weighed, measured and photographed. These coupons were then placed in an ultrasonic cleaner with Alkanox soap and DI water for one hour and dried, reweighed and measured. The locations and materials of construction for these coupons, as well as the calculated corrosion/erosion rates from weight losses in thousandths of an inch per year (mpy) are presented in Table 4-26. The visual changes noted for the coupons are summarized in Table 4-27.

Based on the results presented in these tables, it is apparent that corrosion/erosion caused by the MCL solution on Inconel 600 and 316 stainless steel is practically negligible. Also, corrosion/erosion rates for Carpenter Alloy 20 were $13.2 \mathrm{mpy}$ at the inlet to the first acid wash centrifuge 
TABLE 4-26. CORROSION/EROSION RATES IN MCL TEST LOOP

\begin{tabular}{|c|c|c|c|}
\hline Coupon ID & Location & Material & Corrosion/Erosion Rate, mpy \\
\hline$C C-101$ & P-101 outlet I & Inconel 600 & 0.23 \\
\hline CC-204 & F-202 inlet & 316 SS & 0.025 \\
\hline CC-205 & C-201 inlet & 316 SS & 0 \\
\hline CC-206 & C-202 inlet & 316 SS & 0 \\
\hline$C C-207$ & C-203 inlet & 316 SS & 0 \\
\hline$C C-208$ & C-204 inlet & 316 SS & 0 \\
\hline CC-209 & C-205 inlet & 316 SS & 0 \\
\hline$C C-301$ & c-301 inlet & CARP 20 & 13.2 \\
\hline$C C-302$ & C-302 inlet & CARP 20 & 4.4 \\
\hline CC -303 & C-303 inlet & 316 SS & 0.024 \\
\hline$C C-401$ & T-401 inlet & 316 SS & 0.072 \\
\hline$C C-402$ & T-402 inlet & 316 SS & 0.11 \\
\hline CC -403 & C-401 inlet & 316 SS & 0.057 \\
\hline CC -404 & $\mathrm{~F} 401 / 402$ inlet & 316 SS & 0.039 \\
\hline CC -406 & F430/404 inlet & 316 SS & 0.59 \\
\hline CC -601 & F-601 inlet & CARP 20 & 0.024 \\
\hline$C C-602$ & F-602 inlet & 316 SS & 0.017 \\
\hline
\end{tabular}


TABLE 4-27. VISUAL INSPECTION OF EROSION COUPONS

Coupon ID

\section{Visual Changes}

CC-101 Grey in color, edges appear to be rounded

CC-204 Slight discoloring, no other visual change

CC-205 Very black surface color, no other visual change

CC-206 Slight brownish gold coloring, slight rounding of edges

CC-207 Bronze in color, no other visual change

CC-208 Bronze in color, no other visual change

CC-209 Gold in color, some of the coloring is chipped from surface

CC-301 Dull grey color and surface appears to be etched

CC-302 Dull grey color, no other visual change

CC-303 Bright clean surface, looks new

CC-401 Brownish color, no other visual change

CC-402 Bright clean surface, no other visual change

CC-403 Black in color, no other visual change

CC-404 Light grey color, no other visual change

C.C-406 Dull dark grey surface, looks burnt

CC-601 Bright light grey surface, no other visual change

CC-602 Deposit adhered to surface, one side only. Other side bright and clean 
(C-301), and 4.4 mpy at the inlet to the second acid wash centrifuge $(C-302)$. These rates are comparable to that caused by water corrosion of carbon steel at $10 \mathrm{mpy}$. Therefore, all the coupon materials tested should be suitable as materials of construction at the specific test locations of the MCL plant.

Four Rohrback corrosometer probes were installed in the MCL test 100p. However, one of the probes $(\mathrm{CC}-203)$ was installed in the first vacuum filter slurry tank (T-203), which was not put into service. Another probe (CC-603) was installed in the acid waste treatment iron addition tank (T-601), which was also not put into service. Probe CC-102 (Inconel 600) was originally located in the bottom head of the T-102 dissolver tank, but had to be relocated to the top head (dry coal/caustic zone) to allow the tank pump around loop to return to the bottom head. As a result, the measured corrosion/erosion rate was effectively zero. Probe CC-604 (316 stainless steel) was located in the T-602 waste water treatment 1 ime tank. The calculated corrosion/erosion rate based on probe readings was $0.27 \mathrm{mpy}$. This suggests very low corrosion/erosion rates for 316 stainless stee 1 in the limed T-602 waste water environment. 


\section{EQUIPMENT EVALUATION/MODIFICATION/OFF-LINE TESTING}

The MCL integrated test circuit, as configured during Phase I, had demonstrated that it can be successfully operated on a short-term integrated basis at a total kiln solids feed rate (coal plus caustic) of up to 50 $1 \mathrm{bs} / \mathrm{hr}$. Most of the equipment operated as designed. In most cases, the equipment problems that were experienced were a function of equipment size and would not be a problem at commercial scale. For example, the flow rate in many of the lines was only $0.1 \mathrm{gpm}$. The piping used for these lines ( $1 / 2$ inch) was greatly oversized for this flow rate because any smaller piping would easily become plugged with coal particles. Thus, the flows in these lines were very low. Although the pumps, centrifuges, and vacuum filters purchased were the smallest available, they still were in most cases oversized for this application. The problems experienced with this equipment would probably not occur at higher flow rates or at commercial scale. For a more detailed description of the six unit operational systems that make up the MiCL process, see Section 2 of this Final Keport.

Most of the equipment (rotary kiln, water and acid washing centrifuges, the caustic regeneration system, and the waste water treatment system) operated well during Phase I and required only minor changes (i.e., cleaning of equipment, change-out of mixer propellers, change-out of oil, replacement of rupture discs with over-pressure sensing switches, and replacement of heaters, insulation, valves, pipe fittings, thermocouples, seals, and pump rotors and stators) in order to be operated on a long-term continuous bas is during Phase II. For a detailed list of the equipment repairs, maintenance and refurbishment performed to bring the MCL equipment to a condition that would support the original intended equipment use see Section 3 of the Phase II topical report entitled "Topical Report for Test Circuit Maintenance, Refurbishment, Modification, and Off-Line Operation". The objective of Phase II was to upgrade equipment to original performance levels without the addition of major capital equipment items but rather with the replacement of parts and materials. The overall strategy was to allow for the earliest startup of integrated operations during Phase II with a minimum 
of up front costs attributed to plant modifications. Only those

modifications that were required in order that the plant could be operated in an integrated continuous manner were implemented while other modifications that would improve plant operation were deferred until needed. Priority design modifications deemed critical for integrated operation were those required to bring the vacuum filters on-line (including kiln modifications) and those that would allow the evaporator to operate at design rates.

During Phase I testing, the vacuum filter system experienced intermittent flows to and from the filters, solids buildup in the filter pan, and blinding of the cloth on the filter drum, which resulted in the vacuum filters being bypassed for most of Phase I testing. Also during Phase I testing, the evaporator experienced limited throughput, low overall heat transfer coefficients, and periodic surges or upsets. Following is a discussion of the critical modifications made to the test circuit during Phase II. When these modificatir were completed, a series of off-line tests were performed to verify the operation of the vacuum filters and the evaporation unit. Based on the results obtained from the off-line tests, continuous integrated operation of the plant was resumed during Phase II.

\subsection{REACTOR SECTION CRITICAL MODIFICATIONS}

\subsubsection{Installation of Breaker Bar in Kiln Discharge Chute}

During Phase I operations, some of the larger lumps of coal/caustic mixture exiting the kiln caused bridging and plugging above the jaws of the crusher/delumper. This caused intermittent surges of coal/caustic solids into the dissolver tank, upsetting the flow to the wash section filters. A kiln discharge rotating breaker bar was designed, fabricated, and installed above the crusher/delumper to prevent large particles from hanging up and blocking the flow of coal into the delumper. The problem of bridging and plugging in the kiln discharge chute may not occur in large commercial scale equipment. The breaker bar worked well during Phase II integrated testing. This modification was made in concert with modifications to the vacuum filtration system (see Section 5.2) to allow continuous operation of the rotary drum vacuum filters. 


\subsubsection{Modification of the Ki]n Gas Scrubbing System}

During Phase I test operations, the kiln vent gas scrubbing system emitted a visible plume and noxious odors. In order to prevent this and to have a healthier working environment, an aqueous sulfuric acid packed column scrubber system (fan, acid holding tank, packed column, acid circulation pump, instrumentation) was designed and installed downstream of the existing aqueous caustic venturi scrubber system but ahead of the activated carbon bed. The acid scrubber removes hydrocarbon compounds not soluble in caustic solution. The acid scrubber worked well during Phase II testing.

\subsection{VACUUM FILTRATION SYSTEM CRITICAL MODIFICATIONS}

Numerous times during early Phase I plant operation, one rotary drum filter was put into service. At no time was the operation of the filter acceptable. In nearly all cases a large quantity of solids built up in the filter pan under the filter drum, eventually leading to blinding of the drum against the solids. It was determined that two major problems were occurring.

First, the flow rates in the vacuum filters were too low. (The suspension of the coal solids in the liquid is facilitated by liquid velocity.) As the slurry entered the large filter pan, settling occurred as evidenced by the solids building up in the bottom of the filter pan rather than on the drum. As the solids settled, the liquid was rapidly removed through the filter medium forcing the liquid level in the filter pan down, allowing the liquid vacuum seal to be broken. A complete loss of vacuum resulted.

Second, coal/caustic solids were delivered from the kiln into the dissolver/quench tank in clumps rather than in an even manner. As a result, for some short period, the liquid level in the tank would remain constant, the liquid level control would be satisfied and the pump at the tank outlet (which was tied to the liquid level control) would stop. Conversely, a clump of solids would fall into the tank, the level control would sense an increased level and the pump would run as fast as required to bring the level down to the set point. The recipient of this start/stop pump activity was 
the filter pan which would alterrately overflow and then starve the filter. The offending pump, which was sized for the design flow rate only, was too small to allow a pump-around loop permitting the filter pan to overflow continuously via a spiliback line to the dissolver/quench tank (thus preventing both starvation and settling). Both of these problems probably would not occur in large commercial scale equipment.

After it was discovered that the two above problems were at the root of the filter non-performance, the two filters were bypassed during the remainder of Phase I testing in order to operate the rest of the equipment in the tesi circuit. The following modifications were made to the vacuum filters during Phase II to overcome these problems.

\subsubsection{Modification of Vacuum Filter Plumbing to Allow for High Feed Flow Rates}

The first vacuum filter is fed from the kiln product dissolver/quench tank. During brase I testing, this fiow was quite small and intermittent in nature due to the problems discussed above. Although the filters are the smallest rotary drum units commercially available, they were still oversized for this plant. Many of the problems associated with the filters were a result of the oversized filters. The coal/caustic feed slurry had too much residence time in the filter pan because of low flow rates allowing for settling of coal in the pan. The result was that coal could not be kept in surpension in the filter feed pan which caused plugging and frequent disruptions in filter operation.

In order to bring the filters on-line, a constant high flow feed system for the vacuum filters was uesigned in Phase II which more closely matched the capacity of the filters. By replumbing the system, the flo' rates to the filters were greatly increased (a spillback to the dissolver taik was providef along with filtrate recycle). This increased the velocities in the feed and recycle lines to prevent plugging in the iines and the filter feed pan. Figure 5-1 shows the modified piping system for the first vacuum filter F-201. The excess material not drawn away by the filter is now recycled back to the dissolver tank, T-101, via a spillback line. The slurry drawn onto the filter is separated and dewatered, with the coal proceeding to the next stage as designed and che filtrate collected in the filtrate tank. Any 


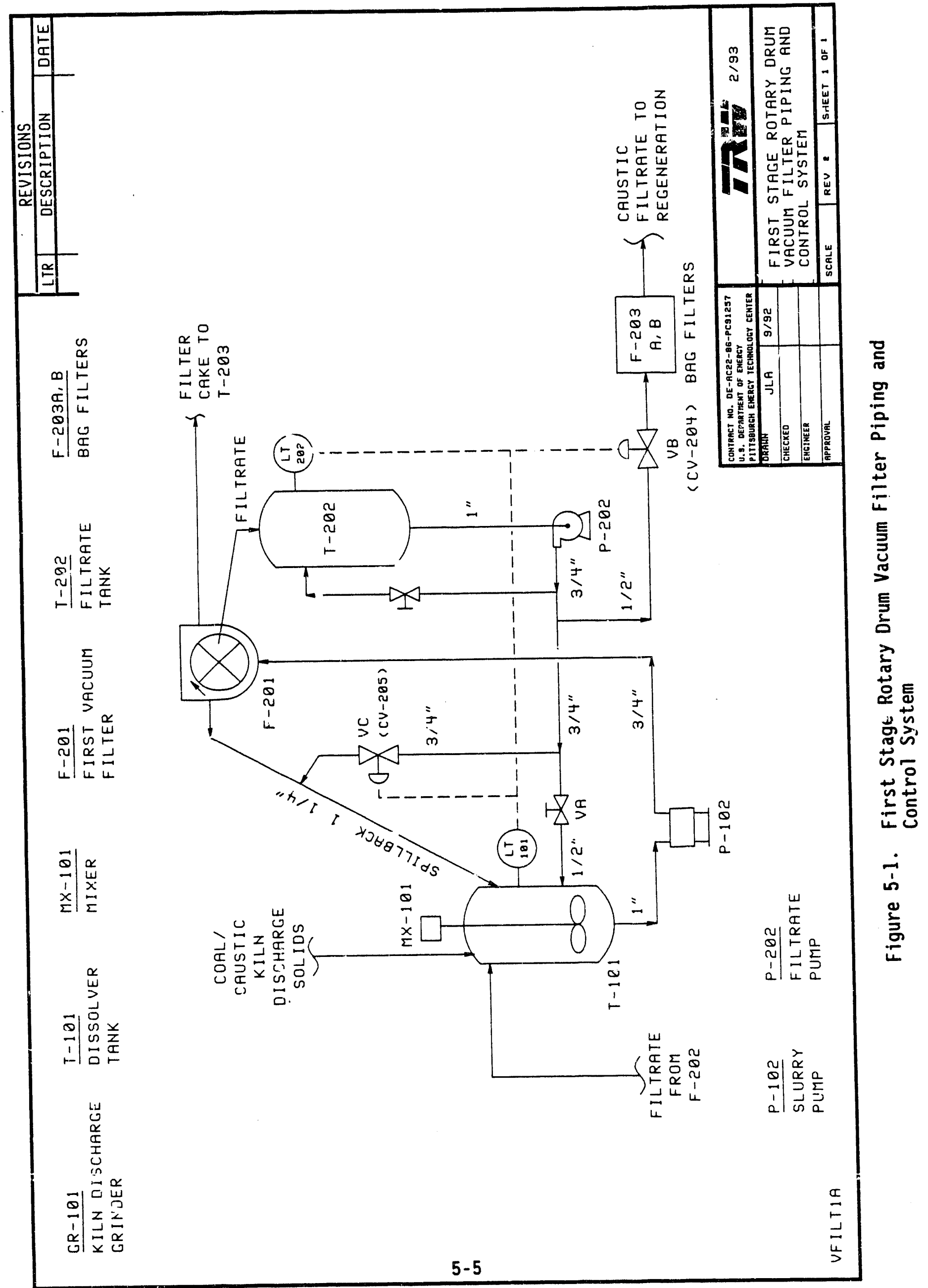


excess filtrate drawn through the filter cloth is recycled back to the dissolver tank from the filtrate tank, T-202 (see Figure 5-1), to maintain steady tank levels and a high feed flow rate. Similar plumbing modifications were made to the second vacuum filter, F-202. These modifications were made in concert with the installation of a kiln discharge breaker bar in the kiln discharge to prevent the intermittent disruption of the flow of solids to the dissolver/quench tank (see Section 5.1.1).

In the modified system, the filter feed pump is operated at much higher but steady flow rates to create turbulence in the filter feed pan to prevent coal settling. The existing first vacuum filter feed pump, P-102, was replaced and the new pump was rebuilt to provide a maximum 5 GPM discharge. The feed tubing was increased from $1 / 2$ inch to $3 / 4$ inch, and the spillback 1 ine was increased from $3 / 4$ inch to $1-1 / 4$ inches. A progressive cavity pump was rebuitit to increase its capacity to 5 GPM and installed to feed the second vacuum filter. Larger impellers were installed in both filtrate pumps to increase the discharge pressure and flow.

\subsubsection{Automation of the Vacuum Filtration System}

Control valves were installed on the net filtrate from the system (CV-204) and on the filtrate recycle (CV-205) to the dissolver tank to control the levels in the dissolver and filtrate tanks and the flow of recycled filtrate (see Figure 5-1). Control valve CV-205 controls the amount of filtrate recycled back to dissolver tank T-101, and CV-204 controls the amount of filtrate sent to caustic regeneration. A similar set of control valves (CV-206 and CV-207) was installed for the second vacuum filter (see Figure 5-2). The level switches in filtrate tanks T-202 and T-204 were replaced with bubbler level indicators.

Two PID control loops were programmed into the Yokogawa computer control system for each vacuum filter to maintain constant levels in the feed and filtrate tanks for each filter. The level indicators in the filtrate and dissolver/feed tanks are fed to the computer which adjusts tine newly installed control valves such that the levels in the tanks ar: maintained at preset levels by recycling some filtrate back to the dissolver/feed tanks and/or removing filtrate from the system. 


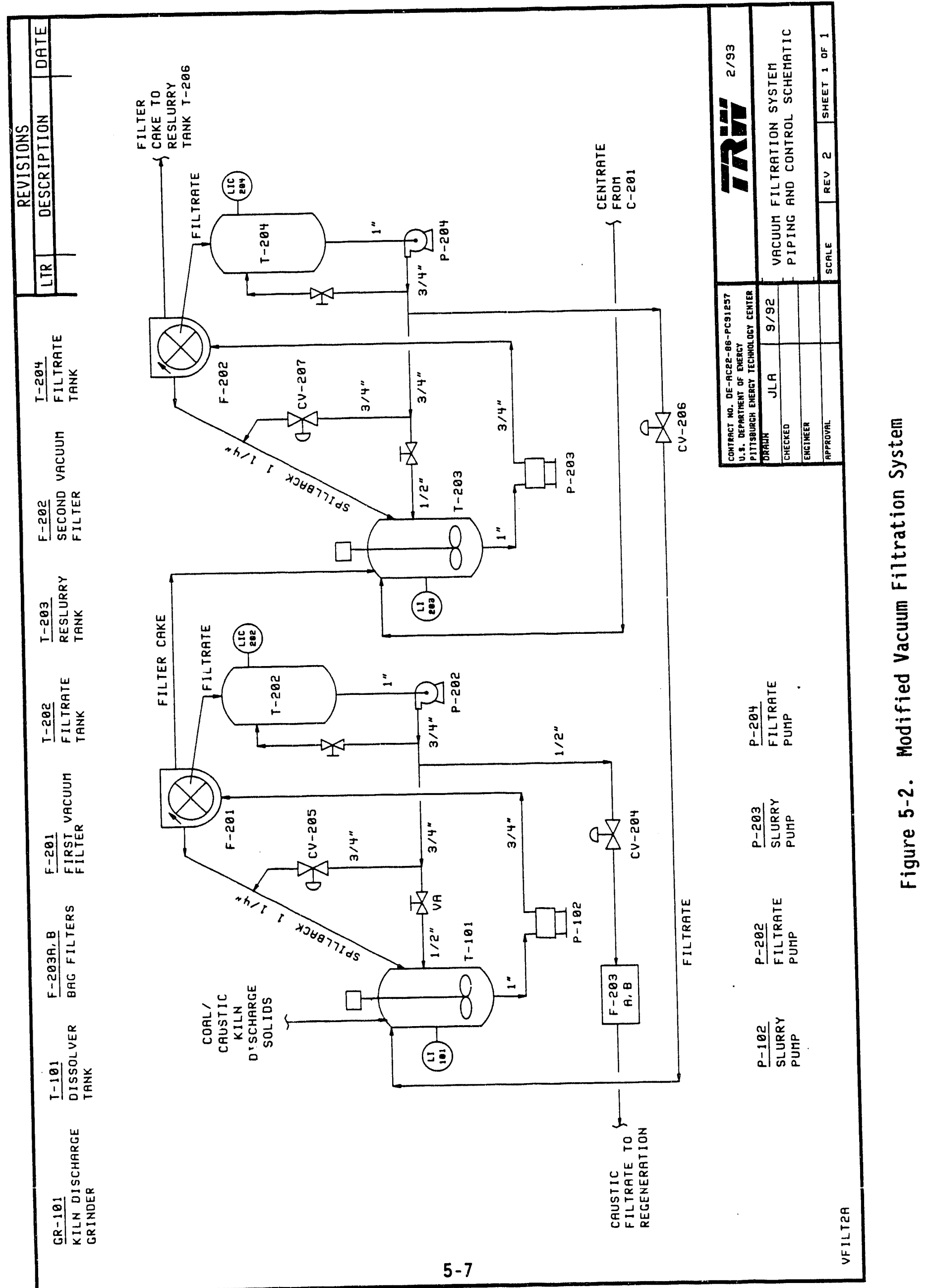




\subsection{WASHING SECTIONS CRITICAL MODIFICATIONS}

\subsubsection{Modification of the Wash Water Feed System to Allow for Higher Temperatures}

The wash water feed system to the water and acid wash sections was modified (addition of a heated tank (T-601), a circulation pump, insulation, level switch) to maintain the wash water temperature at levels up to $180^{\circ} \mathrm{F}$. The system was replumbed so that makeup $D I$ water and recycled condensate from the evaporator are pumped to tank T-601. This tank which has an immersion heater in it is now used to preheat the wash water. A pump-around loop external to this tank was installed to circulate the water in the tank to maintain uniform temperature (see figure 2-26).

In addition, the band heaters and insulation on each of the reslurry tanks in the wash system were upgraded in order to maintain high wash train temperatures (up to $180^{\circ} \mathrm{F}$ ) to facilitate the riquid-solids separations in the filters and centrifuges.

\subsubsection{Modification of the Anti-Foam Injection System}

The method (gravity drip) used during Phase I for injection of anti-foam into the water wash section was not reliable. Foaming in the dissolver and reslurry tanks and the filtrate tanks caused problems with the level sensors which lead to plugging in the bubbler sensors and false readings. Since level measurements in these tanks are now the key to the control of the filtration system, a continuous injection of anti-foam agent was needed. To eliminate plant upsets due to foaming caused by erratic injection of anti-foam solution, a pumped anti-foam injection system with manifolding was connected to dissolver T-101, filtrate tanks T-202 and T-205, and reslurry tanks T-209 and T-211. Flow is contrclled to each stage by using manually set drip valves.

\subsection{EVAPORATION SYSTEM CRITICAL MODIFICATIONS}

The evaporator unit was designed to process $180 \mathrm{lbs} / \mathrm{hr}$ of 30 percent aqueous caustic $(50: 50 \mathrm{NaOH}: \mathrm{KOH})$ or $130 \mathrm{lbs} / \mathrm{hr}$ of 50 percent caustic (50:50 $\mathrm{NaOH}: \mathrm{KOH}$ ) and dry these feeds to give a caustic product containing about eight percent moisture. Several tests were conducted in the caustic 
evaporation/flaking system during Phase I system operation. Due to the problems with the vacuum filters (see Section 5.2 above) which resulted in solutions of only $15 \%$ caustic being sent to the regeneration system, aqueous caustic evaporator feed solutions of $50 \%$ were specially prepared for these evaporator tests.

The limited throughput experienced and the 10w overall heat transfer coefficients strongly indicated tube fouling/plugging. In addition, periodic surges or upsets were experienced. However, visual inspection of the three evaporator tubes after the end of Phase I testing showed only a small amount of scale in any of the tubes. This does not necessarily indicate that scale in larger amounts was not present during operations. The evaporator tubes are thoroughly flushed with water after each run. Therefore, any scale present during operations which is water soluble may have been removed during the post-operation water flush. The following modifications were made to the evaporation system during Phase II to overcome the problems experienced.

\subsubsection{Addition of Caustic Preheater}

The evaporation unit had provided disappointing results including surging feed flow and much lower than expected heat transfer coefficients. The operation of the evaporator at high feed concentrations was reviewed by an evaporator consultant who had worked with TRW on other DOE programs. Based on the his recommendations, a new oil-heated caustic preheater was fabricated and installed upstream of the evaporator. It appeared that a significant fraction of the evaporator tube heat transfer surface was being utilized to preheat the incoming caustic solution to its initial boiling point. While the caustic feed was being preheated to its initial boiling point (before any steam is driven from solution), the liquid velocity in the tubes was extremely low $(<0.05 \mathrm{ft} / \mathrm{sec}$ ) resulting in very low overall heat transfer coefficients $\left(-30 \mathrm{Btu} / \mathrm{hr}-\mathrm{ft}^{2}-{ }^{\circ} \mathrm{F}\right)$ for the evaporator system. During Phase I testing, the caustic feed was heated to 150 to $200^{\circ} \mathrm{F}$ in the regeneration section by immersion coils and band heaters. The normal boiling point of 50 percent sodium hydroxide is $145^{\circ} \mathrm{C}\left(293^{\circ} \mathrm{F}\right)$. The hot oil-heated, double pipe caustic preheat exchanger installed upstream of the evaporator heats the caustic feed to the evaporator to its initial boiling 
point. Thus, the evaporator is now used strictly for boiling. The double pipe exchanger and the short line from it to the evaporation have been insulated. Results of Phase II testing of the caustic evaporator with the feed preheat exchanger in place have indicated a marked improvement in evaporator heat transfer coefficients.

\subsubsection{Upgrade of the Heating and Insulation for Operation With $\mathrm{NaOH}$ Only as the Caustic Feed Material}

The evaporator was originally designed for feed containing a mixture of $\mathrm{KOH}$ and $\mathrm{NaOH}$ rather than pure $\mathrm{NaOH}$. The mixture has a lower melting point than pure $\mathrm{NaOH}$. Thus, the use of pure $\mathrm{NaOH}$ required improvements in the heating and thermal insulation to reduce heat losses in the molten caustic piping to prevent freezing and plugging. The existing electric tracing and insulation were barely adequate for the mixed caustic feed during the Phase I program. Hot oil jacketing and insulation were installed on the molten caustic line (including the flanges and a valve) from the evaporator to the flaker, replacing electrical tracing. $\mathrm{NaOH}$ offers process economies in comparison to a $\mathrm{NaOH} / \mathrm{KOH}$ mixture.

\subsubsection{Modification of the Steam Condensing System}

The original caustic evaporation unit steam condensing system was undersized. Also, the condensate recycle pump was not adequate for the task. The intermittent and surging feed flow observed in the evaporator during Phase I was probably due in part to back pressure in the evaporator created by the undersized vapor line to the condenser. The size of the steam lines was increased from 1 -inch to 2 -inch during Phase II modifications to reduce the back pressure on the evaporator and thus reduce the surging observed in the evaporator. In addition, the second condenser, E-502, the second condensate drum, V-502, and the water cooler, E-503, were disconnected from the system to reduce the back pressure (see Figures 2-19 and 2-20). The condensate pump was replaced to allow recycle of the condensate to the wash train. The condensate is now pumped to tank T-601. This heated tank is now used to preheat the recycled condensate and makeup DI water to the wash system. 


\subsubsection{Reduction of Evaporator Scaling Potential}

The evaporator tubes were inspected internally with a boroscope at the beginning of Phase II. Evidence of a minor amount of scaling was observed, particularly in the lower third of the tubes. Tube scaling was suspected as a cause of the evaporator's performance degradation (decreased heat transfer rates) during Phase I testing. A sample of the scale deposits from one of the three evaporator tubes was taken and submitted for analysis. Analytical results of the scale concluded that the composition was mainly nickel hydroxide and nickel oxide, the base metal of the Inconel tube. Although, no detectable amounts of carbonate were found, it is likely that sodium carbonate $\left(\mathrm{Na}_{2} \mathrm{CO}_{3}\right)$ may have precipitated in the evaporator tubes (and was then removed in the post-operation water flush of the tubes). In order to reduce the carbonate content of the evaporator feed, the evaporator was operated with up to $50 \%$ caustic feeds during Phase II. The equilibrium solubility of sodium carbonate in sodium hydroxide drops to a very low value $(<1 \%)$ at $50 \%$ caustic concentration. Since the caustic concentration from the newly modified vacuum filter system was in the range of 20 to $25 \%$ during Phase II integrated testing, the caustic concentration to the evaporator was increased to $50 \%$ by metering anhydrous fresh caustic flake/beads into the first regeneration reactor, T-401, to precipitate the carbonate which was then removed from the regeneration reactor effluent (evaporator feed) by an existing centrifuge. The screw feeder from tank T-601 was moved to regeneration reactor $T-401$ and calibrated with caustic.

\subsection{OFF-LINE TESTING}

After the above modifications were made, seven off-line filter tests were performed in Phase II to evaluate how filter performance (cake formation, cake discharge, solids and liquid flows, fines losses) is affected by:

1) coal filtration requirements (up to plant design rate of $20 \mathrm{lbs} / \mathrm{hr}$ coal),

2) filter feed stream solids loadings, 3) filtrate caustic concentration, 4) drum speed, and 5) cloth mesh size (to minimize loss of fines to the filtrate). After four successful off-line filter tests using manual control, the filter system was tested in the automatic control mode. The two vacuum filters were successfully operated in series with automatic computer control. 
The filters passed an acceptable percentage (5 to 10\%) of coal feed as fines to the filtrate, were capable of processing $40 \mathrm{lbs} / \mathrm{hr}$ of $1: 1$ caustic:coal kiln solids (20 lbs/hr coal) or $45 \mathrm{lbs} / \mathrm{hr}$ of $2: 1$ caustic:coal (15 $\mathrm{lbs} / \mathrm{hr}$ coal), and produced filtrates with caustic concentrations of up to $-34 \%$. Three filter cloths were used ( 30 mesh, 40 mesh, and 100 mesh) to determine the cloth mesh size which gives the desired filtration rates without an excessive loss of fines to the filtrate.

Results from these filter tests indicated that the piping/control modifications will allow for smooth integrated filter operations, producing a suitable caustic stream for regeneration/evaporation.

A series of off-line evaporation tests were performed to check out the evaporator modifications, to determine the current condition of the evaporator tube heat transfer surfaces (as indicated by the overall heat transfer coefficient), and to establish baseline heat transfer coefficient data with deionized (DI) water and with caustic feed for future reference.

The evaporator test using DI water indicated that the tubes were in a relatively clean condition (and would not need to be chemically or mechanically descaled) and that the limited throughputs and the low overall heat transfer coefficients observed during Phase I testing were probably due to a combination of scaling and the large heat iransfer surface area required for preheating the caustic to its initial boiling point. (Water soluble scale may be present during a run and then removed when the evaporator is flushed with water after each run.) When the new evaporator preheater was put on-line, heat transfer coefficients for boiling DI water were calculated to be in excess of $250 \mathrm{Btu} / \mathrm{hr}-\mathrm{ft}^{2}-{ }^{\circ} \mathrm{F}$. The evaporator performed well with a fresh $50 \% \mathrm{NaOH}$ feed; with regenerated caustic, however, the evaporator continued to experience periodic upsets. Flaked caustic product with a moisture content of 5 to 6 percent was produced.

For a detailed description of the Phase II vacuum filtration and evaporation off-line tests see the Phase II topical report entitled "Topical Report for Test Circuit Maintenance, Refurbishment, Modification, and off-Line Operation". 
Based on the results obtained from the vacuum filtration and caustic evaporation off-line tests, TRW recommended to the DOE project office that the program go forward with the Phase II continuous integrated operation. The results of the Phase II integrated tests (Runs 10 and 11) are incorporated in the discussion of test results in Section 4 of this Final Report. 


\section{PROCESS AND REACTION MODELS}

\subsection{PROCESS MODEL}

A computer process model of the 20 pounds of coal per hour integrated $\mathrm{MCL}$ test circuit to be built was prepared at the beginning of this program. The model was based on the latest test data from the modular test circuit of the previous Gravimelt program. The advantages of generating a-computer model of the process are: 1) to identify each process step in the integrated test circuit, 2) to generate a material balance based on the latest test data which would provide compositions and flow rates for each processing stream, 3 ) to provide guidance during equipment sizing and selection (based on the stream compositions and flow rates), 4) to discover areas where the overall process would be sensitive to economic and operating parameters, 5) to serve as a guide for future MCL testing, and 6 ) to provide a basis for full scale economic studies.

The model's seven page printout, which has already been presented in Section 2 of this report, Test Circuit Description, is repeated in Tables 6-1 through 6-3 (see also Figures 6-1 through 6-3). The model is divided into three parts: 1) the overall material balance (Figure 6-1 and Table 6-1), 2) the water washing subroutine (Figure 6-2 and Table 6-2), and 3) the acid washing subroutine (Figure 6-3 and Table 6-3). Although the model is being presented here in three parts, in actuality, it is all part of one large interconnected Lotus spreadsheet. That is, any change in one part will affect the other two parts.

The printout for the first part of the model (the overall material balance) consists of three pages (Table 6-1). The first page contains two columns of input parameters (top part of page) and a few key calculated values (bottom of page). The input parameters are interconnected to the rest of the process model such that by changing any of the input parameters on this first page, the overall material balance will be recalculated.

The input parameters cover all aspects of the process such as coal feed rate and composition, product coal composition, caustic/coal ratio, kiln vent 


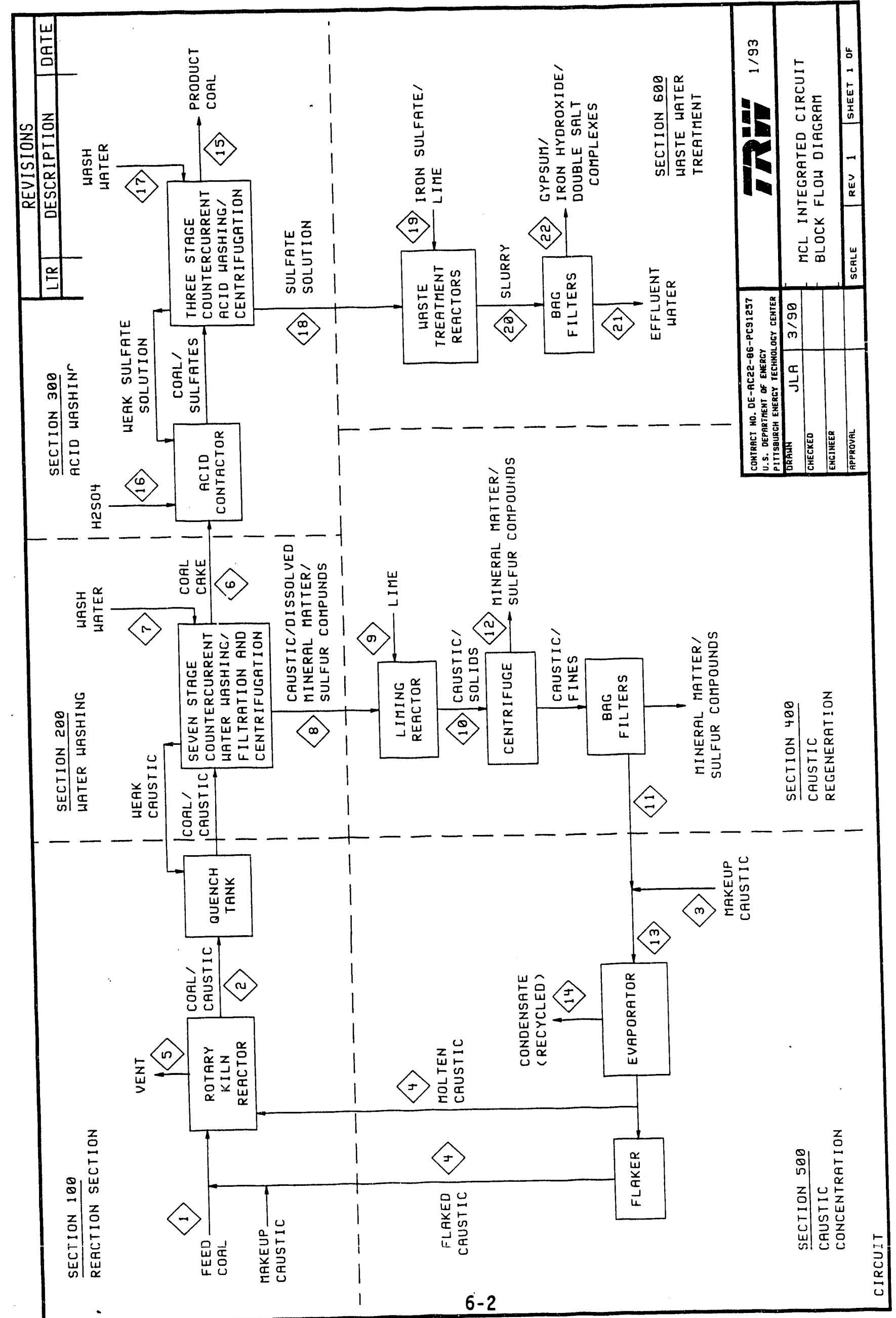




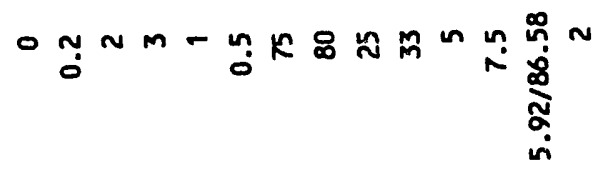

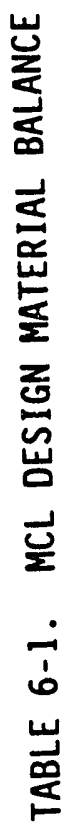

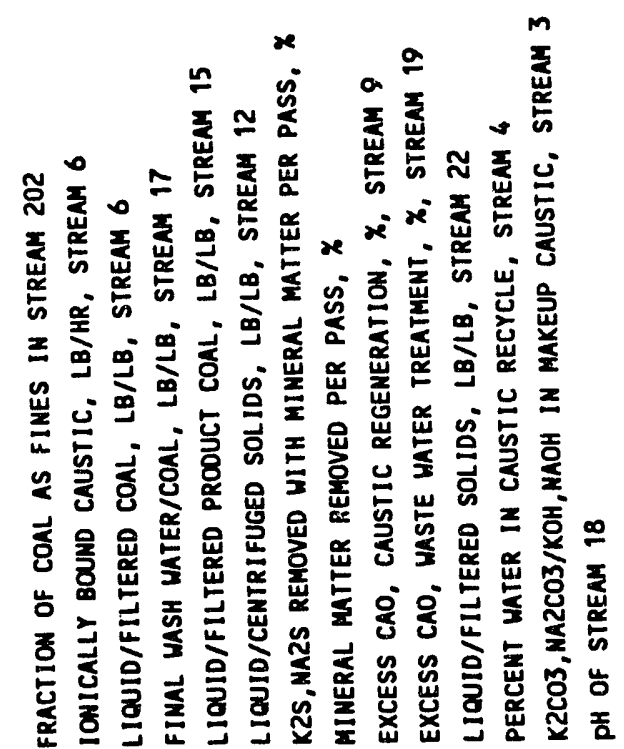

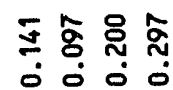

嵌

ำ

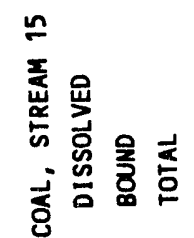

产

宩

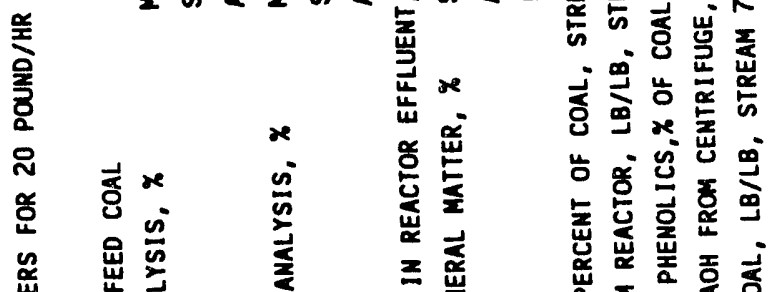

密

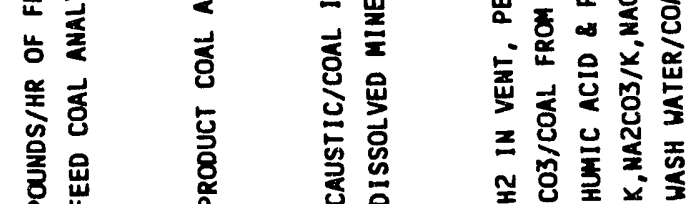

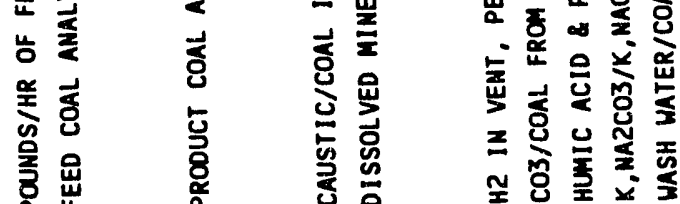

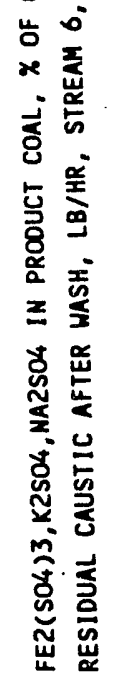




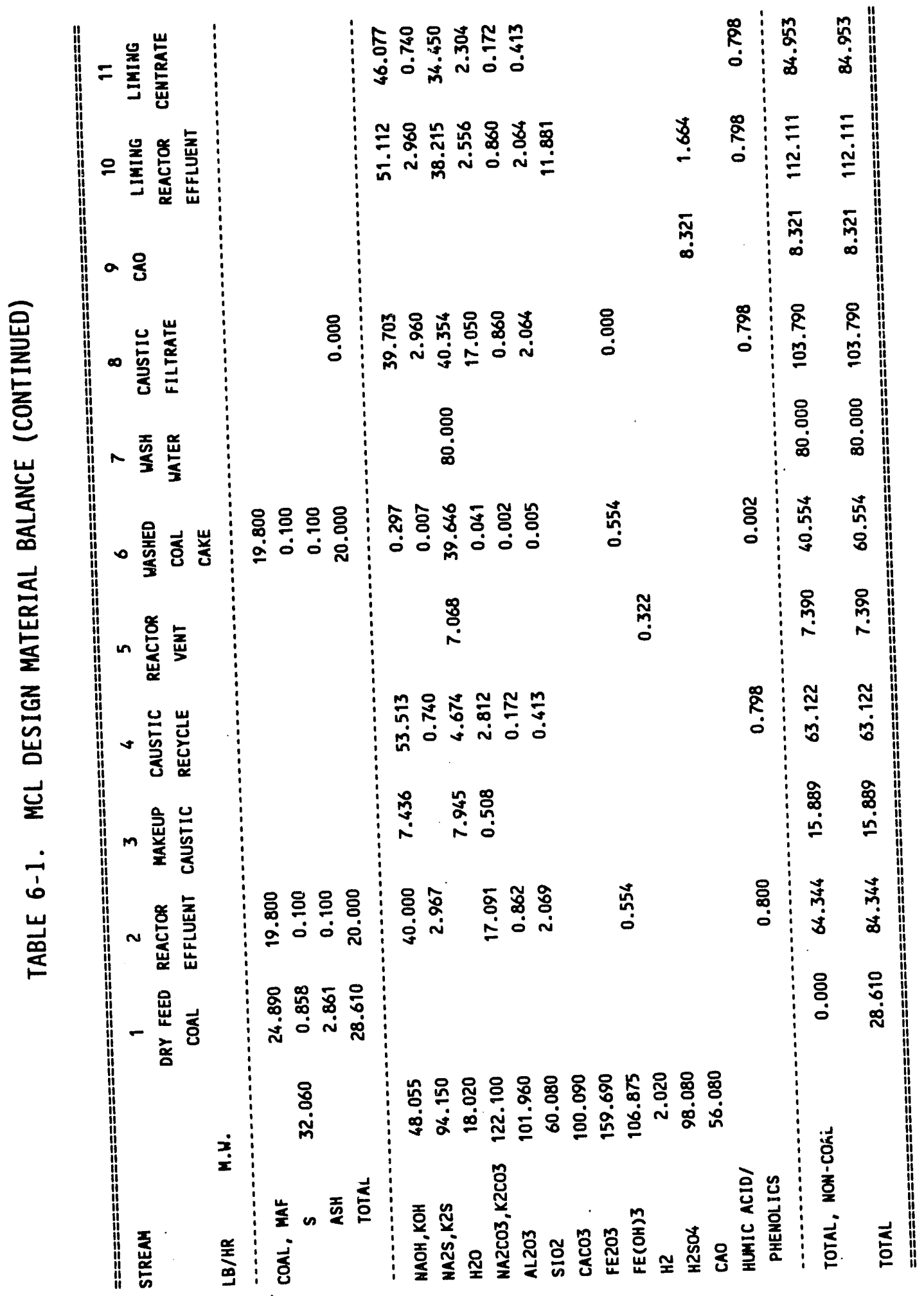




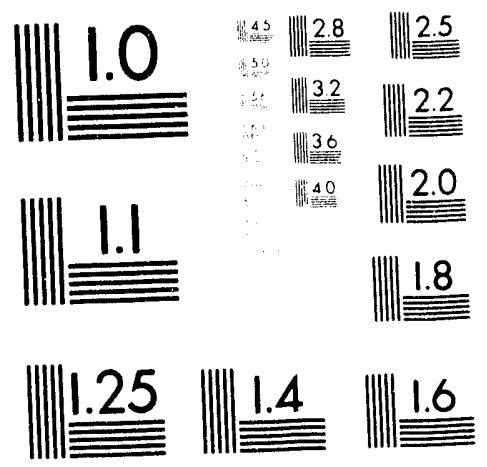



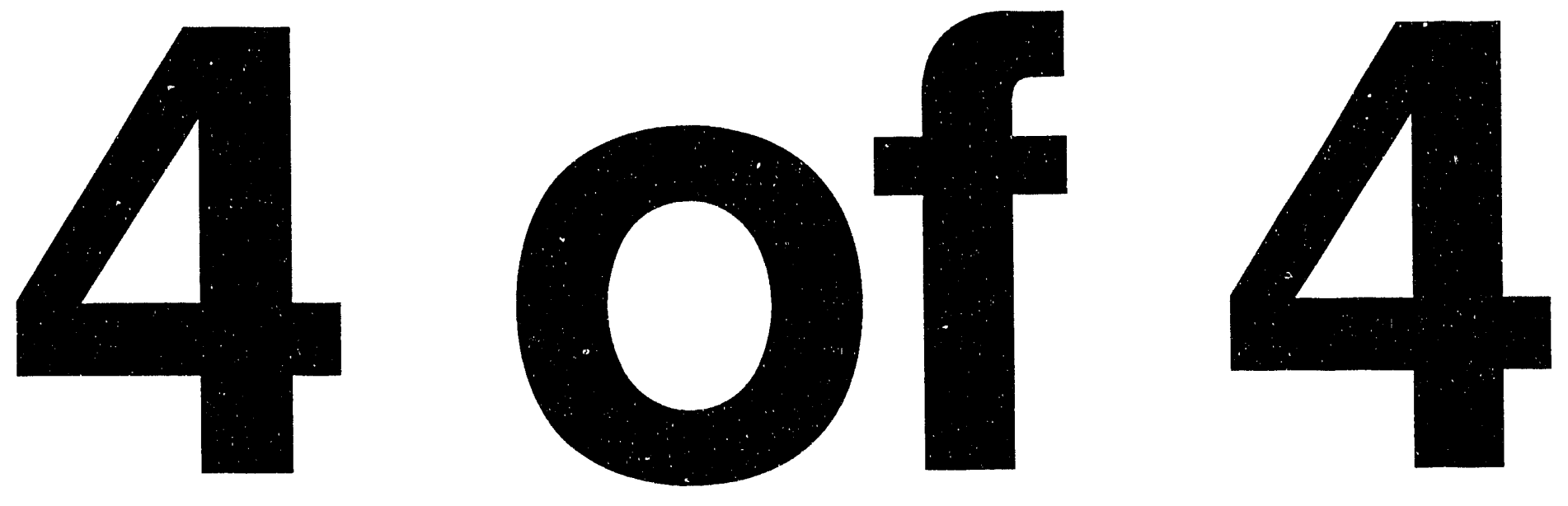


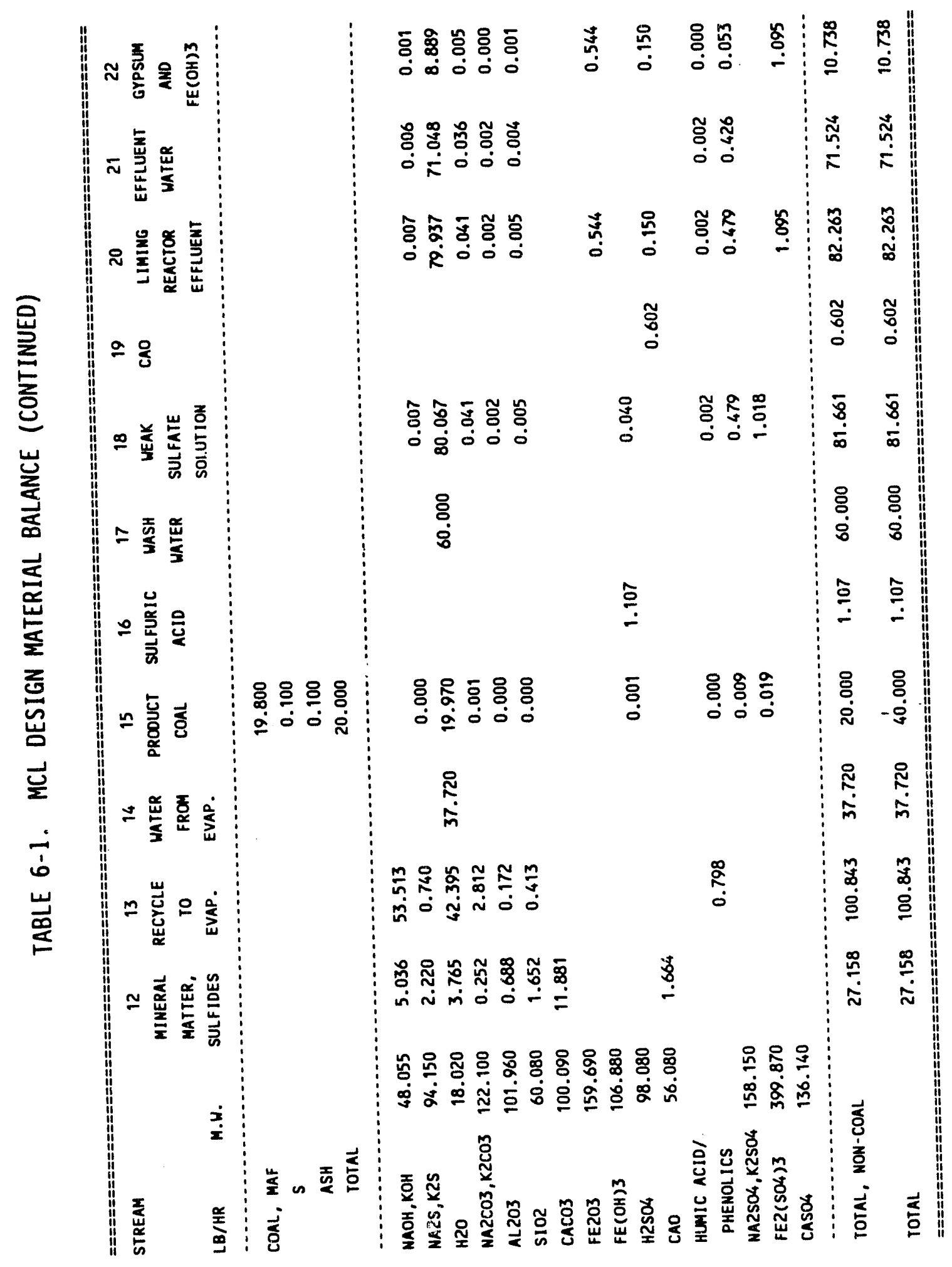




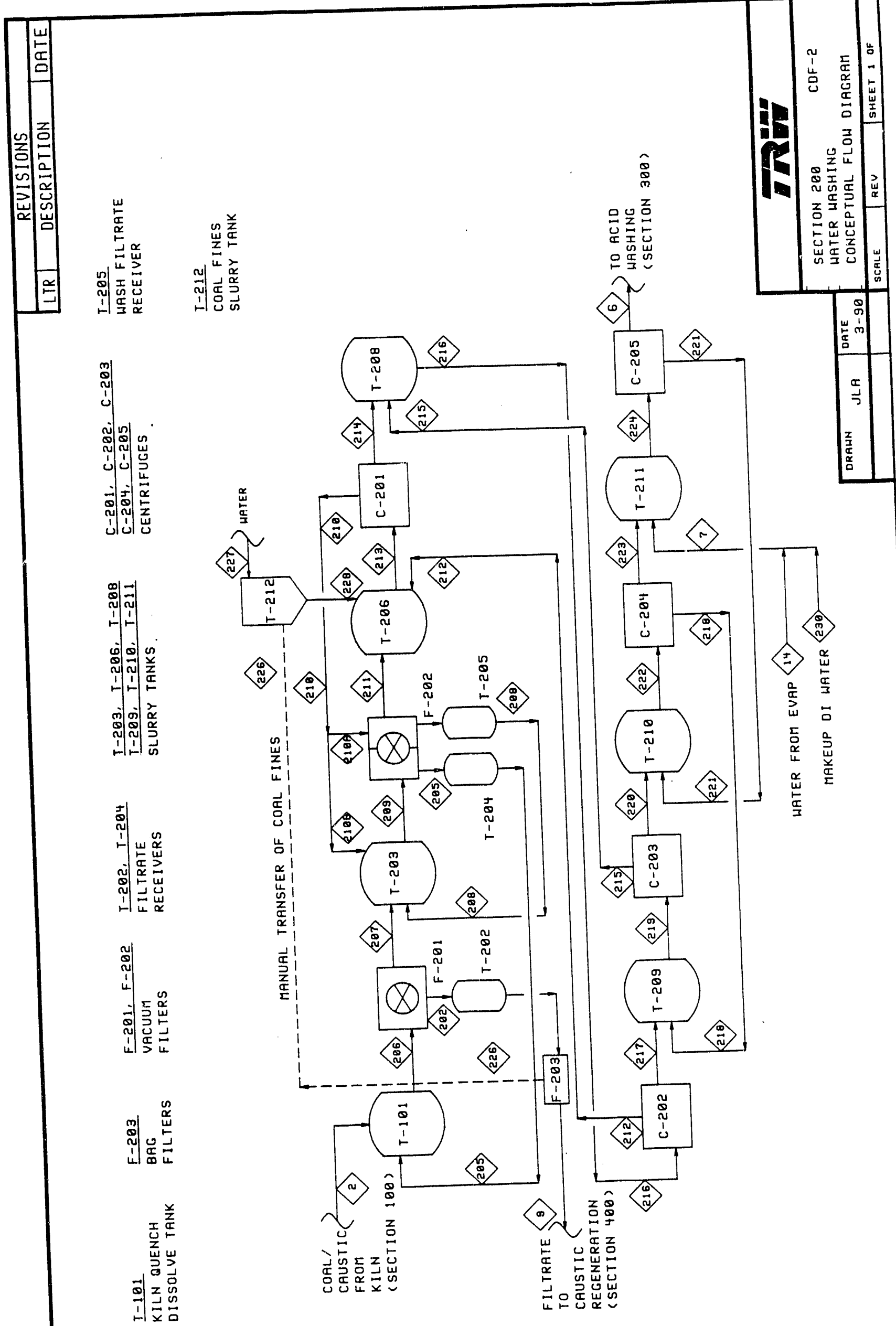




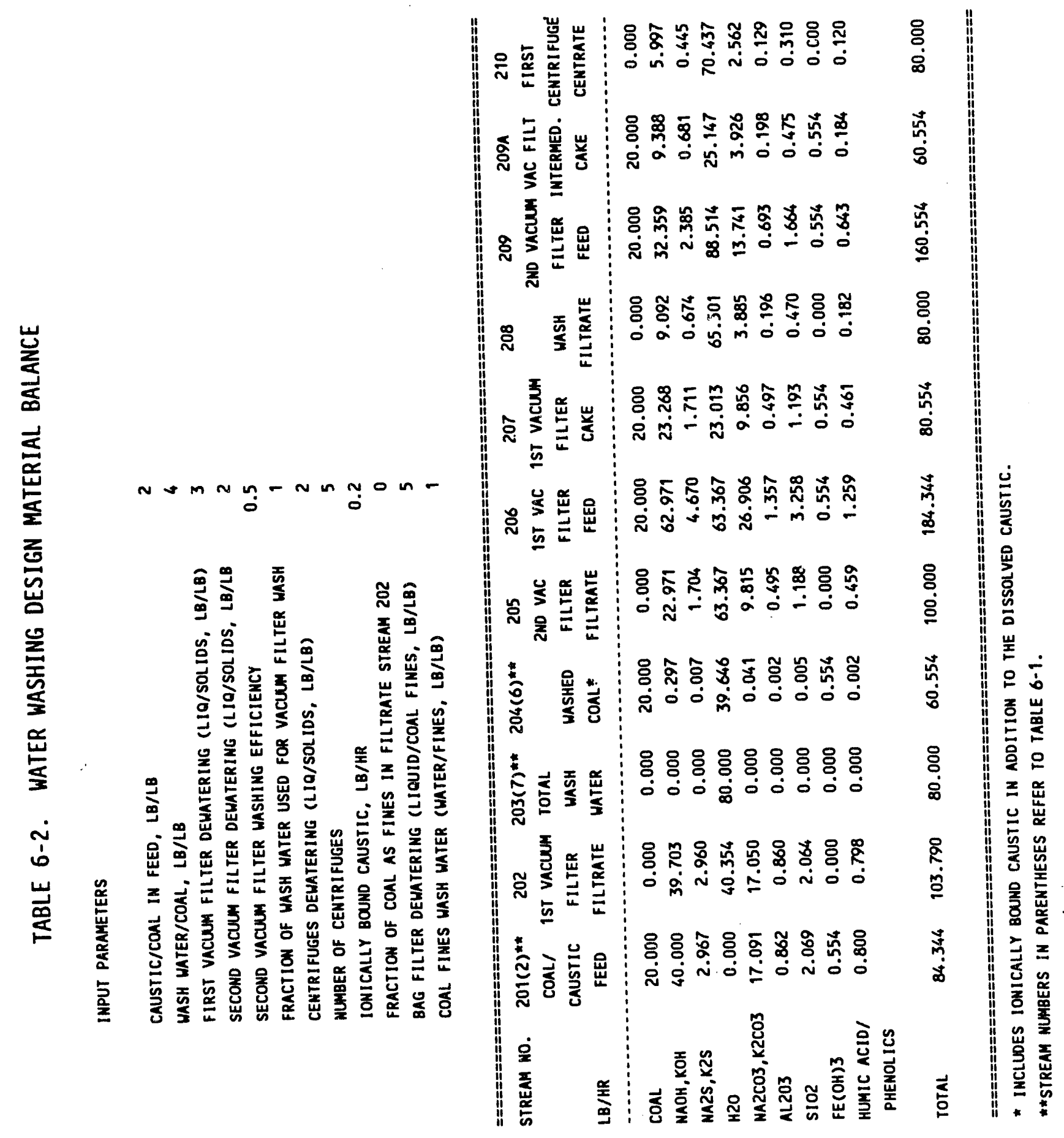




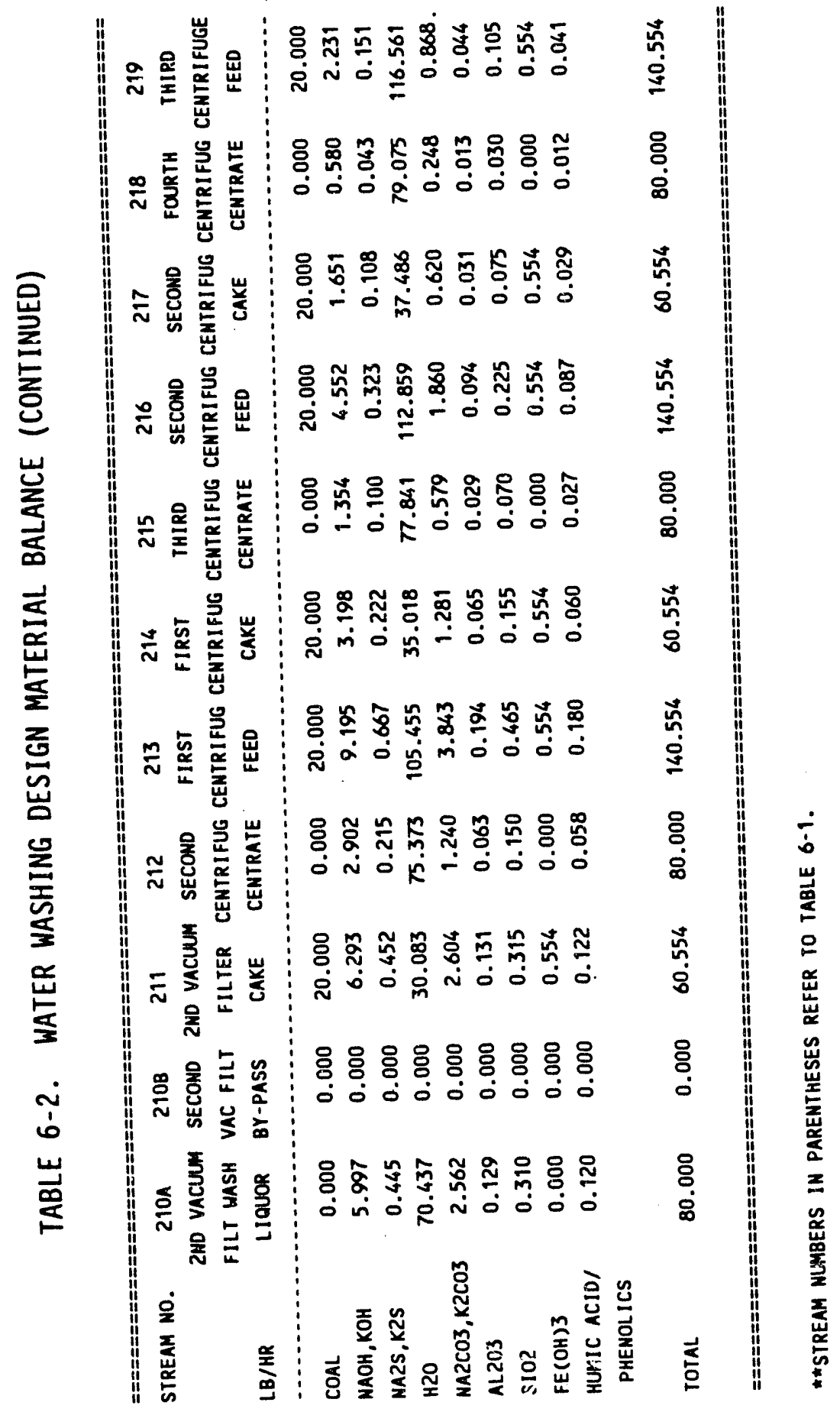




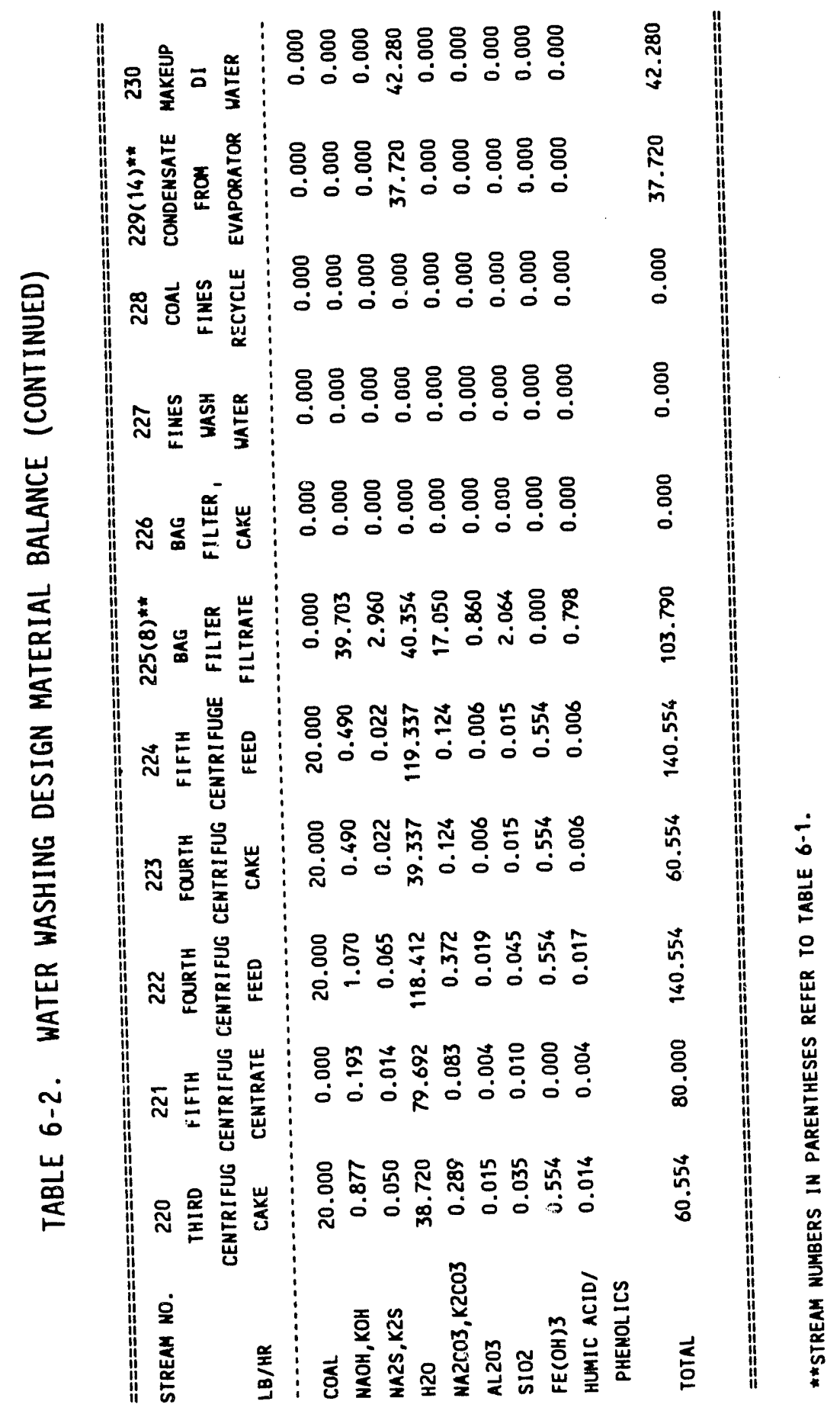




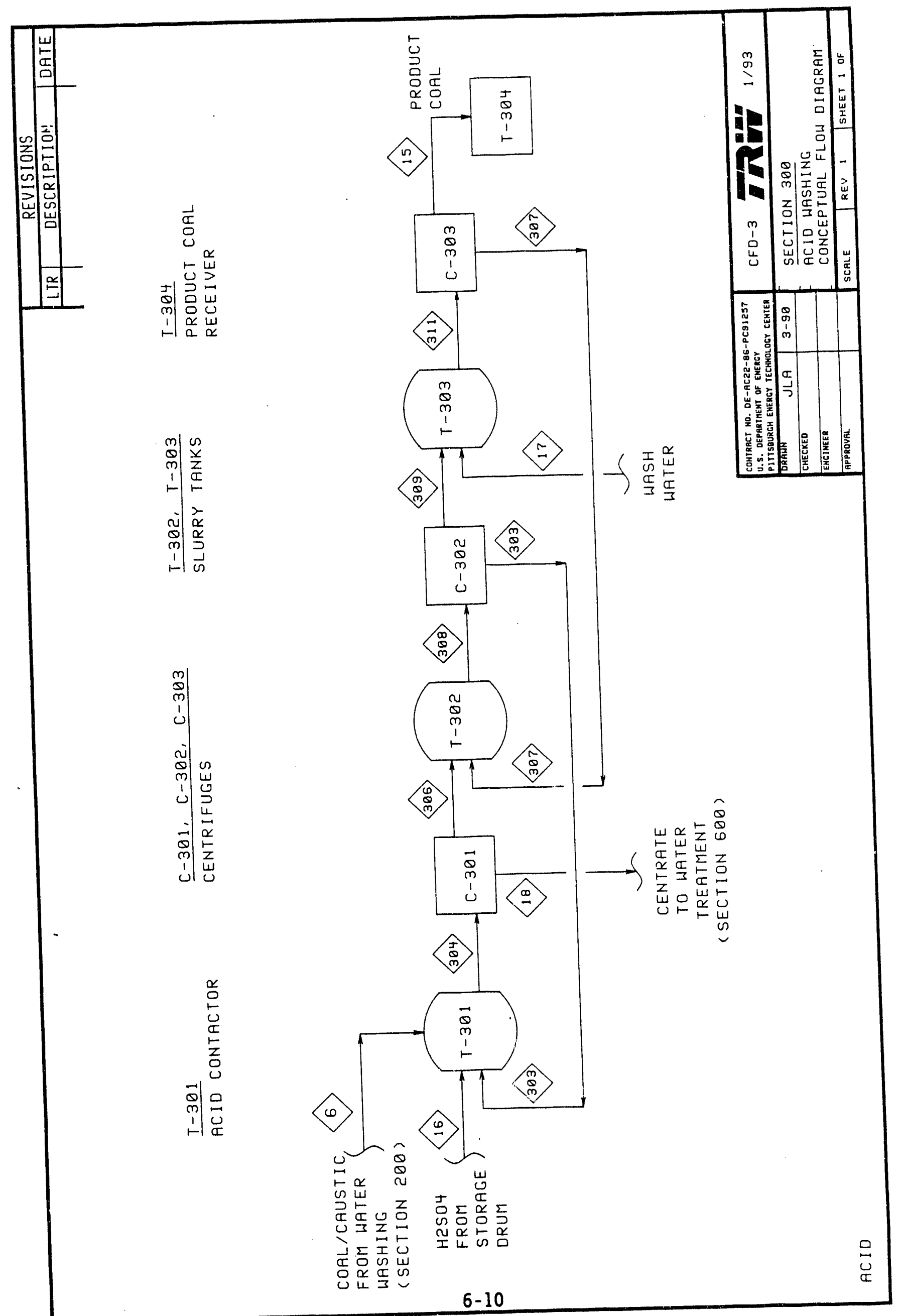




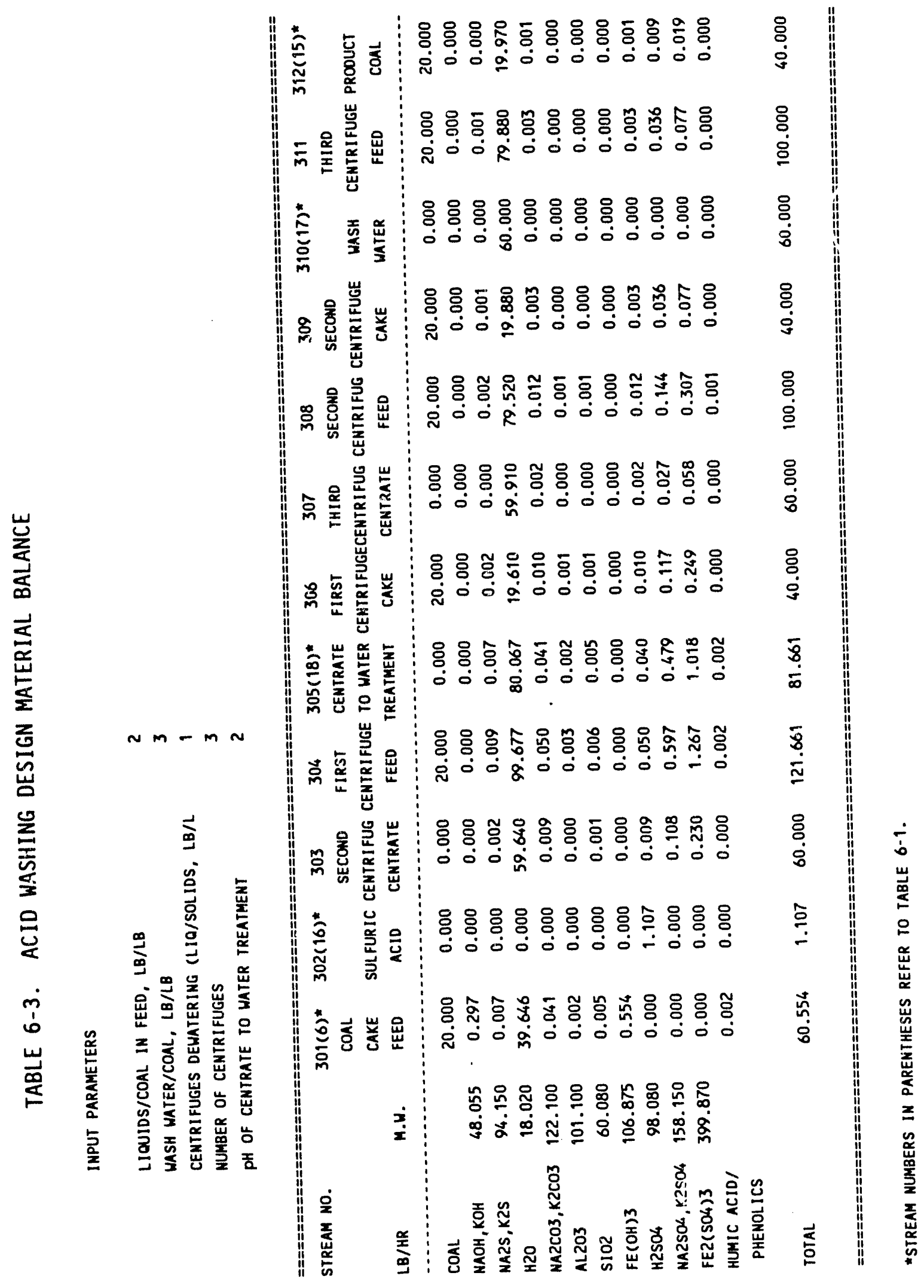


gas $\left(\mathrm{H}_{2}\right)$, carbonate formation in various processing steps, humic acid and phenolics formation, wash water to coal ratio in water and acid washing (each set independentiy), amount of ionically bound caustic, liquids to solids ratios in filter and centrifuge cakes (each set independentiy), percent excess lime in caustic regeneration and waste water treatment (each set independent1y), percent sulfur and mineral matter removed per pass in regeneration (each set independent1y), percent moisture in recycled caustic from evaporator.

Since the water and acid washing systems are rather complicated from a processing viewpoint due to the multiple filters and centrifuges and the countercurrent washing operation, the material balances for these two systems are calculated in two subroutines (Tables 6-2 and 6-3). The compositions and flow rates of the input streams to these subroutines (such as streams 2 and 6) are automatically taken from the overall material balance section of the spreadsheet. Likewise, the output streams from these two subroutines (such as streams 8 and 15) are automatically sent back to the overall material balance section. Some additional input parameters are located at the top of the printouts of these two subroutines.

In addition to the input parameters, the model assumes certain reactions to take place in various processing steps. The reactions used to generate the model are listed in Table 6-4. The extent to which some of these reactions occur (such as the carbonate reaction) can be controlled by the input parameters.

\subsection{REACTION MODEL}

As shown in Section 4.1 of this report, the residual sulfur and the residual ash of the coal product can be correlated with the caustic/coal ratio $(R)$ and the kiln wall temperature $\left(T\right.$ in ${ }^{\circ} C$ ) by regression analysis to obtain:

$$
\begin{aligned}
\% \text { sulfur } & =s_{0}-a_{s} \times R-b_{s} \times T \\
\% \text { ash } & =a_{0}-a_{a} \times R-b_{a} \times T
\end{aligned}
$$


TABLE 6-4. MCL PROCESS MODEL EQUATIONS

Rotary Kiln Reaction Section (Section 100)

$2 \mathrm{NaOH}+\mathrm{MAF}+\mathrm{S} \longrightarrow \mathrm{Na}_{2} \mathrm{~S}+\mathrm{MAF} \cdot \mathrm{O}+\mathrm{H}_{2} \mathrm{O}$

$\mathrm{MAF} \cdot 2 \mathrm{H} \longrightarrow \mathrm{H}_{2}+\mathrm{MAF}$

MAF.C. $\mathrm{O}_{2}+2 \mathrm{NaOH} \longrightarrow \mathrm{Na}_{2} \mathrm{CO}_{3}+\mathrm{H}_{2} \mathrm{O}+\mathrm{MAF}$

$\mathrm{Fe}_{2} \mathrm{O}_{3}+3 \mathrm{H}_{2} \mathrm{O} \longrightarrow 2 \mathrm{Fe}(\mathrm{OH})_{3}$

Water Washing Section (Section 200)

No Reactions

Acid Washing Section (Section 300)

$\mathrm{H}_{2} \mathrm{SO}_{4}+2 \mathrm{NaOH} \longrightarrow \mathrm{Na}_{2} \mathrm{SO}_{4}+2 \mathrm{H}_{2} \mathrm{O}$

$2 \mathrm{Fe}(\mathrm{OH})_{3}+3 \mathrm{H}_{2} \mathrm{SO}_{4} \longrightarrow \mathrm{Fe}_{2}\left(\mathrm{SO}_{4}\right)_{3}+6 \mathrm{H}_{2} \mathrm{O}$

Caustic Regeneration Section (Section 400)

$\mathrm{Na}_{2} \mathrm{CO}_{3}+\mathrm{H}_{2} \mathrm{O}+\mathrm{CaO} \longrightarrow 2 \mathrm{NaOH}+\mathrm{CaCO}_{3} \quad$ (Not Used)

Caustic Concentration Section (Section 500)

No Reactions

Waste Water Treatment Section (Section 600)

$\mathrm{CaO}+\mathrm{H}_{2} \mathrm{SO}_{4} \longrightarrow \mathrm{CaSO}_{4}+\mathrm{H}_{2} \mathrm{O}$

$\mathrm{Fe}_{2}\left(\mathrm{SO}_{4}\right)_{3}+3 \mathrm{CaO}+3 \mathrm{H}_{2} \mathrm{O} \longrightarrow 3 \mathrm{CaSO}_{4}+2 \mathrm{Fe}(\mathrm{OH})_{3}$ 
where values for the regression coefficients for Pittsburgh No. 8 (Powhatan No. 6 mine) coal are:

$\begin{array}{lccccccc}\text { Coal Type } & \underline{\text { Caustic }} & \underline{s}_{0} & \underline{a}_{s} & \underline{b}_{s} & \underline{a}_{0} & \underline{a}_{a} & \underline{b}_{a} \\ \text { Pitt No. 8 } & 50: 50 \mathrm{KOH} / \mathrm{NaOH} & 10.143 & 0.3697 & 0.01975 & 11.870 & 0.7869 & 0.02191 \\ \text { Pitt No. 8 } & \mathrm{NaOH} & 9.785 & 0.6440 & 0.01756 & 2.774 & 0.7416 & 0.00174\end{array}$

The regression analysis excluded data points for runs with shutoff of one or two kiln heaters. Therefore, the correlations represent runs operated with kiln residence time of approximately 2 hours, caustic/coal ratios between 1 and 3 , and kiln wall temperatures between $390^{\circ} \mathrm{C}$ and $441^{\circ} \mathrm{C}$. Within these operational limits, the above correlations and regression coefficients can be used in the process model to determine the sulfur and ash contents of the Pittsburgh (Powhatan mine) coal product after MCL processing. There are insufficient data points to obtain similar correlations with Kentucky No. 9 coal feed because of the fewer number of tests conducted or with the Pittsburgh No. 8 (Blacksville No. 2 mine) coal feed because kiln conditions were not varied during the test (Run 11) conducted with this coal.

For tests with caustic/coal ratios of 2 or greater, it is also shown in Section 4.1 that good correlations can be obtained with the kiln wall temperature alone by regression analysis. In these cases, this values for the regression coefficients $a_{s}$ and $a_{0}$ are zero and the values for the other regression coefficients with Pittsburgh No. 8 (Powhatan No. 6 mine) coal are:

$\begin{array}{lccccc}\text { Coal Type } & \text { Caustic } & \underline{s}_{0} & \underline{b}_{s} & \underline{a}_{0} & \underline{b}_{a} \\ \text { Pitt No. 8 } & 50: 50 \mathrm{KOH} / \mathrm{NaOH} & 11.393 & 0.02478 & 15.941 & 0.03588 \\ \text { Pitt No. 8 } & \mathrm{NaOH} & 11.443 & 0.02466 & 7.010 & 0.01551\end{array}$

The linear dependence of the sulfur and ash contents of MCL coal product on temperature can be explained by using the following reaction models:

$$
\begin{aligned}
d S_{\text {org }} / d t= & -A \exp (-E / R \theta) \cdot \phi \cdot S_{\text {org }} \\
S & =S_{\text {org }}+S_{\text {sulfate }} \\
d A s h / d t & =-A \exp (-E / R \theta) \cdot \phi \cdot \text { Ash }
\end{aligned}
$$


where $t$ is the reaction time, $A$ is the frequency factor, $E$ is the activation energy, $R$ is the gas constant, $\theta$ is the reaction temperature in ${ }^{\circ} \mathrm{K}$ (an average of $30^{\circ} \mathrm{K}$ lower than the kiln wall temperature), $\phi$ is a molten caustic penetration factor or effectiveness factor that varies between 0 and $1.0, S_{\text {org }}$ is the organic sulfur content of the coal, and Ash is the ash content of the coal. For a caustic/coal ratio greater or equal to 2 , it is assumed that all coal particles are in intimate contact with molten caustic and $\phi=1.0$. For a caustic/coal ratio less than $2, \phi$ will have a value less than 1.0. Also, based on the data presented in Section 4.2 , the amount of pyritic sulfur in MCL coal is effectively zero, whereas the amount of sulfate sulfur in $M C L \operatorname{coal}\left(S_{\text {sulfate }}\right)$ appears to be invariant of processing conditions and has an average value of $0.28 \%$. Integration of equations [6-3] and [6-5] lead to the following results:

$$
\begin{aligned}
S_{\text {org }} / S_{\text {org }, i} & =\exp \{-A \cdot t \cdot \phi \cdot \exp (-E / R \theta)\} \\
\text { Ash } / A s h_{i} & =\exp \{-A \cdot t \cdot \phi \cdot \exp (-E / R \theta)\}
\end{aligned}
$$

where $S_{\text {org, }} i$ represents the initial value of organic sulfur in feed coal (2.54\% for Pittsburgh No. 8, Powhatan No. 6 mine), and Ash; represents the initial value of 2 sh in feed coal $111.57 \%$ for Pittsburgh No. 8, Powhatan No. 6 mine). The values of $A$ and $E$ in equations [6-6] and [6-7] can be obtained from equations [6-1] and [6-2] and their associated regression coefficients, by first deriving a linear approximation for the term $\exp (-E / R \theta)$ :

$$
\begin{aligned}
\exp (-E / R \theta) & =\exp \left\{\left(-E / R \theta_{0}\right)\left[1+\left(\theta_{0}-\theta\right) / \theta_{0}\right]\right\} \\
& =\exp \left(-E / R \theta_{0}\right)\left\{1-\left(E / R \theta_{0}^{2}\right)\left(\theta_{0}-\theta\right)\right\}
\end{aligned}
$$

where $\theta_{0}$ is a constant temperature term chosen so that $\left(\theta_{0}-\theta\right) / \theta_{0}<<1.0$. In the operating region of interest, $\theta_{0}$ is selected to be $400^{\circ} \mathrm{C}$. After substituting the expression [6-8] into [6-6] and [6-7], the terms that do not contain $\theta$ in [6-6] and [6-1] are equated, and the terms that contain $\theta$ in [6-6] and [6-1] are also equated. These provide two simultaneous equations to determine the $A$ and $E$ values for the organic sulfur reaction, assuming that the reaction time is 2 hours and using only data with caustic/coal ratio equal or greater than 2.0 , so that $\theta=1.0$. The same 
procedure is used to determine the $A$ and $E$ values for the ash reaction. The frequency factor and Arrhenius activation energy values determined in this manner for Pittsburgh No. 8 (Powhatan No. 6 mine) coal are:

\begin{tabular}{|c|c|c|c|c|}
\hline Coal Type & Caustic & Reaction & $\frac{\text { Frea Factor }}{\left(h r^{-1}\right)}$ & $\frac{\text { Activation Energy }}{(\mathrm{kcal} / \text { inole })}$ \\
\hline $\begin{array}{l}\text { Pitt No. } 8 \\
\text { Pitt No. } 8 \\
\text { Pitt No. } 8 \\
\text { Pitt No. } 8\end{array}$ & $\begin{array}{c}50: 50 \mathrm{KOH} / \mathrm{NaOH} \\
\mathrm{NaOH} \\
50: 50 \mathrm{KOH} / \mathrm{NaOH} \\
\mathrm{NaOH}\end{array}$ & $\begin{array}{l}\text { Org Sulf } \\
\text { Org Sulf } \\
\text { Ash } \\
\text { Ash }\end{array}$ & $\begin{array}{l}1.4784 \times 10^{9} \\
5.867 \times 10^{8} \\
5.7335 \times 10^{6} \\
1.0509 \times 10^{4}\end{array}$ & $\begin{array}{l}28.451 \\
27.136 \\
20.223 \\
11.629\end{array}$ \\
\hline
\end{tabular}

These determined values can be used in equations [6-6] and [6-7] to compute the organic sulfur and the ash contents of the MCL coal product. The total sulfur content of the MCL coal product can then be determined by adding $0.28 \%$ sulfate sulfur to the organic sulfur content. In Table 6-5, the computed values of the sulfur and ash contents of the MCL coal product are compared with the experimentally determined values. These comparisons show fairly good agreements between the reaction model predicted sulfur and ash values and the corresponding experimental values in all cases in the operating region of $\mathrm{kiln}$ wall temperature $\geq 413^{\circ} \mathrm{C}$ and caustic/coal ratio $\geq 2.0$.

In determining the frequency factor $A$, the effective $k i$ in residence time is assumed to be two hours. If it is determined in future analysis that the effective kiln residence time were to be less than two hours, then the frequency factor would be increased proportionally because the product A.t $t$ is the term determined from matching the reaction model to the experimental correlation. This adjustment would not change the computed values in Table 6-5 because the product $A \cdot t$ is used in all the computations. 
TABLE 6-5. COMPARISON OF REACTION MODEL AND EXPERIMENTAL SULFUR AND ASH CONTENTS OF MCL COAL PRODUCT

Pittsburgh No. $8 ; 50: 50 \mathrm{KOH} / \mathrm{NaOH}$

Kiln Wall, ${ }^{\circ} \mathrm{C}$ React Temp, ${ }^{0} \mathrm{~K}$ Org Sulf,\% Tot Sulf,\% Expt Sulf,\% (Run)

\begin{tabular}{lllll}
413 & 656.16 & 0.946 & 1.23 & \multicolumn{1}{c}{$1.31(5 \mathrm{~A} 1)$} \\
427 & 670.16 & 0.535 & 0.82 & $0.77(2 \mathrm{C}), 0.64(7 \mathrm{C})$, \\
432 & 675.16 & 0.409 & 0.69 & $0.56(70), 0.59(9 \mathrm{~A})$ \\
438 & 681.16 & 0.281 & 0.56 & $0.71(3 \mathrm{~B} 1), 0.72(3 \mathrm{~B})$ \\
& & & & $0.65(9 \mathrm{Al}), 0.49(9 \mathrm{~B})$,
\end{tabular}

Pittsburgh No. 8; $\mathrm{NaOH}$

Kiln Wall, ${ }^{\circ} \mathrm{C}$ React Temp, ${ }^{\circ} \mathrm{K}$ Org Sulf,\% Tot Sulf,\% Expt Sulf,\% (Run)

$\begin{array}{ccccc}413 & 655.91 & 0.990 & 1.27 & 1.32(5 \mathrm{~B} 1) \\ 415.5 & 658.66 & 0.908 & 1.19 & 1.25(4 \mathrm{C} 2) \\ 420 & 662.91 & 0.785 & 1.06 & 1.22(4 \mathrm{C} 3)(1.20(5 \mathrm{~B} 2) \\ 427 & 670.16 & 0.585 & 0.87 & 1.19(5 \mathrm{BS}) \\ 438 & 692.16 & 0.154 & 0.43 & 0.40(9 \mathrm{C})\end{array}$

Pittsburgh No. $8 ; 50: 50 \mathrm{KOH} / \mathrm{NaOH}$

\begin{tabular}{|c|c|c|c|}
\hline Kiln Wall, ${ }^{\circ} \mathrm{C}$ & React Temp, ${ }^{0} \mathrm{~K}$ & Ash, \% & Expt Ash,\% (Run) \\
\hline $\begin{array}{l}413 \\
427\end{array}$ & $\begin{array}{l}656.16 \\
670.16\end{array}$ & $\begin{array}{l}1.41 \\
0.63\end{array}$ & $\begin{array}{c}1.23(5 \mathrm{~A} 1) \\
0.64(2 \mathrm{C}), 0.68(7 \mathrm{C}),\end{array}$ \\
\hline $\begin{array}{l}432 \\
438\end{array}$ & $\begin{array}{l}675.16 \\
681.16\end{array}$ & $\begin{array}{l}0.44 \\
0.28\end{array}$ & $\begin{array}{c}0.79(3 B 3) \\
0.23(3 B 1), 0.23(3 C 1) \\
0.33(9 A 1), 0.27(9 B)\end{array}$ \\
\hline
\end{tabular}

Pittsburgh No. 8; $\mathrm{NaOH}$

\begin{tabular}{|c|c|c|c|}
\hline Kiln Wall, ${ }^{\circ} \mathrm{C}$ & React Temp, ${ }^{0} \mathrm{~K}$ & Ash, \% & Expt Ash,\% (Run) \\
\hline $\begin{array}{c}413 \\
415.5 \\
420 \\
427 \\
438\end{array}$ & $\begin{array}{l}656.16 \\
658.66 \\
662.91 \\
670.16 \\
692.16\end{array}$ & $\begin{array}{l}0.69 \\
0.62 \\
0.53 \\
0.39 \\
0.13\end{array}$ & $\begin{array}{c}0.67(5 B 1) \\
0.54(4 C 2) \\
0.52(4 C 3), 0.58(5 B 2) \\
0.45(5 B 5) \\
0.15(9 C)\end{array}$ \\
\hline
\end{tabular}


APPENDIX A

MCL RUN DATA SUMMARY

$A-1$ 
MCL RUN DATA SUMMARY (10/24/1989)

RUN NO.

COAL SEAM

RUN DATE

TIME

$\mathrm{KOH} / \mathrm{NaOH}$

CAUSTIC/COAL

KILN WALL TEMP, $C$

ZONE 1

ZONE 2

ZONE 3

ZONE 4

ZONE I SENS TEMP, $C$

KILN RES TIME, hrs

KILN RPM

AUGER SPEED, \% MAX

AUGER SEC F/SEC B

AVG FEED, LB/HR

WASH WATER, LB/HR

PRODUCT COAL:

$$
\text { S, \% (dry basis) }
$$

Ash, \% (dry basis)

BTU/1b (dry basis)

Vols, \% (dry basis) Moisture, \% w/w

No. of $S$ Data Pts

REMOVAL:

S, \% (MAF basis)

Ash, \% (dry basis)

BTU/Ib, \% (dry basis)

Vols, \% (MAF basis) ib S02/MMBTU, \%

Ib SO2/MMBTU, \%(ROM)

REMARKS

S Analysis Data

Ash Analysis Data

Vol Analysis Data

Moisture Anal Data

\begin{tabular}{|c|c|c|c|}
\hline $\begin{array}{r}1 A \\
\text { PITT } 8 \\
1 / 23 / 89 \\
0400-1430 \\
1 \\
1\end{array}$ & $\begin{array}{r}1 \mathrm{~B} \\
\mathrm{PITT} 8 \\
1 / 23-24 / 89 \\
1430-1630 \\
1 \\
1.5\end{array}$ & $\begin{array}{r}1 C \\
\text { PITT } 8 \\
1 / 24-26 / 89 \\
1630-300 \\
1 \\
1.5\end{array}$ & $\begin{array}{r}10 \\
\text { PITT } 8 \\
1 / 26-27 / 89 \\
0300-1015 \\
1 \\
2\end{array}$ \\
\hline $\begin{array}{r}390 \\
390 \\
390 \\
390 \\
\text { No Data } \\
1.5 \\
2 \\
30 \\
20 \mathrm{~F} / 10 \mathrm{~B} \\
19.2 \\
120\end{array}$ & $\begin{array}{r}390 \\
390 \\
390 \\
390 \\
\text { No Data } \\
1.5 \\
2 \\
30 \\
20 \mathrm{~F} / 10 \mathrm{~B} \\
19.6 \\
120\end{array}$ & $\begin{array}{r}410 \\
410 \\
410 \\
410 \\
\text { No Data } \\
1.5 \\
2 \\
30 \\
20 F / 10 \mathrm{~B} \\
19.5 \\
120\end{array}$ & $\begin{array}{r}410 \\
410 \\
410 \\
410 \\
\text { No Data } \\
1.5 \\
2 \\
30 \\
20 \mathrm{~F} / 10 \mathrm{~B} \\
20.6 \\
120\end{array}$ \\
\hline 2.32 & $\begin{array}{l}1.93 \\
2.08\end{array}$ & $\begin{array}{l}1.43 \\
1.32\end{array}$ & $\begin{array}{l}1.39 \\
1.12\end{array}$ \\
\hline 32.77 & $\begin{array}{r}34.88 \\
51.59 \\
9\end{array}$ & $\begin{array}{r}27.4 \\
55.1 \\
8\end{array}$ & $\begin{array}{r}30.36 \\
55.17 \\
9\end{array}$ \\
\hline 79.95 & $\begin{array}{l}59.18 \\
82.02\end{array}$ & $\begin{array}{l}69.99 \\
88.59\end{array}$ & $\begin{array}{l}70.89 \\
90.32\end{array}$ \\
\hline 23.14 & $\begin{array}{l}18.40 \\
54.75 \\
73.28\end{array}$ & $\begin{array}{l}36.39 \\
66.47 \\
80.20\end{array}$ & $\begin{array}{l}29.66 \\
67.47 \\
80.75\end{array}$ \\
\hline & TRW CP & TRW CP & TRW CP \\
\hline $\begin{array}{l}\text { TRW CP } \\
\text { TRW CP }\end{array}$ & $\begin{array}{l}\text { TRW CP } \\
\text { TRW CP } \\
\text { TRW CP }\end{array}$ & $\begin{array}{l}\text { TRW } C P \\
\text { TRW CP } \\
\text { TRW } C P\end{array}$ & $\begin{array}{l}\text { TRW } C P \\
\text { TRW CP } \\
\text { TRW CP }\end{array}$ \\
\hline
\end{tabular}

* Residence times for all runs were calculated based on 1 imited coal mass balance, feed rate and product discharge rate data from several runs. Also, nitrogen flow was in cocurrent direction with coal/caustic flow in Runs 1 to 6 , and only 14 mesh coal was used as coal feed in these runs. 
MCL RUN DATA SUMMARY

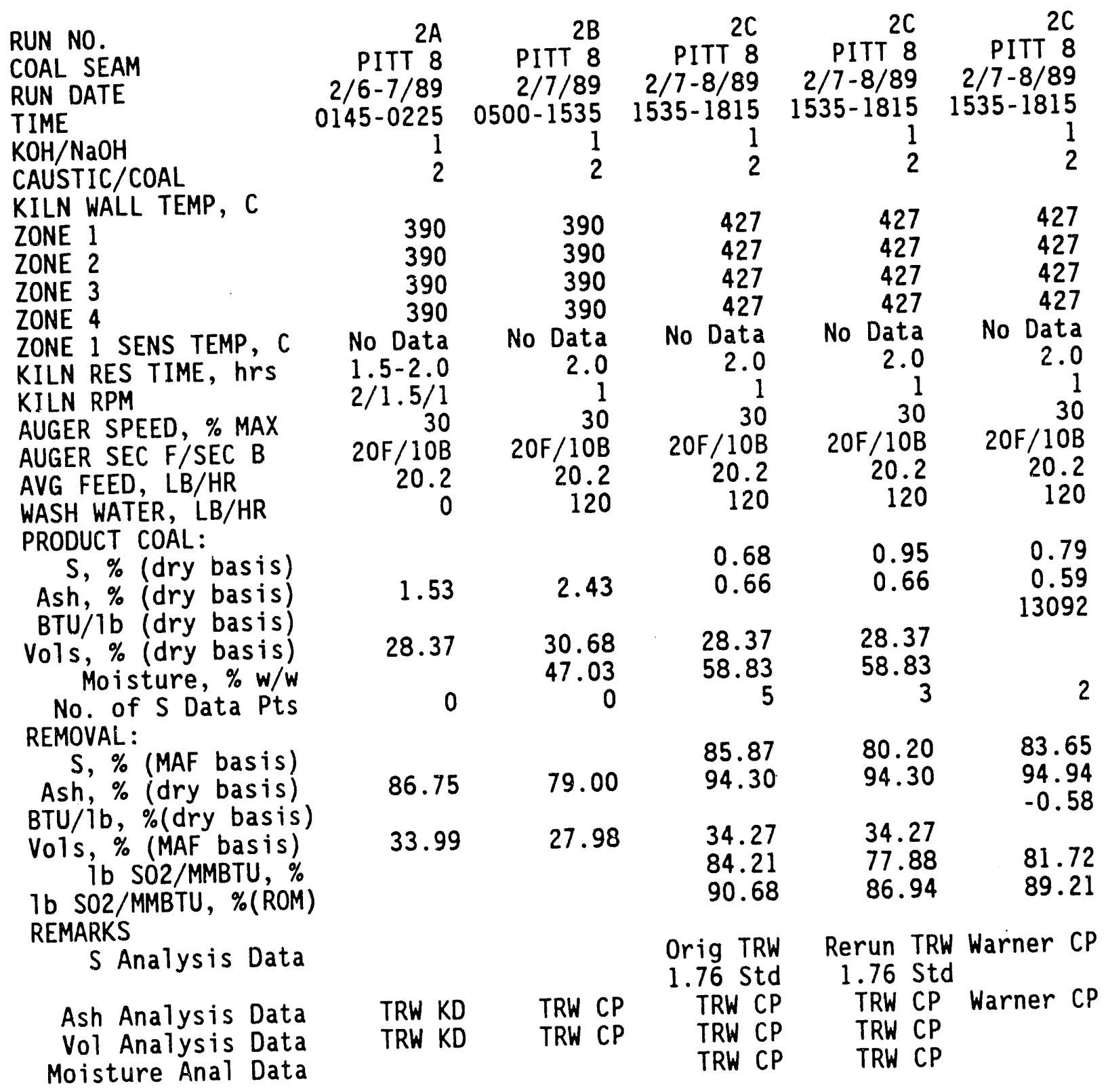


MCL RUN DATA SUMMARY

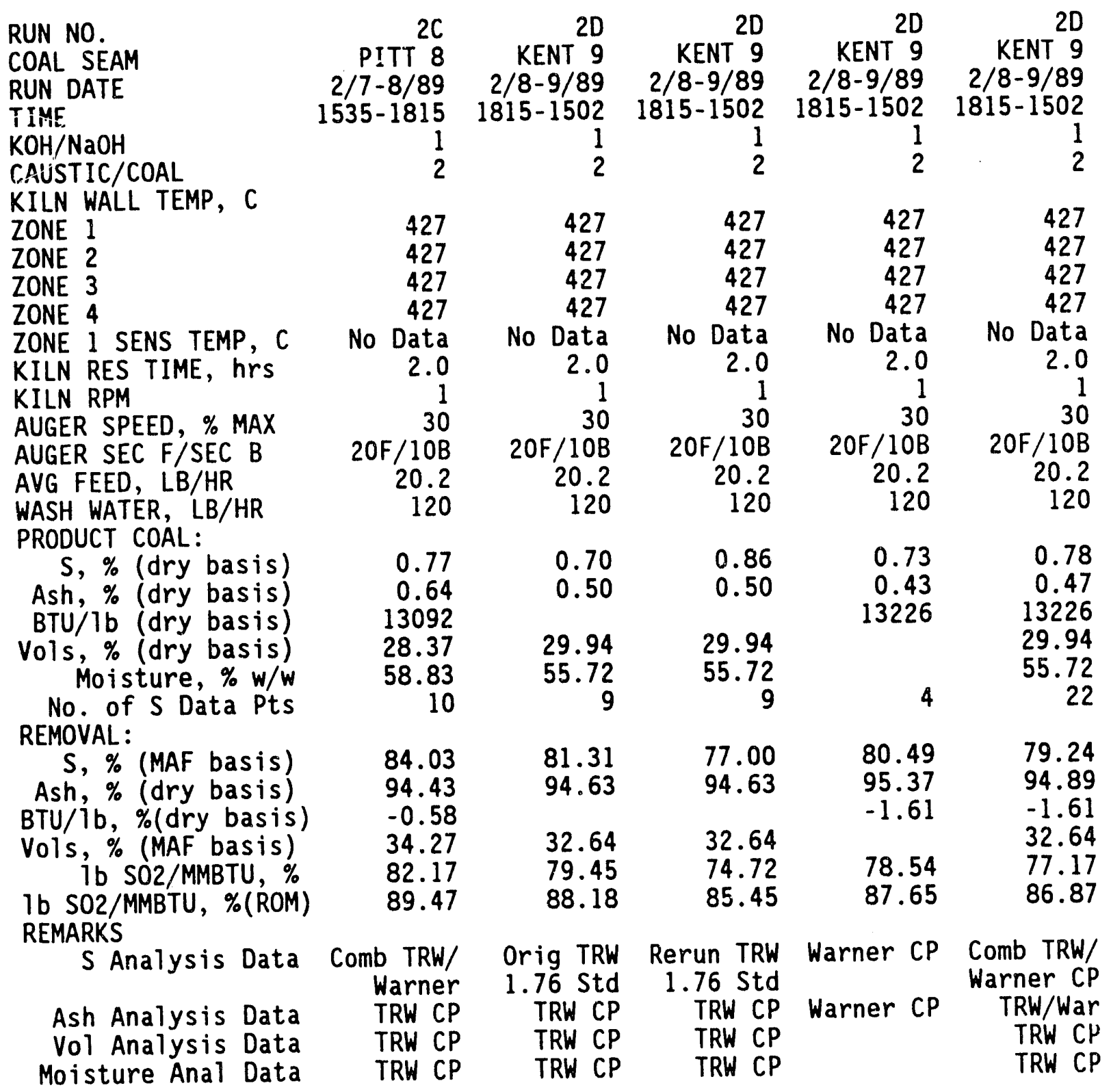


MCL RUN DATA SUMMARY

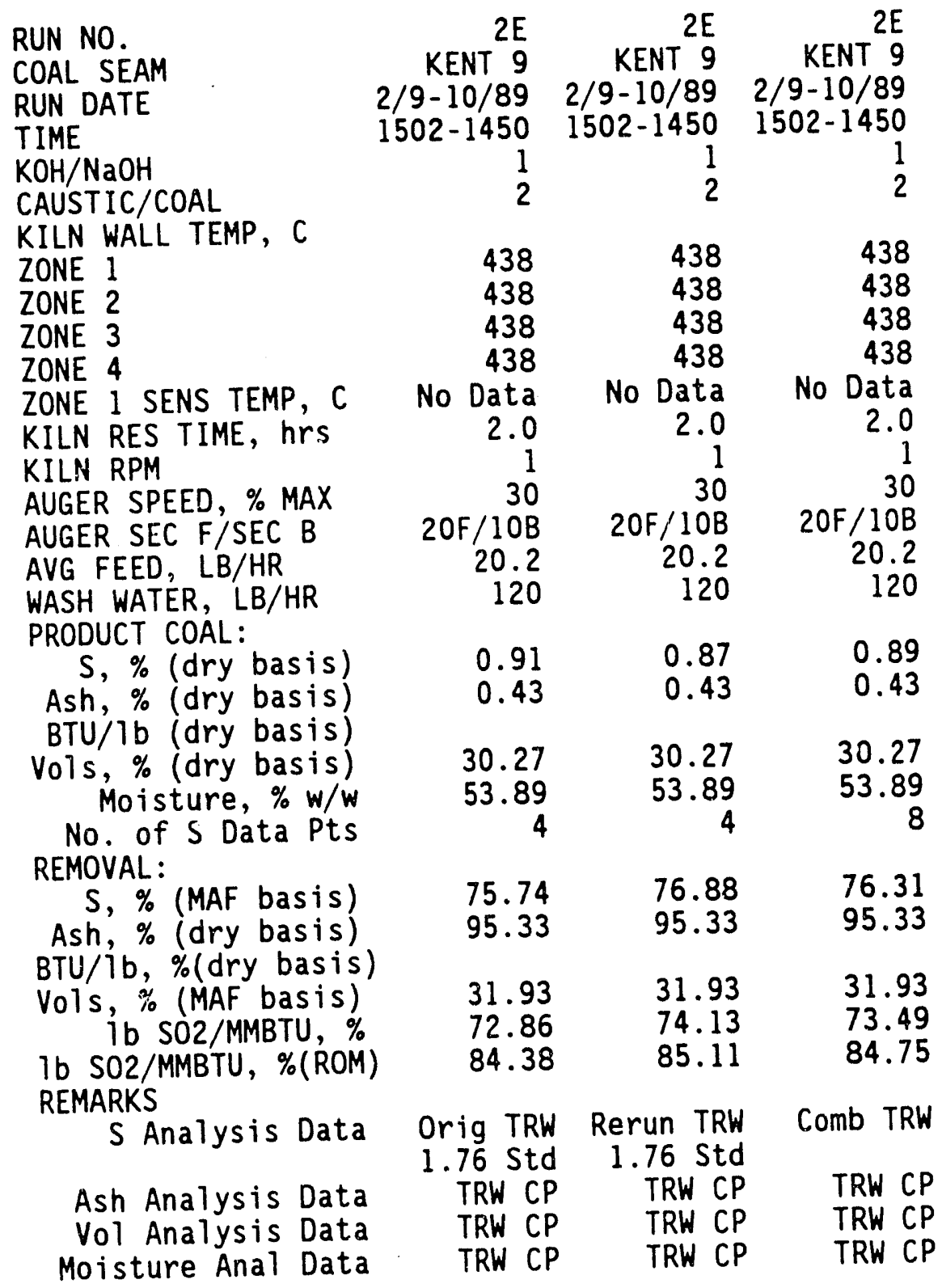


MCL RUN DATA SUMMARY

RUN NO.

COAL SEAM

RUN DATE

TIME

$\mathrm{KOH} / \mathrm{NaOH}$

CAUSTIC/COAL

KILN WALL TEMP, $C$

ZONE 1

ZONE 2

ZONE 3

ZONE 4

ZONE 1 SENS TEMP, $C$

KILN RES TIME, hrs

KILN RPM

AUGER SPEED, \% MAX

AUGER SEC F/SEC B

AVG FEED, LB/HR

WASH WATER, LB/HR

PRODUCT COAL:

S, \% (dry basis)

Ash, \% (dry basis)

BTU/1b (dry basis)

Vols, \% (dry basis) Moisture, \% w/w

No. of S Data Pts REMOVAL:

$$
\text { S, \% (MAF basis) }
$$

Ash, \% (dry basis)

BTU/1b, \%(dry basis)

Vols, \% (MAF basis) ib SO2/MMBTU, \%

Ib S02/MMBTU, \%(ROM)

REMARKS

S Analysis Data

Ash Analysis Data

Vol Analysis Data

Moisture Ana1 Data

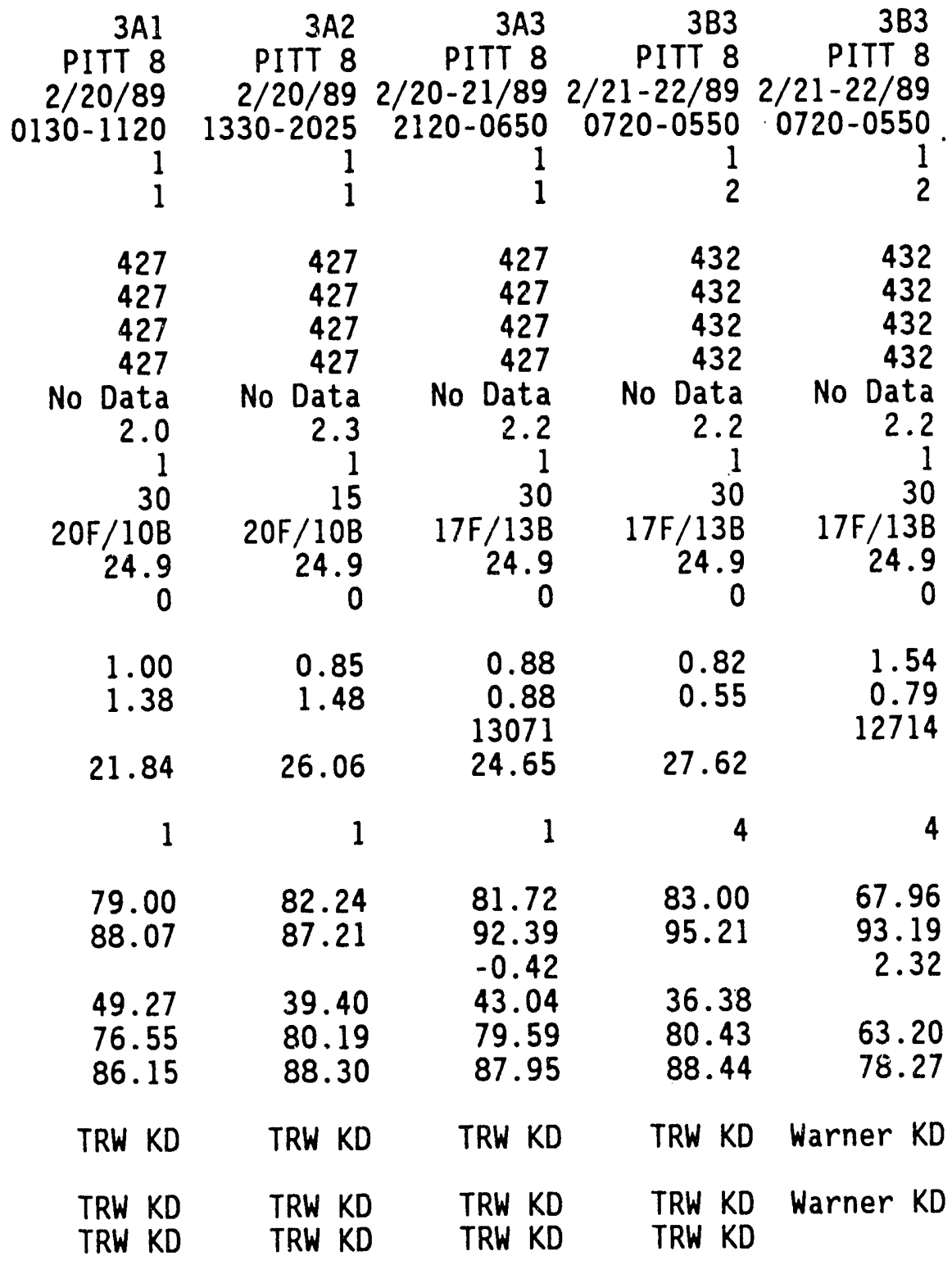

* TRW analysis of the $3 B 3 \mathrm{KD}$ samples indicates sulfur contents of 1.25 , $0.39,1.23$ and $0.395 \mathrm{wt} \%$. Warner analys is of the corresponding samples indicates sulfur contents of $1.14,2.26,1.21$ and $1.53 \mathrm{wt} \%$. 
MCL RUN DATA SUMMARY

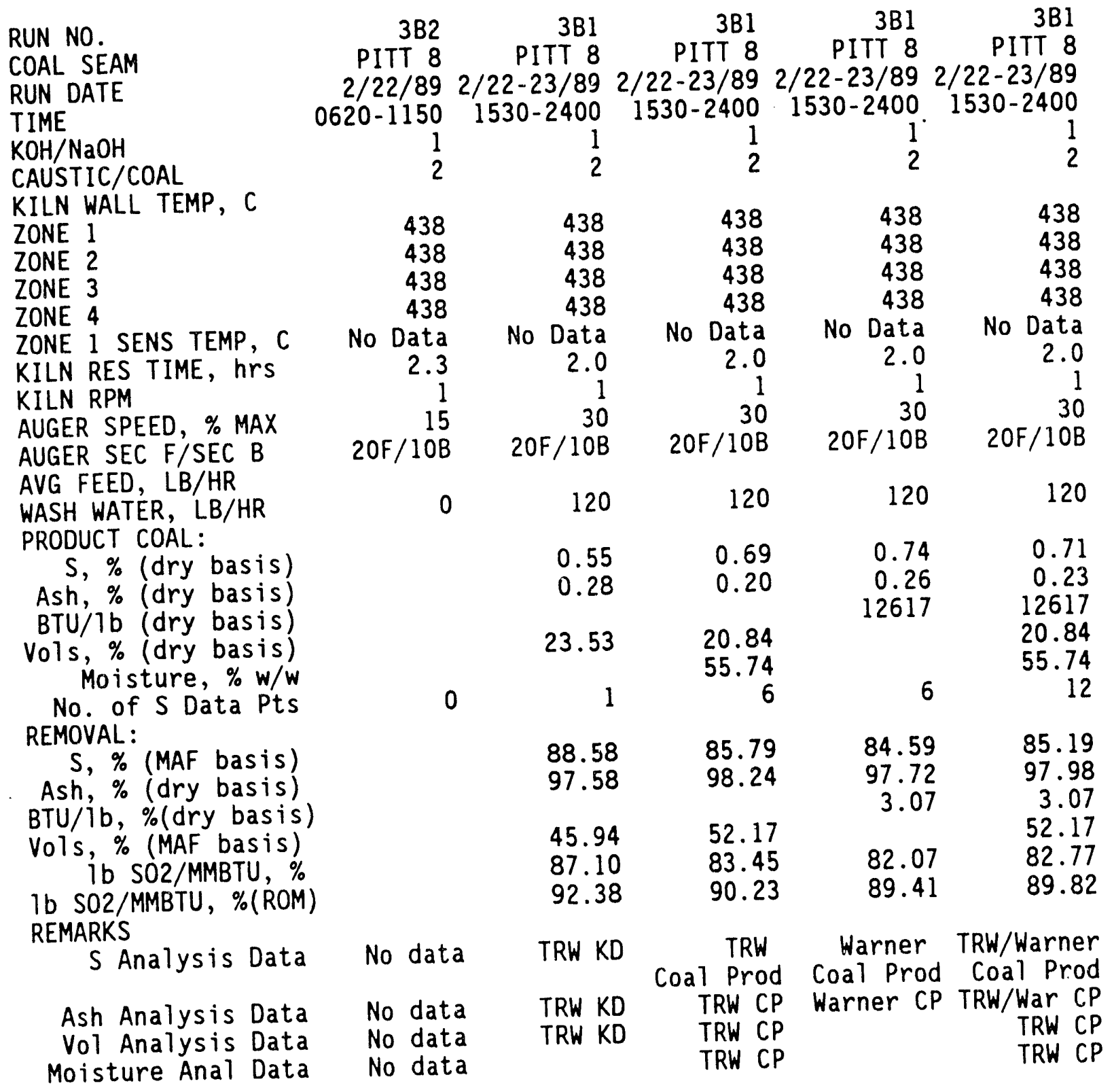


MCL RUN DATA SUMMARY

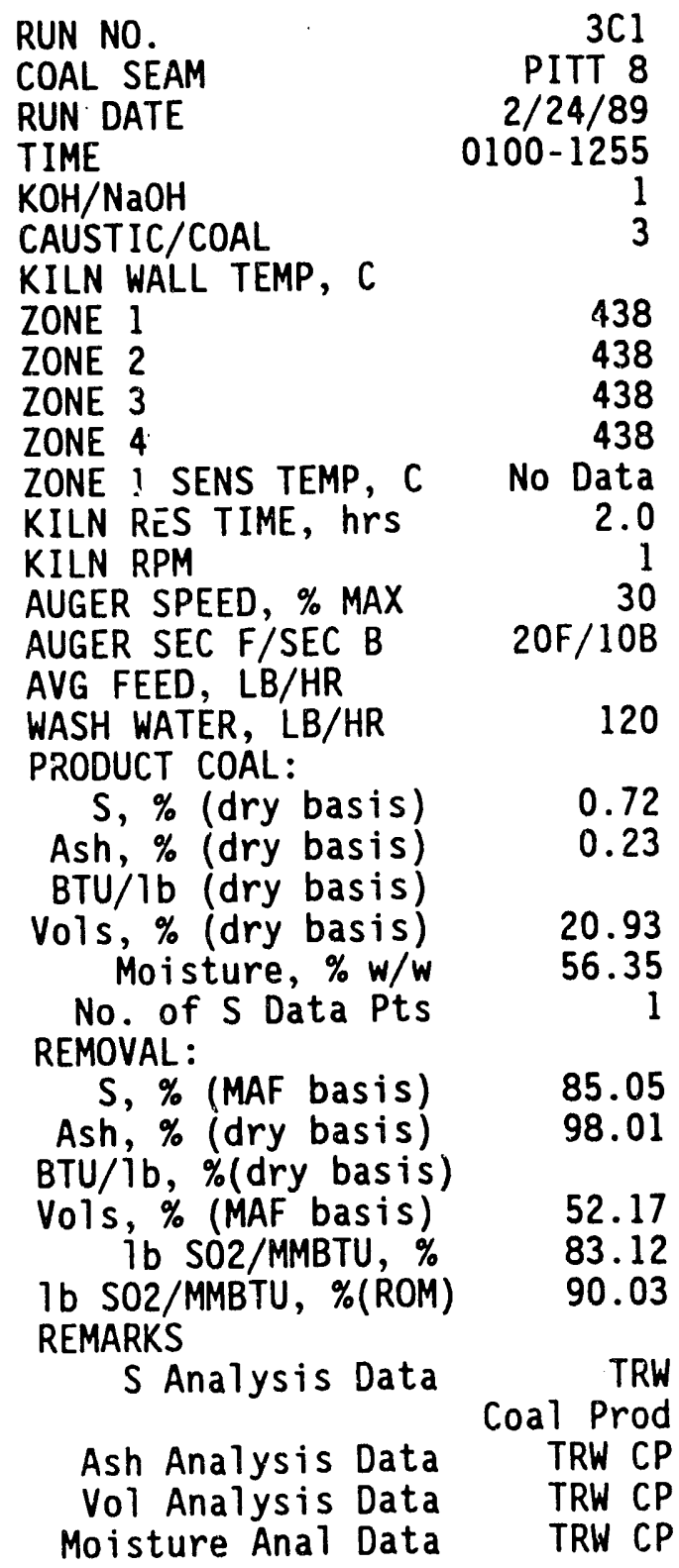


MCL RUN DATA SUMMARY

RUN NO.

COAL SEAM

RUN DATE

TIME

$\mathrm{KOH} / \mathrm{NaOH}$

CAUSTIC/COAL

KILN WALL TEMP, C

ZONE 1

ZONE 2

ZONE 3

ZONE 4

ZONE 1 SENS TEMP, $C$

KILN RES TIME, hrS

KILN RPM

AUGER SPEED, \% MAX

AUGER SEC F/SEC B

AVG FEED, LB/HR

WASH WATER, LB/HR

PRODUCT COAL:

S, \% (dry basis)

Ash, \% (dry basis)

BTU/1b (dry basis)

Vols, \% (dry basis) Moisture, \% w/w

No. of S Data Pts

REMOVAL:

$$
S, \% \text { (MAF basis) }
$$

Ash, \% (dry basis)

BTU/1b, \%(dry basis)

Vols, \% (MAF basis) ib SO2/MMBTU, \%

ib S02/MMBTU, \%(ROM)

REMARKS

S Analysis Data

Ash Analysis Data

Vol Analysis Data

Moisture Anal Data

\begin{tabular}{|c|c|c|c|c|}
\hline $\begin{array}{r}4 A \\
\text { PITT } 8 \\
3 / 6 / 89 \\
0130-1020 \\
0 \\
0\end{array}$ & $\begin{array}{r}4 A \\
\text { PITT } 8 \\
3 / 6 / 89 \\
0130-1020 \\
0 \\
0\end{array}$ & $\begin{array}{r}4 C 1^{\star} \\
\text { PITT } 8 \\
3 / 7 / 89 \\
0700-1200 \\
0 \\
2\end{array}$ & $\begin{array}{r}4 C 1 * \\
\text { PITT } 8 \\
3 / 7 / 89 \\
0700-1200 \\
0 \\
2\end{array}$ & $\begin{array}{r}4 C 1^{*} \\
\mathrm{PITT} 8 \\
3 / 7 / 89 \\
0700-1200 \\
0 \\
2\end{array}$ \\
\hline $\begin{array}{l}410 \\
410 \\
410 \\
410 \\
266 \\
2.0\end{array}$ & $\begin{array}{l}410 \\
410 \\
410 \\
410 \\
266 \\
2.0\end{array}$ & $\begin{array}{l}421 \\
410 \\
410 \\
410 \\
290 \\
2.0\end{array}$ & $\begin{array}{l}421 \\
410 \\
410 \\
410 \\
290 \\
2.0\end{array}$ & $\begin{array}{l}421 \\
410 \\
410 \\
410 \\
290 \\
2.0\end{array}$ \\
\hline $\begin{array}{r}1 \\
30 \\
20 F / 10 \mathrm{~B} \\
19.4 \\
0\end{array}$ & $\begin{array}{r}1 \\
30 \\
20 \mathrm{~F} / 10 \mathrm{~B} \\
19.4 \\
0\end{array}$ & $\begin{array}{r}1 \\
30 \\
20 \mathrm{~F} / 10 \mathrm{~B} \\
20.7 \\
120\end{array}$ & $\begin{array}{r}1 \\
30 \\
20 \mathrm{~F} / 10 \mathrm{~B} \\
20.7 \\
120\end{array}$ & $\begin{array}{r}20 \mathrm{~F} / 10 \mathrm{~B} \\
20.7 \\
120\end{array}$ \\
\hline $\begin{array}{r}4.30 \\
15.22\end{array}$ & $\begin{array}{r}4.03 \\
13.69 \\
12597\end{array}$ & 0.79 & $\begin{array}{r}0.75 \\
0.64 \\
14241\end{array}$ & $\begin{array}{r}0.77 \\
0.64 \\
14241\end{array}$ \\
\hline 30.28 & & $\begin{array}{l}23.73 \\
54.16\end{array}$ & & $\begin{array}{l}23.7 \\
54 .\end{array}$ \\
\hline 2 & 1 & 2 & 3 & \\
\hline $\begin{array}{l}0 \\
0\end{array}$ & $\begin{array}{r}3.30 \\
0 \\
3.22\end{array}$ & 83.54 & $\begin{array}{l}84.44 \\
94.47 \\
-9.41\end{array}$ & \\
\hline $\begin{array}{r}21.55 \\
0 \\
38.56\end{array}$ & $\begin{array}{r}2.48 \\
42.41\end{array}$ & $\begin{array}{l}45.34 \\
83.07 \\
90.00\end{array}$ & $\begin{array}{l}84.00 \\
90.55\end{array}$ & \\
\hline
\end{tabular}

TRW KD Warner KD

TRW KD Warner KD

TRW KD
TRW CP Warner CP TRW/Warner Coal Prod Warner $C P$ Warner $C P$

TRW CP TRW CP
TRW CP

TRW CP

REMARKS: This Run $4 \mathrm{Cl}$ * data set corresponds to the first 3 samples obtained from the run while the Run $4 C 1$ set refers to the average of all 8 samples. TRW analys is of the Run $4 C 1^{*}$ samples shows $5.6,0.43$ and $4.47 \%$ ash The high ash data are not used to calculate the average ash content in the the Run $4 \mathrm{Cl}^{*}$ or Run $4 \mathrm{Cl}$ data set. 
MCL RUN DATA SUMMARY

RUN NO.

COAL SEAM

RUN DATE

TIME

$\mathrm{KOH} / \mathrm{NaOH}$

CAUSTIC/COAL

KILN WALL TEMP, $C$

ZONE 1

ZONE 2

ZONE 3

ZONE 4

ZONE 1 SENS TEMP, $C$

KILN RES TIME, hrs

KILN RPM

AUGER SPEED, \% MAX

AUGER SEC F/SEC B

AVG FEED, LB/HR

WASH WATER, LB/HR

PRODUCT COAL:

S, \% (dry basis)

Ash, \% (dry basis)

BTU/1b (dry basis)

vols, \% (dry basis) Moisture, \% w/w

No. of S Data Pts REMOVAL:

$$
\text { S, \% (MAF basis) }
$$

Ash, \% (dry basis)

BTU/ib, \% (dry basis)

Vols, \% (MAF basis)

$$
\text { ib SO2/MMBTU, \% }
$$

Ib S02/MMBTU, \%(ROM)

REMARKS

$S$ Analysis Data

Ash Analys is Data

Vol Analys is Data

Moisture Anal Data
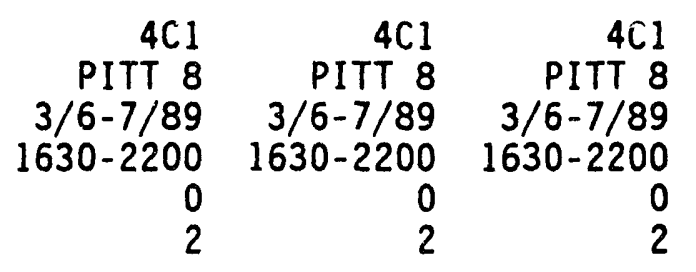

$4 C 2$
PITT 8
$3 / 7-8 / 89$
$2200-1430$
0
2
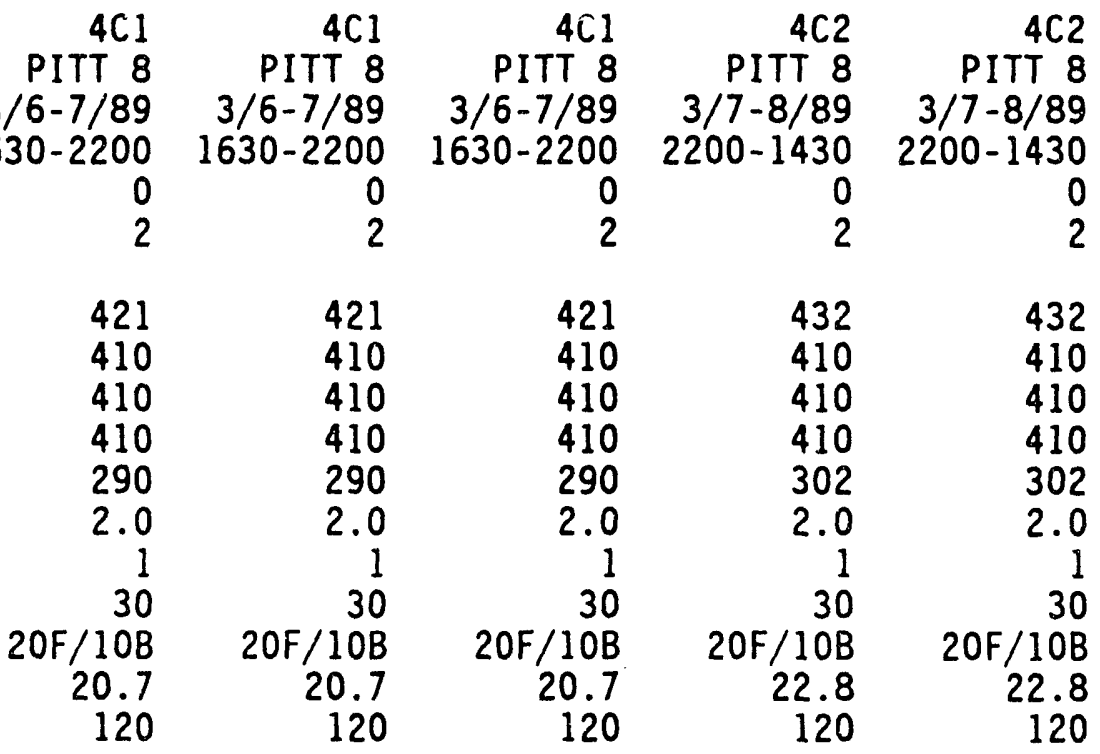

30

$10 \mathrm{~B}$
20.7

120

1.16

0.53

0.97

0.74

$24.6 \mathrm{E}$

52.45

8

14226

75.80

95.44

43.23

75.09

85.29

TRW CP

Warner

79.72

94.47

$-9.30$

79.17

87.70

TRW CP Warner CP

TRW CP

TRW CP
421

410

410

410

290

2.0

$20 \mathrm{~F} / 10 \mathrm{~B}$

20.7

120

1.14

0.68

76.30

94.17

$-9.30$

43.23

75.64

85.62

24.65

52.45

13

432

410

410

410

302

2.0

1

$20 \mathrm{~F} / 10 \mathrm{~B}$

22.8

120

1.25

0.54

1.04

23.91

52.38

10

432

410

410

410

302

2.0

30

$20 \mathrm{~F} / 10 \mathrm{~B}$

22.8

120

14219

73.89

78.43

95.44

94.55

$-9.24$

44.93

73.12

77.81

84.12

86.90

TRW CP Warner CP

TRW/War

TRW CP

TRW CP
TRW CP Warner CP

TRW CP

TRW CP
Coal Prod 
MCL RUN DATA SUMMARY

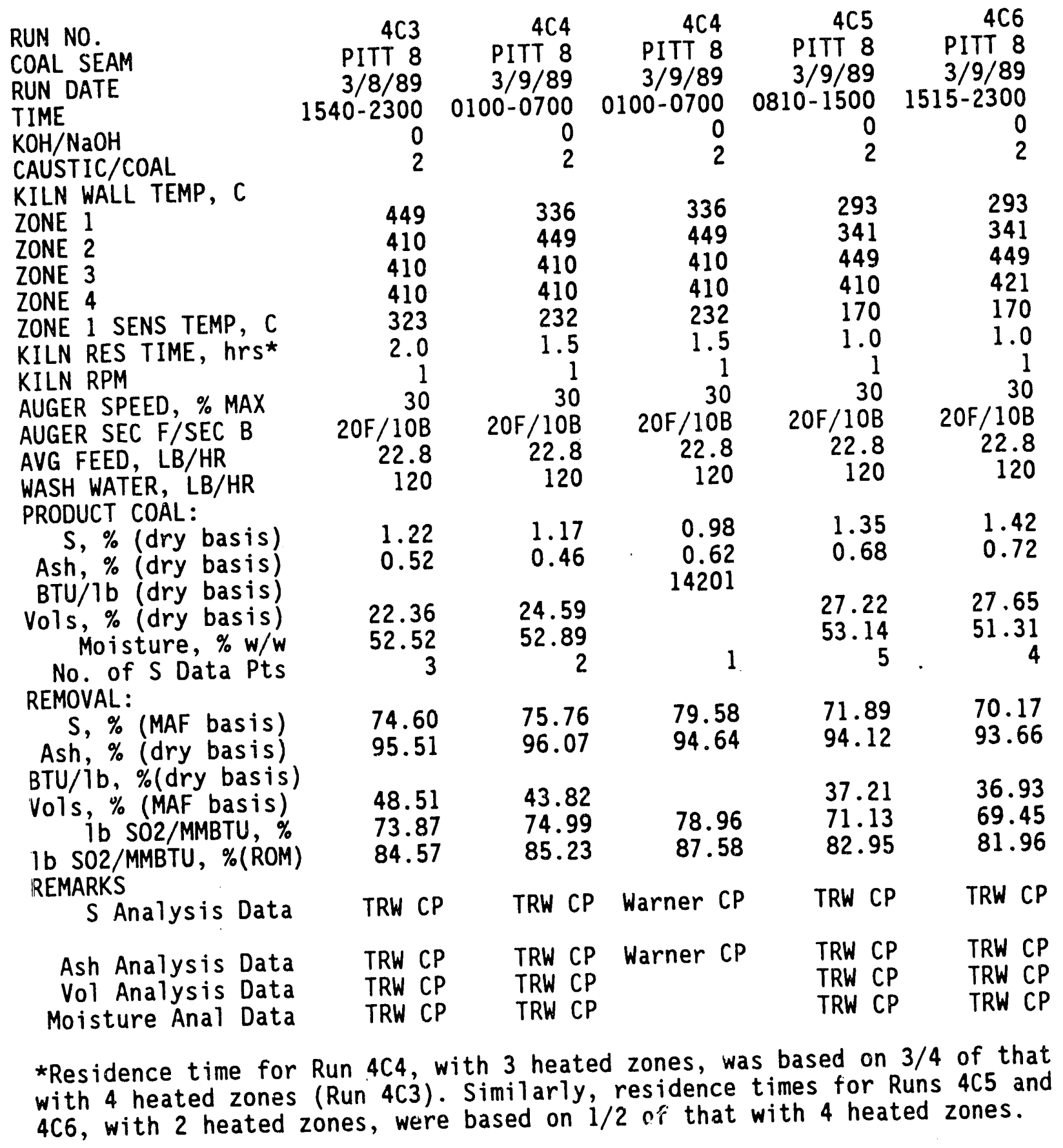


MCL RUN DATA SUMMARY

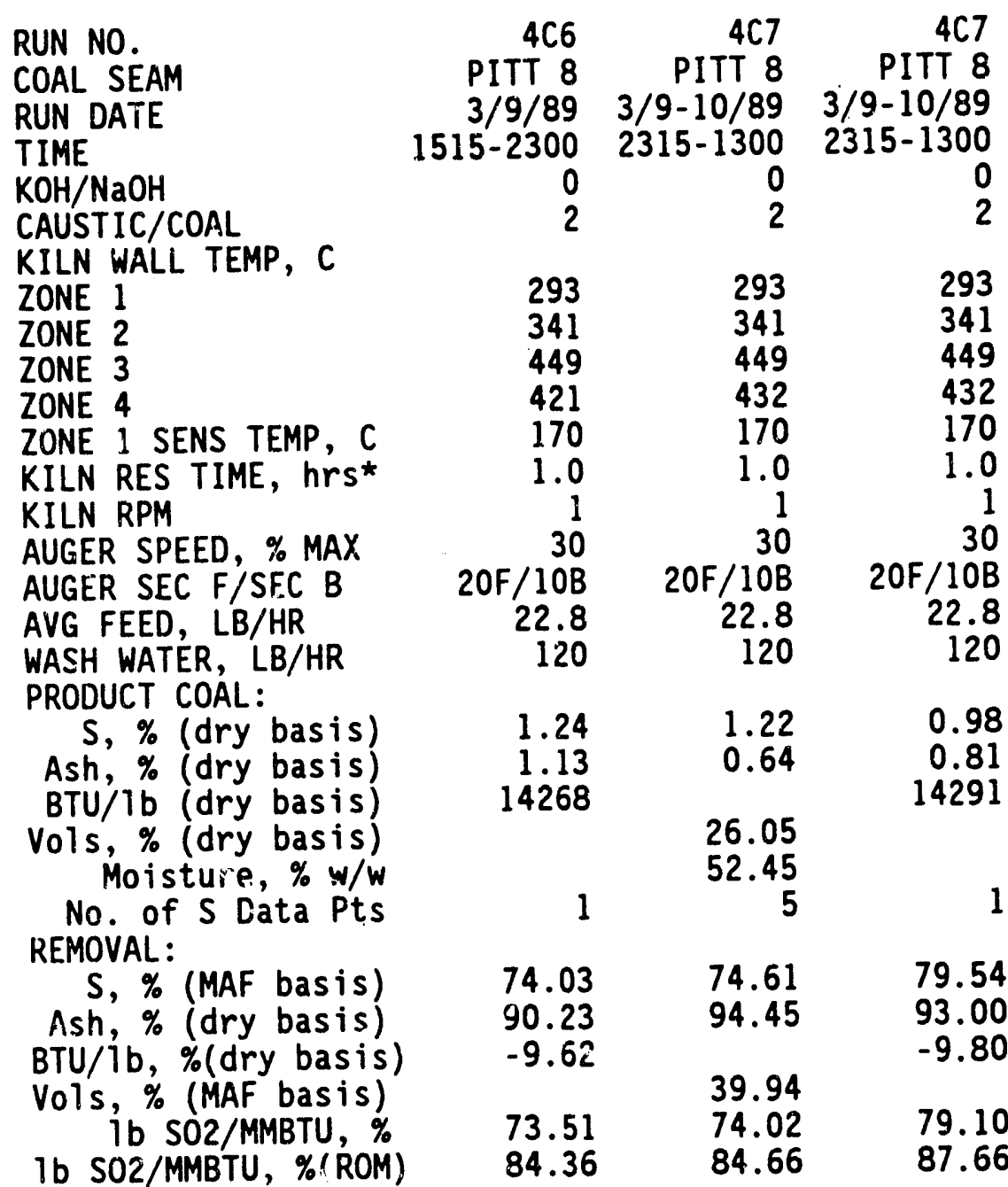

REMARKS

$S$ Analysis Data Warner CP TRW CP Warner CP

Ash Analysis Data Warner CP TRW CP Warner CP

Vol Analysis Data $\quad$ TRW CP

Moisture Anal Data TRW CP

*Residence times for Runs $4 C 6$ and $4 C 7$, with 2 heated zones, were based on $1 / 2$ of that with 4 heated zones (Run $4 C 3$ ). 
MCL RUN DATA SUMMARY

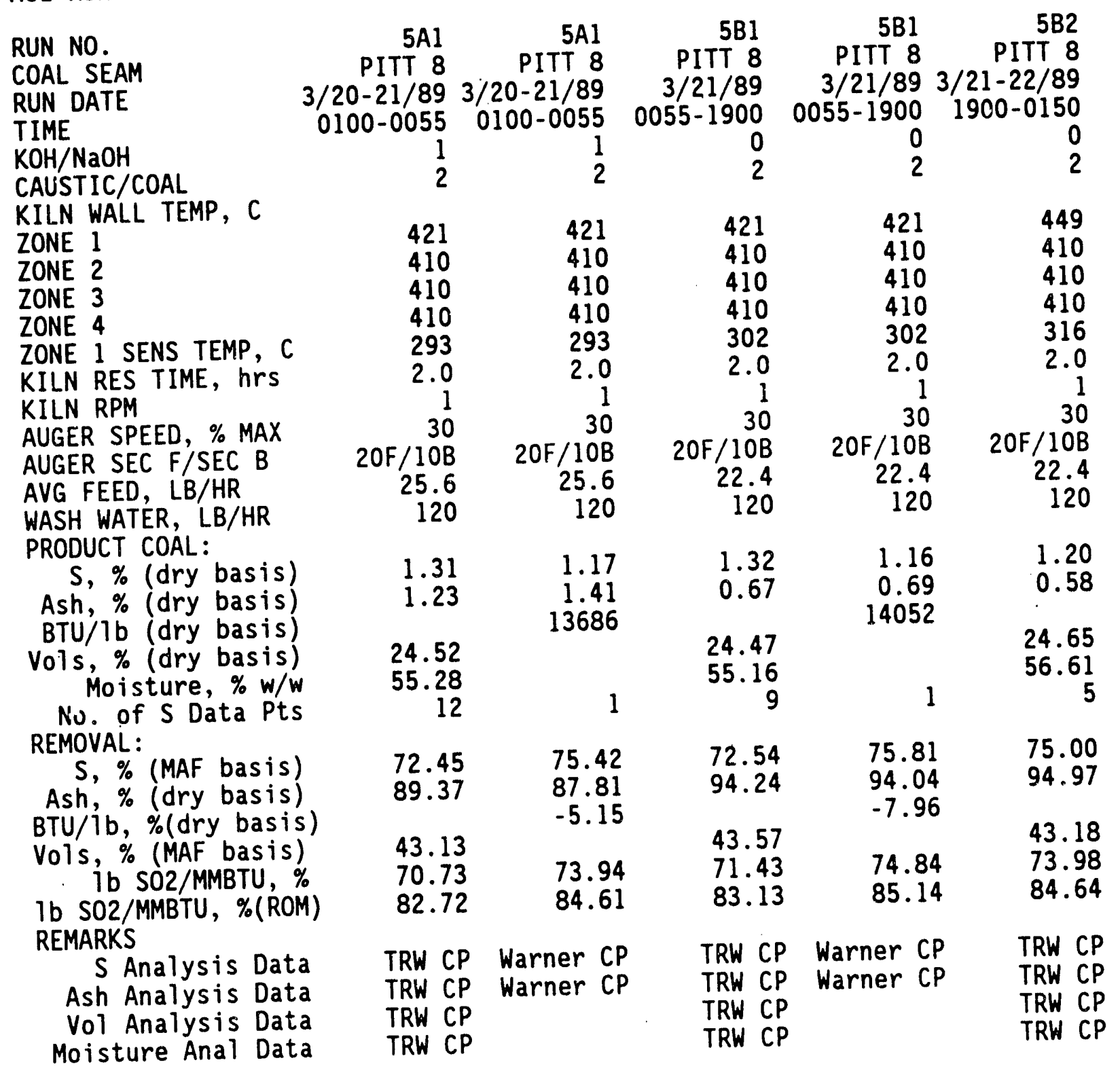


MCL RUN DATA SUMMARY

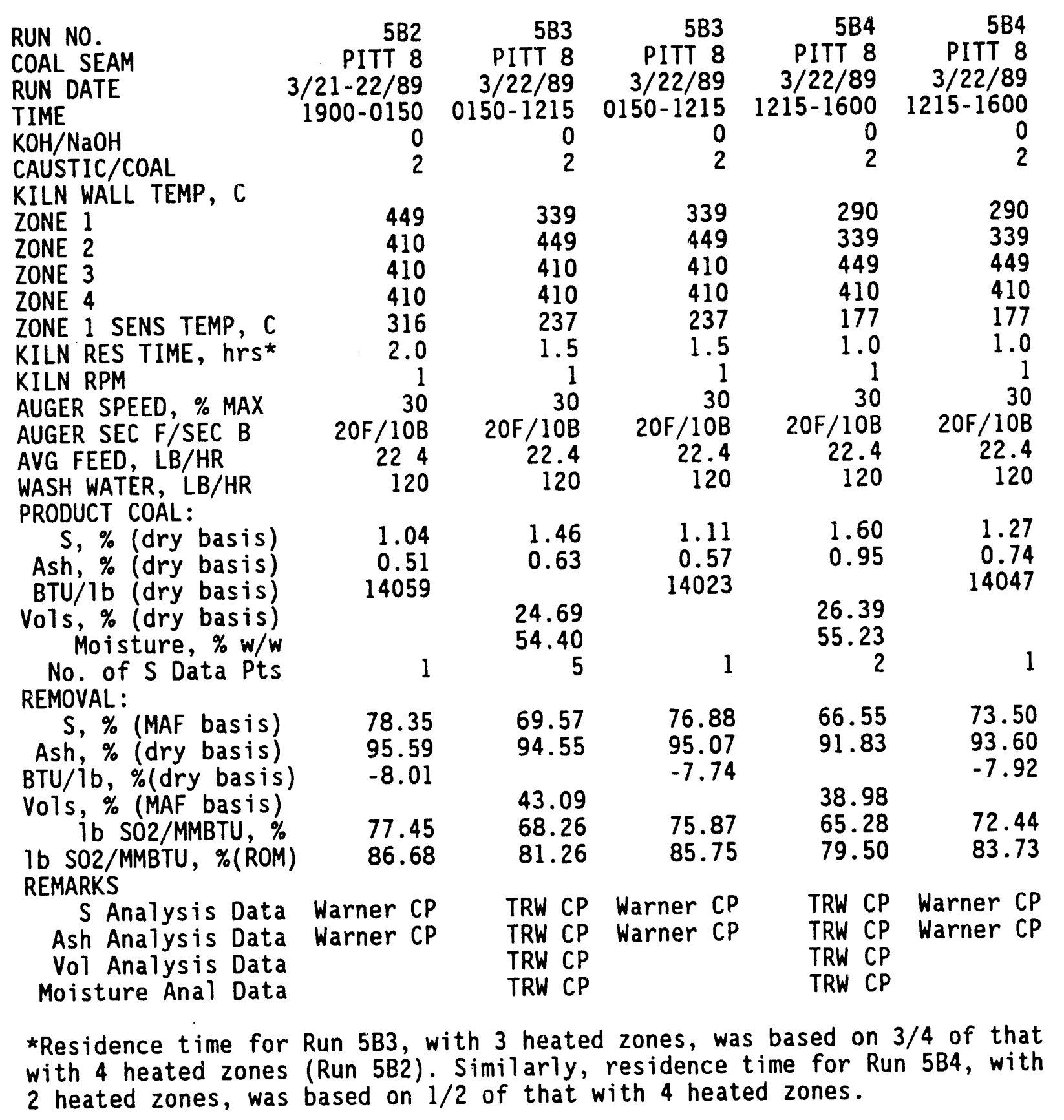


MCL RUN DATA SUMMARY

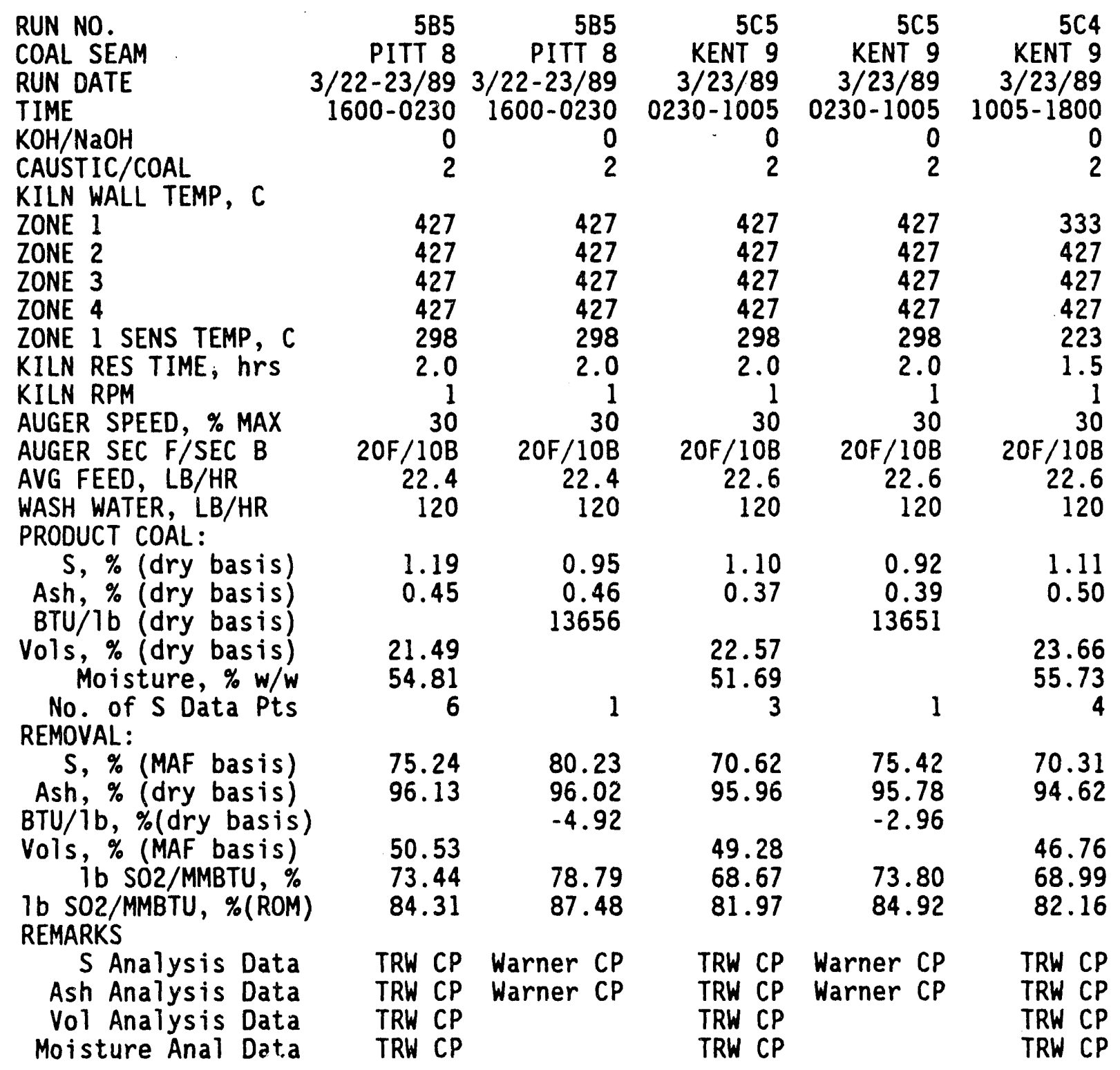


MCL RUN DATA SUMMARY

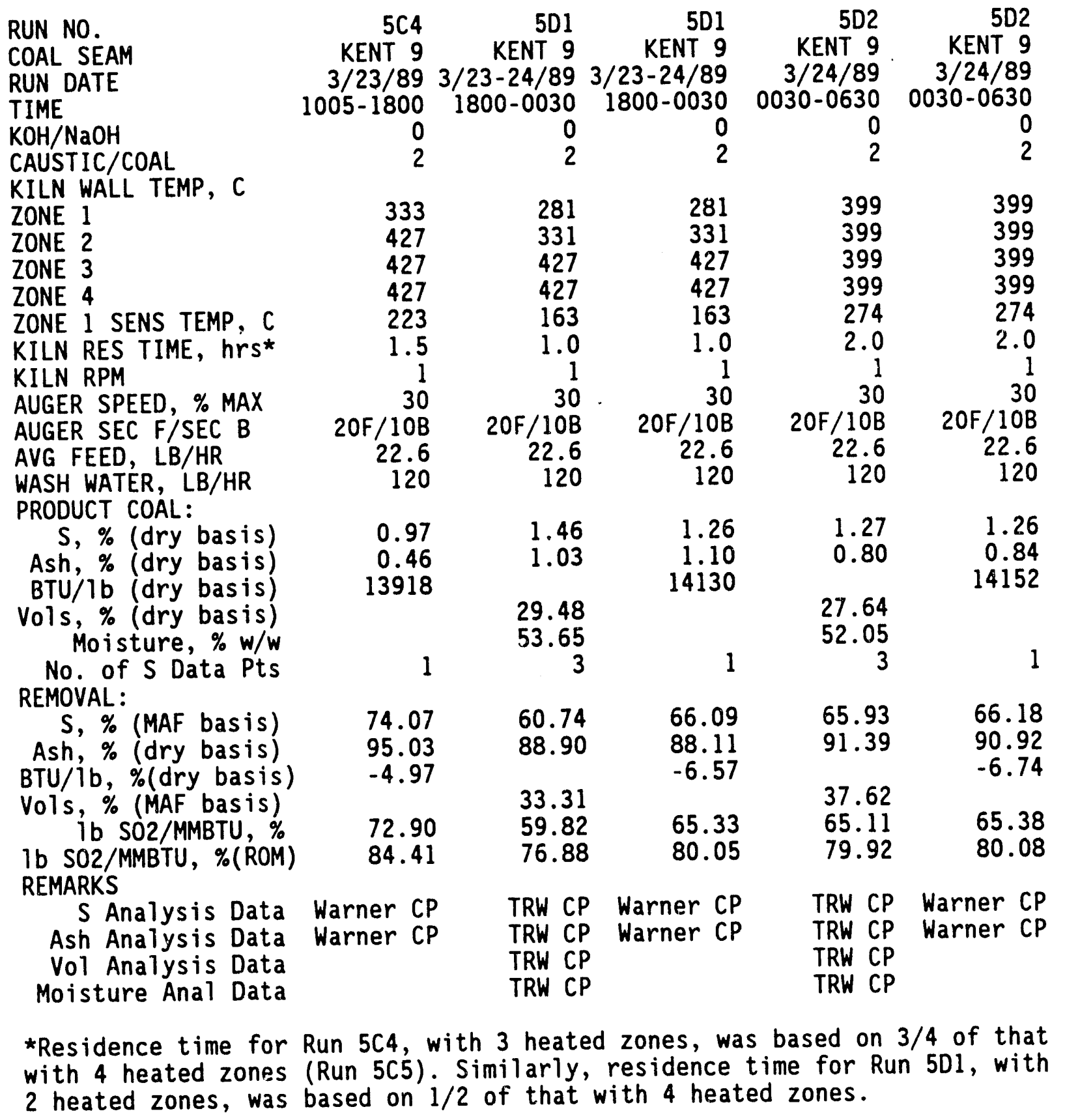


MCL RUN DATA SUMMARY

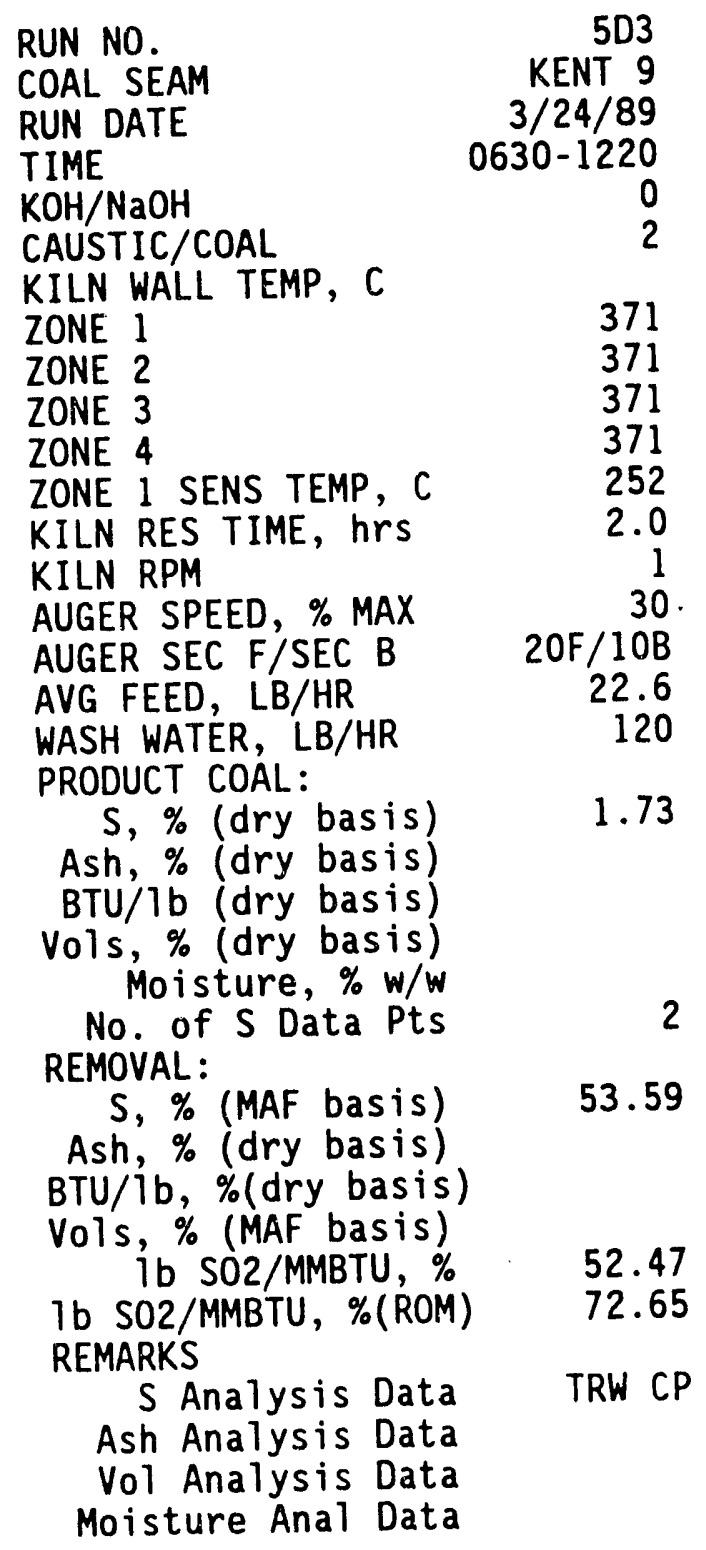


MCL RUN DATA SUMMARY

\begin{tabular}{|c|c|c|c|c|c|}
\hline $\begin{array}{l}\text { RUN NO. } \\
\text { COAL SEAM } \\
\text { RUN DATE } \\
\text { TIME } \\
\text { KOH/NaOH } \\
\text { CAUSTIC/COAL } \\
\text { KILN WALL TEMP, C }\end{array}$ & $\begin{array}{r}6 A 1 \\
\text { PITT } 8 \\
4 / 3-4 / 89 \\
0400-0000 \\
0 \\
1\end{array}$ & $\begin{array}{r}6 A 1 \\
\text { PITT } 8 \\
4 / 3-4 / 89 \\
0400-0000 \\
0 \\
1\end{array}$ & $\begin{array}{r}6 \mathrm{BI} \\
\mathrm{PITT} 8 \\
4 / 4 / 89 \\
0130-1645 \\
0 \\
1.5\end{array}$ & $\begin{array}{r}6 B 1 \\
\text { PITT } 8 \\
4 / 4 / 89 \\
0130-1645 \\
0 \\
1.5\end{array}$ & $\begin{array}{r}6 B 1 \\
\text { PITT } 8 \\
4 / 4 / 89 \\
0130-1645 \\
0\end{array}$ \\
\hline & 427 & 427 & 427 & 427 & \\
\hline & & & & & \\
\hline & & & & & \\
\hline & & $4 i$ & & & \\
\hline SENS & & & & & \\
\hline 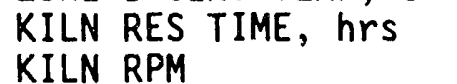 & & & & & \\
\hline$\% \operatorname{MAX}$ & 30 & & & 30 & \\
\hline$C F / S E C B$ & $20 F / 10 B$ & $20 F / 10 B$ & $20 \mathrm{~F} / 1$ & $20 \mathrm{~F} /$ & $20 \mathrm{~F} / 10$ \\
\hline $\begin{array}{l}\text { ED, LB/HR } \\
\text { ATER, LB/HR }\end{array}$ & & $\begin{array}{r}24.5 \\
100\end{array}$ & & & \\
\hline & & & & & \\
\hline S, \% (dry basis) & 1 & 2 & & & \\
\hline $\begin{array}{l}\text { Ash, \% (dry b } \\
\text { BTU/1b (dry }\end{array}$ & & & & $\begin{array}{r}0.64 \\
13948\end{array}$ & \\
\hline Vols, \% (d & 2 & 27.97 & & & 29. \\
\hline $\begin{array}{l}\text { Moistur } \\
\text { No. of } S \\
\text { REMOVAL: }\end{array}$ & & 2 & & & \\
\hline $\begin{array}{c}\text { S, \% (MAF basis) } \\
\text { Ash, \% (dry basis) } \\
\text { BTU/ib, \%(dry basis) }\end{array}$ & $\begin{array}{l}66.43 \\
90.15\end{array}$ & $\begin{array}{l}4.74 \\
9.20\end{array}$ & $\begin{array}{l}7.67 \\
4.51\end{array}$ & $\begin{array}{l}71.86 \\
94.47 \\
-7.16\end{array}$ & $\begin{array}{l}7.6 \\
1.2\end{array}$ \\
\hline $\begin{array}{l}\text { Vols, } \% \text { (MAF basis) } \\
\text { ib SO2/MMBTU, } \% \\
\text { 1b SO2/MMBTU, \%(ROM) } \\
\text { REMARKS }\end{array}$ & $\begin{array}{l}35.93 \\
64.98 \\
79.32\end{array}$ & $\begin{array}{l}35.12 \\
42.41 \\
66.00\end{array}$ & & $\begin{array}{l}70.50 \\
82.58\end{array}$ & 31. \\
\hline $\begin{array}{l}\text { S Analysis Data } \\
\text { ish Analysis Data } \\
\text { ol Analysis Data } \\
\text { isture Anal Data }\end{array}$ & $\begin{array}{l}\text { TRW CP } \\
\text { TRW CP } \\
\text { TRW CP } \\
\text { TRW CP }\end{array}$ & $\begin{array}{ll}\text { TRW } & K D \\
\text { TRW } & K D \\
\text { TRW } & K D\end{array}$ & $\begin{array}{l}\text { TRW CP } \\
\text { TRW CP } \\
\text { TRW CP } \\
\text { TRW CP }\end{array}$ & $\begin{array}{l}\text { Warner CP } \\
\text { Warner } C P\end{array}$ & $\begin{array}{l}\text { TRW } \\
\text { TRW }\end{array}$ \\
\hline
\end{tabular}


MCL RUN DATA SUMMARY

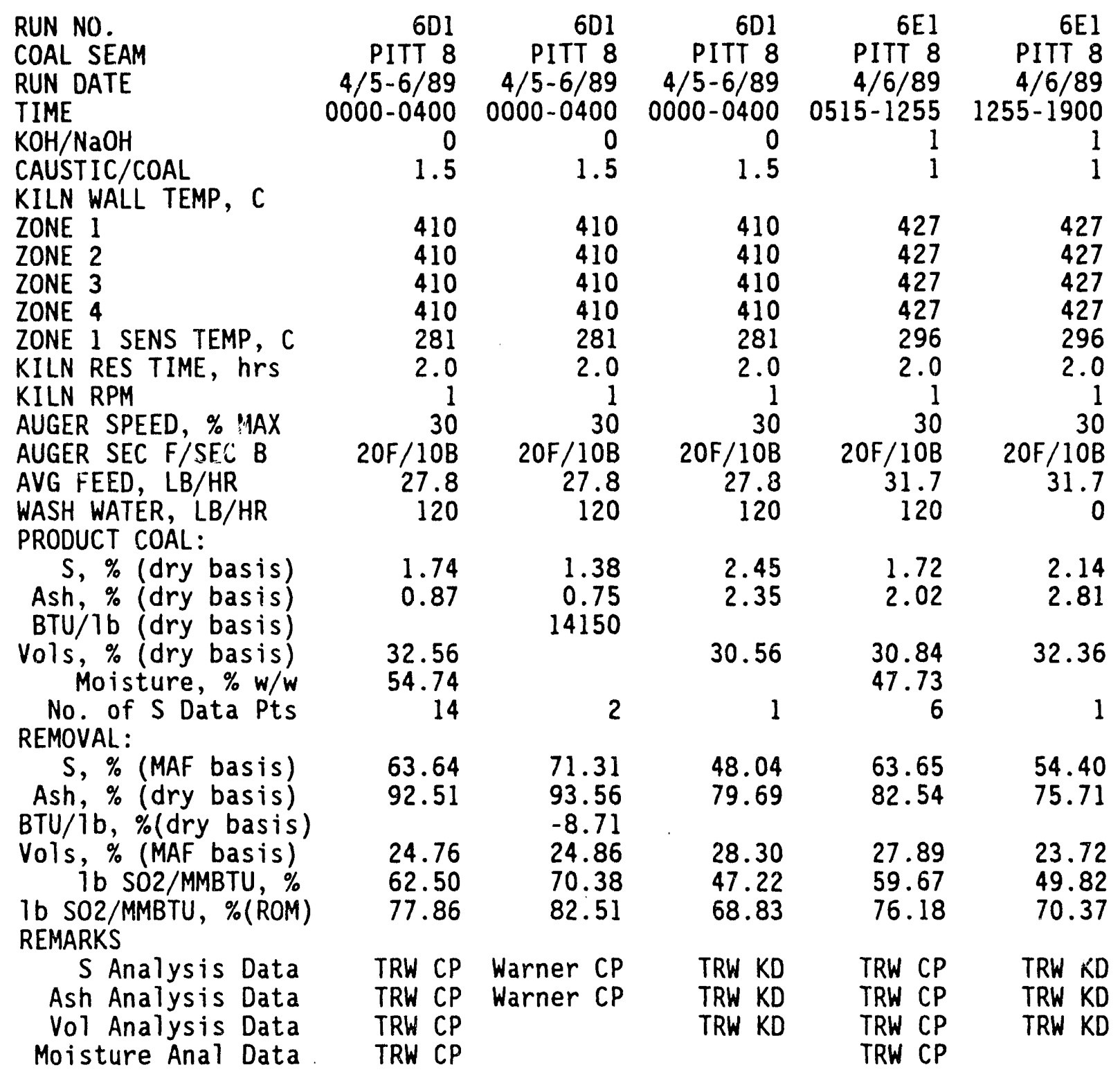


MCL RUN DATA SUMMARY

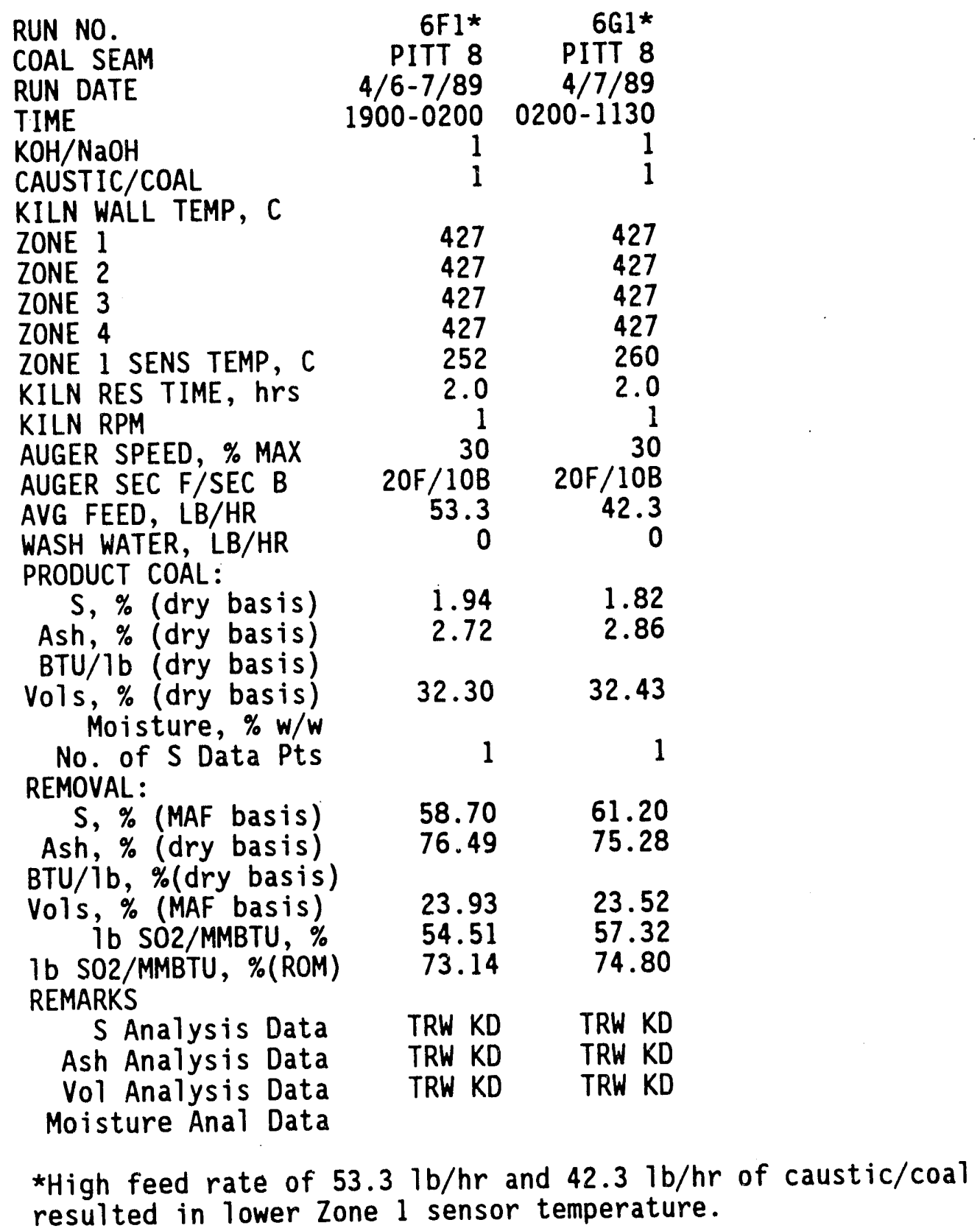


MCL RUN DATA SUMMARY

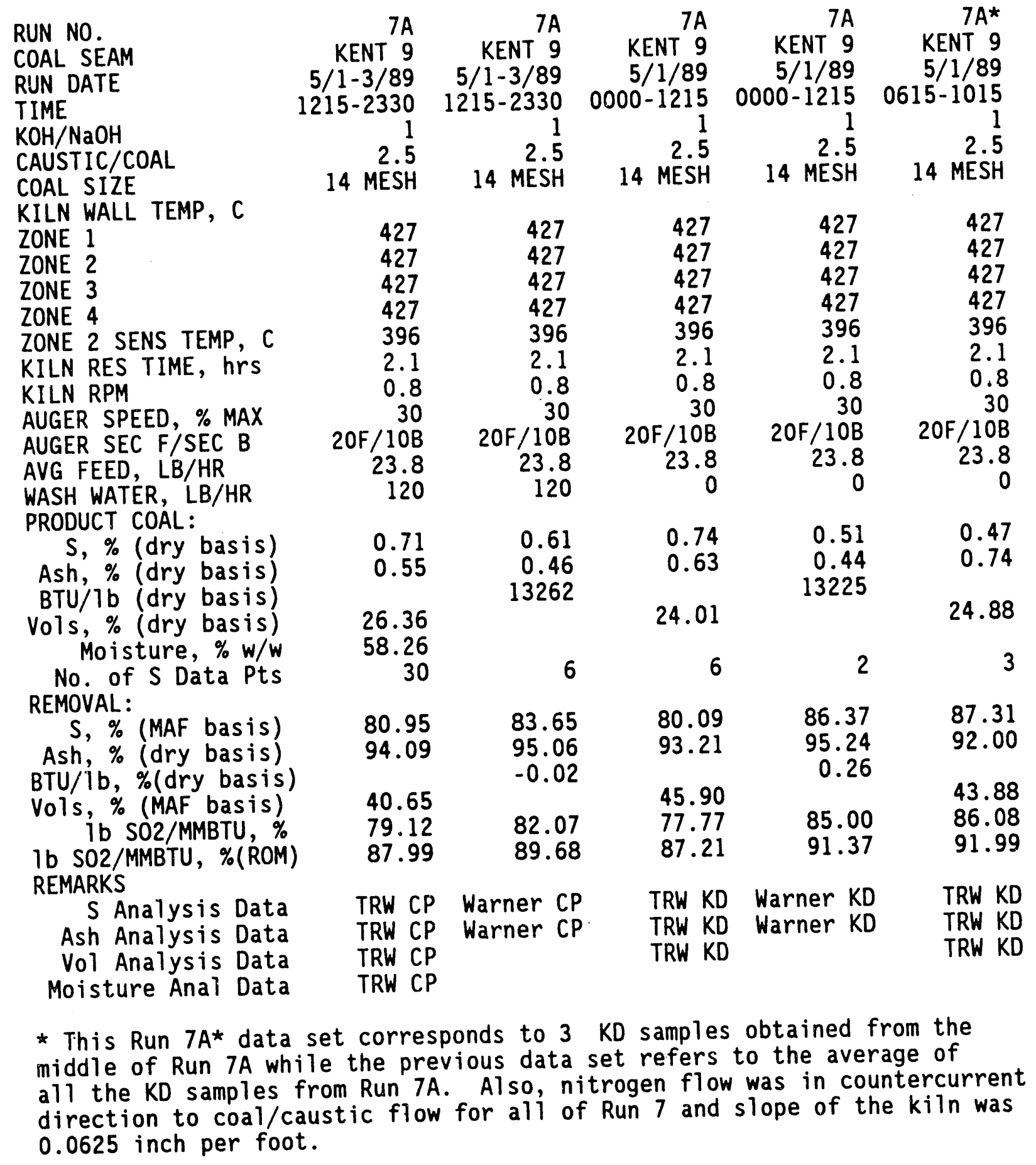


MCL RUN DATA SUMMARY

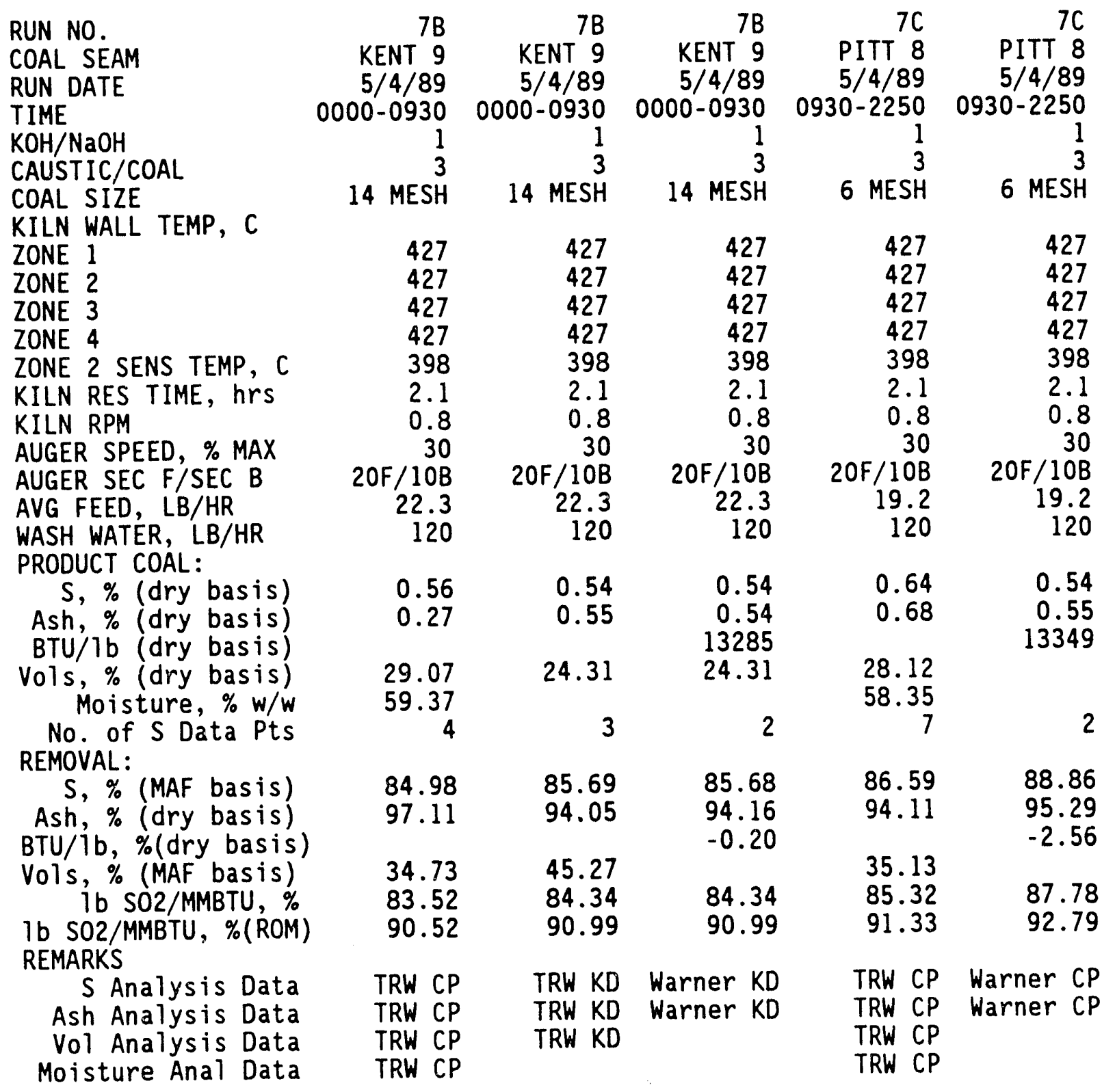


MCL RUN DATA SUMMARY

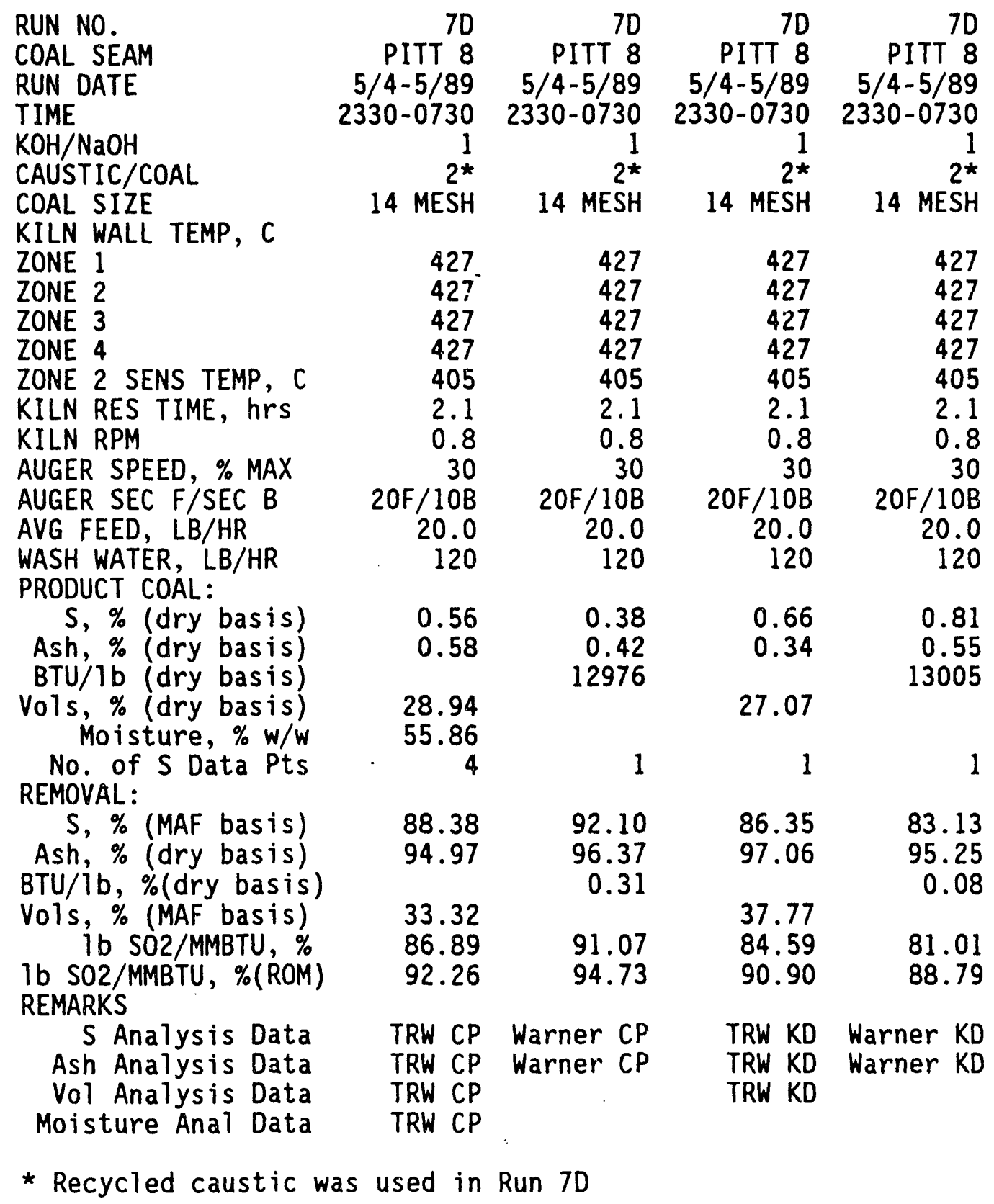


MCL RUN DATA SUMMARY

RUN NO.

COAL SEAM

RUN DATE

TIME

$\mathrm{KOH} / \mathrm{NaOH}$

CAUSTIC/COAL

COAL SIZE

KILN WALL TEMP, $C$

ZONE 1

ZONE 2

ZONE 3

ZONE 4

ZONE 2 SENS TEMP, $C^{*}$

KILN RES TIME, hrs

KILN RPM

AUGER SPEED, \% MAX

AUGER SEC F/SEC B

AVG FEED, LB/HR

WASH WATER, LB/HR

PRODUCT COAL:

S, \% (dry basis)

Ash, \% (dry basis)

BTU/1b (dry basis)

Vols, \% (dry basis) Moisture, \% w/w

No. of S Data Pts REMOVAL:

$$
\text { S, \% (MAF basis) }
$$

Ash, \% (dry basis)

BTU/Ib, \%(dry basis)

Vols, \% (MAF basis) ib S02/MMBTU, \%

Ib S02/MMBTU, \%(ROM) REMARKS

$S$ Analysis Data

Ash Analysis Data

Vol Analysis Data

Moisture Anal Data
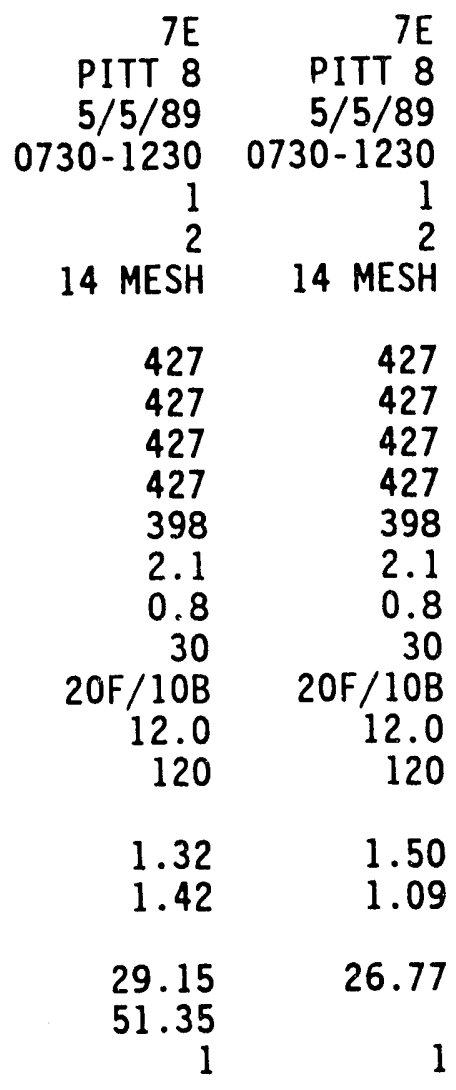

$72.27 \quad 68.59$

$87.73 \quad 90.58$

$32.26 \quad 38.00$

$69.86 \quad 65.75$

$82.20 \quad 79.77$

TRW CP TRW KD

TRW CP TRW KD

TRW CP TRW KD

*After noticing large decrease in sensor temperature at the beginning of Run $7 E$, the auger was run fast forward for a short period during the middle of run, which brought the Zone 2 sensor temperature back to the normal level. However, the low initial sensor temperature al so suggested improper feed conditions or improper movement of the coal/caustic mixture inside the kiln. 
MCL RUN DATA SUMMARY

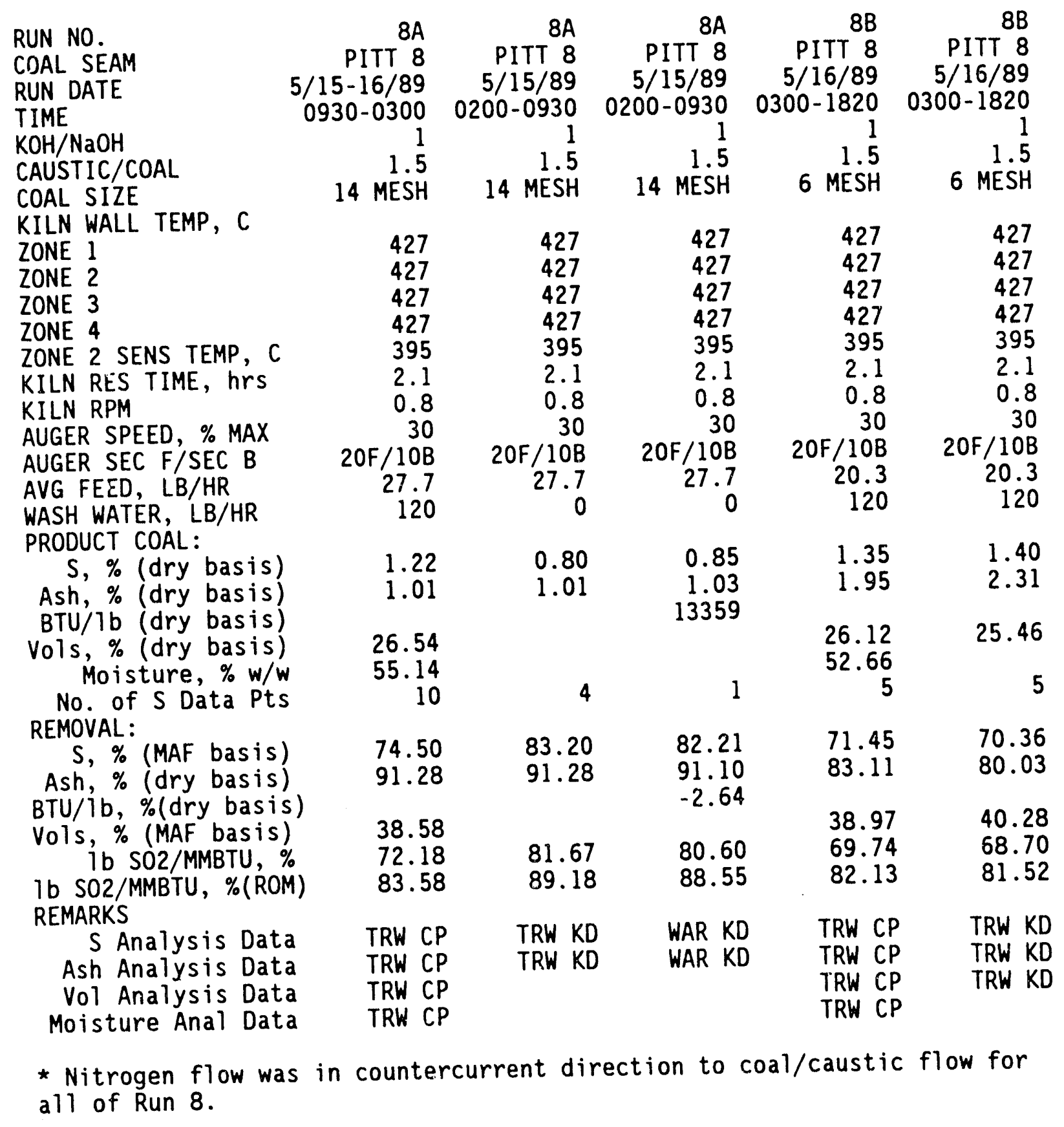


MCL RUN DATA SUMMARY

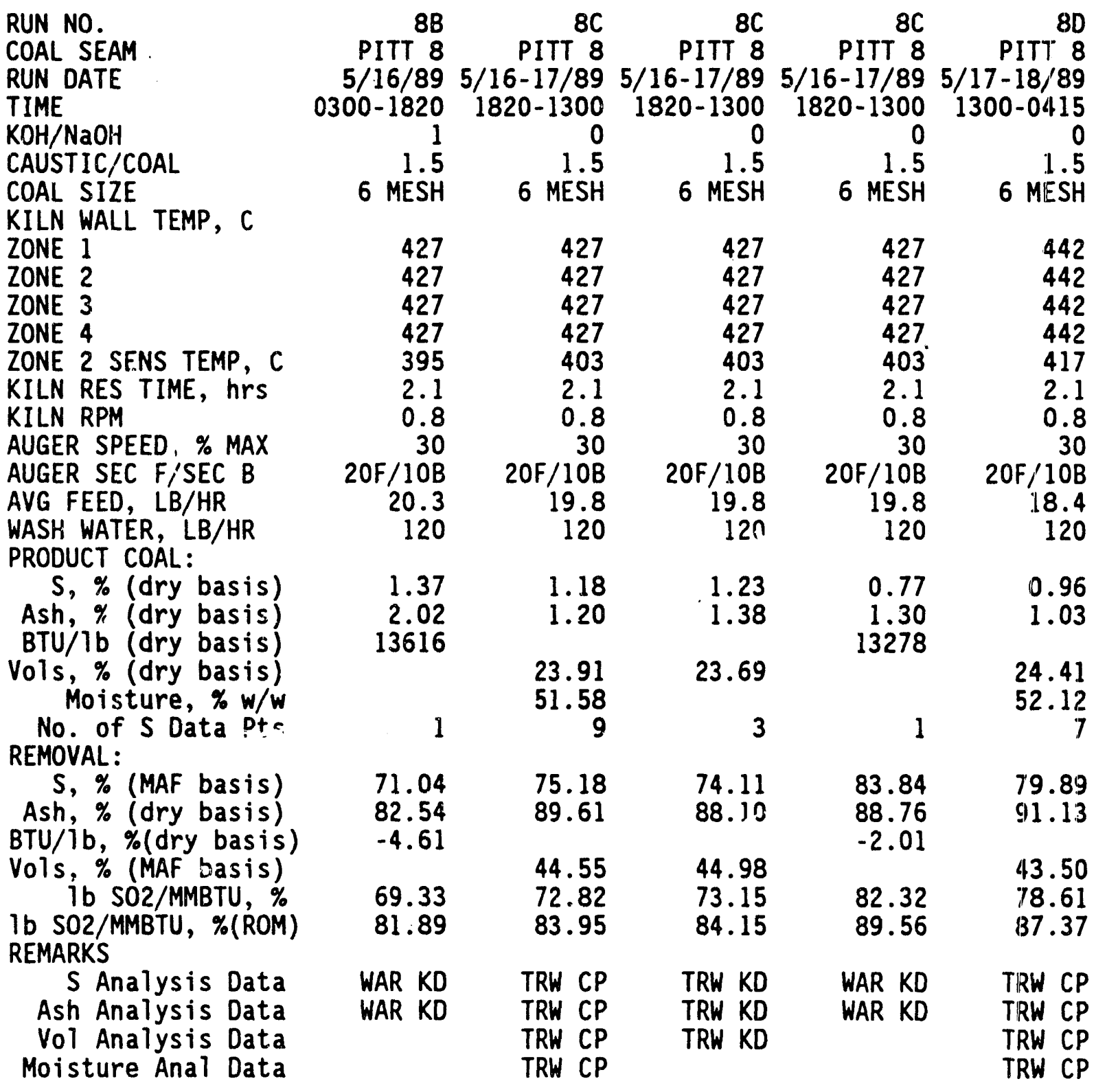


MCL RUN DATA SUMMARY

\begin{tabular}{|c|c|c|c|c|c|}
\hline $\begin{array}{l}\text { RUN NO. } \\
\text { COAL SEAM } \\
\text { RUN DATE } \\
\text { TIME } \\
\text { KOH/NaOH } \\
\text { CAUSTIC/COAL } \\
\text { COAL SIZE }\end{array}$ & $\begin{array}{r}80 \\
\text { PITT } 8 \\
/ 17-18 / 89 \\
1300-0415 \\
0 \\
0 \\
1.5 \\
6 \text { MESH }\end{array}$ & $\begin{array}{r}8 E \\
\text { PITT } 8 \\
5 / 18 / 89 \\
0415-2000 \\
0 \\
1.5 \\
6 \text { MESH }\end{array}$ & $\begin{array}{r}8 E \\
\text { PITT } 8 \\
5 / 18 / 89 \\
0415-2000 \\
0 \\
1.5 \\
6 \text { MESH }\end{array}$ & $\begin{array}{r}8 E \\
\text { PITT } 8 \\
5 / 18 / 895 \\
0415-2000 \\
0 \\
1.5 \\
6 \text { MESH }\end{array}$ & $\begin{array}{r}8 \mathrm{~F} \\
\text { PITT } 8 \\
5 / 18-19 / 89 \\
2000-1300 \\
0 \\
2 \\
14 \mathrm{MESH}\end{array}$ \\
\hline $\begin{array}{l}\text { KILN WALL TEMP, C } \\
\text { ZONE } 1 \\
\text { ZONE } 2 \\
\text { ZONE } 3 \\
\text { ZONE } 4 \\
\text { ZONE } 2 \text { SENS TEMP, C } \\
\text { KILN RES TIME, hrs } \\
\text { KILN RPM } \\
\text { AUGER SPEED, \% MAX } \\
\text { AUGER SEC F/SEC B } \\
\text { AVG FEED, LB/HR } \\
\text { WASH WATER, LB/HR }\end{array}$ & $\begin{array}{r}442 \\
442 \\
442 \\
442 \\
417 \\
2.1 \\
0.8 \\
30 \\
20 \mathrm{~F} / 10 \mathrm{~B} \\
18.4 \\
120\end{array}$ & $\begin{array}{r}412 \\
412 \\
412 \\
412 \\
389 \\
2.1 \\
0.8 \\
30 \\
20 \mathrm{~F} / 10 \mathrm{~B} \\
18.3 \\
120\end{array}$ & $\begin{array}{r}412 \\
412 \\
412 \\
412 \\
389 \\
2.1 \\
0.8 \\
30 \\
20 \mathrm{~F} / 10 \mathrm{~B} \\
18.3 \\
120\end{array}$ & $\begin{array}{r}412 \\
412 \\
412 \\
412 \\
389 \\
2.1 \\
0.8 \\
30 \\
20 \mathrm{~F} / 10 \mathrm{~B} \\
18.3 \\
120\end{array}$ & $\begin{array}{r}412 \\
412 \\
412 \\
412 \\
384 \\
2.1 \\
0.8 \\
30 \\
20 \mathrm{~F} / 10 \mathrm{~B} \\
17.7 \\
120\end{array}$ \\
\hline $\begin{array}{l}\text { PRODUCT COAL: } \\
\text { S, \% (dry basis) } \\
\text { Ash, \% (dry basis) } \\
\text { BTU/1b (dry basis) }\end{array}$ & $\begin{array}{r}0.80 \\
1.00 \\
13694\end{array}$ & $\begin{array}{l}1.46 \\
1.18\end{array}$ & $\begin{array}{l}2.20 \\
1.47\end{array}$ & $\begin{array}{r}0.76 \\
0.73 \\
14001\end{array}$ & $\begin{array}{l}0.97 \\
0.54\end{array}$ \\
\hline $\begin{array}{l}\text { BTU/1b (dry basis) } \\
\text { Vols, \% (dry basis) } \\
\text { Moisture, \% w/w } \\
\text { No. of S Data Pts } \\
\text { Demoval. }\end{array}$ & 13694 & $\begin{array}{r}26.45 \\
51.34 \\
5\end{array}$ & 25.21 & 14001 & $\begin{array}{l}26.19 \\
54.03\end{array}$ \\
\hline $\begin{array}{l}\text { REMOVAL: } \\
\text { S, \% (MAF basis) } \\
\text { Ash, \% (dry basis) } \\
\text { BTU ilb \% (dry basis) }\end{array}$ & $\begin{array}{l}83.26 \\
91.36 \\
-5.21\end{array}$ & $\begin{array}{l}9.49 \\
9.82\end{array}$ & $\begin{array}{l}53.86 \\
87.29\end{array}$ & $\begin{array}{l}84.14 \\
93.69 \\
-7.57\end{array}$ & $\begin{array}{l}79.77 \\
95.33\end{array}$ \\
\hline $\begin{array}{c}\text { BTU/1b, \%(dry dasis) } \\
\text { Vols, \% (MAF basis) } \\
\text { ib SO2/MMBTU, \% } \\
\text { lb SO2/MMBTU, \%(ROM) }\end{array}$ & ) & & $\begin{array}{l}41.40 \\
52.21 \\
71.78\end{array}$ & $\begin{array}{l}83.45 \\
90.23\end{array}$ & $\begin{array}{l}39.67 \\
79.11 \\
87.66\end{array}$ \\
\hline $\begin{array}{l}\text { S Analys is Data } \\
\text { Ash Analys is Data } \\
\text { Vol Analysis Data } \\
\text { Moisture Anal Data }\end{array}$ & $\begin{array}{l}\text { WAR KD } \\
\text { WAR KD }\end{array}$ & $\begin{array}{l}\text { TRW CP } \\
\text { TRW CP } \\
\text { TRW CP }\end{array}$ & $\begin{array}{ll}\text { TRW } & K D \\
\text { TRW } & \text { KD } \\
\text { TRW } & \text { KD }\end{array}$ & $\begin{array}{l}\text { WAR KD } \\
\text { WAR KD }\end{array}$ & $\begin{array}{l}\text { TRW CP } \\
\text { TRW CP } \\
\text { TRW CP } \\
\text { TRW CP }\end{array}$ \\
\hline
\end{tabular}


MCL RUN DATA SUMMARY

RUN NO.

COAL SEAM

RUN DATE

TIME

$\mathrm{KOH} / \mathrm{NaOH}$

CAUSTIC/COAL

COAL SIZE

KILN WALL TEMP, C

ZONE 1

ZONE 2

ZONE 3

ZONE 4

ZONE 2 SENS TEMP, C

KILN RES TIME, hrs

KILN RPM

AUGER SPEED, \% MAX

AUGER SEC F/SEC B

AVG FEED, LB/HR

WASH WATER, LB/HR

PRODUCT COAL:

S, \% (dry basis)

Ash, \% (dry basis)

BTU/1b (dry basis)

Vols, \% (dry basis) Moisture, \% w/w

No. of S Data Pts

REMOVAL:

S, \% (MAF basis)

Ash, \% (dry basis)

BTU/ib, \% (dry basis)

Vols, \% (MAF basis) ib SO2/MMBTU, \%

ib S02/MMBTU, \%(ROM)

REMARKS

S Analysis Data

Ash Analys is Data

Vol Analysis Data

Moisture Anal Data
$8 \mathrm{~F} \quad 8 \mathrm{~F}$

PITT \& PITT 8

$5 / 18-19 / 89$ 5/18-19/89

2000-1300 2000-1300

$\begin{array}{rr}0 & 0 \\ 2 & 2\end{array}$

14 MESH 14 MESH

$412 \quad 412$

$412 \quad 412$

$412 \quad 412$

$412 \quad 412$

$384 \quad 384$

$2.1 \quad 2.1$

$0.8 \quad 0.8$

$30 \quad 30$

$20 \mathrm{~F} / 10 \mathrm{~B} \quad 20 \mathrm{~F} / 10 \mathrm{~B}$

$17.7 \quad 17.7$

$120 \quad 120$

$1.01 \quad 0.92$

$0.31 \quad 0.52$

26.23

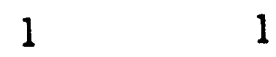

$79.02 \quad 80.84$

$97.32 \quad 95.51$

$-8.92$

39.72

$78.28 \quad 80.22$

$87.18 \quad 88.32$

TRW KD WAR KD

TRW KD WAR KD 
MCL RUN DATA SUMMARY

\begin{tabular}{|c|c|c|c|c|c|}
\hline $\begin{array}{l}\text { RUN NO. } \\
\text { COAL SEAM } \\
\text { RUN DATE } \\
\text { TIME } \\
\text { KOH/NaOH } \\
\text { CAUSTIC/COAL } \\
\text { COAL SIZE } \\
\text { KILN WALL TEMP, C }\end{array}$ & $\begin{array}{r}9 A \\
\text { PITT } 8 \\
5 / 30 / 89 \\
0515-1730 \\
1 \\
2.5 \\
14 \text { MESH }\end{array}$ & $\begin{array}{r}9 A \\
\text { PITT } 8 \\
5 / 30 / 89 \\
0515-1730 \\
1 \\
2.5 \\
14 \text { MESH }\end{array}$ & $\begin{array}{r}9 A \\
P I T T 8 \\
5 / 30 / 89 \\
0230-0515 \\
\ddot{1} \\
2.5 \\
14 \mathrm{MESH}\end{array}$ & $\begin{array}{r}9 A 1 \\
\text { PITT } 8 \\
5 / 30-31 / 89 \\
1730-0430 \\
1 \\
2.5 \\
14 \mathrm{MESH}\end{array}$ & $\begin{array}{r}9 A 1 \\
\text { PITT } 8 \\
5 / 30-31 / 89 \\
1730-0430 \\
1 \\
2.5 \\
14 \mathrm{MESH}\end{array}$ \\
\hline $\begin{array}{l}\text { ZONE } 1 \\
\text { ZONE } 2 \\
\text { ZONE } 3 \\
\text { ZONE } 4 \\
\text { ZONE } 2 \text { SENS TEMP, C } \\
\text { KILN RES TIME, hrs } \\
\text { KILN RPM } \\
\text { AUGER SPEED, \% MAX } \\
\text { AUGER SEC F/SEC B } \\
\text { AVG FEED, LB/HR } \\
\text { WASH WATER, LB/HR } \\
\text { PRODUCT COAL: }\end{array}$ & $\begin{array}{r}427 \\
427 \\
427 \\
427 \\
395 \\
2.1 \\
0.8 \\
30 \\
20 F / 10 B \\
24.8 \\
120\end{array}$ & $\begin{array}{r}427 \\
427 \\
427 \\
427 \\
395 \\
2.1 \\
0.8 \\
30 \\
20 F / 10 B \\
24.8 \\
120\end{array}$ & $\begin{array}{r}427 \\
427 \\
427 \\
427 \\
395 \\
2.1 \\
0.8 \\
30 \\
20 F / 10 B \\
24.8 \\
0\end{array}$ & $\begin{array}{r}438 \\
438 \\
438 \\
438 \\
407 \\
2.1 \\
0.8 \\
30 \\
20 \mathrm{~F} / 10 \mathrm{~B} \\
17.2 \\
120\end{array}$ & $\begin{array}{r}438 \\
438 \\
438 \\
438 \\
407 \\
2.1 \\
0.8 \\
30 \\
20 F / 10 B \\
17.2 \\
120\end{array}$ \\
\hline $\begin{array}{l}\text { S, \% (dry basis) } \\
\text { Ash, \% (dry basis) } \\
\text { BTU/1b (dry basis) }\end{array}$ & $\begin{array}{l}0.59 \\
0.27\end{array}$ & $\begin{array}{r}0.88 \\
0.84 \\
13079\end{array}$ & $\begin{array}{l}0.97 \\
0.74\end{array}$ & $\begin{array}{l}0.65 \\
0.33\end{array}$ & $\begin{array}{r}0.72 \\
0.66 \\
13049\end{array}$ \\
\hline $\begin{array}{l}\text { Vols, \% (dry basis) } \\
\text { Moisture, \% w/w } \\
\text { No. of S Data Pts } \\
\text { REMOVAL: }\end{array}$ & $\begin{array}{r}25.97 \\
58.83 \\
7\end{array}$ & 22.60 & 26.84 & $\begin{array}{r}25.63 \\
57.39 \\
5\end{array}$ & 22.28 \\
\hline $\begin{array}{c}\text { S, \% (MAF basis) } \\
\text { Ash, \% (dry basis) } \\
\text { BTU/ib, \%(dry basis) }\end{array}$ & $\begin{array}{l}87.80 \\
97.68\end{array}$ & $\begin{array}{l}81.62 \\
92.74 \\
-0.48\end{array}$ & $\begin{array}{l}79.67 \\
93.65\end{array}$ & $\begin{array}{l}86.57 \\
97.11\end{array}$ & $\begin{array}{l}84.99 \\
94.34 \\
-0.25\end{array}$ \\
\hline $\begin{array}{l}\text { Vols, \% (MAF basis) } \\
\text { ib SO2/MMBTU, \% } \\
\text { Ib SO2/MMBTU, \%(ROM) } \\
\text { REMARKS }\end{array}$ & $\begin{array}{l}40.35 \\
86.31 \\
91.92\end{array}$ & $\begin{array}{l}47.79 \\
79.49 \\
87.89\end{array}$ & $\begin{array}{l}38.05 \\
77.29 \\
86.59\end{array}$ & $\begin{array}{l}41.08 \\
84.90 \\
91.08\end{array}$ & $\begin{array}{l}48.62 \\
83.18 \\
90.07\end{array}$ \\
\hline $\begin{array}{l}\text { S Analys is Data } \\
\text { Ash Analysis Data } \\
\text { Vol Analysis Data } \\
\text { Moisture Anal Data }\end{array}$ & $\begin{array}{l}\text { TRW CP } \\
\text { TRW CP } \\
\text { TRW CP } \\
\text { TRW CP }\end{array}$ & $\begin{array}{l}\text { WAR CP } \\
\text { WAR CP } \\
\text { WAR CP }\end{array}$ & $\begin{array}{l}\text { TRW } \\
\text { KD } \\
\text { TRW } \\
\text { TRD } \\
\text { TRD }\end{array}$ & $\begin{array}{l}\text { TRW CP } \\
\text { TRW CP } \\
\text { TRW CP } \\
\text { TRW CP }\end{array}$ & $\begin{array}{l}\text { WAR CP } \\
\text { WAR CP } \\
\text { WAR CP }\end{array}$ \\
\hline
\end{tabular}


MCL RUN DATA SUMMARY

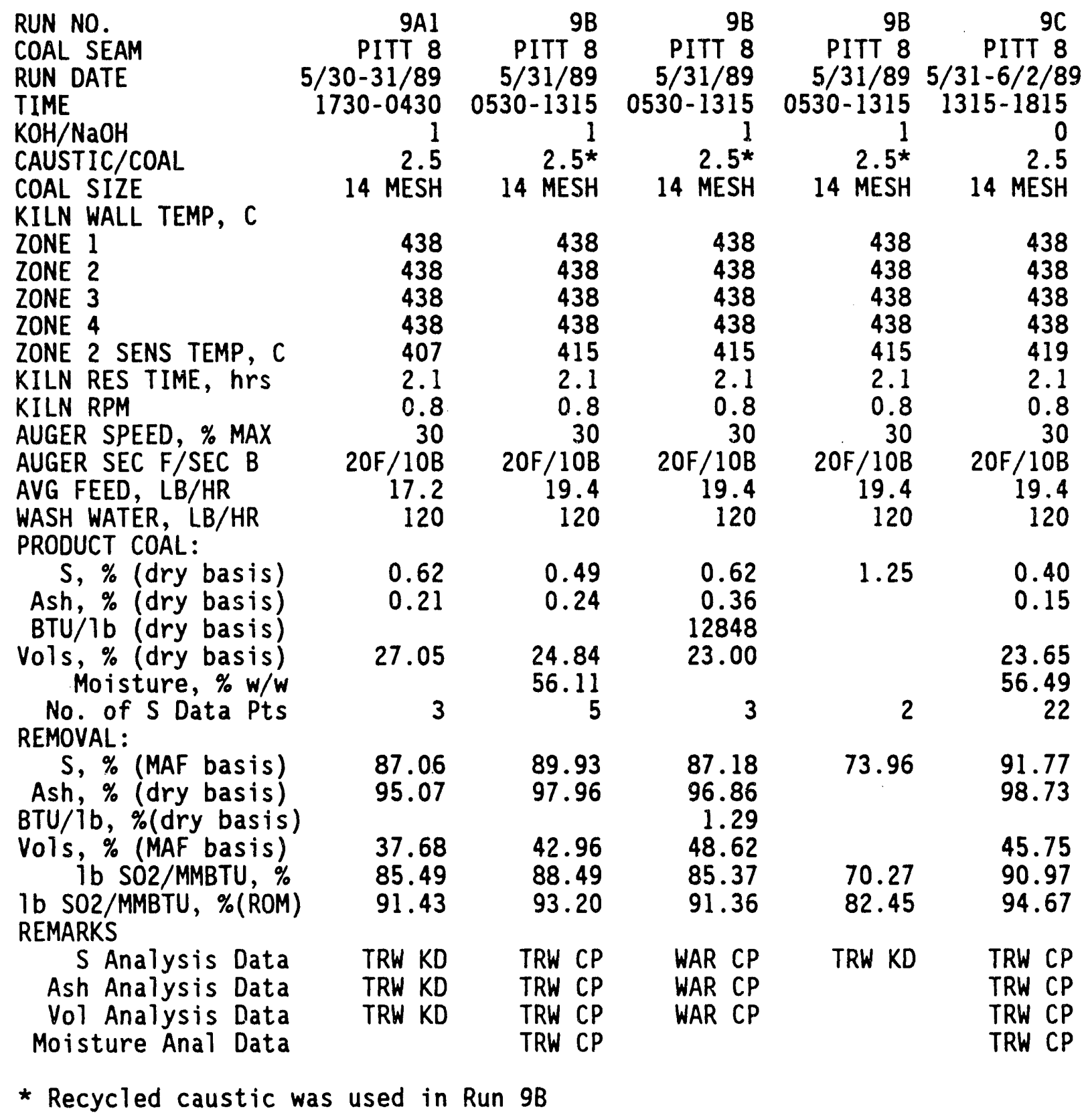


MCL RUN DATA SUMMARY

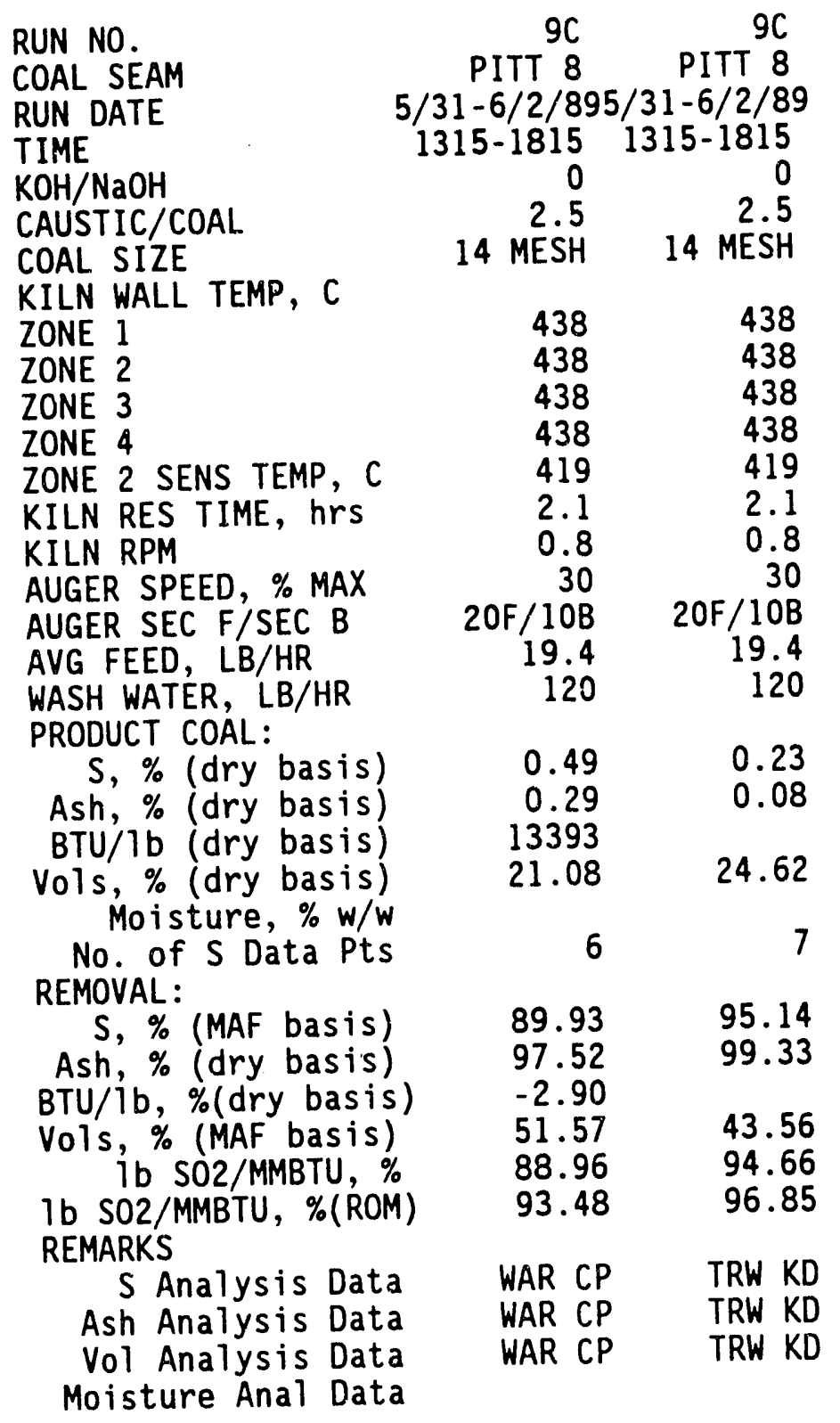


MCL RUN DATA SUMMARY

RUN NO.

COAL SEAM

RUN DATE

TIME

$\mathrm{KOH} / \mathrm{NaOH}$

CAUSTIC/COAL

KILN WALL TEMP, $C$

ZONE 1

ZONE 2

ZONE 3

ZONE 4

ZONE 2 SENS TEMP, $C$

KILN RES TIME, hrs

KILN RPM

AUGER SPEED, \% MAX

AUGER SEC F/SEC B

AVG FEED, LB/HR

WASH WATER, LB/HR

PRODUCT COAL:

S, \% (dry basis)

Ash, \% (dry basis)

BTU/1b (dry basis)

Vols, \% (dry basis) Moisture, \% w/w

No. of S Data Pts

REMOVAL:

S, \% (MAF basis)

Ash, \% (dry basis)

BTU/ib, \% (dry basis)

Vols, \% (MAF basis) ib SO2/MMBTU, \%

Ib S02/MMBTU, \%(ROM)

REMARKS

$$
\text { S Analysis Data }
$$

Ash Analysis Data

Vol Analysis Data

Moisture Anal Data

\begin{tabular}{|c|c|c|c|}
\hline $\begin{array}{r}10 A \\
\text { PITT } 8^{\star} \\
6 / 3 / 91 \\
0150-1530 \\
0 \\
2\end{array}$ & $\begin{array}{r}10 \mathrm{~B} \\
\text { PITT } 8 * \\
6 / 3-4 / 91 \\
1530-0640 \\
0 \\
2\end{array}$ & $\begin{array}{r}10 C \\
\text { PITT } 8^{*} \\
6 / 4-5 / 91 \\
0640-0125 \\
0 \\
2\end{array}$ & $\begin{array}{r}10 D \\
\text { PITT 8* } \\
6 / 5-7 / 91 \\
0125-1000 \\
0 \\
2\end{array}$ \\
\hline $\begin{array}{l}380 \\
380 \\
380 \\
380\end{array}$ & $\begin{array}{l}380 \\
380 \\
380 \\
380\end{array}$ & $\begin{array}{l}360 \\
360 \\
360 \\
360\end{array}$ & $\begin{array}{l}400 \\
400 \\
400 \\
400\end{array}$ \\
\hline $\begin{array}{r}2.0 \\
1 \\
30 \\
20 \mathrm{~F} / 10 \mathrm{~B} \\
40.9 \\
120\end{array}$ & $\begin{array}{r}2.0 \\
1 \\
30 \\
20 \mathrm{~F} / 10 \mathrm{~B} \\
27.3 \\
120\end{array}$ & $\begin{array}{r}2.0 \\
1 \\
30 \\
20 \mathrm{~F} / 10 \mathrm{~B} \\
27.3 \\
120\end{array}$ & $\begin{array}{r}2.0 \\
1 \\
30 \\
20 F / 10 B \\
27.3 \\
120\end{array}$ \\
\hline $\begin{array}{l}1.58 \\
0.86\end{array}$ & $\begin{array}{l}1.49 \\
0.78\end{array}$ & $\begin{array}{l}1.35 \\
1.52\end{array}$ & $\begin{array}{r}1.04 \\
0.82 \\
13650\end{array}$ \\
\hline 37.15 & 34.48 & 34.82 & $\begin{array}{l}30.3 \\
55.2\end{array}$ \\
\hline 1 & 1 & 4 & \\
\hline $\begin{array}{l}66.99 \\
92.57\end{array}$ & $\begin{array}{l}68.90 \\
93.26\end{array}$ & $\begin{array}{l}71.82 \\
86.86\end{array}$ & $\begin{array}{l}78.28 \\
92.91 \\
-4.87\end{array}$ \\
\hline $\begin{array}{l}14.15 \\
64.72 \\
79.16\end{array}$ & $\begin{array}{l}20.39 \\
66.73 \\
80.35\end{array}$ & $\begin{array}{l}19.00 \\
69.85 \\
82.20\end{array}$ & $\begin{array}{l}29.85 \\
76.78 \\
86.29\end{array}$ \\
\hline $\begin{array}{ll}\text { TRW } & \text { KD } \\
\text { TRW } & \text { KD } \\
\text { TRW } & \text { KD } \\
\text { TRW } & \text { KD }\end{array}$ & $\begin{array}{ll}\text { TRW } & \text { KD } \\
\text { TRW } & \text { KD } \\
\text { TRW } & \text { KD } \\
\text { TRW } & \text { KD }\end{array}$ & $\begin{array}{ll}\text { TRW } & C P \\
\text { TRW } & C P \\
\text { TRW } & C P \\
\text { TRW } & C P\end{array}$ & $\begin{array}{ll}\text { TRW } & C P \\
\text { TRW } & C P \\
\text { TRW } & C P \\
\text { TRW } & C P\end{array}$ \\
\hline
\end{tabular}

*The Pittsburgh No. 8 coal (Powhatan No. 6 mine) used for Run 10 was 3 years old and thus weathered (highly friable). 
MCL RUN DATA SUMMARY

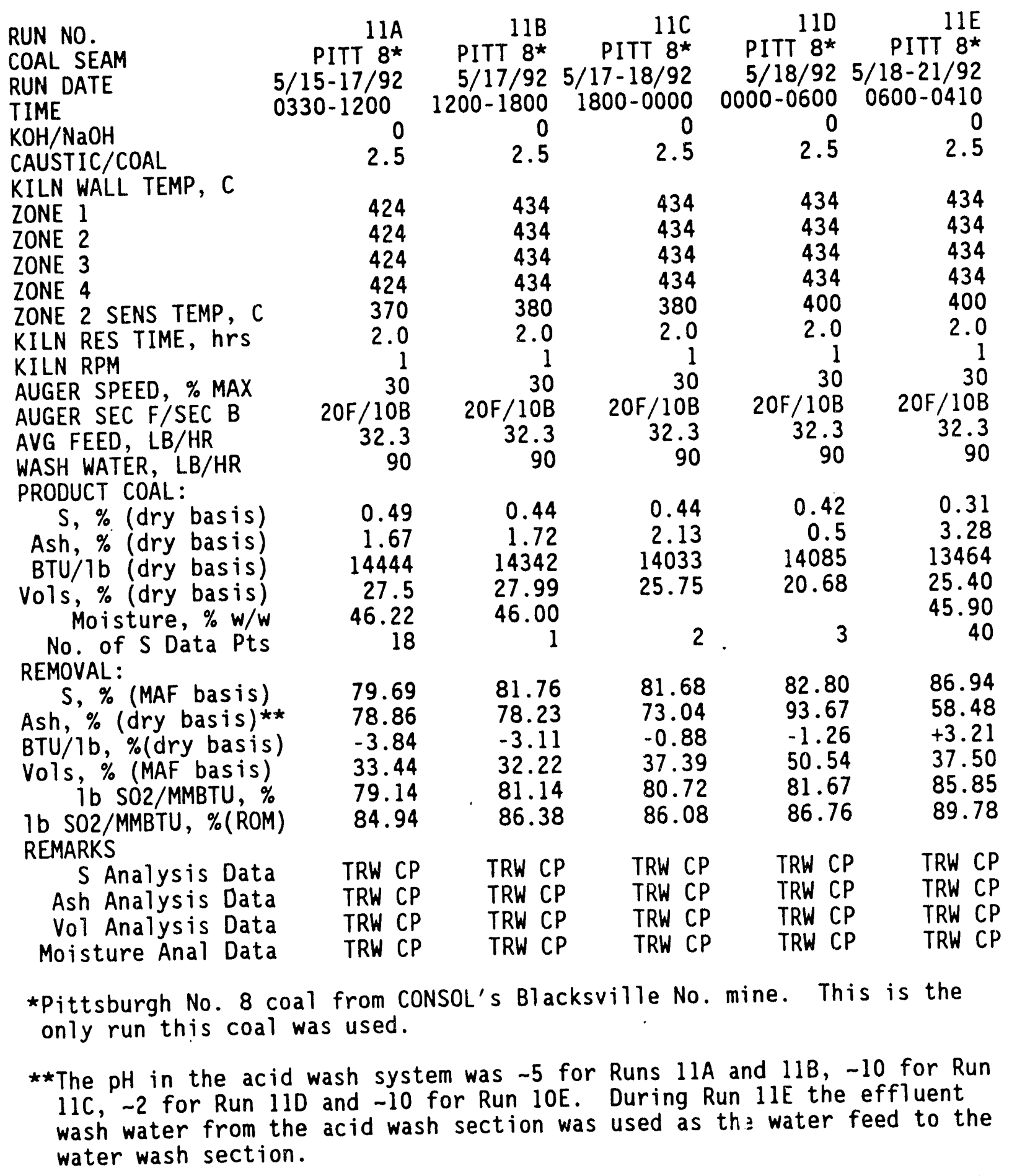


APPENDIX B

PRODUCT COAL ANALYSIS DATA 


\section{B-2}


COAL PRODUCT DATA TABULATION

SHAKEDOWN; 12/12 - 12/16/88

SHAKEDOWN

1 12-Dec 00

2 12-Dec 02

$3 \quad 12-$ Dec 04

$4 \quad 12-$ Dec 06

$5 \quad 12-$ Dec 08

$6 \quad 12-$ Dec 10

7 12-Dec 12

8 12-Dec 14

$9 \quad 12-\mathrm{Dec} \quad 16$

$10 \quad 12$-Dec 18

$11 \quad 12-\mathrm{Dec} \quad 20$

$12 \quad 12-\mathrm{Dec} \quad 22$

13 13-Dec 00

14 13-Dec 02

15 13-Dec 04

$16 \quad 13-\mathrm{Dec} \quad 06$

$17 \quad 13-D e C \quad 08$

$18 \quad 13-$ Dec 10

19 13-Dec 12

$20 \quad 13-\mathrm{Dec} \quad 14$

$21 \quad 13-D e c \quad 16$

$22 \quad 13-D e c \quad 18$

23 13-Dec 20

$24 \quad 13-D e c \quad 22$

$25 \quad 14-\mathrm{Dec} \quad 00$

$26 \quad 14-\mathrm{Dec} \quad 02$

27 14-Dec 04

28 14-Dec 06

29 14-Dec 08

$30 \quad 14-\mathrm{Dec} 10$

$31 \quad 14-\mathrm{Dec} \quad 12$

$32 \quad 14-D e c \quad 14$

$33 \quad 14-\mathrm{Dec} \quad 16$

$34 \quad 14-$ Dec 18

35 14-Dec 20

$36 \quad 14-\mathrm{Dec} \quad 22$

$37 \quad 15-\mathrm{Dec} \quad 00$

$38 \quad 15-$ Dec 02

$39 \quad 15-\mathrm{Dec} \quad 04$

$40 \quad 15-\mathrm{Dec} \quad 06$

41 15-Dec 08

42 15-Dec 10 
COAL PRODUCT DATA TABULATION

SHAKEDOWN; $12 / 12-12 / 16 / 88$

SHAKEDOWN

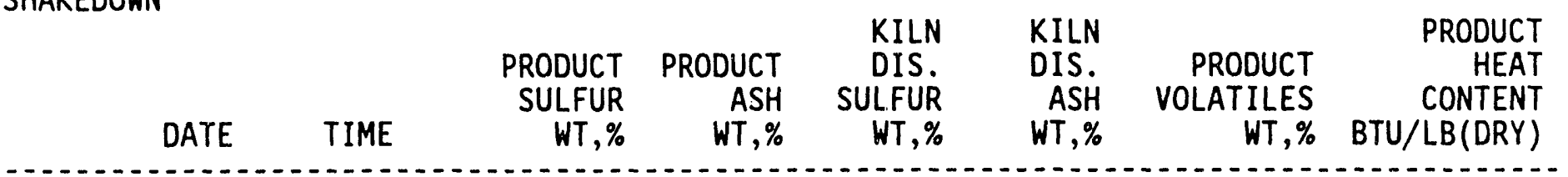

$43 \quad 15-\operatorname{Dec} \quad 12$

44 15-Dec 14

45 15-Dec 16

$46 \quad 15-\mathrm{Dec} \quad 18$

47 15-Dec 20

48 15-Dec 22

49 16-Dec 00

50 16-Dec 02

51 16-Dec 04

$2.40 \% \quad 1.79 \%$

$2.44 \% \quad 1.82 \%$

$2.37 \% \quad 2.24 \%$

$2.52 \% \quad 2.67 \%$

$2.56 \% \quad 2.69 \%$

$2.56 \% \quad 3.11 \%$

$2.55 \% \quad 3.13 \%$

$2.62 \% \quad 3.72 \%$

$2.71 \% \quad 4.54 \%$
$40.47 \%$

$40.19 \%$

$41.20 \%$

$41.59 \%$

$42.40 \%$

$39.37 \%$

$41.53 \%$

$41.10 \%$

$40.65 \%$

58 16-Dec 18

$59 \quad 16-\mathrm{Dec} \quad 20$

60 16-Dec 22

61 16-Dec 00 
COAL PRODUCT DATA TABULATION

RUN NO. $1 ; 1 / 23-1 / 27 / 89$

RUN NO. 1

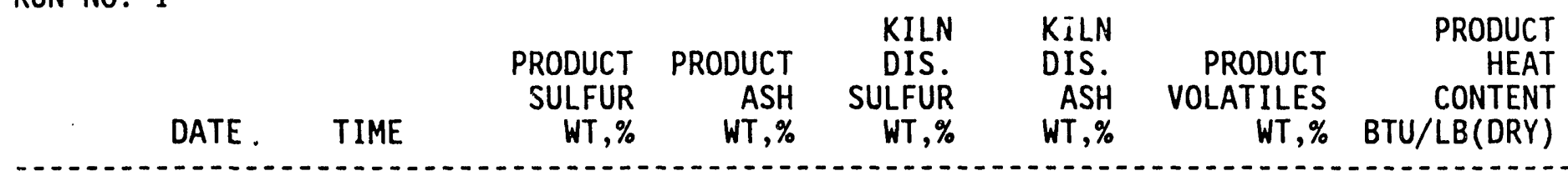

$123-J a n \quad 00$

2 23-Jan 02

3 23-Jan 04

4 23-Jan 06

5 23-Jan 08

$623-J a n \quad 10$

$723-J a n \quad 12$

8 23-Jan 14

$923-J a n \quad 16$

$10 \quad 23-J a n \quad 18$

$1123-J a n \quad 20$

12 23-Jan 22

13 24-Jan 00

14 24-Jan 02

15 24-Jan 04

16 24-Jan 06

17 24-Jan 08

18 24-Jan 10

19 24-Jan 12

20 24-Jan 14

21 24-Jan 16

22 24-Jan 18

23 24-Jan 20

24 24-Jan 22

25 25-Jan 00

26 25-Jan 02

27 25-Jan 04

28 25-Jan 06

29 25-Jan 08

30 25-Jan 10

31 25-Jan 12

32 25-Jan 14

$33 \quad 25-J a n \quad 16$

34 25-Jan 18

35 25-Jan 20

36 25-Jan 22

37 26-Jan 00

38 26-Jan 02

39 26-Jan 04

40 26-Jan 06

41 26-Jan 08

42 26-Jan 10

$\begin{array}{ll}1.76 \% & 2.40 \% \\ 2.20 \% & 2.71 \% \\ 2.05 \% & 2.41 \% \\ 1.82 \% & 1.87 \% \\ 1.91 \% & 2.02 \% \\ 1.93 \% & 2.19 \% \\ 2.00 \% & 2.07 \% \\ 1.87 \% & 1.58 \% \\ 1.79 \% & 1.43 \% \\ 1.61 \% & 1.65 \% \\ 1.37 \% & 1.47 \% \\ 1.40 \% & 1.18 \% \\ 1.49 \% & 1.16 \% \\ 1.41 \% & 1.21 \% \\ 1.36 \% & 1.16 \% \\ 1.43 \% & 1.27 \% \\ 1.39 \% & 1.45 \%\end{array}$

$34.41 \%$

$37.02 \%$

$35.23 \%$

$35.54 \%$

$32.99 \%$

$33.49 \%$

$34.57 \%$

$34.67 \%$

$35.98 \%$

$25.16 \%$

$26.95 \%$

$27.15 \%$

$32.31 \%$

$27.55 \%$

$24.53 \%$

$25.88 \%$

$27.29 \%$ 
COAL PRODUCT DATA TABULATION

RUN NO. $1 ; 1 / 23-1 / 27 / 89$

RUN NO. 1

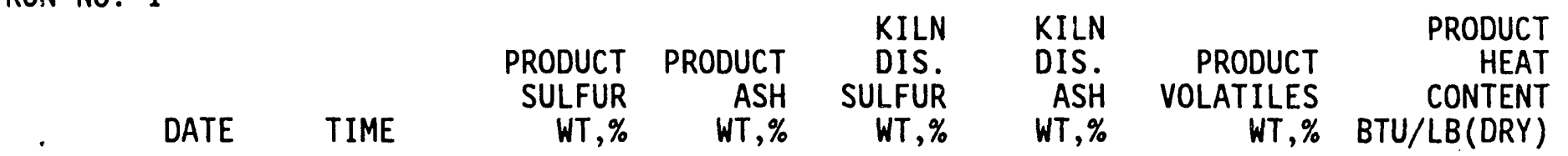

$\begin{array}{lllll}43 & 26-J a n & 12 & & \\ 44 & 26-J a n & 14 & 1.07 \% & 0.86 \% \\ 45 & 26-J a n & 16 & 1.17 \% & 0.97 \% \\ 46 & 26-J a n & 18 & 1.39 \% & 1.19 \% \\ 47 & 26-J a n & 20 & 1.60 \% & 1.17 \% \\ 48 & 26-J a n & 22 & 1.58 \% & 1.09 \% \\ 49 & 27-J a n & 00 & 1.52 \% & 1.08 \% \\ 50 & 27-J a n & 02 & 1.38 \% & 1.17 \% \\ 51 & 27-J a n & 04 & 1.40 \% & 1.17 \% \\ 52 & 27-J a n & 06 & 1.44 \% & 1.37 \% \\ 53 & 27-J a n & 08 & & \\ 54 & 27-J a n & 10 & & \\ 55 & 27-J a n & 12 & & \\ 56 & 27-J a n & 14 & & \\ 57 & 27-J a n & 16 & & \\ 58 & 27-J a n & 18 & & \\ 59 & 27-J a n & 20 & & \\ 60 & 27-J a n & 22 & & \\ 61 & 2 \text { - Jan } & 00 & & \end{array}$

$31.92 \%$

$32.30 \%$

$27.96 \%$

$29.82 \%$

$31.01 \%$

$30.29 \%$

$30.61 \%$

$29.43 \%$

$29.93 \%$ 
COAL PRODUCT DATA TABULATION

RUN NO. $2 ; 2 / 6-2 / 10 / 89$

RUN NO. 2

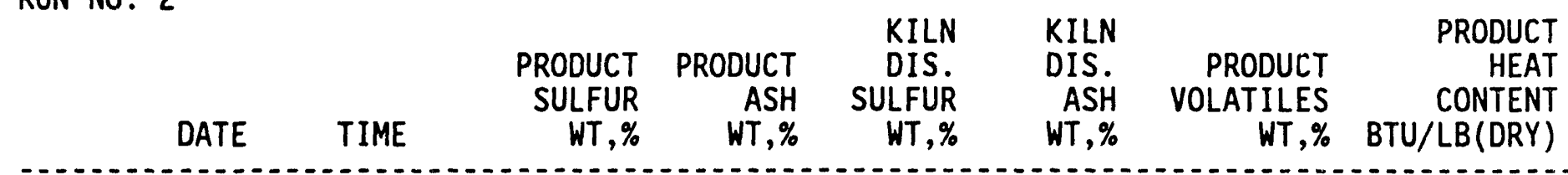

$\begin{array}{rll}1 & 06-\text { Feb } & 00 \\ 2 & 06-\text { Feb } & 02 \\ 3 & 06-\text { Feb } & 04 \\ 4 & 06-\text { Feb } & 06 \\ 5 & 06-\text { Feb } & 08 \\ 6 & 06-\text { Feb } & 10 \\ 7 & 06-\text { Feb } & 12 \\ 8 & 06-\text { Feb } & 14 \\ 9 & 06-\text { Feb } & 16 \\ 10 & 06-\text { Feb } & 18 \\ 11 & 06-\text { Feb } & 20 \\ 12 & 06-\text { Feb } & 22 \\ 13 & 07-\text { Feb } & 00 \\ 14 & 07-\text { Feb } & 02 \\ 15 & 07-\text { Feb } & 04 \\ 16 & 07-\text { Feb } & 06 \\ 17 & 07-\text { Feb } & 08 \\ 18 & 07-\text { Feb } & 10 \\ 19 & 07-\text { Feb } & 12 \\ 20 & 07-\text { Feb } & 14 \\ 21 & 07-\text { Feb } & 16 \\ 22 & 07-\text { Feb } & 18 \\ 23 & 07-\text { Feb } & 20 \\ 24 & 07-\text { Feb } & 22 \\ 25 & 08-\text { Feb } & 00 \\ 26 & 08-\text { Feb } & 02 \\ 27 & 08-\text { Feb } & 04 \\ 28 & 08-\text { Feb } & 06 \\ \text { cy } & 08-\text { Feb } & 08 \\ 30 & 08-\text { Feb } & 10 \\ 31 & 08-\text { Feb } & 12 \\ 32 & 08-\text { Feb } & 14 \\ 33 & 08-\text { Feb } & 16 \\ 34 & 08-\text { Feb } & 18 \\ 35 & 08-\text { Feb } & 20 \\ 36 & 08-\text { Feb } & 22 \\ 37 & 09-\text { Feb } & 00 \\ 38 & 09-\text { Feb } & 02 \\ 39 & 09-\text { Feb } & 04 \\ 40 & 09-\text { Feb } & 06 \\ 41 & 09-\text { Feb } & 08 \\ 42 & 09-\text { Feb } & 10 \\ & \end{array}$

$\begin{array}{ll}0.95 \% & 0.52 \% \\ 0.96 \% & 0.56 \% \\ 0.94 \% & 0.66 \% \\ 0.85 \% & 0.58 \% \\ 0.87 \% & 0.55 \% \\ 0.84 \% & 0.57 \% \\ 0.84 \% & 0.51 \%\end{array}$

$32.32 \%$

13036

$30.27 \%$

$27.80 \%$

$27.88 \%$

$30.25 \%$

$32.00 \%$

$31.20 \%$ 
RUN NO. $2 ; 2 / 6-2 / 10 / 89$

RUN NO. 2

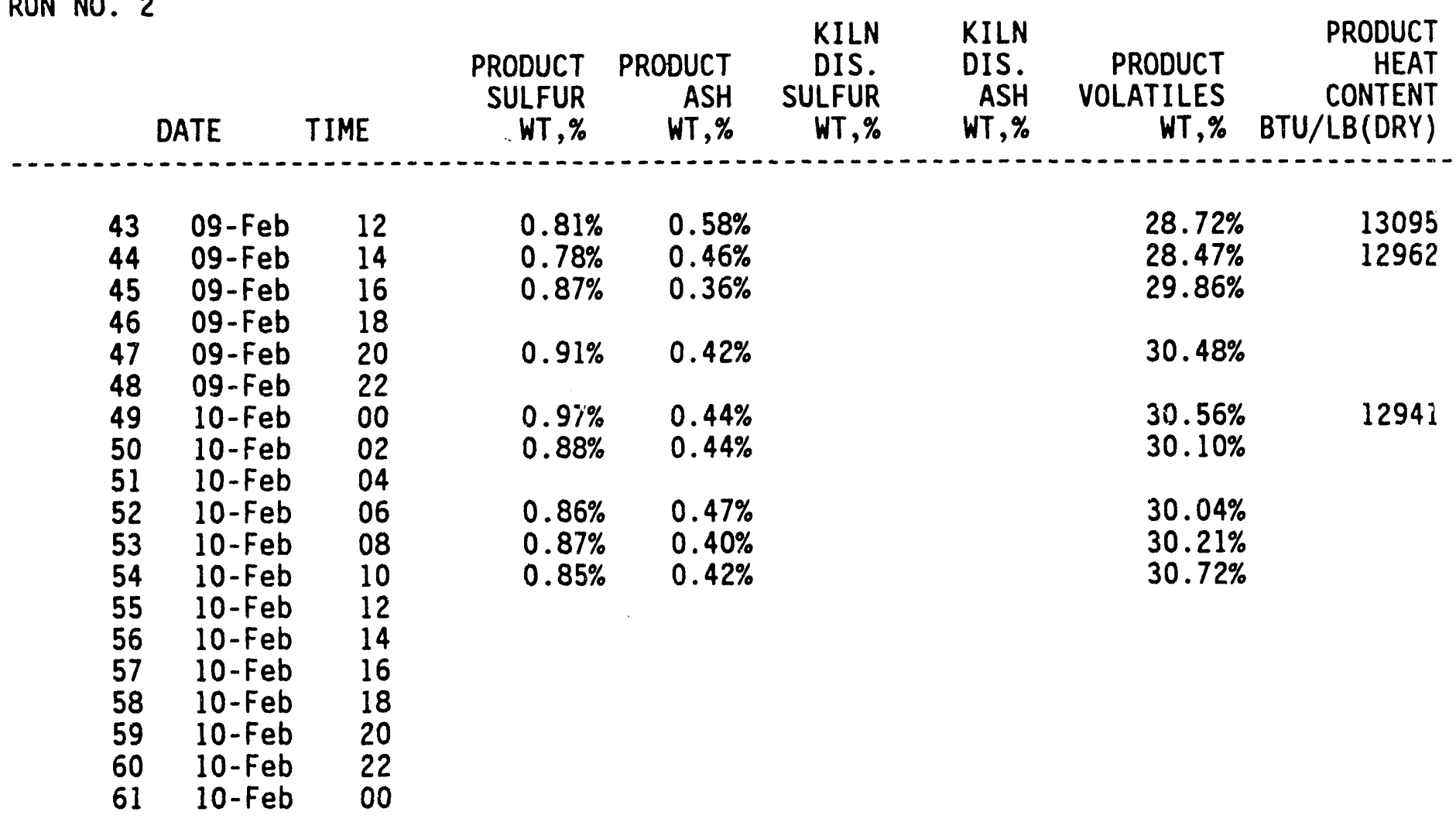


COAL PRODUCT DATA TABULATION

RUN NO. $3 ; 2 / 20-2 / 24 / 89$

RUN NO. 3

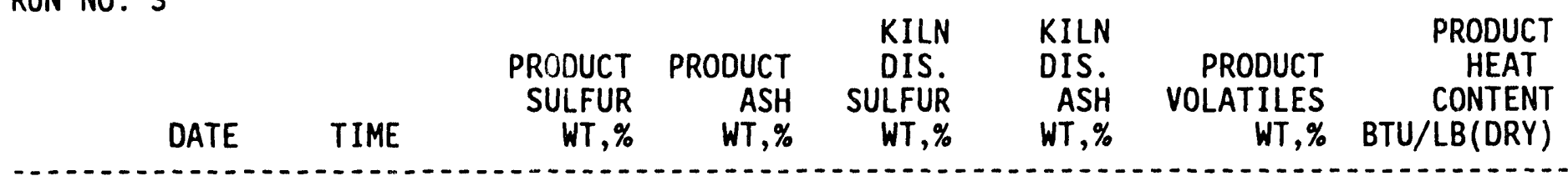

\begin{tabular}{|c|c|c|c|c|c|c|}
\hline $\begin{array}{l}20-\text { Feb } \\
20-\text { Feb } \\
20-\text { Feb } \\
20-\text { Feb } \\
20-\text { Feb } \\
20-\text { Feb } \\
20-\text { Feb } \\
20-\text { Feb } \\
20-\text { Feb } \\
20-F e b \\
20-F e b \\
20-F e b \\
21-F e b \\
21-F e b \\
21-F e b \\
21-F e b \\
21-F e b \\
21-F e b \\
21-F e b \\
21-F e b \\
21-F e b \\
21-F e b \\
21-F e b \\
21-F e b \\
22-F e b \\
22-F e b \\
22-F e b \\
22-F e b \\
22-F e b \\
22-F e b \\
22-F e b \\
22-F e b \\
22-F e b \\
22-F e b \\
22-F e b \\
22-F e b \\
23-F e b \\
23-F e b \\
23-F e b \\
23-F e b \\
23-F e b \\
23-F e b\end{array}$ & $\begin{array}{l}00 \\
02 \\
04 \\
06 \\
08 \\
10 \\
12 \\
14 \\
16 \\
18 \\
20 \\
22 \\
00 \\
02 \\
04 \\
06 \\
08 \\
10 \\
12 \\
14 \\
16 \\
18 \\
20 \\
22 \\
00 \\
02 \\
04 \\
06 \\
08 \\
10 \\
12 \\
14 \\
16 \\
18 \\
20 \\
22 \\
00 \\
02 \\
04 \\
06 \\
08 \\
10\end{array}$ & $0.74 \%$ & $0.17 \%$ & $\begin{array}{l}0.88 \% \\
1.25 \% \\
0.39 \% \\
1.23 \% \\
0.40 \%\end{array}$ & $\begin{array}{l}0.88 \% \\
0.45 \% \\
0.68 \% \\
0.46 \% \\
0.62 \%\end{array}$ & $21.78 \%$ \\
\hline
\end{tabular}


COAL PRODUCT DATA TABULATION

RUN NO. $3 ; 2 / 20$ - 2/24/89

RUN NO. 3

\begin{tabular}{|c|c|c|c|c|c|c|c|c|}
\hline . & ATE & TIME & $\begin{array}{r}\text { PRODUCT } \\
\text { SULFUR } \\
\text { WT, \% }\end{array}$ & $\begin{array}{r}\text { PRODUCT } \\
\text { ASH } \\
\text { WT, \% }\end{array}$ & $\begin{array}{l}\text { KILN } \\
\text { DIS. } \\
\text { SULFUR } \\
\text { WT, } \%\end{array}$ & $\begin{array}{l}\text { KILN } \\
\text { DIS. } \\
\text { ASH } \\
\text { WT, \% }\end{array}$ & $\begin{array}{r}\text { PRODUCT } \\
\text { VOLATILES } \\
\text { WT, } \%\end{array}$ & $\begin{array}{c}\text { PRODUCT } \\
\text { HEAT } \\
\text { CONTENT } \\
\text { BTU/LB(DRY) }\end{array}$ \\
\hline $\begin{array}{r}43 \\
44 \\
45 \\
46 \\
47 \\
48 \\
49 \\
50 \\
51 \\
52 \\
53 \\
54 \\
55 \\
56 \\
57 \\
58 \\
59 \\
60 \\
61\end{array}$ & $\begin{array}{l}23-\text { Feb } \\
23-\text { Feb } \\
23-\text { Feb } \\
23-\text { Feb } \\
23-\text { Feb } \\
23-\text { Feb } \\
24-F e b \\
24-F e b \\
24-F e b \\
24-\text { Feb } \\
24-\text { Feb } \\
24-\text { Feb } \\
24-\text { Feb } \\
24-\text { Feb } \\
24-F e b \\
24-F e b \\
24-F e b \\
24-F e b \\
24-F e b\end{array}$ & $\begin{array}{l}12 \\
14 \\
16 \\
18 \\
20 \\
22 \\
00 \\
02 \\
04 \\
06 \\
08 \\
10 \\
12 \\
14 \\
16 \\
18 \\
20 \\
22 \\
00\end{array}$ & $\begin{array}{l}0.59 \% \\
0.59 \% \\
0.66 \% \\
0.78 \% \\
0.75 \% \\
0.72 \%\end{array}$ & $\begin{array}{l}0.18 \% \\
0.24 \% \\
0.22 \% \\
0.18 \% \\
0.23 \% \\
0.23 \%\end{array}$ & & & $\begin{array}{l}21.64 \% \\
18.51 \% \\
20.62 \% \\
21.24 \% \\
21.23 \% \\
20.93 \%\end{array}$ & $\begin{array}{l}12708 \\
12565 \\
12618 \\
12592 \\
12588\end{array}$ \\
\hline
\end{tabular}


COAL PRODUCT DATA TABULATION

RUN NO. $4 ; 3 / 6-3 / 10 / 89$

RUN NO. 4

\begin{tabular}{|c|c|c|c|c|c|c|c|}
\hline DATE & TIME & $\begin{array}{r}\text { PRODUCT } \\
\text { SULFUR } \\
\text { WT, } \%\end{array}$ & $\begin{array}{r}\text { PRODUCT } \\
\text { ASH } \\
\text { WT, \% }\end{array}$ & $\begin{array}{l}\text { KILN } \\
\text { DIS. } \\
\text { SULFUR } \\
\text { WT, \% }\end{array}$ & $\begin{array}{l}\text { KILN } \\
\text { DIS. } \\
\text { ASH } \\
\text { WT, \% }\end{array}$ & $\begin{array}{r}\text { PRODUCT } \\
\text { VOLATILES } \\
\text { WT, \% }\end{array}$ & $\begin{array}{r}\text { PRODUCT } \\
\text { HEAT } \\
\text { CONTENT } \\
\text { BTU/LB(DRY) }\end{array}$ \\
\hline
\end{tabular}

$1 \quad 06-\operatorname{Mar} \quad 00$

2 06-Mar 02

3 06-Mar 04

4 06-Mar 06

5 06-Mar 08

6 06-Mar 10

7 06-Mar 12

$806-$ Mar 14

9 06-Mar 16

10 06-Mar 18

$1106-$ Mar 20

$1206-$ Mar 22

13 07-Mar 00

14 07-Mar 02

15 07-Mar 04

16 07-Mar 06

17 07-Mar 08

18 07-Mar 10

19 07-Mar 12

$20 \quad 07-$ Mar 14

$21 \quad 07-\operatorname{Mar} 16$

22 07-Mar 18

23 07-Mar 20

$24 \quad 07-\operatorname{Mar} 22$

25 08-Mar 00

26 08-Mar 02

27 08-Mar 04

28 08-Mar 06

29 08-Mar 08

30 08-Mar 10

31 08-Mar 12

32 08-Mar 14

33 08-Mar 16

34 08-Mar 18

35 08-Mar 20

36 08-Mar 22

37 09-Mar 00

38 09-Mar 02

39 09-Mar 04

40 09-Mar 06

41 09-Mar 08

42 09-Mar 10

$0.79 \%$

$0.76 \%$

$0.82 \%$

$1.11 \%$

$1.30 \%$

$1.29 \%$

$1.63 \%$

$1.60 \%$

$1.37 \%$

$1.40 \%$

$1.34 \%$

$1.23 \%$

$1.12 \%$

$1.13 \%$

$1.17 \%$

$1.31 \%$

$1.27 \%$

$1.25 \%$

$1.21 \%$

$1.20 \%$

$0.43 \%$

$0.51 \% \quad 1.19 \%$

$0.52 \%$

$0.65 \%$

$1.64 \%$

$0.69 \%$

$0.63 \%$

$0.53 \%$

$0.46 \%$

$0.46 \%$

$0.51 \%$

$0.57 \%$

$0.54 \%$

$0.54 \%$

$0.49 \%$

$0.54 \%$

$0.53 \%$

$1.14 \%$

$0.41 \%$

$0.84 \%$

$1.41 \%$
$1.74 \%$

$23.95 \%$

$24.53 \%$

$25.51 \%$

$25.99 \%$

$23.25 \%$

$24.89 \%$

$26.37 \%$

$25.62 \%$

$25.15 \%$

$22.96 \%$

$23.71 \%$

$24.46 \%$

$0.54 \%$

$21.49 \%$

$1.45 \% \quad 0.80 \%$

$1.02 \% \quad 0.59 \%$
$22.72 \%$

$24.42 \%$

$22.75 \%$

$24.48 \%$

$22.00 \%$

$22.44 \%$

$24.64 \%$

14116

14306

14302

14217

14191

14213

14224

$24.24 \%$

14201 
COAL PRODUCT DATA TABULATION

RUN NO. 4; 3/6 - 3/10/89

RUN NO. 4

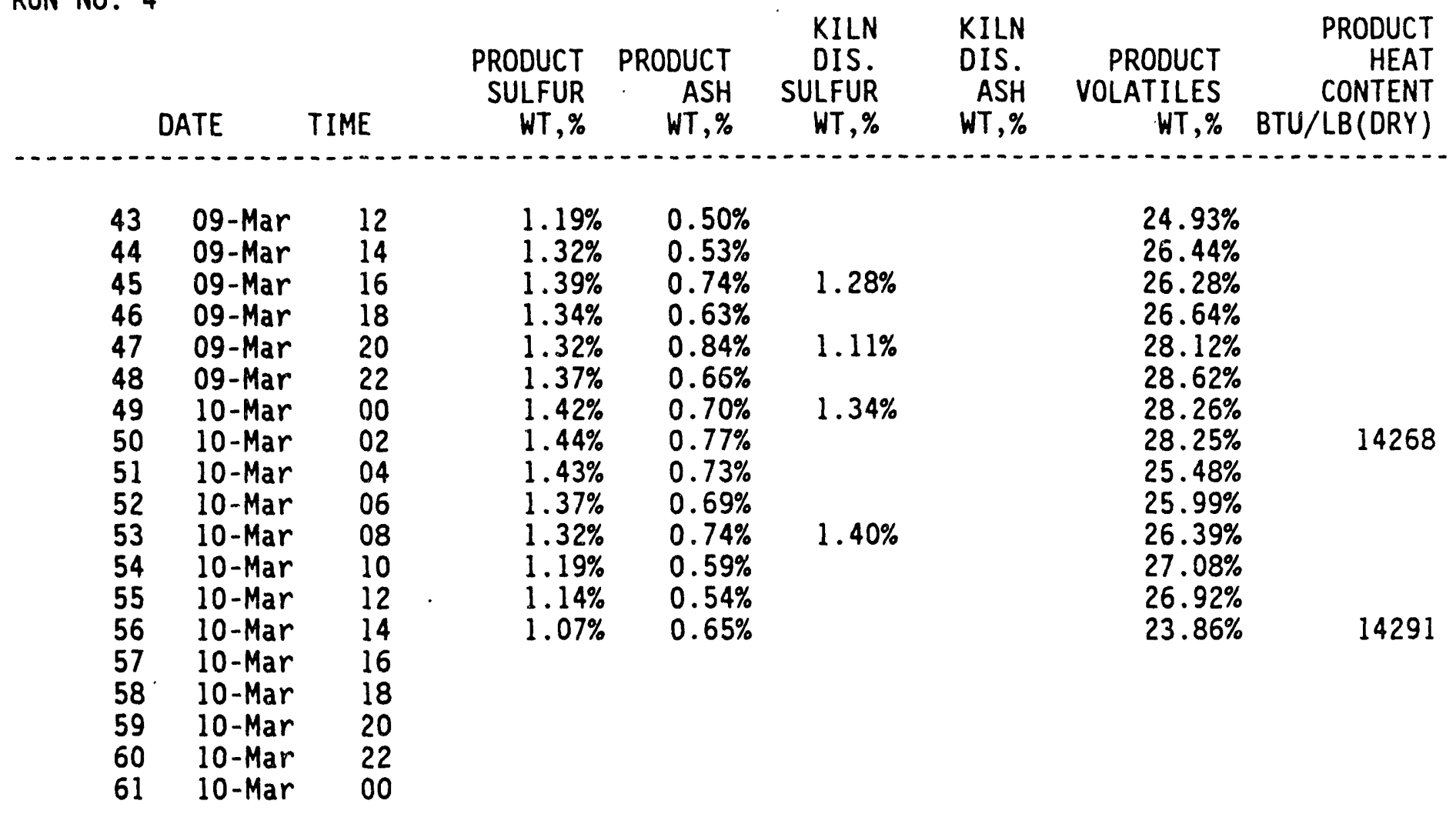


COAL PRODUCT DATA TABULATION

RUN NO. $5 ; 3 / 20-3 / 24 / 89$

RUN NO. 5

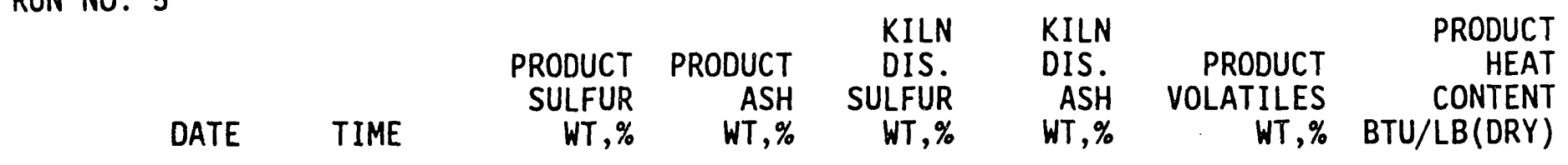

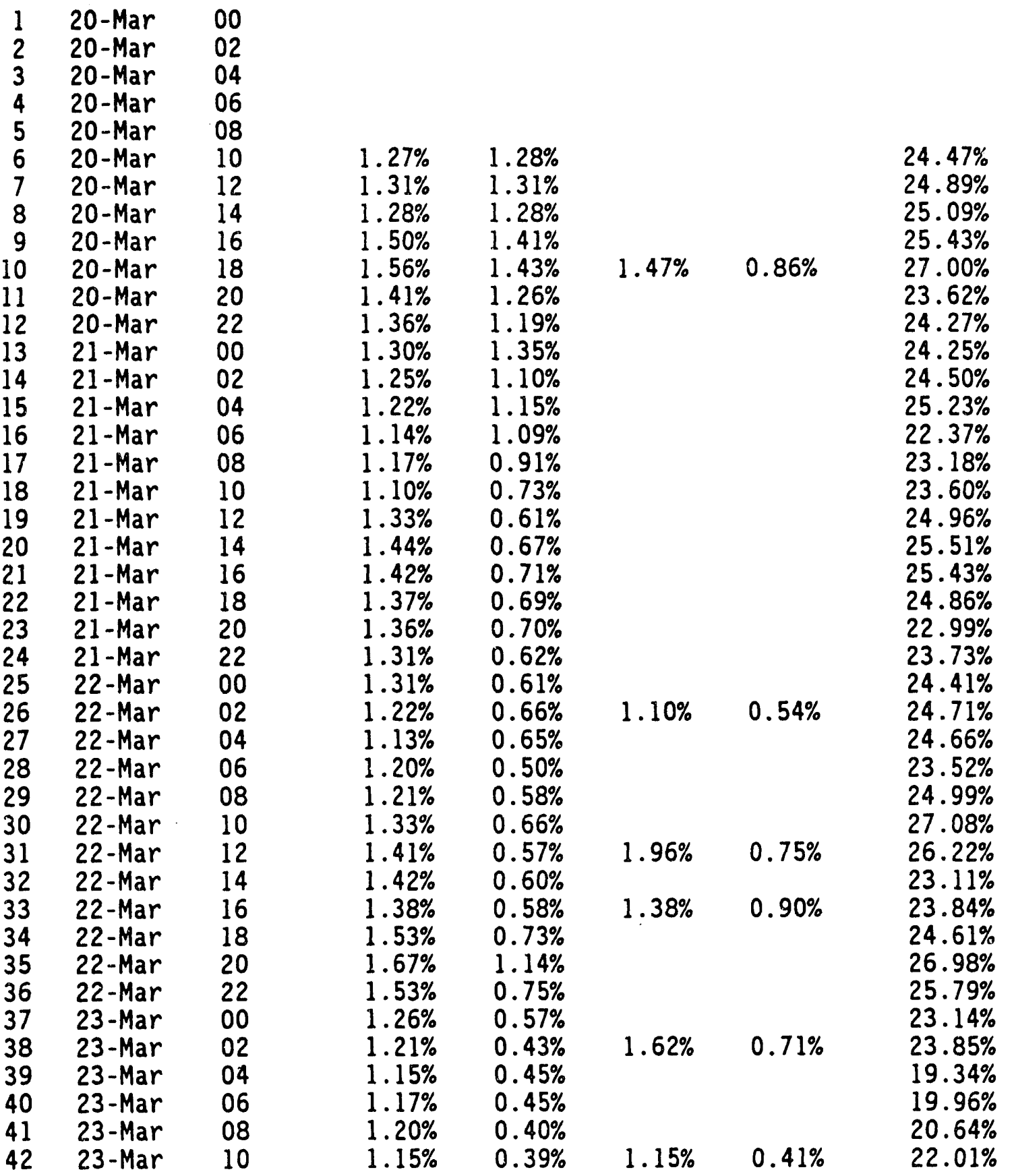

13686

14052

14059

14023

14047

13656 
COAL PRODUCT DATA TABULATION

RUN NO. $5 ; 3 / 20$ - 3/24/89

RUN NO. 5

\begin{tabular}{|c|c|c|c|c|c|c|c|c|}
\hline & ATE & TIME & $\begin{array}{r}\text { PRODUCT } \\
\text { SULFUR } \\
\text { WT, } \%\end{array}$ & $\begin{array}{r}\text { PRODUCT } \\
\text { ASH } \\
\text { WT,\% }\end{array}$ & $\begin{array}{l}\text { KILN } \\
\text { DIS. } \\
\text { SULFUR } \\
\text { WT, \% }\end{array}$ & $\begin{array}{l}\text { KILN } \\
\text { DIS. } \\
\text { ASH } \\
\text { WT, } \%\end{array}$ & $\begin{array}{r}\text { PRODUCT } \\
\text { VOLATILES } \\
\text { WT,\% }\end{array}$ & $\begin{array}{r}\text { PRODUCT } \\
\text { HEAT } \\
\text { CONTENT } \\
\text { BTU/LB(DRY) }\end{array}$ \\
\hline $\begin{array}{l}43 \\
44 \\
45 \\
46 \\
47 \\
48 \\
49 \\
50 \\
51 \\
52 \\
53 \\
54 \\
55 \\
56 \\
57 \\
58 \\
59 \\
60 \\
61\end{array}$ & $\begin{array}{l}\text { 23-Mar } \\
\text { 23-Mar } \\
\text { 23-Mar } \\
\text { 23-Mar } \\
\text { 23-Mar } \\
\text { 23-Mar } \\
\text { 24-Mar } \\
\text { 24-Mar } \\
\text { 24-Mar } \\
\text { 24-Mar } \\
\text { 24-Mar } \\
\text { 24-Mar } \\
\text { 24-Mar } \\
\text { 24-Mar } \\
\text { 24-Mar } \\
\text { 24-Mar } \\
\text { 24-Mar } \\
\text { 24-Mar } \\
\text { 24-Mar }\end{array}$ & $\begin{array}{l}12 \\
14 \\
16 \\
18 \\
20 \\
22 \\
00 \\
02 \\
04 \\
06 \\
08 \\
10 \\
12 \\
14 \\
16 \\
18 \\
20 \\
22 \\
00\end{array}$ & $\begin{array}{l}1.13 \% \\
1.14 \% \\
1.03 \% \\
1.00 \% \\
1.07 \% \\
1.10 \% \\
1.27 \% \\
1.40 \% \\
1.46 \% \\
1.52 \% \\
1.28 \% \\
1.28 \% \\
1.25 \% \\
1.66 \% \\
1.80 \%\end{array}$ & $\begin{array}{l}0.32 \% \\
0.40 \% \\
0.40 \% \\
0.46 \% \\
0.46 \% \\
0.43 \% \\
0.64 \% \\
0.85 \% \\
1.12 \% \\
1.11 \% \\
0.83 \% \\
0.79 \% \\
0.77 \% \\
1.15 \% \\
1.66 \%\end{array}$ & $\begin{array}{l}1.64 \% \\
1.18 \% \\
0.57 \% \\
0.62 \% \\
1.15 \%\end{array}$ & $\begin{array}{l}0.55 \% \\
0.46 \% \\
0.93 \% \\
1.48 \% \\
1.04 \%\end{array}$ & $\begin{array}{l}24.97 \% \\
23.49 \% \\
19.24 \% \\
22.27 \% \\
23.93 \% \\
23.11 \% \\
25.33 \% \\
28.38 \% \\
29.43 \% \\
30.63 \% \\
26.41 \% \\
26.90 \% \\
29.60 \% \\
31.13 \% \\
33.02 \%\end{array}$ & $\begin{array}{l}13651 \\
13918 \\
14130\end{array}$ \\
\hline
\end{tabular}


COAL PRODUCT DATA TABULATION

RUN N0. $6 ; 4 / 3-4 / 7 / 89$

RUN NO. 6

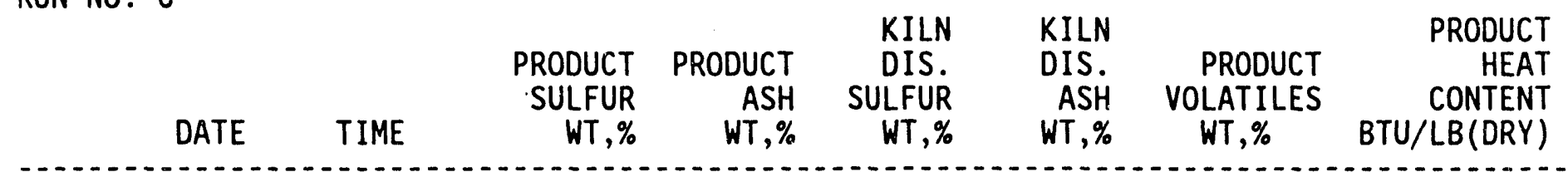

$\begin{array}{cll}1 & 03-\mathrm{Apr} & 00 \\ 2 & 03-\mathrm{Apr} & 02 \\ 3 & 03-\mathrm{Apr} & 04 \\ 4 & 03-\mathrm{Apr} & 06 \\ 5 & 03-\mathrm{Apr} & 08 \\ 6 & 03-\mathrm{Apr} & 10 \\ 7 & 03-\mathrm{Apr} & 12 \\ 8 & 03-\mathrm{Apr} & 14 \\ 9 & 03-\mathrm{Apr} & 16 \\ 10 & 03-\mathrm{Apr} & 18 \\ 11 & 03-\mathrm{Apr} & 20 \\ 12 & 03-\mathrm{Apr} & 22 \\ 13 & 04-\mathrm{Apr} & 00 \\ 14 & 04-\mathrm{Apr} & 02 \\ 15 & 04-\mathrm{Apr} & 04 \\ 16 & 04-\mathrm{Apr} & 06 \\ 17 & 04-\mathrm{Apr} & 08 \\ 18 & 04-\mathrm{Apr} & 10 \\ 19 & 04-\mathrm{Apr} & 12 \\ 20 & 04-\mathrm{Apr} & 14 \\ 21 & 04-\mathrm{Apr} & 16 \\ 22 & 04-\mathrm{Apr} & 18 \\ 23 & 04-\mathrm{Apr} & 20 \\ 24 & 04-\mathrm{Apr} & 22 \\ 25 & 05-\mathrm{Apr} & 00 \\ 26 & 05-\mathrm{Apr} & 02 \\ 27 & 05-\mathrm{Apr} & 04 \\ 28 & 05-\mathrm{Apr} & 06 \\ 29 & 05-\mathrm{Apr} & 08 \\ 30 & 05-\mathrm{Apr} & 10 \\ 31 & 05-\mathrm{Apr} & 12 \\ 32 & 05-\mathrm{Apr} & 14 \\ 33 & 05-\mathrm{Apr} & 16 \\ 34 & 05-\mathrm{Apr} & 18 \\ 35 & 05-\mathrm{Apr} & 20 \\ 36 & 05-\mathrm{Apr} & 22 \\ 37 & 06-\mathrm{Apr} & 00 \\ 38 & 06-\mathrm{Apr} & 02 \\ 39 & 06-\mathrm{Apr} & 04 \\ 40 & 06-\mathrm{Apr} & 06 \\ 41 & 06-\mathrm{Apr} & 08 \\ 42 & 06-\mathrm{Apr} & 10 \\ & & \end{array}$

$\begin{array}{ll}1.50 \% & 1.22 \% \\ 1.52 \% & 1.02 \% \\ 1.69 \% & 1.14 \% \\ 1.70 \% & 1.18 \%\end{array}$

$25.99 \%$

$27.02 \%$

$27.80 \%$

$29.78 \%$

$\begin{array}{ll}1.03 \% & 0.55 \% \\ 1.44 \% & 0.62 \% \\ 1.43 \% & 0.61 \% \\ 1.48 \% & 0.37 \% \\ 1.34 \% & 0.52 \% \\ 1.68 \% & 0.75 \% \\ 1.81 \% & 1.03 \%\end{array}$

$25.44 \%$

$23.77 \%$

$24.98 \%$

$25.64 \%$

$23.47 \%$

$27.34 \%$

$25.44 \%$

13948

$1.45 \% \quad 0.85 \%$

$28.94 \%$

14301

$1.74 \% \quad 0.89 \%$

$32.06 \%$

$32.79 \%$

$39.46 \%$

$1.76 \%$

$0.87 \%$

$0.75 \%$

$1.62 \%$

$33.16 \%$

$1.41 \%$

$0.65 \%$

$27.57 \%$

$31.63 \%$

$1.79 \%$

$31.87 \%$

$1.78 \%$

$0.98 \%$

$37.11 \%$

$1.75 \%$

$0.94 \%$

$31.53 \%$

$1.71 \%$

$0.91 \%$

$0.76 \%$

$32.75 \%$

$1.78 \%$

$0.83 \%$

$1.67 \%$

$0.77 \%$

$29.53 \%$

$35.91 \%$ 
COAL PRODUCT DATA TABULATION

RUN NO. $6 ; 4 / 3-4 / 7 / 89$

RUN NO. 6

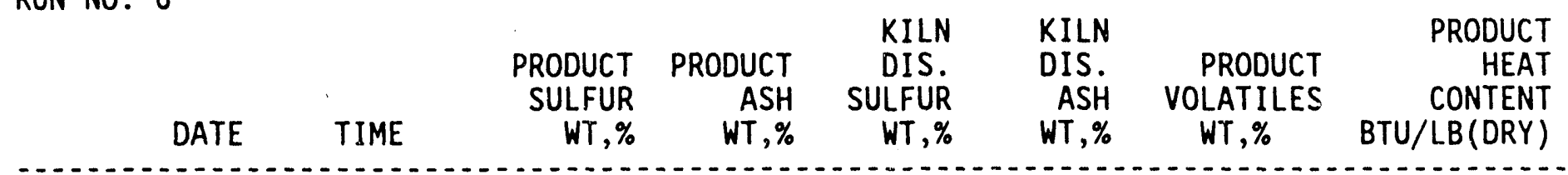

$\begin{array}{lll}43 & 06-A p r & 12 \\ 44 & 06-A p r & 14 \\ 45 & 06-A p r & 16 \\ 46 & 06-A p r & 18 \\ 47 & 06-A p r & 20 \\ 48 & 06-A p r & 22 \\ 49 & 07-A p r & 00 \\ 50 & 07-A p r & 02 \\ 51 & 07-A p r & 04 \\ 52 & 07-A p r & 06 \\ 53 & 07-A p r & 08 \\ 54 & 07-A p r & 10 \\ 55 & 07-A p r & 12 \\ 56 & 07-A p r & 14 \\ 57 & 07-A p r & 16 \\ 58 & 07-A p r & 18 \\ 59 & 07-A p r & 20 \\ 60 & 07-A p r & 22 \\ 61 & 07-A p r & 00\end{array}$

$1.96 \% \quad 1.32 \%$

$1.80 \% \quad 1.97 \%$

$1.72 \% \quad 1.90 \%$

$1.75 \% \quad 2.15 \%$
$31.48 \%$

$31.30 \%$

$26.03 \%$

$30.31 \%$ 
COAL PRODUCT DATA TABULATION

RUN NO. $7 ; 5 / 1$ - 5/5/89

RUN NO. 7

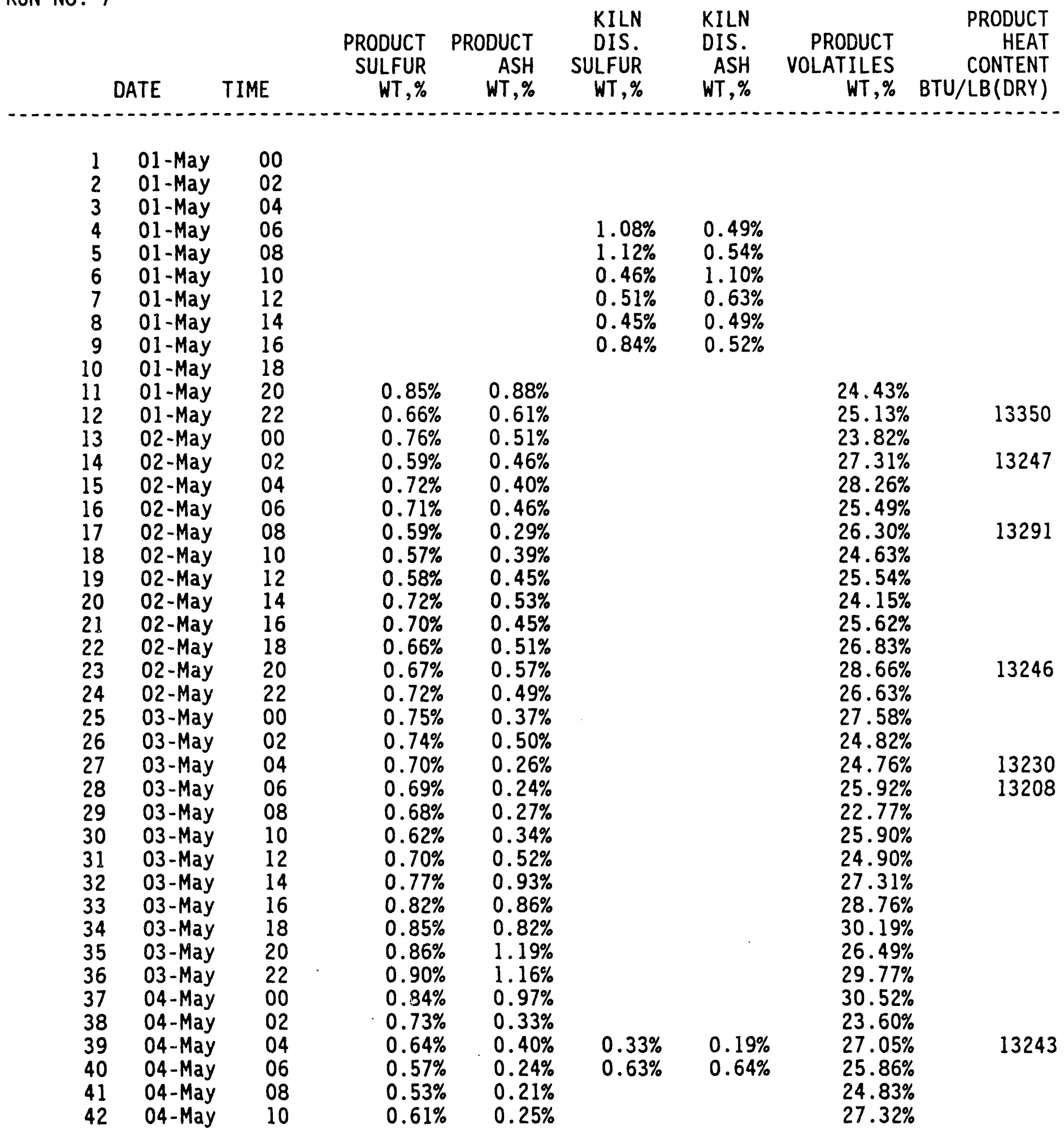


COAL PRODUCT DATA TABULATION

RUN NO. $7 ; 5 / 1$ - 5/5/89

RUN NO. 7

\begin{tabular}{|c|c|c|c|c|c|c|c|c|}
\hline & ATE & TIME & $\begin{array}{r}\text { PRODUCT } \\
\text { SULFUR } \\
\text { WT, \% }\end{array}$ & $\begin{array}{r}\text { PRODUCT } \\
\text { ASH } \\
\text { WT, \% }\end{array}$ & $\begin{array}{l}\text { KILN } \\
\text { DIS. } \\
\text { SULFUR } \\
\text { WT,\% }\end{array}$ & $\begin{array}{l}\text { KILN } \\
\text { DIS. } \\
\text { ASH } \\
\text { WT, \% }\end{array}$ & $\begin{array}{r}\text { PRODUCT } \\
\text { VOLATILES } \\
\text { WT, } \%\end{array}$ & $\begin{array}{r}\text { PRODUCT } \\
\text { HEAT } \\
\text { CONTENT } \\
\text { BTU/LB (DRY) }\end{array}$ \\
\hline $\begin{array}{l}43 \\
44 \\
45 \\
46 \\
47 \\
48 \\
49 \\
50 \\
51 \\
52 \\
53 \\
54 \\
55 \\
56 \\
57 \\
58 \\
59 \\
60 \\
61\end{array}$ & 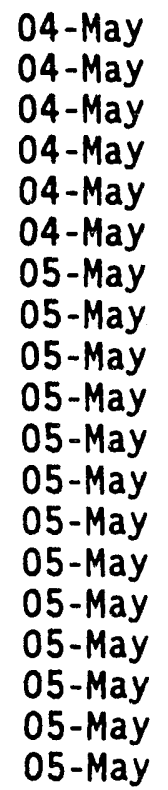 & $\begin{array}{l}12 \\
14 \\
16 \\
18 \\
20 \\
22 \\
00 \\
02 \\
04 \\
06 \\
08 \\
10 \\
12 \\
14 \\
16 \\
18 \\
20 \\
22 \\
00\end{array}$ & $\begin{array}{l}0.62 \% \\
0.54 \% \\
0.52 \% \\
0.57 \% \\
0.61 \% \\
0.65 \% \\
0.62 \% \\
0.68 \% \\
0.70 \% \\
0.68 \% \\
0.52 \% \\
0.50 \% \\
0.40 \% \\
0.82 \% \\
1.32 \%\end{array}$ & $\begin{array}{l}0.25 \% \\
0.32 \% \\
0.29 \% \\
0.38 \% \\
0.43 \% \\
0.56 \% \\
0.48 \% \\
0.73 \% \\
0.92 \% \\
1.27 \% \\
0.56 \% \\
0.55 \% \\
0.32 \% \\
0.90 \% \\
1.42 \%\end{array}$ & $\begin{array}{l}0.66 \% \\
1.50 \%\end{array}$ & $\begin{array}{l}0.34 \% \\
1.09 \%\end{array}$ & $\begin{array}{l}29.53 \% \\
31.74 \% \\
31.30 \% \\
24.87 \% \\
28.79 \% \\
30.16 \% \\
27.28 \% \\
27.71 \% \\
29.59 \% \\
28.45 \% \\
28.28 \% \\
27.19 \% \\
30.07 \% \\
30.21 \% \\
29.05 \%\end{array}$ & $\begin{array}{l}13353 \\
13268 \\
13065 \\
12976\end{array}$ \\
\hline
\end{tabular}


COAL PRODUCT DATA TUBULATION

RUN NO. $8 ; 5 / 15$ - 5/19/89

RUN NO. 8

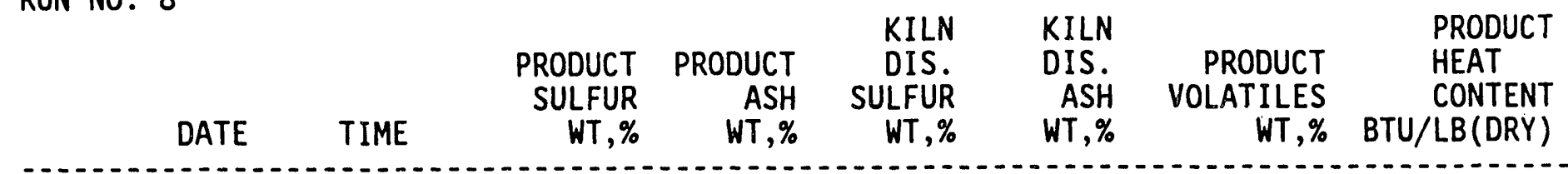

$\begin{array}{rllll}1 & 15-\text { May } & 00 & & \\ 2 & 15-\text { May } & 02 & & \\ 3 & 15-\text { May } & 04 & & \\ 4 & 15-\text { May } & 06 & & \\ 5 & 15-\text { May } & 08 & & \\ 6 & 15-\text { May } & 10 & & \\ 7 & 15-\text { May } & 12 & & \\ 8 & 15-\text { May } & 14 & & \\ 9 & 15-\text { May } & 16 & & \\ 10 & 15-\text { May } & 18 & 1.12 \% & 0.92 \% \\ 11 & 15-\text { May } & 20 & 1.34 \% & 1.14 \% \\ 12 & 15-\text { May } & 22 & 1.38 \% & 1.31 \% \\ 13 & 16-\text { May } & 00 & 1.39 \% & 0.91 \% \\ 14 & 16-\text { May } & 02 & 1.38 \% & 0.91 \% \\ 15 & 16-\text { May } & 04 & 1.20 \% & 0.91 \% \\ 16 & 16-\text { May } & 06 & 1.21 \% & 0.92 \% \\ 17 & 16-\text { May } & 08 & 0.86 \% & 0.76 \% \\ 18 & 16-\text { May } & 10 & 1.07 \% & 0.99 \% \\ 19 & 16-\text { May } & 12 & 1.24 \% & 1.32 \% \\ 20 & 16-\text { May } & 14 & 1.44 \% & 1.68 \% \\ 21 & 16-\text { May } & 16 & 1.52 \% & 2.14 \% \\ 22 & 16-\text { May } & 18 & 1.52 \% & 2.02 \% \\ 23 & 16-\text { May } & 20 & 1.52 \% & 2.64 \% \\ 24 & 16-\text { May } & 22 & & \\ 25 & 17-\text { May } & 00 & & \\ 26 & 17-\text { May } & 02 & 0.76 \% & 1.29 \% \\ 27 & 17-\text { May } & 04 & & \\ 28 & 17-\text { May } & 06 & 0.71 \% & 1.25 \% \\ 29 & 17-\text { May } & 08 & 0.91 \% & 1.33 \% \\ 30 & 17-\text { May } & 10 & 0.87 \% & 0.95 \% \\ 31 & 17-\text { May } & 12 & 0.99 \% & 0.88 \% \\ 32 & 17-\text { May } & 14 & 1.31 \% & 0.94 \% \\ 33 & 17-\text { May } & 16 & 1.52 \% & 1.47 \% \\ 34 & 17-\text { May } & 18 & 1.63 \% & 1.35 \% \\ 35 & 17-\text { May } & 20 & 1.44 \% & 1.37 \% \\ 36 & 17-\text { May } & 22 & 1.33 \% & 1.28 \% \\ 37 & 18-\text { May } & 00 & 1.01 \% & 1.07 \% \\ 38 & 18-\text { May } & 02 & 0.98 \% & 1.01 \% \\ 39 & 18-\text { May } & 04 & 1.03 \% & 1.22 \% \\ 40 & 18-\text { May } & 06 & 0.94 \% & 1.07 \% \\ 41 & 18-\text { May } & 08 & 0.91 \% & 1.04 \% \\ 42 & 18-\text { May } & 10 & 0.87 \% & 1.02 \% \\ & & & & \end{array}$

$\begin{array}{ll}0.96 \% & 1.39 \% \\ 0.66 \% & 1.10 \% \\ 0.53 \% & \\ 1.07 \% & \end{array}$

$25.32 \%$

$25.63 \%$

$28.38 \%$

$26.25 \%$

$27.71 \%$

$24.78 \%$

$26.01 \%$

$25.72 \%$

$29.37 \%$

$26.24 \%$

$24.59 \%$

$25.22 \%$

$25.32 \%$

$29.13 \%$

13359

$2.27 \%$

13616

$1.42 \%$
$1.16 \%$

$1.08 \%$

$1.02 \%$

$26.33 \%$

$22.96 \%$

$23.49 \%$

$24.09 \%$

$27.15 \%$

$22.14 \%$

$22.86 \%$

$24.08 \%$

$24.45 \%$

$23.99 \%$

$22.99 \%$

$24.35 \%$

$22.39 \%$

$30.00 \%$

$26.08 \%$

$20.98 \%$

13694 
COAL PRODUCT DATA TUBULATION

RUN NO. $8 ; 5 / 15$ - 5/19/89

RUN NO. 8

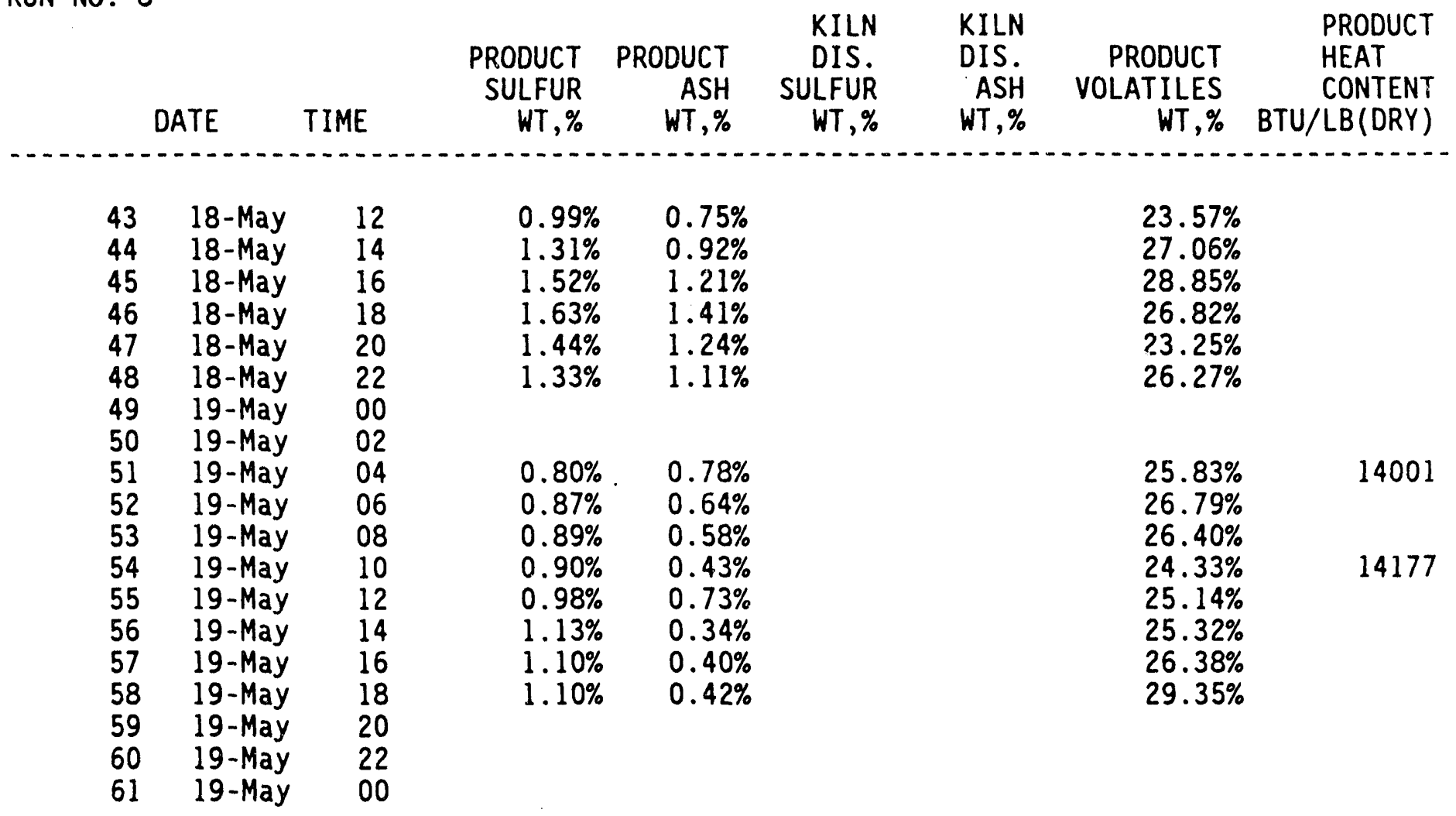


COAL PRODUCT DATA TABULATION

RUN NO. 9; 5/29 - 6/2/89

RUN NO. 9

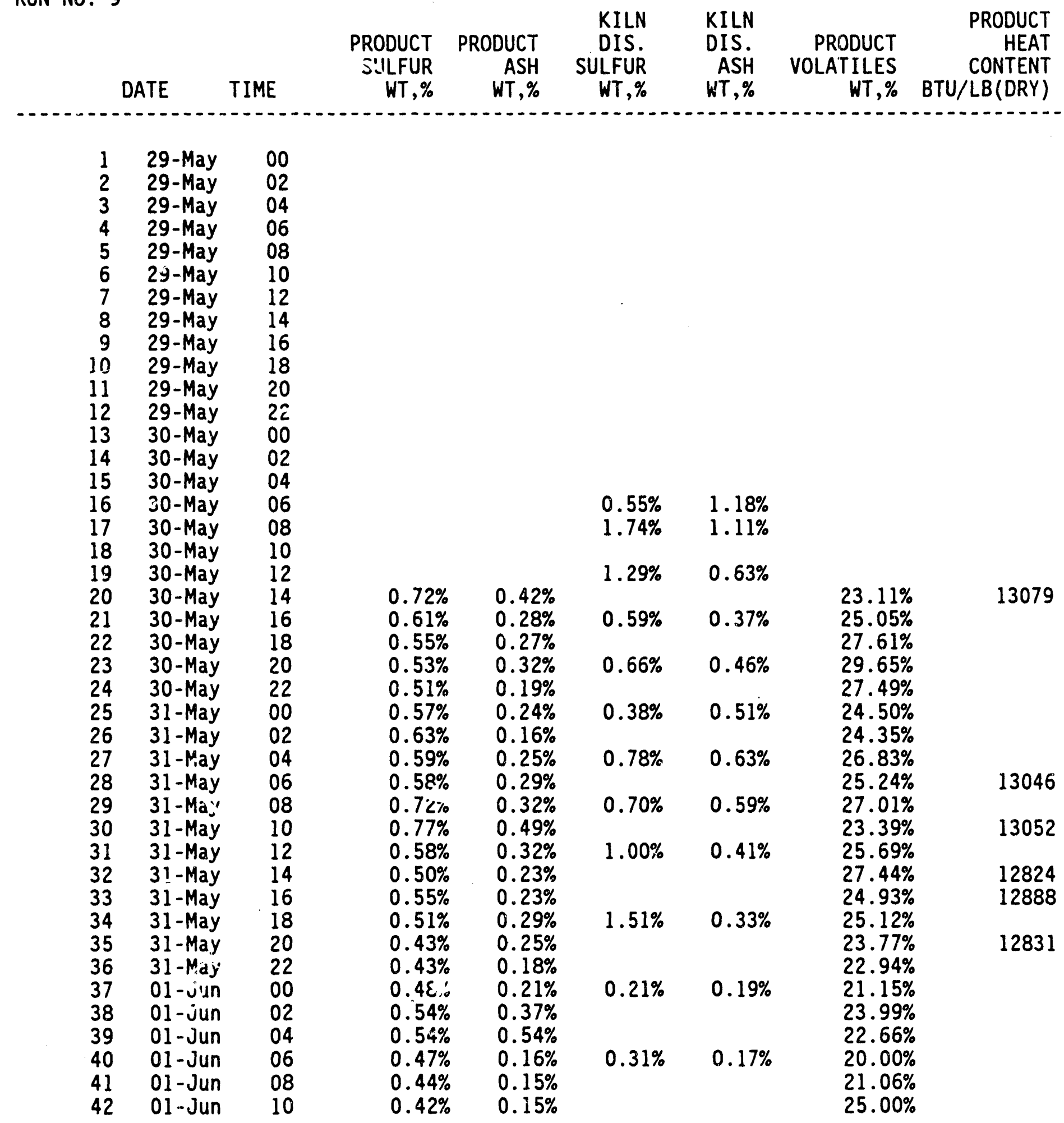


COAL PRODUCT DATA TABULATION

RUN NO. 9; 5/29 - 6/2/89

RUN NO. 9

\begin{tabular}{|c|c|c|c|c|c|c|c|c|}
\hline & ATE & TIME & $\begin{array}{r}\text { PRODUCT } \\
\text { SULFUR } \\
\text { WT, \% }\end{array}$ & $\begin{array}{r}\text { PRODUCT } \\
\text { ASH } \\
\text { WT, \% }\end{array}$ & $\begin{array}{l}\text { KILN } \\
\text { DIS. } \\
\text { SULFUR } \\
\text { WT, \% }\end{array}$ & $\begin{array}{l}\text { KILN } \\
\text { DIS. } \\
\text { ASH } \\
\text { WT,\% }\end{array}$ & $\begin{array}{r}\text { PRODUCT } \\
\text { VOLATILES } \\
\text { WT, \% }\end{array}$ & $\begin{array}{r}\text { PRODUCT } \\
\text { HEAT } \\
\text { CONTENT } \\
\text { BTU/LB }(D R Y)\end{array}$ \\
\hline $\begin{array}{l}43 \\
44 \\
45 \\
46 \\
47 \\
48 \\
49 \\
50 \\
51 \\
52 \\
53 \\
54 \\
55 \\
56 \\
57 \\
58 \\
59 \\
60 \\
61\end{array}$ & $\begin{array}{l}01 \text {-Jun } \\
01 \text {-Jun } \\
01 \text {-Jun } \\
01 \text {-Jun } \\
01 \text {-Jun } \\
01 \text {-Jun } \\
02 \text {-Jun } \\
02 \text {-Jun } \\
02 \text {-Jun } \\
02 \text {-Jun } \\
02 \text {-Jun } \\
02 \text {-Jun } \\
02 \text {-Jun } \\
02 \text {-Jun } \\
02 \text {-Jun } \\
02 \text {-Jun } \\
02 \text {-Jun } \\
02 \text {-Jun } \\
02 \text {-Jun }\end{array}$ & $\begin{array}{l}12 \\
14 \\
16 \\
18 \\
20 \\
22 \\
00 \\
02 \\
04 \\
06 \\
08 \\
10 \\
12 \\
14 \\
16 \\
18 \\
20 \\
22 \\
00\end{array}$ & $\begin{array}{l}0.33 \% \\
0.29 \% \\
0.31 \% \\
0.32 \% \\
0.34 \% \\
0.37 \% \\
0.39 \% \\
0.39 \% \\
0.43 \% \\
0.41 \% \\
0.39 \% \\
0.37 \% \\
0.38 \% \\
0.37 \% \\
0.38 \% \\
0.39 \%\end{array}$ & $\begin{array}{l}0.10 \% \\
0.09 \% \\
0.17 \% \\
0.12 \% \\
0.05 \% \\
0.04 \% \\
0.10 \% \\
0.20 \% \\
0.08 \% \\
0.02 \% \\
0.06 \% \\
0.16 \% \\
0.13 \% \\
0.14 \% \\
0.14 \% \\
0.11 \%\end{array}$ & $\begin{array}{l}0.18 \% \\
0.20 \% \\
0.24 \% \\
0.15 \% \\
0.31 \%\end{array}$ & $\begin{array}{l}0.07 \% \\
0.06 \% \\
0.08 \% \\
0.08 \% \\
0.10 \%\end{array}$ & $\begin{array}{l}21.81 \% \\
23.27 \% \\
21.92 \% \\
22.24 \% \\
21.85 \% \\
25.75 \% \\
26.64 \% \\
24.27 \% \\
21.20 \% \\
22.91 \% \\
24.70 \% \\
22.26 \% \\
22.03 \% \\
25.98 \% \\
30.09 \% \\
27.78 \%\end{array}$ & $\begin{array}{r}13340 \\
\\
13269 \\
13459 \\
13465\end{array}$ \\
\hline
\end{tabular}


COAL PRODUCT DATA TABULATION

RUN NO. $10 ; 6 / 03-6 / 07 / 91$

RUN NO. 10

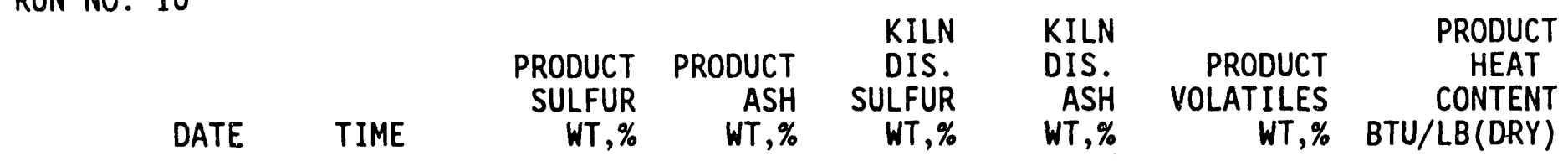

103 -Jun 00

2 03-Jun 02

3 03-Jun 04

4 03-Jun 06

5 03-Jun 08

6 03-Jun 10

7 03-Jun 12

8 03-Jun 14

903 -Jun 16

10 03-Jun 18

$1103-J u n \quad 20$

12 03-Jun 22

13 04-Jun 00

14 04-Jun 02

1504 -Jun 04

16 04-Jun 06

17 04-Jun 08

18 04-Jun 10

19 04-Jun 12

20 04-Jun 14

$2104-J u n \quad 16$

2204 -Jun 18

23 04-Jun 20

24 04-Jun 22

25 05-Jun 00

26 05-Jun 02

27 05-Jun 04

28 05-Jun 06

29 05-Jun 08

30 05-Jun 10

31 05-Jun 12

32 05-Jun 14

33 05-Jun 16

$34 \quad 05$-Jun 18

35 05-Jun 20

36 05-Jun 22

37 06-Jun 00

38 06-Jun 02

39 06-Jun 04

40 06-Jun 06

41 06-Jun 08

42 06-Jun 10

$1.58 \quad 0.86$

$1.49 \quad 0.78$

$1.35 \quad 1.52$

34.82

$1.02 \quad 0.42$

$1.04 \quad 0.89$

32.05

13764 
COAL PRODUCT DATA TABULATION

RUN NO. $10 ; 6 / 03-6 / 07 / 91$

RUN NO. 10

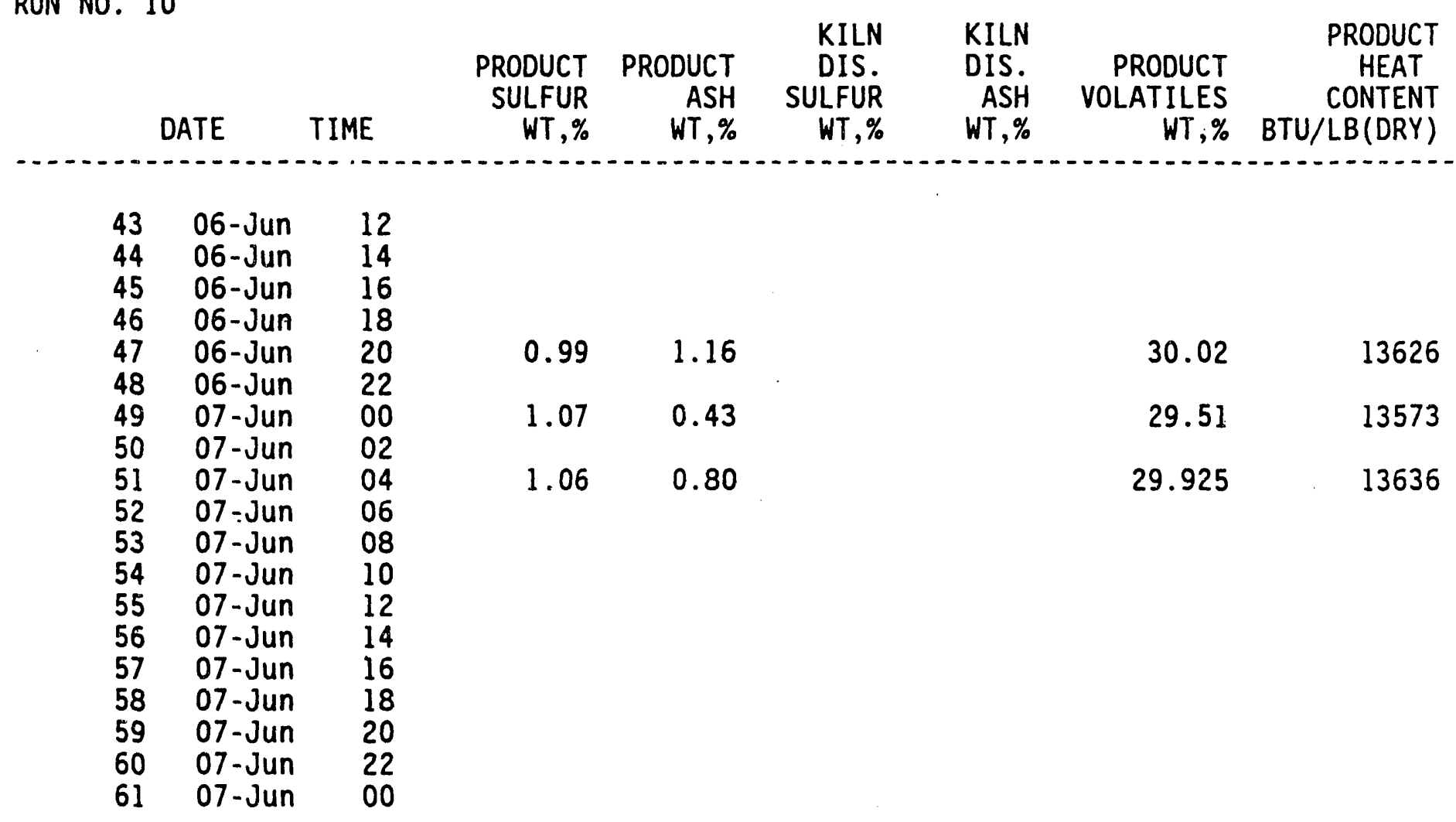


COAL PRODUCT DATA TABULATION

RUN NO. $11 ; 5 / 14$ - 5/21/92

RUN NO. 11

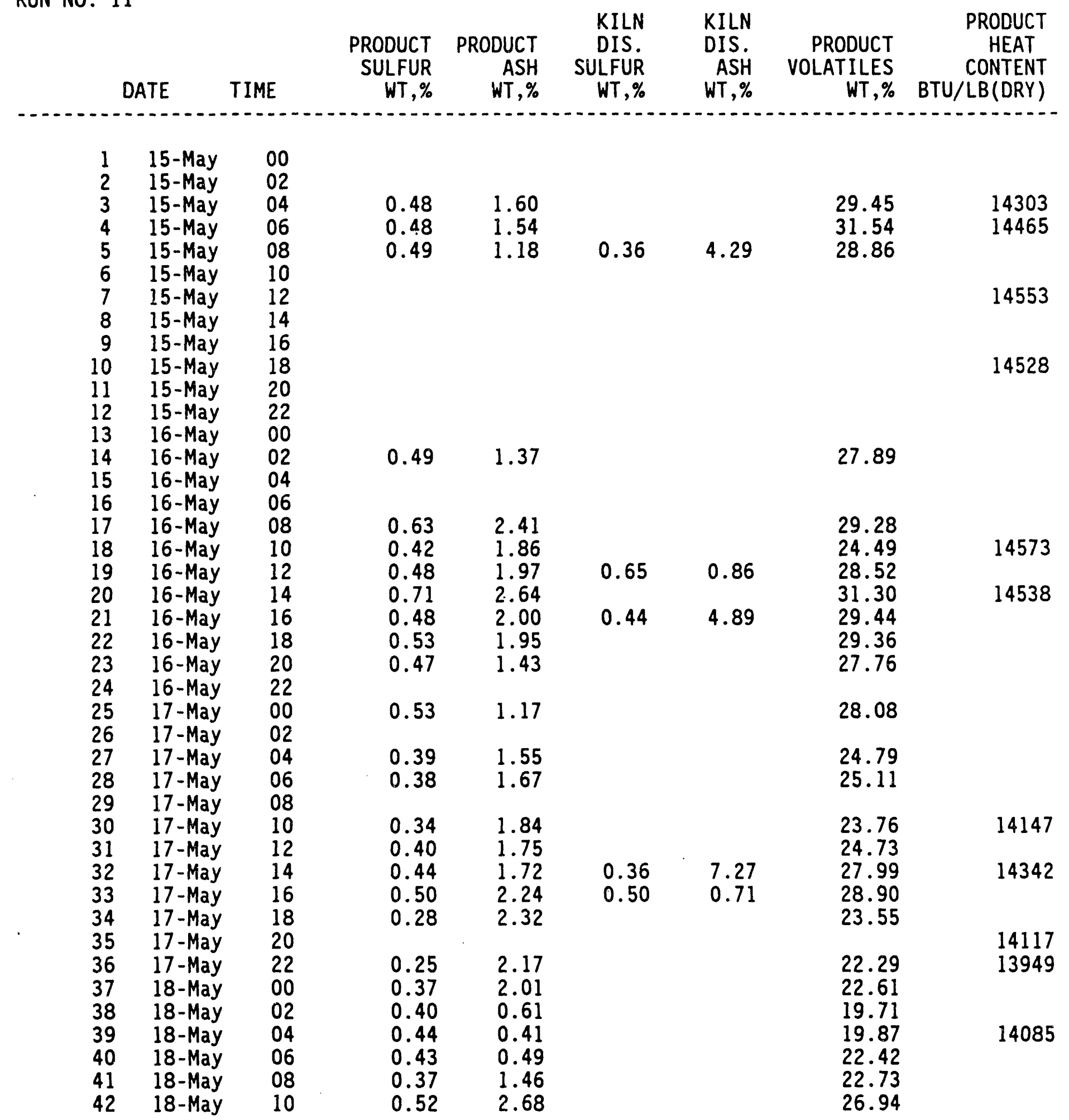


COAL PRODUCT DATA TABULATION

RUN NO. $11 ; 5 / 14$ - 5/21/92

RUN NO. 11

\begin{tabular}{|c|c|c|c|c|c|c|c|c|}
\hline & ATE & TIME & $\begin{array}{r}\text { PRODUCT } \\
\text { SULFUR } \\
\text { WT, \% }\end{array}$ & $\begin{array}{r}\text { PRODUCT } \\
\text { ASH } \\
W T, \%\end{array}$ & $\begin{array}{l}\text { KILN } \\
\text { DIS. } \\
\text { SULFUR } \\
\text { WT,\% }\end{array}$ & $\begin{array}{l}\text { KILN } \\
\text { DIS. } \\
\text { ASH } \\
\text { WT, \% }\end{array}$ & $\begin{array}{r}\text { PRODUCT } \\
\text { VOLATILES } \\
W T, \%\end{array}$ & $\begin{array}{c}\text { PRODUCT } \\
\text { HEAT } \\
\text { CONTENT } \\
\text { BTU/LB(DRY) }\end{array}$ \\
\hline $\begin{array}{l}43 \\
44 \\
45 \\
46 \\
47 \\
48 \\
49 \\
50 \\
51 \\
52 \\
53 \\
54 \\
55 \\
56 \\
57 \\
58 \\
59 \\
60 \\
61 \\
62 \\
63 \\
64 \\
65 \\
66 \\
67 \\
68 \\
69 \\
70 \\
71 \\
72 \\
73 \\
74 \\
75\end{array}$ & $\begin{array}{l}\text { 18-May } \\
18-\text { May } \\
18-\text { May } \\
18-\text { May } \\
18-\text { May } \\
18-\text { May } \\
19-\text { May } \\
19-\text { May } \\
19-\text { May } \\
19-\text { May } \\
19-\text { May } \\
19-\text { May } \\
19-\text { May } \\
19-\text { May } \\
19-\text { May } \\
19-\text { May } \\
19-\text { May } \\
19-\text { May } \\
20-\text { May } \\
20-\text { May } \\
20-\text { May } \\
20-\text { May } \\
20-\text { May } \\
20-\text { May } \\
20-\text { May } \\
20-\text { May } \\
20-\text { May } \\
20-\text { May } \\
20-\text { May } \\
20-\text { May } \\
21-\text { May } \\
21-\text { May } \\
21-\text { May }\end{array}$ & $\begin{array}{l}12 \\
14 \\
16 \\
18 \\
20 \\
22 \\
00 \\
02 \\
04 \\
06 \\
08 \\
10 \\
12 \\
14 \\
16 \\
18 \\
20 \\
22 \\
00 \\
02 \\
04 \\
06 \\
08 \\
10 \\
12 \\
14 \\
16 \\
18 \\
20 \\
22 \\
00 \\
02 \\
04\end{array}$ & $\begin{array}{l}0.51 \\
0.34 \\
0.31 \\
0.23 \\
0.16 \\
0.13 \\
0.13 \\
0.21 \\
0.22 \\
0.14 \\
0.11 \\
0.14 \\
0.26 \\
0.39 \\
0.45 \\
0.50 \\
0.36 \\
0.41 \\
0.37 \\
0.27 \\
0.28 \\
0.33 \\
0.44 \\
0.51 \\
0.51 \\
0.38 \\
0.25 \\
0.23 \\
0.27 \\
0.39 \\
0.52\end{array}$ & $\begin{array}{l}3.02 \\
3.08 \\
3.82 \\
3.19 \\
3.03 \\
3.21 \\
2.95 \\
3.33 \\
2.80 \\
2.82 \\
1.54 \\
3.04 \\
3.54 \\
3.76 \\
4.37 \\
3.88 \\
3.48 \\
3.74 \\
3.55 \\
3.22 \\
2.97 \\
3.09 \\
3.82 \\
3.53 \\
3.46 \\
3.48 \\
3.91 \\
3.58 \\
3.84 \\
4.52 \\
4.47\end{array}$ & 0.09 & & $\begin{array}{l}27.50 \\
30.88 \\
26.13 \\
27.64 \\
25.89 \\
26.66 \\
29.07 \\
26.80 \\
23.29 \\
21.11 \\
21.89 \\
24.80 \\
25.58 \\
25.19 \\
25.78 \\
24.82 \\
25.74 \\
24.74 \\
23.93 \\
26.50 \\
27.65 \\
23.58 \\
24.82 \\
24.17 \\
25.54 \\
27.01 \\
25.91 \\
23.78 \\
25.84 \\
23.51 \\
25.93\end{array}$ & $\begin{array}{l}14284 \\
13635 \\
13162 \\
13015 \\
13206 \\
13139 \\
13601 \\
13793 \\
13729 \\
13892 \\
13133 \\
13133 \\
13312\end{array}$ \\
\hline
\end{tabular}




\title{
ERRATA
}

\author{
MOLTEN-CAUSTIC-LEACHING (GRAVIMELT) SYSTEM INTEGRATION PROJECT \\ FINAL REPORT \\ CONTRACT NUMBER DE-AC22-86-PC91257
}

1. Referring to page 1-6, fourth paragraph, second sentence: the use of four weights of wash water per weight of coal was given as an example. From a cost optimization standpoint this is the operating condition TRW would like to be at in a full scale MCL plant. However, TRW did not attempt to achieve this low a water-to-coal ratio in the integrated MCL facility for the following reasons:

1) The size of the piping in slurry service in the MCL wash circuit (0.5-inch tubing) precluded operations with the high solids loadings and low flow rates that could be expected for the coal slurries at low wash water to coal ratios. Coal slurries containing an "effective solids loading" of 25 to $30 \%$ solids can bs expected to plug the small diameter piping in the MCL wash circuit. (MCL coal can absorb moisture into its internal pore structure which would increase the "effective" solids content of this slurry for transport purposes relative to a slurry containing an equal weight percent of impermeable solids.) In addition, at low wash water to coal ratios, slurry flows will be only -0.1 GPM reducing slurry velocities to $<0.3$ $\mathrm{ft} / \mathrm{sec}$ in the small diameter tubing which is very likely to allow coal to fall out of suspension in the tubing, thereby increasing the likelihood of tube plugging.

2) TRW was not able to operate the wash train with both vacuum filters on-line for more than a few hours at a time (at which time the cloth on the drum of the second filter blinded). However, the wash train worked well for excended periods of time with just a single vacuum filter on-line. This operating anomaly has not been explained to TRW's complete satisfaction. TRW has "theorized" that the extra residence time provided by the second filter system has allowed some components to precipitate from the "live" caustic solution which then act to blind the filter cloth. In any case, operation of the wash train with just a single filter on-line upstream of the centrifuges raises the practical lower limit on the wash water to coal ratio in the wash train (at a caustic:coal ratio to the kiln of $2.5: 1$ as in Run 11) to $-9: 1$. This lower limit on the water to coal ratio is determined by the necessity for the caustic-containing centrate from the first centrifuge in the wash train to have a specific gravity less than that of the coal to assure that a liquid-solids separation can be made. The specific gravity of the centrate was conservatively estimated to become less than that of the coal when the caustic content of the centrate drops below $\sim 10 \% \mathrm{NaOH}$.

2. On pages 1-8 and 2-38 reference is made to commercial applications for MCL treated coal which would not necessarily require a low ash product. Three such commercial applications are use of the coal in existing boilers equipped with ash handling equipment, use of the $M C L$ Coal as a 
water cleanup agent, and use in home heating in countries such as Czechoslovakia and Poland where a high heating value is not required.

3. On page 1-11 reference is made to the advantages of a caustic to coal ratio of one. Operation of the MCL process should be conducted with the minimum caustic:coal ratio that will give the desired product quality (sulfur, ash). The amount of caustic utilized in the process is a very strong cost driver from both a capital and energy standpoint. Most of the tests that were conducted in the MCL Integrated Test Facility were conducted at caustic:coal ratios in excess of $2: 1$. It was found that this high a ratio was needed in the 14 -inch diameter kiln reactor to produce compliance coal. TRW is optimistic that an improved larger scale reactor design, particularly one having the ability to prevent deposits from forming on the walls, will allow operation at reduced caustic:coal ratios and thereby achieve large cost savings.

4. Near the top of page $2-47,145^{\circ} \mathrm{F}$ should be $145^{\circ} \mathrm{C}$.

5. Expanding on the discussion of Run 11 on page 4-36, TRW has attached Figure 3-4 from the "Topical Report on Test Circuit Operation" to this errata sheet. This figure illustrates the $\mathrm{pH}$ swings in the acid wash section.

6. Several places in Section 4 (such as page 4-67) reference is made to kiln conditions. The kiln internal temperature variations are caused by the periodic buildup of coal/caustic solids on the internal walls of the kiln. However, even with this variation in kiln internal temperature, TRW was able in Run 11 to consistently make a low sulfur coal product: sulfur $=0.30 \% \pm 0.15 \%$. TRW is not entirely happy with the performance of the internal auger but feels that for the kiln size (14-inch diameter) used, the auger may be about as practical and as effective a wall scraping device as can be employed. It is not at all certain that an internal auger will be used in a scaled up MCL kiln. Once large diameter kilns are used many more options become available for wall scraping devices.

7. With reference to the last paragraph on page 2-13, the first priority of the first fully integrated continuous test run (Run 11) was to demonstrate fully integrated, long duration operation with recycled caustic. It was felt that the use of the molten caustic recycle line, which had undergone no testing prior to this run, introduced a potentially risky processing step, which could interrupt operations if not successful, and was therefore not consistent with the goals established for Run 11. Therefore, the decision was made to not attempt to use the molten caustic recycle line during Run 11.

8. Surface area and ash analysis data which have already been submitted as an errata sheet under separate cover are also included with this errata sheet. 


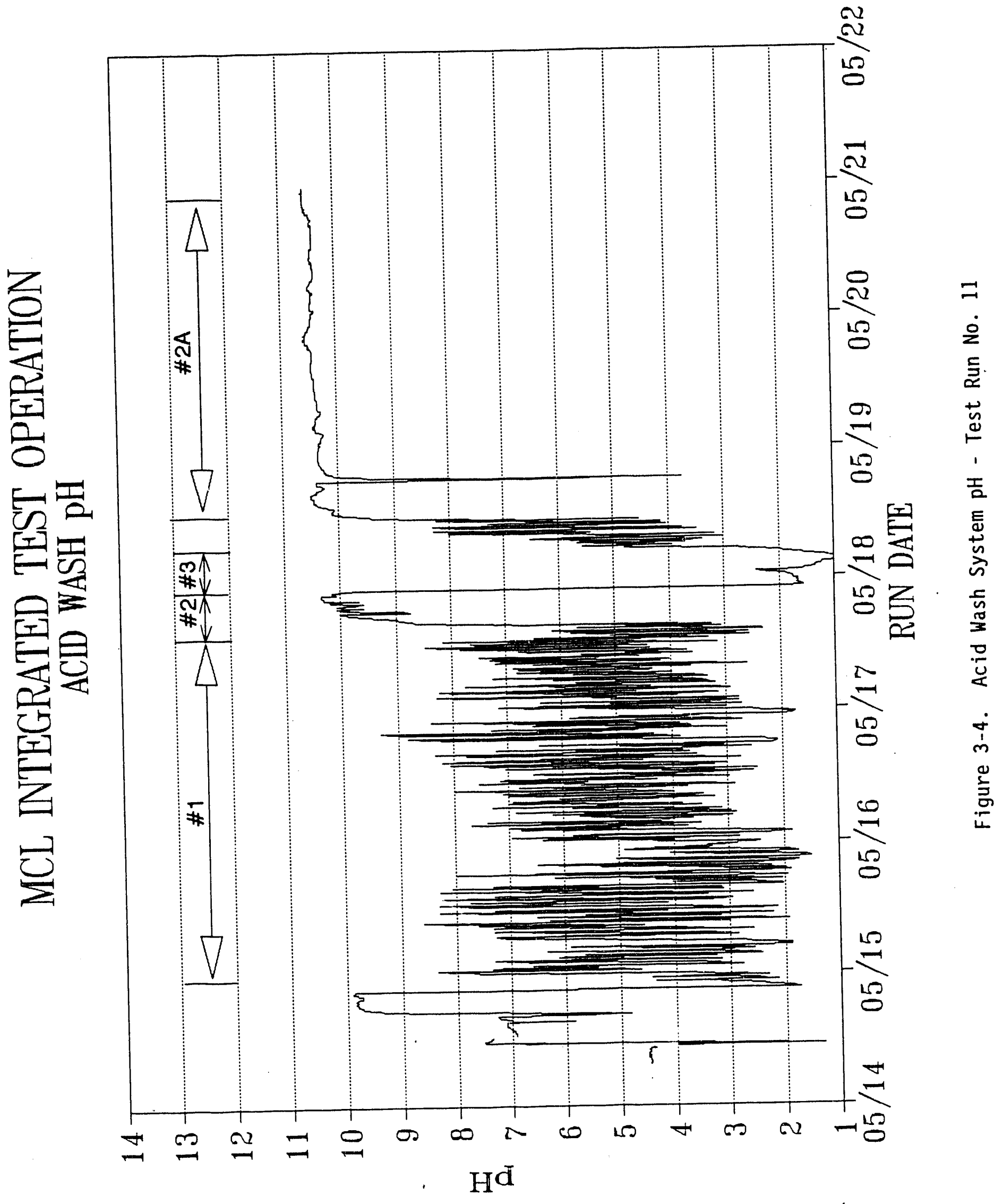




\section{ERRATA}

\section{Molten-Caustic-Leaching (Gravimelt) System Integration Project Final Report}

Contract Number DE-AC22-86-PC91257

\subsection{SURFACE AREAS AND ASH ELEMENTAL ANALYSES OF SELECTED PRODUCT COAL SAMPLES}

Surface area and pore size measurements as well as ash mineral composition analyses were obtained on selected Integrated Test Circuit product cual samples. Surface area and pore size measurements, presented in Table 1, show low surface areas for samples taken from run $11 \mathrm{~A}$ and a relatively high surface area for coal taken from run 3B1. The samples from run 11 were high in ash relative to that from run $3 B 1$ as these were taken during plant operation which did not utilize the acid wash section while the 3B1 sample was taken during the week in which that acid wash section was utilized. Apparently, the pore structure of. Gravimelt coal is created by the "etching" of caustic and subsequently, acid, into micro vains of mineral matter forming a micro pore structure and any ash that is not removed during this process reduces surface area.

Ash mineral composition of selected coal samples are shown in Table 2 where it can be seen that approximately $30 \%$ of the residual ash in non-acid treated samples is sodium (computed as sodium oxide). TRW has recently patented a Gravimelt process variation in which carbonic acid (carbon dioxide and water) is utilized to remove remaining ash and sodium from coal (U.S. Patent No. 5,059,307 granted October 22, 1991). Utilizing TRW funds, we evaluated the treatment of plant washed coal (no acid 
wash) with carbonated water as shown in the last example on Table 2. It can be seen that the ash level is further reduced by $40 \%$ and the combined sodium and potassium oxide content is reduced to a level below that of the starting coal.

Clearly, the carbonation of the last water wash step is desirable in order to produce a low ash and low sodium product coal. This should also produce a higher pore volume in the coal. The water solution containing dissolved sodium carbonate and coal mineral matter can then be limed and centrifuged to produce dilute sodium hydroxide for recycle to the plant. 


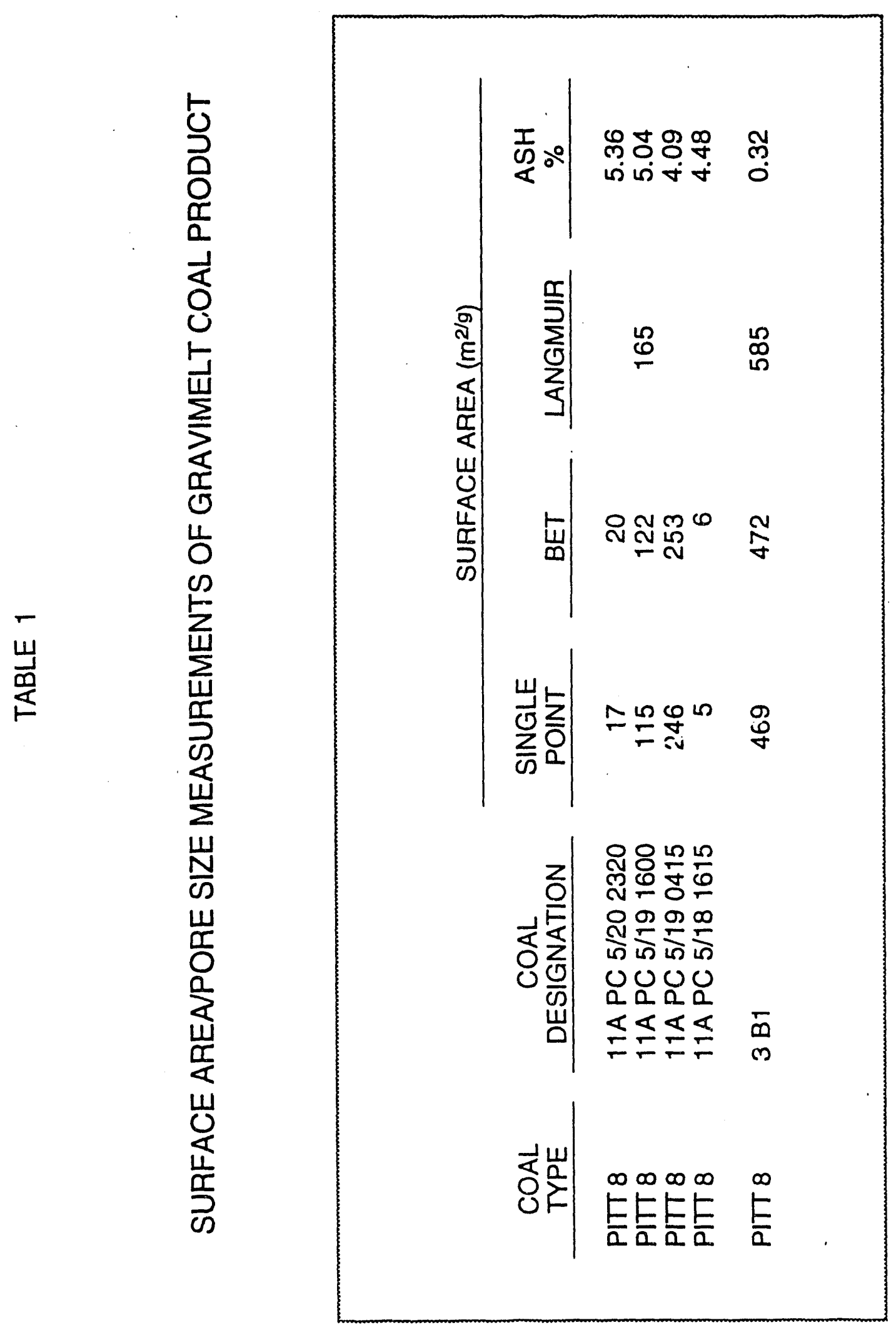




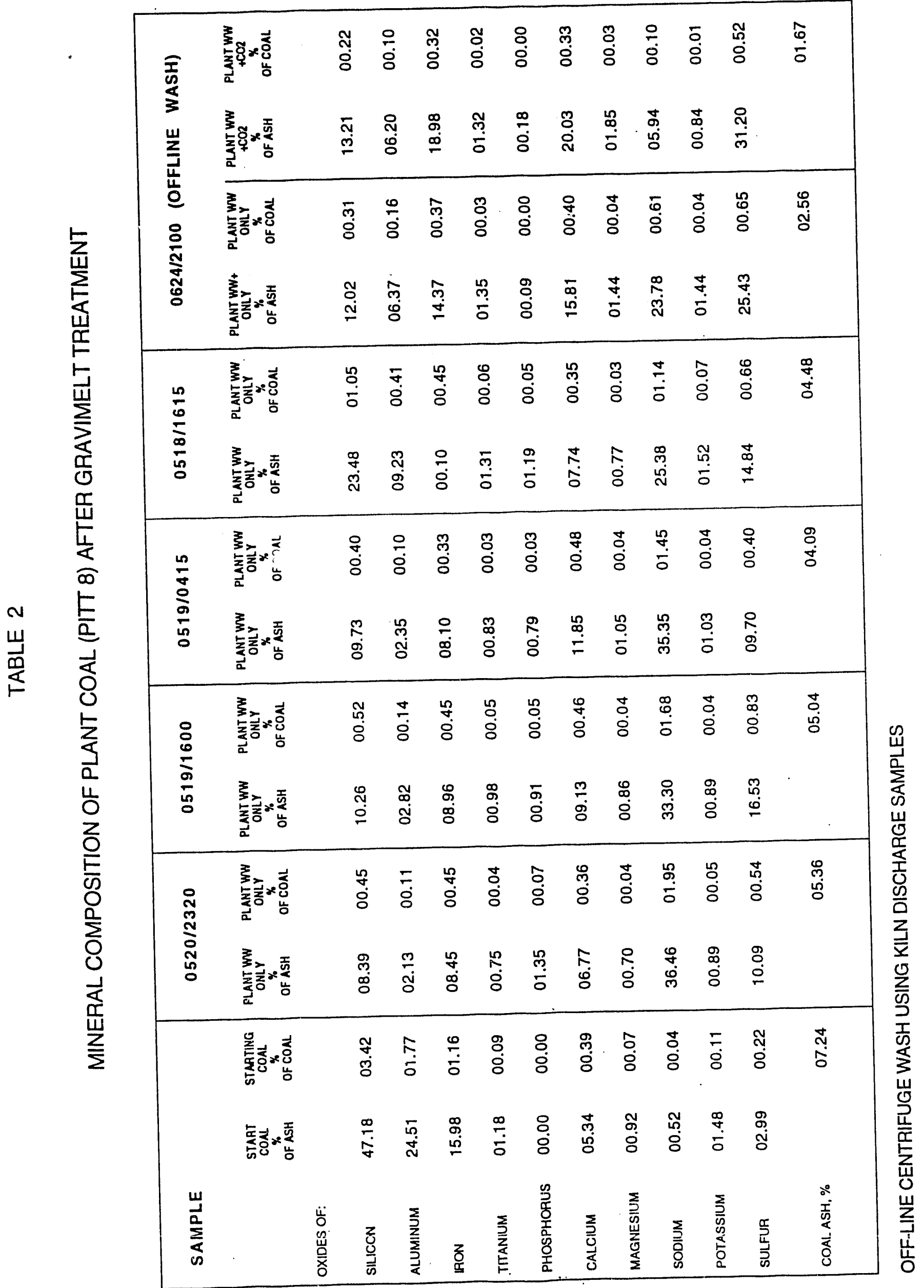



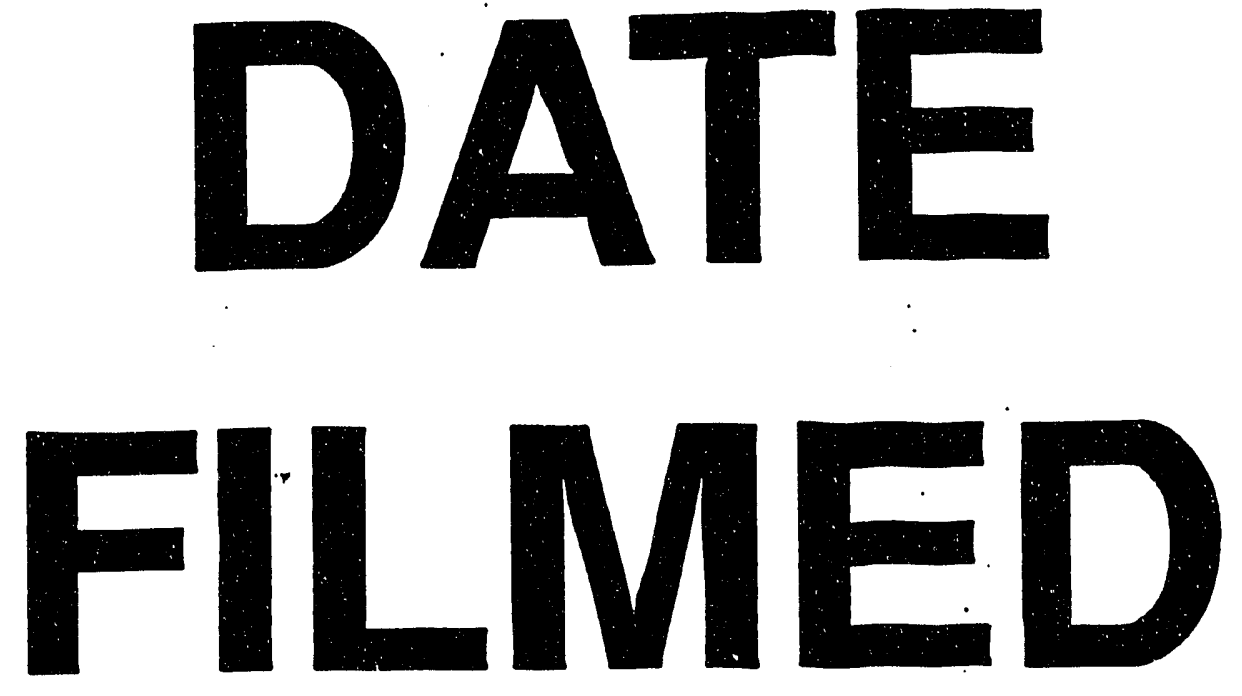

$12 / 15 / 93$
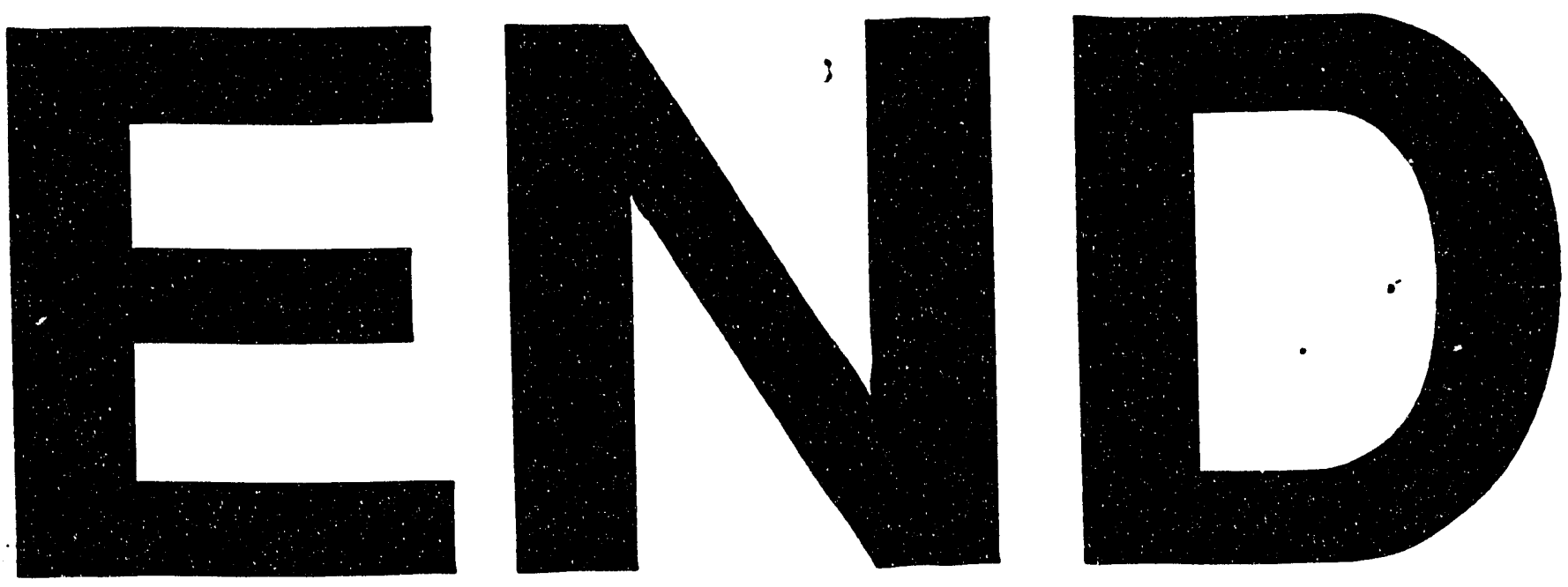